NATL INST OF STANDARDS \& TECH R.I.C.

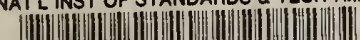

\title{
An Investigation of Horizontal Flow Boiling of Pure and Mixed Refrigerants
}

Howard D. Ross

U.S. DEPARTMENT OF COMMERCE

National Bureau of Standards

National Engineering Laboratory

Center for Building Technology

Building Equipment Division

Gaithersburg, MD 20899

August 1986

Issued November 1986

$$
\begin{array}{ll}
A C & \text { In part by: } \\
100 & \text { 'artment of Energy } \\
.1156 & \text { Ridge National Laboratory) } \\
86-3450 & \text { on, DC } 20585 \\
1986 &
\end{array}
$$




\section{AN INVESTIGATION OF HORIZONTAL FLOW BOILING OF PURE AND MIXED REFRIGERANTS}

Howard D. Ross

U.S. DEPARTMENT OF COMMERCE

National Bureau of Standards

National Engineering Laboratory

Center for Building Technology

Building Equipment Division

Gaithersburg, MD 20899

August 1986

Issued November 1986

Sponsored in part by:

U.S. Department of Energy

(via Oak Ridge National Laboratory)

Washington, DC 20585

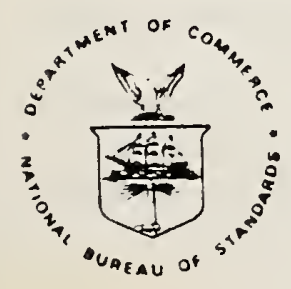

U.S. DEPARTMENT OF COMMERCE, Malcolm Baldrige, Secretary NATIONAL BUREAU OF STANDARDS. Ernest Ambler. Director 

The research involved determining experimental heat transfer coefficients (HTC) and analyzing the predictive ability of available models for both pure and mired R152a/R13B1 refrigerants. Over 1,000 data points were collected, covering a range of pressure, composition, quality, and heat and mass flux.

A current controversy regarding suppression of nucleate boil ing was resolved in favor of traditional theory. A suppession criterion for pure refrigerants predicted quantitatively the quality at which suppres sion occurs. The method was extended to mired refrigerants and partial ly verified.

01 der pare fluid heat transfer correlations 1 acked general val idity. The complete Chen correlation and many suggested variations were tested. The original correlation overestimates the nucleate boil ing contribution. In the nucleate boiling regime, the method of Stephan and Abdel sal am (SA) was validated. In the evaporative regime, Bennett and Chen's (BC) Prandtl nuber correction predicted HTC's for pure Iefrigerants. The method incorporates the suppression criterion to determine when to invoke the Prandtl correction. It is better grounded in theory than recent regression-based correlations. The method predicted the val ues of this and other independent data well. 
The circumferential variation in HTC is opposite for mistures than observed for pure fluids, suggesting the existence of a circumferential gradient in concentration and interfacial temperature. The measured values in both the nucleate boiling and the evaporative regimes showed a degradation in heat transfer. In the nucleate boiling regime, mass transfer resistance caused the degradation. In the evaporative regime, the reduction may be due to mass transfer resistance suppressing nucleate boiling for the mixture but not for the pare component.

None of the mixtures' calculation methods achieved closure with measured values to the same degree as was achieved with pure fluids. Closure, however, was typical of the literature for mistures. In the nucleate boiling regime, the method of Thome achieved the best agreement. It however predicted the opposite quality dependence.. In the evaporative regime; the best fit was achieved by the evaporative portion of Chen's original equation which neglects any mixture effect and suggests the absence of nucleate boiling. 
4.3 Summary and Analysis of Experimental Evidence:

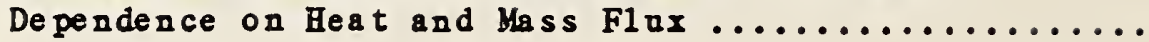

4.4 Summary of Experimental Evidence: Dependence of

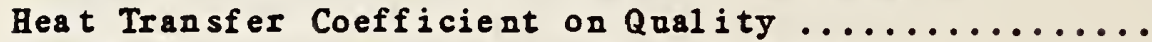

4.5 Summary of Experimental Evidence: Effect of

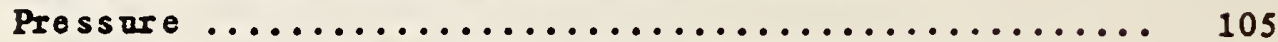

4.6 Stmmary of Experimental Evidence: Presence of

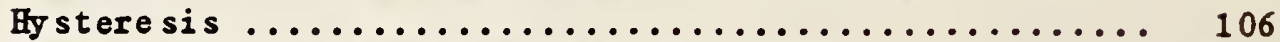

4.7 Summary of Literatore Review: Mirtures ............. 106

4.8 Comparison of Experimental Results to Theory ......... 108

4.8.1 Application of Conventional Theory to

Refrigerants and Refrigerant Mirtares ......... 108

4.8.2 Experimental Results: Pare Refrigerants...... 112

4.8.3 Experimental Results: Mirtares............. 116

4.9 Concl nsions and Recommendations................ 118

5. A FUR THER EXAMINATION OF THE DATA $\ldots \ldots \ldots \ldots \ldots \ldots \ldots \ldots \ldots 14$

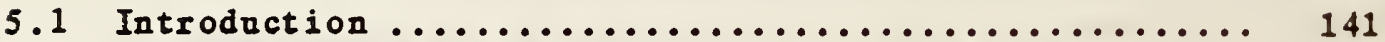

5.2 Circomferential Variation in Heat Transfer in

Horizontal F1 on Boil ing ...................... 141

5.3 Effect of Equilibriom Quality on Mirture Heat

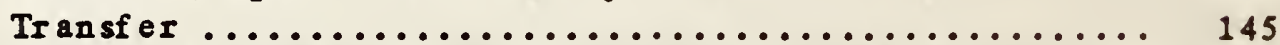

5.4 Departure from Nacleate Boil ing (DNB) Events........ 147

5.5 Comparison Between Pure and Mired Refrigerants....... 148

5.6 Pressure Drop in Horizontal Fl ow Boiling of Pare

and Mized Refrigerants ..................... 150

6. PREDI CTION OF PURE REFRIGERANT HEAT TRANSFER $\ldots \ldots \ldots \ldots \ldots \ldots 1$

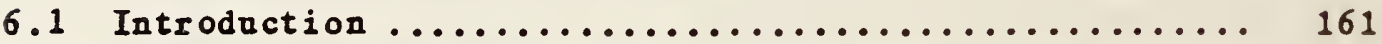

6.2 Correlation for Annular Fl ow Boiling ............. 162

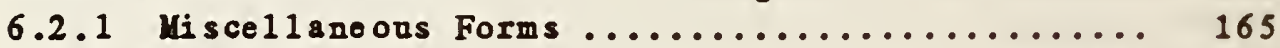

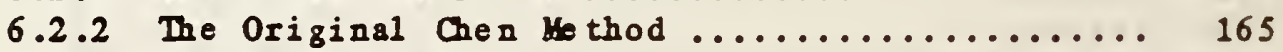

6.2.2.1 Closed Form Solution for Chen's

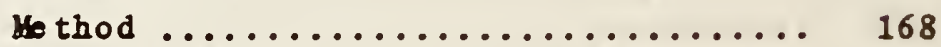

6.2.3 Comparing Chen's Me thod to Experimental

Data: Literature Review ................. 169

6.2.4 Modification to Nacleate Boiling

Contribution in Chen's thod ............. 170

6.2.5 Modification of Forced Conrection

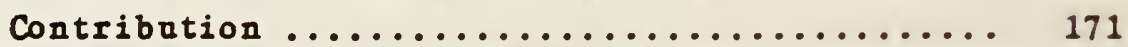

6.2.6 Modification of Suppression Fact or .......... 172

6.2.7 0ther Modifications to Chen's thod ......... 173

6.2.8 Application of Modification to Chen's Mothod .. 173

6.3 Experimental Results: Camparison to Measured Data ... 175

6.3.1 Comparison to Rig \#1, Rl52a Data ............ 176

6.3.2 Comparison to Rig \#2, Preheat Data ........... 177

6.3.3 Comparison to Rig \#2, Test Section Data....... 178

6.4 Discussion of Findings ....................... 178

6.4.1 Nacleate Boil ing Dominated Flon Situations.... 178 


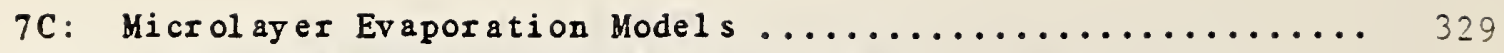

7D: Fur ther Comments on Shock's Analysis ................ 333

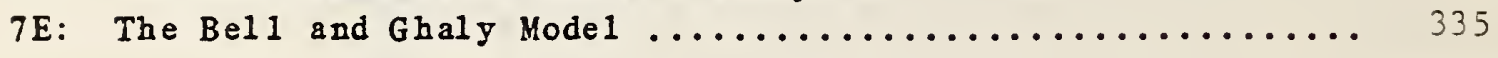

REFERENCES 


\section{LIST OF TABLES}

1-1: Flow Boiling Experiments with Pare Refrigerants: Literature Review

2-1: Comparison of Equation of State Values and Mole Fraction Weighting of Pure Components

3-1 a: Hardware Differences Between Rig \#1 and Rig \#2

3-1b: Data Reduction Differences Between [Ra83] and This Thesis (Rig \#1)

3-2: Effect of Pressure Taps on Heat Transfer Coefficient

5-1: Sumary of Experimental Data

6-1: Some Simple Correlations for Flow Boiling of Pure Refrigerants 6-2: Simple Correlations for Average Heat Transer of F1 ow Boiling Refrigerants

6-3: Comparison of Predictions to Data: Mass Fractional Deviation

6-4: Summary of Chen-Styled Me thods

7-1: Pool Boiling Correction Factors for Binary Mirtures

7-2: Summary of Assumptions in Mirtares' Models

7-3: Comparison of Mixed Refrigerant Correlations and Experimental Data 


\section{LIST OF FIGURES}

1-1: Regimes of Two Phase Fl ow (Reprinted from [La62]).

1-2: Temperature-Composition Diagrams for Test Fluids in Terms of weight and Molar Fractions.

1-3: Heat Balance on Control Volume in Annular Flow of Mistures.

1-4: Effect of 0 il on Heat Transfer Coefficients of Refrigerants.

2-1: Comparison of Transport Properties as Determined by Mole Fraction Weighting and Miring Rules.

3-1: Typical Test Rig in the Literatare.

3-2: Experimental Test Rig \#1.

3-3: Experimental Test Rig \#2

3-4: Thermocouple Lead Arrangement.

3-5: Thermocouple Mounting Scheme Including Electrical Isolation.

3-6: Tall and Instream Temperature Measurement.

3-7: The Simulation of Pressure Taps.

3-8: Calculated Pressure Drop via Martinelli-Nel son Method.

3-9: Effect of Instream Thermocouples on Single Phase Measurements.

3-10: Effect of Instream Thermocouples on Evaporative Beat Transfer Coefficient.

3-11: Sampling Technique to Determine Mirture Composition.

3-12a: Data Redaction Scheme for Pare Refrigerants.

3-12b: Data Redaction Scheme for Mirtures.

3-13: Condensation Curves for Various Compositions.

3-14: Typical Outpat from Test Run Data Redaction.

3-15: Comparison of Predicted to Measured Data for Single Phase Heat Transfer Coefficients.

3-16: Pressure Drop: Predicted versus Measured. 
3-17: Effect of Preheat Flax on Test Section Wall Temperature Measurements.

3-18: Effect of Preheat Flux on Heat Transfer Coefficients for Mixtures.

4-1: Criterion for the Onset of Nucleate Boiling.

4-2: F1 ow Chart for Determining Suppression Beat F1ux.

4-3: Effect of Pressure and Mass Flux on Suppression Heat Flux (R152a).

4-4: Calculated Suppression Heat F1 ux for Mixtures--Isolated Bubble The ory.

4-5: Calculated Suppression Heat F1 ux for Mixtures--Pool Boiling The ory.

4-6: Effect of Heat F1ux on Pure R152a at Low Prẹssure.

4-7: Effect of Pressure on Pure R152a.

4-8: Effect of Heat Flax on Pure R13B1

4-9: Effect of Heat flax on Pare R152a and R152a/R13B1 Mixtare:

Preheat Data.

4-10: Effect of Heat FIuz on Pure R13BI and RI52a/Rl3BI Miztare:

Preheat Data.

4-11: Effect of Heat Flax on Puxe R152a--Test Section Data.

4-12: Effect of Mass Flax: Test Section Data.

4-13a: Effect of Step Change in Heat F1 ax: Pure Refrigerants.

4-13b: Effect of Step Change in Heat F1ax: Mirtures.

4-14: Effect of Heat and Mass FIar on 0.662 wt. R13BI Mizture.

4-15: Effect of Heat and Mass F1 ax on 0.706 wt. R13B1.

4-16: Effect of Heat and Mass FI ux on 0.75 wt. R13Bl.

4-17: Effect of Beat and Mass FIux on 0.833 wt. R13BI.

4-18: Rig \#2 Test Section Data.

5-1: Variation in Measured Heat Transfer Coefficient.

5-2: Variation in Measured Heat Transfer Coefficient for Mixtures. 
5-3: Effect of Composition on Top-Bottom Wall Temperature Difference.

5-4: Indications of Probable Film Boiling for Pure R13Bl and a Mixture.

5-5: Probable Film Boiling in the Data of [Mi81].

5-6: Comparison Between Mirtures and Pure Fluids in Evaporative Flow.

5-7: Comparison Between Predicted and Measured Pressure Drop for Mistures.

6-1: Comparison of Shah's Method with Experimental Data.

6-2: Comparison of Empirical and Analytic Functions in Chen's Method.

6-3: Comparison of Cher-Styled Me thods to Experiments.

6-4: Comparison of Forster-Zuber and Stephan-Abdelsalam Me thods.

6-5: Comparison of Complete Correlation with Test Section Data.

6-6: Comparison of Camplete Correlation with Selected Rig \#1 Data

6-7: Comparison of Complete Correlation with Others' Data.

7-1: Isolated Bubble Growing in a Superheated Binary Liquid.

7-2: Pool Boiling Experiments of Happel and Stephan with Binary Mirtares.

7-3: Comparison of Mixtare Correction Factors.

7-4: Comparison of Preheat Data to Pool Boiling Models.

7-5: Comparison of First Thermocouple Station Preheat Data to Pool Boiling Models.

7-6: Comparison of Rig \#1 Data to Thome's and Bell and Ghaly's Me thods.

7-7: Comparison of Measured Test Section Data to Evaporative Only Me thods.

8-1: Proposed New Test Rig.

4C-1: Visual ization Me thods.

7C-1: Microlayer Evaporation Models.

7E-1: The Bell and Ghaly Method. 


\section{English}

T

P

$M$

$\mathrm{X}$

$\mathrm{Y}$

R

$\mathrm{C}_{\mathrm{p}}$

$\mathrm{q}$

$\mathrm{x}$

G

$\dot{m}$

h

$\Delta \mathrm{h}_{\mathrm{v}}$

$s$

L

E

(TD)

(GAUGE)

$A_{c}$

As

$\mathrm{v}$

2

F

$S$

d*

\section{Greek}

$a$

$\sigma$

$\mu$

$\gamma$

$\Delta$

$\varepsilon$

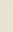

$\mathrm{D}^{2}$

$\delta$

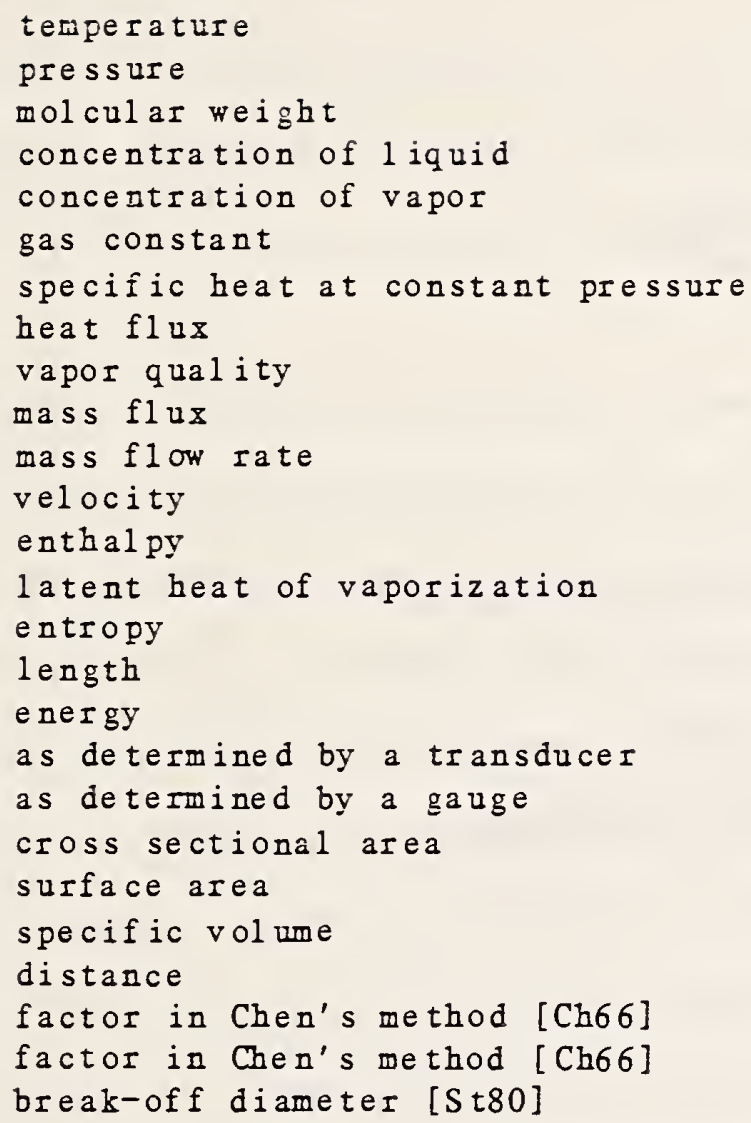

\section{Dimensionless Numbers}
$\operatorname{Re}$
Reynolds Numbers GD/ $\mu$
PI
Co
Prandt 1 Number $H_{\mathrm{p}} / \lambda$
$\mathrm{I}_{t t}$
Convection Number
Martinelli Parameter
Lewis Number $a_{T} /{ }^{a} D$
Sc
Schmidt Number $H_{/} / \mathrm{Pa}_{D}$

heat transfer coefficient

surface tension

viscosity

kinematic viscosity

conductivity

change in property

eddy diffusivity or void fraction

density

two phase multiplier [Ma48]

thickness (length) of layer 


\section{Superscripts}

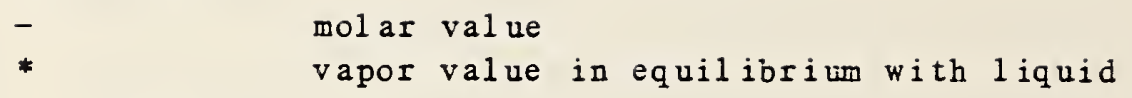

\section{$\underline{\text { Subscripts }}$}

\begin{tabular}{|c|c|}
\hline $\mathrm{T}$ & thermal \\
\hline $\mathrm{D}$ & $\operatorname{mass}$ \\
\hline SL & sensible heat, liquid \\
\hline SV & sensible beat, vapor \\
\hline G & gas \\
\hline $\mathrm{L}$ & 1 iquid \\
\hline LO & liquid only \\
\hline A & component A \\
\hline B & component B \\
\hline DEW & dew point \\
\hline BUB & bubbi e point \\
\hline LIN & as determined by a 1 inear relation \\
\hline 0, TOT & overall, total \\
\hline $\mathrm{C}$ & due to Chen \\
\hline $\mathrm{BC}$ & due to Bennett and Chen \\
\hline FC & forced convective \\
\hline TPF & two phase, frictional component \\
\hline i & component i \\
\hline j & component $j$ \\
\hline I & reduced \\
\hline c & critical \\
\hline sat & saturation \\
\hline in & inl et \\
\hline out & outlet \\
\hline $\mathrm{f}$ & fluid \\
\hline sc & subcooled \\
\hline w & wa11 \\
\hline $\mathrm{pr}$ & preheat \\
\hline a & acceleration component \\
\hline $\mathrm{nbc}$ & nucleate boiling contribution \\
\hline e & evaporative contribution \\
\hline $\mathrm{n}$ & nucleate boiling relation used in Chen's method \\
\hline poo1 & pool boiling \\
\hline egb & equilibrium value \\
\hline id & ideal value (mole fraction weighted) \\
\hline $\mathrm{v}$ & vapor \\
\hline act, meas & actual, measured value \\
\hline 20 & two phase \\
\hline m & mixture \\
\hline
\end{tabular}




\section{PREAMBLE}

This report is ossentialiy the dissertation of Howard Ross which vas submitted to the Oniversity of Maryland in partial fulfillment of his PhD. Iequirement s. The following are the acknowledgents fram that docment.

Special thanks mast go first to my advisor, Marino di Marzo, for his spirited and insightfol advice in the preparation of this report. Also, deep gratitude is expressed to Reinhard Radermacher for his generous and patient counsel in the lab. Sponsors are usualig noted in the carsory fashion. Howerer, in this case, David Didion provided support on both a technical and personal level for wich the athor is grateful. Appreciation is given to Dave Tard for accommodating my anusul schedule and demands in operating the brine cooling system. Ertra effort was required in typing this report. My special thanks go to Laurie Tatkins for enduring the several drafts, and Gail Crum and Mary Baier for typing the final. I al so thank everyone in the group at NBS, especial 1y Mark McLinden, Graham Morrison, and Brian Heber for mang fruitful technical discussions. 

CHAPTER 1: BACKGROUND AND SCOPE

\subsection{Introduction and Goals}

The interest in two phase gas-liquid flow is increasing dramatically. In 1966 , less than 60 papers were published; in each of the last three years (1982-84), more than 1000 papers have appeared in the technical literature. Surprisingly, however, the study of flow boiling of mixtures, a commonly occurring process, has received relatively little attention; a recent 1 iterature survey [St82] turned up only six papers. While in fact there may be twice that number, the availability of existing data and models is scarce. Of those studies many were with aqueous solutions, which due to certain properties of water, may be inapplicable for other fluids.

The boiling/evaporation of mixtures is a very common industrial application. In distilleries and in reboilers of which there are tens of thousands in use, the evaporation of a mixture is inherent in the process. More recently, the use of refrigerant mixtures as the working fluid in heat pumps and refrigerator/freezers has shown theoretical promise [St80, Sc85] which has been verified experimentally [Di84]. One of the serious barriers to the use of these refrigerant mixtures is the current lack of understanding of the heat transfer process in refrigeration equipment, specifically the impact of mixtures on the heat transfer coefficient and therefore the heat exchanger size in evaporators and condensers. 
The principal goal of this study therefore is to experimentally determine heat transfer coefficients under a wide range of conditions, to assess and recommend models and correlations for their predictions, and to examine specific physical processes governing the beat transfer.

The need for such work has been expressed widely in the literature:

"No other tests [of the Chen correlation for fluids

with $P r>1]$ have been reported and this is a gap which should be filled". - recent flow-boiling review keynote paper at int' 1 Heat Transfer Conference [Bu82].

"It is clear that several possible methods are now available for convective vaporization. It is essential in order for them to be tested, the present lack of data for local convective heat transfer coefficients in multicomponent systems should be remedied". [Sa82].

"Further experiments with other mixtures are still necessary. Some of the assumptions [in the models] seem to be very far going and require further experimental scrutiny". -- recent boiling of mixtares review keynote paper at Int'l Heat Transfer Conference [St82].

This study attempts to respond directly to the se requests.

\subsection{Text Organization}

In the remainder of this chapter, background is given regarding the boiling/evaporation process in flow boiling, especially annular flow. The general complications introduced by the use of mixtures are also discussed.

In Chapter 2, a review of the physical property determination is given. The use of a special equation of state for determining the rmodynamic properties is described. Transport property correlations for pure fluids and rules for determining the se properties for mixtures are 
given; these rules take into account the non-idealities of mixing. Nonideal mixing properties are shown to reduce heat transfer coefficients, both in single and evaporative two phase flow.

In Chapter 3 , the experimental test rigs used in the heat transfer coefficient investigation are discribed. Particular attention to potential errors is included, as well as the testing protocol and results of various quality assurance tests. A total of 1459 data points are collected in this effort; about $15 \%$ are not in the annular flow regime and are eliminated from further consideration.

Chapter 4 discusses a current controversy in the literature, the suppression of nucleate boiling. For many years, increased heat transfer at high vapor quality was attributed to the thinning of the liquid film and the acceleration of the vapor core. Recently a much different phenomena, an enhancement of nucleate boiling, has been suggested in this region. The literature is reviewed critically and new experimental data are analyzed in favor of traditional theory. A sup pression criterion is applied and validated for pare fluids. Extensions to mixed fluids are hypothesized and partially validated.

In Chapter 5, the general results of the various experimental tests are further discussed. A circumferential variation in heat transfer coefficient was observed in horizontal flow boiling of mixtures as compared to pure fluids. A reason for the new variation is hypothesized. The effect of quality on the mixtures' heat transfer coefficient is 
discussed. Finally, sudden departure from nucleate boiling (DNB) were observed in some of the data. The implication of DNB events for heat pumps are discussed.

Chapter 6 reviews the available models and correlations for predicting heat transfer coefficients with pare fluids. In particular, the Chen correlation [Ch66] is reviewed and anslyzed, as it is the most widely

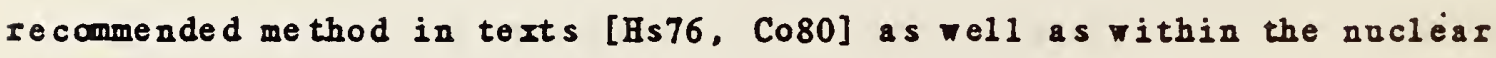
industry in general [Tr78]. The method has been dismissed bistorically within the refrigeration industry because of its poor predictive ability with refrigerants. Recent modifications of the correlation and the application of the method to refrigerants are examined. It is shown that portions of the correlation may be used successfuly ith refrigerants. A new procedure, more analytical, based on recent correlations, is devel oped.

The modelling and correlations for pool and flom boiling of mixtaros is the subject of Chapter 7. The available methods proposed for estimating heat transfer coefficients are roviewed critically and compared to the experimental data. None of the methods produced agreement ith the data as closely as was achieved for pare flutds. however, closure was achieved to the same degree as is found cur rently in the mixtures' literature.

Conclusions and recommendations regarding flom boiling models for pure and mized refrigerants are discussed in Chapter 8 . It is 1 ikely 
that the se first studies of mixtures will lead to further experimental work As such, several recommendations for test fluids, experimental apparatus and testing protocol are al so made in Chapter 8.

\subsection{Nomenclature}

There has been a recent attempt at standardized nomenclature in two phase literature. As such, the athor has used, wherever possible, the latest international comention. In particalar, the following symbols should be noted:

$$
\begin{aligned}
& \mathbf{a}=\text { heat transfer coefficient } \\
& \mathbf{\Delta h}_{\mathbf{b}}=\text { latent heat of vaporization } \\
& \mathbf{a}_{\mathbf{T}}=\text { thermal diffusivity } \\
& \mathrm{a}_{\mathbf{D}}=\text { mass diffusivity } \\
& \mathbf{X}=\text { molar concentration } \\
& \mathbf{I}=\text { vapor quality } \\
& \lambda=\text { thermal conductivity }
\end{aligned}
$$

The general nomenclature is given in the foreward to this report. When exceptions are made, the tert $\mathbf{\text { il }} 1$ define the variable directly.

\subsection{Test Fluids}

The test fluids used in this investigation are pare R152a (CH3CHF2), pure R13B1 (CF3Br) and mixtures of various compositions of these fluids. The two refrigerants are recommended as a mirtare by their manufacturer for heat pump use due to their relatively wide difference in boil ing point $\left(35^{\circ} \mathrm{C}(4.7\right.$ bar). In addition, the mixture can be used in existing machinery without major modification. Some tests have been performed with pure R22 (CC1 HF2), a much more widely otil ized refrigerant. The R22 was used to ensure the experimental testing rig was operating correctly. 


\subsection{General Description of Forced Convection Boiling (Flow Boiling) of Pure Fluids}

Figure 1-1 [La62] shows the typical flow pattern development of a moving fluid being boiled and then evaporated. In most of the applications for refrigerants, the annular flow pattern (sections $E$ and F) is the one of interest, as refrigerants commonly enter the evaporator at a vapor quality of about twenty percent and quickly develop into annalar flow.

For low heat flux, the heat transfer coefficient changes along the length of the tube as follows: when the fluid is a single phase 1 iquid (section A on Figure 1-1), the heat transfer coefficient is approximately constant; it changes only as the thermodynamic and transport properties of the 1 iquid change with temperature. In Section B, subcooled boiling occurs, i.e., vapor forms at portions of the tube wall despite the fact that the bulk fluid temperature remains below the saturation temperature. In this flow section, the thermodynamic quality, $x$, defined as $\left(h-h_{L s a t}\right) / \Delta h_{y}$ is still less than zero, and the heat transfer coefficient owing to developing turbulence increases linearly. When $x$ equals zero (between Sections $B$ and C), saturated nucleate boiling begins, and the value of ais relatively constant. At a quality of a few percent, an annular flow pattern forms (sections E and F). In annular flow with heat addition, the quality increases, stripping the thickness of the 1 iquid layer. Vapor generation in this region is commonly considered to be by evaporation primarily at the 1 iquid/vapor interface, rather than by nucleate boiling at the wall. If nucleate boiling is completely absent, "suppression of nucleate boiling" is said to have taken place. The suppression process is the subject of some 
recent controversy discussed in a later chapter. Since the 1 iquid film becomes thinner and thinner with increasing quality and since the vapor core velocity increases sharply, heat is conducted more readily through the liquid, and the heat transfer coefficient increases. At some point, the 1 iquid $\mathrm{f}$ ilm is entirely evaporated and dryout (a1so known as boiling crisis) occurs: since vapor is a much poorer thermal conductor than 1 iquid, the heat transfer is suddenly and severely diminished. As the quality approaches one, single phase vapor flow occurs, and the heat transfer coefficient again is relatively constant, changing only as fluid properties change.

The heat transfer coefficient depends not only on quality, but on mass and heat flux. As in single phase flow, an increase in mass velocity. causes an increase in turbulence and may cause a consequent increase in the heat transfer coefficient. It should al so be noted that at higher mass flux the dependence on quality becomes much stronger. 1 For the case of increased heat flux, nucleate boiling at the wall may occur in more locations, increasing the heat transfer coefficient. For very high heat flux, no annular flow may be established and sudden reductions in a values may occur at low quality or even in the subcooled boiling regime. This phenomenon is known as a sudden departure from nucleate boiling and is caused by sudden flashing of vapor all along the tube wall (i.e., film boiling).

\footnotetext{
${ }^{1}$ If the vapor generation process is dominated by nocleate boiling, then the heat transfer coefficients become nearly independent of mass flux. This is one way among several to examine the physical process occurring inside the tube.
} 
As previously noted, this study concentrates on the annular flow region prior to dryout. Some other comments are al so appropriate: Figure 1-1 shows the flow patterns in a vertically-oriented tube. In a horizontal tube, the liquid film can be considerably asymetric due to gravity. The thick liquid film on the bottom of the tube tends to reduce the local a values considerably; however the liquid film on the top and sides is thinner in the horizontally-oriented tube, increasing the heat transfer coefficient. To the author's knowledge only one group used the same tube, fluid, and experimental appartus to measure the heat transfer coefficient in both orientations: Lavin and Young showed that a horizontal was fifty percent larger typically than a vertical, when the heat transfer coefficients at a locatior were averaged all around the tube ( $a$-avg equals [ $\alpha$-top plus $a$-bottom plus $a$-leftside plus $a$-rightside]/4). [La66]. Actual average values are difficult to obtain in this a symmetric case.

This simplistic explanation of the flow pattern and heat transfer Iegimes masks many of the complicating features of real annular flow boiling. First in any annular two phase flow, the vapor-liquid interface is wavy. Film thicknesses at a fired spatial location may vary over time by a factor of twenty due to wave passage. The wave changes the turbulent structure of the film and may induce or retard nucleate boiling at the wall. The presence of nucleate boiling may, in turn, break-up the viscous sublayer in the 1 iquid film. Information about the turbulence structare at the vapor-1 iquid interface is unavailable, though there are suggestions that the turbulence may be damped in this 
region. The waves themselves are frequently sheared, and liquid droplets entrained in the vapor core. A large fraction of the total liquid flow may be entrained in this manner. Rates of entrainment and droplet deposition are, for the most part, poorly quantified. These rates in fact control filn thicknesses and are the key to any eventual analytical mode1. Liquid film models derived from the momentum and energy equations have poorly predicted heat transfer rates [Co80]. The 1 iterature suggests the problem is due to interfacial turbulence damping, but recently it has been suggested that the root cause is poor understanding of entrainment [He84]. In any case, these multiple problems have made realistic analytical model development a rather distant hope.

\subsection{The Differences Between Mixtures and Pure Fluids}

In this section a review is presented of the additional characteristics which must be considered when a fluid is a non-azeotropic mixture. The review is not intended to be comprehensive, but stresses the most important features of non-azeotropic refrigerant mixtures as applied to fl ow boiling.

Figure 1-2 displays a temperature-composition phase diagran for the R13B1/R152a mixture used in this report. It is typical of many nonazeotropic mixtures. Examination of the figure reveals immediately the two most important mixture features:

1. The evaporation process in non-isothermal. 
2. As the fluid begins to evaporate, vapor is formed preferentially of one component (the more volatile or "1 ight" component).

The non-isothermal nature of the mixture is advantageous in terms of use in a counter-flow heat exchanger (a higher effectiveness is possible since a constant temperature difference can be maintained throughout the exchanger). On the other hand, the non-isothermal nature al so causes only a portion of heat input to a flowing mixture to be used for vapor generation; the remainder is required to heat sensibly the 1 iquid and vapor streams. Figure 1-3 shows a control volume for a flowing evaporating fluid (ignoring momentarily nucleate boiling). For vapor generation to occur, the vapor already formed must be further heated, and the liquid heated as well to remain in near-equilibrium at their interface (where the evaporation is taking place). From a heat balance on the control volume, over a distance $d z$ :

$$
\mathrm{q}=\mathrm{q}_{\mathrm{SL}}+\mathrm{q}_{\mathrm{e}}+\mathrm{q}_{\mathrm{SV}}
$$

or

$$
d h=(1-x) C p_{L} d T_{L}+\left(\Delta h_{v}\right) d x+x C p_{v} d T_{v}
$$

where $x$ is the mean quality over the interval. In the case of pure fluids the first and third terms in the right side are equal to zero (neglecting the superheating requirements for the 1 iquid and other

\footnotetext{
$1_{\text {The term }} 1$ ight component is a misnomer. In this study, Rl3BI is more volatile but is the more dense of the two refrigerants.
} 
non-equilibrium effects). In the case of mixtures, the sensible heating may represent more than $20 \%$ of the overall heating required.

The second feature shown on the previous page, the composition difference between vapor and 1 iquid, reveals that physical properties, both thermodynamic and transport, vary substantially throughout the evaporation process even in the absence of pressure drop. For example, with pure fluids, one tends to think of 1 iquid density as constant under these conditions. With mixtures however, with one component stripped preferentially away from the 1 iquid layer during evaporation, the 1 iquid density may vary by $50 \%$ or more, even without pressure drop from inlet to outlet of an evaporator tube. Other thermodynamic properties such as latent heat of vaporization also possess this complicating feature. Thus thermodynamic properties must be reevaluat ted continuously during the evaporation process.

A great difficulty appears with mirtures in that the addition of a second component into a pure fluid may have spectacular effects on surface tension or viscosity. Precise prediction of these properties is impossible in many cases. Surface tension directly affects the nature of nucleate boiling, yet may be impossible to even estimate since general mixing rules are unavailable.

In addition to the property complications, the vapor-liquid composition difference introduces mass transfer resistance. The interfacial composition is different from the bulk 1 iquid and vapor streams. In 
condensation various calculation methods have been developed to account for this problem, with rather extreme as sumptions used in common practice. The addition of mas transfer resistance suggests that mass diffusivities should be known; yet these are rarely known for refrigerants. In turbulent flow one needs to estimate the eddy (mass) diffusivity, a process which itself is uncertain, and made even more complicated in the presence of nucleate boiling.

Given the above consideration along with those described earlier for pure fluids, it is easy to understand the current futility of analyti- . cally modeling the the heat transfer process from first principles. Instead, the literature has proceeded with correlations or simple models which account for as many physical phenomena as their authors deemed possible. 'To the author's knowledge, no published verification of their proposed models has taken place prior to this report.

\subsection{Literature Review of Experiments of Annular Flow Boiling of Mixtures}

A number of texts provide a general review of annular flow boiling experiments for pure fluids [Co80, Hs76]. Table 1-1 lists experimental investigations with refrigerants. Very few experiments however have been conducted under similar conditions for mixtures. Some early experiments used cal orimetric methods to determine overall heat transfer in reboilers [Bo51] or long steam heated evaporators [Mc42]. The se experiments did not provide sufficient information for evaluation of local conditions or physical processes governing the heat transfer. 
Shock investigated an ethanol-water mixture in a vertical tube [Sh73]. The inlet conditions were subcooled and the maximum outlet quality he tested was 0.16. This quality is less than the inlet to most heat pump evaporators. The flow patterns he investigated rarely included annular flow. The ethanol-water mixture has a very non-ideal (see Chapter 2) property behavior. The tube used in his experiments was specially plated with a thin nickel film, removing many cavity sizes and possible nucleation sites. His experiments therefore are not particularly relevant to this report. However, Shock performed very substantial analytic modelling in this effort, and several subsequent publications. These efforts will be referred to frequently throughout this report.

Toral modified the test loop used by Shock [To79]. A copper tube was vertically oriented, and the working fluid of ethonol-cyclohexane was selected due to its near-ideal property behavior. Wall thermocouples were spaced very closely so that the position of the onset of nucleate boiling could be located. They were however, mounted only on one side of the tube and symmetry assumed. He found a deterioration of heat transfer due to mass transfer resistance and that nucleate boiling was the dominant mechanism for his testing conditions. Maximum outlet quality was 0.30 . He compared his experimental results to the Chen correlation; poor prediction was attributed to the poor prediction of nucleate boiling. Surprisingly, the correlation underpredicted the experimental results. This result, as will be shown in Chapter 7, is unusual since mass transfer resistance is not accounted by the 
correlation. Like Shock, Toral performed substantial theoretical studies and they will be quoted frequently.

Bennett recently tested mixtures of ethylene-glycol and water, again in a vertical tube [Be80]. The test section was very short $(L / D \sim 3)$ and inlet flows were in the two phase region, provided by preheaters. Only a single wall thermocouple was used in all the measurements, again with symmetry assumed. Several hundred data points were taken over a wide range of conditions. Bennett developed a modification to Chen's correlation to account for mixture effects. They predicted his data to a mean deviation of $\pm 15 \%$.

Chaddock and Mathur investigated refrigerant-oil mixtures in a serpentine horizontal copper tube [Ma79]. The full quality range was covered. One potential complication with the study was the impact of oil on properties such as surface tension. The authors noted a dependence of the heat transfer coefficient on heat flux, indicating a nucleate boiling contribution (neglected in the authors' final correlation). Nucleate boiling behavior with refrigerant-oil mixtures defies current understanding [Bu79]; pool boiling experiments with these mixtures show rather erratic results (see Figure 1-4). In the study of Mathur and Chaddock, the addition of oil produced an increased beat transfer at 1 ow qualities and a decrease at high qualities, the latter due to the 1 iquid composition being oil-rich. 
Mishra, et a1., investigated mixtures of $\mathrm{R} 12 / \mathrm{R} 22$ over a 1 imited composition range in a horizontal stainless steel tube [Mi81, Va79]. Two phase inlet conditions were maintained by a preheater. No tests of pure R22 were conducted. The authors used pure fluid correlations and revised the exponents of the correlations tofit most of their experimental results to $\pm 25 \%$.

Singal, et al., experimented with an R13/Rl2 mixture, again with a horizontal tube over a 1 imited quality range [Si83]. The authors again correlated their results with a different pure fluid correlation. They concluded that the $\alpha$ was decreased for mixtures compared to pure R13 whenever the quality was less than 0.3 , and that with a further increase in vapor quality an increased heat transfer coefficient was observed when mixtures were compared to pure RI3.

Radermacher, Ross and Didion investigated a mixture of R152a/R13B1 selected for its wide range of boiling points [Ra83]. This work has been reanalyzed and is presented in detail as part of this report. 
Table 1. Flow Boiling Experiments with Pare Refrigerants: Literature Review

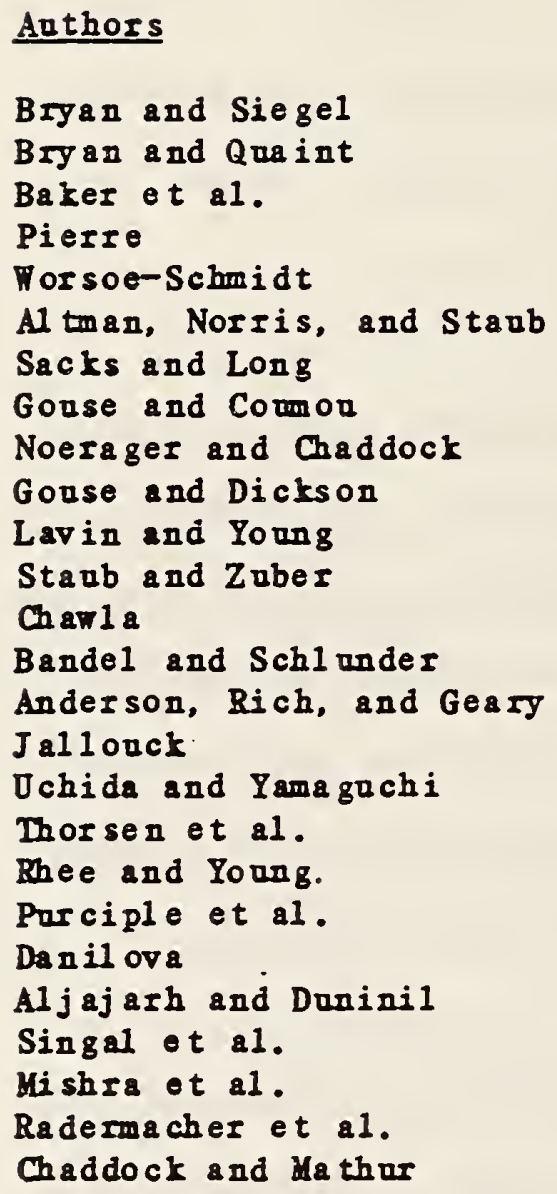

Refrigerant

$\underline{\operatorname{Ref}}$.

Ba1

R11

B12

R1 2

B. 2

R22

R21

R113

B1 2

R113, R11

$\mathrm{B} 12, \mathrm{B22}$

R22

R11

R1 2

R22

R11

B21 2

B113

B12,

B11, B12, $\mathbb{B} 13$

R11

$\mathrm{B} 12, \mathrm{~B} 22, \mathrm{~B} 502, \mathrm{~B} 13 \mathrm{BL}$

BD3

BD 2

$\mathrm{B22}, \mathrm{BC} 52 \mathrm{2}, \mathrm{BL} 3 \mathrm{BL}$

B22
B 55

BI51

Ba53

Pi56

T060

Al 60

Sa61

G065

Cb6 6

G066

La66

St6 6

Ch6 7

Ba7 4

An6 6

$\mathrm{J}$ a 74

Dc66

Th70

$\mathrm{Rh} 74$

Pu72

Da6 9

A17 7

Si 83

Mi 81

$\mathrm{Ra} 83$

Ch7 9 


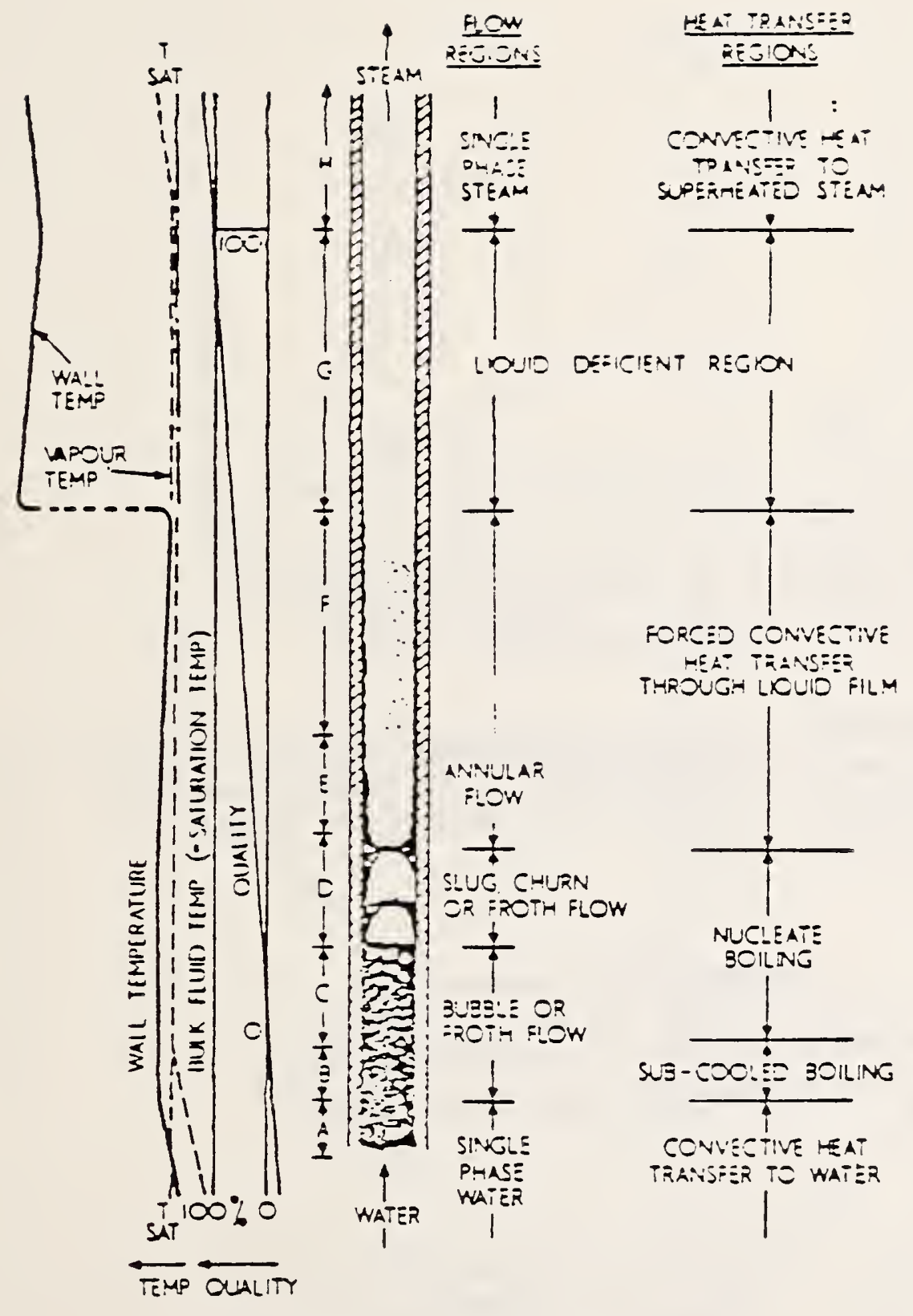

REJ wES OF THO. DUASE FIOW

$\frac{\Delta \equiv 25-23962}{F+G-251}$

Figure 1-1: Regimes of Tro brase Tlow (Reprinted from (La62) 
CONCENTRATION: wt. $152 \mathrm{a}$

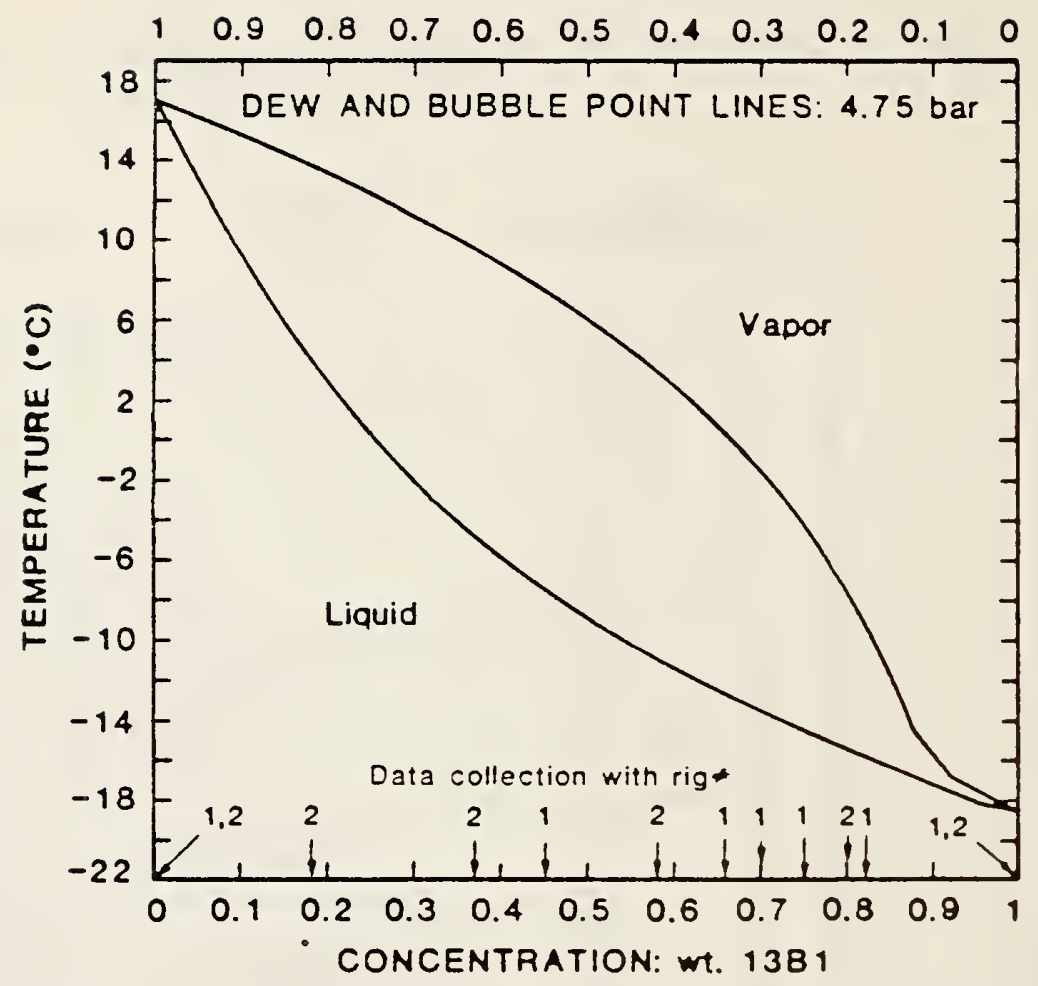

Figure 1-2: Temperature-Concentration Diagrams for Test Fluids in terms of Weight and Molar Fractions. Shown also are the feed concentrations used in the experiments

CONCENTRATION: moles 152 a

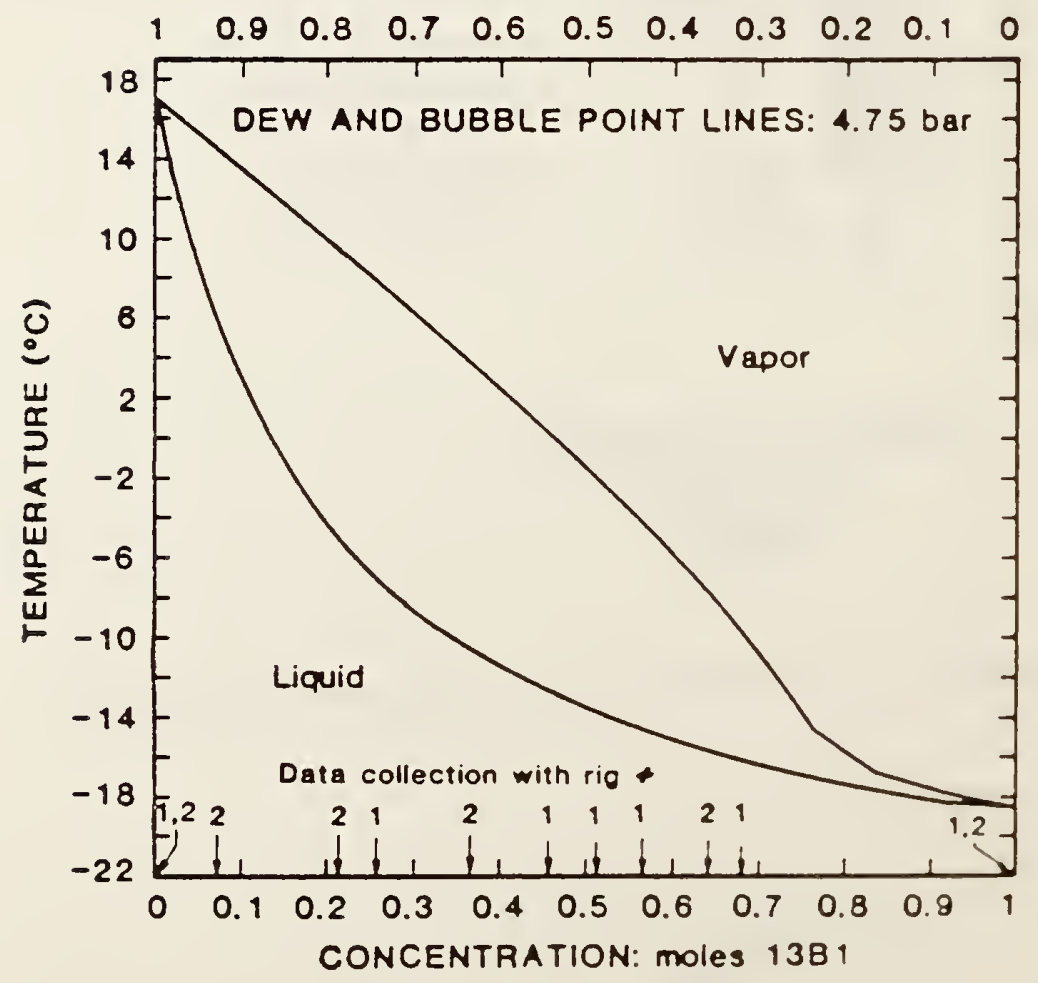




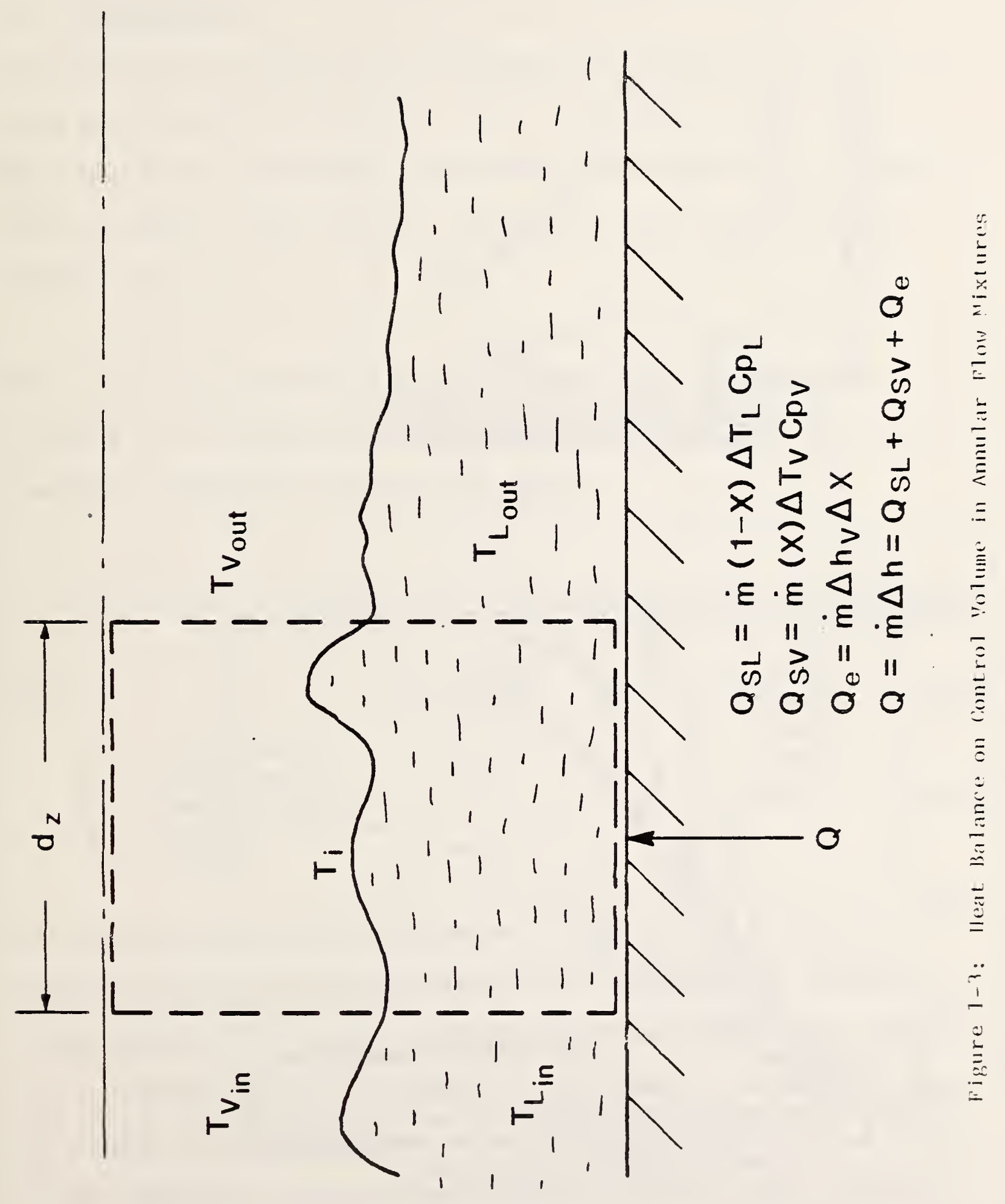



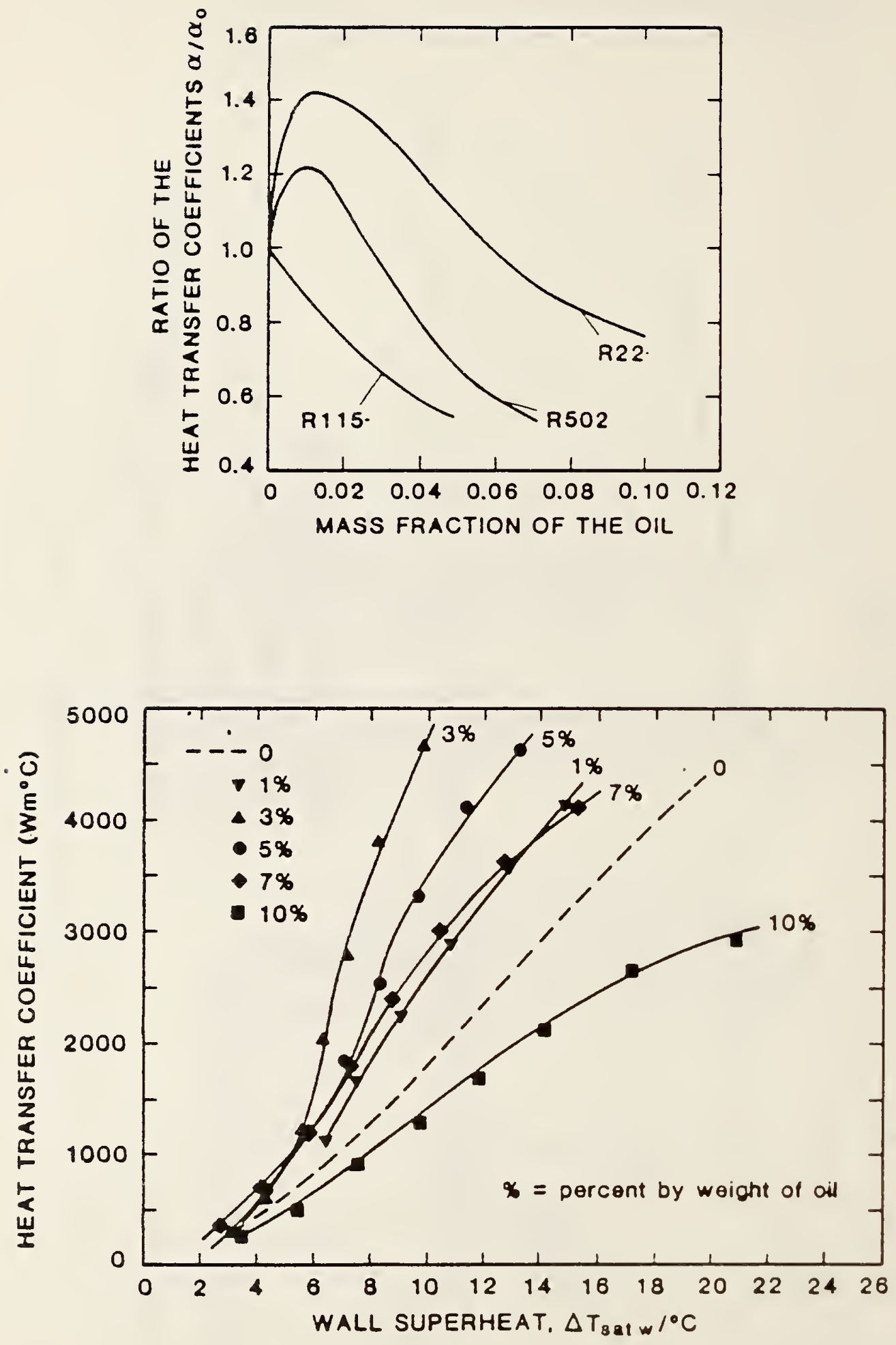

Figure 1-4: Effect of Oil on Heat Transfer Coefficients of Refrigerants. Small additions cause increase, however further additions cause decrease. 


\section{CHAPTER 2: PROPERTY DETERMINATION}

\subsection{Introduction}

Determination of transport and thermodynamic properties for mirtures is much more difficult for mixtures than for pore fluids. This is due to more complicated theoretical considerations of molecular interactions as well as the very common lack of experimental data of the mirture's properties.

The most frequent method for estimating many of the properties of mirtures is to weight the mixture property by the mole fraction of the individual components comprising the mirture:

$$
P_{m}=\bar{X}_{A} P_{A}+\left(1-X_{A}\right) P_{B}
$$

where

$$
\begin{aligned}
& \mathbf{P}=\text { any property } \\
& \mathbf{X}=\text { mole fraction } \\
& \mathbf{A}=\text { component A } \\
& \mathbf{B}=\text { component } \mathrm{B} \\
& \mathbf{M}=\text { mixtare }
\end{aligned}
$$

This approach has several deficiencies. First of all, spectacular deviations from this presumed behavior have been observed. Examples include surface tension (e.g., ethanol water) and $v$ iscosity of 1 iquids (e.g., rater-N, N-dimithy lacetamide). Fur themore, a misture may exist of a liquid at a pressure mich is at or above the critical point of one of the components. In this region, specific heat and 1 iquid viscosity are either undefined or infinite. Most engineering work is done aray from the critical point; however, this point is exceeded with one of the 
refrigerants of this report, R13B1, as it operates in a mixture in the condenser of real heat pumps. ${ }^{1}$ Thus, the problem is not simply an academic one.

The refrigerant mixture of R152a and R13B1 presents a particular problem in that both molecules are polar (RI3BI is weakly polar; R152a strongly polar). Deviations from the ideal mixing rule of equation (2-1) can therefore be expected. Extensive P, T, v, X measurements of the mixture have been made by Morrison [Mo82]. However, no transport property measurements of the mixture have been found in the literature.

\subsection{Thermodvnamic Properties and the Equation of State}

In order to determine the themodynamic properties of a mixture, an equation of State (EOS) is required. The most commonly used one in industry is the Red1ich-Kwong-Soave (R-K-S) equation:

$$
\frac{P v}{R T}=\frac{v}{v-b}-\frac{a}{(v+b) R T}
$$

Mizing coefficients used in determing a and ban be estimated from the molecular structure of the components. The difficulty with the R-K-S equation is that it is not applicable to the liquid phase. In practice a 1 ibrary of 1 iquid properties is used in conjunction with the R-K-S two phase and vapor phase predictions. Al ternataly, curvefit or semi empirical equations can be witten for the 1 iquid phase and pieced to

\footnotetext{
${ }^{1}$ Measurements in this report were conducted below the critical point of
} either component. 
the $R-K-S$ model. Unf ortunately, discontinuities appear in certain properties (e.g., $C_{p L}$ ) with this approach. Extrapolation near the critical point also produces significant error.

Morrison has applied the DeSantis equation of state to the R152a/R13Bl misture. This EOS is continuous in both the 1 iquid and vapor phases [Mo82] :

$$
\frac{P v}{R T}=\frac{1+y+y^{2}-y^{3}}{(1-y)^{3}}-\frac{a}{(v+b) R T}
$$

where

$$
y=\frac{b}{4 v}
$$

The first term on the right hand side of equation (2-2) accounts for molecular repulsive forces and the second term accounts for attractive forces. As can be seen in the equation, the term b must have the units of molecular volume. It is a means of adjusting the closest approach distance between two molecules. As the temperature of a real fluid is raised, this distance becomes smaller (since molecular kinetic energy increases with increasing temperature). Thus, the variable b is a function of temperature. The term a accounts for the non-spherical nature of the forces between molecules because the refrigerants are polar. Again as temperature is raised, the directional attractions of the molecules are reduced, as well as the average attractive force. Thus, a has also a temperature dependence. Morrison determined 
empirically the values of "a" and " $b$ " from the pure R13B1 and R152a data se ts published in in ASHRAE Tables of Refrigerants after these data sets checked with his own measurements.

In the case of mixtures, the terms "a" and "b" become:

$$
\begin{aligned}
a & =\sum_{i} \sum_{j} \quad \bar{x}_{i} \bar{x}_{j} a_{i j} \\
b & =\sum_{i} \sum_{j} \bar{x}_{i} \bar{x}_{j} b_{i j} \\
a_{i j} & =\left(1-f_{i j}\right) \quad\left(a_{i} a_{j}\right)^{1 / 2} \\
b_{i j} & =\left[\left(b_{i}^{1 / 3}+b_{j}^{1 / 3}\right) / 2\right]^{3}
\end{aligned}
$$

These account for molecular interaction between molecules of different components. The term, $f_{i j}$, is an empirically determined mixing coefficient which might account for both the nor-spherical nature of the molecules and possible interactions such as hydrogen bonding.

The solution scheme, and actually the computer code, used in this report, is taken directly from Morrison [Mo84]. It requires an input pressure, temperature and overall composition and outputs enthalpy, entropy, specific volume, and composition of each of the phases as well as the overall mixture. Al so output are molar quality and 1 iquid and vapor specific heats.

The code requires several internal iterative loops. The form of equation (2-2) is $f$ if th order and does not have an analytic solution. 
In general, given $T$ and $X_{i}$, one determines bubble and dew point pressures by iteration. If the given pressure is higher than $P_{B U B}$ the mixture state is subcooled; if $P<P_{\text {DEW }}$ it is superheated vapor. In the two phase region, given $T$ and $P$, one guesses $\bar{X}_{L}$, and determines by iteration Pguess. When the difference between $P$ and $P$ guess is sufficiently small, the solution is considered closed. Pguess is itself the subject of certain conditions. For mixtures, the chemical potential of each component in each phase must be equal: this requirement determines the final pressure guess.

The computer code for the equation of state is not arranged to handle pure refrigerants easily. Furthermore, the data reduction time can be rednced substantially by using curvefits to the property table data. These curvefits are:

$$
\begin{array}{lll}
\text { (13B1) } & 1 / \mathrm{T}=-.04-.0004656 \mathrm{ln}(\mathrm{P} / 21867.08454) & \mathrm{T}\left[{ }^{\circ} \mathrm{K}\right], \mathrm{P}[\mathrm{bar}] \\
(152 \mathrm{a}) & 1 / \mathrm{T}=.004039-.00038091 \mathrm{n}(\mathrm{P}) & \mathrm{T}\left[{ }^{\circ} \mathrm{K}\right], \mathrm{P}[\mathrm{bar}]
\end{array}
$$

Most experimental tests with mixtares for this report were run at pressures between 4.4 and 5.0 bar. Table 2-1 displays the calculated densities for the 1 iquid and vapor phases, as well as the latent heat of vaporization at a pressure of 4.75 bar. Values of the thermodynamic properties may vary by up to $15 \%$ from a mole fraction weighting of the pure components' values at the same pressure. 


\subsection{Transport Properties}

The determination of varions transport properties for mirtures is complicated by the requiroment of using propermixing rules. The miring roles are taken inevitably fram the idely referenced tert by Reid, Prauszitz and Sherwood [Re79]. The mixing rules wich they recomend, and which are used in this report, have not been verified experimentally for the particular R13B1/R152a combinstion.

Transport property data for the pure refrigerants are available from two sources: ASHRAE [As81] and HTFS [Ht83].1

The ASHRAE tables are incomplete in their transport properties; the HTFS data only as used in this report. All correlation coefficients $\left(\mathbb{R}^{2}\right)$ were greater than 0.99 for the pure fluid curvefits.

(a) Thermal Conductivity (Liquid Phase)

The curvefit equation for the pure refrigerants was:

$$
\lambda_{L}=\mathrm{A}+\mathrm{BT}_{\mathrm{I}}+\mathrm{CT}_{\mathrm{I}}^{2}+\mathrm{DT}_{\mathrm{I}}^{3} \quad\left[10^{-3} \nabla / \mathrm{M} / \mathrm{K}\right]
$$

\begin{tabular}{rrrrrrrr} 
& \multicolumn{1}{c}{$\mathrm{A}$} & \multicolumn{1}{c}{$\mathrm{B}$} & $\mathrm{C}$ & \multicolumn{1}{c}{$\mathrm{D}$} & $\mathrm{T}_{\text {Imin }}$ & $\mathrm{T}_{\text {ImaI }}$ \\
(13B1) & 150.396 & -77.341 & -107.302 & 81.272 & 0.6 & 0.96 \\
$(152 \mathrm{a})$ & 244.932 & -122.821 & -177.969 & 133.061 & 0.6 & 0.88
\end{tabular}

The former has an error in R152a spocific heat [R283, Mo84a] and the latter in latent heat [Mo84b]. 
Reference [Re79] recommends the following mixing rule, with a marimum error of $4 \%$ :

$$
\lambda_{m}=\left[(1+C) W_{A}-C W_{A}^{2}\right] \lambda_{A}+\left[(1-C) w_{B}+C W_{B}^{2}\right] \lambda_{B}
$$

where

$$
\begin{aligned}
\lambda & =\text { thermal conductivity } \\
C & =0.72 \text { (empirical constant) } \\
W & =\text { weight fraction }
\end{aligned}
$$

Figure 2-1 shows the results of applying the mixing rule at various compositions. It can be seen that there is a substantial deviation from the ideal mixing $I$ ule of equation (2-1).

\section{(b) Thermal Conductivity (Vapor Phase)}

The vapor conductivity of each of the pure refrigerants was estimated from the HTFS data as:

$$
\begin{aligned}
& \lambda_{\mathrm{V}}=\mathrm{A}+\mathrm{BT} \mathrm{T}_{\mathrm{I}}+\mathrm{CT}_{\mathrm{I}}^{2}+\mathrm{DT}_{\mathrm{I}}^{3} \quad\left[10^{-3} \mathrm{w} / \mathrm{k}\right]
\end{aligned}
$$

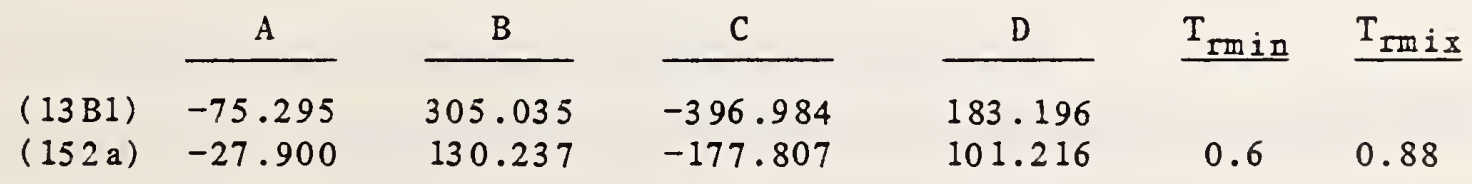

The mixing rule in $[\operatorname{Re} 79]$ (maximum error $=5 \%$ ) which was selected was: 


$$
\lambda_{m}=\sum_{i=1}^{2} \frac{\bar{Y}_{i} \lambda_{i}}{\sum_{j=1}^{2} \bar{Y}_{i} A_{i j}}
$$

where

$$
\begin{aligned}
A_{i j} & =\frac{\left[1+\left(\lambda_{i} / \lambda_{j}\right)^{1 / 2}\left(M_{j} / M_{i}\right)^{1 / 4}\right]^{2}}{\left[8\left(1+\left(M_{i} / M_{j}\right)\right]^{1 / 2}\right.} \\
\frac{M}{Y} & =\text { molecular weight }
\end{aligned}
$$

\section{(c) Liquid Viscosity}

The pore liquid viscosities were determined from:

$$
\begin{aligned}
& \mu_{13 \mathrm{~B} 1}=4935.37126-43.7022 \mathrm{~T}+0.1379 \mathrm{~T}^{2}-0.00015209 \mathrm{~T}^{3} \\
& \mathrm{~T}\left[{ }^{0} \mathrm{~K}\right], \mu\left[10^{-6} \mathrm{~Pa}-\mathrm{sec}\right] 220<\mathrm{T}<300 \\
& \mu_{152 \mathrm{a}}=\mathrm{A}+\mathrm{BT}_{\mathrm{I}}+\mathrm{CT}_{\mathrm{I}}^{2}+\mathrm{DT}_{\mathrm{I}}^{3} \\
& \mathrm{~A}=387.5886 \quad \mathrm{~B}=1238.98 \quad \mathrm{C}=2864.765 \quad \mathrm{D}=1313.244 \\
& 0.6<\mathrm{T}_{I}<0.88
\end{aligned}
$$

and the mixing rule used was (maximum error at $\pm 15 \%$ ) described in terms of the kinematic viscosity: 


$$
\nu_{m}=\phi_{A} \nu_{A}^{B}{ }^{B *} B+\phi_{B} \nu_{B} e^{\phi_{A} R_{L}^{*}}
$$

where

$$
\begin{aligned}
\nu & \equiv \text { kinematic viscosity }(1 \text { iquid })=\mu / e \\
\phi & \equiv \text { volume fraction } \\
\mathbf{R}_{A}^{*} & \equiv 0.27 \ln \frac{\nu_{B}}{\nu_{A}}+\left(1.3 \ln \frac{\nu_{B}}{v_{A}} 0.5\right.
\end{aligned}
$$

\section{(d) Yapor Viscosity}

The pare component vapor viscosities were estimated from:

$$
\mu_{\nabla}=A+B T_{I}+C T_{I}^{2}+O T_{I}^{3} \quad\left[10^{-6} \mathrm{~Pa}-\sec \right]
$$

\begin{tabular}{cccccccc} 
& $\mathrm{A}$ & $\mathrm{B}$ & $\mathrm{C}$ & \multicolumn{1}{c}{$\mathrm{D}$} & $\mathrm{T}_{\text {Im in }}$ & $\mathrm{T}_{\text {Imax }}$ \\
$(13 \mathrm{BI})$ & -26.308 & 132.34 & -166.51 & & 81.7996 & 0.6 & 0.96 \\
$(152 \mathrm{a})$ & -10.320 & 60.803 & -74.075 & 38.667 & 0.6 & 0.88
\end{tabular}

The same miring rule as used for vapor conductivity was used to determine vapor $\nabla$ iscosity.

\section{(e) Liquid Mass Diffusivity (Diffusion Coefficient)}

No measurements of the 1 iquid mass diffusion coefficient have been taken on any refrigerant pair. Fur thermore, [Re79] makes no clear recommendation regarding this property, especially for polar mirtures.

Randlikar et a1. [Ka75] attempted to predict the mass diffusion coefficient for an R22/R22 mixture, using a formula suggested in [Re79]. The 
method requires the misture's 1 iquid viscosity, an accurate equation of state, and generous assumptions regarding activity coefficients, ideal gas behavior, and interaction parameters. Their approach and result may be viewed as an order of magnitude approximation. For their misture at $0^{\circ} \mathrm{C}$ and 0.6 mole $\mathrm{R} 22$,

$$
{ }^{a} D \simeq{ }^{a} T^{160}
$$

In tarbulent flow, the following assumption is sometimes made for the eddy diffusivity:

$$
\varepsilon_{D}=\varepsilon_{T}
$$

Since the film flow is turbulent and since the presence of nucleate boil ing may destroy the viscous sublayer, the actual diffusivity may be anywhere between these values ( 1 and 60 ).

In the models described in Chapter 7 , a decision was made to assume parametric values of ap to try to bound the results.

\section{(f) Surface Tension}

Surface tension of the pure components was estimated via the following relation:

$$
\sigma=\mathrm{A}+\mathrm{BT}+\mathrm{CT}^{2}+\mathrm{DT}^{3} \quad \mathrm{~T}\left[{ }^{\circ} \mathrm{K}\right], \tau[\mathrm{mN}-\mathrm{m}]
$$




\begin{tabular}{|c|c|c|c|}
\hline A & B & C & D \\
\hline 37.969 & -.01575 & $-6.73 E-4$ & $1142 \mathrm{E}-9$ \\
\hline 52.828 & -.12150 & $-2.30 E-4$ & $4349 \mathrm{E}-10$ \\
\hline
\end{tabular}

For the surface teasion of the mixture, [Re79] provides 1ittle guidance. Mole fraction weighting was assumed, regarding equation (2-1). Since the pure components are not particularly disparate, this assumption should lead to 1 ittle error.

\subsection{The Effect of Non-Ideal Property Behavior on the Heat Transfer Coefficient}

Transport properties of mixtures are rarely determined by ideal mixing, i.e., simple mole weighting of the component properties. Mixtures tend to have higher Iiquid viscosities and 1 ower 1 iquid thermal conductivities then would be suggested by ideal mixing. The impact of these physical physical property tendencies are evalnated below.

In single phase heating, the following equation is widely accepted to be of sufficient accuracy for predicting heat transfer coefficients:

$$
a_{L}=\frac{\lambda}{L}_{D} \frac{G D}{\mu}^{0.8} \frac{\mu_{L} c_{p_{L}}}{\lambda_{L}} \cdot 4
$$

Consider first, a as calculated from ideal mixing

$$
a_{i d L}=\frac{G^{0.8}}{D^{0.2}} C_{p_{i d L}} 0.4 \lambda_{i d_{L}} 0.6 \mu_{i d L}-0.4
$$


and for a misture using the appropriate miring rules

$$
a_{m}=\frac{G^{0.8}}{D^{0.2}} C_{P_{m L}} 0.4 \lambda_{m_{L}} 0.6 \mu_{m_{L}}-0.4
$$

Dividing (2-3) into (2-4)

$$
\frac{a_{\text {ml }}}{a_{i d}}={\frac{c_{p_{m L}}}{c_{p i d}}}^{0.4} \frac{\mu i d}{\mu m}^{0.4} \frac{\lambda m}{\lambda i d}^{0.6}
$$

Now, it is known

$$
\frac{c_{p_{m}}}{c_{p_{i d}}} \approx 1, \frac{\mu i d}{\mu m}<1 \frac{\mu m}{\mu_{i d}}<1 \text { from mixing rules }
$$

Theref ore

$$
\frac{a_{m_{L}}}{a_{i d}}<1 \text { al ways }
$$

Thus, the mixture's single phase heat transfer coefficient should be less than that predicted by a mole fraction weighting of the pure fluid properites. The analysis can now be extended to forced convection evaporation flows.

For evaporating flows, the following correlation form appears of ten in the 1 iterature. 


$$
a_{2 \phi}=a_{L} A\left(X_{t t}\right)^{-B}
$$

So, assuming ideal mizins rules

$$
a_{i d_{2} \phi}=a_{i d_{L}} A X_{t t_{i d}}{ }^{-B}
$$

and for actual mixing

$$
a_{i d_{2 \phi}}=a_{i d_{L}} A X_{t t_{i d}}-B
$$

Dividing $(2-7)$ into $(2-8)$

$$
\frac{a_{m_{2} \phi}}{a_{i d_{2}}}=\frac{a_{m_{L}}}{a_{i d_{L}}} \frac{x_{t t_{i d}}}{x_{t t_{m}}}
$$

Now,

$$
\mathrm{z}_{\mathrm{tt}}=\left(\frac{1-\mathrm{x}}{\mathrm{x}}\right)^{0.9}\left(\frac{\rho_{\mathrm{v}}}{\rho_{\mathrm{L}}}\right)^{0.5}\left(\frac{\mu_{\mathrm{L}}}{\mu_{\mathrm{v}}}\right)^{0.1}
$$

so,

$$
\frac{X_{t t i d}}{X_{t t m}}=\left(\frac{\rho_{\mathrm{Lm}}}{\rho_{\mathrm{Lid}}}\right)^{0.5}\left(\frac{\rho_{\mathrm{vid}}}{\rho_{\mathrm{vm}}}\right)^{0.5}\left(\frac{\mu_{\mathrm{Lid}}}{\mu_{\mathrm{Lm}}}\right)^{0.1}\left(\frac{\mu_{\mathrm{vm}}}{\mu_{\mathrm{vid}}}\right)^{0.1}
$$

Substituting $(2-10)$ and $(2-5)$ into (2-9) 


$$
\begin{aligned}
& \frac{a_{\mathrm{m} 2 \phi}}{a_{i d} \phi}=\left(\frac{C_{\mathrm{PLm}}}{C_{\mathrm{PLid}}}\right)^{0.4}\left(\frac{\mu_{\mathrm{Lid}}}{\mu_{\mathrm{m}}}\right)^{0.4}\left(\frac{\lambda_{\mathrm{m}}}{\lambda_{\mathrm{id}}}\right)^{0.4}\left(\frac{\rho_{\mathrm{Lm}}}{\rho_{\mathrm{Lid}}}\right)^{0.5 \mathrm{~B}}\left(\frac{\rho_{\mathrm{vid}}}{\rho_{\mathrm{vm}}}\right)^{0.5 \mathrm{~B}}\left(\frac{\mu_{\mathrm{Lid}}}{\mu_{\mathrm{Lm}}}\right)^{0.5 \mathrm{~B}} \\
& \left(\frac{\mu_{\mathrm{vm}}}{\mu_{\mathrm{vid}}}\right)^{0.5 \mathrm{~B}}
\end{aligned}
$$

Grouping terms:

$$
\begin{aligned}
& \frac{a_{\mathrm{m} 2 \phi}}{a_{\mathrm{id} 2 \phi}}=\left(\frac{C_{\mathrm{PLm}}}{C_{\mathrm{PL}}}\right)^{0.4}\left(\frac{\lambda_{\mathrm{gL}}}{\lambda_{\mathrm{id}}}\right)^{0.4}\left(\frac{\mu_{\mathrm{Lid}}}{\mu_{\mathrm{Lm}}}\right)^{0.4+.1 \mathrm{~B}}\left(\frac{\mu_{\mathrm{Vm}}}{\mu_{\mathrm{Vid}}}\right)^{0.1 \mathrm{~B}} \\
& \left(\frac{\rho_{\mathrm{Lm}}}{\rho_{\mathrm{Lid}}}\right)^{0.5 \mathrm{~B}}\left(\frac{\rho_{\mathrm{Vid}}}{\rho_{\mathrm{Vm}}}\right)^{0.5 \mathrm{~B}}
\end{aligned}
$$

Away from the critical point the density ratios are $\approx 1$ and $\mu_{\mathrm{vm}} / \mu_{\mathrm{v} i d} \approx 1$ for R152a/R13B1 so, $a_{m 2 \phi} / a_{i d 2 \phi}<1$ always. The evaporative heat transfer coefficient for the mixture is less than would be calculated from ideal property behavior.

Now, near the critical point, $\rho_{\mathrm{Lm}} / \rho_{\mathrm{Lid}}>1$ and $\rho_{\mathrm{Vm}} / \rho_{\mathrm{Vid}}\langle 1$, and the se tend to balance the other terms, so nothing definitive can be said. Furthermore near the critical point, estimation of transport properties is exceptionally difficult.

The extrcise on the previous page, which to the author's knowledge, has not appeared in the literature, simply shows that even in the absence of the mass transfer resistance, assuming ideal miring may lead to serious overestimation of heat transfer in both single and two phase mirtures. 


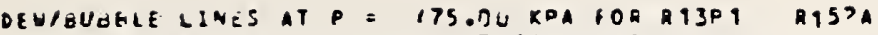

LJOUID PROPERTIES EYALUATIG AT (TBUR,I):

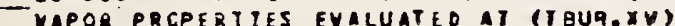

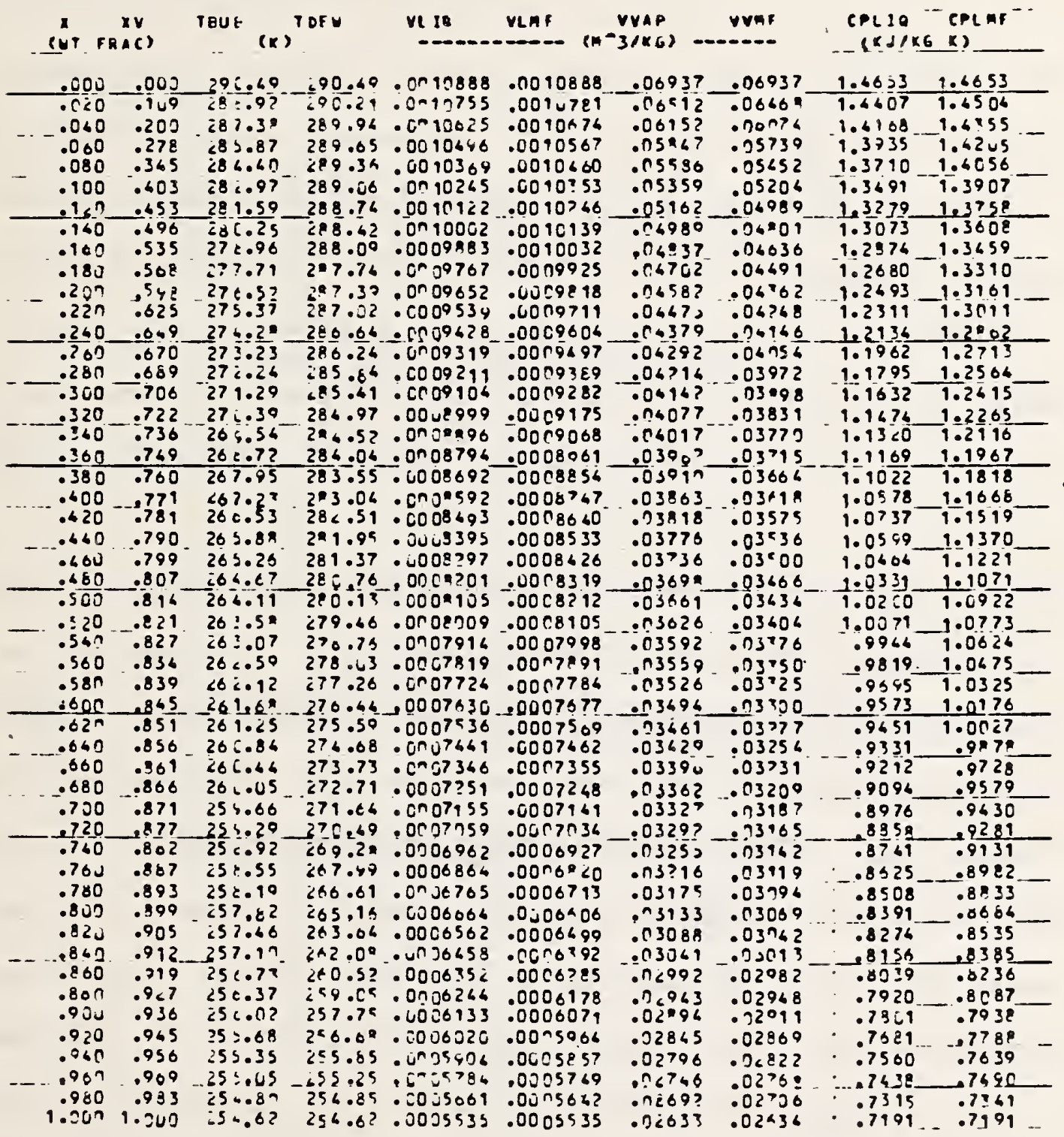

Table 2-1: Comparison of EOS values and mole fraction weighting of pure components. 

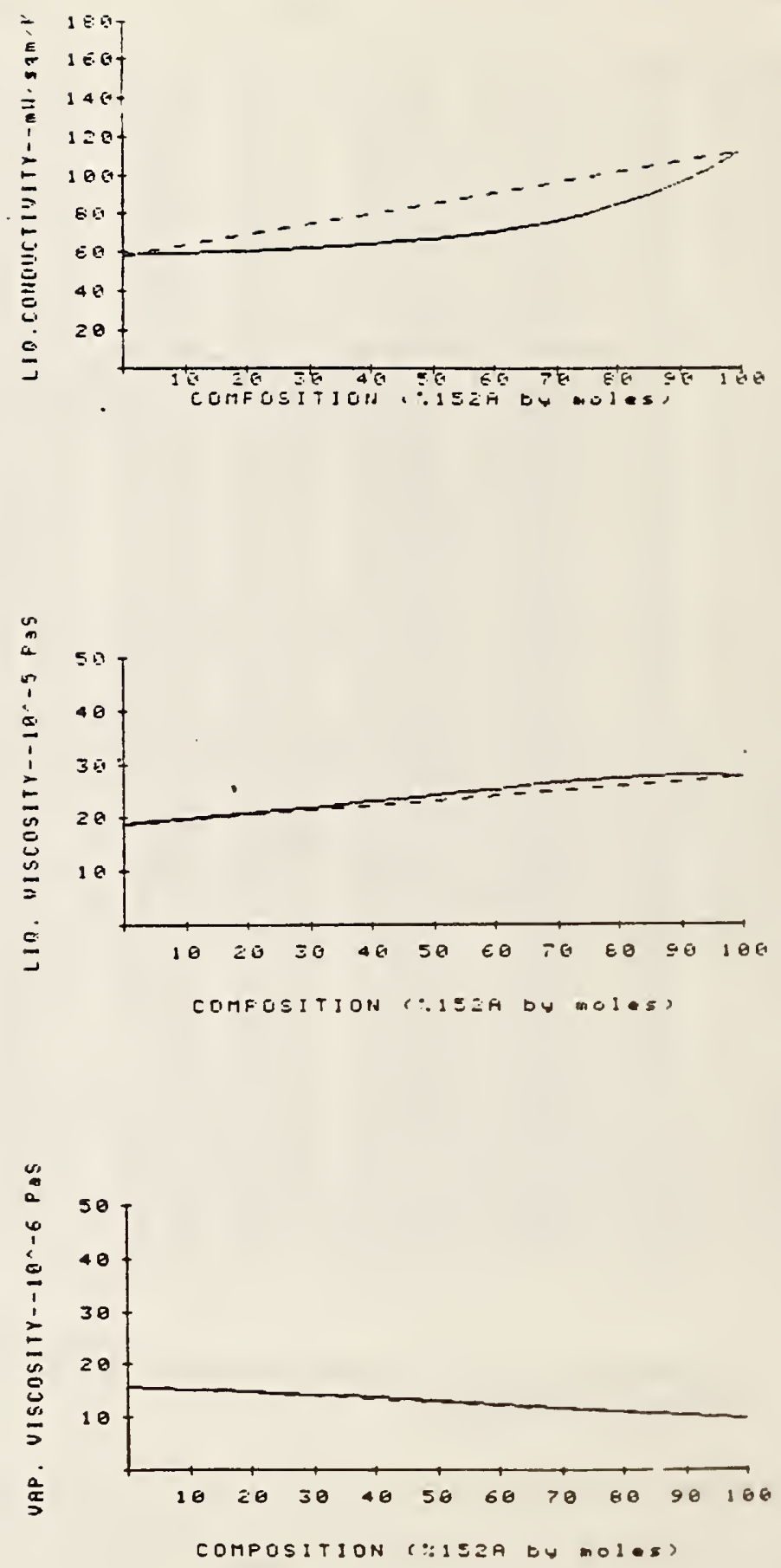

Figure 2-1: Comparison of Transport Properties as determined by mole fraction weighting and mixing rules 


\section{CHAPTER 3: EXPERIMENTS}

\subsection{Introduction and Historical Review}

This chapter describes in detail the experimental apparatus, instrmentation, and data reduction methods used in this report. The remainder of this section reviews the types of experimental test rigs Which have appeared in the literature. Sections 3.2 through 3.4 describe the detailed features of the test rigs. Section 3.5 displays the tests done to provide some assurance of the quality of the data. Problems which were encountered are discussed in Section 3.6. Finally a sumaty of the deta is provided in Section 3.7 .

Most experimental rigs described in the literature have been designed as shown in Figure 3-1. Fluid was circulatedvia a punp or compressor throggh a smooth heated circular tube where it was boiled/eraporated, and then reliquified in a condenser. Preheaters and afterheaters and sometimes pressurizers were employed to control entering or exiting conditions. Pressure taps were installed along the test section, and these pressure readings were used to determine the local saturation temperature of the moving fluid. Wall temperatures were determined by a series of thermoconples attached to the tube wall by solder [Bas\}, Al60], spot weld [Ch79, Mi81], braze [Sa61] or mechanical clip [Di66, Ra83, Ab82]. The test section itself was electrically heated [Ch77, Ch66a, St66, G065, Di66, Mi81] or heated isothemally by fast flowing water [A160, An66] or by a condensing fluid surrounding the tube [Ba53]. In any case, the heat flux was considered wel1-known and the local heat transfer coefficient was then experimentally de termined by the equation: 


$$
a=\frac{\dot{q} / A_{s}}{T_{w}-T_{f}}
$$

The test section in some investigations was made of glass so that $v$ isual observation of flon patterns could be made [St66, Di66]. However, the use of glass had a serious deficiency, forcing its eventual abandonment. A plating process was ased to provide a continuons metal film on the inside of the glass test section. The metal film served as an excellent electrical resistance heater, however non nniformities in its surface caused the surface roughness (de spite attempts at milling smooth) to cavitate and swirl the flow and angment the heat transfer. The variable surface thickess al so caused nonuniform heat generation. Most studies instead used thin wall metal tubes in wich the temperature drop through the $\mathbf{w l l}$ is quite smal1. 'Flow visualization' with metal tubes can be attempted through deduction, either from void fraction measurements, or from the appearance of large differences betweon top and bottom ral 1 temperature measurements indicating stratified flow, [Cb66a].

\subsection{General Description of Test Rigs Used In This Investigation}

Through the course of this investigation, two experinental rigs were built and atilized. ${ }^{1}$ Both employed a horizontal stainless stoel tube (1ength 2.7 meters; inside diameter .9 cm; out side diameter $.95 \mathrm{~cm}$ ) which was electrically heated.

\footnotetext{
${ }^{1}$ Prel iminary results of tests conducted ith Rig \#l have beon pablishod in [Ra83]. The athor wishes to stress that the construction and original data collection described in [Ra83] was sharod with his advisor for the experimental portion of the report. Since then, several improvements have been made independently in the data roduction techniques as well as analysis, interpretation and presontation.
} 
Rig \#1 is described in Figure 3-2. A semi-hermetic, oil free pump delivered subcooled 1 iquid refrigerant to the test section. Heat was generated in the tube wall by applying a DC voltage difference along the tube. The test section itself was heavily insulated (approximately 15 cm radial thickness) to reduce heat gain from the surroundings; the minimal heat gain was accounted by calibration (described later). The vapor generated in the test section was reliquified in an oversized condenser/receiver. The pump then drew on the liquid reservoir in the condenser and the cycle completed. Inlet subcooling and flow rate were controlled by valves in the 1 iquid 1 ine. Subcooling and pressure level could also be modified by altering the condenser temperature (by changing flowrate or supply temperature on the brine side of the condenser).

Thermocouple stations were located at axial positions shown in Figure 32b. At each station, thermocouples were clamped at $90^{\circ}$ intervals around the outer tube circumference. Instream thermocouples were centered in the flow, extended and pointed upstream for a distance of at least $2 \mathrm{~cm}$, at the single phase 1 iquid inlet and two phase outlet. Pressures were al so measured at these locations but not in the heated section. Flaid temperatures in the heated section were estimated from an assumed pressure drop distribution and thermodynamic equilibrium. Sight glasses located at the test section inlet and outlet allowed visual verification of the flow pattern. Flow rates were determined by means of a calibrated tarbine meter in the subcooled liquid line. 
One of the features of Rig \#1 was the use of a uniform heat flux along a fixed tube 1 ength. With a given heat and mass flox and degree of inlet subcooling, the outlet quality is fixed at:

$$
\mathrm{x}_{\mathrm{out}}=\frac{\Delta \mathrm{h}_{\mathrm{act}}-\Delta \mathrm{h}_{\mathrm{sc}}}{\Delta \mathrm{h}_{\mathrm{v}}}
$$

where

$$
\begin{aligned}
& \Delta \mathrm{h}_{\text {act }}=\frac{\dot{q} D_{\sigma} L}{\dot{m}}=\text { heat added to refrigerant by } D C \text { power supply } \\
& \Delta \mathrm{h}_{s c}=\mathrm{C}_{\mathrm{PL}_{\mathrm{L}}} \Delta \mathrm{T}_{\mathrm{sc}}=\mathrm{C}_{\mathrm{p}_{\mathrm{L}}}\left(\mathrm{T}_{\mathrm{bub}}-\mathrm{T}_{\text {inlet }}\right)=\begin{array}{l}
\text { heat needed to raise sub- } \\
\text { cooled liquid to saturation }
\end{array} \\
& \text {. } \\
& \text { or bubble point }
\end{aligned}
$$

Generally, $\Delta \mathrm{h}_{\mathrm{v}} \gg \Delta \mathrm{h}_{\mathrm{sc}}$, i.e., the liquid is only slightly subcooled, so that:

$$
\mathrm{x}_{\mathrm{out}} \cong\left(\frac{\dot{q}}{\dot{\mathrm{m} \Delta h} \mathrm{v}_{\mathrm{v}}}\right) \mathrm{D}_{\mathrm{oL}}
$$

At a specified heat and mass flax, the outlet quality is then determined by the tube length. To reach high exit qualities with 1 ow heat flux a tube length of more than 20 meters is required in some cases. This length is impractical clearly for experimental purposes.

In order to obtain data over the fall quality range at the required heat and mass fluxes and without using a tube length greater than 5 meters 
(available space), a second experimental rig was constructed. It is displayed in Figure 3-3. The principal change is to employ two distinct heating sections, the first of wich serves as a preheater. The tube itself was continuous, but heated separately by two independent DC power supplies. The first serving as a preneater provides partially evaporated fluid to the new shorter test section. The quality at the test section inlet is:

$$
\mathrm{x}_{\text {in }}=\frac{\Delta \mathrm{h}_{\mathrm{pI}}-\Delta \mathrm{h}_{\mathrm{sc}}}{\Delta \mathrm{h}_{\mathrm{v}}}
$$

where

$$
\begin{aligned}
& \Delta h_{p r}=\frac{q_{p h} D_{0} L_{p h}}{\dot{m}} \\
& L_{p I}=\text { length of preheat section }
\end{aligned}
$$

Upon entering the test section, the fluid is further evaporated by heat provided from a second power supply so that

$$
x_{\text {out }}=z_{\text {in }}+\frac{\Delta h_{T S}}{\Delta h_{v}}
$$

with

$$
\Delta \mathrm{h}_{\mathrm{TS}}=\frac{\dot{\mathrm{q}}_{\mathrm{TS}} \mathrm{D}_{\mathrm{\sigma}} \mathrm{L}_{\mathrm{TS}}}{\dot{\mathrm{m}}}
$$


$\mathrm{L}_{\mathrm{TS}}=$ length of test section

By using a large, at times unrealistic, preheat flux, qualities at the test section inlet could be made greater than with Rig \#1. In the test section itself, lower more reai istic heat fluxes were used to further vaporize the fluid. The test section itself was.6 m in length, so that quality changes across the test section were relatively small. To 'build up' the entire quality range of interest, 205 to $90 \%$ vapor, several tests had to be run at a given mass flux. In each test, the amount of preheat was selected to provide a different inlet quality to the test section. While the use of a preheat section allowed the full quality range to be investigated, the time for data collection was increased greatly (factor 8) over that which would be necessary if a long uniformly heated tube was used. It al so requires much stricter reproducability of flow rates, heat fluxes and composition. Recommendations regarding improved experimental design are made at the end of this report.

While Rig \#2 differed conceptually only in its use of a preheater, several changes were also made in the instrumentation and data reduction techniques. The following sections describe both rigs in great detail as such the discussion is somewhat fragmentary. A sumary of their differences is provided in Table $3-1$.

The sections are organized as follows: 


\subsection{Testing Protocol}

3.4 Measurement and Data Redaction Technique
(a) Wail temperature
(b) Mass flow Iate
(c) Heat flux
(d) Pressure
(e) Instream temperature (inlet-outlet)
(f) Data acquisition system
(g) Sampling for composition
(h) Calculated fluid temperature
(i) Overall logic: data reduction scheme

\subsection{Quality Assurance Tests}
(a) Single phase heating tests
(b) Energy balance
(c) Pressure drop: measured vs. predicted
(d) Reproducability
(e) Effect of Preheater

\subsection{Testing Protocol}

With the uniformly heated test rig, Rig \#1, a series of tests were performed at the following conditions:

\begin{tabular}{|c|c|c|c|}
\hline COMPOSITION & $\begin{array}{c}\mathrm{G}=\dot{\mathrm{m}} / \mathrm{A}_{\mathrm{c}} \\
\left(\mathrm{kg} / \mathrm{m}^{2} / \mathrm{sec}\right)\end{array}$ & $\underset{\left(\mathrm{q} / \mathrm{q} / \mathrm{s}_{2}\right)}{ }$ & $\begin{array}{c}P \\
(b a r)\end{array}$ \\
\hline $\mathrm{R} 152 \mathrm{a}$ & $200-700$ & $10-40$ & $1.2-2.4$ (a) \\
\hline $\mathrm{R} 13 \mathrm{~B} 1$ & $400-1200$ & $20-40$ & $5.7-7$ \\
\hline .833 w R13BI & $200-600$ & $30-40$ & $7-9$ \\
\hline .750 wt $\mathrm{R} 13 \mathrm{Bl}$ & $200-600$ & $30-40$ & $5.7-6.6$ \\
\hline .706 wt R13 B1 & $200-550$ & $30-40$ & $4-7$ \\
\hline .662 wt R13BI & $200-550$ & $30-40$ & $4-7$ \\
\hline .454 wt R13B1 & $150-300$ & $20-40$ & $5-7$ \\
\hline
\end{tabular}

The selected mixtare compositions span those recommended by the refrigerant supplier for use in heat pumps. Most of the mass fluxes were typical of those which might be employed in heat pumps. The heat 
flaxes were representative of those found in evaporators in today's beat pumps. Rather than controlling pressure, outlet temperatures were controlled to be those tyoical in the desired application. Onfortunately, boiling phenomena is al so a complicated function of pressure, so that a strict physical comparison and interpretation of the heat transfer coefficients between pure fluids and mixtures is complicated, if not impossible.

With the second rig, the following conditions were selected:

COMPOSITION

$$
\left(\mathrm{kg} / \mathrm{m}^{\mathrm{G}} / \mathrm{sec}\right)
$$

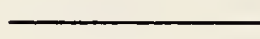

\section{R152a}

R13 B1

.80 wt R13B1

$.58 w \mathrm{t} \mathrm{R} 13 \mathrm{~B} 1$

.37 w $R 13 B 1$

$.18 \times \mathrm{t} \mathrm{R} 13 \mathrm{~B} 1$

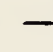

$$
100-300
$$$$
200-500
$$$$
200-500
$$$$
200-500
$$$$
100-400
$$$$
100-300
$$

$$
\dot{\mathrm{q}}_{\mathrm{ph} / \mathrm{Ac}}
$$$$
\left(\mathrm{kW} / \mathrm{m}^{2}\right)
$$

$\dot{q}_{t s / A c}$

$\left(\mathrm{kW} / \mathrm{m}^{2}\right)$

$P_{\text {t s }}$

bar

$10-95$
$10-50$
$10-70$
$10-70$
$10-80$
$10-90$

$10-20$
10
10
$20-30$
10
10

$4.70-4.80^{\mathrm{a}}$

$4.70-4.80$

$4.70-4.80$

$4 \cdot 70-4 \cdot 80^{\mathrm{a}}$

$4.70-4.80$

$4.70-4.80$

a: isolated tests at other pressures

In all Rig \#2 cases, pressure was controlled at a fired level typical of that which might be found with mixtures in a beat pamp. This experimental pressure was selected based on the range of condenser temperatures which could be provided by the brine system.

\subsection{Measurement and Data Reduction Techniques}

Heat transfer coefficients are calculated from: 


$$
a=\frac{\dot{q} / A_{s}}{T_{w}-T_{f}}
$$

The measurement of these and other critical variables are discussed in de tail below.

\section{4.a) Wall temperature, $T_{W}$}

Wall temperature measurements were made with $0.25 \mathrm{~mm}$ thermocouples (T/C) which had been silver soldered and flattened to provide good thermal contact. Silver soldering was selected so that the T/C junction would survive dryout accidents. The T/C leads were arranged as shown in Figure 3-4 to reduce any 'fin' effect conduction gains. The T/C junction and wires were isolated electrically from the tube by a very thin layer of teflon tape ( $<.01 \mathrm{~mm})$. Good thermal contact was maintained by cl amping the thermocouple to the tube (see Figure 3-5). Inside wall temperatures were calcalated from the measured outside temperatures by use of the steady-state radial, one dimensional ( 1 - D) conduction equation with uniform heat generation and assuming adiabatic conditions on the outside of the tube.

With Rig \#1, wall temperature differences were in fact measured. Specifically the difference betweer the instream outlet temperature and the local wall temperature were used; this temperature difference itself was referenced to a thermocouple submerged in a slush ice bath--see Figure 3-6. The use of temperature differences is generally considered more accurate than absolute temperature measurements. However, the 
potential stability of the measured outlet temperature may be questioned. Entrained droplets strike and depart this instream thermocouple so that the $T / C$ sees alternately vapor and droplet temperatures and may read something in between depending on its response time. In the case of pure fluids the measured outlet temperature was stable. This stability results from the fact that saturation temperatures change relatively little in the axial direction in the absence of pressure drop, no change would occur. Droplets and vapor tend to be near this saturation temperature. In the case of mixtures, however, entrained droplets may have a much different temperature since the bubble point temperature varies axially since evaporation is non-isothermal even in the absence of pressure drop. Thus, temperature fluctuations in outlet T/C measurements were greater for mixtures than for pure fluids, and. wall temperature measurements which were referenced to the outlet $\mathrm{T} / \mathrm{C}$ Ieflect the se fluctuations.

One other potential difficulty with the Rig \#1 wall temperatures is the analog-to-digital ' $\mathrm{mV}$ to ${ }^{\circ} \mathrm{C}$ ' conversion. The usual industry technique is to utilize the curvefit formulas of NBS monograph 125 [NB]; the published errors in the se formulae is $\pm .3^{\circ} \mathrm{C}$. Near room temperature, where most of the Rig \#1 data was taken, the error is in fact much smaller $\left(<.1^{\circ} \mathrm{C}\right)$. However, at colder conditions $\left(-20^{\circ} \mathrm{C}\right)$, the error can in fact be $0.6^{\circ} \mathrm{C}$.

Rig \#2 employed a different measurement and data reduction technique for wall temperatures. Each wall thermocouple was referenced strictly to an electronic ice bath temperature (precision of electronic ice bath $\pm .02^{\circ} \mathrm{C}$ 
typically, \pm .05 maximum). In this way, the wall temperatures were freed from instream fluctuations. Secondly, the use of an electronic ice bath reduced uncertainties regarding the quality of the slush ice bath. The use of more closely spaced test section therwocouple stations (see Figure 3-3b) provided redundance to verify the goodness of the measured data. Finally, data was reduced with a more accurate curvefit to the ' $m V$ to ${ }^{\circ} \mathrm{C}$ ' data of the NBS Monograph; the 4 th order curvefit generated by the author has a maximum error of $\pm .01^{\circ} \mathrm{C}$ over the $100^{\circ} \mathrm{C}$ temperature range of interest.

The higher precision of the data collected with Rig \#2 was necessary because of the 1 owe heat fluxes employed in the test section of Rig \#2. The data of Rig \#1 is still of satisfactory quality.

Corrections for heat gain from the room and for the teflon tape Iesistance and any contact resistances were made by the following proceaure. Single phase 1 iquid tests were run at high flow rate and zero heat input. Under these conditions, the fluid inlet-outlet temperatures rise was very small $\left(.2^{\circ} \mathrm{C}\right)$. If there was no heat gain from the room and if the $\mathrm{T} / \mathrm{C}^{\prime} \mathrm{s}$ were in perfect contact with the fluid, the wall $\mathrm{T} / \mathrm{C}$ readings would match exactly the local fluid temperature. These single phase tests were run at various fluid temperatures covering the range used in later tests. The $\Delta T$ between the fluid and room ranged from 0 to $50^{\circ} \mathrm{C}$ ). At the maximum $\Delta T$ between filuid and room a heat gain of about $200 \mathrm{w} / \mathrm{m}^{2}$ was measured. This can be compared to the lowest heat flux employed of $10,000 \mathrm{w} / \mathrm{m}^{2}$. Based on the measured $\Delta \mathrm{T}$ of an actual boiling 
test, this heat gain was subtracted away from the measured wall temperatures to give the value of $T_{w}$ used in equation $(3-1)$.

\section{b) Mass flow rate}

Though mass flow rate is not necessary to determine local $a$, it is required to calculate local equilibrium quality. In order to determine mass flow rate, a turbine meter was used in the subcooled 1 iquid 1 ine about 50 diameters upstream of the test section. With Rig \#1 the signal was sent to a pulse counter which took readings once per second. With the second rig, the signal was sent additionally to a counter integral to the data acquisition system. The two counters agreed to within $1 \%$ over the range of interest.

The turjine meter/pul se counters were calibrated with water at near room temperature (scale and electronic stopwatch technique). Temperature corrections were not made, as they are very minor $(<<15)$ [Ma83]. The turbine meter response is also flat over a wide range of Reynolds num bers so that a viscosity correction was unnecessary. It was discovered from energy balance tests that near the upper end of its rated flow Iate the spinning rate of the tarbine meter was less than expected. Measurements in this flow rate range were avoided.

Calibration of the meter was made before the Rig \#1 tests and before the Rig \#2 tests (approximately 1 year apart). The change in calibration was less than 1\%, the estimated accuracy of the measurement. 
Finally with Rig \#1, a rotameter was installed to provide visual confirmation of flow rate, as well as redundancy in measuremert. It fias removed from Rig \#2 after it developed an internal leak whict nas difficult to repair.

\section{c) Heat flux}

Heat was generated by DC power supplies as noted previously:

- Rig \#1 and Preheat Section of Rig \#2: Dynapower Corporation (0-60v, 0-300a)

- Rig \#2 Test Section: Rapid Electric Company (0-10v, 0-150a)

The DC power supplies were al so checked for the addition of AC ripple current. Though the Dynapower Corporation claimed the ripple to be $<5 \%$ at full load, its value was found to be $50 \%$ at part 1 oad. A bank of capacitors was used to reduce its ripple to the point that the $\mathrm{AC}$ contribution to the total heat flux was less than $1 \%$ [Ra83]. The integrity of the capacitors was checked throughout the measurement program. AC rippled with the Rapid Electric Company power supply was measured to be less than $1 \%$.

Heat flux measurements (i.e., electrical input) were made with digital voltmeter integral to the data acquisition systems. Voltage drop across the test section and preheat section was measured at the busbars. Current was calculated by measuring the voltage drop across a calibrated resistor in series with the test section. Early in the testing program, the electrical resistance of the stainless steel tube was checked and its resistance determined to be 1 inear with distance suggesting the 
absence of local hot spots. The variation of electrical resistivity with temperature as calculated to be 1 ess than $1 \%$, the estimated accuracy of the heat flux measurement [Ra83].

\section{d) Loca1 pressure}

Local pressure values are needed to estimate the local fluid temperature of equation $(3-1)$. In studies reported in the 1 iterature, it has been common to place pressure taps near thermocouple stations so that in the vicinity of the wall temperature measurements, one could calculate the local fluid saturation temperature. A decision was made to avoid this approach for the following reasons: (a) the stainless steel tube was thin and pressure taps might have intruded into the flow stream: (b) the only means to fị the pressure tap "leads would be șoldering; the solder would have provided a preferential electrical path (a local shunt) creating a local irregularity in heat flux; (c) pressure drops were relatively sma11, and errors would be small in estimating 1 ocal pressure from overall measured val ues of pressure.

To the anthor's knowledge, no determination of the effect of pressure taps on measured heat transfer coefficients has appeared in the literature. As such, an experiment was devised to make such a determination. A test was run with refrigerant R22 as the evaporating f1uid. Pressure was measured at the inlet and outlet, with boiling taking place very near the inlet. A linear pressure drop was assumed to occur between the location of $z=0$ (saturation boiling point location) and the outlet. The test was repeated twice with the local a varying about $1 \%$. 
Following these tests, holes were drilled and hypodermic needles silver soldered in place about $5 \mathrm{~cm}$ dowstream of each thermocouple station (see Figure 3-7); the opening at the needle top was sealed with epoxy. The needles served to simulate small pressure taps. The R22 test was then repeated with identical conditions and data reduction. The results of the comparison are shown in Table 3-2; in general the introduction of the pressure taps reduced the $a$ by $10 \%$. No change in pressure or heat flux was measured. The a result was surprising in that if any change occurred it was anticipated that an increased $\alpha$ would be observed due to increased nucleation at the tap locations or increased film turbulence due to tap intrusion. Instead, the reduction might be explained either by: the needles served as fins to add heat from the room, causing the wall thermocouples to read somewhat higher than anticipated; or the pressure tap intrusion, if it extended into the flow, increased the upstream film thickness sightly. The solder may also offer 1 ower electrical resistance, so that the heat flur in the other parts of the tube might have been slightly raised. It is not clear which, if any, of the se mechanisms caused the observed change, but the change is apparent. In any case, further tests were made without this type of experimental arrangement.

Figure 3-2b shows the means by which pressure was measured for Rig \#1. At the outlet of the test section a tap was made through the busbar by spark erosion so that the hole would be exceptionally smooth. A calibrated pressure transducer and gauge were connected. At the test section inlet, a similar tap was made and a pressure gauge installed. 
Inlet and outlet differential pressure was also measured both with a . 139 bar (2 psi) pressure transducer [Sensotec] and a .6895 bar (10 psi) gauge. The absolute pressure devices were calibrated with a dead load tester; the size of the pressure gauges however prevented readings from being more precise than \pm .0689 bar $( \pm 1$ psi). The outlet pressure which was used in the data reduction was that of the pressure transducer accurate to .006895 bar ( $\pm .1 \mathrm{psi}$ ).

The . 139 bar ( 2 psi) differential pressure transducer unfortunately had insufficient range. The .6895 bar (10 psi) differential pressure gauge had sufficient range but could be read. to only .017 bar $( \pm .25$ psi) at 1 P $>139$ bar (2 psi). A decision was made in the original data reduction [Ra83] to use the differential pressure gauge results in all cases. The inlet pressure was then calculated as

$$
P_{\text {in }}=P_{\text {out }}(T D)+\Delta P(\text { GAUGE })
$$

where the designations (TD) and (GAUGE) represent measurements by the transducer and gauge, respectively.

For Rig \#2, pressures were measured as shown in Figure 3-3b. Pressure transducers were employed at the preheat inlet and test section outlet. Differential pressure transducers were used across the test section with a range $=.139$ bar $(2$ psi)) and across the whole tube with a range $=.345$ bar ( 5 psi). While the pressure tap at the test section inlet may be questioned due to the $R 22$ test results described above, care was 
taken to reduce its potential impact. First, the hole was made by spark erosion so that there would be no intrusion into the flow. Secondly, the tap was located in the busbar so that there was no problem with electrical shunting. Thirdly, no thermocouple stations were placed in the immediate area of the pressure tap. For Rig \#2, pressures were then obtained from

$$
\begin{aligned}
& \left(\text { Preheat) } P_{\text {in }}=P_{\text {out }}(T D)+\Delta P_{5}(T D)\right. \\
& \text { (Test Section) } P_{\text {in }}=P_{\text {out }}(T D)+\Delta P_{2}(T D)
\end{aligned}
$$

where $\Delta \mathrm{P}_{5}$ (TD) and $\Delta \mathrm{P}_{2}$ (TD) represent the measurements by the .345 bar ( 5 psi) and . 139 bar (2 psi) differential pressure transducer.

The differential pressure transducers were calibrated in an upward and dow nward traverse bellowmeter and a mercury manometer. Unfortunately, this could only be done at atmospheric pressure. Any errors which might develop by the use of the transducers at higher absolute pressure could not be quantified. Estimated accuracy is $+/-.005$ bar.

Given these overall pressure drops, local pressure values, i.e., the pressures at the thermocouple stations need still to be estimated. In [Ra83], the estimation was done by: (a) neglecting the single phase pressure drop in the region between the subcooled inlet and the saturated boiling point location (BPL), i.e., the position where $x=0$; and (b) assuming a 1 inear pressure drop betwen the BPL and the outlet. 
The implications of the latter, more critical assumption can be examined by the following study.

Figure 3-8 displays the results of applying a widely recommended pressure drop correlation, that of Martinelli-Nelson modified by Chisholm [Ma48,Ch67a], to the typical flow conditions employed in Rig \#2. The correlation requires a numerical integration, and steps of $\Delta x=3 \%$ in the preheat section and $\Delta x<1 \%$ in the test section were taken. The BPL was determined by an energy balance, and is denoted in the Figure by an arrow. The series of curves represent different levels of preheat flux, i.e., different overall $\Delta x^{\prime} s$ for a given flow rate. In all cases the test section flux was fixed at $10 \mathrm{~kW} / \mathrm{m}^{2}$. It can be seen that as the change in quality between inlet and outlet increases, the preheat section pressure drop becomes sharply non-1inear. However, in the test section, with the relatively small $\Delta x$, the pressure drop is nearly linear. These results imply that the assumption of a linear pressure drop in the test section for Rig \#2 would be quite valid, but the same assumption in the preheat section at large $\Delta x$ is somewhat questionable. The linear pressure drop assumption in [Ra83] then may be questionable since (1) flow rates were bigh increasing the non-1inearity and (2) the distance between pressure taps was larger in Rig \#l than with Rig \#2.

These errors are balanced by the neglect of single phase pressure drop and by the fact that at large flow rates x tended to te small with Rig $\# 1$. 
In order to quantify these 1 inear pressure drop errors a sensitivity analysis was run with varying pressure drops. The linear pressure drop assumption typically caused about a 5\% error in a for Rig \#1; errors could be as high as $15 \%$. The error in preheat a of Rig \#2 would be roughly $1 / 5$ smaller, since the Rig \#2 preheater is $20 \%$ shorter than the Rig \#1 test section.

A few alternatives are available to the linear pressure drop assumption. The pressure drop correlation could be used to predict local pressure values. This procedure however requires numerical integration, increasing computation time dramatically in the case of mistures where an iterative scheme is required because of the nature of the EOS code. It al so requires normalization of the results to the measured pressure drops. A more convenient approach, and the one used in the final data reduction of Rig \#1, was to weight the 1 inear pressure drop assumption depending on the overall $\mathbf{x}$ :

$$
\begin{aligned}
& \text { For } \mathrm{x}_{\text {out }}<.4 \quad \Delta \mathrm{P}=.85 \Delta \mathrm{P}_{\mathrm{LIN}} \quad 0<\mathrm{z}<\mathrm{x}_{\mathrm{out}} / 2 \quad(3-8 \mathrm{a}) \\
& =P_{\text {out }}-P_{\text {zout } / 2} \quad x_{\text {out } / 2} \leq x<x_{\text {out }} \\
& .4 \leq \mathrm{x}_{\text {out }}<1 \quad \Delta \mathrm{P}=.7 \Delta \mathrm{P}_{\mathrm{LIN}} \quad 0<\mathrm{x}<\mathrm{x}_{\text {out }} / 2 \quad(3-8 \mathrm{~b}) \\
& =P_{\text {out }}-P_{\text {zout } / 2} \mathbf{x}_{\text {out }} / 2 \leq x<x_{\text {out }}
\end{aligned}
$$

where $\Delta \mathrm{P}_{\mathrm{LIN}}=$ the pressure drop predicted by assuming a linear relation between BPL and the outlet

$$
\mathbf{x}_{\text {out }}=\text { the outlet quality }
$$

$\mathbf{x}_{\text {out } / 2}=$ the outlet quality divided by 2 . 
The effect of this approach is to fit the curves on Figure $3-8$ in a piecewise 1 inear fashion.

While not ezact, the pressure drop is corrected in the proper direction and reduces the overall error. The same approach was used in the preheat section of Rig \#2; the direct 1 inear pressure drop assumption was retained in the test section of Rig \#2, since errors were negligible.

\section{e) Instream Temperature (Inlet-Outlet)}

With Rig \#1, unshielded thermocouples were installed in the flow stream and supported by a brass collar (see Figure 3-6). The hole made by the thermocouple was sealed with epoxy. This technique provided an accurate measurement of the instream temperature however, the epory seal tended to fail over time, causing 1 eaks and refrigerant $10 \mathrm{ss}$ (the latter being particularly critical with mixtures since one component is lost preferential1y).

With Rig \#2, shielded thermocouples were soft-soldered in place (see Figure 3-6b). These thermocouples eliminated the leak problem but provide poorer contact with the flow stream. They al so tended to fail inexplicably, therefore neither approach was particularly advantageous.

An attempt was made to insert an instream $T / C$ at the test section inlet. This thermocouple would have provided information about the true temperature rise across the test section. The thermocouple, unfortunately. affected both the single phase and two phase wall thermocouple readings. 
Figure 3-9a shows the a calculated for single phase 1 iquid heating R22 tests. The thermocouple was inserted into the flow stream nea bottom. As can be seen, a wide circumferential scatter is observed. Figure 3-9b shows a similar test with the thermocouple removed. A series of tests were al so conducted under flow boiling conditions. Figure 3-10 shows the results of these tests with and without the thermocouple in place. Again, data scatter is reduced without the thermocouple. In order to preserve the purity of the wall temperature measurements, the Rig \#2 tests were run without the instream thermocouple at the test section inlet.

This finding of the effect of instream thermocouples may be significant. The literature abounds with test rigs employing instream $T / C^{\prime} s$; it has . been assumed of ten that the large turbulence in the flow is sufficient to overwhelm any disturbance introduced by the disturbance of the ther mocouple. While this may be true with large tube diameters and smaller instream thermocouples, it was not the case here.

\section{f) Data acquisition system}

Data collection for both rigs involved the use of a Hewlett-Packard (HP) Series 80 computer connected to a data acquisition system (Rig \#1: F1uke and Rig \#2: HP $3497 \mathrm{a}$ ). Automatic scanning of all thermocouples, pressure transducers and flow meters was done. Data was saved when steady-state was reached, i.e., when instream temperatures and pressures variation dissipated. This requirements was satisfied typically one hour after a change in mass or heat flux was made. With the second rig, 
pressure control frequently added even more time between tests. Hith Rig \#1, the result of 10 scans which took place at 1 minute intervals were stored. With Rig \#2, seven to fifteen scans separated by 40 second intervals were stored. These results were later averaged, their standard deviation determined, and used in further data reduction.

\section{g) Sampl ing}

Mixture composition was determined by withdrawing a liquid sample, expanding it to a complete vapor state and analyzing the vapor sample by gas chromatography. Sample bottles are shown in Figure 3-11. The addition of a pressure gauge to the sample bottle helped determine if the sample had been completely vaporized in the expansion process (one could check the measured pressure at room temperature to see if the sample was well into the vapor region as predicted by the equation of state).

However, sampling techniques associated in Rig \#2 introduced unnecessary error. In what was thought an improvement, the sampling 1 ines were purged of air before taking a final sample. This was accomplished as discussed in Figure 3-11; some vapor may have been trapped in the process. This vapor, preferentially of the more volatile component, may have caused variation in the results. Later sampling done without air purging proved very reproducible. Sampling errors on the order of 0.55 could have been avoided. On the other hand, the technique was able to show that virtually no air had dissolved in the refrigerant based on the GC analysis. 


\section{h) Calculated fluid temperatures}

The only quantity missing in the determination of the $a$ is the fluid temperature. This bad to be estimated for reasons cited above.

For pure refrigerants the saturation temperature of each thermocouple station was calculated from property tables, given the local pressure as found by the procedures previously used. Sometimes researchers Ieport their results based on $T_{f}=T_{s a t}(P)$; other times it is based on $T_{f}=T_{\text {meas }}=T_{\text {bulk }}$. In this report, all values are reported on the basis of $T_{f}=T_{\text {sat }}(P)$.

For mixtures, the standard approach in the literature is to base $T_{f}$ in equation (3-1) on $T_{\text {eq }}$, the thermodynamic equilibrium temperature. The equilibrium temperature was calculated from the equation of state since at each thermocouple station, pressure, enthalpy and original overall composition are known.

i) Overall data reduction scheme

The above detailed discussion is necessarily fragmentary. Figure 3-12 is a flow chart of the data reduction scheme. As the figures are for the most part self-explanatory, only a few comments are provided here, principally on the mixtures' algorithms. All data reduction was performed on HP series 80 computers. The Equation of State (EOS) necessary for the misture work, is fifth order in nature with several internal iterative loops. The code was developed, by the EOS author [Mo82], 
and used here without change. 1 It requires as input $T, P$ and overall molar composition $\left(\overline{\mathrm{X}}_{\mathrm{O}}\right)$ and outputs overall $\overline{\mathrm{h}}, \overline{\mathrm{v}}, \overline{\mathrm{s}}, \overline{\mathrm{X}}, \overline{\mathrm{Y}}, \overline{\mathrm{C}}_{\mathrm{P}_{\mathrm{L}}}$ and $\overline{\mathrm{C}}_{\mathrm{p}_{\mathrm{Y}}}$ on a molar basis. Rutime for the equation of state alone is 1-2 minutes on the HP Series 80 computer. As seen in Figure 3-12, two iterative loops involve the equation of state. The first is to determine the BPL; a bisection search method was used to determine a two phase quality very close to zero. Closure was reached typically in 6 iterations. The second iterative loop involved calculating the equilibrium temperature at each $T / C$ station. There a secant method was used to reduce the number of iterations to $3-5$.

Overall the program took 30-45 minutes to reduce the data from a single test, calculate the local $a^{\prime} s$, and print the results in tabular and graphical form. By contrast, the data reduction for pure fluids took 510 minutes on the Series 80 computer, most of which was for printing time.

Typical outputs of each run are shown in Figure 3-14; a complete set is available upon request. Appendix $3 \mathrm{~A}$ contains a sumary of the measured results which can be used by other researchers.

${ }_{1}$ The code results were compared by the author of this report with a
separate code developed by [Mc85] with virtually identical results. 


\subsection{Quality Assurance}

\section{5.a) Single phase heating tests}

In order to verify temperature measurements, several single phase 1 iquid heating tests were made. The results of those with Rig \#1 are shown on Figures 3-13a and 3-13b. The measured val aes kave been compared with the well-known equation: 1

$$
a_{L}=.023 \frac{\lambda_{L}}{D}\left(\operatorname{Re}_{L}\right)^{0.8}\left(\operatorname{Pr}_{L}\right)^{0.4}
$$

as well as the more accurate equation [Pe70]:

$$
\begin{aligned}
& a_{L}=\frac{(f / 8) \operatorname{Re}_{L} \operatorname{Pr}_{L}}{k_{1}+k_{2} \operatorname{Pr}_{L}(f / 8) 1 / 2\left(\operatorname{Pr}_{L}^{2 / 3}-I\right.} \\
& \text { with } \quad \begin{array}{l}
f=\left(1.8510 g_{10} \operatorname{Re}_{L}-1.64\right)^{-2} \\
k_{1}=1+3.4 f \\
k_{2}=11.7+1.8 \operatorname{Pr}_{L}^{1 / 3}
\end{array}
\end{aligned}
$$

All tests show good agreement. These tests were for Rig \#1; a few tests with Rig \#2 and R22 or R152a showed similar agreement.

b) Energy Balance

In order to assure that the instrumentation was behaving correctly, an energy balance was made between fluid temperature rise and energy input. Specifically, a comparison was made be tween

\footnotetext{
${ }^{1}$ This equation has been credited variously in the literature to Dittus and Boelter, McAdams, Colburn, or Kraubold (F.R.G.). The author is not sure where credit belongs but will refer to it in this paper as DittusBoelter equation.
} 


$$
\Delta E_{f}=\dot{m} C_{p_{L}}\left(T_{\text {out }}-T_{i n}\right)=\text { energy gained by the fluid }
$$

$\Delta E=\dot{q} \pi D L=$ electrical heat input

The quantity $\left|\frac{\Delta E_{f}-\Delta E}{\Delta E}\right|$ was less than $7 \%$ with single phase heating R22 tests with Rig \#1 and 1 ess than 5\% with R22 and R152a tests with Rig

\#2. Energy balances quoted in the literature by other researchers are:

\section{REFERENCE}

A160

An 67

Ch66 a

\section{ENERGY BALANCE}

$\pm 5 \%$

$\pm 10 \%$

$\pm 2 \sigma_{0}^{1}$

In the process of these tests, the [As81] value for $\mathrm{Cp}_{\mathrm{L}}$ of $\mathrm{R} 152 \mathrm{a}$ was shown to be in error by $15 \%$. Independent work by [Mo85] later confirmed this finding.

\section{c) Pressure drop}

The most widely recommended two phase pressure drop correlation at low pressures is that of Martinelli-Nelson [Ma48], with various autbors suggesting modifications [Ch67a, Hs76]. In their original development, Martinelli and cow orkers used aimensional analysis and a large data base to predict isothermal twocomponent (e.g., air-water) pressure drop.

\footnotetext{
${ }^{1}$ In [Ch66a], measured outlet $\mathrm{T}$ and calculated outlet $\mathrm{T}$ based on pressure measurements disagreed, however.
} 
Later Martinel $1 \mathrm{i}$ and Nel son extended the approach to evaporating steamwater systems. Refrigerants were not included in the original Martinelii efforts. A literature search of the application of the technique to refrigerants revealed the following:

$\begin{array}{llr}\text { REFERENCE } & \text { REF. } & \left(\Delta P-\Delta P_{\text {exp }}\right) / \Delta P_{\text {exp }} \\ \text { An67 } & \text { R11 } & +10-30 \% \\ \text { Cb66 a } & \text { R12 } & -30-50 \% \\ \text { Si83 } & \text { R12 } & +50 /-20 \% \\ \text { A8 82 } & \text { R12 } & +1-25 \% \\ \text { A160 } & R 22 & 0-20 \% \\ \text { An67 } & R 22 & -20 \%\end{array}$

In horizontal evaporating $f 10 w$, the pressure drop is composed of two terims,

$$
\Delta P=\Delta P_{f}+\Delta P_{a}
$$

Where

$$
\begin{aligned}
& \Delta P_{f}=\text { pressure drop due to friction } \\
& \Delta P_{a}=\text { pressure drop due to flom acceleration }
\end{aligned}
$$

Martinelli and conorkers developed an empirical procedure to predict the frictional pressure drop. 


$$
\Delta P_{f}=\Delta P_{L} \emptyset_{L}^{2}
$$

where

$$
\begin{aligned}
\theta_{L}^{2} & =1+\frac{C}{X_{t t}}+\frac{1}{X_{t t}^{2}} \\
X_{t t} & =\left(\frac{1-z}{x}\right)^{.9}\left(\frac{\rho_{\mathrm{V}}}{\rho_{L}}\right)^{.5}\left(\frac{\mu_{L}}{\mu_{v}}\right)^{1} \\
\Delta P_{L} & =\begin{array}{l}
\text { Pressure drop, as calculated for single phase 1iquid flow, } \\
\text { for that portion of the f1ow which is 1iquid. }
\end{array} \\
& =\frac{f}{2} \frac{L}{D} \frac{G^{2}(1-x)^{2}}{\rho_{L}} \\
L & =1 \text { length of tube }
\end{aligned}
$$

and

$$
\mathrm{C}=\left(\frac{\rho_{\mathrm{L}}}{\rho_{\mathrm{V}}}\right)^{1 / 2}+\left(\frac{\rho_{\mathrm{V}}}{\rho_{\mathrm{L}}}\right)^{1 / 2} \quad \mathrm{Ch} \text { isholm }[\mathrm{Ch} 65]^{1}
$$

The pressure drop due to acceleration can be derived from momentum considerations to yield

$$
\Delta P_{a}=G\left(\frac{x^{2} v_{v}}{\varepsilon}+\frac{(1-x)^{2} v_{L}}{1-\varepsilon}\right)
$$

${ }^{1}$ An alternate procedure which has been suggested is to calculate a 'property index' $\left(\mu_{L} / \mu_{v}\right) \cdot 2\left(\rho_{v} / \rho_{L}\right)$, for the fluid of interest. Then find the water pressare which gives the same value of the inder and use the water/steam densities in (3-11d). For the refrigerants used here, very nearly the same results occur. 
assuming a quality of zero at the inlet.

To apply equations $(3-10),(3-11)$ and $(3-12)$ correctly, a stepwise integration must be performed. This requirement derives from the fact that $x$ changes as the flow proceeds downstream. The frictional pressure drop is a fairly strong function of quality (as was shown on Figure 38). At each step, the inlet, mean, and outlet qualities were calcu1ated. Void fractions were estimated from Martinel1i-Nelson [Ma48]. Equation (3-11) was applied at the mean quality equation (3-12) was applied at the step's outlet and inlet qualities, and the results subtracted:

$$
\Delta \mathrm{Pa}_{\mathrm{j}}=\Delta \mathrm{Pa}_{\mathrm{j} \text { out }}-\Delta \mathrm{Pa}_{\mathrm{j} \text { in }}
$$

The total pressure drop was the sum of the individual steps. The predicted results were compared with the pressure drop measurements of Rig \#2 for al1 pure f1uid tests. Figure 3-16 shows the results of a comparison between measurement and prediction. Results were considered quite satisfactory.

d) Preheater effect

A preheat section is commonly used to help set a desired quality of the f1 uid under investigation independent of the heat flux within the test section. 
Consequently, in most cases the fluid experiences an abrupt change in heat flux upon leaving the preheater and entering the test section, which may affect the entrainment and therefore the wall temperature readings. The influence of sudden change in heat flux at wall thermocouples was tested at higl flow rates, high qualities and low test section heat fluxes. For one set of experiments with pure R152a, no heat was supplied to the test section, while the beat flux of the preheater was changed between $30 \mathrm{~kW} / \mathrm{m}^{2}$ and $90 \mathrm{~kW} / \mathrm{m}^{2}$. For a mass flow rate of about $400 \mathrm{~kg} / \mathrm{sqm} / \mathrm{s}$, no significant change was found in readings of the test section wall temperatures. Nevertheless, all wall temperatures were an average of $0.25 \mathrm{~K}$ higher than the saturation temperature of the fluid calculated from the pressure drop; the value was the same for all thermocouples at a particular station. The data shown in figure 3-17 are for a thermocouple group which is 50 diameters downstream of the preheater. The fact that the wall temperatures are slightly higher than the saturation value, can be explained by the superheated liquid leaving the preheater, which is significantly but not completely cooled by further evaporation driven by both the existing superheat and the pressure drop.

The test with $75 \mathrm{~kW} / \mathrm{m}^{2}$ preheater heat flux was repeated with mass flow rates 1 ower and higher than the one previously chosen. The deviation of the wall temperature readings becomes smaller with bigher flow rates. This behavior might be expected because ith increasing flow rates turbulence and the pressure drop increases causing higher evaporation 
rates and a faster decrease of the superheat available in the liquid phase.

A second set of experiments was conducted with a 0.37 wt 13 B1 mixture. A fixed heat flux in the test section and a fixed mass flow rate was maintained but heat flux was varied in the preheater. At the same time, the degree of subcooling of the fluid entering the preheater was changed. Therefore, when a large degree of subcooling was set, a large amount of preheat flux was required in order to obtain a given quality at the preheater outlet. Figure 3-18 shows the results of these tests. The heat transfer coefficient measured with the test section is obviously a function of the quality, but not of the amount of preheat used. Lower case letters in Figure 3-18 refer to tests where the 1 iquid was subcooled by $5 K$, while the upper case letters refer to tests with a considerably more subcooled 1 iquid $\left(25^{\circ} \mathrm{K}\right)$ entering the preheater. The difference in preheat fluses in order to obtain comparable qualities is between 10 to $20 \mathrm{~kW} / \mathrm{m}^{2}$ or 20 to 30 percent.

e) Reproducibility

Reproducibility of two phase flow results is rarely discussed in the literature. In the case of refrigerants, only two values could be found: $\pm 4 \%[\mathrm{Sa} 61]$ and $\pm 10 \%$ [A160].

Some Rig \#1 tests were repeated at various points in the day and sometimes from day to day. Agreement of Rig \#1 heat transfer coefficients was within $\pm 2 \%$ for tests done one day apart and $\pm 5 \%$ for tests done one 
week apart. Eighteen tests with pure R152a were repeated approximately 3 weeks apart for Rig \#2. Preheat measured values were compared. In the original tests, test section heat flures were $10 \mathrm{~kW} / \mathrm{m}^{2}$; ir the later tests, the test section was set at $20 \mathrm{~kW} / \mathrm{m}^{2}$. Of the 54 data points, all agreed within $8 \%$; most agreed much closer (about $4-5 \%$ ). Most of the variation with Rig \#2 was due to differences in mass flux. A few checks were made with mixtures, with similar findings.

\section{$3.6 \quad$ Problems}

Three problems occurred during the data collection with Rig \#2. First, the measured and calculated temperatures at the outlet for one set of tests with R13B1 disagreed by about $1^{\circ} \mathrm{K}$ (at high flow rate, high quality). This set of data was not used in the further data analysis. ${ }^{1}$

Second, the electrical isolation failed during some of the misture tests at the top thermocouples in each of the preheat thermocouple stations. At the time, the preheat data was considered superfluous and repairs were not made in order to continue test section data collection. Later it was realized that the preheat data was a substantial resource. This data is included here by averaging only the side and bottam stations. The estimated effect of excluding the top station from the average is to increase the mixture's $100 a 1$ a by $5 \%$. The dependence of heat transfer on circumferential location is discussed in Chapter 5. The third problem was the failure of the electric icepoint reference for the last

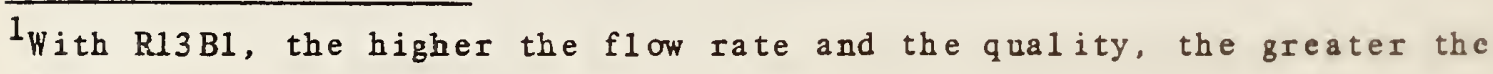
error. 
series of tests, those with 0.58 wt 13B1. To bypass this problem, the data acquisition system was used directly with internal (softhare) temperature compensation. For the tests the uncertainty in temperature is $\pm 0.1^{\circ} \mathrm{K}$, according to Hewlett Packard specifications. As such, test section heat fluxes were maintained at higher values for the se tests to reduce the overall uncertainty in the heat transfer coefficient.

\subsection{Summary of Experimental Data}

A total of 1459 data points were collected with R152a/R13B1 in the fol1owing proportions: R152a:409, R13B1:170, Mixtures:880. The data are tabulated with relevant variables in Appendix $3 \mathrm{~A}$.

Approximately $20 \%$ of the data is not in the annular flow regime and is not included in further analysis.

The tested range of relevant variables includes:

Heat F1 ux: $10-95 \mathrm{~kW} / \mathrm{m}^{2}$

Mass Flux: $\sim 150-1200 \mathrm{~kg} / \mathrm{m}^{2} / \mathrm{sec}$

Composition: $0.0-1.0$, several intermediate values

Pressure: $1.7-9.0$ bar

Quality: $0.0-1.0$

Martinelli Parameter $\left(1 / x_{t}\right): 0.3-35$

Subcooled Liquid Reynolds Number: 3000-50000

Prandt 1 Number: $\quad$ 3-4

Al1 parameters, except Prandtl Number, varied by nearly an order of magnitude. The data base then provides a strong basis for analysis of physical phenomena as well as the heat transfer coefficient. 
Table 3-1a: Hardware Differences Between Rig \#1 and Rig

\begin{tabular}{|c|c|c|}
\hline & Rig \#1 & Rig \#2 \\
\hline $\mathrm{T}_{\mathrm{w}}$ & o $\Delta T$ between $T_{W}$ and $T_{f}$ & $\begin{array}{l}\text { - individual } \mathrm{T} / \mathrm{C} \text { with } \\
\text { redundant stations }\end{array}$ \\
\hline$T_{\text {reference }}$ & - slush ice bath & o electronic ice point \\
\hline$P_{\text {out }}$ & - $P_{T D}$ and $P_{G A U G E}$ & o same \\
\hline$P_{\text {in }}$ & $\circ \mathrm{P}_{\mathrm{GAUGE}}$ & - $P_{T D}$ and $P_{G A U G E}$ \\
\hline$\Delta P_{\text {tot }}$ & - $\Delta P(2 \mathrm{psi})$ and $\mathrm{P}_{\mathrm{GAUGE}}$ & $\begin{array}{l}\text { - } \Delta \mathrm{P}(5 \mathrm{psi}) \Delta \mathrm{P}(2 \mathrm{psi}) \\
\text { and } \Delta \mathrm{P}_{\mathrm{GAUGE}}\end{array}$ \\
\hline Heat Flux & 0 uniform heat & $\begin{array}{l}\text { - preheater and test sec- } \\
\text { tion heater }\end{array}$ \\
\hline$T_{f}$ & $\begin{array}{l}\text { - unshielded, inlet and } \\
\text { outlet }\end{array}$ & $\begin{array}{l}\text { - shielded, inlet and } \\
\text { outlet }\end{array}$ \\
\hline D. A. S. & О Fluke - НP85 & О НР3497 - НР86B \\
\hline Sampl ing Technique & $\begin{array}{l}\text { o no air purge single } \\
\text { bottles }\end{array}$ & $\begin{array}{l}\text { - air parge, multiple } \\
\text { samples, pressure gauge }\end{array}$ \\
\hline Fl ow Me tering & $\begin{array}{l}\text { - Rotameter Turbine } \\
\text { meter }\end{array}$ & - Turbine meter \\
\hline Condenser & - Temp. controlled & $\begin{array}{l}\text { Temp. and flow } \\
\text { controlled (via bypass) } \\
\text { to give improved } \\
\text { stability and pressure } \\
\text { control }\end{array}$ \\
\hline
\end{tabular}


Table 3-1b: Data Reduction Differences Between [Ra83] and This Thesis (Rig \#1

$\mathrm{T}_{\mathrm{w}}$
$\mathrm{P}_{\text {1 ocal }}$

$\mathrm{T}_{\mathrm{f}}$
- NBS Honograph

- 1 inear pressure drop based on $\Delta P$ gauge

- 1 inear between $\mathrm{T}_{\text {out }}$ (measured) and $\mathrm{T}_{\mathrm{B} U \mathrm{~B}}$ o same

- piecewise 1 inear $\Delta P$, based on cal ibrated $\Delta P_{T D}$ if within range, el se $\Delta P_{\text {GAUGE }}$

- equilibrium temperature based on improved equation of state 
Table 3-2: Effect of Pressure Taps on Heat Transfer Coefficient

\begin{tabular}{|c|c|c|c|c|c|c|c|}
\hline Bef ore & (After) & Before & (After) & Before & (After) & Before & (After) Taps \\
\hline 4096 & $(3546)$ & 4636 & $(4346)$ & 3665 & (3069) & 0.00 & $(0.00)$ \\
\hline 4494 & $(3830)$ & 4687 & $(4511)$ & 3654 & $(3404)$ & 0.10 & $(0.10)$ \\
\hline 4278 & $(3574)$ & 4380 & (3915) & 4233 & $(4062)$ & 0.18 & $(0.18)$ \\
\hline 4221 & $(4068)$ & 4448 & $(3393)$ & 4096 & $(3620)$ & 0.26 & $(0.26)$ \\
\hline 4488 & $(4040)$ & 4352 & (3393) & 4091 & $(3416)$ & 0.34 & $(0.34)$ \\
\hline 4584 & $(3438)$ & 4233 & (3359) & 3807 & (3 126) & 0.42 & $(0.42)$ \\
\hline 5402 & $(3801)$ & 4454 & (4079) & 3989 & (3938) & 0.50 & $(0.50)$ \\
\hline 5504 & $(4698)$ & 4749 & $(4516)$ & 4153 & $(4034)$ & 0.58 & $(0.57)$ \\
\hline 5379 & $(6656)$ & 5612 & $(4182)$ & 5033 & (4925) & 0.66 & $(0.65)$ \\
\hline
\end{tabular}

Refrigerant: 'R22

Mass f1ux: $228 \mathrm{~kg} / \mathrm{m}^{2} / \mathrm{sec}$

Heat flux: $29318 \mathrm{~W} / \mathrm{m}^{2}$ 


\section{TYPICAL TEST RIG}

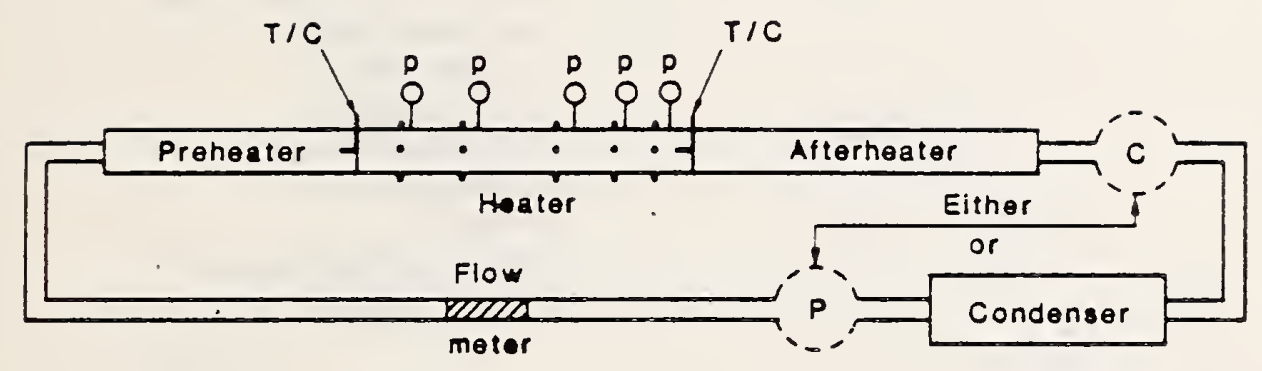

Figure 3-1: Typical Test Rig in the Literature: P=Pump; $\mathrm{C}=$ Compressor; $\mathrm{p}=\mathrm{pressure}$ tap and gauge 

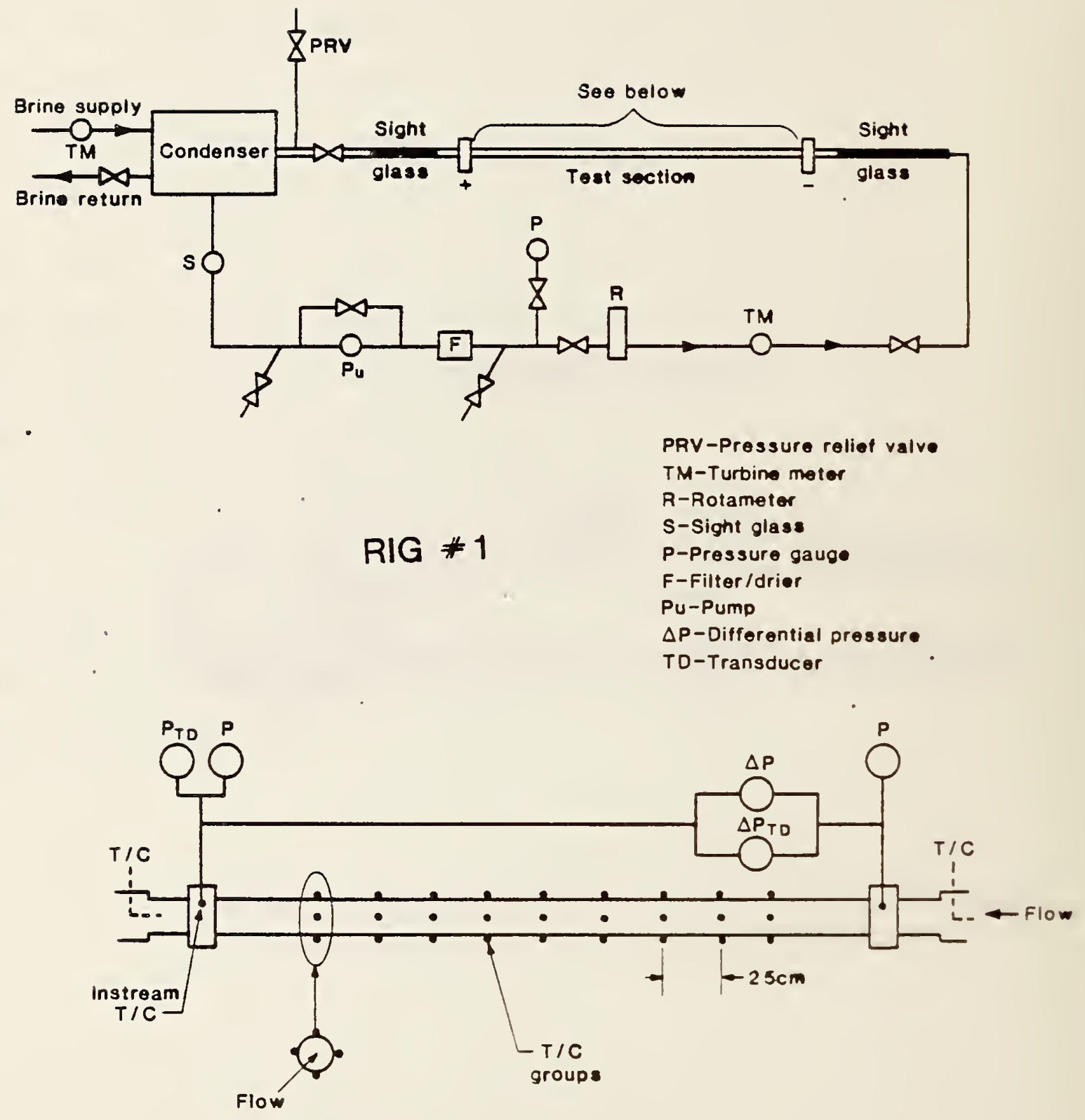

FIGURE TEST SECTION RIG \#I

Figure 3-2: Experimental Test Rig "\#1: Uniform Heating. No Preheat Section. 


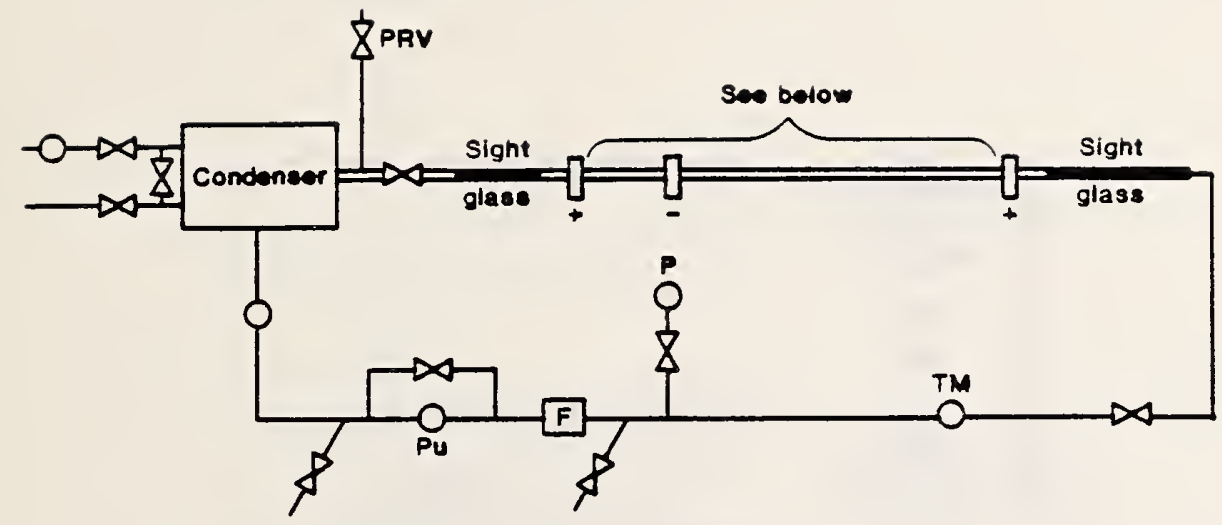

RIG $* 2$

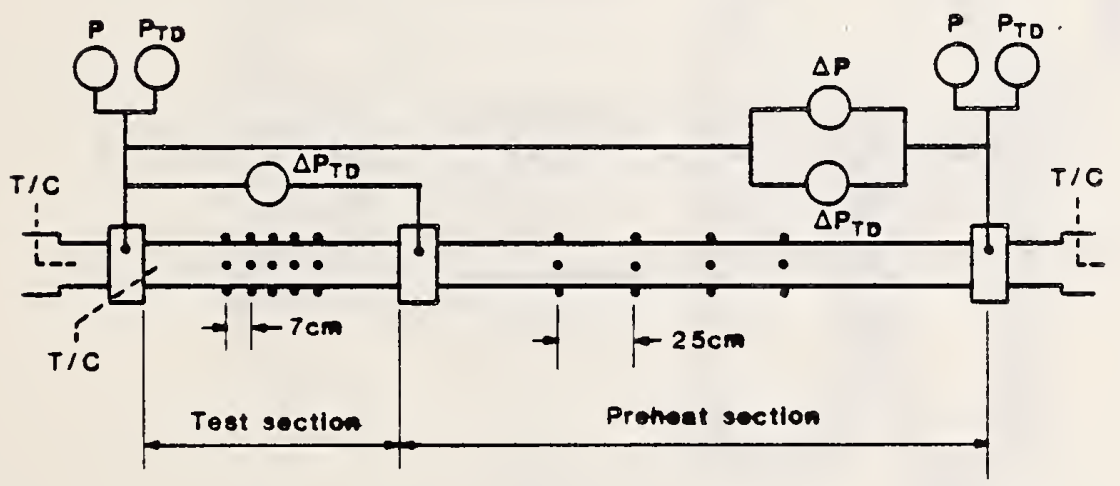

Figure 3-3: Experimental Test Rig 非: Preheat and Test Sections employed via separate DC power supplies. 


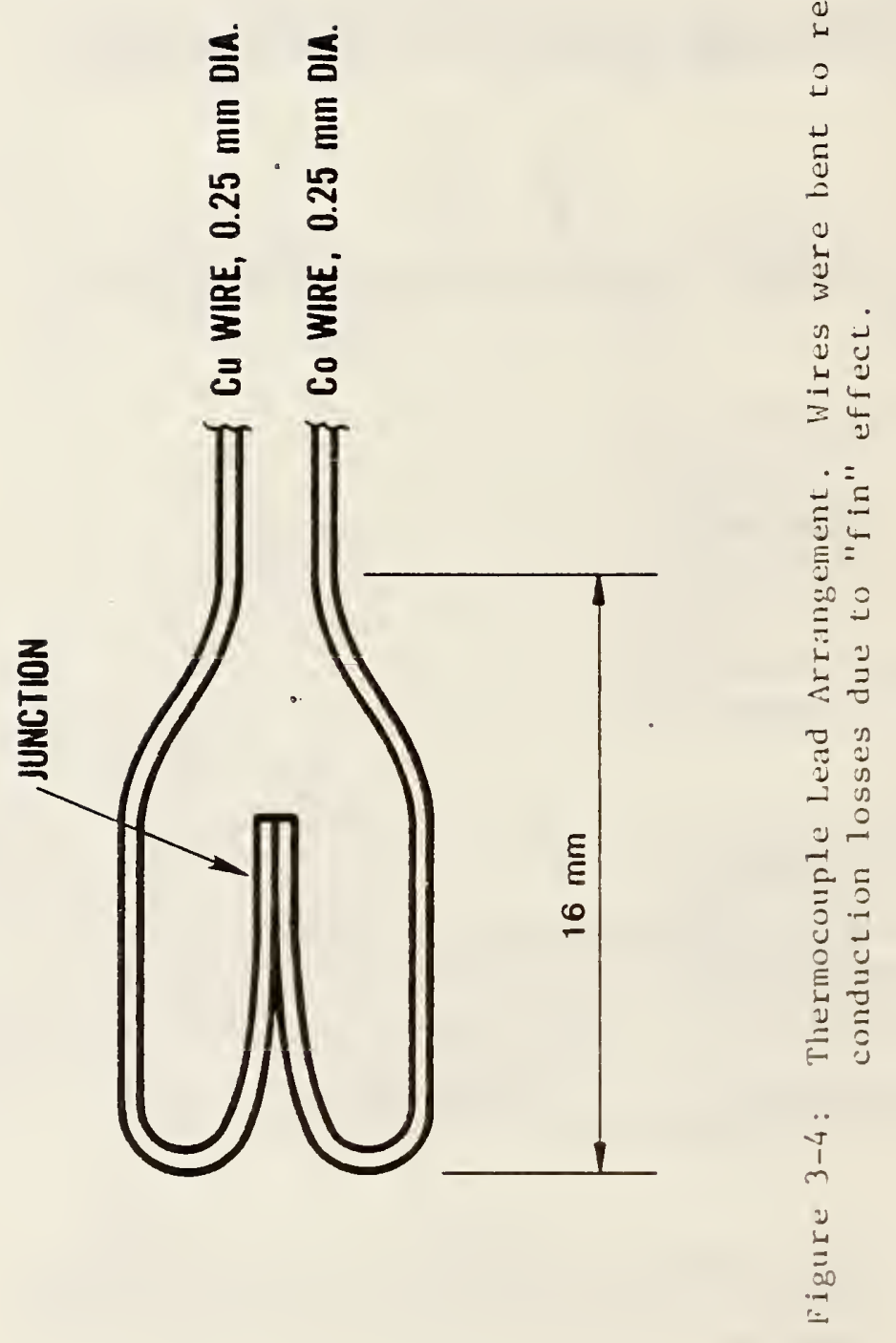



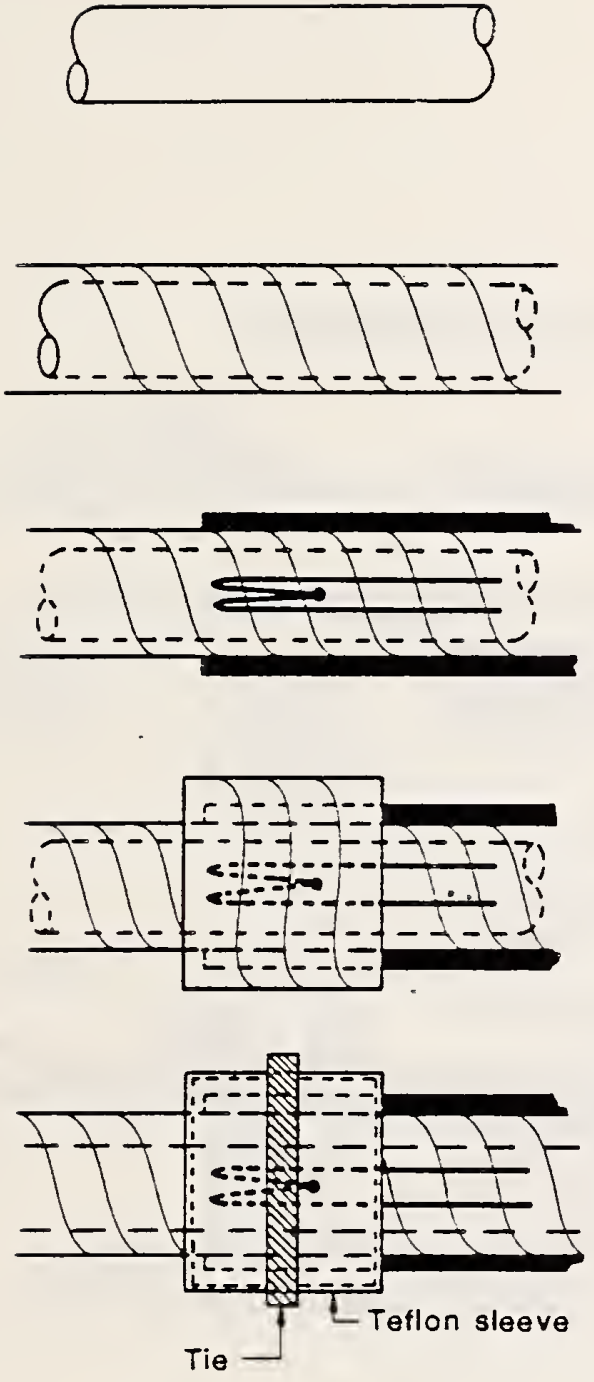

Plain tube

\section{Teflon tape}

wrapped around

entire tube

\section{Thermocouples}

placed around top

and sides of tube

\section{Second teflon tape} wrapped around tube, covering thermocouples

Stiff teflon sleeve placed over assembly and tied down to insure pressure. Teflon tape under T/C squeezed to $0.1 \mathrm{~mm}$ thickness 
TEMPERATURE MEASUREMENT

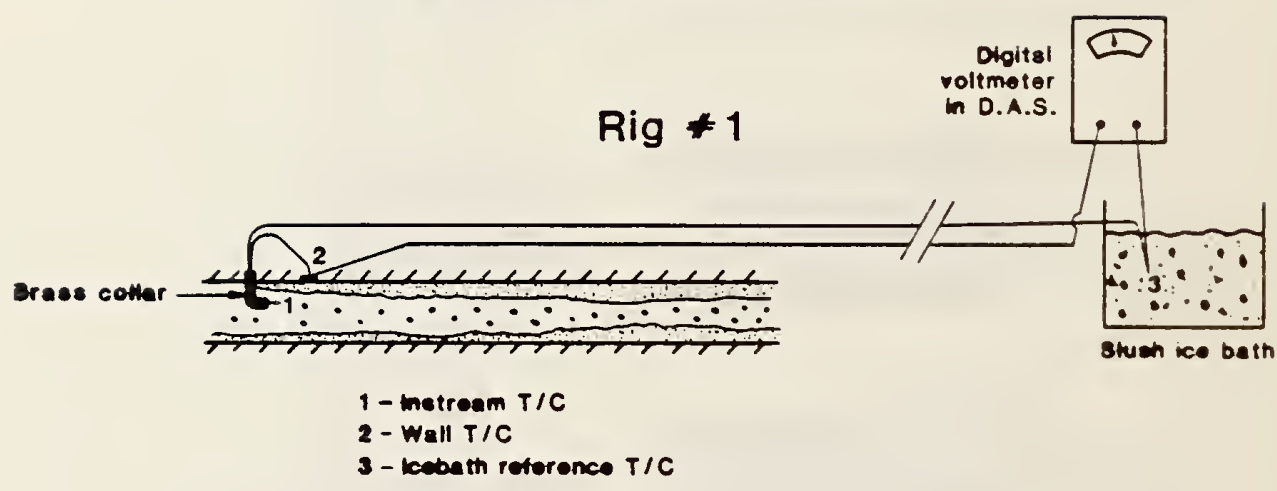

Rig $* 2$

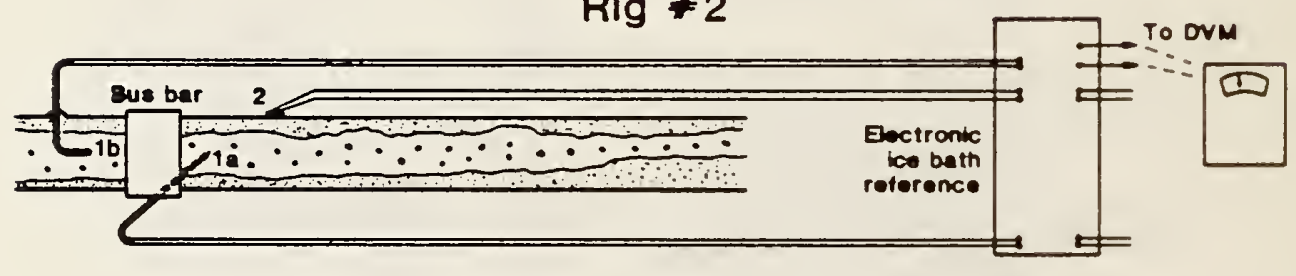

Figure 3-6: Wall and Instream Temperature Measurement: DVM= Digital Volt Meter; DAS: Data Acquisition System; Unshielded Thermocouples were used with Rig "1. Shielded thermocouples were used with Rig " 2 . 


\section{FIGURE SIMULATING PRESSURE TAPS}

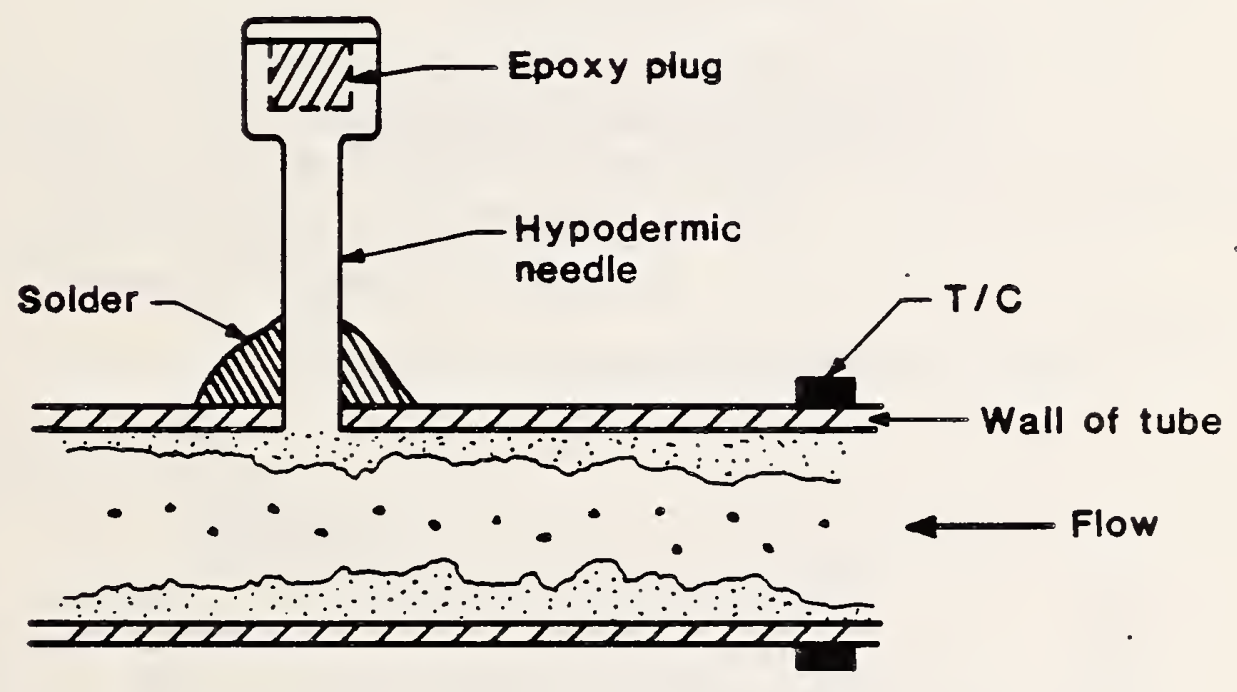

Figure 3-7: The Simulation of Pressure Taps: Taps mounted about $5 \mathrm{~cm}$ downstream of wall thermocouple measurement stations. 

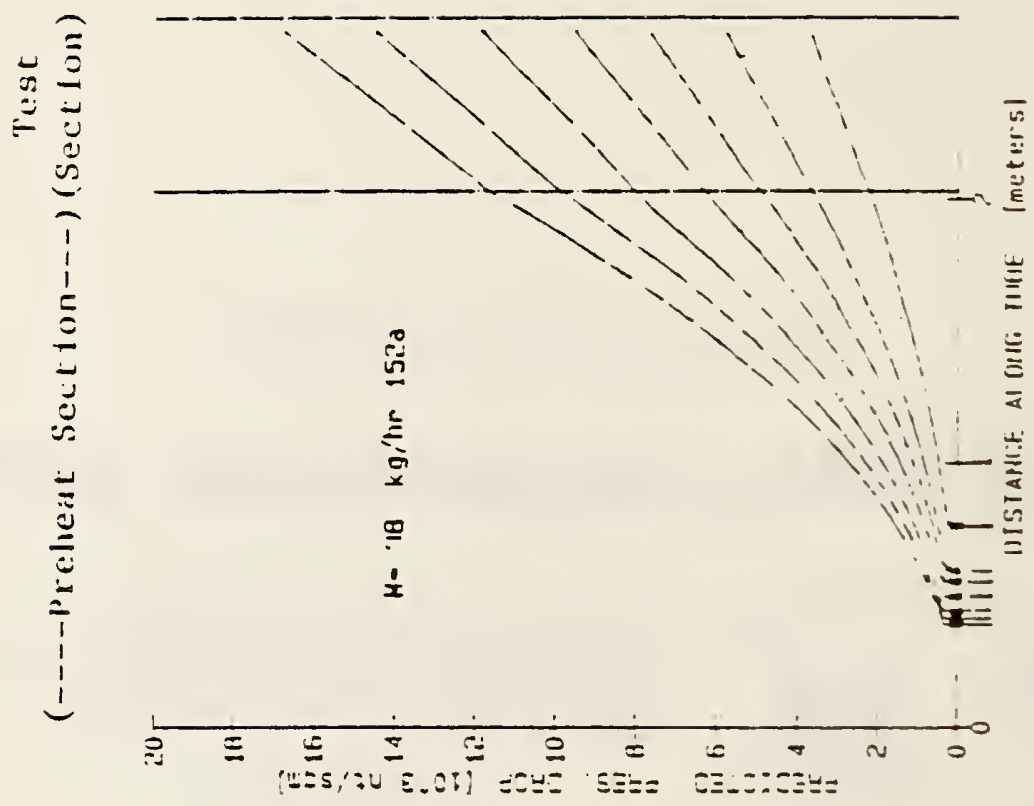

-

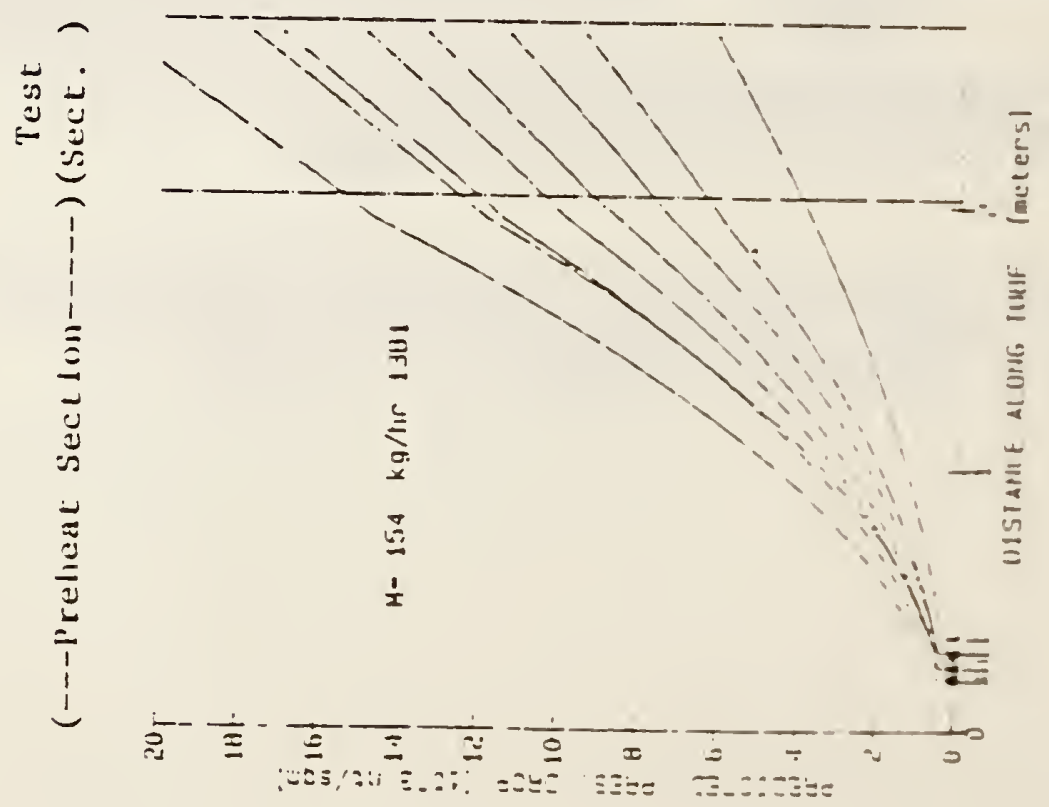




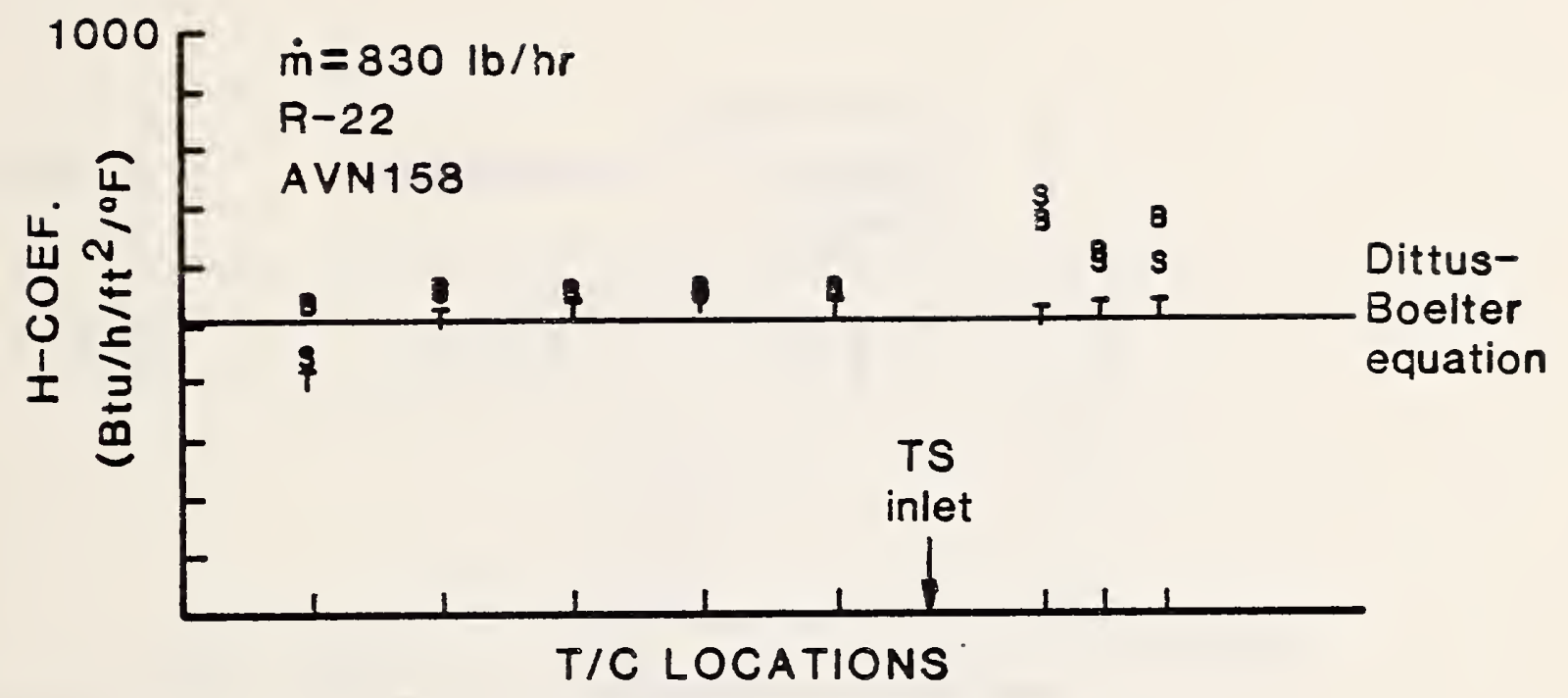

Figure 3-9: EFFECT OF INSTREAM THERMOCOUPLE:

Single phase (liquid) heating test with and without instream thermocouple at test section inlet

(T-Top, B-Bottom, S-Sides)

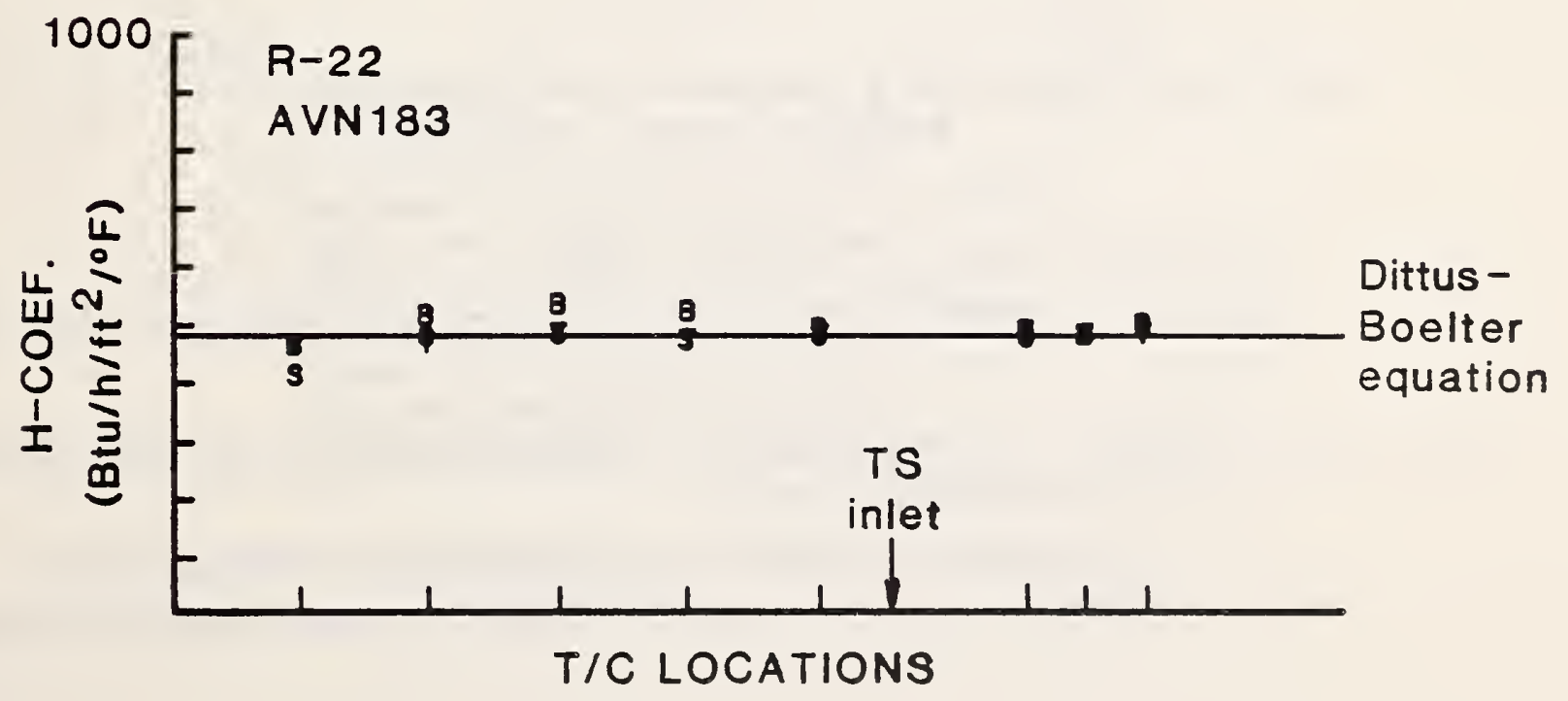



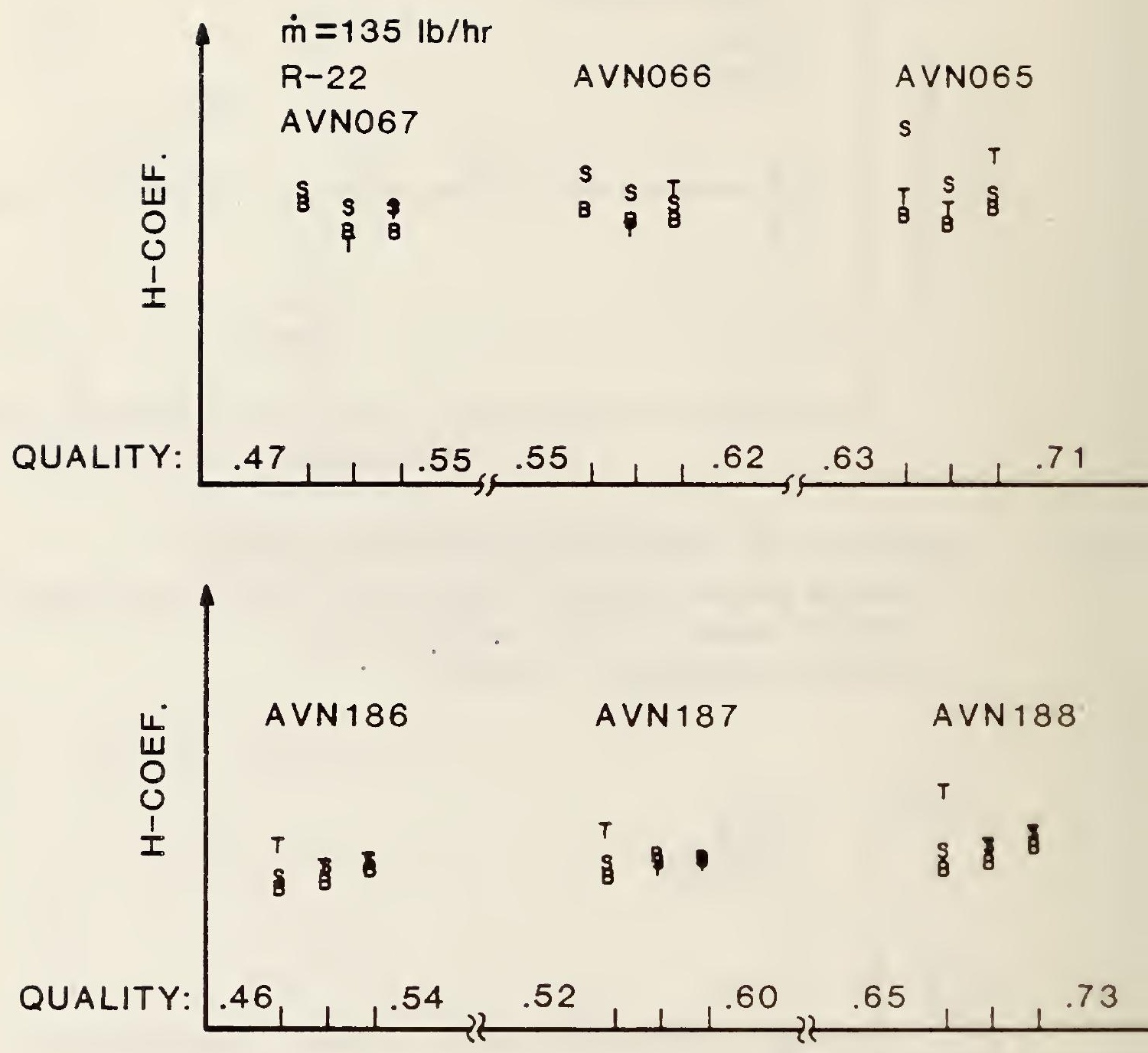

Figure 3-10: EFFECT OF INSTREAM THERMOCOUPLE ON EVAPORATIVE HT. TRANSFER COEFF.

Top set of curves: with instream thermocouple Bottom set of curves: without instream thermocouples 


\section{SAMPLING TECHNIQUE}

Tost rlg tine (Hquid)

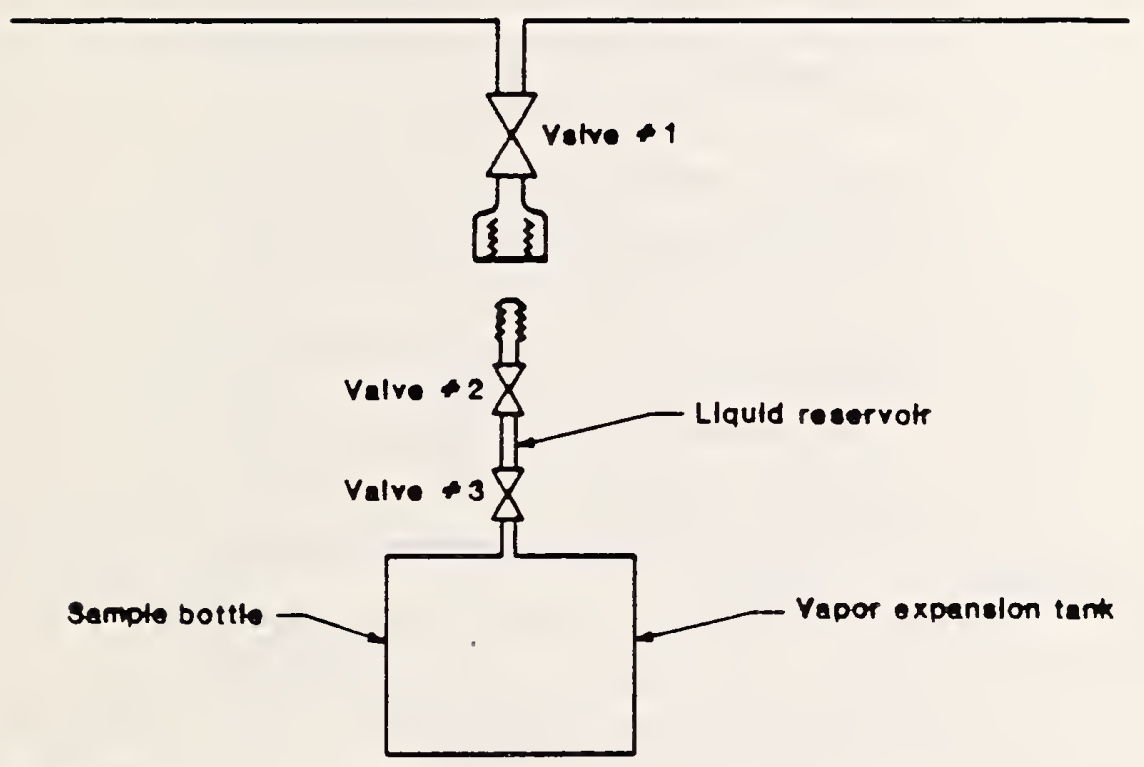

Procedure:

1. Evacuate eample bottle Including vapor a llauld reservotrs; cloes valves

2. Attach bottle to test rlo (all valves closed)

3. Open valves 1 \& 2 to fll Hquild section

4. Close valves 1 \& 2 ; remove bottle from test ro

5. Open valve 3 to expand llquid Into vapor

6. Bring bottle to gas chromatograph for enalyale

Error: Between etepe 2 and 3, valve 1 opened with bottle only loosely attached. Purges alr between valves $\$ 1$ and $\$ 2$, but introduces vapor rather than llquid into this sectlon.

Figure 3-11: Sampling Technique to determine mixture composition 


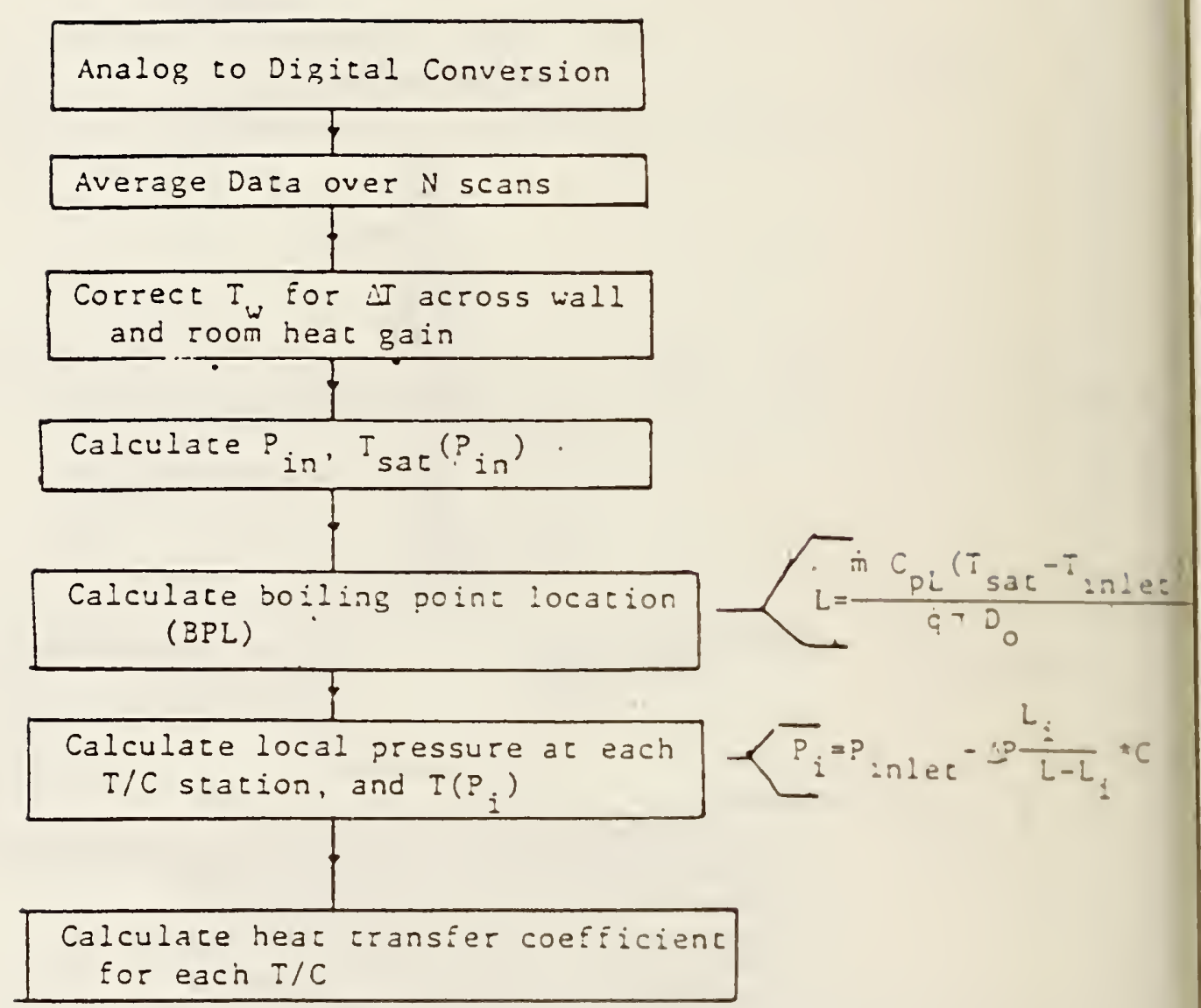

Figure 3-12a: Data Reduction Scheme for Pure Refrigerants 


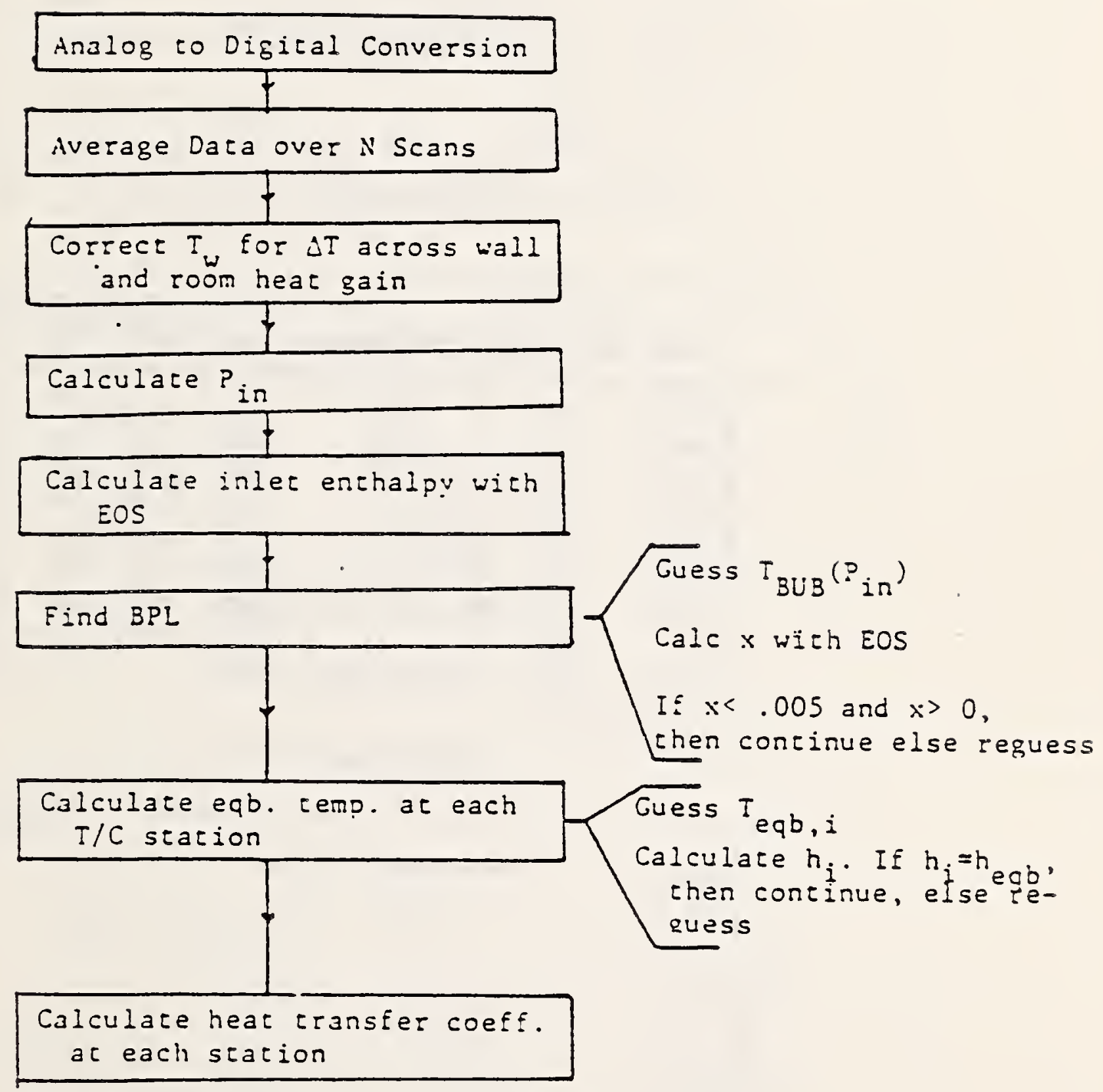

Figure 3-12b: Data Reduction Scheme for Mixtures 

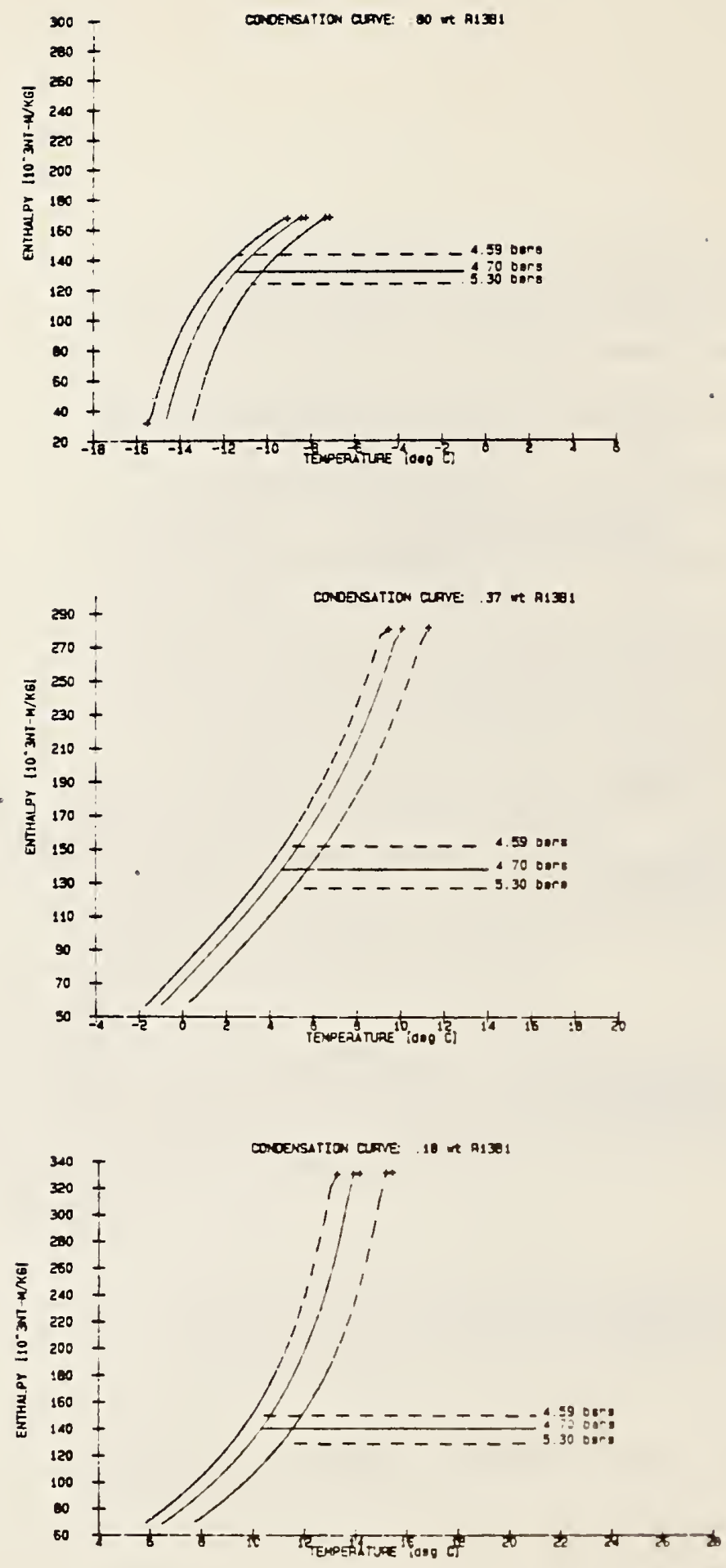

Figure 3-13: Condensation Curves ( $T$ vs. H) for "arious Compositions. A poor guess of $\mathrm{dH} / \mathrm{dT}$ may lead to divergence. 


\section{$\operatorname{ANM4OE}$}

REFRIG:MIXT. CONCENT(WTI52a $=.630$ FIOH: ANYULR $04: 04: 20: 53: 32$.

\begin{tabular}{|c|c|c|c|c|c|c|c|c|c|c|}
\hline & 20.4 & 20.1 & 18.8 & 10.2 & 9.9 & 9.9 & 9.8 & 10.0 & & \\
\hline \multirow[t]{3}{*}{-14.2} & 17.5 & 18.8 & 18.2 & 9.5 & 9.7 & 9.6 & 9.6 & 9.6 & 8.1 & 7.7 \\
\hline & 17.6 & 17.9 & 17.8 & 9.5 & 9.6 & 9.5 & 9.5 & 9.4 & & \\
\hline & 17.9 & 17.8 & 18.8 & 9.6 & 9.7 & 9.5 & 9.6 & 9.5 & & \\
\hline
\end{tabular}

$\begin{array}{lrrrrrrrr}\text { AuxT: } & 22.1 & -21.7 & -13.9 & -12.3 & 8.0 & -26.3 & -7.9 & \text { deg C } \\ \text { PRES: } & 4.77 & .02 & .015 & 4.70 & 5.08 & 4.84 & .08 & \text { bars } \\ \text { HEAT: } & 124.08 & 23.8 & 45.2 & 2.60 & 51708 & 6800 & \\ \text { FLOH: } & 67 & 144 & 80.4 & & & & & \end{array}$

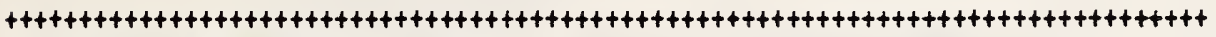
HEAT TRANSFER COEFFICIENT ( $/$ /sqa/K)

H. $152 \mathrm{a}$

TOP LSIDE BOTM RSIDE AVG MassQuality VapConp LiqConp Eqb.T

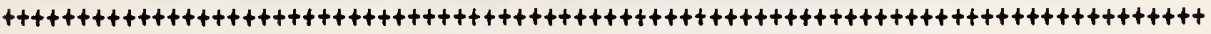

$\begin{array}{lllllllll}3119 & 3805 & 3784 & 3676 & 3596 & .35 & .41 & .75 & 3.83\end{array}$

$\begin{array}{lllllllll}3474 & 3811 & 4117 & 4142 & 3886 & .48 & .46 & .79 & 5.22\end{array}$

$\begin{array}{lllllllll}4231 & 4442 & 4607 & 4223 & 4376 & .59 & .50 & .82 & 6.51\end{array}$

$\begin{array}{lllllllll}2777 & 3841 & 3981 & 3813 & 3603 & .70 & .54 & .85 & 7.52\end{array}$

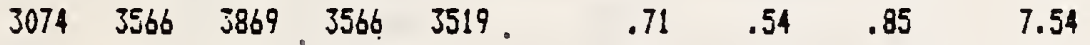

$\begin{array}{lllllllll}3254 & 3820 & 3980 & 3829 & 3721 & .71 & .54 & .85 & 7.56\end{array}$

$\begin{array}{lllllllll}3522 & 3746 & 3937 & 3788 & 3748 & .71 & .54 & .85 & 7.58\end{array}$

$\begin{array}{lllllllll}3129 & 3853 & 1223 & 4038 & 3811 & .71 & .54 & .85 & 7.60\end{array}$

TESTSEC AV6: 3680 ENERGY BALAMCE: $1.17^{\circ} 7.66$

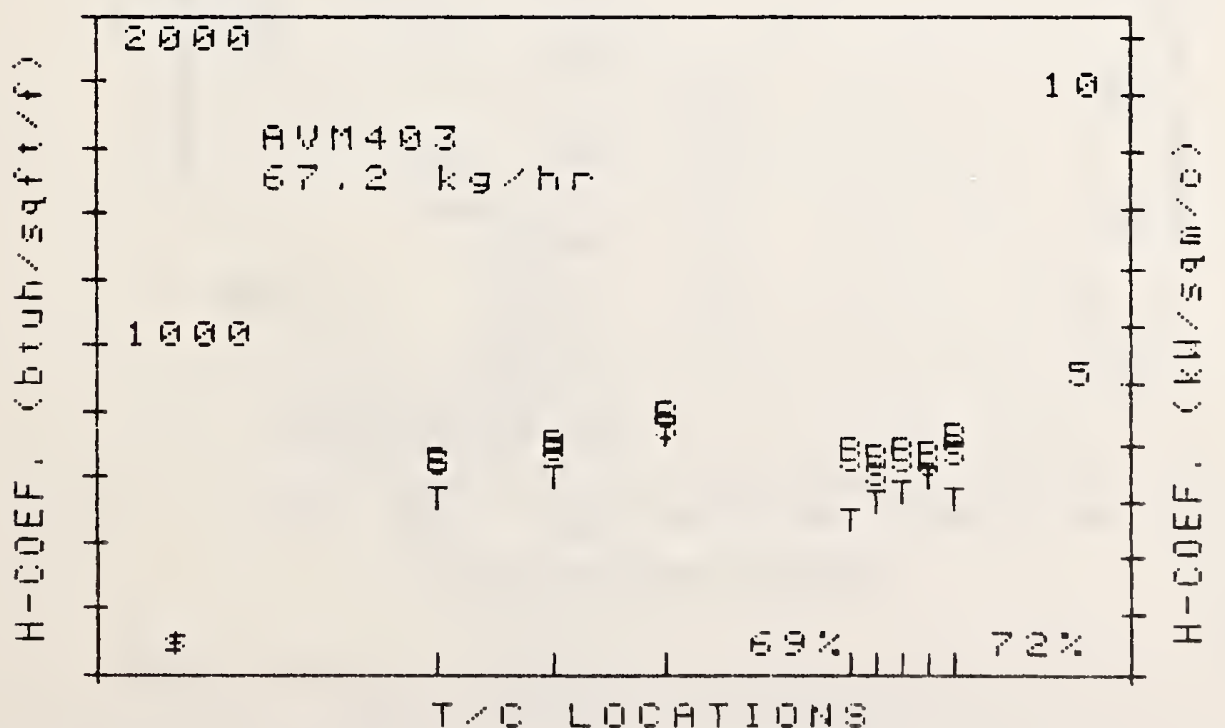

Figure 3-14: Typical Output from Test Run Data Reduction. T: Top; B: Bottom; S: Side average 
h- PREDICTED - Dittus \& Boetter $\left[w / m^{2} /{ }^{\circ} k\right)$

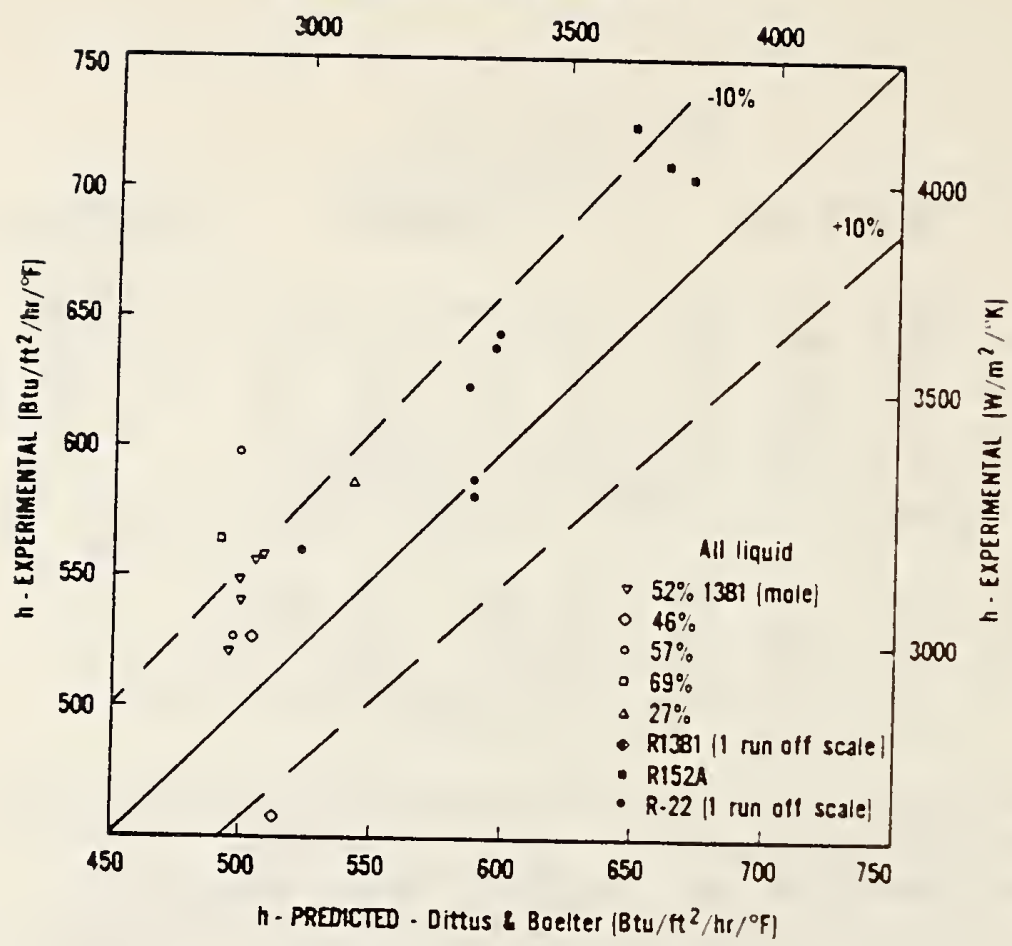

h - MEDICTED - Petukhor $\left[W / m^{2} /{ }^{\circ} \mathrm{K}\right]$

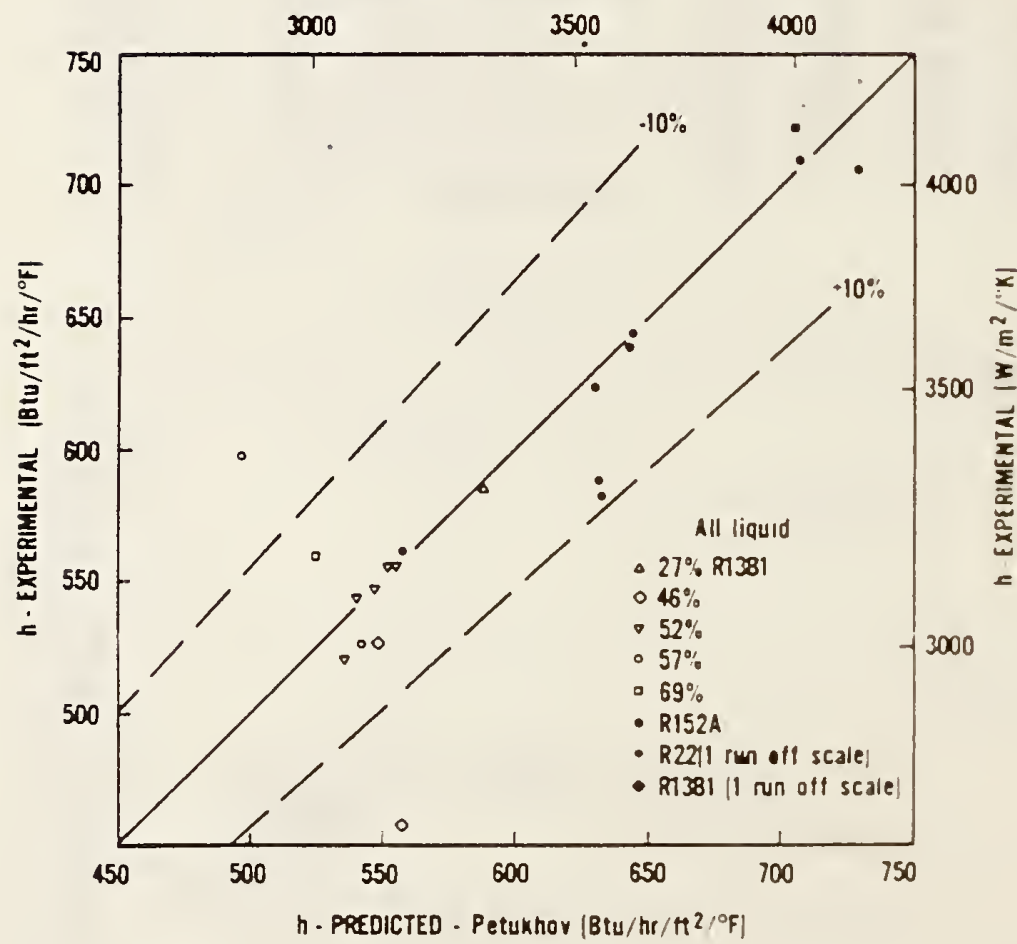

Figure 3-15: Comparison of Predicted to Yeasured Data for Single Phase Heating Tests 

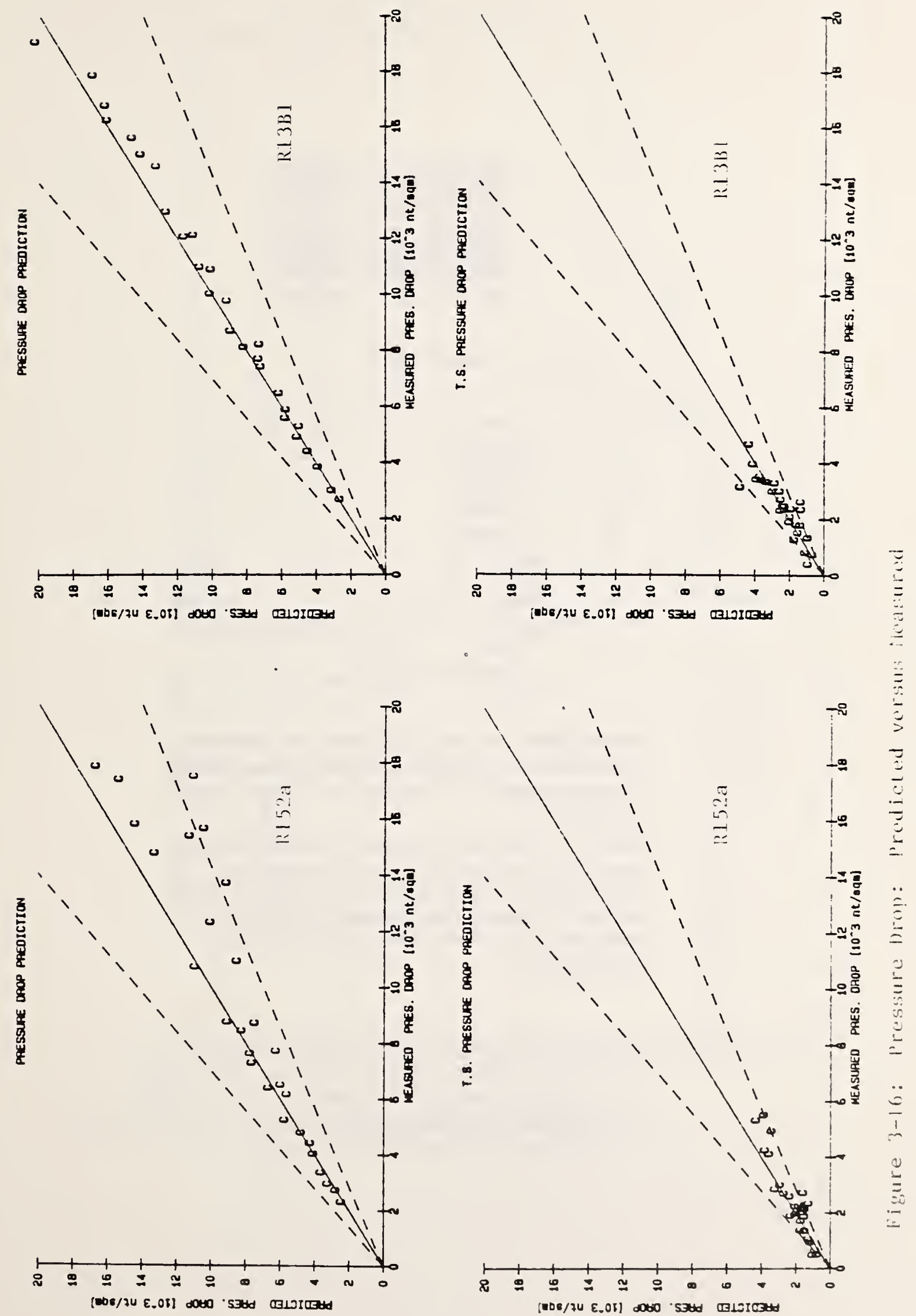


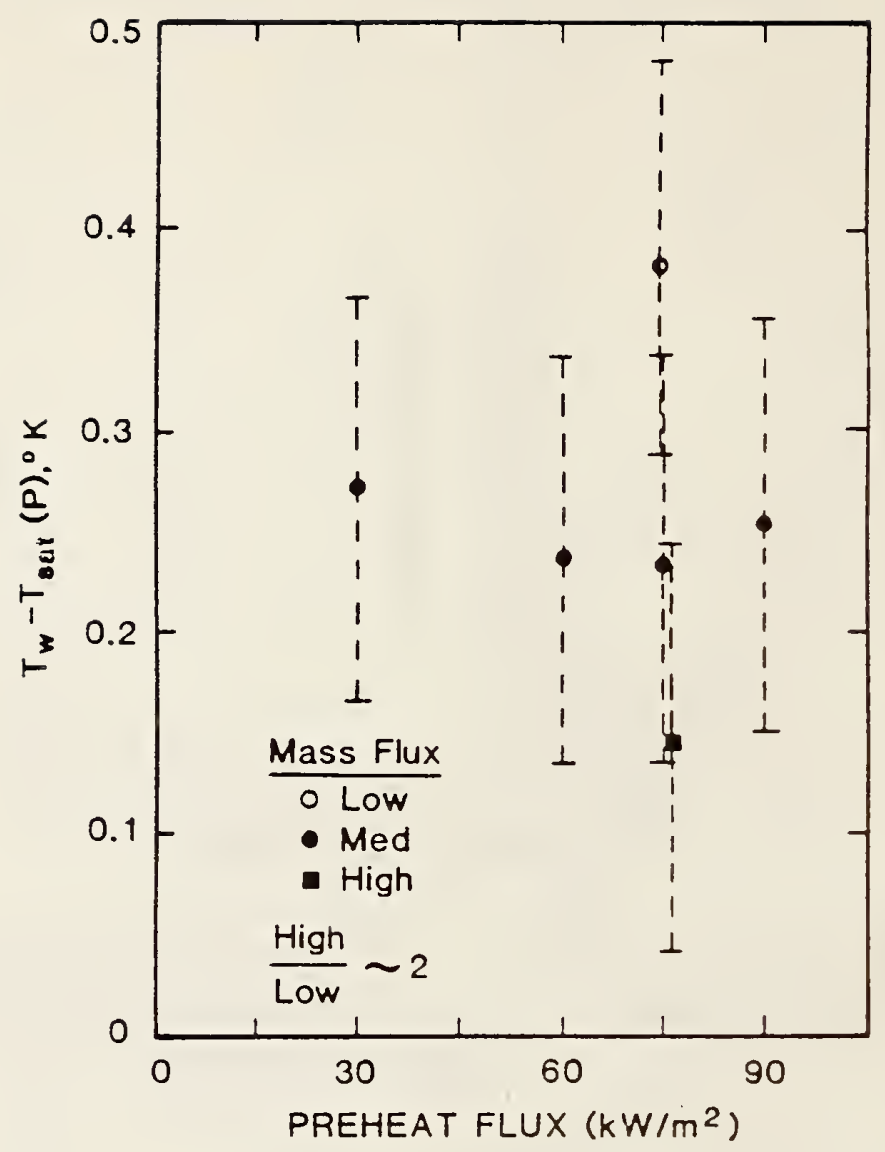

- Values shown are for T/C station 50 diameters downstream of preheat. No heat in test station. Top and bottom T/C agreed with each other within $0.1^{\circ} \mathrm{C}$.

- Data at $15 \mathrm{~kW} / \mathrm{m}^{2}$ (preheat) showed distinct top/bottom difference even 50 diameters downstream. This is due to thicker liquid film at bottom. (Continued evaporation occurs due to pressure drop.) Thus flow patterns persist downstream.

\footnotetext{
Figure 3-17: Effect of Preheat Flux on Test Section Wall Temperature Measurements. The large error bars are due to the use of the DAS internal temperature compensator, rather than the normal ice point reference.
} 


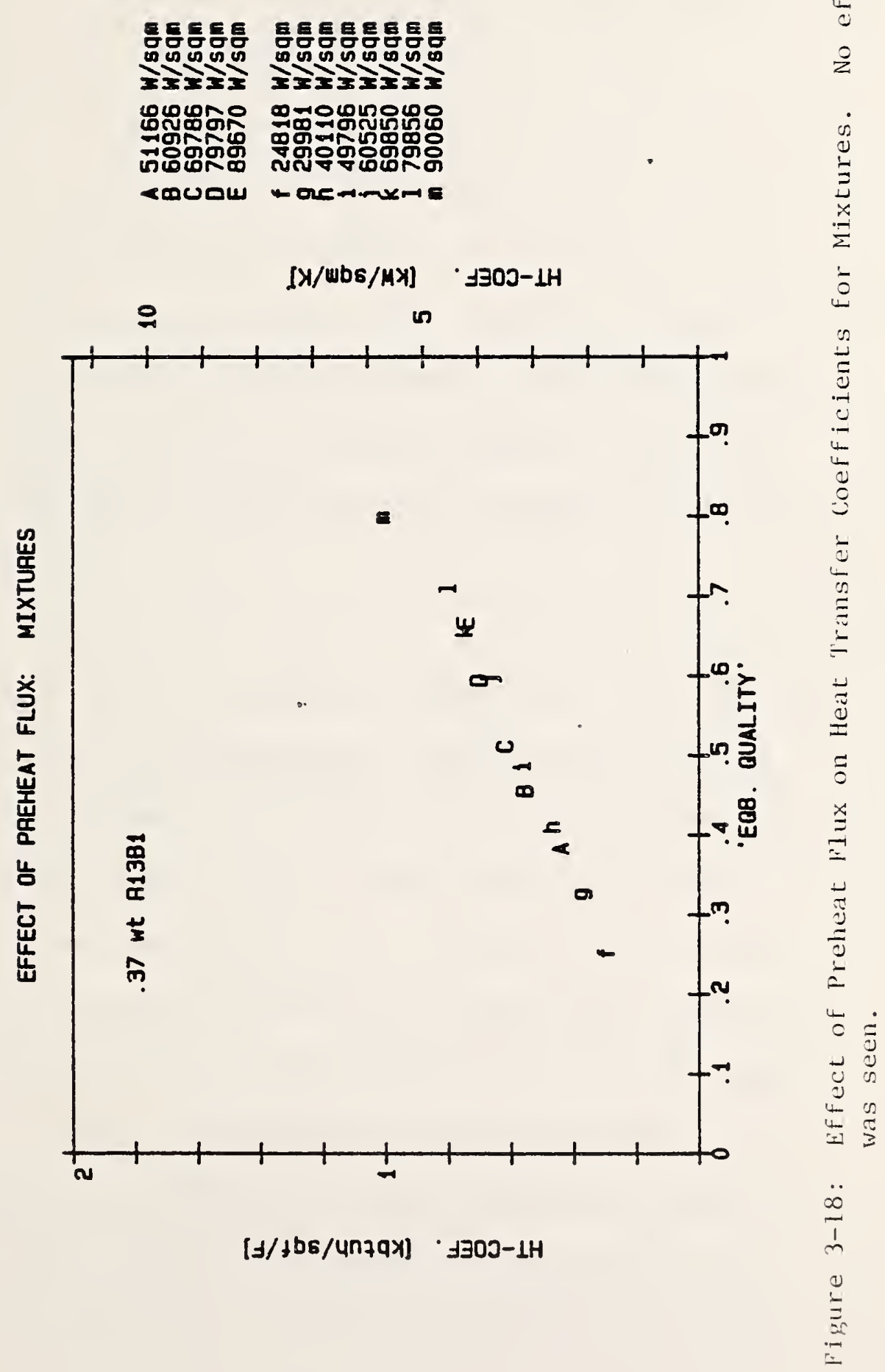


- 
CHAPTER 4: ON THE SUPPRESSION OF NUCLEATE BOIL ING

\subsection{Background}

The most common explanation of the physical mechanism of heat transfer in annular flow boiling is that of a superposition of a forced convection evaporative process and a nucleate boiling process. With increasing vapor quality the liquid fin thins and the core vapor accelerates (as required by continuity). Heat transfer to the core is improved by this acceleration and the thinning of the 1 iquid film also serves to lessen its conductive resistance. Heat transfer is thought to improve sufficiently and to occur with such rapidity that bubble growth disappears. At this point, the nacleate boiling process is said to be suppressed, and vapor generation is due strictly to evaporation from the vapor-1 iquid interface.

It is critical to know if nucleate boiling is suppressed. First, if the. process becomes purely convective/evaporative, then the heat transfer coefficient should be independent of wall heat flux, and depend on 1 ow and fluid parameters (e.g., mass flow rate, eddy diffusivity, Prandt I number), as in single phase shear-driven flow. In this case, the heat transfer process might be modelled strictly from single phase considerations. Also, correlations for annular film condensation might be applicable to the evaporative case, in the absence of nucleate boiling. If, on the other hand, nacleate boiling exists, from a sufficient number of sites then the 1 iquid film viscous sublayer may be destroyed, and the fluid flow and heat transfer processes become more difficalt to predict. 
In the case of mixtures, chaotic concentration profiles may occur when nucleate boiling is not suppressed.

For heat transfer purposes, it is the boiling site density which is particularly important. The existence of ar isolated, metastable bubble is not significant. Throughout this chapter, the former is of interest. However, as a starting point, the prediction of the existence of any individual bubbles is required.

4.1.1 Conventional Theory of Onset and Suppression of Nucleate Boiling The onset and suppression of nucleate boiling are effectively the same problem, differing only in the direction from which the heat flus required to sustain nucleation/bubble growth is approached. Classical theory for the growth of a bubble begins with a force balance.

The maintenance of a spherical bubble requires, from a force balance. the 1 iquid immediately surrounding the bubble to be superheated by an amount :

$$
T_{L}-T_{V}=\frac{2 \sigma}{I_{c} \frac{\partial P_{s a t}}{\partial T}}
$$

where $r_{c}$ is the radius of the bubble. Upon applying the ClausiusClapeyron relation and assuming the inside bubble temperature to be saturated, equation (4-1) becomes: 


$$
T_{L}-T_{s a t}=\frac{2 \sigma}{I_{c}} \frac{\Delta h_{v}}{T \Delta v_{v}}
$$

The same criterion can be applied to a heated surface with a vapor bubble, assumed to be hemispherical, developing from a surface cavity of mouth radius, $I_{c}$. In the case of a heated surface, however, the liquid temperature surrounding the bubble will not be uniform generally, but instead diminish with distance from the heated surface. Also, the bubble shape will be approximateiy that of a trancated sphere with radius of curvature $r_{c}$, rather than completely spherical. Both of these effects were accounted by Hsu [Hs62], followed by others. First from pare geometrical considerations, the height, $y_{B}$, and radius of curvature, $I_{B}$, of a spherical truncated bubble are related to the mouth radius by:

$$
y_{B}=(1+\cos \theta) r_{c}=c_{1} r_{c} \text { and } r_{B}=\frac{1}{\sin \theta} r_{c}=c_{2} r_{c}
$$

so that equation $(4-2)$ becomes:

$$
T_{L}-T_{s a t}=\frac{2 \sigma}{\left(c_{2} / c_{1}\right) y_{B}} \frac{\Delta h_{v}}{T \Delta v_{v}}
$$

Secondly, Hsu assumed bubble grow th to be possible only if the 1 iquid temperature at the bubble cap was superheated to satisfy (4-2). For a 1 inear temperature field as might be reasonably approrimated across a thin thermal boundary layer, the wall heat flux and temperature field are represented by: 


$$
q_{w}=\frac{\lambda_{L}}{\delta}\left(T_{w}-T_{s a t}\right)
$$

and

$$
T(y)=T_{w}-\frac{T_{w}-T_{S a t}}{\delta} y=T_{w}-\frac{q_{w}}{\lambda_{L}} y \quad 0 \leq y \leq \delta
$$

where $y$ is the distance from the wall and $\delta$ is the boundary layer thickness, the temperature at the edge of which the condition is assumed sa turated.

Equations (4-3) and (4-5) may be plotted for a given beat flux and film thickness, as shown on Figure 4-1, and most importantly the range of cavity sizes which may be active (i.e., those from which ebullition is possible). can be determined. At cavities outside the range shown on Figure 4-1, the 1 iquid is not sufficiently superheated for bubble growh to occur. A large superheat is necessary to activate the more numerous small cavities. The superheat may derive from either a large wall heat flux or a low liquid thermal conductivity (see equation 4-5). For large cavities the superheat requirement is small, but this superheat must be maintained far from the beated surface for the bubble to grow to its critical radius (hemispherical shape).

In the case of annular flow boiling, measured wall superheats have been sufficient seemingly to initiate bubble growth even when none has been observed [La62]. Collier and Pulling proposed to explain the apparest 
contradiction by noting the sublayer thickness in turbulent films is smaller than for stagrant pools [Co64]. They assumed that ow ins to turbulence, the 1 iquid outside the viscous sublayer was at or close to saturation. The temperature drop occurs then across only the small viscous sublayer, or:

$$
\begin{aligned}
& q_{w}=\frac{K}{\delta_{v s}}\left(T_{w}-T_{s a t}\right) \\
& T(y)=T_{w}-\frac{q_{w}}{\lambda_{L}} y
\end{aligned}
$$

where $\delta_{v s}$ is the thickness of the viscous sublayer.

Collier and Pulling recommended using a dimensionless viscous sublayer thickness of $y^{+}=7$. In the single phase turbulent flow literature, the viscous sublayer thickness has been presented in values from $y^{+}=5$ to $y^{+}=10$. In fact turbulent eddies carrying cool fluid from the vapor1 iquid interface may penetrate the sublayer down to $\mathrm{y}^{+}=1$ [La62]. More relevantly, Bejan has studied analytically buckling and rolling of 1 iquid 1 ayers in shear-driven flow (as occurs in annular flow boiling). He determined the value of $y^{+}=7.62$ as the viscous sublayer thickness which persists regardless of possible buckling or rolling [Be82].

Several years later, Collier suggested a modification to the viscous sublayez approach, relating the point of suppression to the Martinelli parameter, $\mathbb{X}_{t t}[\mathrm{Co80}]$. This parameter can be related however to the 
viscous sublayer thickness, as both are subject to flow conditions. The viscous sublayer is a function of wall shear stress, which itself is a function of frictional pressure drop. Finally, Martinelli et al predicted frictional pressure drop for two phase flows through the parame ter $x_{t \pm \cdot} \cdot 1$

\subsubsection{New Alternate Theory: Enhancement of Nucleate Boiling} Recently, the conventional explanation has been questioned initially by Mesler [Me76, Me77] and subsequently by Beattie et al [Be79,Be84]. Mesler has suggested that the heat transfer process, rather than becoming convective/evaporative at high quality, is due even in thin tarbulent films to nucleate boiling. He has suggested that the high heat transfer rates experimentally measured with thin films (high compared to pools or thick films) is due to an enhancement of nucleate boiling with thin films. The high heat transfer rates seen with thin films are hypothesized to be due to evaporation of the thin 1 iquid microlayer and rapid replenishment of the microlayer. The replenishment process with thin films is improved over usual pool boiling. With thin films, the bubble ruptures the film surface and vapor escapes through the top of the broken bubble, causing the liquid film to be Ieestablished quickly. An improved replenishment process which is Ielated to film thickness might account for the observed improvement is heat transfer with increasing quality.

${ }^{1}$ Collier's recent suppression criterion is reviewed in Appendix 4-A, as are other proposed criterion. 


\subsubsection{Suppression of Boiling with Organic Fluids}

A second issue was raised by Toral [To79], who suggested that complete suppression of nucleate boiling will not occur under common conditions in annular flow boiling of organic fluids. These fluids of which refrigerants are included, have a thermal conductivity much 1 ower than water, and as such will tend to yield high wall superheats, sufficient for nucleation.

\subsubsection{Problem Resolution Me thods}

The issues then are:

(1) can the physical process by which vapor is generated be entirely evaporative, or is it best described by nucleate boil ing theory?

(2) is it possible for organic fluids, specifically refrigerants, with their relatively 1 ow thermal conductivity, to be vaporized by an entirely evaporative mechanism in annular f1 ow?

(3) can conventional suppression theory or various other suppression criteria be verified (and modified for mirtures) to quantify the point at which nucleate boiling is absent?

(4) are there unique mechanisms which occur with nonazeotropic mixtures? 
The following techniques might be employed to resolve the problem:

A. Visual Evidence (e.g., vapor generation without bubble presence or bubble presence with thin films).

B. Experimental Evidence

1. Dependence of the heat transfer coefficient on heat fluxes and mass flux.

2. Effect of pressure on heat transfer coefficient.

3. Presence of hysteresis.

4. Dependence of heat transfer coefficient on quality.

5. Predictive ability of evaporative or pool boiling models to flow boiling data.

The following discussion critically analyzes the literature for pure and mixed fluids. Detailed reviews are available in Appendix $4 \mathrm{C}$ through $4 \mathrm{~F}$. New experimental evidence for single and binary refrigerants is presented. The new criterion for determining the suppression point for mixtures is hypothesized. The discussion will attempt to concentrate on refrigerants, but will cite several studies from other literature in response to the cautionary note and advice of Butterworth and Shock [Bu82]. It also serves as an interaction to the data base developed for this report, and as such several graphs are presented to display the data.

\subsection{Summary of Visual Evidence}

Several visualization studies of flow boiling of pure fluids have been done to determine flom pattern and bubble existence; the major studies 
are discussed in detail in Appendix 4C. Most of the stadies have been with water, bot some with refrigerants. The stadies employed trans parent metal coating, heater strips on one side, or glass tubes. Often the surfaces had been milled smooth to ensure nniform heat generatior, horever, this process removes potential nucleation sites, preventing generalization of results. In one case an artificial nucleation site was added to the surface in order to itness boiling in a thin $f$ ilm flow. This study done by Mesler led him to formalate his al ternate the ory.

Nearly al 1 isuslization stadies show some isolated bobbles within the 1 iquid $f$ il m, the nuber of sited babbles diminishes with increasing quality. The anthors attribute the continued vapor generation to evaporation from the vapor-1 iquid interface. It is possible bowerer that eballition continued in smal 1 cavities or that babbles were so short. lived as to escape notice even with high speed films or stil 1 photographs, thas $\nabla$ isual evidence is not itself definitive.

The study by Hewitt et al [He84] showed activation of a site whenever beat transfer through the film was inhibited by wave passage, suggesting film thickness was an important though not solely definitive criterion. The Hewitt et al study al so observed that vapor velocity had a strong influence on the observation of nucleation. The stady of boiling fram an artificial site did not have a higher vapor velocity and does not correspond directly to the physical case of tarbolent flow boiling in tubes. 
Many visualization studies used tubes which bad been milled smooth or which were of materials without large cavity sizes; this could inhibit bubble growth. Bowever, they suggest that vapor generation can take place in the absence of such cavity size availability, suggesting that a mechanism other than nucleate boiling is the cause of such vapor generation.

\subsection{Summary and Analys is of Experimental Evidence: Dependence on Heat and Mass FIux}

Appendix 4D describes in detail the studies which examined the dependence of the heat transfer coefficient on heat and mass flux. When no dependence on heat flux is observed $(\alpha \neq a(q))$, nucleate boiling is considered suppressed, and $a=a(G)$. On the other hand, when nacleate boiling dominates, the heat transfer coefficient is a strong function of heat flux and a weak funtion of mass flux. This, the dependence of $a$ on $G$ or $Q$ may define the dominant heat transfer mechanism, and if the heat transfer coefficient is independent of heat flux $(a \neq a(q))$, then the sole mechanism is usually considered evaporative.

A recent study by Aounallah et al [A082] showed clearly $a \neq a(q)$ for a range of qualities, heat and mass flax values. Care was taken in their experiment to ensure that measurements at the same spatial location were compared. In direct response, Beattie and Green [Be84] cited work by Bertoletti et al [Be64] with a similar experimental apparatus and also using water as the working fluid; the Bertoletti data showed a strong dependence on heat flux and was correlated well by a pool boiling correlation. 
The results of Aonnal $1 \mathrm{ab}$ et al appear to contradict those cited by Beattie and Green (the Bertoletti et al experiments). However, the apparent discrepancy may be resolved by examining the test conditions used by the two groups. The latter, where nucleate boiling was observed to be dominant, invol ved higher pressures and heat fluxes where conventional theory suggests a small superheat requirement. The conventional theory therefore 811 ow s both experimental observations to be $v a l$ id. The much higher heat flures empl oyed by Bertoletti et al most 1 ikely produced a vapor generation process dominated by nucleate boil ing.

In a separate pablicatior, Beattie and Law ther [Be79] describe their own successful work in predicting pressure drop. at high quality by theorizing the existence of attached bubbles within a 1 iquid film. They point to their success as a proof of bubble existence.

The heat flux level of the Beattie and Law ther experiment was al so very high, since their observations were made in a critical heat flur experiment. Here again, the existence of attached surface bubbles are entirely possible, and explained by the conventional theory.

Mesler has examined many studies of nucleate boiling in thin films, noting high heat transfer rates and a dependence on heat flar. However, the boiling studies cited by Meslex involved slow moving films. For example, the referenced Toda and Dchida study [To73] invol fed 1 aminar or near 1 aminar flows. The study by Fletcher et al (Ieferenced by [Me77]) was designed specifically to avoid high vapor velocities, which Hewitt et al [He63] observed to be important in the suppression process. Thus the 
Ieferenced studies are not strictly comparable to the case of the tarbulent flow s associated with and ar flow boil ing.

\subsection{Summary of Experimental Evidence: Dependence of Hest Transfer Coefficient on Quality}

The inverse Martinelli parameter $\left(1 / X_{t t}\right)$ is sometimes referred to as a surrogate for quality. Because of its successful employment in prossure drop prediction, several authors have used it in heat transfer coefficient prediction. The idea was first advanced by Dengler and Addoms (Appeadir 4D) based on their experimental observations [De56]. Mesler has reviewed the [De56] data, and upon careful examination, showed that the data is not closely correlated by the ase of $1 / x_{t t}$. He further attempts to shor on theoretical grounds thet the general approach of using $a=a_{L 0} f\left(1 / X_{t t}\right)$ is insppropriate. However, these objections to the use of $X_{t t}$ as a correlating parameter do not soem varranted. As shown in Appendix 4B, the Mesler anglysis of $a / a_{L}=f\left(1 / X_{t}\right)$ inadver tently neglects the fact that same parameters he considered constant do indeed $v$ ary.

The enhanced nucleate boiling theory cannot explain a phencenen obsorved in several experiments, that of gradal reduction in beat transfer coefficient despite increasing quality. At times the measured reduction disappears as quality is further increased. Such behavior, originally attributed to an entrance length effect [Go66] has been seen at L/D ratios greater than 100, with pore refrigerants, water and with refrigerant mirtures [Ch67, Ma76, Ra83]. The experiments show in the nucleate 
boiling dominated region, a decreases with increasing quality. Sucb observations are in sharp contradiction to the hypothesis of Mesler, which requires a to increase with reduced film thickness, i.e., an increased quality.

\subsection{Summary of Experimental Evidence: Effect of Pressure}

Pool boiling experimental heat transfer coefficients increase with increasing pressure. This often seen observation is accounted in equation (4-1) since $\partial P_{s a t} / \partial T$ increases generally with pressure. Additionally, surface tension, at least for refrigerants, decreases with increasing temperature (related directly to saturation pressure). Both of these phenomena tend to reduce the superheat requirement so that more sites are activated for a given superheat as pressure is increased.

Conversely, in the case of forced convection/evaporation, the heat transfer coefficient may decrease with increasing pressure [De56]. The vapor density increases with pressure, so that at a given core vapor mass flux, the vapor velocity decreases. The reduced vapor velocity diminishes the level of shear at the liquid-vapor interface, inhibiting beat transfer through the liquid film. The correlating parameter, $1 / X_{t t}$, decreases with increasing pressure so that again, the predicted a would decrease.

The opposite behavior associated with boiling vs. convection/evaporation might serve then as a line of demarcation between the two mechanisms. The data of Toral with organic mixtures showed a proportional dependence 
between $a$ and pressure, even at 'high quality' $(x=.3)$. This suggested the dominance of nucleate boiling in his experiments.

\subsection{Summary of Experimental Evidence: Presence of Hysteresis}

Hysteresis has been found sometimes in the pool boiling of pure fluids. Different superheat requirements were needed to initiate boiling when an experiment was conducted first with increasing and then with decreasing heat fluxes. Murphy and Bergles [Mu72] suggested that in subcooled flow boiling, high heat fluxes activated small cavities and bubbles from these cavities then migrated and activated 1 arge cavities. These large cavities remained active while the heat flux is reduced. On the upward heat flux traverse, the large cavities were considered fully wetted. It was noted that in subcooled flow boiling, a reduced hysteresis effect should be expected due to the steeper temperature profile; large cavities with trapped vapor may remain inactive due to the profile.

The presence of hysteresis then might confirm the presence of nucleate boiling. No parallel process occurs with convective evaporative flows.

\subsection{Summary of Literature Review: Mixtures}

The introduction of a second component has several consequences in the analysis of the onset and suppression of nucleate boiling, as described in detail in Appendix $4 \mathrm{E}$.

First, the terms of the applicable equation (4-1) are changed in value. Surface tension may be drastically affected by even small additions of a 
second component (e.g., figare 1-4 with refrigerant and oil). The theoretical val de of $\mathrm{dP}_{\text {sat }} / \mathrm{dT}$ is less for mixtures than for an equivalent pure fluid, suggesting an increased superheat requirement, how ever, the actaal superheat requirement may be less than for either pare camponent, due to the change in surface tension

Tora1 [To79] has attempted a theoretical study of the effect of turbolence damping near the 1 iquid/vapor interface in thin film, shear driven flow. He concludes that with organic fluids and organic flaid mirtures sufficient superheat $\$ i 11$ be available to initiate nacleation ander common conditions of heat and mass flur. If tarbulence damping exists, nucleation $\nabla i 11$ be even more 1 ike $1 \mathrm{y}$, as an additional resistance erists to transfering the heat aw ay from the wall region However, the boundary conditions used by Toral are flawed (see Appendix 4E). This conclusion may then be questioned, though not necessarily rejected $\nabla i$ th his problem being correctly reposed.

Thame and Shock recently reviewed the effect of composition on the ONB point and boiling site density [Th82]. While fewer sites are active With mirtures, the difference is not systematical1y related to $|\bar{Y}-\bar{X}|$, though mass diffusion is a likely contributor to change in boiling site density. In some onset of nucleate boiling studies, mass diffusion seen as an important factor and in others, the ONB point was anaffected by composition. 
To date, no one has analyzed, either theoretically or experimentally, the nature of the concentration profile in flow boiling with the presence of nucleate boiling, and the consequent mass transfer resistances in the 1 iquid and vapor streams. With ebullition in flow boiling, the 1 iquid surrounding the bubbles in the wall region will be depleted of the more volatile component. This will tend to increase the superheat requirement, i.e., make it easier to suppress bubble growth. However, at the same time, any resistance near the film interface (turbulence damping, both mass and thermal diffusion) will inhibit surface evaporation, so that more superheat might be available near the wall. A further complication is that the conductivity of the two fluids might be disparate, so that upon depletion of the more volatile component around a bubble, heat might be conducted more readily or with more difficulty through the viscous sublayer. The ultimate effect of these multiple competing processes has not been studied analytically for flow boiling.

\subsection{Comparison of Experimental Results to Theory}

\subsubsection{Application of Conventional Theory to Pure Refrigerants and Refrigerant Mixtures}

The Hsu/Collier and Pulling suppression criterion was applied to the two pure refrigerants ased in this report. The pressure gradient, aceded to determine the wall shear stress and subsequently the thickness of the viscous sublayer, was estimated using the Martinelli-Nelson/Chishol correlation. Contact angle was assumed to be $35^{\circ}$, typical for refrigerants [St82]. A flow chart of the calculation is given in Figure 4-2. 
Measured mass fluxes and pressures were used. Properties were estimated using the equations of Chapter 2. Assuming all cavities to be available, the criterion suggests a very small superheat requirement.

Instead, a heat flux needed to activate a critical cavity size of $1.0 \mu m$ was calculated. Both Polley [Po82] and Stephan [St80] have offered this size as a rough guideline for refrigerants. Cavities of size greater than 1.0 um were assumed to exist either in an insufficient number to affect the heat transfer, or to be fully wetted by refrigerant. This assumption then modifies the bssic suppression criterion, so that a greater wall superheat is needed to initiate boiling than if all sizes were available in large numbers and were onwetted.

Three factors complicate the analysis, leading to substantial uncertainty. First, the estimate of the critical viscous sublayer thickness is not exact. It depends both on the determination of pressure drop and on a selected critical value (Collier and Palling's $\mathrm{y}^{+}=$ 7). Secondly, the selection of a critical cavity size may be in error. Collier has suggested $0.5 \mu \mathrm{m}$ as a rough guideline for refrigerants [Co80]. This leads to a larger estimate of the suppression heat flax. Thirdly, vaporization may take place nearer the bubble base even in the absence of vaporization at the bubble cap. The required heat flux in this case rould be less than as calculated. These complications lead to an estimated uncertainty of about $+/-40 \%$. Despite the large uncertainty, it will be shown that the criterion can be used with success. 
Figure 4-3 displays samplo rosults of the procedure, showing the effects of changes in pressure and mass flax. As pressure is 1 owered or as uass flux is raised, the criterion predicts a greater supprossion beat flor If the experimental heat flur was bel ow the calculated 'suppession beat flux' $\nabla a 1$ ue, the heat transfer coefficient should be independent of heat flux and instead depend proportionately on mass flux and quality. Conversely, if the criterion prodicts sufficient heat flux, a dopendence on heat flur should be observed.

A similar analysis can be applied to mirtures and was applied bere to the R13B1/R152a mixture. The governing oquation is offoctively the same for mirtures, except as noted in Section 4.7. Properties such as $\Delta \bar{v}_{v}$ and $\Delta \bar{h}_{\nabla}$ were eral wa ted at different compositions, so

$$
\begin{aligned}
\Delta \bar{h}_{\nabla} & \cong \bar{h}_{\nabla}(\bar{Y} *)-\bar{b}_{1}\left(\bar{X}_{B}\right) \\
\text { and } \Delta \bar{\nabla}_{v} & \cong \bar{\nabla}_{\nabla}(Y *)-\bar{\nabla}_{1}\left(\bar{X}_{B}\right)
\end{aligned}
$$

The stppression critorion was then appliod, assuning the liquid layer vas vel 1-mizod, ie., withont an mass transfer rosistance. It vas therefore implicitly assumed that the turbalence in the 1 iquid film supplied a ufficient concentration of the more volatile component to the brbble interface. This is a very conservativo assuption for pro dicting suppression, since boiling site density is likely to be reducod by the mirtare. The above treatmont then considers the wixtures as an equivalent pure fluid. Tro possiblo approachos to correct for mirtare effects on boiling site density wero al so bypothosized. An exact solation is available in the literature for the grow thate of a isolated 
spherical bobble located in quiescent, uniformly superheated 1 iquid. When the basic equations are solved, a reduction in bubble growth rate for a mirture over that of an equivalent pure fluid can be calculated. Chapter 7 discusses this problem in more detail; the reduction due to mass transfer resistance, is given in equation (7-0). Then this factor, $C_{b u b}$, is applied to the suppression criterion, the required heat flur to sustain ebulition is raised, typicalig by about $2 \% \pi i t h a_{T} / a_{D}=5$ or 40-80\% with $a_{T} / a_{D}=60$ as shown in Figure 4-4. The concentrations shown are 'feed concentrations', i, $\theta_{0}$, the initial concentration of a subcooled 1 iquid being evaporated/boiled. At the 1 arger val ue of $a_{T} / a_{D}$ the mixture effect mag be sufficient to increase the suppression heat flur above other pare components. The assumption with this approach is that mass diffusion is the sole reason for reducing boil ing site density.

An al ternate correction factor can be calculated from the 1 iterature on pool boiling of mirtures. As described in Chapter 7, equation (7-0) underpredicts the meansred reduction in heat transfer coefficient for the pool boiling of mirtures. A variety of empirically based correction factors are available for predicting the reduction (Table 7-1). None of the methods of Table 7-1 has been tested for flon boiling of mixtures. However, the method of Stephan and Korner [St69] has been used $\nabla$ idely with some success in pool boiling. When it is used, the increase in the suppression heat flux, $Q_{\text {sup }}$ may be sufficiently substantial to raise the suppression heat flux for mixtures above that of either pure component (figure 4-5). 


\subsubsection{Experimental Results: Pure Refrigerants}

The first experimental results to be examined are those for pore R152a at 1 ow pressure; the tests were conducted with Rig \#1. If measured data taken at the same mass flux and pressure but different heat flux levels yield identical heat transfer coefficients, then boiling is completely suppressed. Unfortunately, pressure was not held strictly constant with Rig \#1; however in some cases, the pressure variation was small enough to allow such comparisons. The suppression point can also be inferzed approximately by the dependence of da/dx on quality. When da/dx becomes strongly positive, forced convection/evaporation is dominant. Complete or near complete suppression should occur in this range. Figures 4$6 a, b, c$ plot the effect of heat flux at constant flow rate. A strong heat flux dependence is observed at low qualities, but this effect is reduced with increasing quality. Shown also is the prediction of the suppression criterion for the heat flur 1 evel required to initiate boiling as a function of quality and the given flow rate. When the criterion states that boiling should be suppressed, the measured data are shown in upper case; lower case letters indicate the criterion predicts sufficient heat fluz to sustain boiling of a $1 \mu$ m cavity.

The criterion predicts quatitatively the quality at wich camplete suppression occurs. Apparent discrepancies are seen in only two cases (runs OLDH201 and OLDH203) where the error is within the range of uncertainty in the method. 
Both the figures and the criterion show that as mass fluz is increased, suppression occurs at lower quality. A strong dependence on mass flur is observed suggesting a convective/evaporative process to be dominant.

Figure 4-7 shows the effect of pressure on the heat transfer coefficient. The experimental value is greater at high pressure initialiy, bot the difference is reduced or disappears at high quality. These results indicate nucleate boiling, easier to achieve at high pressure, to be dominant at 1 ow quality. The boiling process then diminishes in favor of forced convection evaporation at high quality. The transition point is a function of heat flux: the lower the heat flux, the 1 ower the quality at which nucleate boiling diminishes. The initial decrease of the heat transfer coefficient despite increasing quality has been observed by several other researchers [Ch67, Ch66a]. Mesler's proposed explanation of the heat transfer process cannot predict this observation; it in fact suggests the opposite behavior.

These results are consistent with the traditional theory. They suggest that nucleate boiling can be suppressed even at significant heat flux levels ith relatively low conductive fluids such as refrigerants; however suppression becomes much more difficult as pressure is increased.

Similar Rig \#1 tests were done with pure R13B1 at the high pressure level since the condenser could not be made colder to reduce pressure. The results on Figare 4-9 show little quality dependence and suggests 
nacleate boiling domination. These results are consistent ith the criterion.

Al 1 pare refrigerant tests with Rig \#2 were done at a pressure aronnd 4.75 bar (+.2/-.02 preheat, $+/-.05$ test section). Measurements were made in the proheat section where heat flur lovels were varied over a wider range (10-95 kW/ sqm R152a; 10-55 kW/sqm R13B1). Representative preheat data is shown on Figures 4-9 and 4-10. Here a clear dependence on heat $f 1 \mathrm{nz}$ is observed, indicating a strong nucleate boiling contribation. ${ }^{1}$ The suppression criterion predicts that at this pressure 1 evel the actual heat fluxes vere suficient to initiate nucleate boil ing. There is a very slight dependence on wass flax.

The test section data is for 1 ow heat flax bat high vapor quality. The date is shown on Figures 4-11 and 4-12. Tests were done with R1s2a and 10 and $20 \mathrm{kT} / \mathrm{sqm}$ in the test section at throe different mass fluxes. Figure 4-11 a show that only a weak dependence on heat flar occurs. The dependence on mass fluI is seon clearly in Figure 4-12. The depondonce on quality increases with increasing mass flar as was observed with Ris \#1 and by others in the literature [An66]. These results sug80st the dominant vapor generation mechaniom is by evaporation, but that complete suppression may not be achieved. The criterion predicts sofflciont beat flux to prevent complete suppression

\footnotetext{
${ }^{1}$ The only substantial contradiction to this trend is ith heat flar above $50,000 \nabla / \mathrm{m}^{2}$, which gielded 1 arer heat trasfor coefficient than expected. The high heat flur initiated a departare fras aucleate boiling (DNB), with film boiling being the probablo heat transfer regime. DNB events were observed with pure R13B1 in other tests around the same heat flur.
} 
The test section data for R13Bl shows a dependence on mass flux at high quality, but not at low quality. Nucleate boiling dominates in the low quality region but the process is controlled by convection/evaporation at high quality. This conclusion is made tentatively however. The outlet temperature and pressure measurements for R13B1 tended to disagree (Chapter 3). Shown here are the results based on pressure measurements. The temperature based results show less mass flax dependence. In either case the dependence on mass flux is less than 0.8 which has been widely used in correlating a in pure evaporative flow. This suggests that some boiling is present, and is in agreement with the suppression criterion.

Fina11y, Rig \#2 a1 so allows a unique examination of the effect of a step change in heat flax, since preheat and test section heat fluxes were set independently. If the process were completely independent of heat flux, then one would expect the measured heat transfer coefficient in the test section to be greater than that measured in the preheat section, doe to a continued increase in vapor quality. If on the other hand, heat flux was dominant, then a large change in heat transfer coefficient should accompany a large step change in heat flax.

One can compare the ratio of measured heat transfer coefficients between the last preheat measuring station and the test section ith the ratio of preheat to test section heat fluxes. Figure 4-13a plots such a conparison. Note that as the preheat flux is raised relative to the test section, the heat transfer coefficient ratio increases also, but to a 
lesser degree. These comparisons suggest that both mechanisms, boiling and evaporation, are contributing to the beat transfer (at this pressure leve1), though evaporation is dominant. It thus serves as a further verification of the suppression criterion.

\subsubsection{Experimental Results: Mirtures}

Four suppression methods were tried for mirtares. The first treats the mirture as an EPF. The next three account for mass transfer resistance in various ways. The 'exact' and SK methods were displayed on Figures 4-4 and 4-5, respectively. The last way is empirical, based on a pool boiling method of Thome. As will be shown in Chapter 7 , this method predicts the measured heat transfer coefficient best for a large number of cases.

Figures 4-14 through 4-17 show the effect of heat and mass flux on the heat transfer coefficient at fixed flow rate and initial composition for Rig \#1. A strong dependence on heat flax is clearly observed; a weaker dependence on mass flax is also seen. The dependence on pressure appears greater than the dependence on mass flur; this is an indication that boiling is not only present, bot dominant.

All criteria predict sufficient heat flux to sustain ebullition. The only exception is Thome's method wich predicts suppression at the lower weight compositions of R13B1, and high quality and mass flux. The data

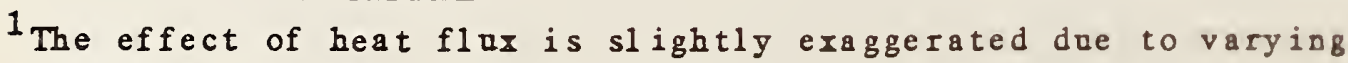
pressure from test to test.
} 
indicate the Thome prediction is in error in these cases. However, the error is within the range of uncertainty of the method.

The Rig \#1 tests were conducted with various pressure levels. In Rig \#2, pressure was maintained generally around 4.75 bar level. The preheat tests were conducted again with heat fluxes varied between 10 and $90 \mathrm{~kW} / \mathrm{sqm}$. As before, a dependence on heat flux was observed indicating nucleate boiling to be present (representative Figures 4-9 and 4-10). Al1 suppression criteria predicted sufficient superheat availability. There is only a weak dependence on mass flux.

Some hysteresis tests were al so performed with 0.80 wt R13B1. Measurements were taken with preheat fluxes raised (to a maximum of $40 \mathrm{~kW} / \mathrm{sqm}$ ) and then lowered. No change in heat transfer coefficient was observed in the preheat section or the test section (at $10 \mathrm{~kW} / \mathrm{sqm}$ ). Despite the lack of hysteresis, some effect of preheat flax can be observed.

The preheat results suggest therefore that boiling is not suppressed. For all concentrations, the suppression criteria are verified for the case of nucleate boiling existence.

Representative test section data ( 1 ow heat flux, high quality) is shown on Figure 4-18. The EPF method predicts $Q_{w}>Q_{\text {sup }}$ (boiling possible) at all compositions and flow rates. The 'exact' method with a Lewis number equal to 5 predicts complete suppression only at the highest mass 
flow rates and 1 ow compositions of R13B1. The exact method with Lewis equal to 60 and the empirical methods of Stephan and Forner and of Thome predict suppression at all compositions at high flow rates. The neasured data show a clear and strong dependence on mass flux. This indicates that convection/evaporation is strong in this region, but does not necessarily imply that boiling is absent.

As was done for pure components, the new approach of the effect of a step change in heat flux was examined. At low heat flux, the data shows a positive da/dx so that evaporation is much more effective, and could support the notion of complete suppression.

These results show that forced convection/evaporation effects dominate in the test section, but are inconclusive regarding complete suppression of nucleate boiling. If the fact that forced convection/evaporation is dominant is extrapolated to suggest complete suppression, then the EPF methods is incorrect. The exact (Lewis $=60$ ) and the empirical methods would then be validated. Evidence based on heat transfer correlations suggest complete suppression does occur with mirtures at these conditions. This evidence is discussed in Chapters 5 and 7 . Measurements at lower heat flux could clearly confirm this conjecture; however the accuracy of such measurements would have been poor.

\subsection{Conclusions and Recommendations}

1. Conventional suppression theory is supported by experimental tests as we11 as a critical review of the literature. Contradictor 
findings in the literature can in fact be explained by conventional theory. The alternate hypothesis of enhanced nucleate boiling with annular flow boiling is not supported analytically or experimenta11y.

2. At pressure, flow and heat flux levels of most residential heat pump evaporators, complete suppression of nucleate boiling is not commonly observed with pure refrigerants. However, as pressure is 1 owered, nucleate boiling may be absent even with 1 ow conductive fluids such as refrigerants.

3. The conventional suppression criterion is verified quantitatively for pure refrigerants. For mixed refrigerants the criterion was modified to include mass transfer resistance effects. The result is to 1 ower the heat flux at which complete suppression occurs. Methods were hypothesized from 'ezact' and pool boiling theory. The se methods were validated in a qualitative sense. It may be possible to have complete suppression for mixtures and not for either pure component.

4. The conclusions of Toral regarding suppression of flowing mirtures may or may not be valid. The problem should be reposed with the correct number of boundary conditions. Analytic development is al so needed which accounts for a revised 1 iquid concentration profile in the presence of nucleate boiling. Experimental data 
should be taken at sufficiently low pressure that one might expect complete suppression.

5. Incipient and suppressed boiling experiments need to be conducted for mized fluids in flow boiling. The literature is particularly sparse in this critical area. 


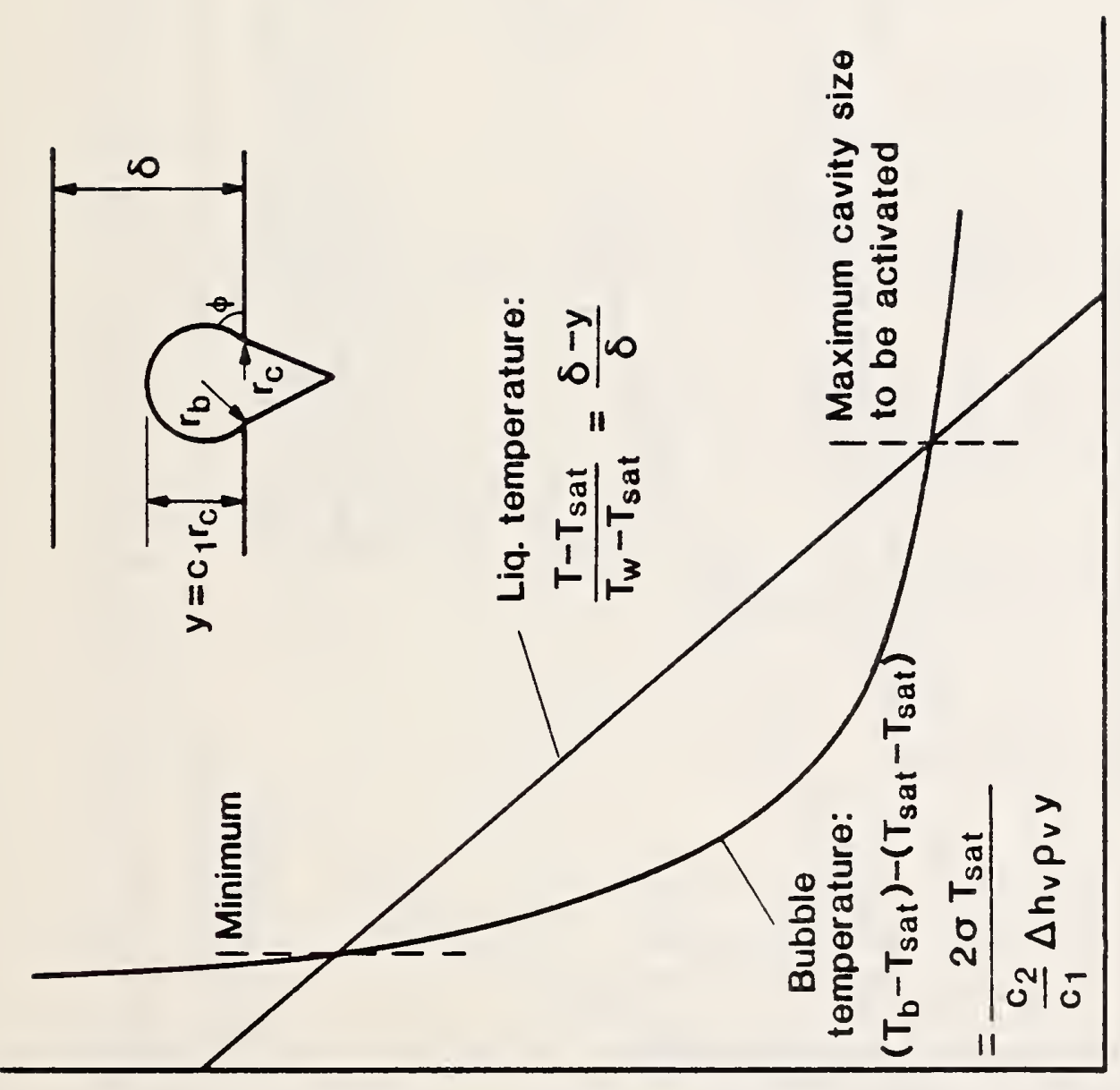

ปิ

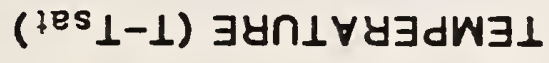

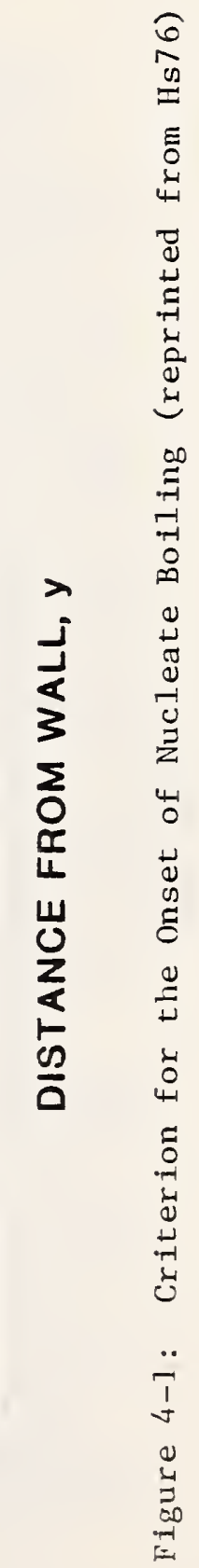




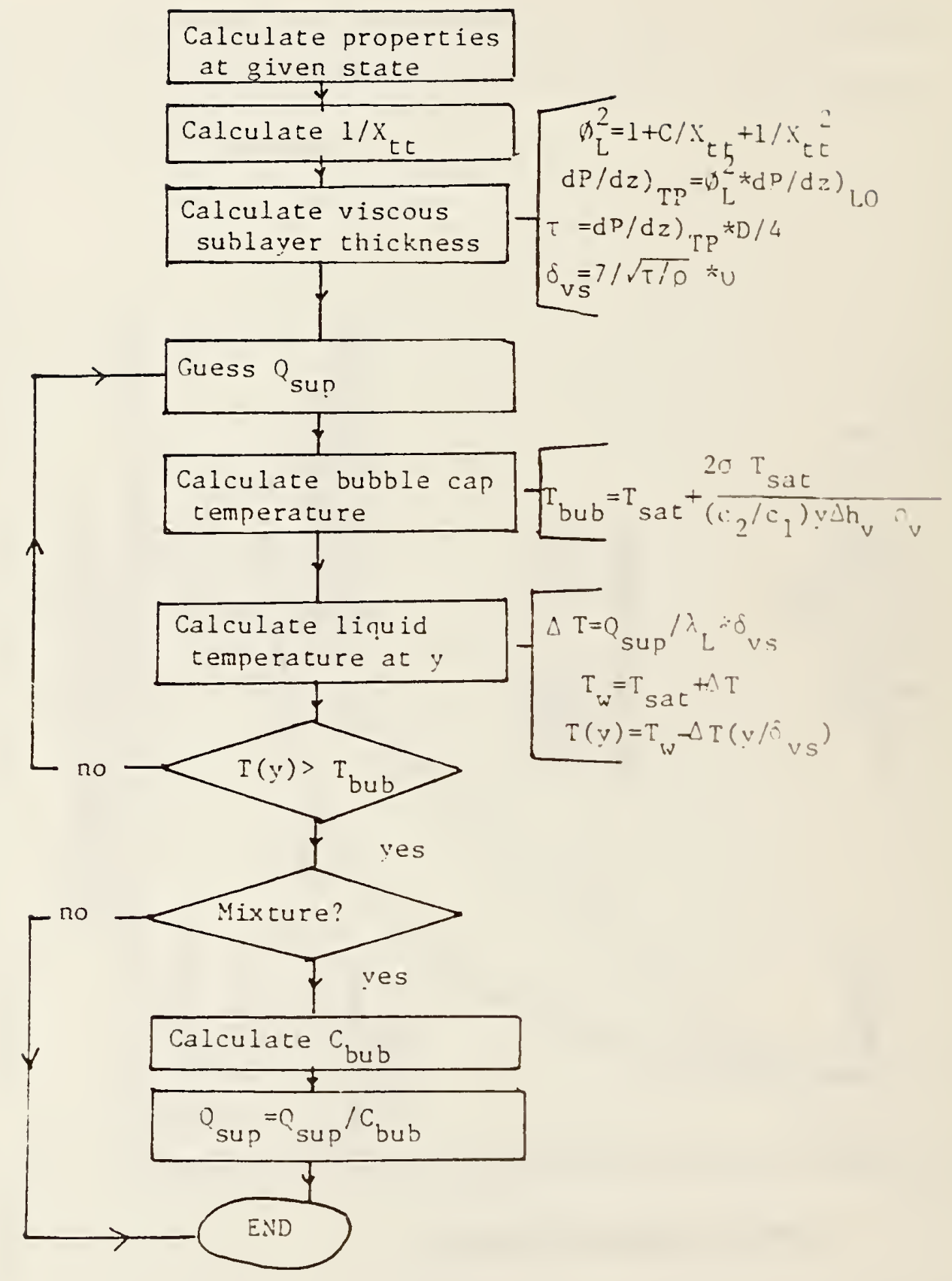

Figure 4-2: Flow Chart for Determinins. Sundression Heat Plux 


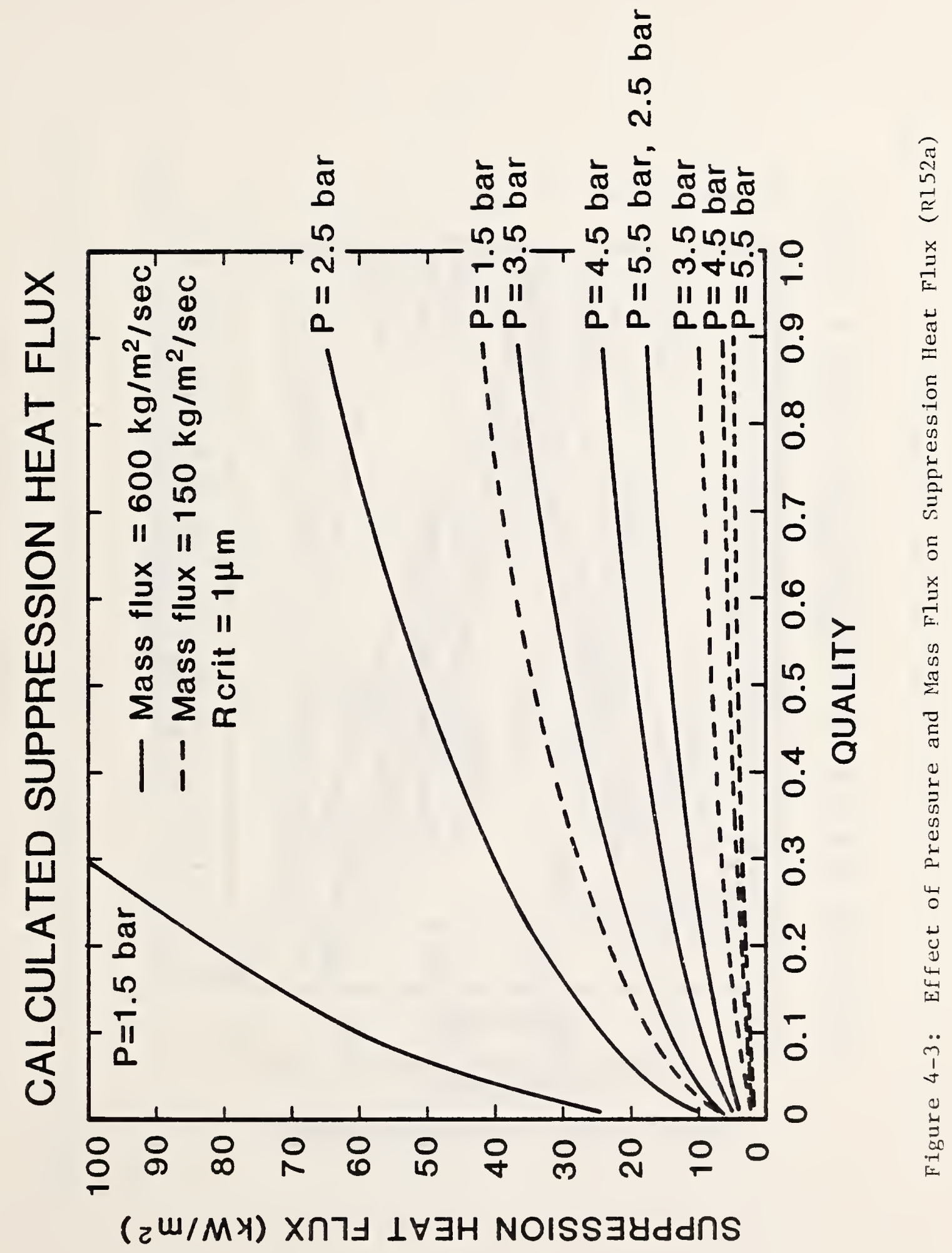




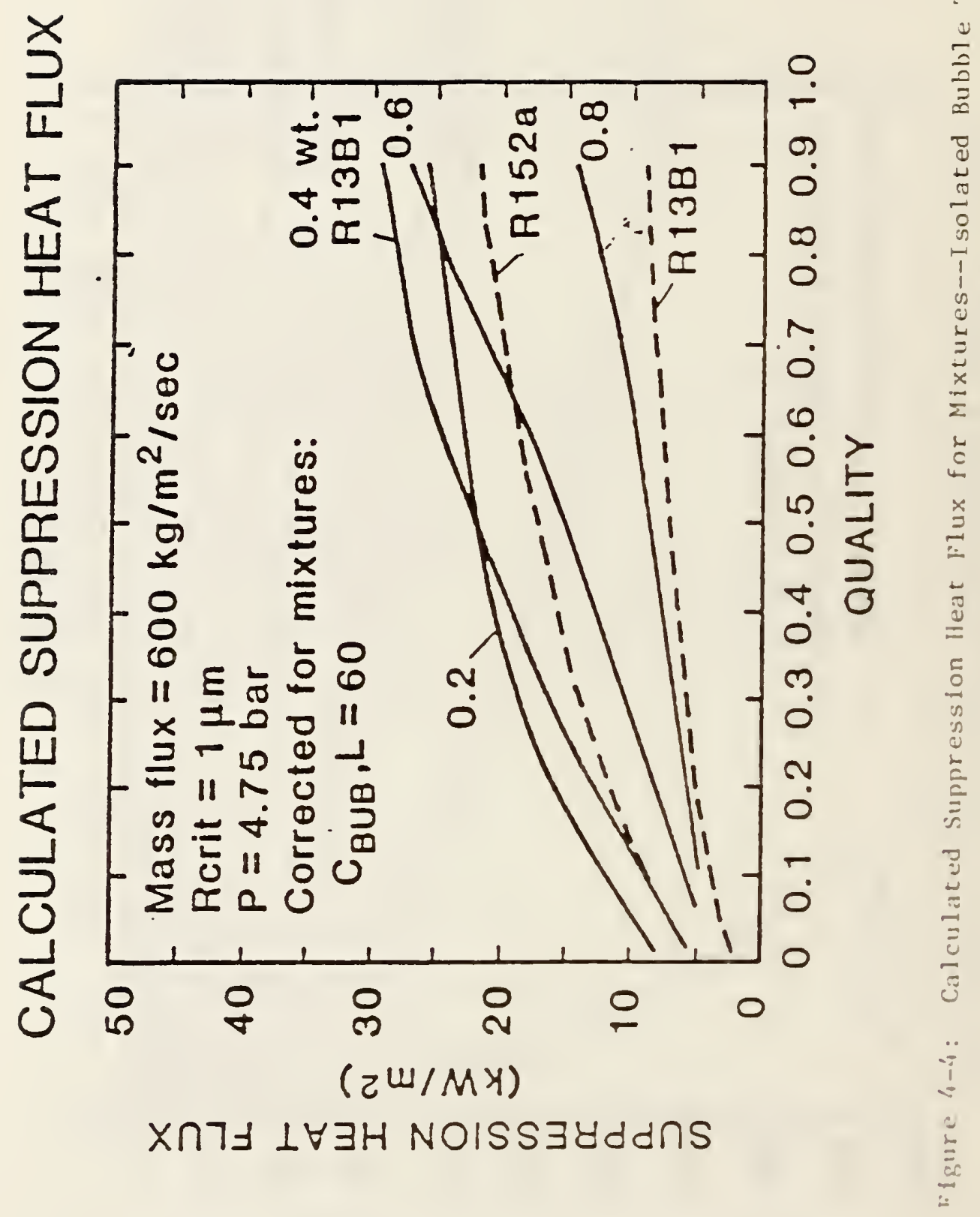




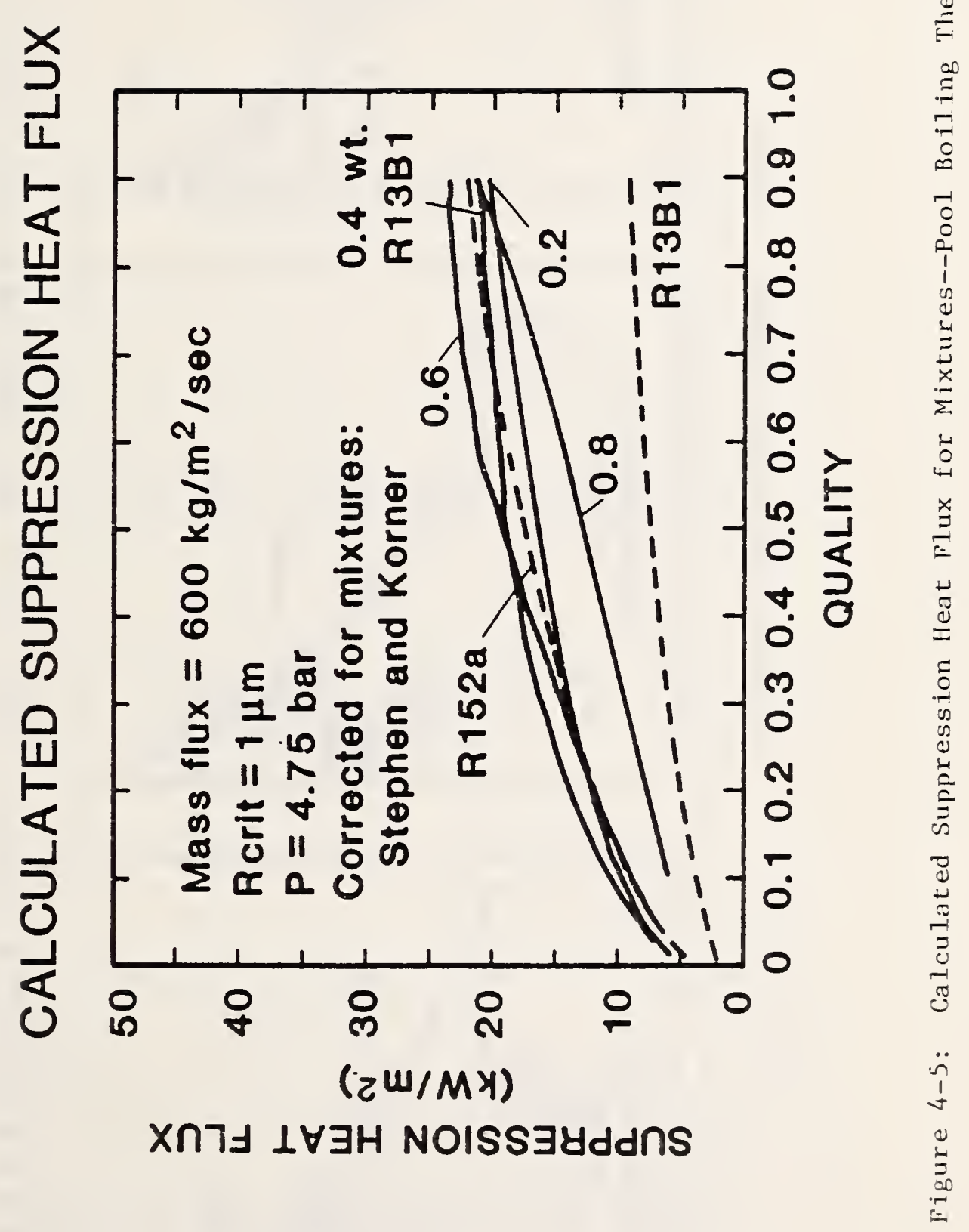


(X/
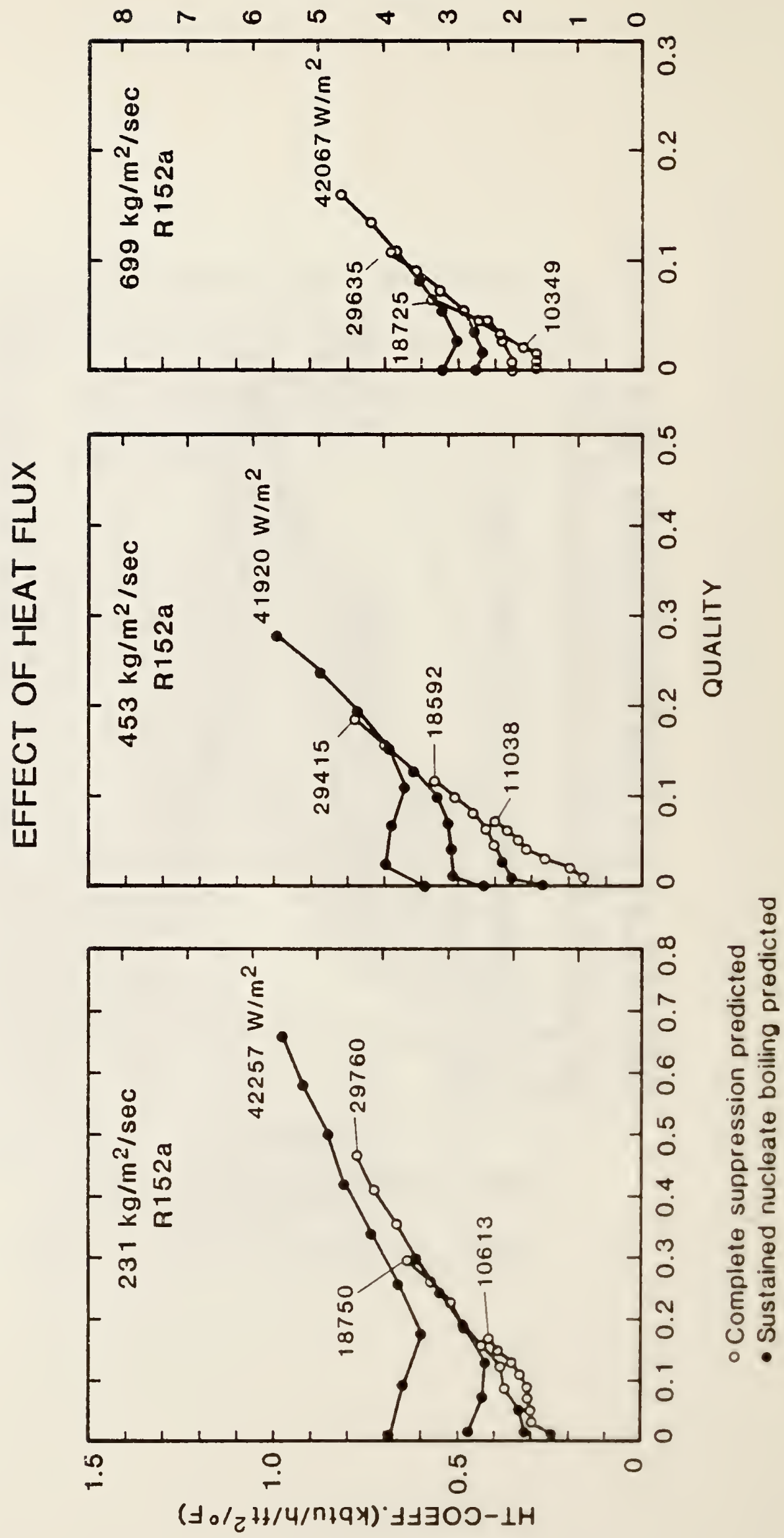

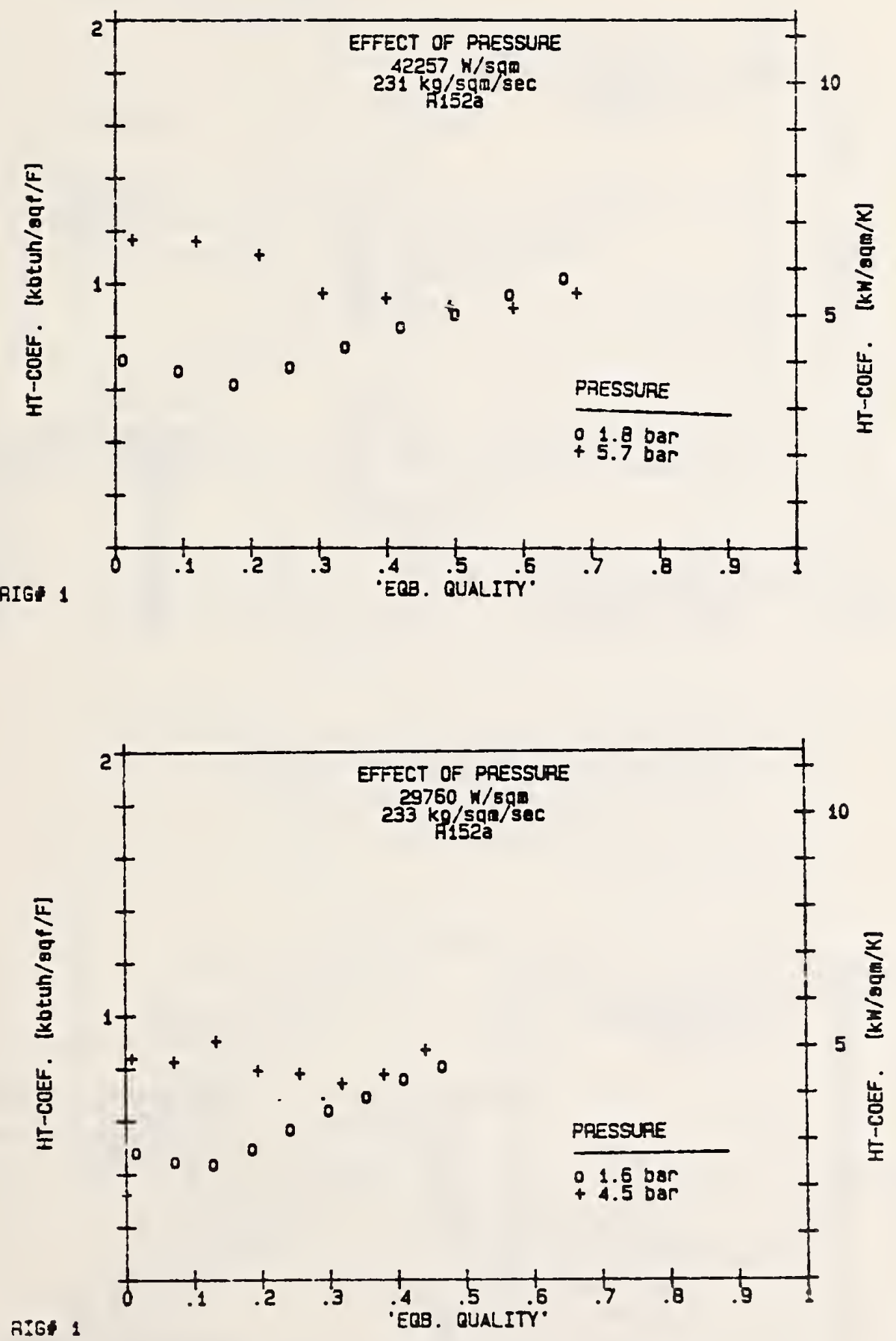

Figure 4-7: Effect of Fressure on Pure Rl52a. Nucleate boiiing dominates at high pressure, even at large quality. 


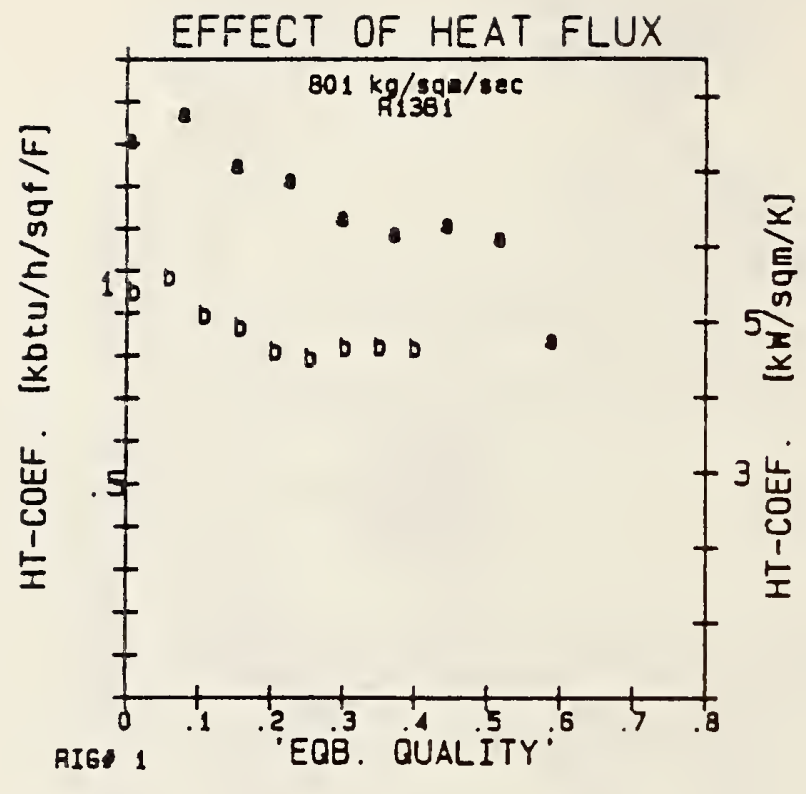

REAT FUUX

- $42203 \mathrm{H} / \mathrm{sqa} 16.4 \mathrm{bor})$ a 00285 b $29330 \mathrm{~m} / \mathrm{sq}$ (5.7 $\mathrm{ber})$ 200266

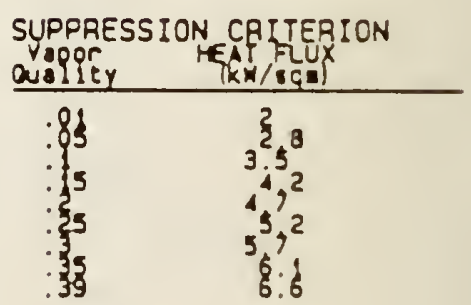

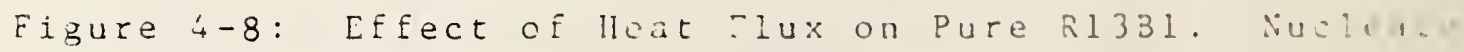
boiling comináles.

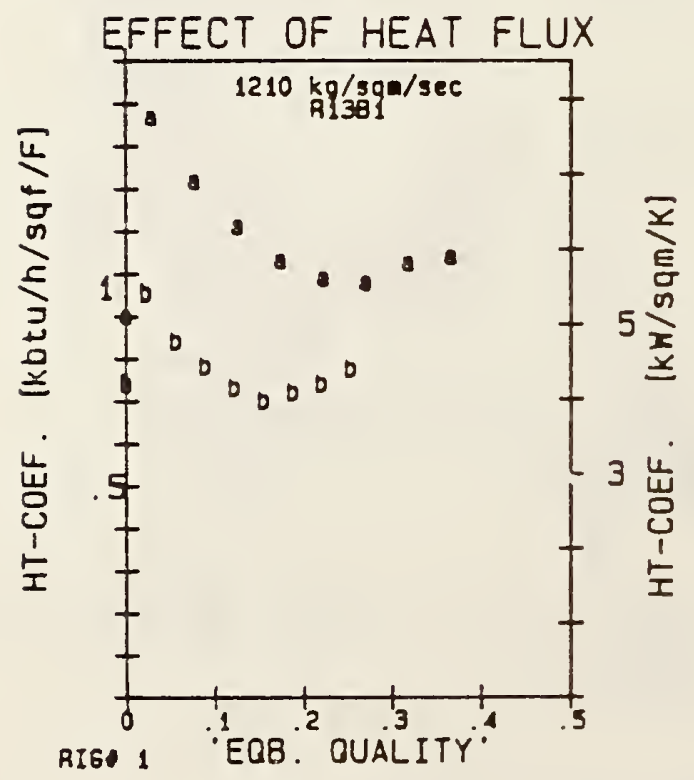

HEAT RUUX

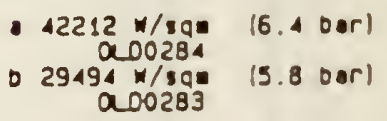

SUPFPESSION CRITER!ON guapor ik $/$ /squ

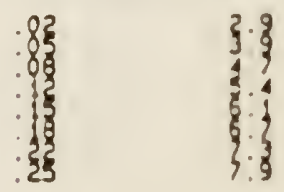




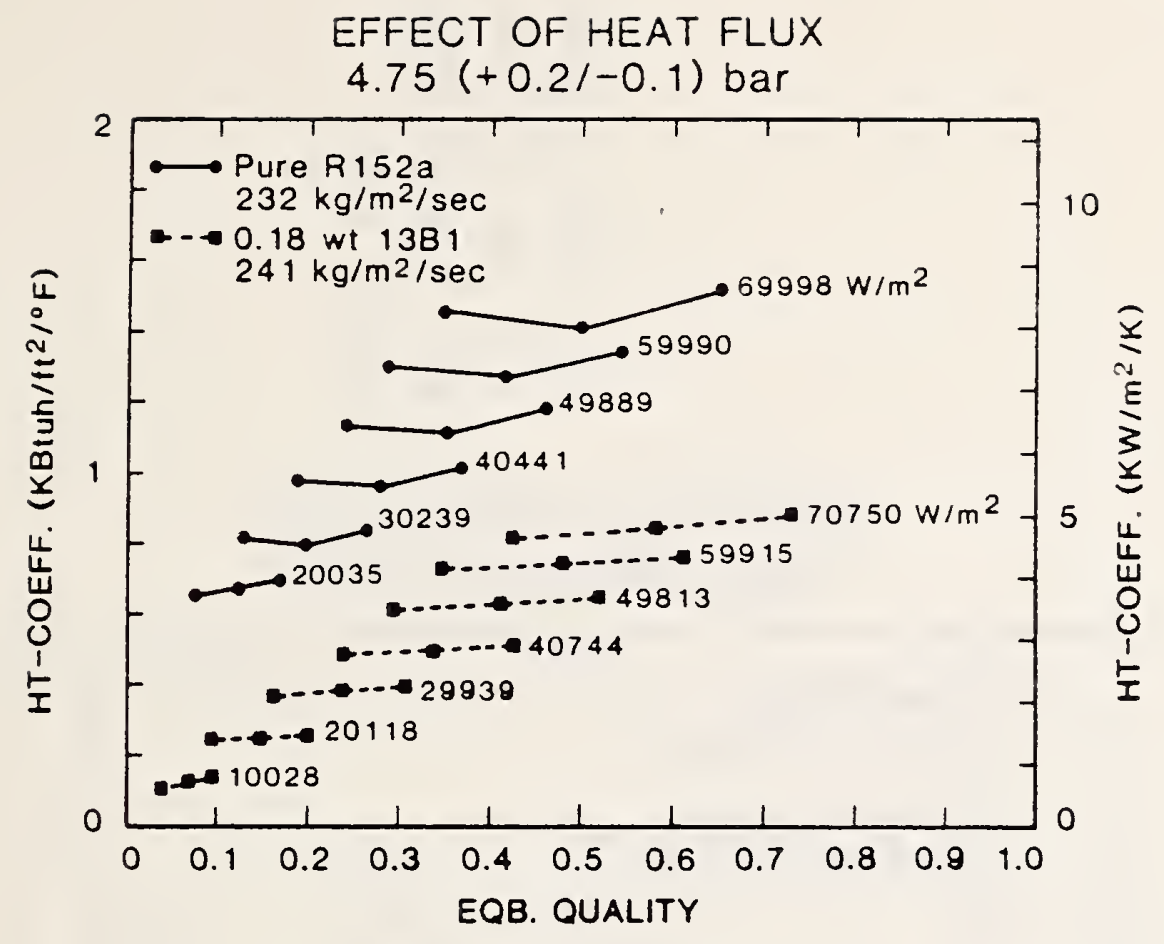

Eigure 4-9: Effect of Heat Flux on Pure R152a and R152a/R13BI Mixture: Preheat Data

EFFECT OF HEAT FLUX

$4.75(+0.2 /-0.1)$ bar

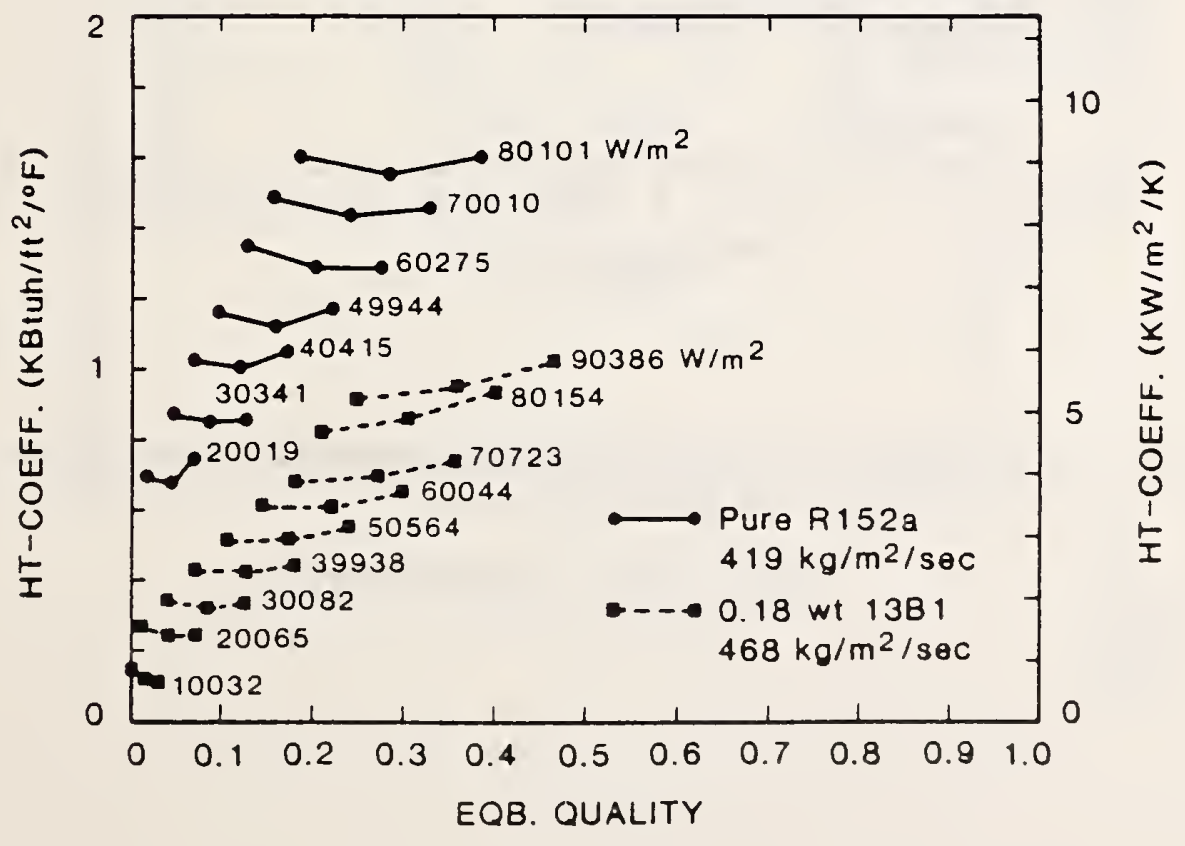


EFFECT OF HEAT FLUX

$4.75(+0.2 /-0.1)$ bar

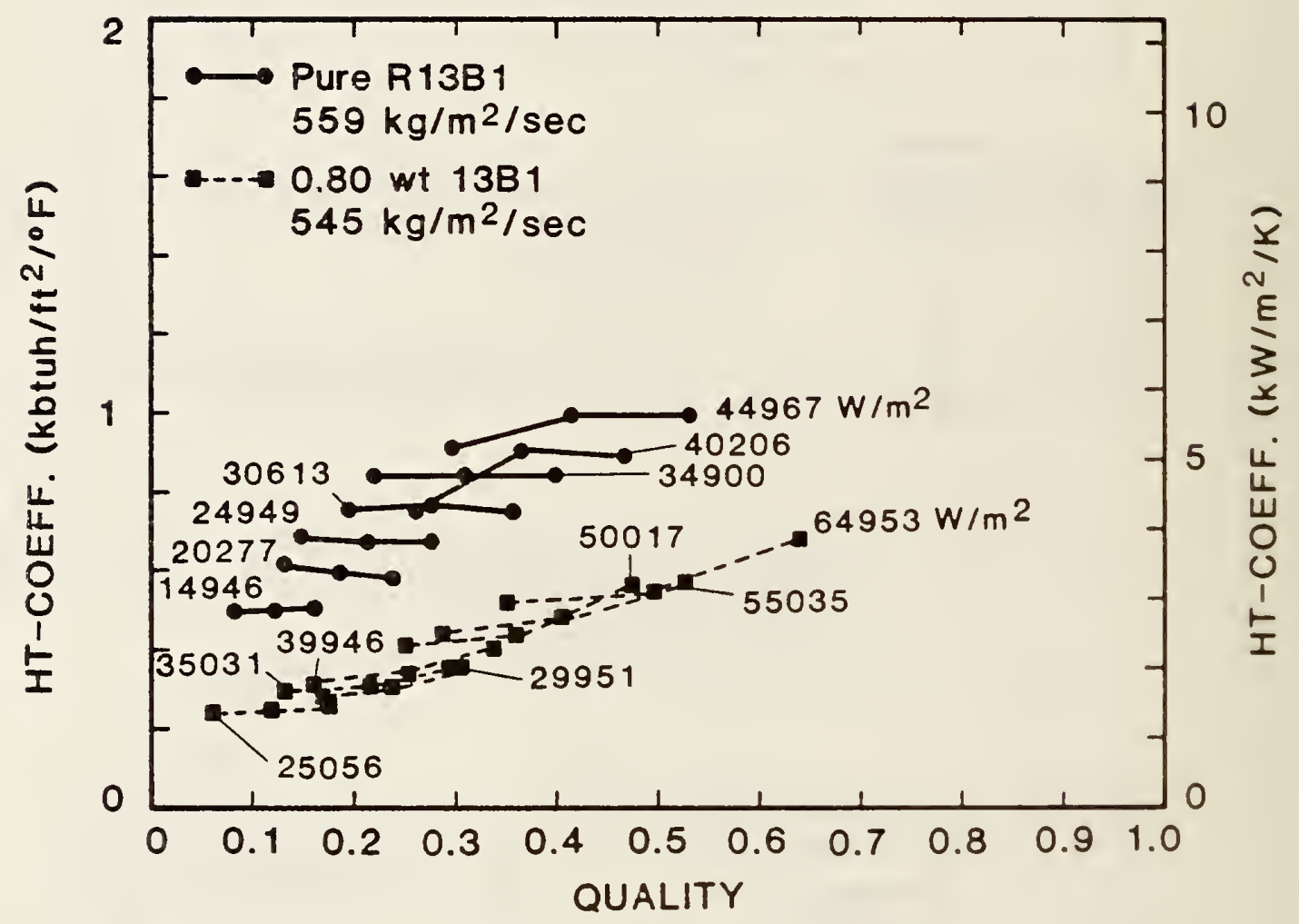

Figure 4-10: Effect of Heat Flux on Pure RI3BI and R152a/R13BI Mixture: Preheat Data 

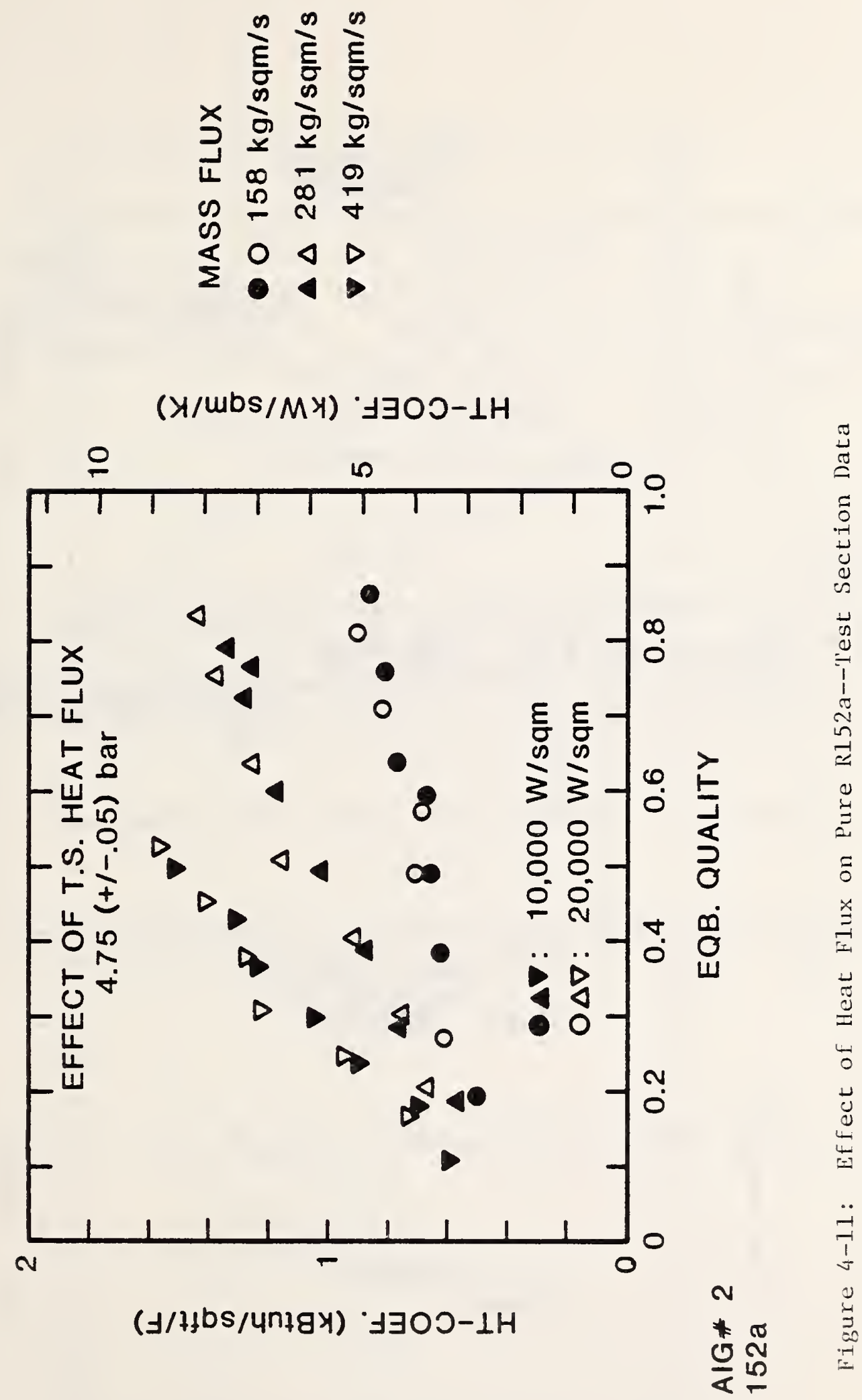


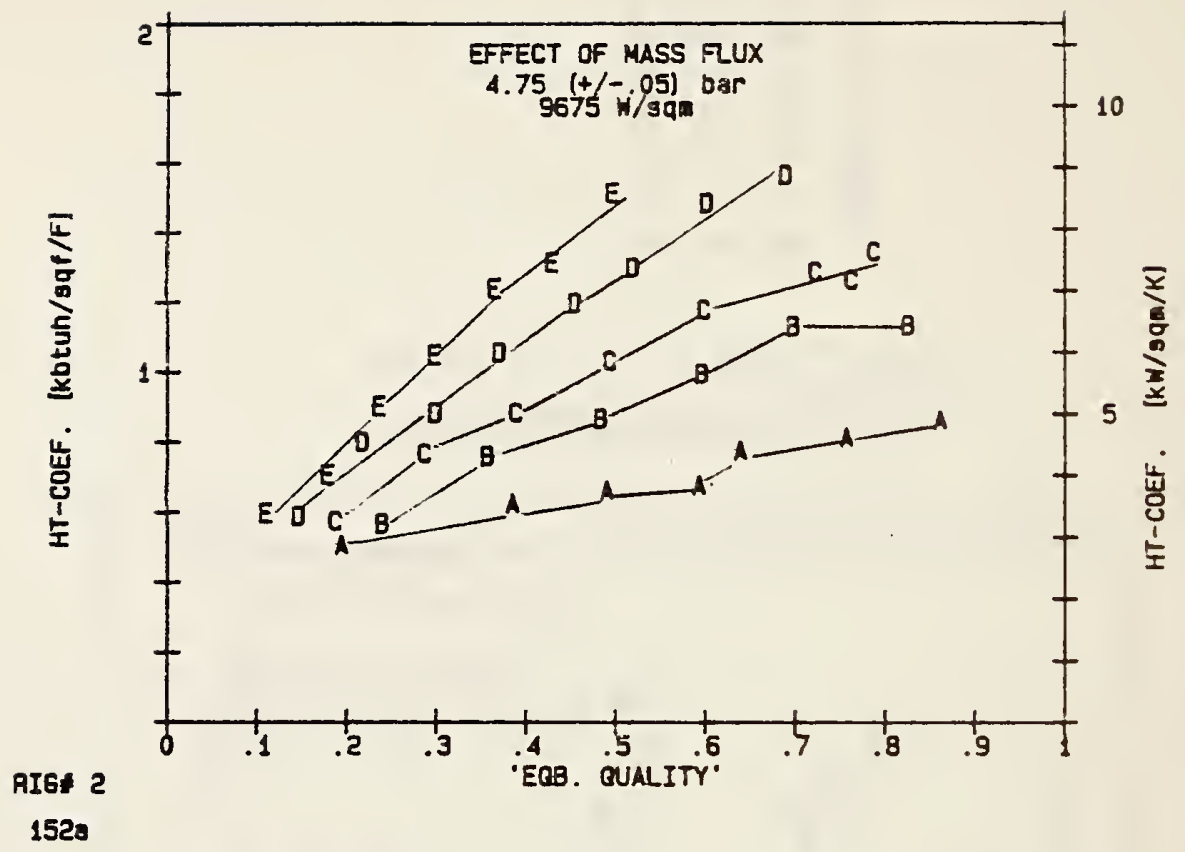

MASS RUX

1 $158 \mathrm{~kg} / \mathrm{sqn} / \mathrm{sec}$ 列 $339 \mathrm{~kg} / \mathrm{sam} / \mathrm{sec}$ $419 \mathrm{~kg} / \mathrm{sq}$ .

Figure 4-12: Effect of Mass Flux: Test Section Data

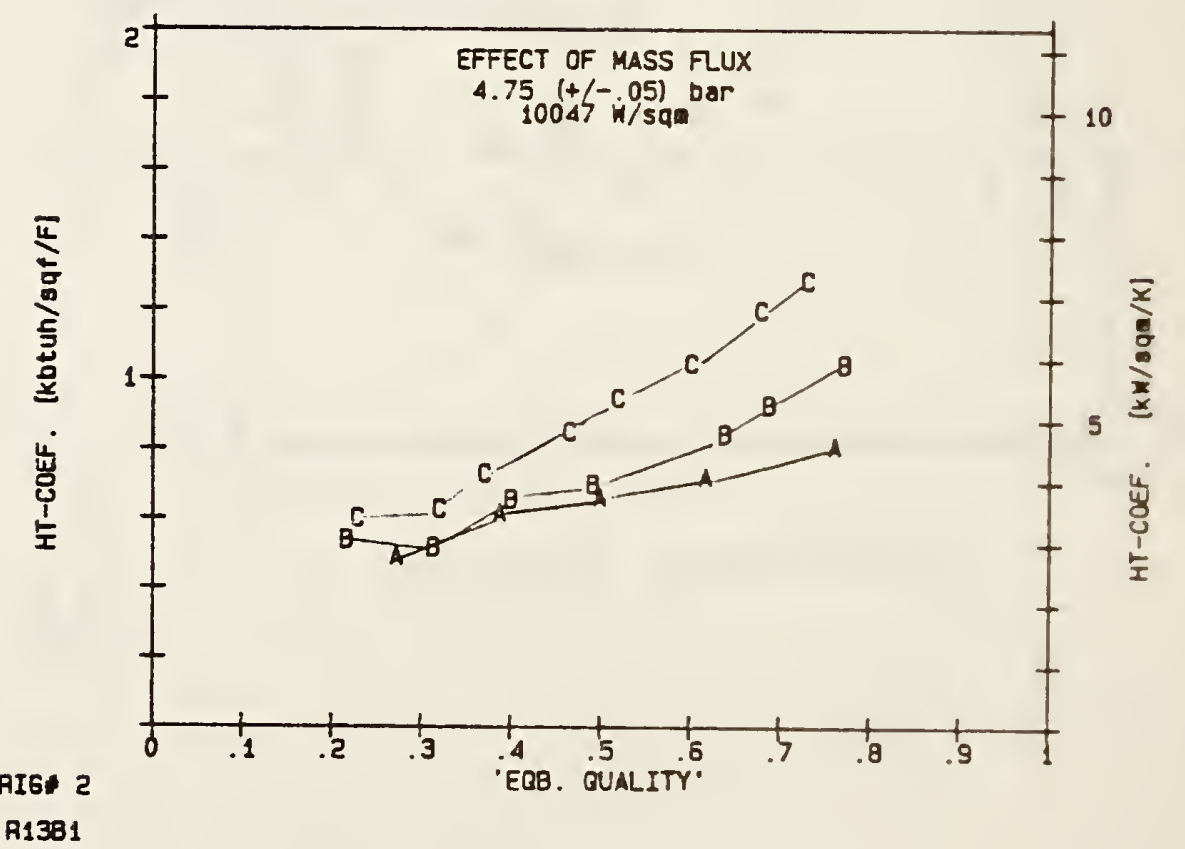

MASS FLUX

A $353 \mathrm{~kg} / \mathrm{s0 \textrm {m } / \mathrm { se }}$

C $559 \mathrm{~kg} / \mathrm{sqa} / \mathrm{se}$ 


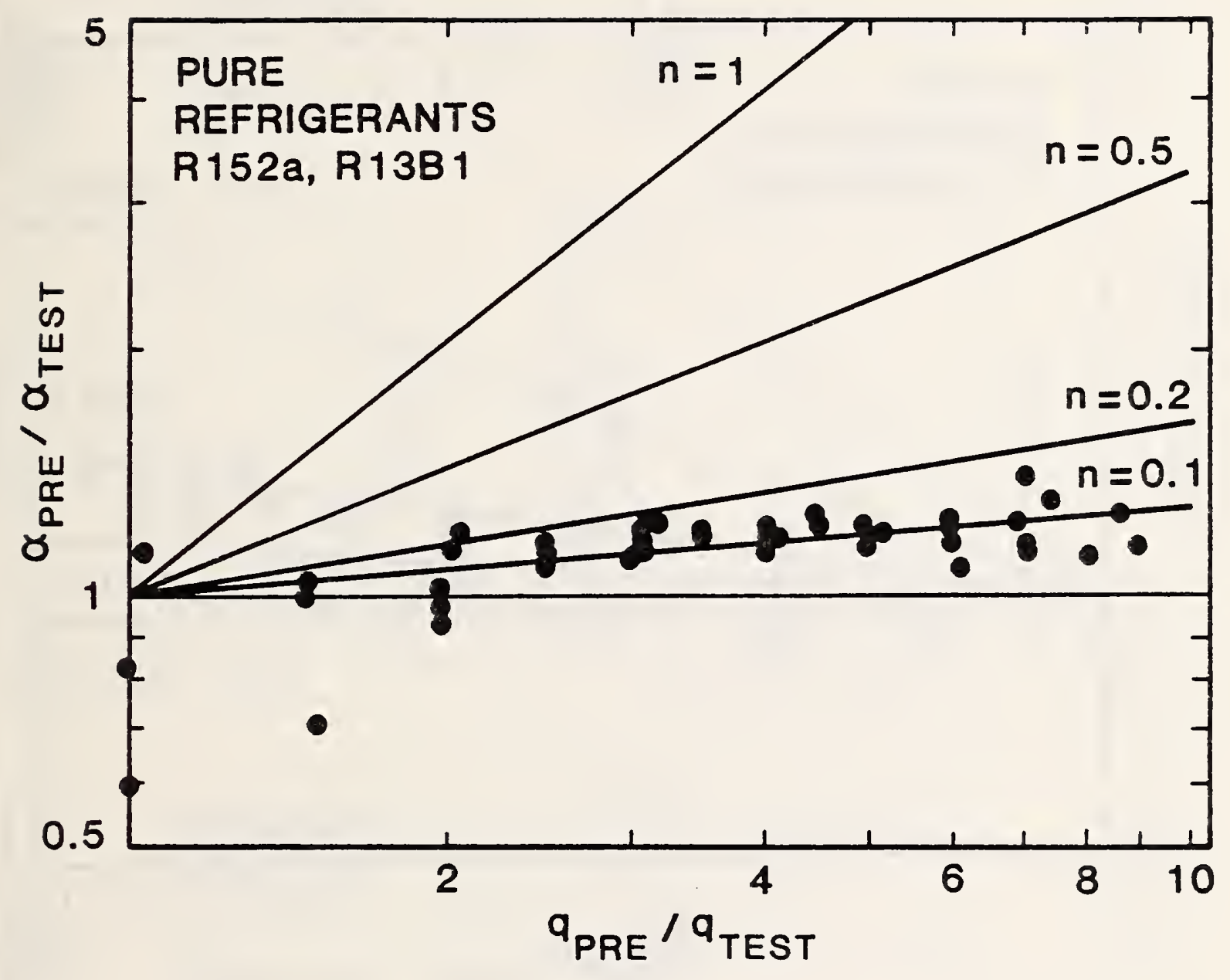

Figure 4-13a: Effect of Step Change in Heat Flux: Pure Refrigerants 


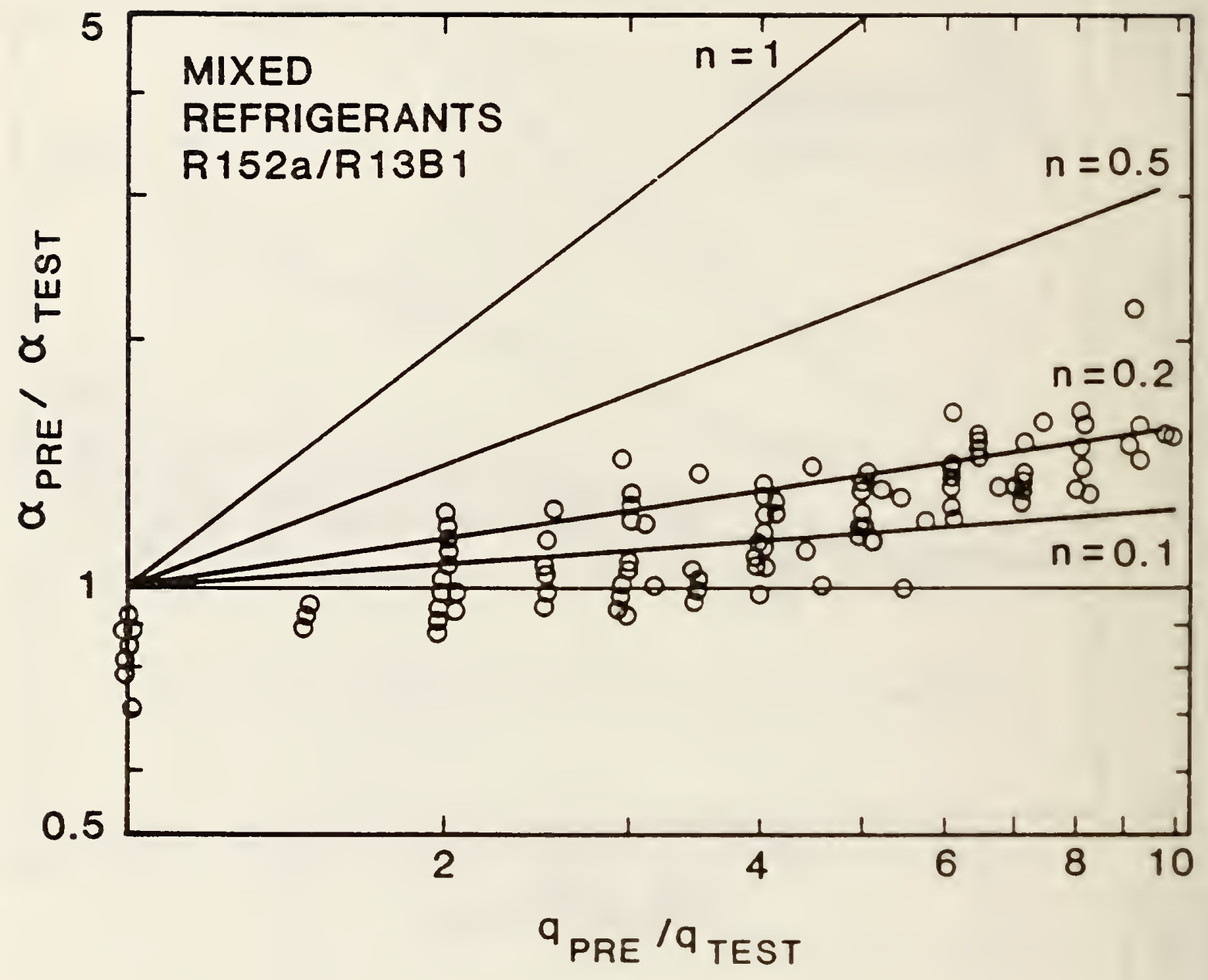

Figure 4-13b: Effect of Step Change in Heat Flux: Yirtures 

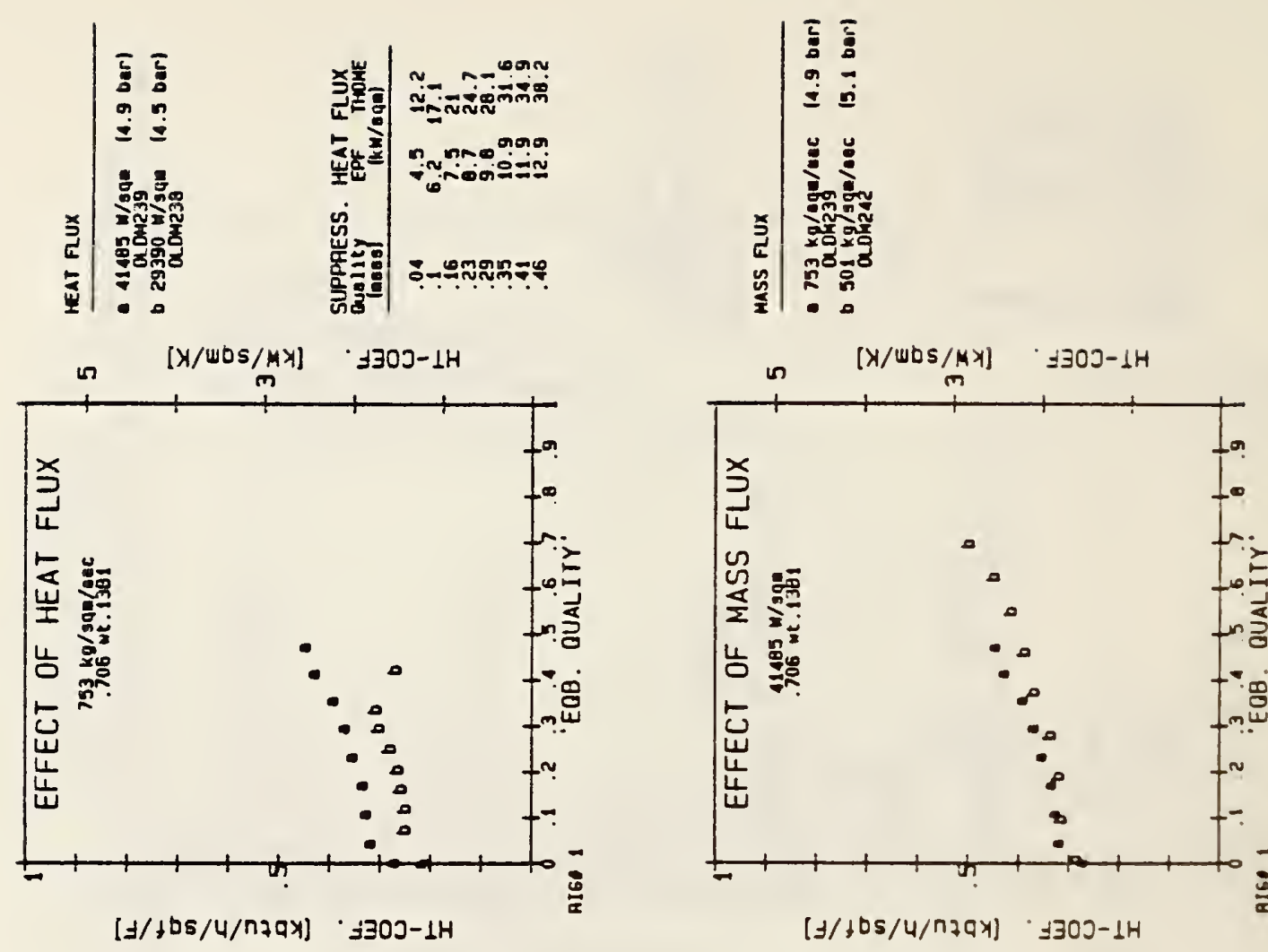

a
0
0
0
$\pi$
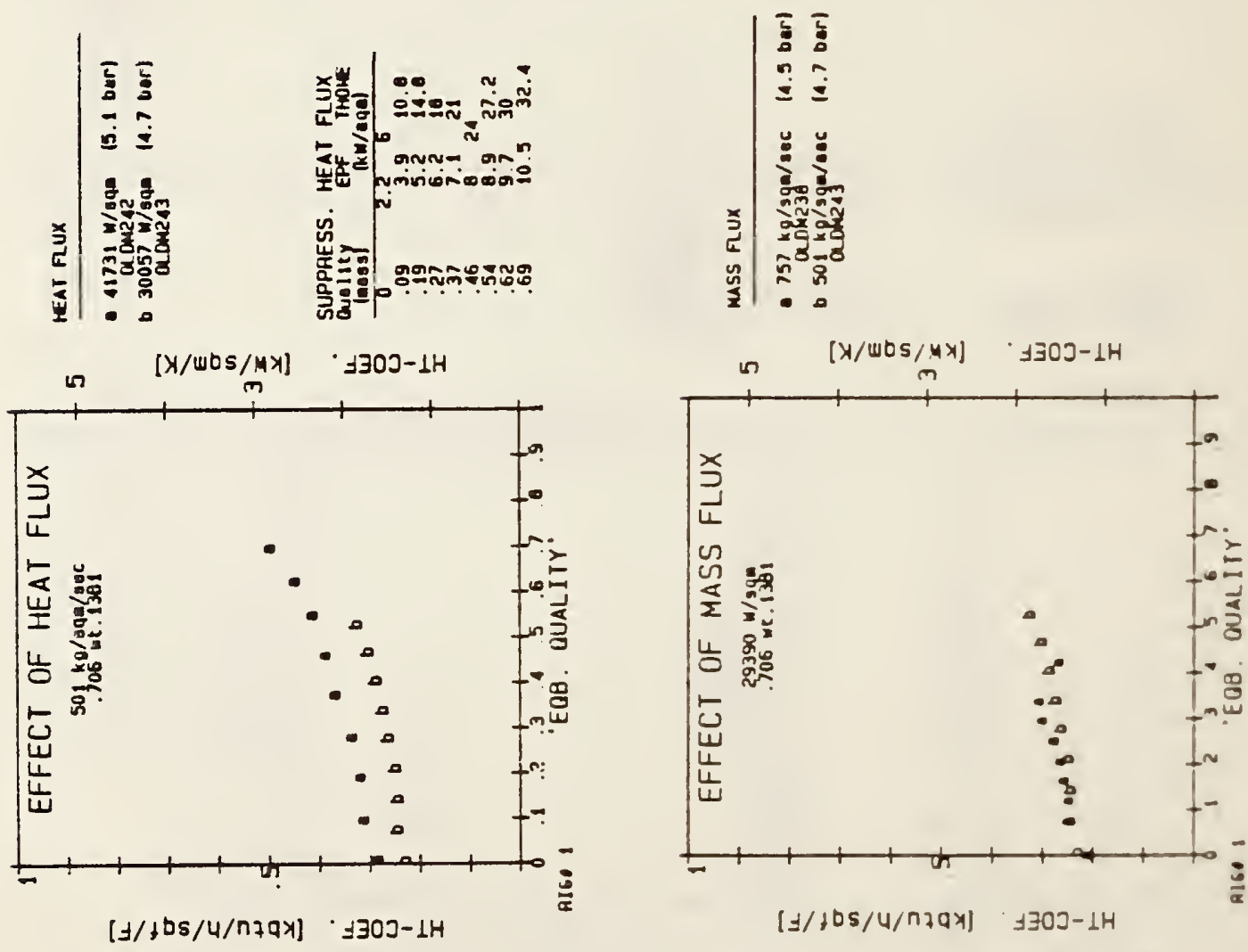

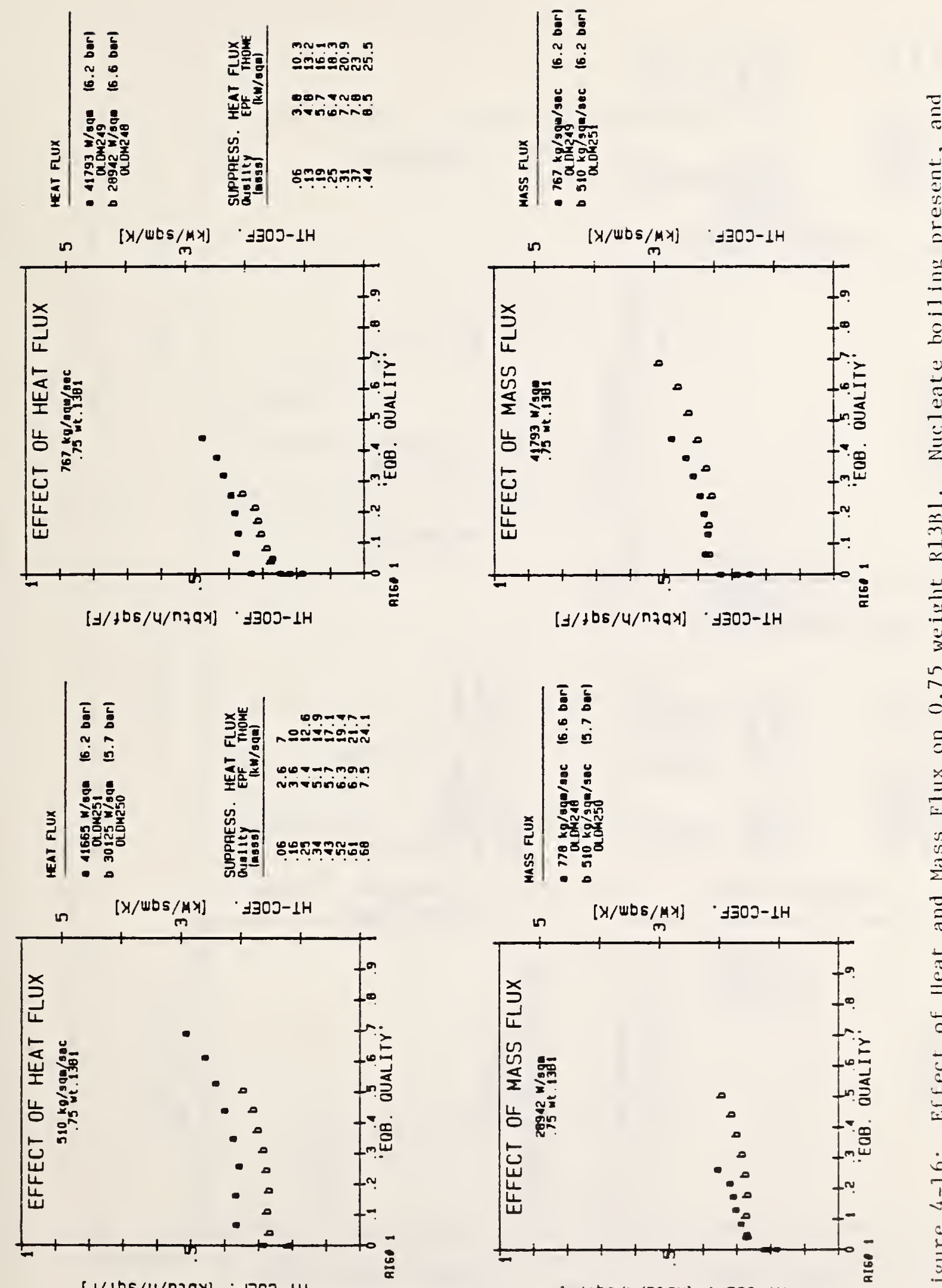

$[H / 108 / 4 /$ ก $29 x] \cdot \pm 30 J-1 H$

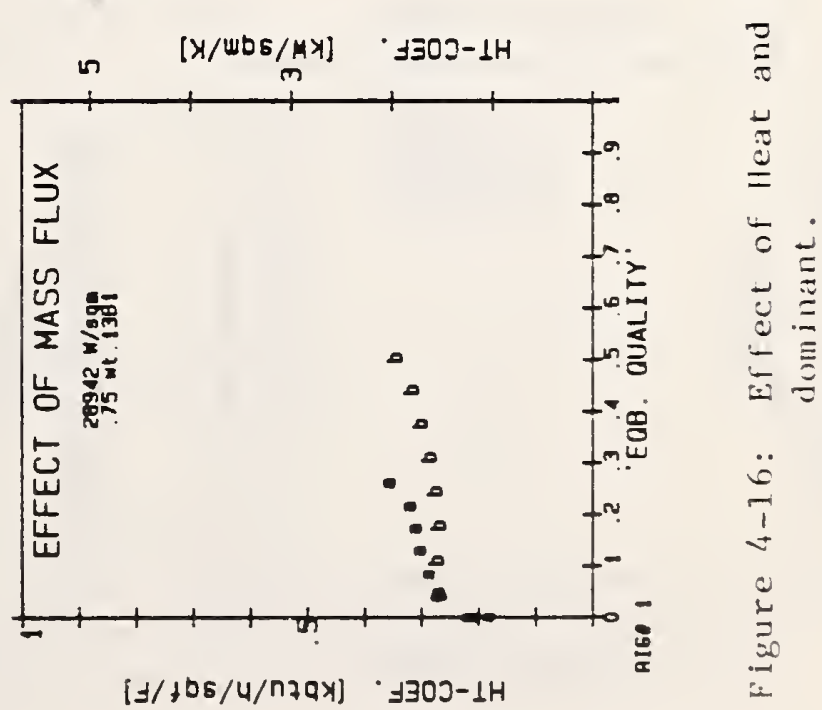



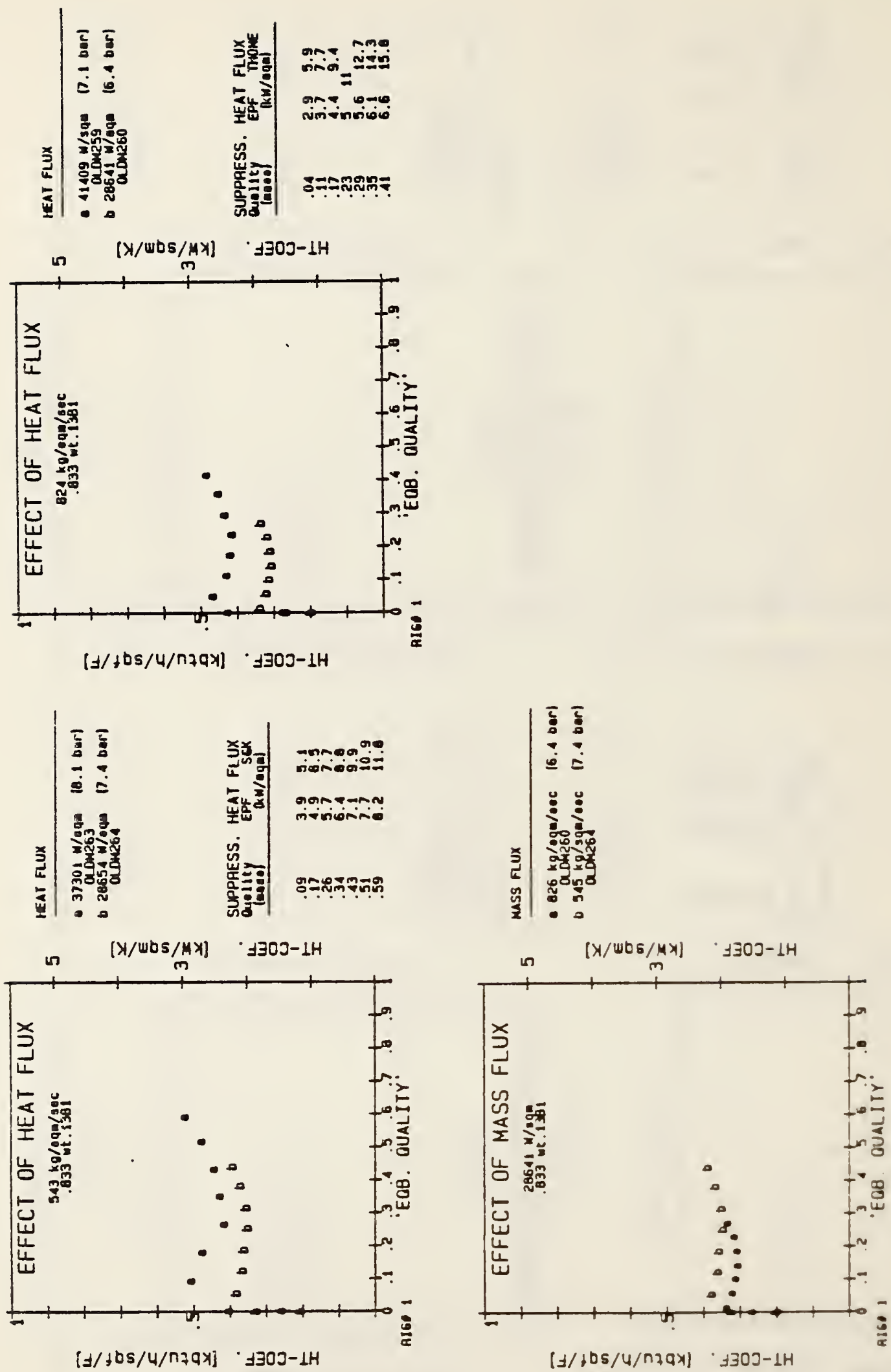

$[y / t 05 / 4 / n z a x] \cdot j 30 J-1 H$ 

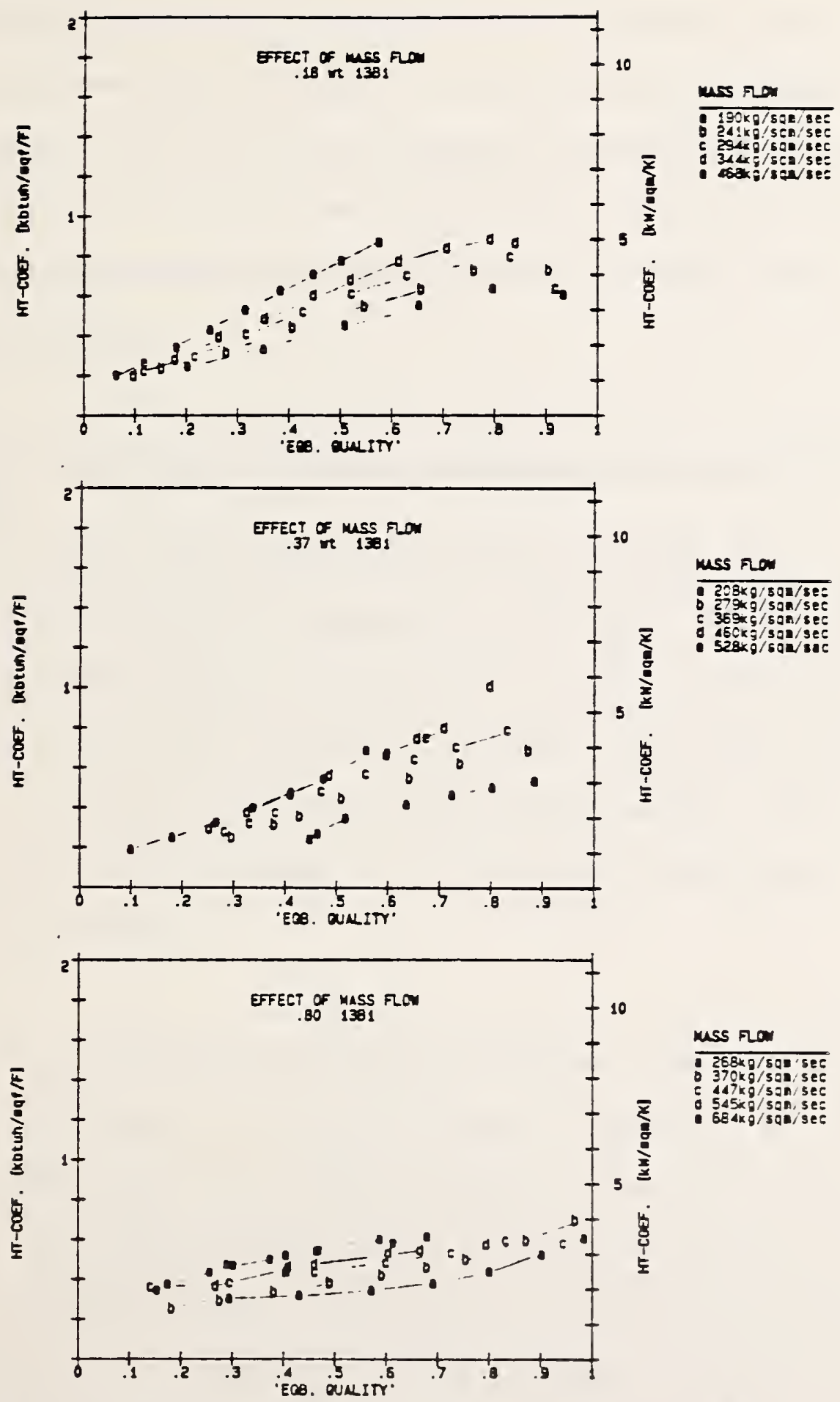

Figure 4-18: Rig非2 Test Section Data. Effect of Mass Flux; Heat Flux $=10 \mathrm{~kW} / \mathrm{m}^{2}$. Forced Convection/evadoration dominates. 

CHAPTER 5: A FURTHER EXAMINATION OF THE DATA

\subsection{Introduction}

The last chapter revealed some of the features of the experimental data base. They are summarized in Table 5-1. In this chapter, some additional features of the data base are discussed, including new physical phenomena which were observed, and an analytical discussion of the use of equilibrium temperature in the definition of the mixture heat transfer coefficient.

\subsection{Circumferential Variation in Heat Transfer in Horizontal Flow Boiling}

In horizontal annular flow boiling the 1 iquid film thickness is a symmetric around the inner circumference of the tube. Due to gravity, the film at the tube botton is thicker than at the tube top. The effect of this thick film for pure fluids is to produce a lower heat transfer coefficient at the trabe bottom due to increased resistance. This behavior has been observed widely in the 1 iterature [Ch66a, Ra83], and was seen with both rigs.

In the case of horizontal flow boiling of mixtures, a new opposite phenomena was observed, i.e., the heat transfer coefficient at the tube bottom was higher than at the top by an amount far outside experimental uncertainty. The observation as made consistently with both rigs; the rigs utilized different tubes, thermocouples, and test section heaters, and thus should not be a function of these apparatus. The wall temperature measurements determine the circumferential variation and are not dependent on the data analysis technique. The effect was observed with 
and without a preheat section, with a variety of heat fluxes, flow rates, and mixtare compositions, and over a range of pressures (left uncontrolled in the tests with the first rig).

Figure 5-1 displays a typical data set (Rig \#1) for the pare fluids. The heat transfer coefficient at the bottom is seen to be 1 ower than at the tube top, except near $I=0.8$, where dryout is beginning. In contrast, Figure 5-2 displays similar plots for mixtures. The reversal is noted at qualities greater than 0.2 . Identical trends were observed with Rig \#2 in both the preheat and the test sections.

One night question why this finding has not been discussed before in the 1 iterature. Section 1.7 discusses previous flow boiling of mirture investigations. Three of the previous studies [Sh73, To79, Be80] involved vertical flow with only one thermocouple mounted around the tube circum ference. Both the lack of instrumentation and the flow orientation preclude the possibility of observation.

Conjecture can be made regarding the reason for the reversal. Possibilities include:

(a) Increased turbulence at botton of tube

(b) Nacleate boiling at bottom of tube, and not at the top

(c) F1 ow pattern differences between mirtures and pure fluids

(d) Film boiling at tube top

(e) Different concentrations at tube bottom and top 
The first two reasons may be dismissed in that they should appear for the pure fluids as well. Further, in the case of mixtures, nucleate boiling is more difficult to sustain, so it is unlikely that this is the cause. If any trend should appear in the nucleate boiling dominated region, it is that a greater disparity between top and bottom might occur with mixtures, not a reversal. The third reason may be dismissed in that flow patterns were observed at the outlet. Based on the se observations, a greater disparity again might have been expected at a fixed quality. The effect of quality is discussed in the next section.

Film boiling is al so a possibility since it will occur first at the tube top. It is not 1ikely since: (a) the wall temperatures were relatively stable; (b) the wall temperatures at the top were not widely different than at the bottom of the tube; (c) the wall temperatures were axially consistent even at 1 ow quality. Still, film boiling is a possibility which cannot be entirely dismissed.

The 1 ast possibility is believed to be the root cause of the reversal: the higher heat transfer at the tube bottom is due to the relatively greater amount of the more volatile component (R13B1) at the bottom of the tube. The gravity-driven drainage of bulk liquid to the tube bottom provides a larger amount of fluid upon which to draw. Initially vaporization occurs at both tube top and bottom into the annular vapor core. The top portion of the tube, with its thinner film (and initially higher heat transfer rate) vaporizes most of the more volatile component. At some point it becomes starved of this component and the vaporization 
rate is diminished. In the bottom portion of the tabe, vaporization is initially small then increases relative to the tube top; since it has a greater balk amount of fluid, it becomes depleted of the more volatile component at a slower rate than the tube top.

There exists then a competition between the depletion of more volatile component and film thickness. If this explanation is correct, one might expect to see in the early portions of the vaporization process a top greater than abot, as both trbe top and bottom have similar compositions. Then as the top becomes depleted of the more volatile component, the two values should merge and then reverse. Figure 5-2 verifies this conjectare.

An attempt was made to correlate the topto-bottom wall temperature difference with local vapor-1 iquid composition difference. The latter quantity is correlated with local mass transfer resistance. As seen on Figure 5-3, no clear correlation was seen in the test section data. The relatively small $\Delta \mathrm{T}$ is due to the low heat flux in this region (constant wall flax and pressure). A similar plot was made with initial composition as the independent variable. Here, there is a general trend that the greater the initial composition of the more volatile R13B1, the greater the observed $\Delta T$. This suggests that the tube bottom has readily available an amount of more volatile component at the higher initial composition, while the top has been depleted. 
The finding of the circumferential variation is a further difficulty for those interested in modelling the heat transfer process from first principles. In addition to axial and radial gradients in composition, there appears to exist a circumferential variation in composition. By necessity then, a varying interfacial temperature exists. Fur thermore, circumferential diffasion might exist due to the existence of the composition gradient. This latter effect is likely small; if it were large, then the gradient would disappear, as would the reversal in heat transfer coefficient.

\subsection{Effect of Equilibrium Quality on Mixture Heat Transfer}

When the thermodynamic equilibrium quality, xeqb, is less than zero, some vapor may be present due to subcooled flow boiling. The actual quality then is higher than $z_{\text {eq }}$ when $x_{\text {eqb }}$ is small. At values of $x_{\text {eqb }}$ $\approx 0.7$, dryout may occur with the remaining 1 iquid entrained in the vapor core. The 1 iquid droplets may persist beyond the point where $x_{e q b}=1$, due to relatively poor heat transfer through the vapor, so that at large $x_{\text {eqb }} z<x_{\text {eqb }}$. At values of $x_{\text {eqb }}$ up to 0.2 , 1 ittle vapor was seen for some mixtures at the outlet sight glass. Al so dryout was not of ten measured until $x_{\text {eqb }}$ was near or exceeded 1. Inaccuracy of the EOS might explain these observations. Alternatively, the above classic description of quality dependence may need to be altered for mixed fluids. Mirtures have a bigher onset of nucleate boiling point than do some fluids. With less subcooled boiling, the actual y may lag reqb. This effect would explain both the $1 \mathrm{ack}$ of vapor at 1 ow $x_{\text {eqb }}$ and the 1 ack of dryout at high $x_{\text {eqb }}$. Also the addition of a second less volatile 
component may simply delay dryout dae to mass transfer resistance (see Appendix TC). In either case, the quality dependence may be structurally different for mixtures as campared to pure fluids.

In the last chapter the measured heat transfer coefficient of mixtures was proportional to both heat flux and quality. This trend is also opposite that commonly observed for pure fluids. The dependence on heat flus requires the existence of nacleate boiling. In the nucleate boiling regime, a weak inverse proportionality between quality and heat transfer coefficient is asually observed for pure fluids [St82].

The quality dependence da/dx varies with heat flux, composition and quality itself. As seen in Figure 4-20, a larger da/dr is observed at higher heat flux and quality. Again, this is the opposite behavior as that seen with pore fluids.

In this case, the reason might be the use of an equilibrium temperature in the defining relation for the heat transfer coefficient:

$$
a=q /\left(T_{w}-T_{e q b}\right)
$$

If this equation is now partially differentiated by dx to determine the rate of change with quality:

$$
\frac{\partial a}{\partial x}=\frac{-q}{\left(T_{w}-T_{e q b}\right)^{2}} \frac{\partial T_{w}}{\partial x}-\frac{\partial T_{e q b}}{\partial x}
$$




$$
\frac{\partial T_{e q b}}{\partial x}>\frac{\partial T_{W}}{\partial x}
$$

The quantity $\partial \mathrm{T}_{\text {equb }} / \partial \mathrm{x}$ is a function of composition. It is largest when the dew and bubble point temperature difference is largest (at $\Delta X=1$, $\left.\Delta \mathrm{T}_{\text {eqb }}=\mathrm{T}_{\mathrm{DEW}}-\mathrm{T}_{\mathrm{BUB}}\right)$. Thus, it may be that the apparent dependence on quality is largest in the regions where the dew-bubble temperature difference is largest. In examining the data, a trend of this nature is apparent. The quality dependence then may be an artifact of the defining relation, rather than representative of the heat transfer regime as it is in flow boiling of pure fluids.

\subsection{Departure from Nucleate Boiling (DNB) Events}

In some of the data taken with Rig \#1, DNB events were observed along the tube top. The events occurred generally at large values of heat flux, pressure and concentrations of R13B1. Some of the erratic behavior of R13B1 can be attributed to this occurrence (Figure 5-4). Surprisingly, in the case of mirtures, the bottom and side heat transfer coefficients seem to be 1 ittle affected by the behavior near the tube top.

Soine available data in the literature were examined for the occurrence. The data of [Mi81] for pure R12 and a misture of R12/R22 shows the same behavior (Figure 5-5). The authors note the 'tube wall temepratures exhibited large fluctuations which indicated the unstable behavior of 
boiling. . the scatter of the tube $\mathbf{a l 1}$ tempertare is wider in the case of miztures than $\mathbf{w i t h}$ pure R12.' They did not attribute the scatter to DNB events, and analyzed their data as if it were in an annlar flow pattern with conventional nucleate boiling and ovaporative heat transfer contribations. Same data of [Ma76] al so seems to erbibit scatter at 1 ow $f 10$ rate and high heat $f(a x$, and is suspected of similar erents.

For standard heat pump applications $w$ ith R13B1/R152a and with other Iefrigerant s, there is a possibility that the hoat transfer process will be diminished in the first row of coils (where $q$ is 1 argest). In this caso, the addition of a less volatile component may actualiy augment the observed heat transfer. DNB events were never observed with pure R152a, the less volatile component of the tested minture.

In the following chapters the tests suspected of foaturing DNB events are not incloded in the comparison to the predictive correlations.

\subsection{Comparison Betreon Pare and Mired Refrigerants}

In Chapter 7 the measured hest transfer coefficients for mirtures are compared to predicted val ues based on treating tho wirture as an equivalent pare fluid. In the process of that analyis, a canprison wil 1 be made between pare fluids and mirtures when the heat transer is dominated by nucleate boiling. In that case, the comparison is made at identical pressures and heat flares. In the case of flom boil ing whoro flon rate has a sizeable influence on the measured beat transfer coefficient, the comparison is more difficult. 
When there is an influence of flow rate, one can compare fluids on any of the following basis:
(a) same mass flow rate
(b) same molar flow rate
(c) same volume flow rate
(d) same Reynolds/Prandtl number

The mass flow rate comparison is the one with which most engineers are acquainted. On the other hand, chemists and physicists work in molar quantities, and might claim that the weight of a molecule matters less than the number of molecules which flow. The application to a heat pump suggests the same volume flow rate: compressors are effectively constant volume devices. In defense of the last item, single phase scaling 1 aws suggest $R e$ and $P r$ should be used. The se however are derived from the single phase Dittus-Boelter relation; on two phase flow, other parameters affect the results.

Figure 5-6 displays the test section data for both the pare and mixed refrigerants, at identical values of pressure, heat flux and the Martinelli parameter. The measured heat transfer coefficient has been normalized by $a_{\text {LO }}$ so that mass flux effects are eliminated from the comparison. The heat transfer coefficient for the mirtures are less than for the pure refrigerants. This finding suggests that there is a distinct mirture effect, possibly due to mass transfer resistance. Shown also on the figure are two correlations to be discussed in Chapters 6 and 7. They are both for pure fluids. The Prandtl number 
term is included whenever it is suspected that nucleate boiling has not been suppressed. As can be seen, it appears that nucleate boiling is suppressed for the mixtures, but not for the pure fluids. The presence of nucleate boiling for the pure fluids might also explain their larger heat transfer coefficients. This result is consistent with the suppression criterion prediction of Chapter 4.

\subsection{Pressure Drop in Horizontal Flow Boiling of Pure and Mired Refrigerants}

It has been suggested recently that conventional pressure drop prediction methods must be modified to account for mirture effects [Si83a]. Others claim that there is no physical reason for requiring such a change. Arguments might be made for either case. All prediction methods use the quantity $\mathbf{r}_{\text {eqb }}$ in determining pressure drop. For pure fluids at equilibrium qualities above zero, the vapor quality difference, $x_{a c t}-x_{e q b}$, is usually small in the andar flow regime. It is well established in nucleate boiling of nonazeotropic mirtures, mass transfer resistance leads to degraded heat transfer. This phenomena could change the vapor generation rate, such that the vapor qua 1 ity difference, $x_{a c t}-I_{e q b}$, is dissimilar between pure fluids and mirtures. The dissimilarity could lead to poor prediction using pure fluid methods. On the other hand, it has been established analytically that mass transfer resistance is of minor importance when nucleate boiling has been suppressed and the vapor generation process becomes evaporative on1y in turbulent annular f1 ow [Sh77]. 
The Martinelli-Nelson me thod [Ma48], as modified by Chisholm [Ch67] was selected due to its simple application and success with the pure fluids. The method requires stepwise integration as was described in Chapter 3 . For the tests conducted here, the inlet conditions were only slightly subcooled, and single phase pressare drop was neglected. The entire pressure drop was assumed to occur between the calculated position of $I_{\text {eqb }}=0$ and the outlet.

Figure 5-7 displays the comparison between prediction and measurement for the mixtures' data. Agreement is satisfactory, and may be compared to Figure 3-12. No correction for composition is required for this Iefrigerant. 


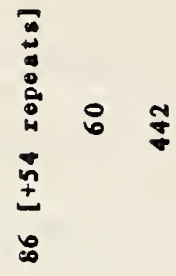

2 里

일 씰 일

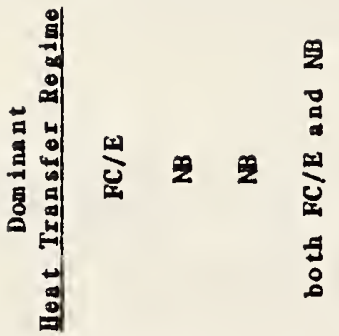

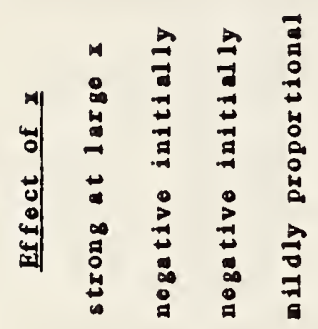

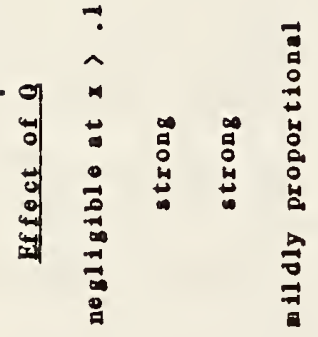

芒

$\frac{1}{0}$

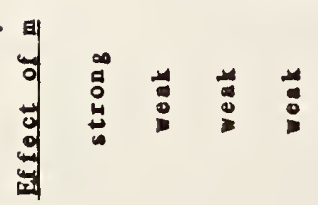

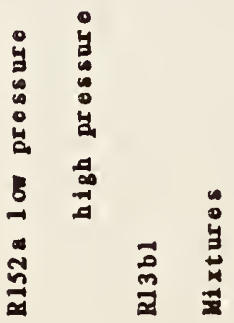

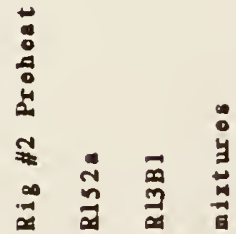

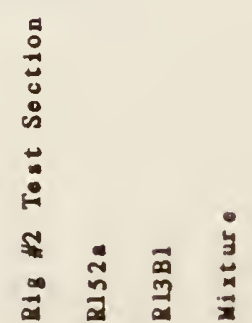

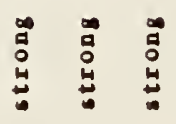

$\vdots \frac{1}{z}$ 


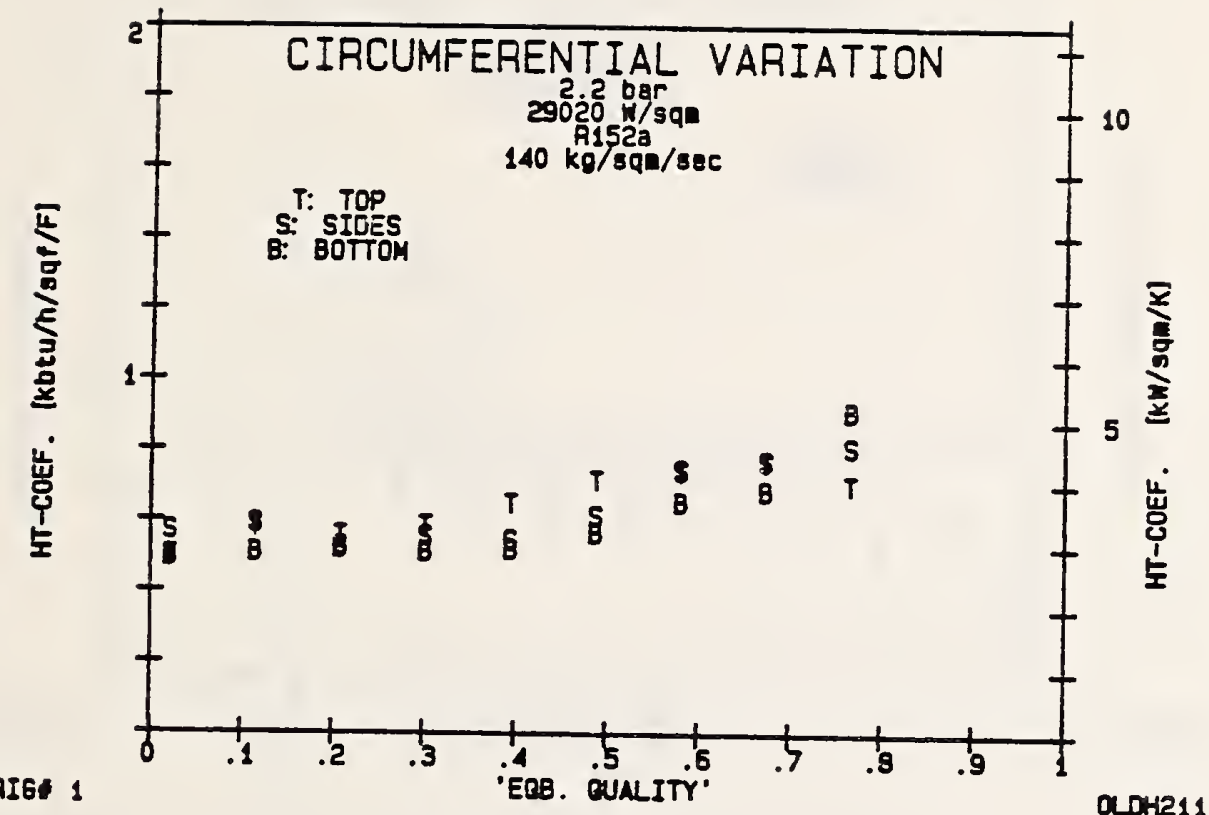

Figure 5-1: Variation in Measured heat Transfer Coefficient. Heat transfer at tube bottom lower than at top due to thicker film. (a) R152a; (b) RI3BI

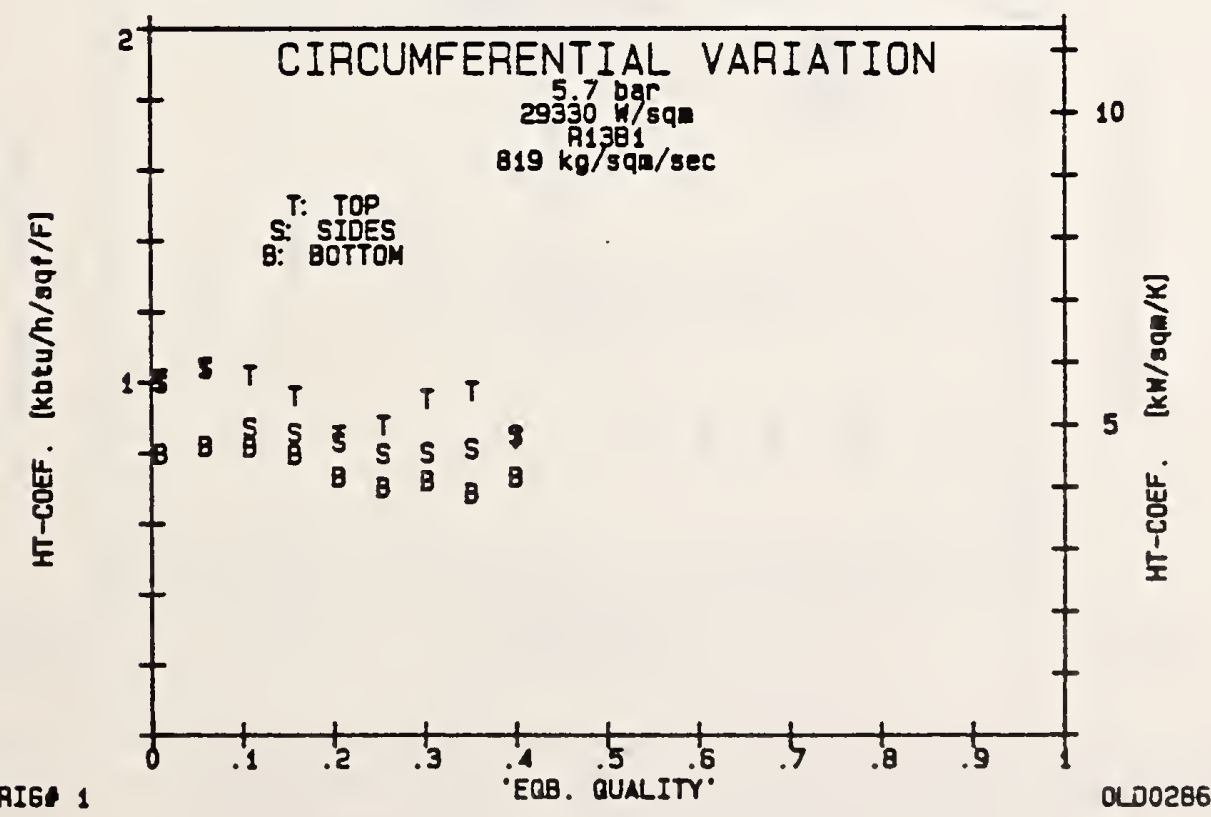




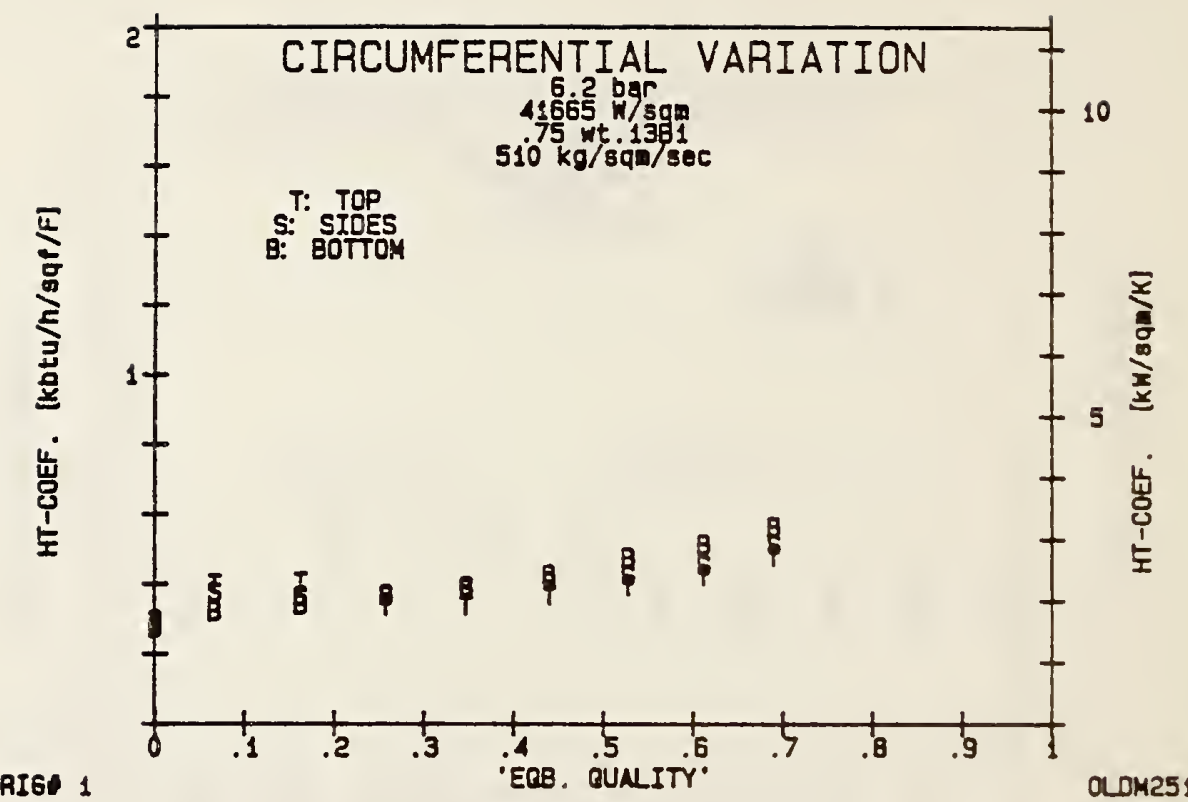

Figure 5-2: Variation in Measured Heat Transfer Coefficient. Heat transfer at tube bottom higher than at top, presumably due to greater availability of more volative component.

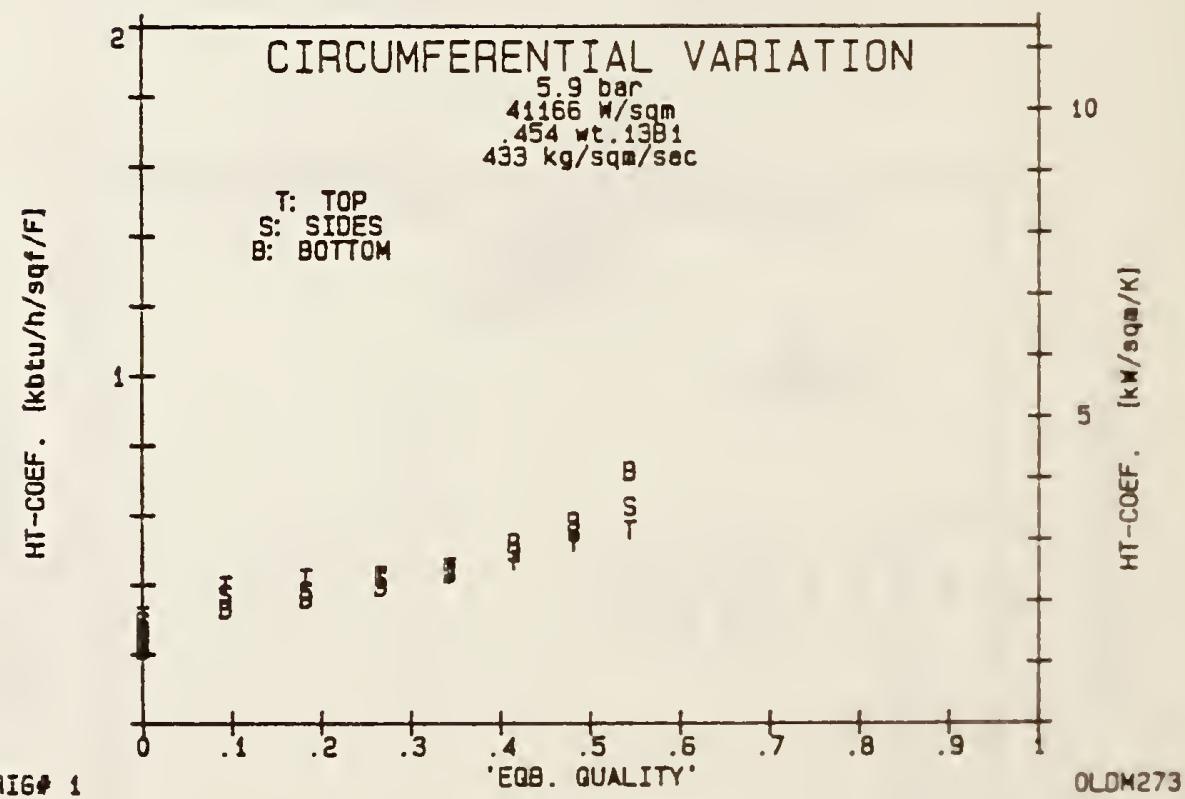



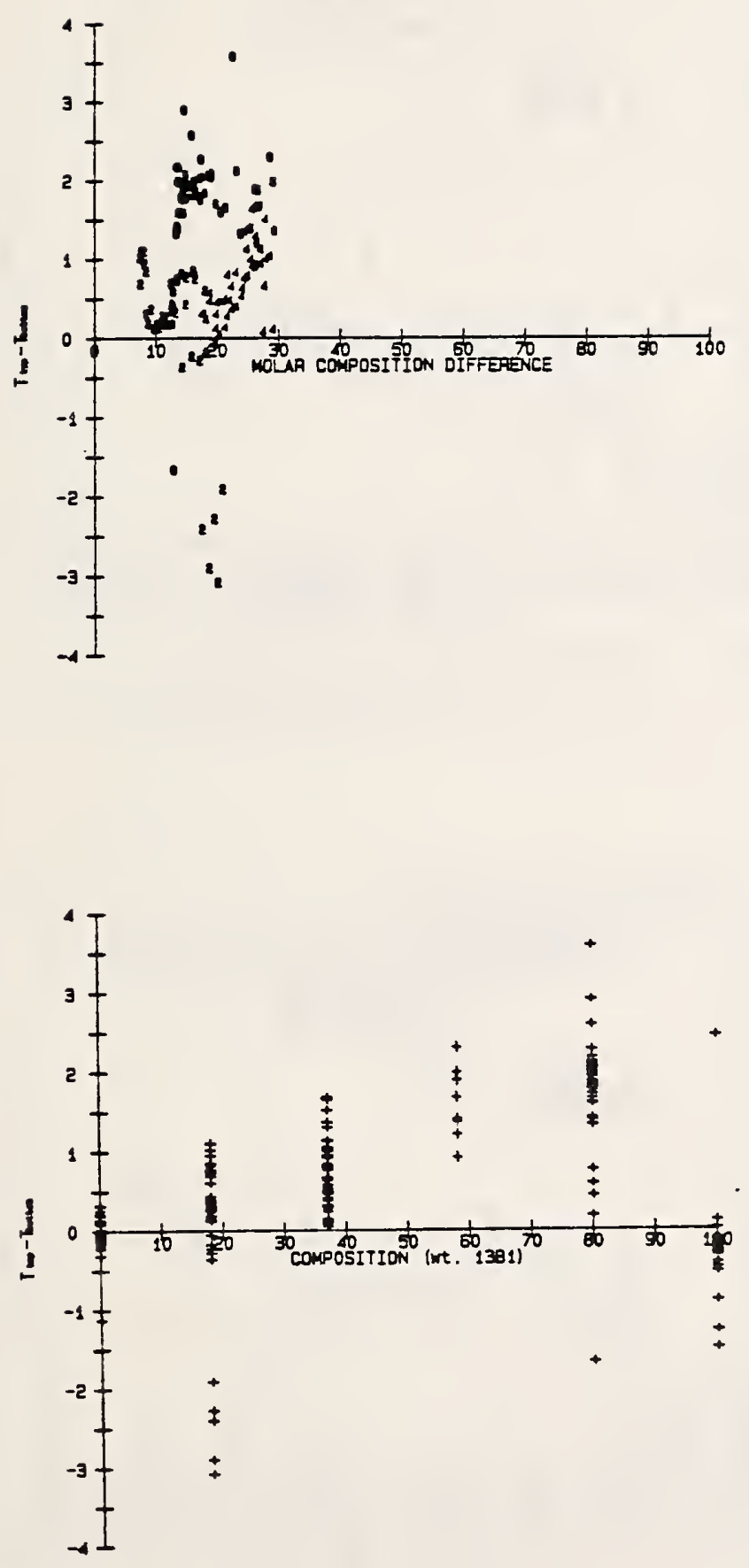

Figure 5-3: Effect of Composition on Top-Bottom Vall Temperature Difference. (a) Effect of Local Mnlar Composition Difference; (b) Effect of Feed Composition. Outlying Points are due to stratified flow. 


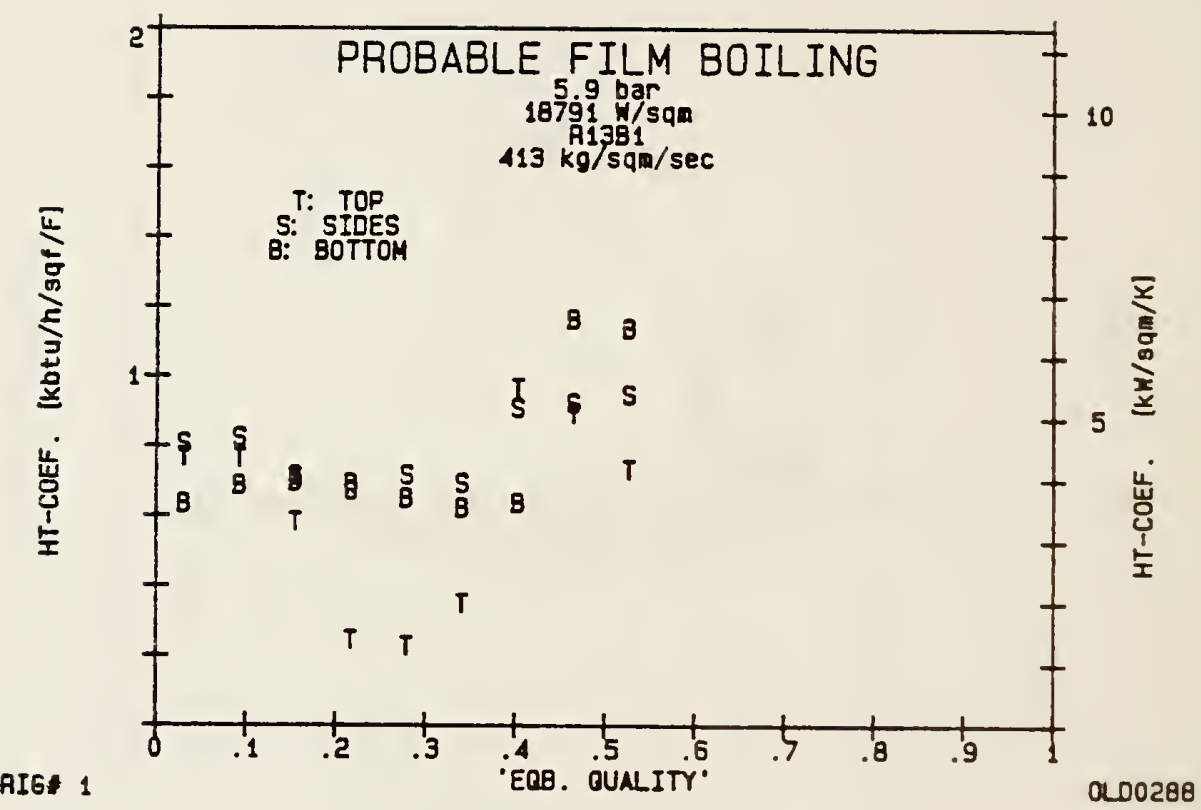

Figure 5-4: Indications of probable film boiling for pure R13BI and a mixture.

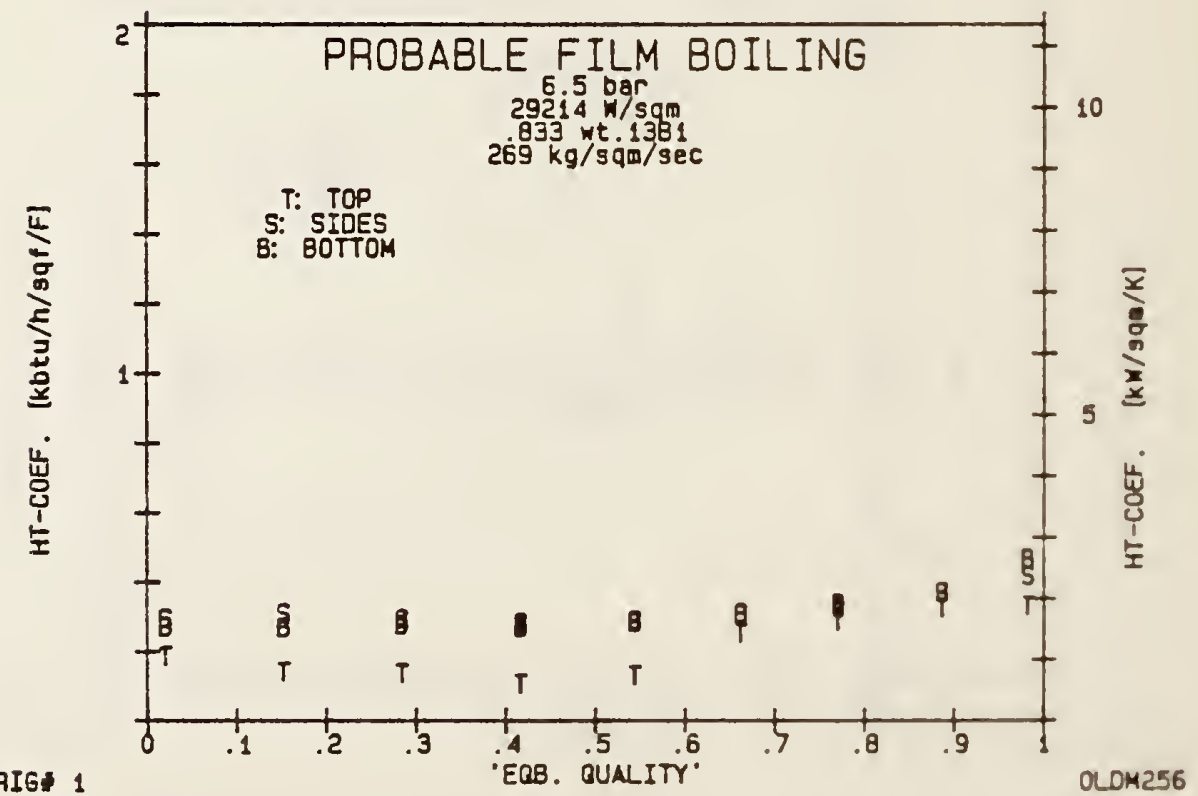




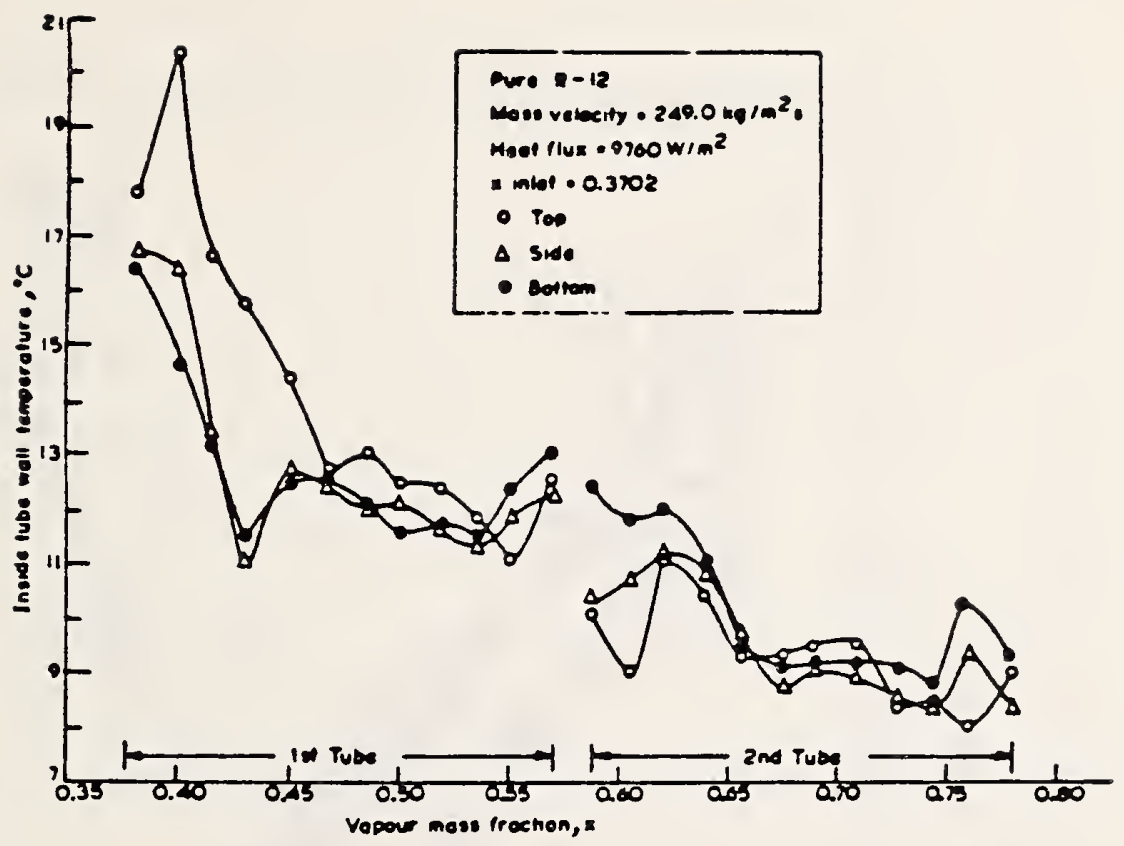

(a) Pure retrigeront 12

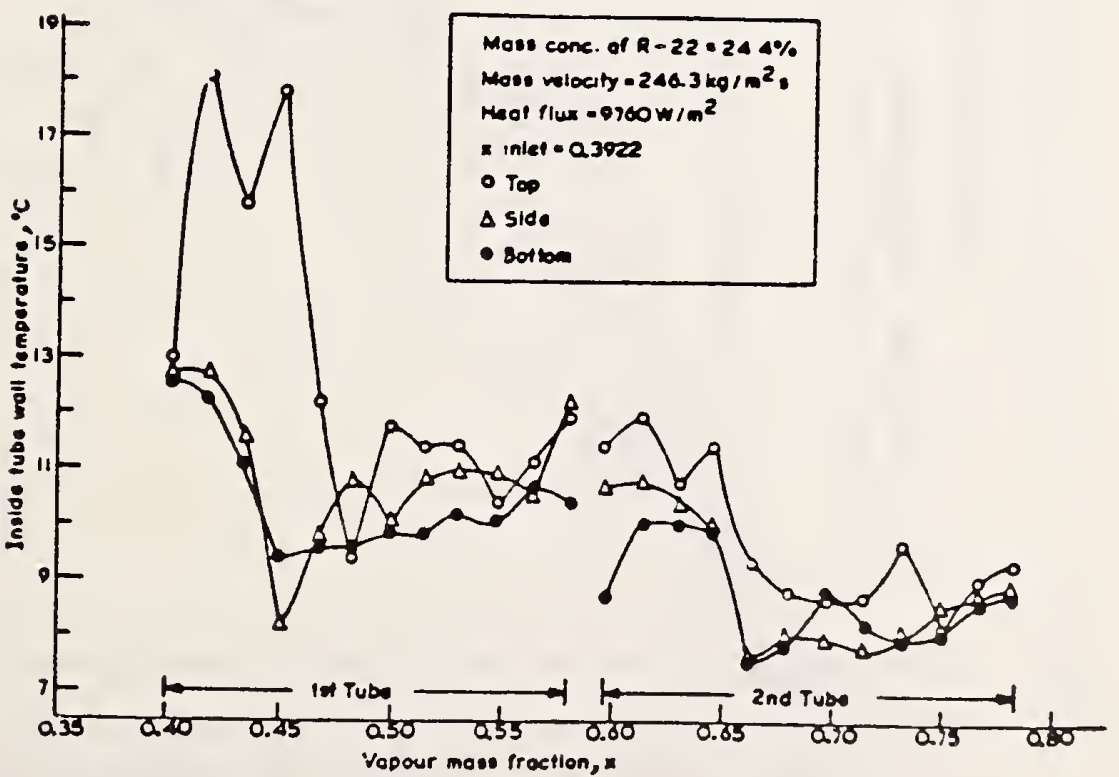

(b) Binory mixlure

Figure 5-5: Probable Film Boiling in the Data of (Misi) 


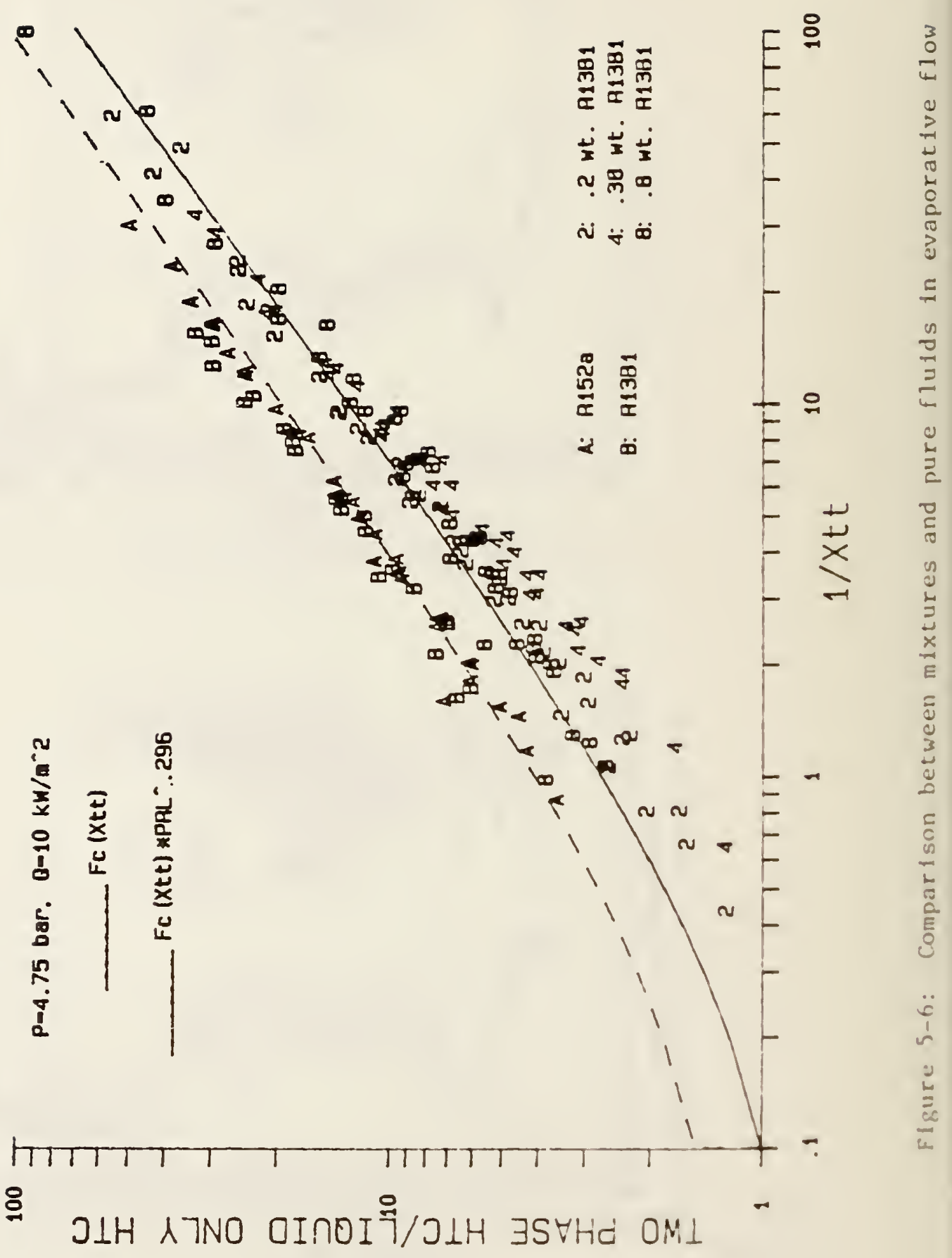




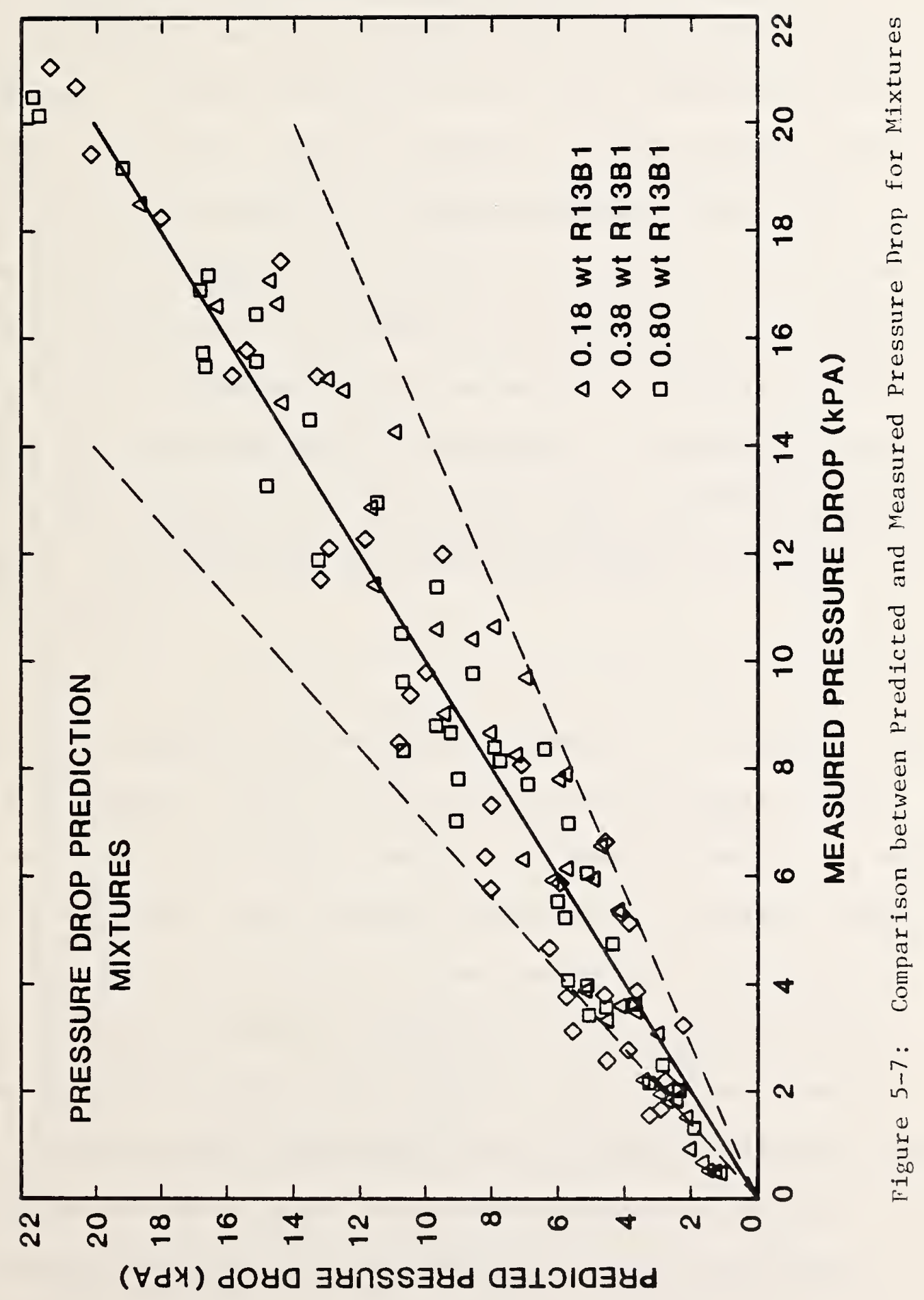



CHAPTER 6: PREDICTION OF PURE REFRIGERANT HEAT TRANSFER

\subsection{Introduction}

In order to understand the effect of miztures on flow boiling heat transfer, one needs to be able to predict flow boiling behavior with pure components. If such a prediction can be made accurately with a single model/correlation, then the analysis of mirtare behavior can begin by treating the mixture as an 'Equivalent Pare Fluids (EPF)', i.e., one which has properties determined by appropriate mixing rules, bot which is modelled as if the properties belonged to a pare fluid. If the EPF correlation underpredicts the measured heat transfer for miztares, then some additional effect attributable to the mirture is augmenting the heat transfer. If, on the other hand, the EPF correlation overpredicts, the mixture has some inhibiting feature, e.g., mass transfer resistance.

The modelling of annular flow boiling heat transfer is not simple. As discussed in Chapter 1 , there are many complicating features. Some predictive estimation is needed, however, for design purposes. As such, many correlations have been developed which attempt to consider some of the physical aspects of the flow. In the remainder of this chapter, the se correlations are discussed (Section 6.2) and compared with the experimental results of this report (Section 6.3). The best features of varinos correlations are incorporated into an overall calculation procedure (Section 6.4) which yields excellent agreement with the data. It is then compared favorably with data provided by other researchers. Conclusions and recommendations are described in Section 6.5. 


\subsection{Correlations for Annular Flow Boiling}

In the earlier chapters, the mechanisms of vapor generation in annular flow boiling were seen to be: (a) nucleate boiling at the wall characterized experimentally by a strong dependence of $a$ on beat flar, or (b) evaporation from the vapor-liquid interface characterized by a strong dependence on flow parameters and vapor quality, or (c) a combination of (a) and (b). In the case of refrigerants, both mechanisms commonly contribute, with the more dominant contribution switching from (a) at low quality to (b) at high quality for fired heat and mass flux.

Correlations have attempted to accont for the simulaneous contirubtion of nucleate boiling and evaporation in an additive sense:

$$
a / a_{L O}=A_{1}\left(\frac{q}{m \Delta h_{v}}\right)^{C_{1}}+A_{2}\left(x_{t t}\right)^{C_{2}} \text {, with } A_{1}=0 \text { sometimes (6-1s) }
$$

or, a multiplicative sense,

$$
\alpha=A_{3} \cdot{ }^{C_{3}}{ }_{q}^{C_{4}}{ }_{d}^{C_{5}}
$$

or, a combination of the two approaches ( $S=S(F)$ ):

$$
\alpha=a_{L 0} F+a_{p o o l} S
$$

Forms (6-1a) and (6-1b) have been used widely in refrigeration indusir: form (6-1c) was developed originally by Chen [Ch66] and is comonly 
advocated in the nuclear industry. All of the forms have the problem of fail ing to predict accurately in all circumstances. All forms show distinct contributions of nucleate boiling (seen in the inclusion of $q$ or $a_{n}$ ) and forced convective evaporation (seen in either $x_{t t}$ and/or $a_{L 0}$ ).

Form (6-1a) has the problem that it particularly has not proven general. In some cases, although a dependence on heat flux could be seen from the data, no improvement was achieved by including it as a correlating parameter, i.e., setting $A_{1}=0$ and defining $A_{2}$ and $C_{2}$ empirically produced the same or better goodness of fit as including it [ $\mathrm{Ch} 79$ ]. This result may merely demonstrate the inadequacy of the correlating parameter or the ability to develop a false correlation. A second difficulty arises in the use of $a_{L 0}$, the single phase heat transfer, as a normalizing parameter. The nucleate boiling contribution is then ${ }^{a_{L O} A_{1}}\left(q / m \Delta h_{v}\right)$, which af ter inserting the usual correlations for $a_{L O}$, e.g., Dittus-Boelter, indicates nucleate boiling heat transfer to depend on the diameter for no apparent physical reason. Furthermore, the specific fluid-surface combination has been proven important in nucleate boiling heat transfer. The physical influence of the surface is lost in the empirical constants $A_{1}$ and $C_{1}$. Still, agreement of form (6-1a) correlations with the data upon which they were developed is \pm 25 percent typically. This result is somewhat misleading since: (a) the same correlation applied to a different refrigerant/surface yields poor agrement; (b) the value of $x_{t}$, developed to correlate pressure drop with flow parameters, yielded agreement for that parpose of only \pm 30 percent; and (3) the Dittus-Boelter equation correlated to measurement 
data to \pm 13 percent [Sh73]. Despite these problems, the refrigeration industry has typically applied form (6-1) correlations, and several correlations of this type were examined. Table 6-1 1ists the form (6-1) correlations.

Form (6-1b) is applicable only to average heat transfer coefficients. Butterworth and Shock [Bn82] have published a summary table of values for $C_{3}, C_{4}$, and $C_{5}$ which is reproduced here as Table 6-2. The table shows most of the experimental data to be characterized more often by nucleate boiling, i.e., a fairly weak dependence on flow rate $(0.1$ to 0.25 , excepting Danilov) and a dependence on heat flax of exponent 0.5 to 0.7 , typical of pool boiling experiments. Form $(6-1 b)$ al so has the problem of a boiling dependence on tube diameter.

For annular flow, the most widely accepted correlations in the nuclear industry was suggested by Chen [Ch66]. It was developed originally from a large set of data compiled from several authors' experiments; the data are exclusively for vertical flows of water and various organic compounds. The refrigeration industry has rejected its use principally because it has proven inaccurate for refrigerants evaporating in horizontal tubes, and perhaps because of the iterative nature of its solution for the case of constant wall flux. Various researchers in other fields have attempted to improve the methods as will be described. 


\subsubsection{Miscellaneous Forms}

Some other correlations have appeared in the 1 iterature and have on occasion predicted refrigerant HTC's with some accuracy. These are the methods recently advanced by Shah [Sh76, Sh82], Dembi et al [De78], and Kandliker [Ka84]. Shah's method was checked with the results shown on Figure 6-1. Due to its suppression criterion which does not include mass flux effects, it selects the wrong heat transfer regime and predicts poorly.

As shown in Appendix 6A, the approach recently has been to compile large sets of data from various experiments and use regression analysis to fit them. Extension of the methods to other data has been disappointing. Furthermore, the regression analysis may lead to correlations which ignore physical features in the data. The approach of this report is to examine the older methods developed from theory and analyze or extend them.

\subsubsection{The Original Chen Equation}

Rosenhow originally suggested that in the case of flow boiling with nucleation, that the forced convection/evaporative and nucleate boiling contributions might be additive. Following this 1 ine, Chen postalated:

$a=a_{e}+a_{n b c}$

$$
=a_{L O^{F}}+a_{n} S
$$


The evaporative portion, $\alpha_{e}$, was related in the fashion similar to form $(6-1):$

$$
\alpha_{e}=a_{L 0} F_{c}\left(X_{t t}\right)
$$

where $F_{c}\left(X_{t t}\right)$ is an empirical function of the Martinelli parameter:

$$
\begin{aligned}
& F_{c}\left(X_{t t}\right)=2.35\left(\frac{1}{X_{t t}}+.213\right) .736 \text { for } \frac{1}{X_{t t}}>0.1 \\
& \text { ELSE } F_{c}=1
\end{aligned}
$$

It accounts for the increase in heat transfer hich accompaies an increase in quality when the flow is in a porely evaporative mode. While $F_{c}\left(X_{t t}\right)$ was developed empirically, Chen al so showed that, through a Reynolds analogy,

$$
F_{c}\left(O_{L}^{2}\right)=\left[\left(\frac{d p}{d z}\right)_{2 D} /\left(\frac{d p}{d z}\right)_{L 0}\right] \cdot 445=\left[O_{L_{t t}}^{2}\right] \cdot 445
$$

so that one finds F related to the pressure drop ratio. This latter ratio is itself correlated by $x_{t t}$ as shown in the Martinelli-Nelson method. The empirical $F_{c}\left(X_{t t}\right)$ of equation $(6-7 a)$ agrees closely with equation (6-7b) over most of the probable range of $I_{t}$ values when the original Martinelli-Nel son method is used [Be80].

The nucleate boiling contribution of equation (6-4) was postulated as: 


$$
a_{n b c}=a_{n} s_{c}
$$

where $a_{n}$ is derived from a pool boiling correlation. The suppression factor, $s_{c}$, accounts for the general suppression of nucleate boiling as flow rates and/or quality become large. It is derived from the consideration that the temperature field in flow boiling differs from that of pool boiling. The turbalent natore of the liquid film and the excellent heat transfer to the fast moving vapor core tend to reduce the temperature of the liquid near the wall, effectively preventing bubble growth. The suppression factor, $S_{c}$, was al so determined empirically:

$$
\mathrm{S}_{\mathrm{c}}=1 /\left(1+2.53 \mathrm{E}-6\left(\operatorname{Re}_{\mathrm{LO}} \mathrm{F}_{\mathrm{c}}^{1.25}\right) 1.17\right.
$$

Chen selected the Forster-Zuber pool boiling relation for $a_{n}$ :

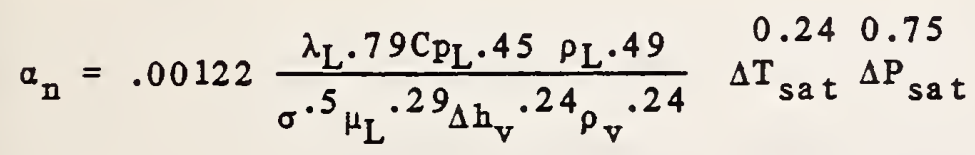

$$
\begin{aligned}
& =\mathrm{K}_{1} \Delta \stackrel{0}{\mathrm{~T}}_{\text {sat }}^{.24} \Delta \stackrel{0}{\mathrm{P}}_{\text {sat }}^{.75}
\end{aligned}
$$

Since, by definition:

$$
\begin{aligned}
\mathrm{q}_{\mathrm{w}} & =\left(a_{e}+a_{n b c}\right) \Delta \mathrm{T}_{s a t}=a \Delta \mathrm{T}_{s a t} \\
& =\left(a_{e}+a_{n} S\right) \Delta T_{s a t}
\end{aligned}
$$

one can sabstitate $(6-6 c)$ into $(6-7 b)$ to get: 


$$
q_{w}=\left(a_{e}+K_{1} s \Delta T_{s a t}^{0.24} \Delta P_{s a t}^{0.75}\right) \Delta T_{s a t}
$$

The solution of the equation for $q_{w}$ reugires iteratively guessing values of $\Delta \mathrm{T}_{\text {sat }}$, calculating the nucleate boiling contribution, and either testing that the proper $\Delta T$ yields the given heat flux or interpolating graphically toward it [Co80].

\subsubsection{Closed Form Solution for Chen's Me thod}

Instead of iterating, a simple closed form solution can be found from the following: for small $\Delta T / T$ as is common for evaporating refrigerant applications, Clausius-Clapeyron can be nsed to eliminate the pressure difference term:

$$
\Delta P_{\text {sat }}^{0.75}=\Delta T_{\text {sat }}^{0.75}\left(\frac{\Delta \mathrm{h}_{\mathrm{v}}}{\mathrm{T}_{\text {sat }} A v_{\mathrm{v}}}\right)^{0.75}
$$

Now if equation (6-9) is substituted into equation (6-8), then

$$
q_{w}=\left(a_{e}+B_{2} \Delta T_{s a t}^{0.99}\right) \Delta T_{s a t}
$$

where

$$
\mathrm{R}_{2}=\mathrm{K}_{1} \mathrm{~S}\left(\frac{\Delta \mathrm{h}_{\mathrm{v}}}{\mathrm{T}_{\mathrm{sat}} \Delta \mathrm{v}_{\mathrm{v}}}\right)^{0.75} \text {, and } \Delta \mathrm{T}=\Delta \mathrm{T}_{\mathrm{sat}} \text { for convenience }
$$

Approximately $\Delta T^{1.99}$ by $\Delta T^{2}$, equation $(6-10)$ becomes a simple quadratic for the unknown $\Delta T$, the solution of which is 


$$
\Delta T=\frac{-a_{e} \pm \sqrt{a_{e}^{2}+4 K_{2} q_{w}}}{2 K_{2}}
$$

Only the positive root is meaningful physically. Substituting this root into $(6-7 a)$ gives

$$
q_{\nabla}=\left[a_{e}+K_{2}\left(\frac{-a_{e}+\sqrt{a_{e}^{2}+4 K_{2} q_{W}}}{2 K_{2}}\right) \Delta T=\left[a_{e}+a_{n}\right] \Delta T\right.
$$

or, since $q_{w}=a \Delta T$, the bracketed term is the overal 1 hoat transfer coefficient; in simplified form:

$$
a=\frac{a_{e}}{2}+\frac{\sqrt{a_{e}^{2}+4 R_{2} q_{r}}}{2}
$$

Al 1 terms in this equation are bnow and a can be found directly, el iminating the iterative process. Though the derivation seems obvions, the author has never seen it in print.

\subsubsection{Comparing Chen's Correlation to Exporimental Data: Literature Rev iew}

When compared against the original data bant, Chen's formulation fit the da ta to a absolute mean deviation of 11 percent. This excellent a groement has been the principal source of the advocacy by the nucloar indestry of Chen's method. How ovor, when compared to measured heat transfor coefficients of 1 ion boil ing refrigerants, the method tends to anderpredict bad1y in most cases [De78, Ch66a,Pa74], or on occasion 
overpredict [0.g., Ch67 data]. Furthermore, the method tends to underpredict the quality dependence. Lastly, though Chen compared his method with five organic fluids, the maximum qality was 0.12 . In this range, nucleate boiling dominates. The method then was not real iy tested in the range of interest to refrigerants. The poor prediction with refrigerants has been attributed by researchers for various Ieasons:

(a) the convective contribution is anderpredicted due to a negligence of a Prandt number effect [Be80];

(b) the nucleate boiling contribution is poorly predicted by the use of the Forster-Zuber correlation. Its contribution is underpredicted [To79] or overpredicted [Ka84] with organic 1 iquids.

The to interpretations may then be directly opposed in try ing to understand the physical heat transfer processes.

6.2.4 Modification to Nacleate Boiling Contribation in Chon's Method Nearly all the terms in Chen's formulation have boen suggested for revision. Butterwor th and Shock [Bu82] sugested that a different pool boiling relation be nsed in equation (6-5). They recomonded a recont correlation by Stephan and Auracher [St81] which includes the fluid's wetting characteristics and was specialized to various fluid classifications (refrigerants, water, cyrogenic fluids, otc.). For tho pool boiling of Iefrigerants, Stephan and Abdel sal am rocamondod [St80]:

$$
\frac{a_{p 001}}{\lambda_{L}}=207\left(\frac{g d}{\lambda_{L} L_{8}}\right)^{.745}\left(\frac{\rho_{v}}{\rho_{L}}\right)^{.581} P_{r_{L}} .533
$$



convection:

$$
\frac{a_{n}}{a_{p 001}}=29\left[\frac{\dot{m} D(1-x)}{\mu_{L}}\right]^{-0.3}\left[\frac{\dot{\underline{m}}^{2}(1-x)^{2}}{\rho_{L}^{2} g D}\right]^{0.2}
$$

where the bracketed terms are Reynolds and Froude numbers, respectively. The forced convection modification is actually based on Chaw $1 a^{\prime}$ s supposition [Ch67].

Either (6-14) or (6-15) could replace the Forster-Zuber relation in Chen's correlation. Alternately, another nucleate boiling correlation could be used, such as that of Vaihinger [Va79] who correlated recently various pool boiling refrigerant data for a wide range of reduced pressures to within \pm 15 percent.

\subsubsection{Modification of Forced Convection Contribution}

Bennett and Chen recommended recentiy a modification of the F function for fluids with $\operatorname{Pr}_{L}>1$ (refrigerants have $2<\operatorname{Pr}_{L}<5$ ) [Be80]. Recalling equation (6-5), they noted the Reynolds analogy used in Chen's original development was valid strictly for $\mathrm{Pr}_{L}=1$. Bennett and Chen proposed various Prandt number corrections, the best fitting their data with

$$
F_{B C}=\left(\frac{P_{L}+1}{2}\right)^{0.445}\left(\frac{(d P / d z) T_{f}}{(d P / d z)_{L O}}\right)^{0.445}
$$




$$
\approx \operatorname{Pr}_{L} 0.296\left(0_{L_{t t}}^{2}\right) .445
$$

The frictional pressure drop ratio is to be predicted on a 'best available' basis; commonly this selection is the Martinelli method (and referred by Bennett and Chen as a reasonable approach).

For $\mathrm{PI}_{\mathrm{L}}>1$, the recommended modification has the effect of increasing the evaporative and decreasing the nucleate boiling contributions. Development of the Prandtl number correction assumed that ebullition destroys the viscous sublayer. If nucleate boiling is suppressed completely, Bennett and Chen recommend deletion of the prandt number correction.

\subsubsection{Modification of Suppression Factor}

An al ternative that has recently been proposed changes the suppression factor. Bennett et al. derived a semi-analytical formulation for $S$, given as [Be80a]:

$$
\begin{aligned}
S_{B} & =\frac{\lambda_{L}}{a_{L 0} F z}\left(1-\exp \left(\frac{-a_{L O} F z}{\lambda_{L}}\right)\right) \\
\text { where } z & =0.041\left(\frac{\sigma}{g\left(\rho_{L}-\rho_{v}\right)}\right)
\end{aligned}
$$

The only empirical value is 0.041 . 


\subsubsection{Other Modifications to Chen's Method}

Po1ley [Po82] has recently developed a modification to Chen's method with the following characteristics:

(a) a simpler pool boiling relation

(b) a criterion to exclude the nucleate boiling contribution if there is insufficient superheat

(c) a suppression factor based on the magnitude of the pool and evaporative contributions, rather than the flow parameters used in Chen's method

The suppression factor was optimized empirically from a large set of water-steam data. The method produced improved agreement with this data base. His equations are given in Appendia 6B.

\subsubsection{Application of Modifications to Chen's Correlation}

The previous sections have described several modifications to Chen's original method; prior to this report, none has been applied independently of the data base used by the authors of these modifications. It is interesting to note that virtually every term except the Dittus-Boel ter equation used in Chen's original me thod has been recommended for change.

Considerable care must be taken in testing the changes suggested in the previous sections, as will be shown. Chen's original superposition employed the Forster Zuber pool boiling relation where $\alpha_{\text {pool }}=a_{\mathrm{FZ}}=$ $f(\Delta T)=f\left(T_{w}-T_{s a t}\right)$. This approach is different systematically than ${ }^{a}$ pool $=f(q)$ as suggested by equation $(6-14)$. The pool boiling heat transfer coefficient of Forster and Zuber is: 


$$
\alpha_{F Z}=C_{F Z} \Delta T^{0.99}
$$

where $C_{F Z}=C_{F Z}$ (properties), and which may be found by comparison to equation (6-10). By examination of equation $(6-10)$ or $(6-11)$, it can be seen that, for a fired total heat flux, the calculated $\Delta T$ is 1 owered by the presence of forced convection over that which would occur in pool boiling alone. Consequently, because of the form of equation (6-11), the nucleate boiling contribution is seen to be 1 ower than for the pool boiling beyond that accounted by the suppression factor. Put another way,

$$
q_{w}=q_{f c}+q_{n b c}=a_{L} F \Delta T+c_{F Z} \Delta T^{1.99} S
$$

so that $q_{n b c}=q_{w}$ only in the case of no flow. In contrast, the approach suggested by the use of equation (6-14) implies $q_{n b c}=q_{w}$. In Polley's development, this problem is resolved due to the reoptimization of the suppression factor.

To use (6-14) in Chen's method, the equation must be reformulated in terms of $a_{S A}=f(\Delta T)$, as follows:

$$
a_{n}=a_{p 001}=a_{S A}=c_{S A^{q}} \cdot 745
$$

where $\mathrm{C}_{\mathrm{SA}}$ can be found by camparison to equation (6-14). For pool boiling alone, 


$$
q_{\text {poo1 }}=a_{S A} \Delta T=\left(c_{S A} q_{p 001} \cdot{ }^{.745}\right) \Delta T
$$

upon eliminating $q_{p o o l}$

$$
a_{S A}=C_{S A}^{3.2916} \Delta T^{2.9216}
$$

This result can now be substituted into equation (6-3) to get

$$
a=a_{L} F+c_{S A} 3.9216 \Delta T^{2.9216} \mathrm{~S}
$$

and

$$
q=a \Delta T=\left(a_{L} F+C_{S A}{ }^{3.9216} \Delta T^{2.9216} S\right) \Delta T
$$

This equation must be solved iteratively for $\Delta T$, and consequently $a$, in the case of constant wall flux. Thus, the suggested modification of Butterworth and Shock, which might yield more accuracy, pays the cost of increased computational diffical ty.

\subsection{Experimenta1 Resu1ts: Comparison to Measured Data}

Recall Table 5-1 for the summary of the data base characteristics.

Table 6-4 displays the many variations of Chen-styled methods which were considered in the analysis.

These characteristics must be considered in the analysis of the predictive ability of correlations. 
Table 6-3 displays the results of comparing many of the methods to the experimental results. All methods are compared to the data according to the mean fractional standard deviation:

$$
\bar{\sigma}=\frac{\sum_{i=1}^{N}\left|\left(a-a_{\text {meas }}\right) / a_{\text {meas }}\right|}{N}
$$

The best two prediction methods are circled in Table 6-j.

\subsubsection{Comparison to Rig \#1, R152a Data (Forced Convection/Evaporation Dominant}

Al1 of the form (6-1) equations predict badly. The method of Traviss et a1. is derived from condensation research. It underpredicts due most likely to different entrainment rates in evaporation and condensation.

Of the many Chen-styled correlations, the evaporative portion of Chen's original formalation with $F_{c}$ being empirical and Chen's original formalation with the reformalated Stephan-Abdelsalam method (based on equation (6-24)) predict best. Figure 6-2 displays the variation of a with quality for some of the Chen-styled correlations. Again, the two methods mentioned above predict the slopes very well, though sometimes the magnitude of $a$ is in error. It is interesting to note that Chen's original method overpredicts the measured values. Overprediction with refrigerants has only occurred in one other instance [Ch67]. 
6.3.2 Comparison of Rig \#2 Preheat Data (Nucleate Boiling Dominant)

This data base consisting of about 200 points, includes beat flures ranging from 10 to $95 \mathrm{~kW} / \mathrm{m}^{2}$ and mass fluxes of 100 to $500 \mathrm{~kg} / \mathrm{sqm} / \mathrm{s}$.

Table 6-3 displays the predictive ability of various correlations against both pure R152a and pure R13B1. None of the form (6-1) correlations do well. Since nucleate boiling is the dominant featare, one might expect pool boiling relations to perform well. The correlation of Stephan and Abdelsalam (equation 6-14) predicts esceptionally well with the data within \pm 20 percent and most predictions within 5 to 10 percent. Since equation (6-14) was developed from a large refrigerant data base, the results are pleasingly consistent. Refrigerant R152a was not considered in the authors' equation development, so the experimental data extends the verification of the predictive ability of the method. The effects of mass flux and quality are not accounted by equation (6-14). Equation (6-15) is the attempt made by Stephan and Auracher to account for mass flux and quality. It however overestimates their effect.

After eliminating the film boiling data for R13Bl, the remaining data of Rig \#1 was seen to be dominated by nucleate boiling. As shown on Table 6-3, the measured values are predicted best by the same pool boiling relations as R152a, adding further verification to the method. 
6.3.3 Comparison of Rig \#2 Test Section Data (Forced Convection Dominant - Some Nacleate Boiling Contribution Possible)

Again, none of the Form (6-1a) methods predict either the slope da/dx nor the magnitude of $\alpha$ we11. The best prediction techniques are those when the Prandt1 number correction suggested by Bennett and Chen is combined with the empirical F function:

$$
a_{e}=a_{L O} F_{c}\left(X_{t t}\right) P_{L} 0.296
$$

and the nucleate boiling contribution is that of Stephan and Abdelsalam (equation 6-24). Figure 6-4 displays the results. There is a larger error, but greater uncertainty with the R13Bl data (section 3.8). If the measured values were based on measured outlet temperature, the RI3BI agrement with the above equation would be superior.

By using equation (6-24) and the Prandt1 correction, the nucleate boiling contribution is nearly negligible. Agrement is excellent even when excluding the nucleate boiling contribution (methods (w) and ( $x$ ) on Table 6-3).

\subsection{Discussion of Findings}

6.4.1 Nucleate Boiling Dominated Fl ow Situations

It was found that little or no mass flux correction was needed to fit ihe data in this situation. Inclusion of the mass flux correction suggested by Chaw 1 a and adopted by Stephan and Anercher degraded the predictive ability of the pool boiling correlation of Stephan and Abdelsalam (equation 6-14). In their original paper, Stephan and 
Auracher eramined oight refrigorant data bases (throe with R11, three with R12, one $\mathbf{\text { ith }} \mathrm{R2} 2$ and one ith R1 14). The RII data was provided by Chaw $1 \mathrm{a}$, so the forced convection modification, derived fram his data, should be expected to fit the data well. Tho of the remaining five data bases are not as well predicted.

Fur ther, Vaihinger noted no influence of mass flur in his results, nor has Moller, has Brauer [Va79]. On the other hand, [Ma79] data clearly has a mass flux effect.

The mass $f 1 u x$ correction (equation 6-15) has a dependence on tabe diameter; though the dependence is weak, there is no physical reason for anf functional relation. Also, the heat transfer coefficient is inversely proportional to quality. It agrees vith various observations in the Iiterature [Ch67, $\mathrm{Ka76}, \mathrm{St82}$ ] of a heat transfor coefficient reaching a minimum before convective/eraporative processes gron and dominate. Such behavior is al so observed with some of the Rig \#1 data when it is dominated by nucleate boiling.

Cherstyled correlations explicitly include forced convection offect, even with small flow rates. The influence of mass flar diminishes as heat flax increases (i.e., as apool increases). It establishes a proper trend, bat never reduces the mass flur effect to zero. It saggests as quality increases, mass flax effects increase in influence, the opposite of Chamla's supposition. 
The predictive ability of the complete Chen-styled correlations developed from the 1 iterature was not good for this heat transfer regime. The best prediction came with using $a_{\text {pool }}=a_{S A}(q)$, i.e., using equation (6-14) in conjunction with equation $(6-3)$. As previously discussed, this is an incorrect application of the pool boiling relation in Chen's method. Furthermore, use of the Forster-Zuber pool boil ing method instead of the Stephen-Abdel salam method in Chen's formulation revealed dramatic differences in the predicted $a$. In their original poo1 boiling form, the methods are compared in Figure 6-3a. Forster and Zuber underpredicts a at large heat flaxes, e. $8, a_{F Z}<a_{S_{A}}$. However when these formulas are used in Chen's method with $\alpha_{\text {pool }}=\alpha_{\text {pool }}(\Delta I)$, their relationship is reversed (Figure 6-3b). The Stephan and Abdelsolam method predicts a larger nucleate boiling contribution. This is due to the methods having a different functional dependence on $\Delta T$.

It is clear that since the original Chen formulation of 1966, progress has been made in predicting pool boiling heat transfer and that the improved capability has as a prerequisite the specification of fluid classifications (and surface characteristics). Given the pool boiling progress, it may be useful to reformulate the suppression factor in Chen's method to improve its overall predictive ability. Any reformulation should allow the forced convection term to diminish in influence to near zero, as shown in the experimental data.

The issue of the influence of mass flux is not settled. It bas been observed to be negligible in some cases and apparently not in others. 
The predictive ability is not available to determine conditions when a mass flux correction term is needed.

\subsubsection{Forced Convection Dominated F1 ow Situations}

None of the form (6-1a) equations fit the data well; this result is not surprising since rarely have they produced satisfactory agreement with data sets other than the ones for which they were originaliy developed.

The original Chen correlation badly predicted da/dx slopes. This is consistent with many other researchers' findings. The nucleate boiling contribution is overestimated in this flow situation. It is interesting to note that the method overpredicts the Rig \#1 data and generally underpredicts the Rig \#2 data. Underprediction of refrigerant heat transfer coefficients is more common, al though the method has overpredicted in at least one instance [De78].

The use of the analytic form of the F-function (equation (6-5)) degraded the predictive ability. The analytic form $F=F_{c}(\Delta P)$ has a higher value than the empirical $F=F_{c}\left(X_{t t}\right)$ at values of $1 / X_{t t}<2$ corresponding to 1 ow quality. It overpredicts in this region. At high quality, the methods nearly agree, and it makes little difference. In general the empirical F was slightly more accurate.

Of the multiple Chen-styled correlations, none fit both the Rig \#1 and Rig \#2 results. Best agreement with Rig \#1 data (R152a only) was achieved including the Stephen-Abdelsalam relation with Chen's original 
formulation (equation $(6-24)$ ). This approach requires iteration, however, adding to the complexity. Further, it produced poor agreement with both Rig \#2 results and importantly the nucleate boiling dominated reuslts previously discussed. In general the agreement with da/dx is good for the Chen predictions without the nucleate boiling contribution.

Best agreement with the Rig \#2 data al so used the Stephan-Abdelsalam method, but included a Prandt number correction suggested by Bennet and Chen. The question then naturally arises as to how to predict the need for the Prandtl number correction. An approach is discussed in the next section.

\subsubsection{A Complete Correlation}

In the previous sections, it was seen that no single correlation fit all the data. However, a particular correlation predicted well in certain situations. In this section, a criterion/procedare will be established which determines when to use a particular correlation.

Following Chaw $1 a^{\prime}$ s suggestion [Ch67], one can determine the heat transfer regime and $\alpha$ by:

$$
\begin{aligned}
a & =a_{\text {pool }} \text { if } a_{p 001}>a_{F C}^{*} \\
& =a_{F C}^{*} \text { if } a_{F C}^{*}>a_{p o o l}
\end{aligned}
$$


The previous sections showed that, at 1 ow pressure, when nacleate boiling $\nabla a s$ completely suppressed, the two best predictive methods were: 1

$$
\left.a_{F C}^{*}=a_{L O} F_{c}\left(x_{t t}\right)+c_{S A} 3.9216 \Delta T^{2.9216 s} \overline{[\sigma}=0.07\right]
$$

and

$$
\left.a_{F C}^{*}=a_{L 0 F} F_{t t}\right)[\bar{\sigma}=.212]
$$

At higher pressures, where same nucleate boiling contribution was observed (Chapter 4), the best predictions were -ith

$$
a_{F C}^{*}=a_{L O F} F_{c}\left(x_{t t}\right) P_{L} 0.296+C_{S A} 3.9216 \Delta T^{2.9216} \mathrm{~s}[\bar{\sigma}=0.055](6-27 \mathrm{a})
$$

and

$$
a_{F C}^{*}=a_{L O} F_{c}\left(x_{t t}\right) P_{I} 0.296[\bar{\sigma}=0.057]
$$

The difference between the two methods is the Prandtl correction, which is substantial. To determine when to include the correction, the suppression criterion of Chapter 4 is recommended as the determinant. If the given $\mathbf{\text { all }} 1 \mathrm{flux}$ is greater than that predicted by the suppression criterion, then equation (6-27a) or (6-27b) should be osed Alternately

The pool boiling relation to be used is that of Stephen and Abdel sal am (equation $(6-14)$ ). 
if the suppression criterion predicts insufficient heat flux, equation $(6-26 a)$ or $(6-26 b)$ should be used.

Al though equation (6-26a) was superior to (6-26b), one might question on theoretical grounds its use. If the data is in fact representative of a completely suppressed nucleate boiling heat transfer regime, then there is no reason to include the nucleate boiling term. In defense of its inclusion, two reasons might be offered:

(a) The original Chen development/optimization presumed contributions from both regimes, so that $F$ was not optimized for complete suppression.

(b) The suppression criterion predicts ebullition from large cavities even with very little superheat available. The large cavities may then be 'active' bat too few in number to destroy the viscous sublayer (and require the Prandtl number correction). The fact that some cavities are active may justify the inclusion of the nucleate boiling term.

If the nucleate boiling term is included, however, there is an inherent contradiction. With more substantial ebullition (e.g., higher $q_{w}$, the Prandt 1 number correction is invoked, and the evaporative contribution is increased at the expense of the nucleate boiling contribution. Thus while one would expect $a_{n}$ to be greater at higher $q_{w}\left(q_{w}\right) q_{\text {sup }}$ ), it may in fact be reduced.

Because of this reason and others to be discussed below, the author prefers the use of equation $(6-26 b)$ in the case when $q_{w}<q_{\text {sup }}$ When combined with the criterion of equation (6-25), $\sigma=0.122$ for Rig $H 1$; 
this value is superior to every other method, and yields better agreement than appears in much of the literature for heat transfer correlations. 1

Equation (6-27b) yields nearly as good agreement as (6-27a). Elimination of the nucleate boiling contribution, though not correct the oretically, offers the practical benefit of a non-iterative solution. The prediction is al so more conservative without the nucleate boiling term. The use then of $(6-26 b)$ and $(6-27 b)$ with the criterion of $(6-25)$ is theref ore recommended.

\subsubsection{A Comparison with 0ther Data}

As a further test of the approach, spot checks were done against other Iefrigerant data in the literature.

Heat transfer coefficients for R2 2 were measured by at least three different authors $\left(\operatorname{Pr}_{L} \cong 2.5\right)$. Their data is shown on Figure 6-6, with equation (6-27b). The need for the Prandtl correction for the Anderson and Mathur/Chaddock data was supported by an examination of their raw data. The Anderson data is predicted very well; it is known that it excludes dryout data. The Mathar/Chaddock data is predicted very well in the 1 ow and middle quality range. At high quality, the approach slightly overpredicts: however this data is for dryout conditions. At low quality, the data exhibits considerable scatter. The scatter is due to the effect of heat flux, neglected by [Ch79]. The use of equation

$\overline{1_{\text {Figure }} 6-5}$ shows the results of using the criteria. 
(6-25) would have improved agrement in this region; however property values were not available to perform the calculation.

Chaddock and Noerager measured $R 12(P I=3.1)$ and $f$ it the average a to $\pm 15 \%$ w th

$$
a=a_{L 0}\left(3 / X_{t t}\right) \quad 0.2<x_{t t}<0.5
$$

Again, their data showed a fairly strong heat flux dependence, so equation (6-27b) is appropriate. Agrement is fair: $-30 \%$ for $x_{t t}=0.2$ and $-2 \%$ at $X_{t t}=0.5$. The underestimates may be due to the effect of heat f1ux.

Recall that only one data set from the literature [Ch67] was badly overpredicted by the Chen correlation. However, the data of Chaw a exhibited no heat flax dependence. In this case, equation (6-26b) is the correct one for use, and reduces the prediction by more than $50 \%$. This will place the prediction in the appropriate range.

\subsection{Closing Remarks: Conclusions and Recommendations}

A large data base was developed $w$ hich included data from all possible heat transfer regimes. A comparison as made between puolished correlations and the data base. All form (6-1) correlations, widely used in the refrigeration industry, predicted poorly. The result is not surprising as they have tended in the past to suffer from a lack of generality. The new Shah correlation al so predicted badly. 
In the nucleate boiling dominated regime, a recent pool boiling correlation (equation (6-14)) predicted exceptionally well. A correction for forced convection was unnecessary and its inclusion degraded the agreement. Therefore, a criterion is needed for predicting the onset and amount of a mass flur influence. The Chen correlation implicitly includes one which is not correct. Forced convection should be allowed to have little or no influence in certain cases.

The forced convection dominated regime was predicted best by Chen's original method modified to include equation (6-14) for the Rig \#1 data. A Prandtl number correction was needed to achieve good agrement with the Rig \#2 data. The discrepancy between the results is believed due to sustained ebullition. A criterion was developed to determine when to apply the Prandtl number correction.

The complete Chen styled correlation is the most widely used method outside the refrigerant industry for predicting heat transfer coefficients. The literature has show it to predict poorly for organic fluids with blame placed and corrections suggested on various terms of the original correlation. None of these corrections however had been checked prior to this report. All changes suggested in the literature were tested, alone and in combination. Certain forms of the correlation predicted well. A procedure was developed to determine when to apply the various forms. It was shown that the superposition principle could be abandoned in favor of a single dominant mode (either nucleate boiling or evaporation). In this respect, it draws apon the success of Shah, 
Chaw 1a, and portions of Polley. It al so el iminates the need for iteration. It includes the recent suggestion by Bennett and Chen for a Prandtl number correction.

If one chose to retain the complete Chen styled correlation, virtally every term in the original formulation (equation (6-3)) conld bo changod to imrpove agreement. As such each term will be discussed soparately.

To date no one has suggested revising the $a_{\mathrm{L}}$ term in equation $(6-3)$. Yet, the Dittus-Boelter relation is accurate to \pm 13 percent, so that agreement between two-phase (evaporative only) data and prediction may be 1 imtied to this range of uncertainty. More accurate singlo-phase relations are arailable, e.8., Petuthor's method [Pe70] which is believed to be accurate to \pm 5 to 6 percent. This could bo used without adding mach complexity since it is nor-iterative.

In this chapter, a closed form solution was derived for Chen's original method. Thus, iterative solutions, as suggested in rocont torts, may bo discarded for conditions ay from tho critical point. Tho original method empl oyed the Forsterzuber pool boil ing correlation. How over, substantial progress has been made in pool boiling sinco tho Forstor Zuber derelopment. It has boen found that tho best correlations for pool boiling are fluid-spocific, i.e., classos of fluid soch as refrigerants require their own empirical correletione. The pool boiling predictive ability of Forster and Zuber's relation is poor whon appl lod to refrigerants. 
The use of an analytic F-function, such as that found through Reynolds analogy, suffers from the practical lack of predicting closely the pressure drop. Available pressure drop correlations such as MartinelliNel son predict refrigerants to at best $\pm 20 \%$. This uncertainty combined with the uncertainty of predicting single-phase heat transfer coefficients yields an overall uncertainty of at 1 eat \pm 25 percent in predicting $a$ in the simplest case of evaporation without nucleate boiling. The use of the empirical F-function in Chen's original supposition produced better agreement with the measured data. Future work might concentrate on improving pressure drop correlations, perhaps treating separately the cases of nucleate boiling and evaporation.

The semi-analytical S-function developed by Bennett et a1., aggravated the overprediction of the nucleate boiling contribution. This represented the first test of their approach. Since Fand $S$ were developed empirically, and since it has been shown that pool boiling predictions are specialized to classes of fluids, a reoptimization of the $F$ and $S$ functions for refrigerants is recommended with:
a) $a_{\text {LO }}$ derived from Petukhov
b) $a_{n}=a_{p o o l}$ from Stephan and Abdelsalam, kept in its original form to escape the need for iteration
c) a criterion included to el iminate forced convection effects $(F=0)$ in some case $s$
d) a criterion included to eliminate nucleate boiling effects $(S=0)$ in some case $s$, and
e) a large data base of similarly determined experimental data (i.e., all with subcooled inlet, or all with two-phase inlet) over a wide pressure and heat flux range.


Finally the experimental data existent in the literature should be more closely analyzed and stratified by experimental technique. If this is done, improved predictive ability will likely be achieved. The author al so hopes that the complete correlation outlined in Section 6.4 .3 will be tested further. 


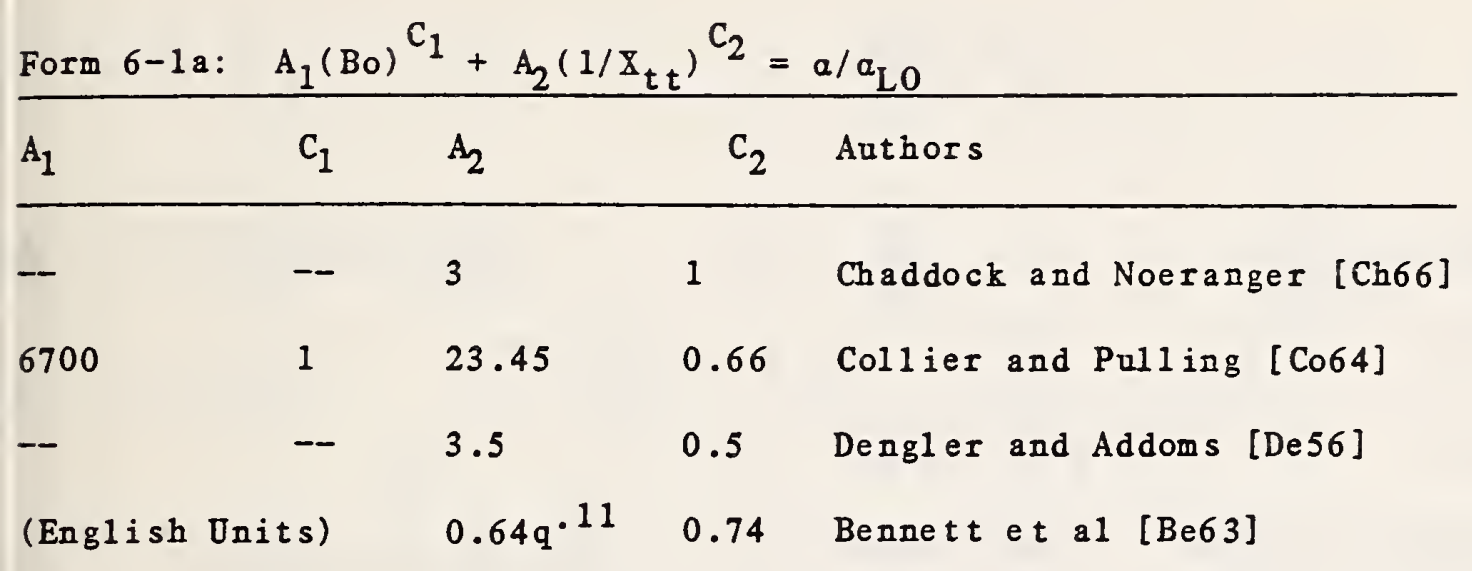

\section{Miscellaneous References and Relevant Refrigerants}

- Lavin and Young [La67]

- Shah [Sh76, Sh82]

- Dembi et al [De78]

- Kandl ikar [Ka84]

- Chaddock and Ma thur [Ch79, Ma79]

- Stephan and Auracher [St81]

- Chaw 13 [Ch67]

- Bende1 and Schlander [Ba74]

- Rhee and Young [Rh72]

- Pierre [Pi56]

- Singal et a1 [Si83]
R12

many

ma ny

many

R22

many

R11

$\mathrm{R} 12$

$\mathrm{R} 12, \mathrm{R} 22$

R12

R13 
Table 6-2: Simple Correlations for Average Heat Transfer of Flow Boiling Refrigerants

$$
a \sim \dot{m}^{-} \mathrm{C}_{3} \mathrm{C}_{\mathrm{u}_{\mathrm{d}}} \mathrm{C}_{\mathrm{s}}
$$

$$
\text { Authors }
$$

Danilova [Da69]

A1 jarrah and Duminil [A177]

Riedle and Purciple [Pu72]

S1 ipevic [S170]

$$
\text { high in }
$$

\begin{tabular}{|c|c|}
\hline & $\mathrm{C}_{4}$ \\
\hline
\end{tabular}

Bogdanov
0.5

0.5

$-1$

$0.25 \quad 0.55 \quad-.2$

0

$0.7-.6$

1.40

$-.54$

$\begin{array}{lll}0.1 & 0.7 & -.54\end{array}$

$0.2 \quad 0.6-.6$

$R 11, R 12, R 22, R 13$

[reprinted from [Bu82]] 
Table 6-4: Smang of Cher-Stylod Mothods

$$
\begin{gathered}
a=a_{L 0^{F}}+a_{n} s \\
a_{L O}=0.023\left(\lambda_{L} / D\right)\left(G(1-x) / \mu_{L}\right) 0.8{P x_{L}}^{0.4}
\end{gathered}
$$

LEHOD

Chen Origianl

Bennett and Chen Original

Bennett and Chen Enpirical $F$

Chen, Anelytic F

Chen Iith Stophan's Pool Boiling ib thod

Chen with Ref ormal ated stephen tethod

Benept $t$ /Chen with Stophn' lothod

Benvet $t /$ Chen ith Refornalated Stephun

\section{Bonnett/Chen vith} Stephan/Chawla

Same as $Q$, bet ith Eaprical $F$

Same as $S$, bet vith

Seme as $\mathrm{K}$, bet vith Analytic S

Chen Originn (Eraporative Contribution orly)

Bennott and Chen (Evaporative Onis)
$\mathbf{P}$

$P_{g}\left(x_{t t}\right)$
$(6-5 a)$

$a_{n}$

$\mathrm{a}_{\mathrm{FZ}}(\Delta \mathrm{T})$

$\mathrm{P}_{\mathrm{g}}\left(0^{2}\right) \operatorname{Pr}_{\mathrm{L}} 0.296$

(6-15)

$P_{\varepsilon}\left(x_{t t}\right) P_{x_{L}} 0.296$

(6-26b)

$P_{q}\left(\theta^{2}\right)$
$(6-5 a)$

$F_{q}\left(I_{t f}\right)$

$F_{g}\left(I_{t t}\right)$

$\left.P_{C}(0) 2\right) 0.296$
$(6-15)$

$F_{q}\left(0_{L}^{2}\right) 0.296$

$(6-15)$

$P_{g}\left(\theta_{1}^{2}\right) 0.296$

$(6-13)$

$\mathrm{P}_{(\sigma-2 i b)}\left(x_{t}\right) P_{L} 0.296$

(6-6a)

$a_{(6-6 a)}(\Delta T)$

${ }_{(6-62)}^{a}(\Delta T)$

${ }_{(6-6 a)}^{a}(\Delta T)$

$a^{a 2}(q)$

$a_{38}(\Delta T)$
$(6-21)$

$a_{(z-13)}^{(q)}$

$a_{2}(\Delta T)$

$(6-21)$

S

$S_{c}(\mathrm{~F})$

$(6-5 c)$

$\mathbf{s}_{\varepsilon}(\mathrm{F})$

$(6-50)$

$S_{c}(\mathrm{P})$

$(6-5 c)$

$s_{(6-5 c)}(B)$

$S_{\varepsilon}(F)$

$(6-5 c)$

$s_{f}(F)$

$(6-5 c)$

$S_{(}(P)$

$(6-5 c)$

$S_{q}($ F )

$(8-5 c)$

$\mathrm{a}_{\mathrm{ga}}(\mathrm{O}){ }^{\circ} \mathrm{C}_{\mathrm{Ch}} \quad \mathrm{S}_{q}(\mathrm{~F})$

$(6-5 c)$
$(6-5)$

a $(\Delta t)$

$\mathrm{S}_{(\mathrm{g}-3}(\mathrm{P})$

$a_{(b-21)}(\Delta T)$

$S_{(}(F)$
$(6-5 c)$

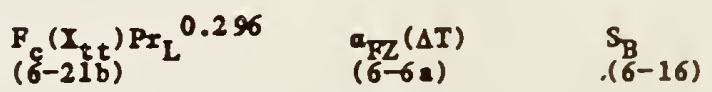

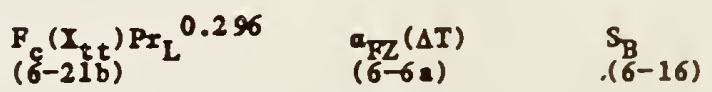

$R_{c}\left(\boldsymbol{x}_{t}\right)$
$(6-5 a)$

$\mathrm{P}_{q}\left(\pi_{t t}\right) \mathrm{Pr}_{\mathrm{L}} 0.296$
$P_{c}\left(x_{t t}\right) P_{L} 0.2956$

As Shown on Tabl 0 6-3

I

J

$\mathbf{I}$

u

$\mathbf{N}$

0

$\mathbf{P}$

a

I

S

I

D

V 


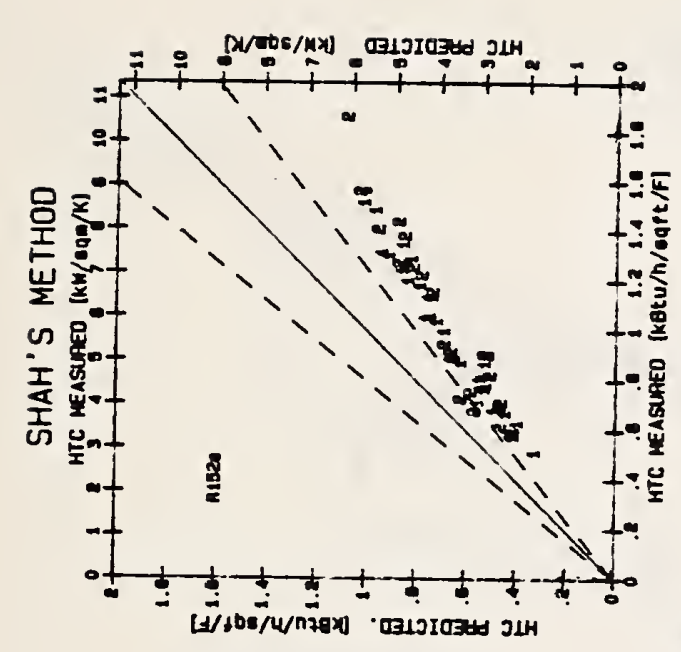

岃

出

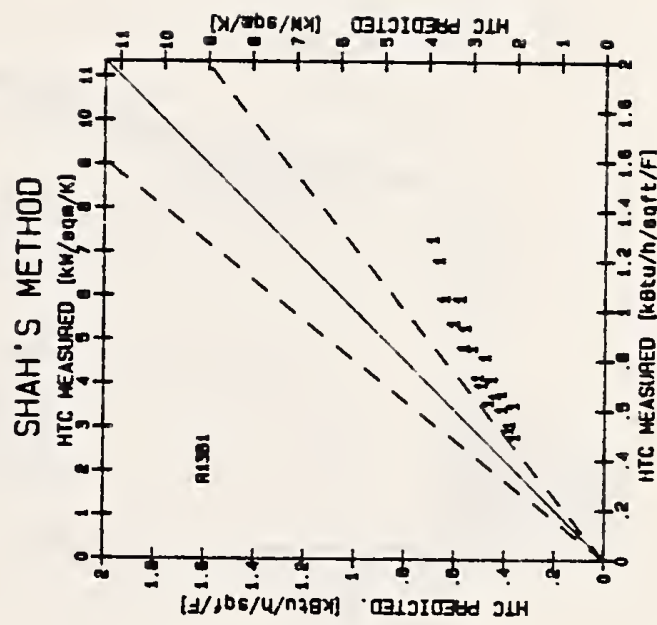

ฮ $\subseteq-1$
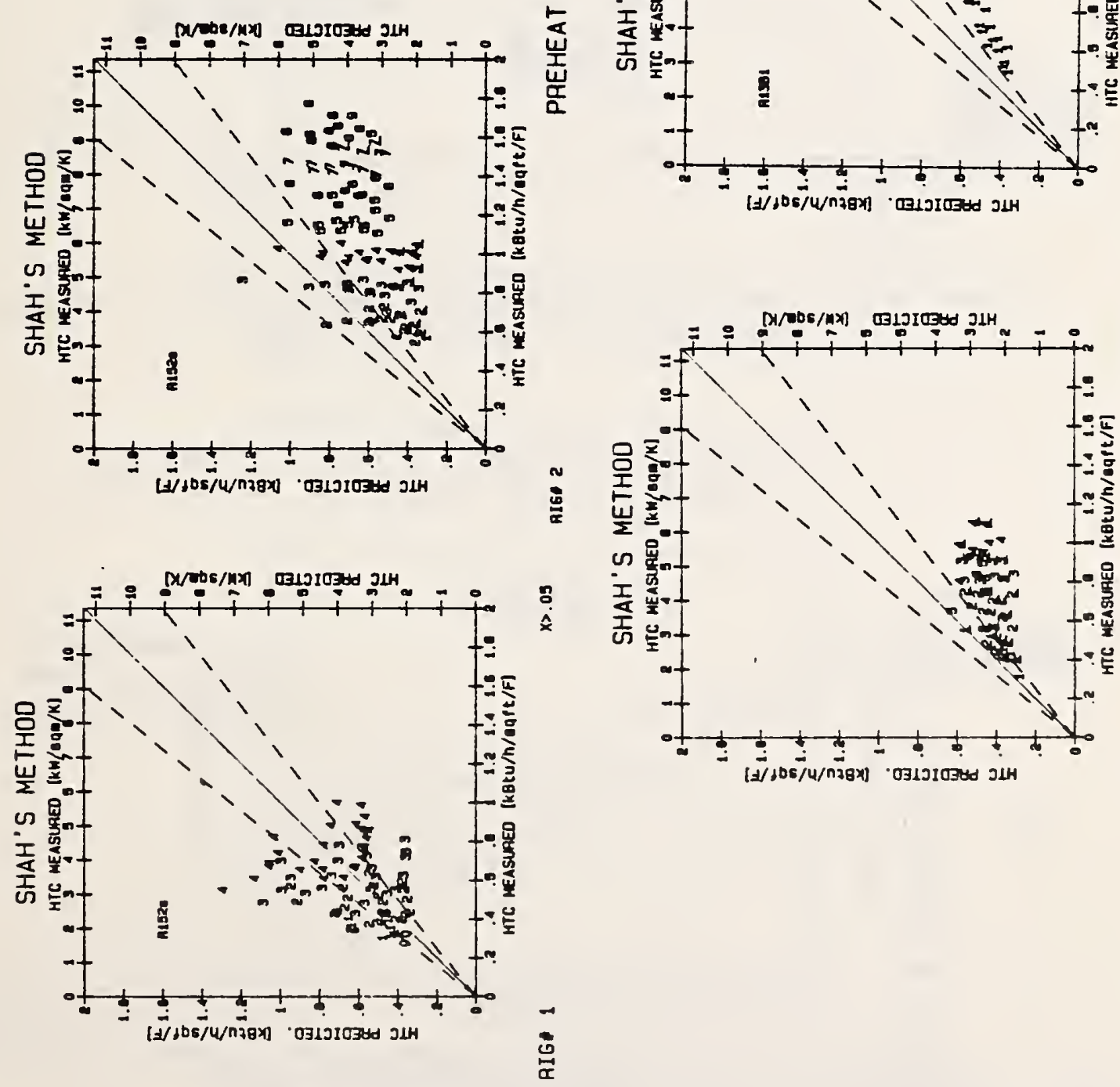

욘

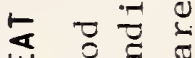

I.

एँ

क 品

¿

吾

L -1

$=$ is

$\Xi$

捠吉

$\pi \Xi$

ఏ્巳巳巳 
F FUNCTION: EMPIRICAL AND ANALYTICAL

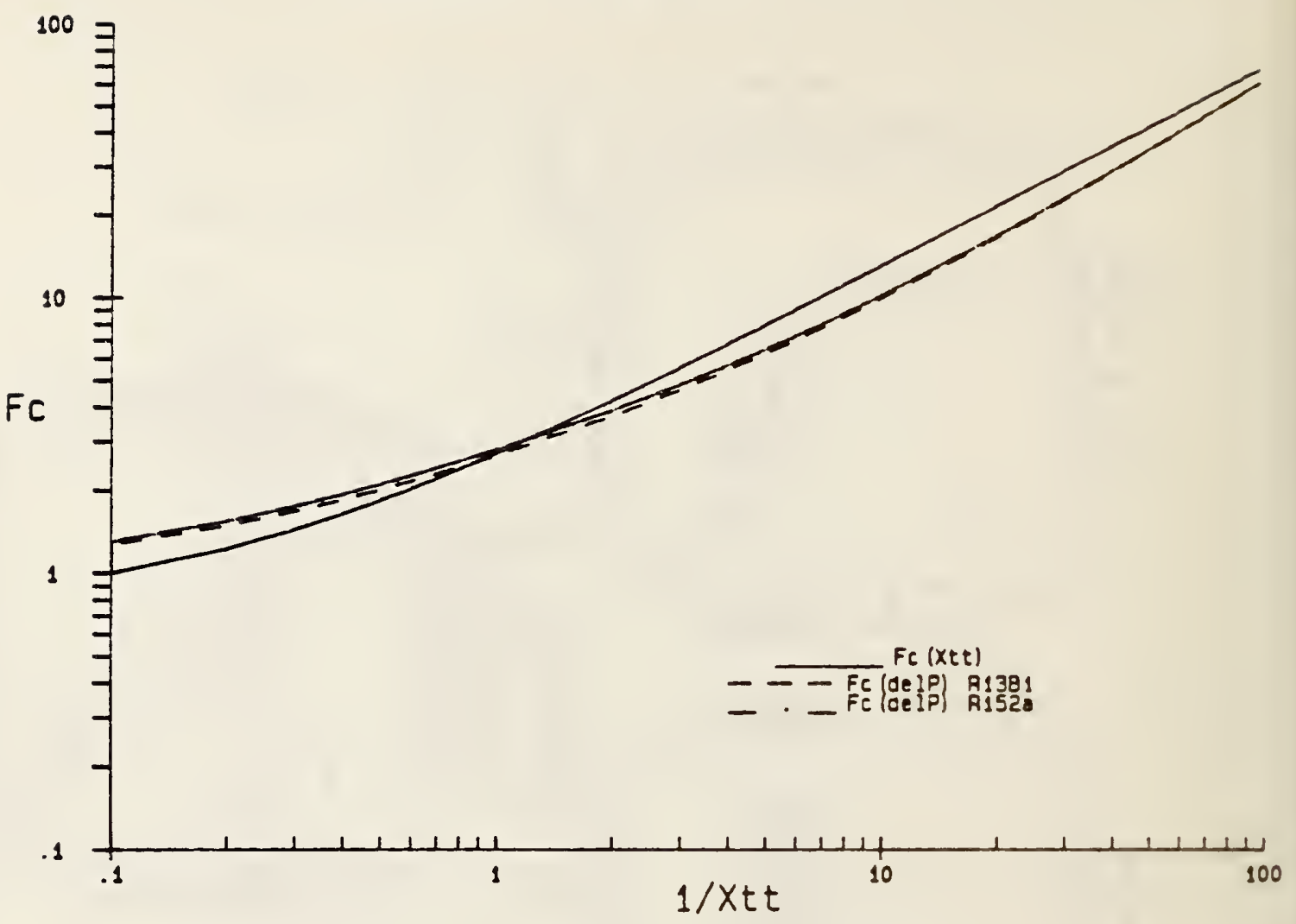

Figure 6-2: Comparison of Empirical and Analvtical Functions in Chen's Method. Pressure $=4.75$ bar.

S FUNCTION: EMPIRICAL AND ANALYTICAL

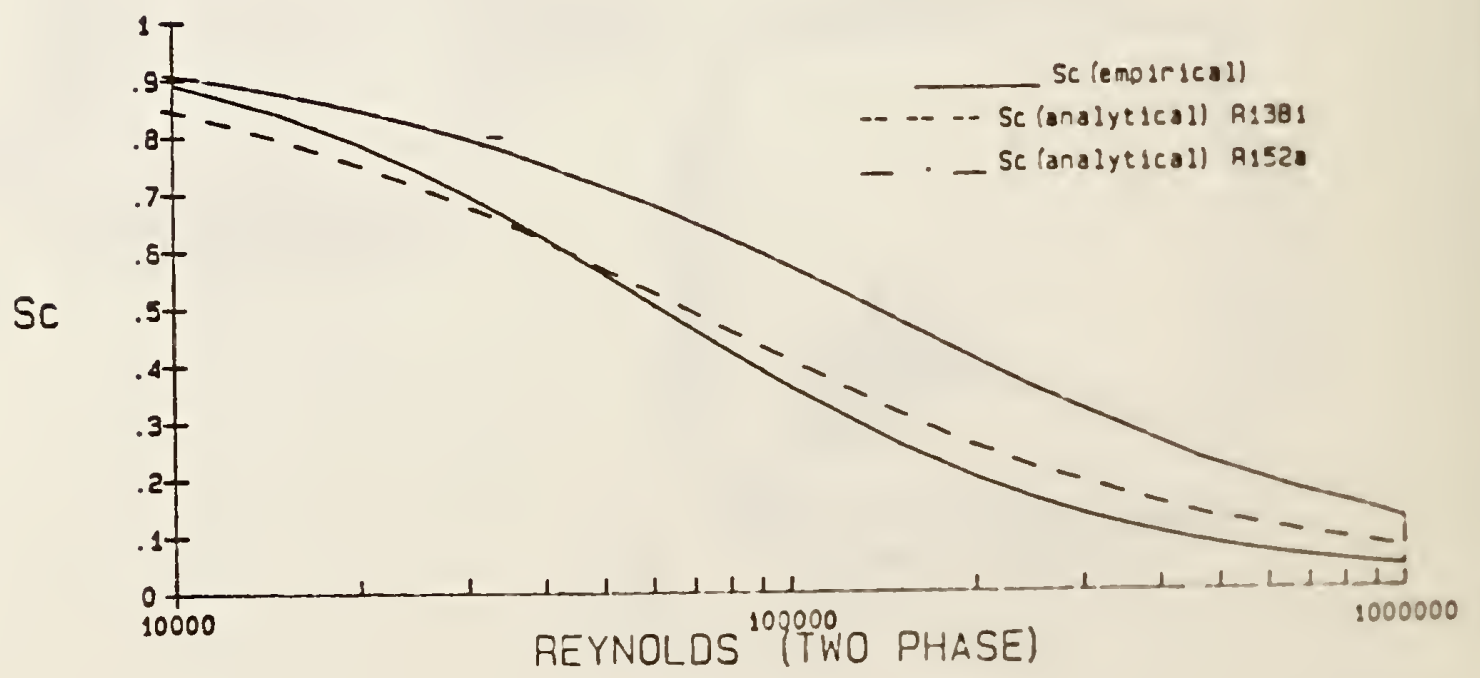



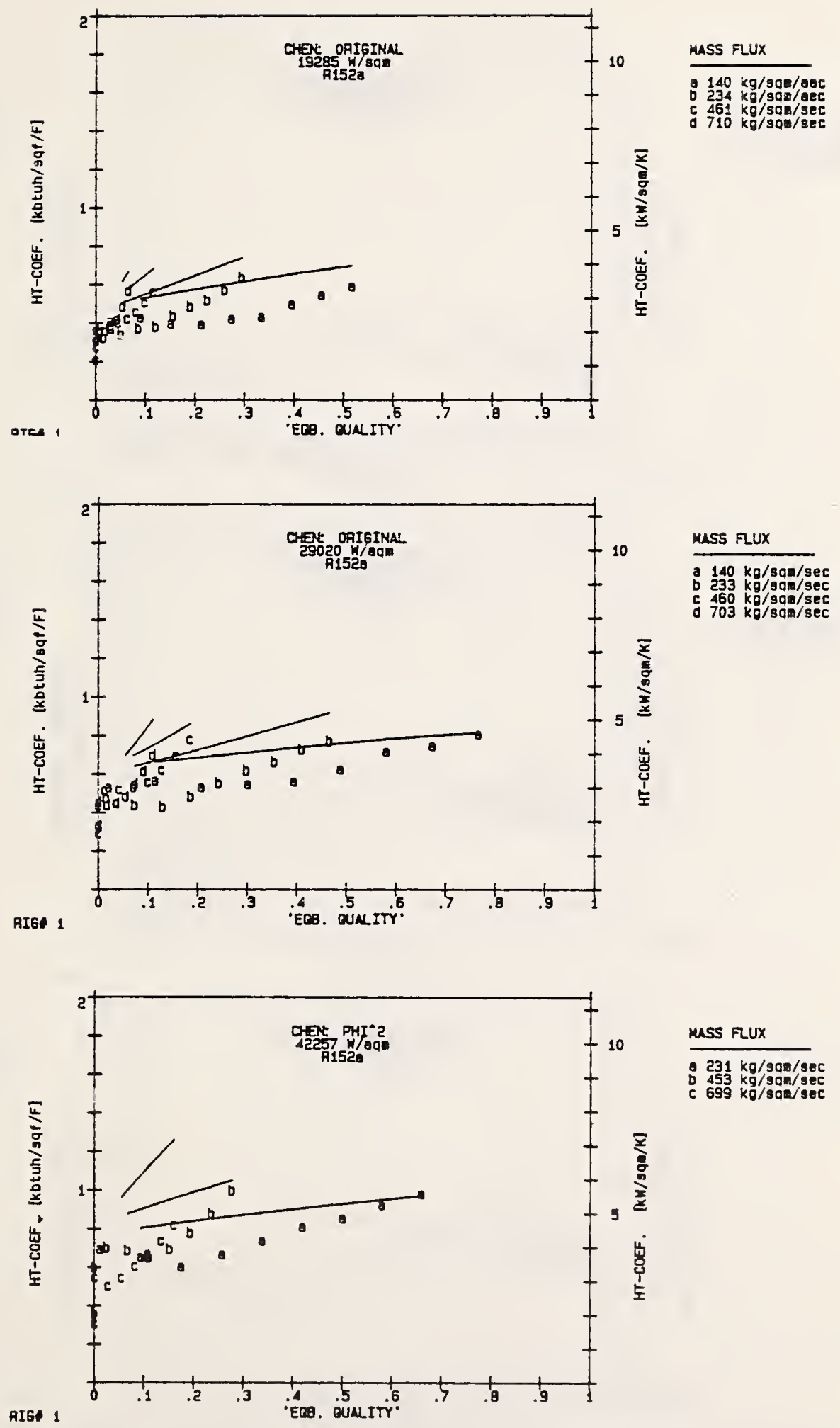

Figure 6-3: Comparison of Chen-Styled Methods to Exneriments. Overprediction is due to nucleate boiling contribution. Use of analytic F-function aggravates overprediction at low $\mathrm{x}$. 

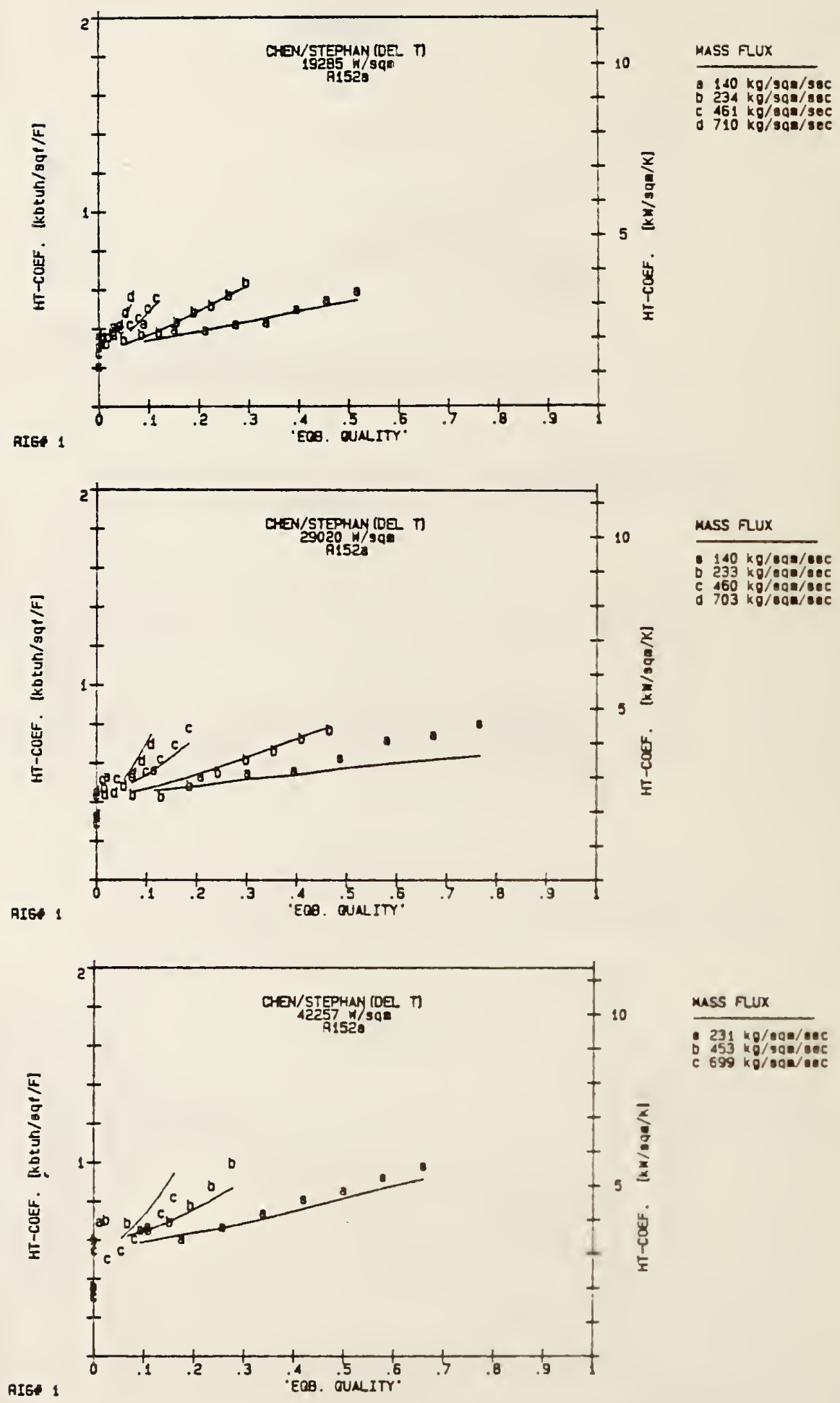

Figure 6-3: Comparison of Chen-Styled Yethods to Experiments. 

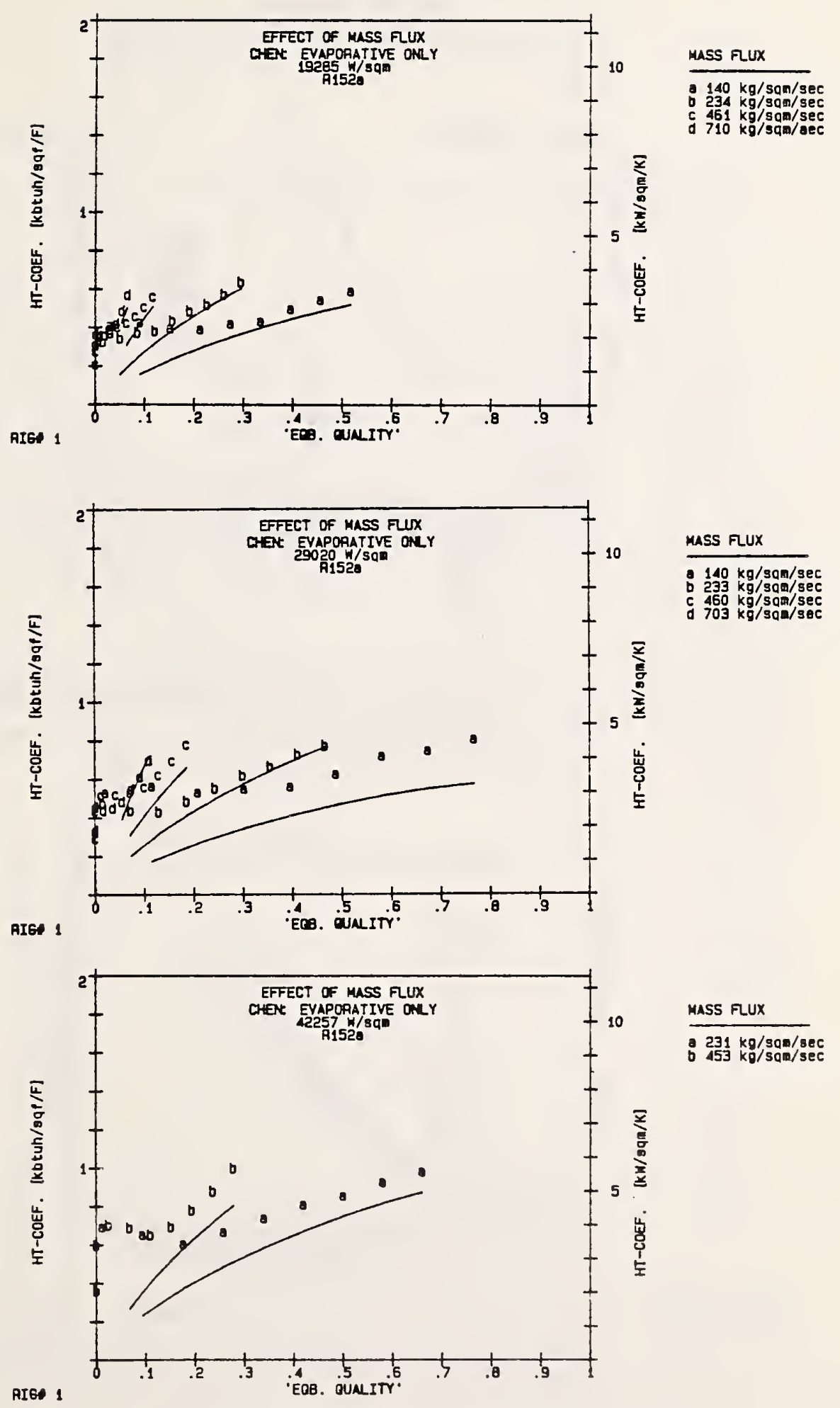

Figure 6-3 (cont): Comparison of Chen-Styled Methods to Experiments. 
POOL: BOILING COMPARISON

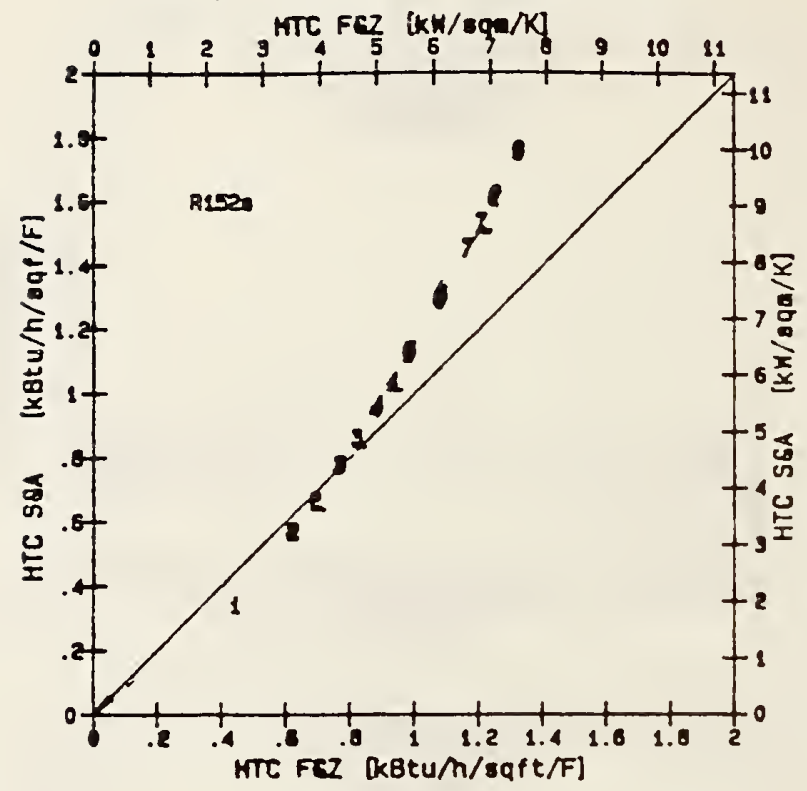

NUC. BOIL. CONTRIBUTION COMPARISON: CHEN

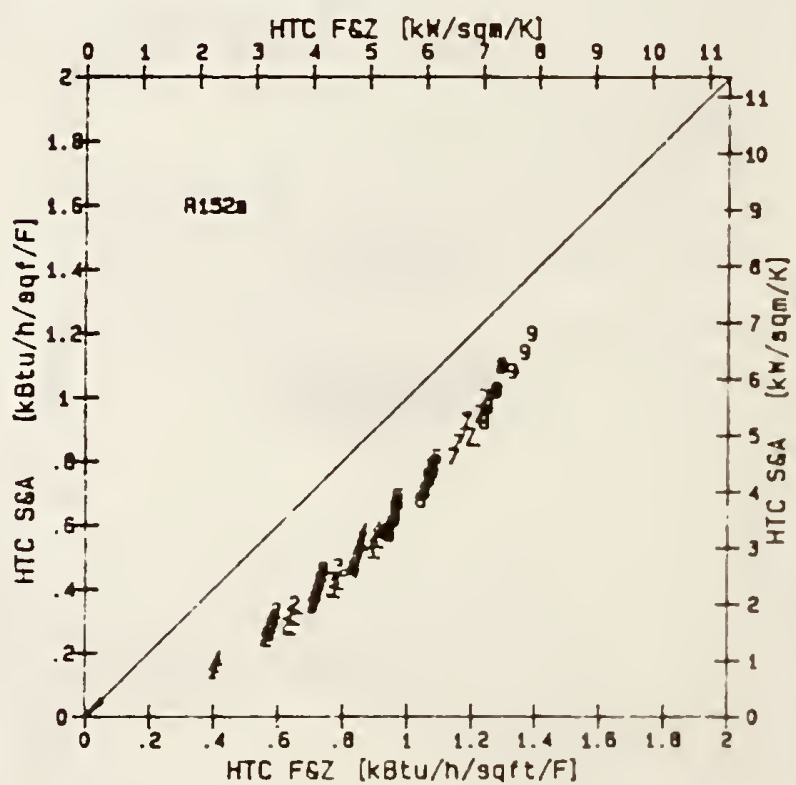

Figure 6-4: Comnarison of SA and Fz Methods. When nut in their original pool boiling form, sA method nredicts creater than FZ. When used in Chen's correlation, the onnosite trend occurs. 

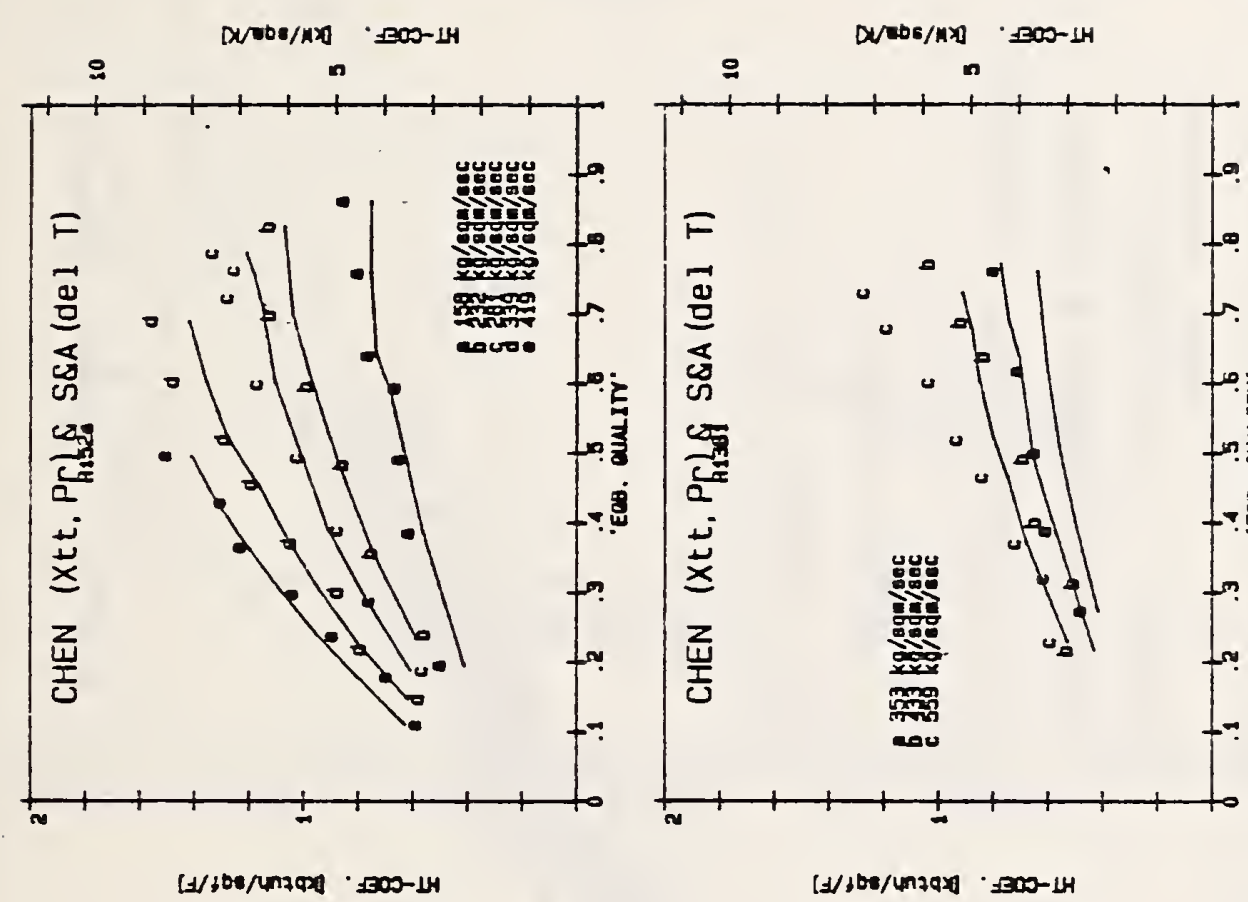

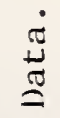

ปี

$\Xi$

U

药

$=$

工

$-\frac{3}{3}$

ธะ

-1.

范

-

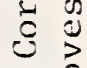

()

岂

हิ

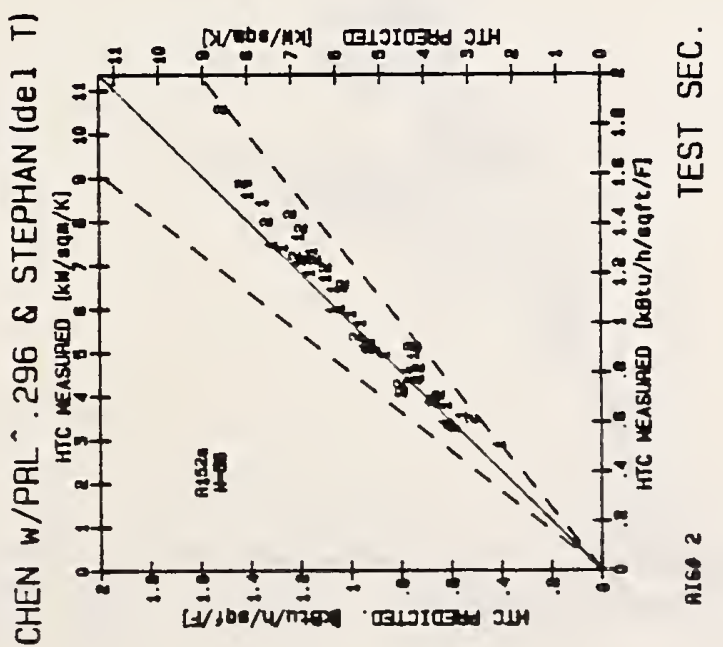

L

के

.

总志

0
1
0
0
0
0
0
0
0 


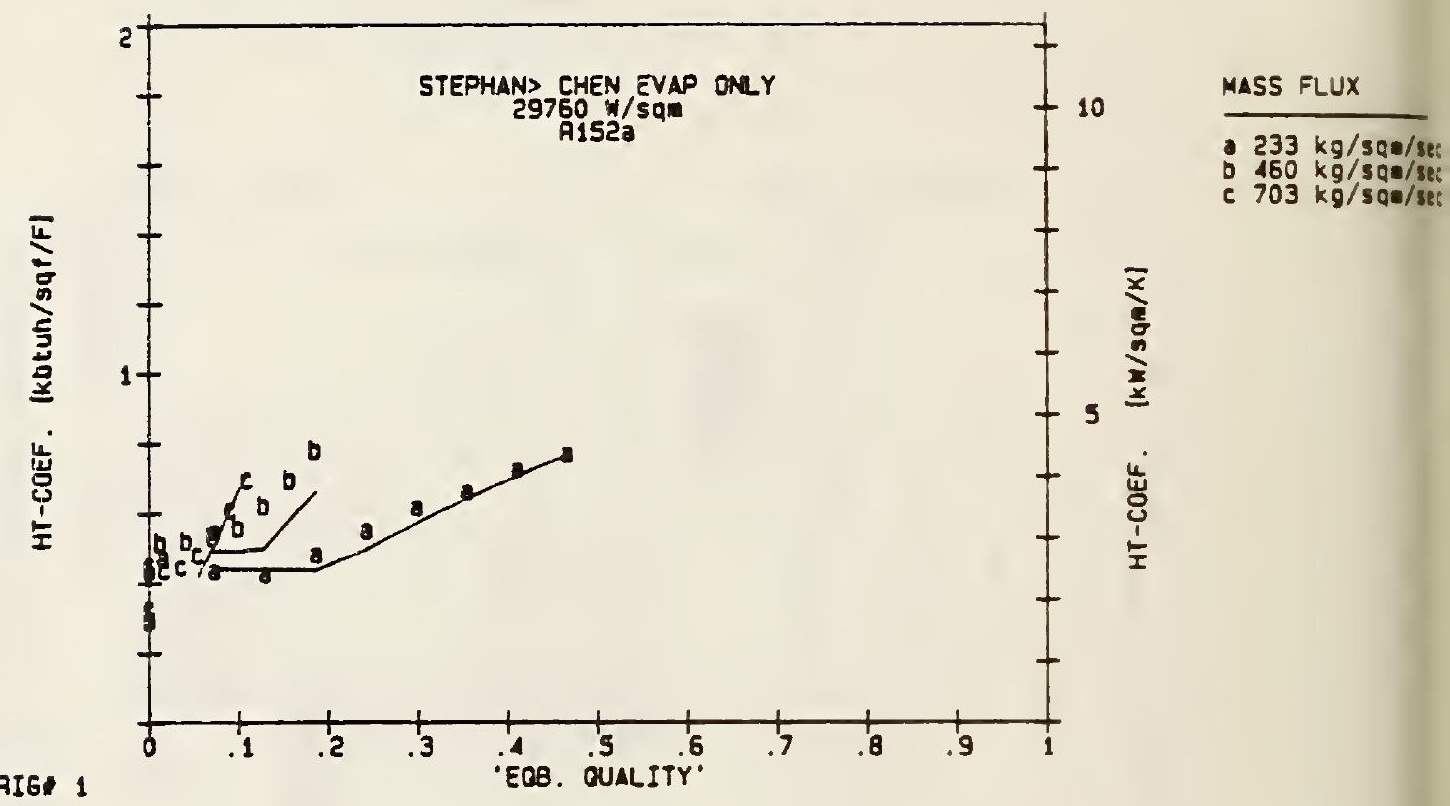

Figure 6-6: Comparison of Complete Correlation with selected Rig非1 data. Chawla's criterion imnroves agreement.

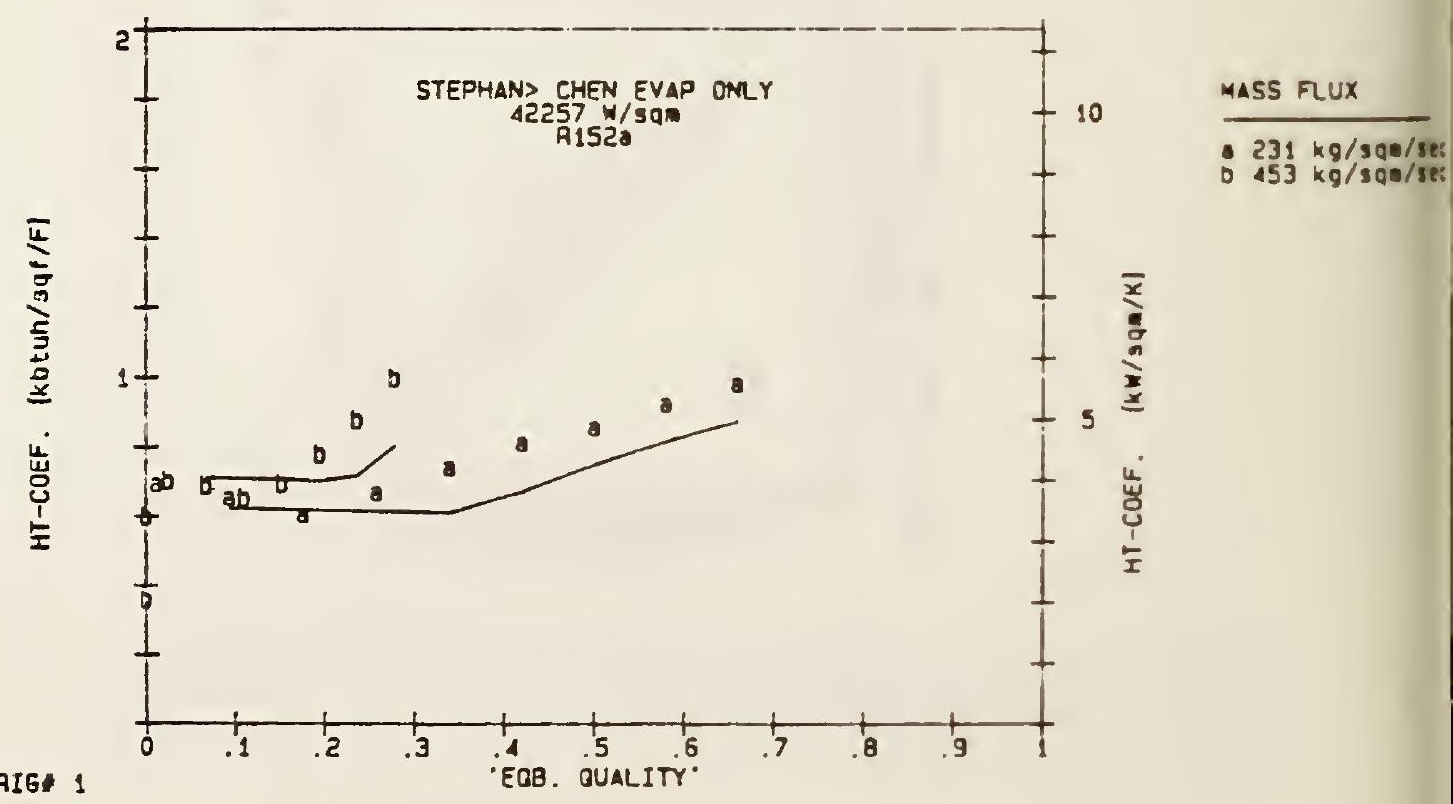




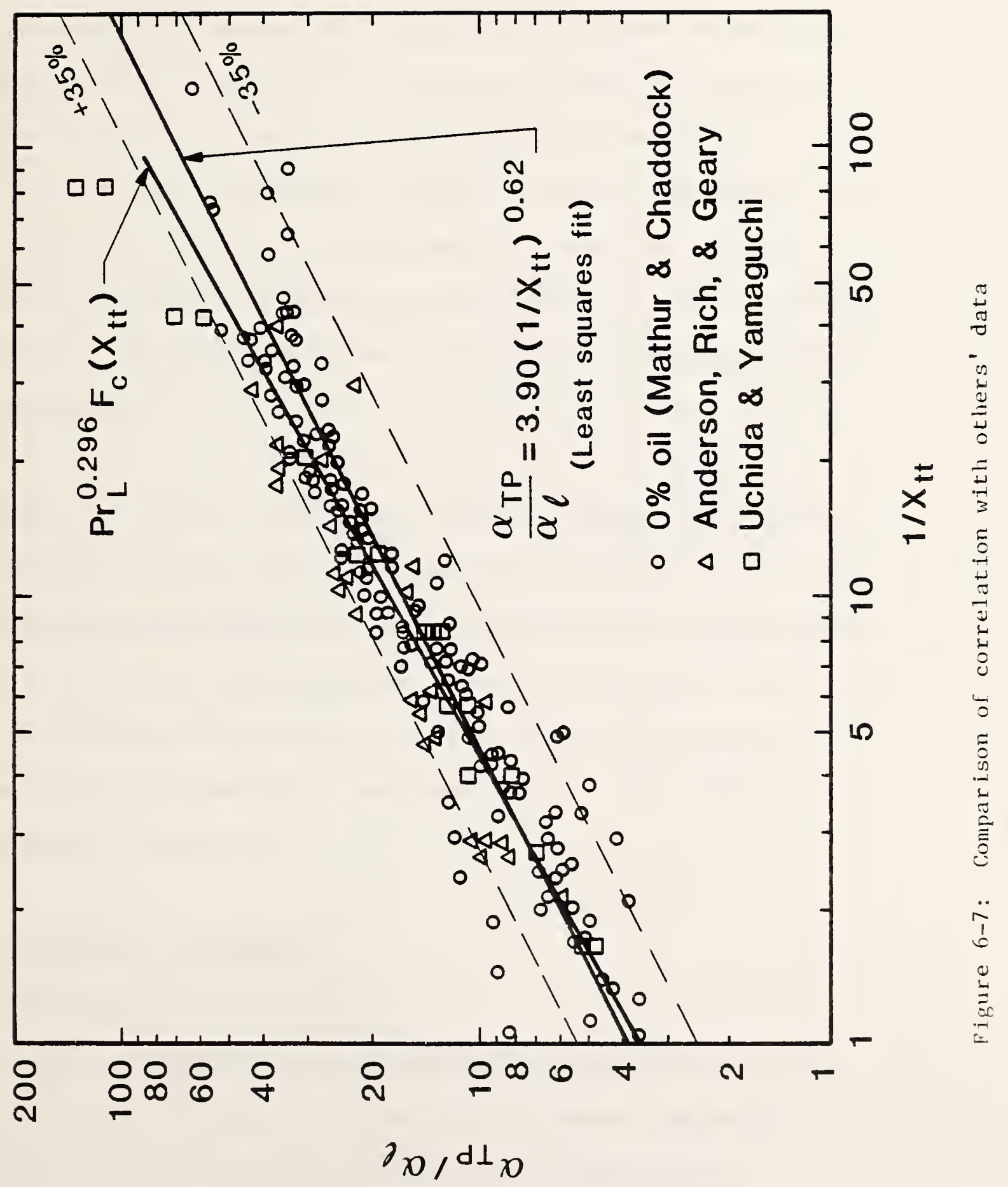



CHAPTER 7: PREDICTION OF HEAT TRANSFER WITH MIXED REFRIGERANTS

\subsection{Introduction}

As has been seen in the previous chapters, heat transfer may be dominated by evaporation from the vapor-liquid interface or by nuclear boiling at the wall surface. Thus, an understanding of each process, and how it is changed by the addition of a second component, is necessary. To date, relatively little work has been done in flow boiling of

mirtures. In contrast, a large experimental base exists for pool boiling of mixtures. As with pare fluids no analytic model is available for predicting heat transfer in pool boiling, and correlations have been devel oped instead.

This chapter will review briefly the analytic modelling of individaal bubbles, discussing their implications on the real situation (section 7.2.1), pool boiling correlations for mirtures (7.2.2), analytic mode1ling of forced convection/evaporation mirtures and relation correlations (7.3). Then complete correlations for flow boiling of mixtures are described (7.4) and compared to experimental data (7.5). The findings are discussed in section 7.6 .

\subsection{Pool Boiling of Mirture}

\subsubsection{Modelling of Single Bubbles}

As discussed in Chapter 1, mixtures differ from pure fluids in two fundamental ways: (1) vapor is formed of a different composition than liquid, and (2) the saturation or bubble point temperature is 
non-isothermal, being a function of local composition. The consequence of these differences can be illustrated qualitatively:

Consider a liquidmixtare of overall composition of $\mathrm{x}_{\mathrm{e}}$ which is superheated to temperature $T_{e}$, i.e., the amount of superheat is $T_{e}$ $T_{b a b}\left(X_{e}\right)$, as shown in Figure 7-1. As a spherical vapor bubble forms and grows $s^{1}$, it is formed preferentially of the more volatile component. Due to mass continuity, the 1 iquid in the region near the bubble has a lower composition than the bulk 1 iquid. The 1 iquid and vapor compositions at the bubble interface are considered to be in equilibriom. As such the interfacial bubble point temperature is not $\mathrm{T}_{b u b}\left(X_{e}\right)$ but $T_{b a b}\left(X_{i}\right)$, and the appropriate driving force [the amount of superheat] is not $T_{e}-T_{b u b}\left(X_{e}\right)$, but $T_{e}-T_{b u b}\left(X_{i}\right)$, the latter being the smaller quantity. This loss of available superheat is commonly accepted as the principal reason for reduced bubble growth rates, and 1 ower nucleate boil ing heat transfer coefficients observed with mirtures as compared to an equivalent pare fluid.

Several authors have analyzed the grow th rate reduction [Sc59, Va67. Sh83]. The results of their analysis, all of which startwith simple heat and balances, is:

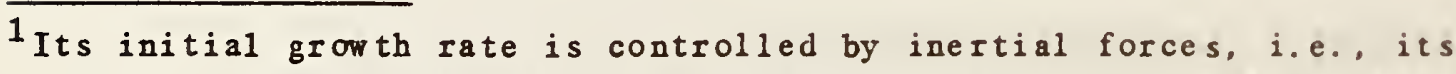
ability to push outwards the surrounding liquid fluid, the process is simply that of an expanding sphere and governed by Rayleigh's equation. Foll owing the initial growth period, the growth of the bubble is controlled by the rate at which heat can diffuse to the bubble interface. In the case of mistures, the growth rate is also influenced by the rate at which the more volatile component can diffuse to the interface. 


$$
\dot{\mathrm{R}}=\frac{\dot{\mathrm{R}}_{\mathrm{EPF}}}{1-\frac{\mathrm{C}_{\mathrm{P}_{\mathrm{L}}} \frac{{ }_{\mathrm{a}} \mathrm{T}}{\Delta \mathrm{h}_{\mathrm{v}}}\left(\mathrm{Y} *-\mathrm{X}_{\mathrm{e}}\right) \frac{\mathrm{dT}_{\mathrm{bnb}}}{\mathrm{a}_{\mathrm{D}}}}{\mathrm{x}_{\mathrm{e}}}}=\mathrm{C}_{\mathrm{BUB}} \dot{\mathrm{R}}_{\mathrm{EPF}}
$$

where $R_{E P F}$ is the growth rate of a bubble of an equivalent pure fluid superheated by $T_{e}-T_{b a b}\left(X_{e}\right)$. The terms of this equation can be examined: as might be expected by the previous discussion, the 1 iquid-vapor composition difference $(Y-X)$ and the ratio of thermal and mass diffusivities ${ }^{2} \mathrm{~T}^{/ \partial_{\mathrm{D}}}$ appear. The term $\mathrm{dT}_{\mathrm{BUB}} / \mathrm{dX} \mathrm{X}_{\mathrm{e}}$ is the slope of the bubble point line. It should be noted that this term and ( $Y-X)$ always have opposite sign, so that the denominator is always greater than 1 , and a reduced grow th rate is predicted. This relation has been verified experimentally [F1 74]. However, the above equation is valid only for the unrealistic situation of an isolated spherical bubble in a uniformly superheated 1 iquid. Several efforts have been made to analyze microlayer evaporation under a binary bubble near a heated wall surface as described in Appendix $7 \mathrm{C}$.

\subsubsection{Boil ing of Mixtures versus Pare Fluid}

Al of the models described in Appendix 7C point to the vapor-1iquid composition difference as a principal factor in the degradation of heat transfer observed in mixtures. This quantity is therefore a 1 ikely candidate to be used as a correlating parameter. Before discussing correlations however, a few other differences between mirtures and pure fluids should be noted. As shown in section 4.4, a larger superheat with a mixture is required to sustain bubble growth from a cavity of 
fixed radius. In a similar fashion, for a fixed superheat, only larger cavities will be activated for mixtures as compared to pure fluids.

Since the population of cavity sizes is

$$
\mathrm{n} \sim \frac{1}{\mathrm{I}}
$$

there will be fewer sites which will be active at a given superheat. This finding suggests again a degradation in heat transfer occurs, due to the reduced number of activated sites with mixtures.

When a site has been activated, observations have shown that the bubble departure size and frequency is less for mixtures than for pare fluids. This leads to less coalescence with neighboring bubbles. The implication of the reduced frequency is again degraded heat transfer.

\subsubsection{Mizture Correlations for Pool Boiling}

None of these detailed points are considered in wixture correlations. Virtually all correlations consist of correction factors to a pool boiling heat transfer coefficient which would be predicted if the fluid were considered to be pure or ideal. Table 7-1 1 ists the correction factors. Note the parameter $|Y-X|$ appears repeatedly. Experiments by Happel and Stephan have shown that the arimum reduction in heat transfer as compared to that predicted by ideal molar mixing occurs when $|Y-X|$ is maximum. They also found no degradation wen the mixtare is at its azeotropic composition, as shown in Figure 7-2. These athors noted 
a pressure dependence on the degradation, as in earlier experiments by Stephan which led to equation (7-24). Shock recently reviewed some of the se correlations, and prioritized their application as:

(1) Use a correlation if it was developed from actual erperimental data for the fluid pair of interest.

(2) If one new experimental data point is available, use equation (7-14) with the constant $A_{0}$ determined from the experimental value.

(3) Use equation (7-13)

(4) Use equation (7-14) with $A_{0}=1.53$

Section 6.3.2 showed that the pure refrigerant data was predicted to $\pm 15 \%$ by the correlation of Stephan and Abdelsalam (equation 6-14). Thas this equation might be used as the basis for analyzing the mirtures' methods shown in Table 7-1. The pure refrigerant data was calculated at the given system pressure and heat flax. An ideal heat transfer coefficient, $a_{i d}$, was then calculated using mole fraction weighting of the pure refrigerant $a^{\prime} s$. This $a_{i d}$ was then corrected for mizture effects by the nse of equations 7-13,7-14, and 7-15. The general curve shapes of these equations is shown on Figure 7-3. Note that the methods produce a maximum degradation in heat transfer at roughly the same molar composition. However the size of the maximum degradation differs considerably between methods. Al so the general shape of the method of Thome predicts a larger degradation over a wider composition range. 
A heat transfer coefficient based on treating the misture as an equivalent pure fluid was calculated with equation 6-14. Note that*EPF is greater than $a_{i d}$. This result is in contrast to that for the evaporative situation as described in Section 2.4. An analysis, similar to that done in Section 2.4, was conducted with equation 6-14, with the result:

$$
\frac{a_{m}}{a_{i D}} \cong \frac{\rho}{v m}_{\rho_{\mathrm{Vid}}}^{.581}{\frac{\rho \rightarrow \mathrm{Lid}}{\rho_{\mathrm{Lm}}}}^{.454}{\frac{\mu_{\mathrm{Lm}}}{\mu_{\mathrm{Lid}}}}^{.533}{\frac{\mathrm{C}_{\rightarrow \mathrm{PLm}}}{\mathrm{C}_{\mathrm{PLid}}}}^{.533}{\frac{\lambda \rightarrow \mathrm{Lid}}{\lambda_{\mathrm{Lm}}}}^{.278}
$$

The last three dimensionless groups have values greater than one. The density ratios have values of about 0.9 or greater, and their opposing effect is thus small. In this case, then, if it were not for other mixture effects, an increased pool boiling coefficient (over an ideal flaid) would be observed.

\subsection{Forced Convection/Evaporation of Mixtures}

\subsubsection{Analytic Mode11 ing}

Shock has examined in detail an ethanol water mixture in turbulent flow, evaporating due to constant wall heat flux [Sh76]. This analysis neglects entrainment phenomena and the presence of waves. Additional assunptions included: a) negligible sensible heating of liquid; b) equil ibrium at the vapor-1 iquid interface; c) neglible axial density and velocity gradients; d) constant shear. The 1 iquid flow velocity profile was approximated via mixing length theory, using eddy diffusivities due to Deissler (near the wa11) and Von Karman. He then assumed the eddy diffusivity for mass to have the same value, i.e. 


$$
\varepsilon_{D} \approx \varepsilon_{M}
$$

Vapor side heat and mass transfer diffusivities were estimated via the Chilton-Colburn analogy, which relates the se coefficients to the friction factor (calculated via Blasius equation):

$$
j_{\text {H }}=j_{D}=f / 2
$$

He then solved the basic continuity and energy equations to determine the interfacial temperature and composition in a stepwise fashion along the length of the tube. He examined changes in the magnitude of the resistances, the heat flar, and the pressure gradient to determine their relative importance. As a base case he assmed no mass transfer resistance, and equilibrium flash vaporization, i.e. $\overline{\mathrm{X}}_{\mathrm{BULK}}=\overline{\mathrm{X}}_{1}, \overline{\mathrm{Y}}_{\mathrm{BULK}}=$ $\overline{\mathrm{Y}} *\left(\mathrm{X}_{\mathrm{B}}\right)$

He found:

(a) mass transfer resistance (MTR) on the 1 iquid side has a negligible effect on the interfacial temperature and composition.

(b) MTR on the vapor side controls what effects do appear;

(c) however, the MTR effect is negligible, i.e. assuming equil ibrium vaporization leads to little error in estimating the temperature drop through the film.

(d) the error introduced by (c) is lessened with reduced heat flux

(e) the inclusion or exclusion of pressure gradient has no effect on the above conclusions, and

(f) very little sensible heating of vapor occurs over the range of tested variables $\left(T_{G} \simeq T_{i}\right)$. 
The effect of finding (c) is that the mixture can be treated as an EPF if the process is strictly evaporative. Any deviation in the beat transfer coefficient from an ideal mole fraction weighting would be caused by nor-ideal property behavior, not mass transfer effects. This finding then is in sharp contrast to the conclusion of the preceeding section on pool boiling of mixtures. This is also in mild contrast to condensation of vapors with noncondensible gases (NG); NG cause severe degradation in condensing coefficient with stagnant vapor. A $20 \%-30 \%$ degradation can al so be found when the vapor stream is in laminar flow. There is anfortanately no literature on turbulent in-tube condensation of vapor with NG. ${ }^{1}$ Recently Stoecker [St85] noted a condensing coefficient for an $R-12 / R-114$ mixture which was 1 ower than either pare refrigerant. He attributed without analysis the degradation to slip, and not mass transfer resistance. ${ }^{2}$ Appendir $7 \mathrm{D}$ has additional comments on Shock's analysis.

7.3.2 Predictive Model1ing for Forced Convective/Evaporation of Mixture (Bell and Ghaly Model)

Given the findings of the previous section, it is not surprising that Shock has recommended the ase of a simplified equilibrium model [Sa82, Sh83]: the Be11 and Ghaly method [Be72]. It was developed originally for film condensation of a superheated vapor. It can however be rederived for forced convection/evaporation, as is done in Appendix $7 \mathrm{E}$.

\footnotetext{
$1_{\text {Webb [We82] examined turbulent flow over a tube and found in }}$ condensation of water vapor with $2 \%$ air (by volume) a $20 \%$ reduction in condensing coefficient.

2 It may be due to non-ideal property behavior, but this possibility was not analyzed.
} 
A summary of the assumptions in the method is:

(1) No sensible heating of the 1 iquid.

(2) Over a small axial distance, the change in balk vapor temperatore is equal to the change in equilibrium temperature.

(3) Mass transfer resistance is neglected. To balance the error of this assumtion, single phase heat transfer coefficients are empl oyed rather than the (higher) two phase values.

The effective heat transfer coefficient is then given by:

$$
\frac{1}{a_{\text {eff }}}=\frac{1}{a_{L O}}+\frac{x C_{P G} d T_{e q b} / d h}{a_{G}}
$$

Assumption (3) was later revised so that two phase heat transfer coefficients are now recommended. For evaporation, the sensible heating of vapor is small, so that the above equation reduces roughly to

$$
\frac{1}{a_{\mathrm{eff}}} \approx \frac{1}{\left.a_{\mathrm{LO}}\right|_{\mathrm{TP}}}=\frac{1}{a_{\mathrm{Lo}}\left(\phi_{\mathrm{Ltt}}^{2}\right) \cdot 445}
$$

The term is identical to Chen's evaporative term for pure component evaporation. Thus, with little sensible heating of the vapor stream the Bell and Ghaly model is actualy a pare fluid liquid film correction.

\subsection{Complete Mirtures' Correlations}

The previous sections have described each heat transfer regime, and methods to calculate a for mixtures. This section describes methods of calculation when both evaporation and nucleate boiling are present. 
7.4.1 Modification to Bell and Ghaly to include Nucleate Boiling Sardesai, Shock, and Butterworth suggested recently a modification to he Be11 and Ghaly method to include nucleate boiling [Sa82]. In this case, equation (7E-1) of Appendix E becomes:

$$
q_{T O T}=a_{L 0}\left(T_{W}-T_{i}\right)+a_{n}\left(T_{W}-T_{b u b}(\bar{X})\right)
$$

where $a_{n}$ is found from the methods described in section 7.2.3. The problem then is to estimate $T_{b a b}(X)$. If one sets $T_{b u b}(X)=T_{i}$, then, equation $(7-1)$ becomes

$$
a_{\text {eff }}=\frac{1}{\frac{1}{a_{L O}+a_{n}}+\frac{q_{G} / q_{T O T}}{a_{G}}}
$$

If instead $T_{b u b}(X)=T_{G}$, then equation $(7-1)$ becomes

$$
a_{\text {eff }}=\frac{1+a_{n} / a_{L O}}{\frac{1}{a_{L O}}+\frac{q_{G} / q_{T O T}}{a_{G}}}
$$

The authors howerer do not suggest when to include the nucleate boiling contribution, or if a suppression factor should be included. If in fact one is included then equation (7-3) becomes very similar to Chen's me thod described in the last chapter. 
7.4.2 Modification to Bennett and Chen's Method to include Mirture Effects

Bennett and Chen developed an extension of their method to include mixture effects [Be80]. Their approach is dissimilar in its assumptions to those advanced in all the previous sections. Specifically, sensible heating of the vapor is neglected, but sensible heating of the 1iquid is accounted. Additionally mass transfer resistance in the 1 iquid (and not in the vapor) is assumed and accounted semi-empirically. Last1y, a much different approach is taken to account for reduced nucleate boiling heat transfer.

In the case of mixtures, the nucleate boiling contribution is considered reduced by the same factor as described in section 7.2 .1 for single bubbles (equation 7-0)

$$
\mathrm{C}_{\mathrm{BUB}}=\frac{1}{1-\frac{\mathrm{C}_{\mathrm{P}_{\mathrm{L}}}}{\Delta \mathrm{h}_{\mathrm{V}}} \frac{{ }^{\mathrm{a}} \mathrm{T}}{{ }^{\mathrm{a}}}\left(\mathrm{Y} *-\mathrm{X}_{\mathrm{e}}\right) \frac{\mathrm{dT}_{\mathrm{BUB}}}{\mathrm{dX}_{\mathrm{e}}}}
$$

so that the nucleate boiling part of equation (6-3) becomes

$$
a_{n}=a_{p o 01} C_{B U B} s
$$

The forced convection term is al so modified to account for mass transfer resistance in the 1 iquid film. It is hypothesized that, due to this resistance, the real interfacial temperature is higher than calculated 
from equilibriom. In this manner, the driving potential, $T_{W}-T_{i}$ is less for mixtures than for an equivalent pure fluid. Thus, for a pure fluid,

$$
\dot{q}_{L}=a_{L 0}\left(T_{W}-T_{e q b}\right)
$$

and for a binary mixture,

$$
\begin{aligned}
& q_{B L}=a_{L 0}\left(T_{W}-T_{i}\right) \\
& =a_{L 0} \frac{\left(T_{W}-T_{i}\right)}{\left(T_{W}-T_{e q b}\right)}\left(T_{W}-T_{e q b}\right) \\
& =a_{L 0} C_{C o n}\left(T_{W}-T_{e q b}\right)
\end{aligned}
$$

The problem remains to eliminate the unknown interfacial temperature.

Appendix $7 \mathrm{~A}$ shows how this elimination was achieved through the introduction of a mass transfer coefficient:

$$
C_{\text {con }}=1+\frac{q y * \frac{d T_{B U B}}{d X_{e}}}{\rho_{L} \beta_{L} \Delta h_{v}\left(T_{W}-T_{e q b}\right)}
$$

where $\quad \beta_{L}=$ mass transfer coefficient

The mass transfer coefficient was assumed to behave as 


$$
\beta_{L}=B \frac{{ }^{a} D}{D} \operatorname{Re}_{L}^{0.8} S^{0.4}
$$

where $B$ was determined empirically to be 0.023 . The athors note the coincidental agreement with the Dittus - Boelter constant. The authors next account for sensible heating of the liquid phase. A derivation of this accounting is given in Appendix 7B; the equations (7-5) and (7-8) should be replaced by an effective heat of vaporization:

$$
\Delta h_{v e f f}=\Delta h_{v}-C_{p_{L}}\left(Y *-X_{e}\right) \frac{d T_{B U B}}{d X_{e}}
$$

and $B=0.015$ gives better agrement with their dates when $\Delta$ veff $_{\text {is }}$ used. The anthor noted little change in agreement resulted with the inclusion or exclusion of equation (7-9).

7.4.3 Other Modifications to Chen's Method to include Mirture Effects Collier and Shock [Co80, Sh73] have suggested modifying the nacleate boiling contribution when mirtares are used. They suggest treating the components as separate resistances in series, and including a correction factor for mixtares:

$$
\frac{1}{a_{\text {pool }} s}=\frac{\bar{x}_{A}}{a_{\text {pool }_{A} s}}+\frac{1-\bar{x}_{A}}{a_{\text {pool }_{B} s}} \frac{1}{C}
$$

where $C$ is found from Table 7-1. Tacitly they assume that sensible beating of 1 iquid and vapor are negligible. Consistent with Shock's earlier findings, they assume the turbulent evaporation process to be unaffected by mixtures so $a_{L O}, F$, and $S$ are unaffected. 


\subsubsection{Other Correlations for F1 ow Boiling of Mixtures}

Varma et al. [Va79] studied a binary mirture of R12/R22, as discussed in Chapter 1. They correlated their data to $\pm 30 \%$ for low mass fractions of R22 and $\pm 15 \%$ for higher mass fractions, using the following form:

$$
\frac{a}{a_{L O}}=8.275{\frac{1}{X_{t t}}}^{.253} \frac{\dot{q}}{{\dot{m} \Delta h_{v}}} \cdot 12\left(1-\left|\bar{Y} \frac{z}{E}-\bar{X}\right|\right)^{-.9}
$$

By its nature, the correlation does not consider the physical processes involved in the flow boiling of mixtures. It is interesting to examine the only term which is explicit to mirtares:

$$
\left(1-\left|\bar{Y}^{*}-\bar{X}_{e}\right|\right)^{-.9}
$$

This term is always greater than unity, suggesting the use of a mixture augments the heat transfer process over an EPF. Their experimental data did show a higher heat transfer coefficient for the mixtare than for pure R12. No tests were done with pare R22. As previously noted, the mixture degrades the heat transfer in nucleate boiling dominated situations. In forced convection dominated situations, the term $\left(1 / x_{t}\right)$ generally appears to the exponent $2 / 3$ to $3 / 4$. Their correlation suggests neither regime. The agreement between (7-11) and the data is therefore not expected to be general. It however should not be dismissed, since the heat and mass flux range they tested is qu:te similar to that used in the test section of rig \#2. 
In the only other known study, Singal et al. measured R13/R12 miztures

[Si83]. Their Iesults produced erratic behavior with quality and composition; there was a clear dependence on mass and heat fluz. Average heat transfer coefficient was compared with the Pierre correlation for average heat transfer coefficient and agreement was poor (wide scatter, both positive and negative).

\subsubsection{Summary of Mixtures' Models}

At this point, the literature may be sumarized along the following 1 ine $s:$

(1) Experiments ith pool boiling of mixtares have shown severe degradation in heat transfer coefficient when compared to ideal or equivalent pure fluid values. The degradation has been accounted by various correction factors. Since pool boiling methods have been extrapolated successfuly in the prediction of flow boiling of pure fluids, the same extrapolation has been hypothesized as valid for mixtures.

(2) Shock's analytic treatment suggests that mass transfer resistance (MTR) and sensible heating are negligible in turbulent flow evaporation. His conclusions are generally supported by condensation research. He concludes that non-mirture methods are adequate for predicting the heat transfer. If MTR is included, Shock suggests the dominant MTR is on the vapor side. In sharp contrast, Bennett and Chen have advanced a true mixtures' model which includes both MTR and sensible heat only on the 1 iquid side; they correlated a large data set $w$ ith their model.

(3) Other authors in the literature have either attempted to use non-mirture relations in the presence of nucleate boiling, or have produced curvefits which are structurally flawed in their interpretation of the physical phenomena.

Table 7-2 1ists the complete mixture methods and their related assumptions. 


\subsection{Comparison to Measured Data}

Table 7-3 displays 46 variations of the models described in the previous sections. Because the mass diffusion coefficient is unknown, parametric runs were made, assuming a range of Lewis numbers. The best methods are discussed in the following sections.

\subsubsection{Comparison to Preheat Data of Rig \#2}

While the mixtures data of this section shows effects of mass flux and quality, it is primarily affected by heat flux, suggesting that it might be best represented by a pool boiling correlation corrected for mirture effects, as discussed in Section 7.2.3.

Figure 7-4 displays some of the measured data superposed on the predicted coefficients. In al1 cases, a severe degradation in heat transfer is observed (about $40-50 \%$ as compared to $a_{i d}$, and $70 \%$ compared to a EPF). Hidden in these figures are the effects of mass flux and quality. Also there is an uncertainty in the actual local liquid composition, given tiat a mass transfer resistance and a nonequilibrium condition are part of the physical process. The se complications can be mitigated by examining only that experimental data closest to equilibriam, i.e. the first thermocouple group in the preheat section. At this group, the vapor quality is lowest, so that the liquid composition will be close to the know subcooled inlet composition. At this point also, the evaporative mode should contribute only weakly, so that mass flax and vapor quality levels should not be significant. Figure 7-5 displays this data, along with the predictive methods. In this 
figure, unlike the previous set, the Stephan and Korner method is evaluated using $A_{0}=2.64 ;$ this value was selected to predict ezactly one of the experimental data points. The vertical scatter is due to the neglect of the mass flux effect. This approach was discussed in Section 7.2, and was in fact successful in evaluating Toral's subcooled flow boiling data [To79].

Table 7-3 show s that the best correlations of the data are a pure evaporative, normirture model $\left(a=a_{L O} F_{C}\left(X_{t t}\right)\right.$ ) and two pool boiling relations. The agreement between the evaporative model and the data may be coincidental; the data itself does not exhibit the strong mass flux dependence predicted by the model. Instead it exhibits a strong heat flur dependence ignored by the evaporative model. Of the pool boiling relations, the method of Thome and the specialized Stephan and Korner methods predict the measured data fairly well. Thome's method is both more conservative and more accurate in the mirture concentrations at Which heat pumps are recommended for operation. It predicts the observed level of degradation at points away from the marimum degradation better than Stephan and Korner.

\subsubsection{Comparison to Rig \#1 Data}

This data set consists of 184 points with equilibrium qualities between .05 and 0.90 . The data includes a wide range in molar composition (though not weight composition as shown in Table 3-1). A variety of pressures were used so that a comparison between runs is difficult; 
however the pressure variation provides an additional parameter upon which to check the ability of the correlations.

The data shows a proportional dependence on heat flux, quality and weakly mass flux. This suggests that nucleate boiling is principally contributing to the vapor generation process; in this manner it is like the previous data set.

All of the pure fluid correlations overpredict the measored valoes by an amount exceeding their overprediction of the pure flaids. This is a first indication of a degraded heat transfer with mirtares.

Of all the correlations, a few stand out for their predictive ability. Since $a$ is a strong function of heat flux, the pool boiling methods were again examined. Here Thome's method is superior to Stephan and Xorner because much of the data has a greater molar composition of R13BI than was the case in Section 7.5.2. The Stephan and Korner method is only superior at small molar compositions of Rl3Bl. While Thome's method yielded the 1 owest mean deviation, it did not represent well the data trends. By its nature, Thome's method produces an inverse relationship between vapor quality and heat transfer coefficient, opposite of the measured observation. It tends to overpredict the measurements at 10 w quality and underpredict at high quality (Figure 7-6). Because of this feature, it predicts the average heat transfer coefficient very well. The two other methods which predict the data with ang accuracy are evaporative only, normirture modeling: Chen's original method 
without Prandtl correction, and Bell and Ghaly's method. They are actually very similar, as is discussed shortly.

\subsubsection{Comparison to Rig \#2: Test Section Data}

This data base consists of 141 points with equilibrium qualities between 0.05 and 0.90 , and shows significant mass flux effects. It therefore is likely dominated by forced convection/eraporation. The poor predictive ability of the pool boiling methods offers further verification of this conclusion.

Several methods shown on Table 7-3 [(B), (J), (CC), (FF), (HH) (II)] yielded mean deviations less than 0.20. The "evaporative only", nonmirture correlation of (B) which is simply equation (6-26b):

$$
a=a_{L 0} F_{c}\left(z_{t}\right)
$$

yielded one of the best agreements. This same equation predicted well for the pare R152A data when nucleate boiling was completely suppressed. However, anlike its anderpredictive tendency with the pare floids it overpredicts the mirtares' data (Figrre 7-7). Slightly better prediction is achieved with methods (BB) and (FF) which do include a mass transfer resistance effect. However the predictive ability of (BB) and (FF) is artificial. First the terms $\mathrm{PI}_{L}, \Delta \mathrm{h}_{\text {eff }}$ and $\Delta \mathrm{T} / \Delta \mathrm{T}_{\mathrm{e}}$ tend to compensate each other and produce a value near 1. The Lewis number of 1 is unrealistic in any case. Finally the term $\Delta T / \Delta T_{e}$ was calcalated based on some nucleate boiling contribution, i.e. the complete Bennett and 
Chen correlation was employed, and the nucleate boiling contribotion subtracted out. This is physically carealistic. For these reasons, methods ( $B B$ ) and (FF) are not recommended.

Method (HH) is a Bell and Ghaly styled method, which redaces effectively to the same method as (B) since the liquid film dominates. It therefore is not really a new approach. It has all the same features as (B) described above. The use of $F_{c}\left(D^{2} L t\right)$ made little difference, since the methods produced similar values in this pressure and quality range. Method (J) yields the best agreement of the complete Bennett/Chearstyled correlations. It however tends to underpredict the dependence on vapor quality and overpredict the nucleate boiling contribution. Agreement at this stage of analysis can only be considered coincidental. The method badly predicted the experimental data in the beat transfer regime dominated by nucleate boiling.

\subsection{Discussion of Findings}

The complete Chen-styled correlations predicted poorly. In general they tended to overpredict the magnitude of $\alpha$, and anderpredict the dependence on quality, da/dx. The overprediction is a principal result of the treatment of the fluid as an equivalent pure fluid with the Forster and Zuber correlation (i.e. calculating ith EPF properties). This results in a large nucleate boiling contribution, as was show in Figure 7-3. Secondly, the term $C_{B U B}$ reduces the nucleate boiling contribution to a lesser ertent than the methods in Table 7-1. 
Fur thermore a structural problem was revealed in the mass transfer resistance inclusion in the evaporative term (equation 7-7). At Lewis numbers of five or greater, as might be common for most organic fluids, the term

$$
C_{B U B}=\Delta T / \Delta T_{e}=\text { equation }(7-5)
$$

could become negative at reasonable values of $\Delta T_{e}$ (close to measured values). A negative value of $\Delta \mathrm{T} / \Delta \mathrm{T}_{\mathrm{e}}$ has no physical meaning. As such it was set to zero in these cases. This caused the method to reduce to a pure nucleate boil ing method, equal to $a_{E P F} c_{B U B} s$. Since the $a_{E P F}$ is too 1 arge, the method tends to overpredict.

Mang perturbations of the Chen-styled correlation were tried, including a few new approaches:

a) Collier and Shock's nucleate boiling contribution was changed so that $a_{A}$ and $a_{B}$ was determined $\nabla$ ia equation $(6-17)$ corrected $\nabla$ ia Thome's me thod:

$$
a_{n} s=\left|\frac{\bar{x}_{A}}{a_{n} s}+\frac{\left(1-\bar{x}_{A}\right)}{a_{B} s}\right|^{-1} c_{\text {IH }}-1
$$

b) Bennett and Chen's method -ithont the Prandt 1 correction: this was tried since pure R152a measured $\nabla a l$ nos were predicted rell without the correction (section 6.4).

The se modifications yielded improvements over the original approaches, but did not give satisfactory agreement. 
In the nucleate boiling regime, pool boiling methods corrected for mirture effects were seen to be the most accurate. They failed however to predict the observed quality dependence. Thome's method is very simple to apply and is the most accarate and conservative, and is thus re commended.

In the forced convection/eraporative regime, a non-mirtare method which assumes nucleate boiling suppression predicted the data best. This resalt is consistent with recent turbalent flow evaporation theory. The best predictor for this data was al so the best predictor for the completely suppressed pure refrigerant data, lending further credibility to Chen's evaporative correlation. Jnlike pare fluids through it tended to over predict the mass flux effect. It is tempting to attribute the overprediction to MTR, though no definitive statement can be made. MTR may however accont in a subtle way for the fact that the measured mixtares data, a/a $a_{10}$ was less than the pare fluid values at the same pressure, heat flux and Martinelli parameter. This was shown on Figure 5-6.

It is known that MTR reduces bubble grow th rates and decreases the heat flur where complete suppression is predicted. The reduced a for mirtures may be the result then of nucleate boiling having been suppressed for the mirtures but not for the pare fluids. With eballition, the viscous sublayer may be destroyed, thus increasing both the evaporative and the nucleate boiling contributions. Such a theory would explain the measured reduction and be consistent ith turbalent flow evaporation theory and the correlation results. 


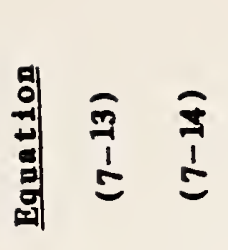

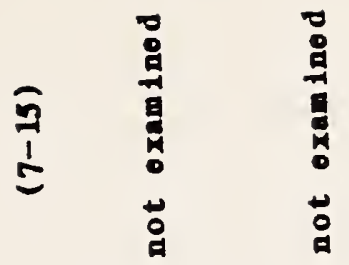
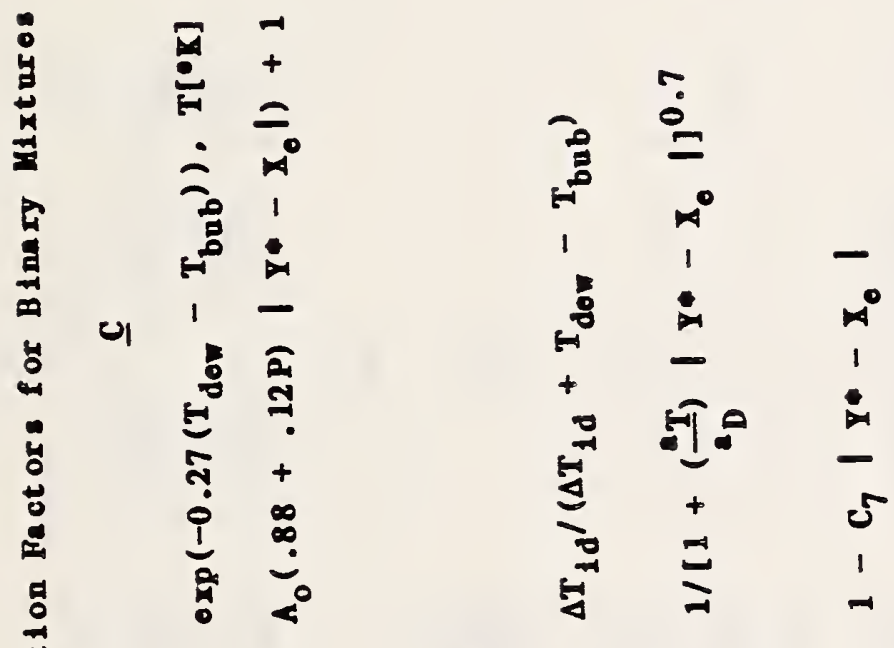

:

:

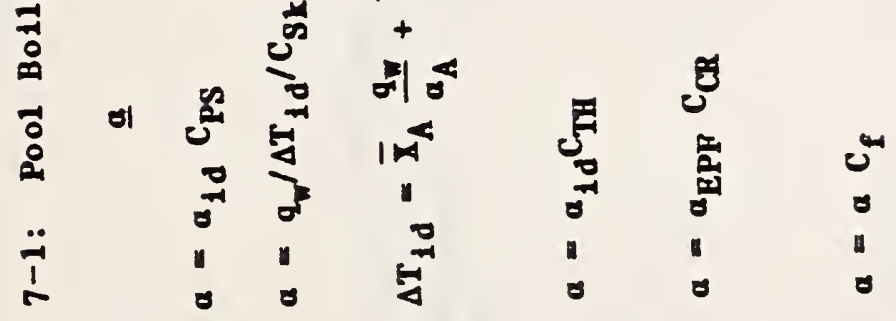

욜

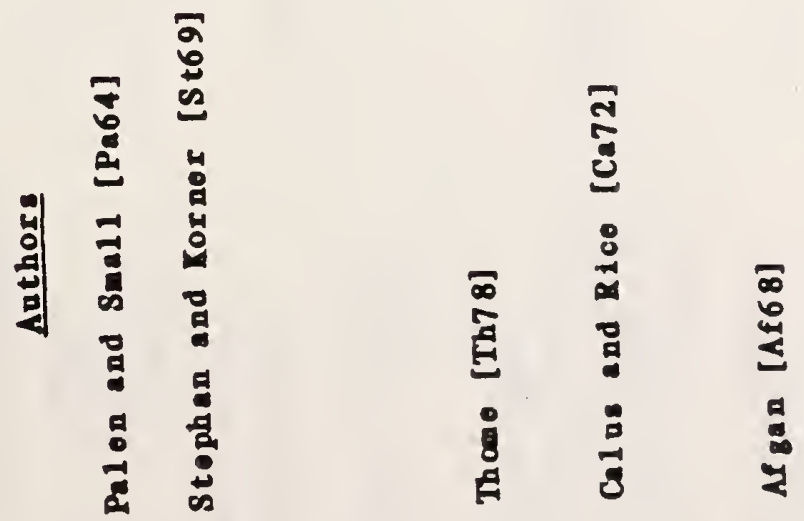




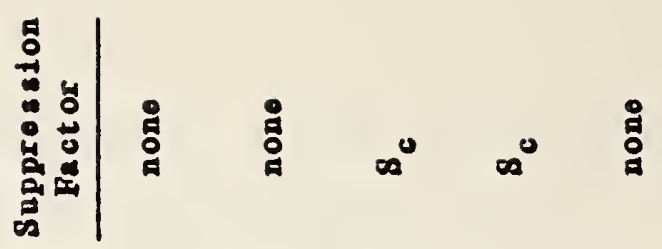

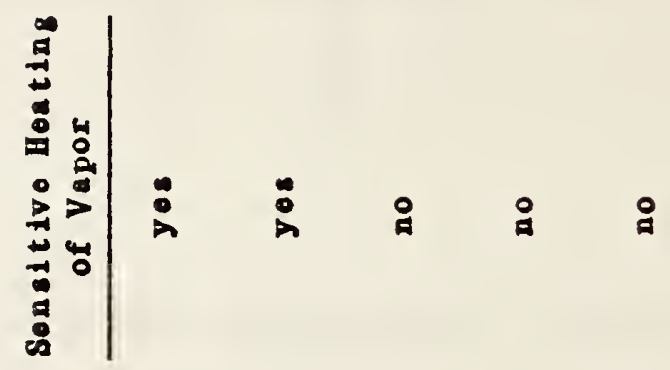

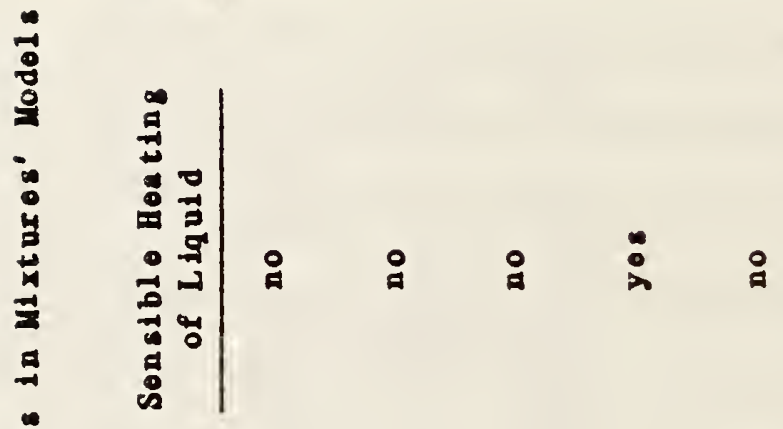

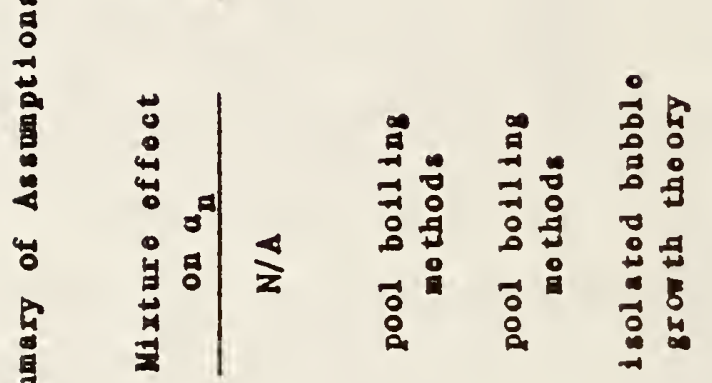

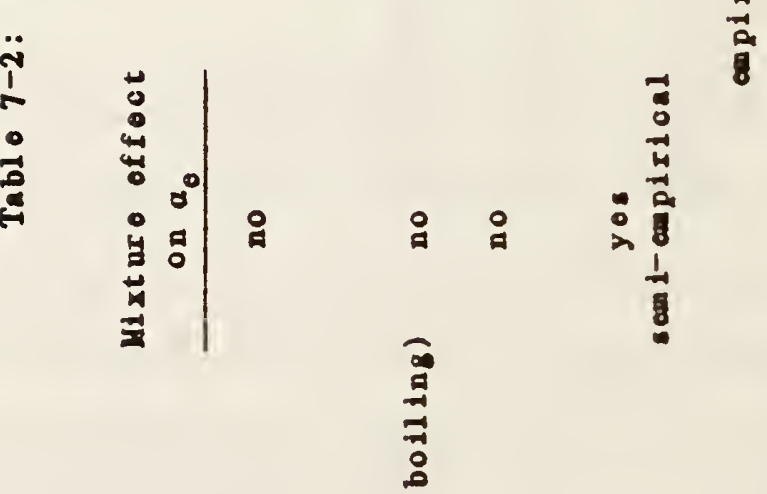

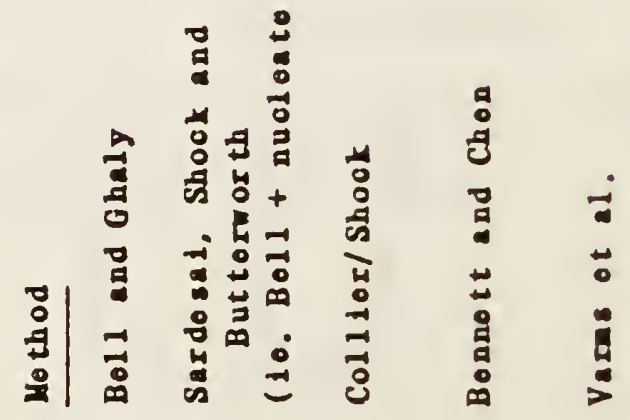



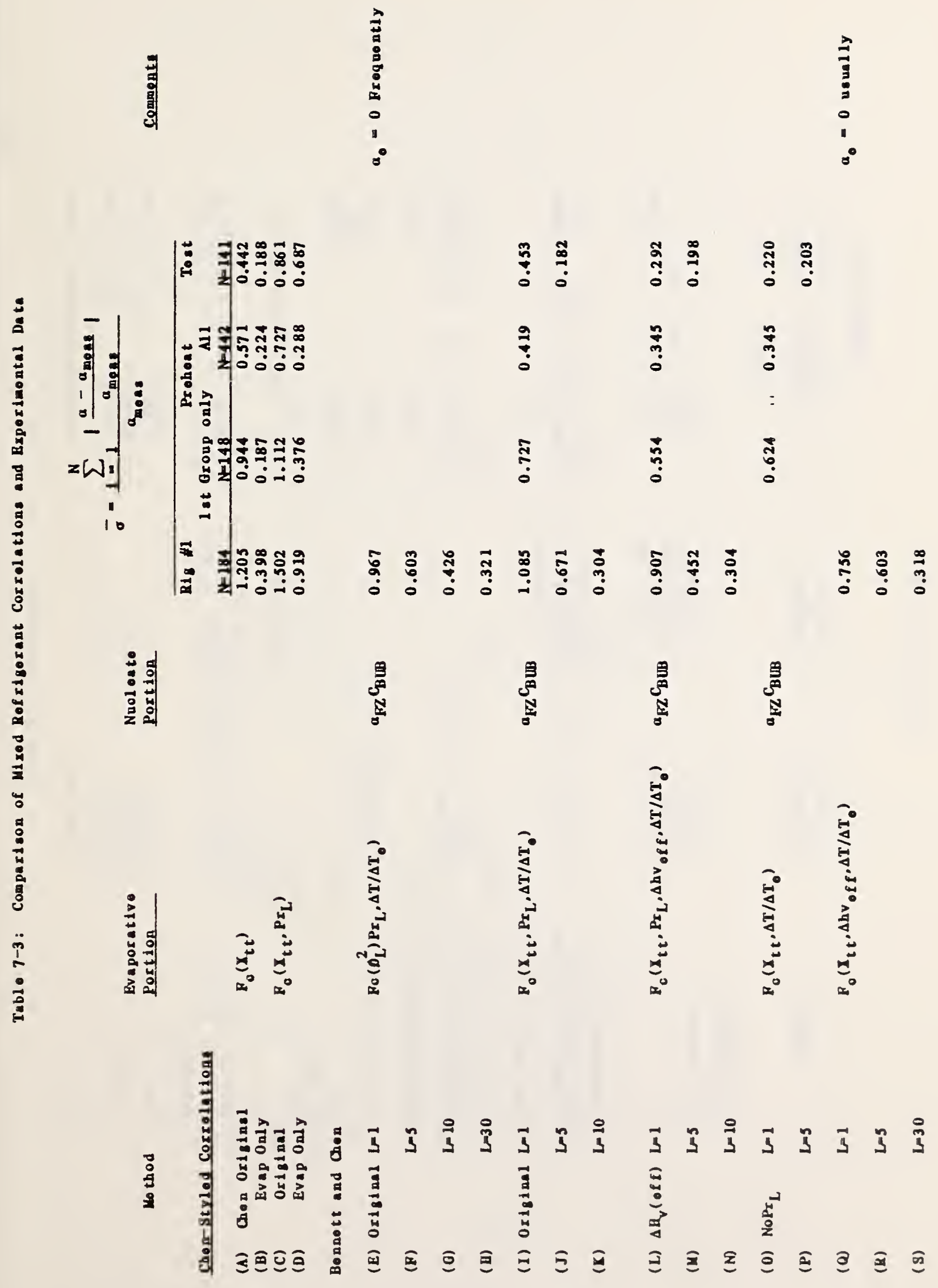

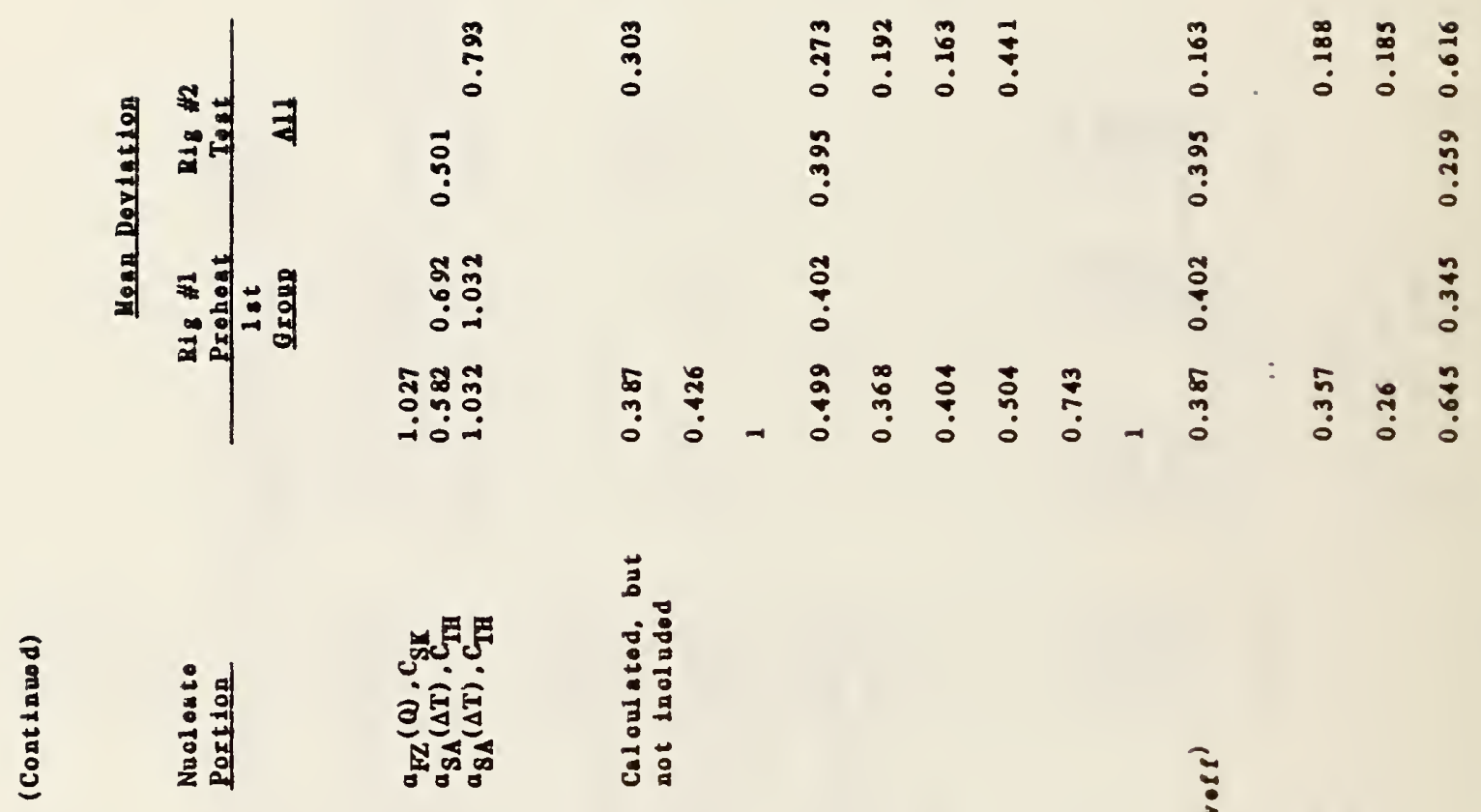

竞
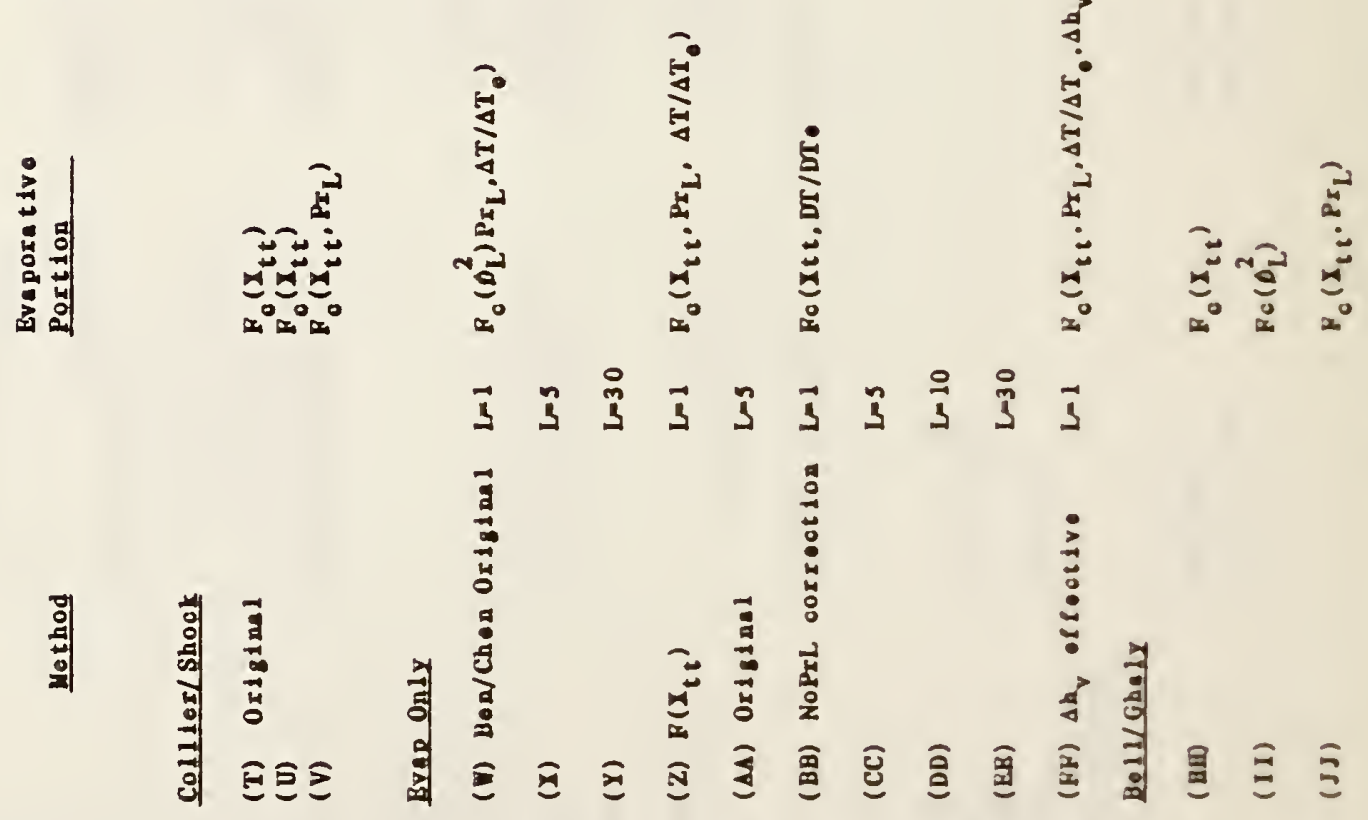


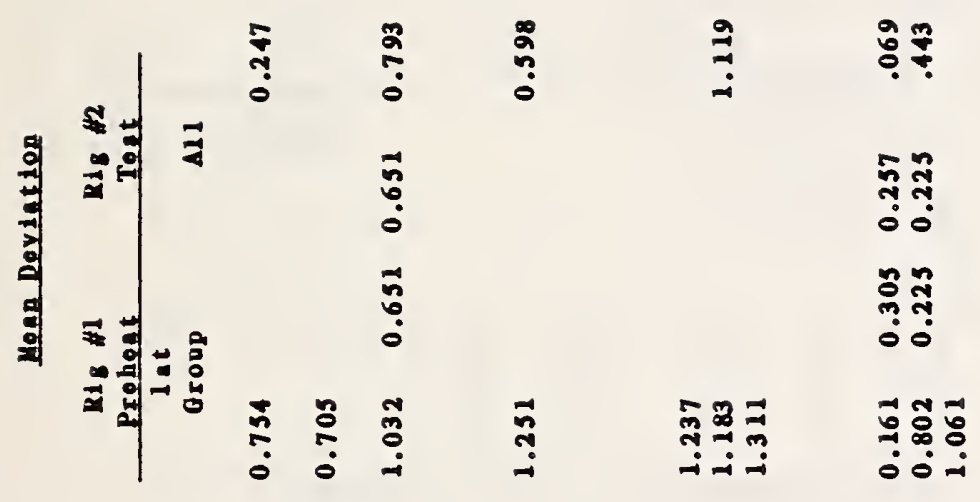

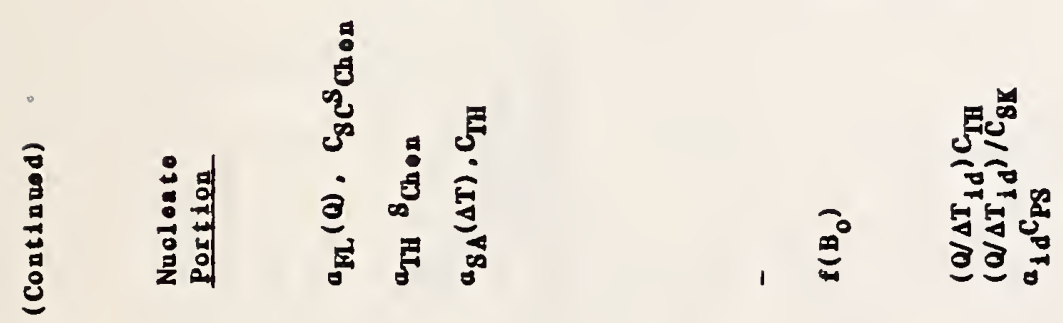

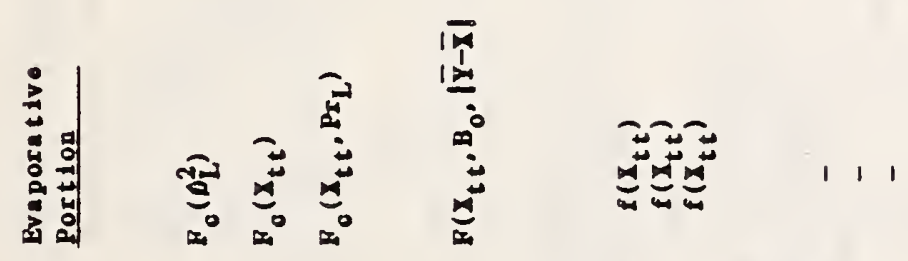

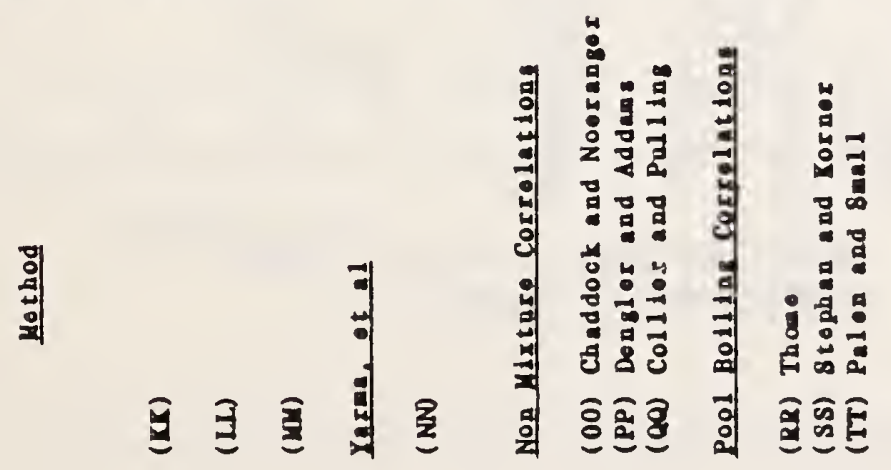


$\rightarrow \quad-$ Mass diffusion boundary layer $\sqrt{a_{0} t}$

$\rightarrow-$ Thermal boundary layer $\sqrt{a_{T^{t}}}$
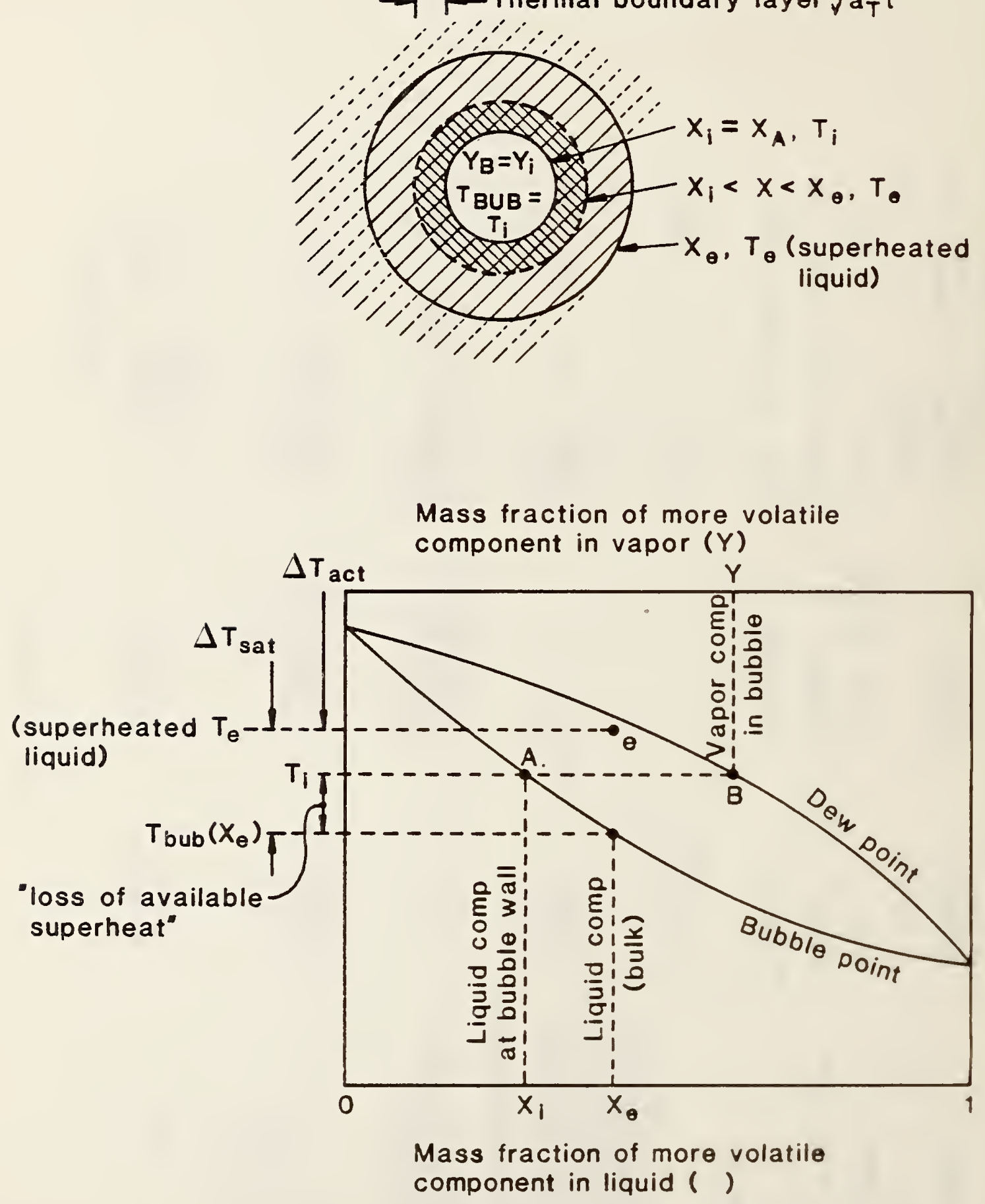

Figure 7-1: Isolated Bubble Growing in a Superheated Binary Liquid. 


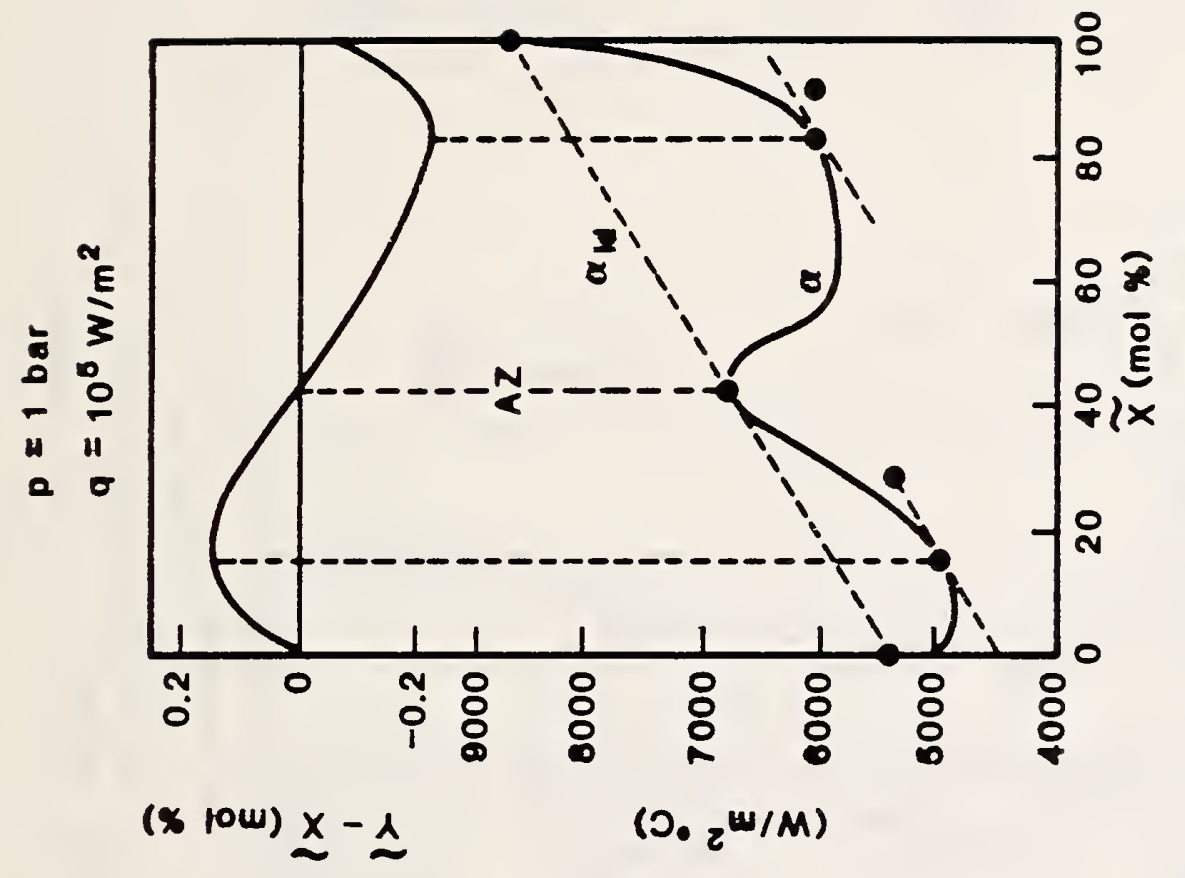

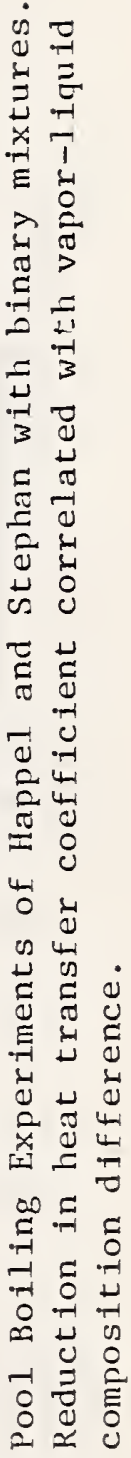
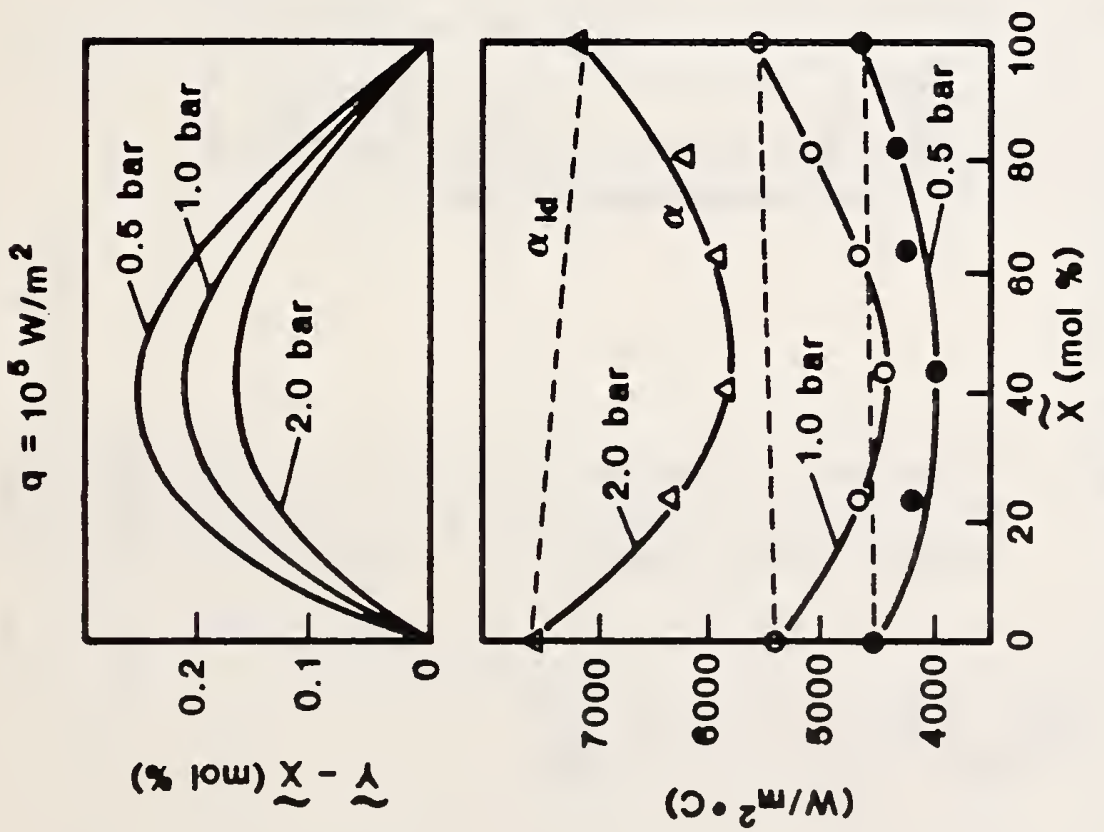

$\frac{9}{5}$

(x fow) $\underline{x}-\underline{\lambda}$

(ว. $z^{m / M)}$ 


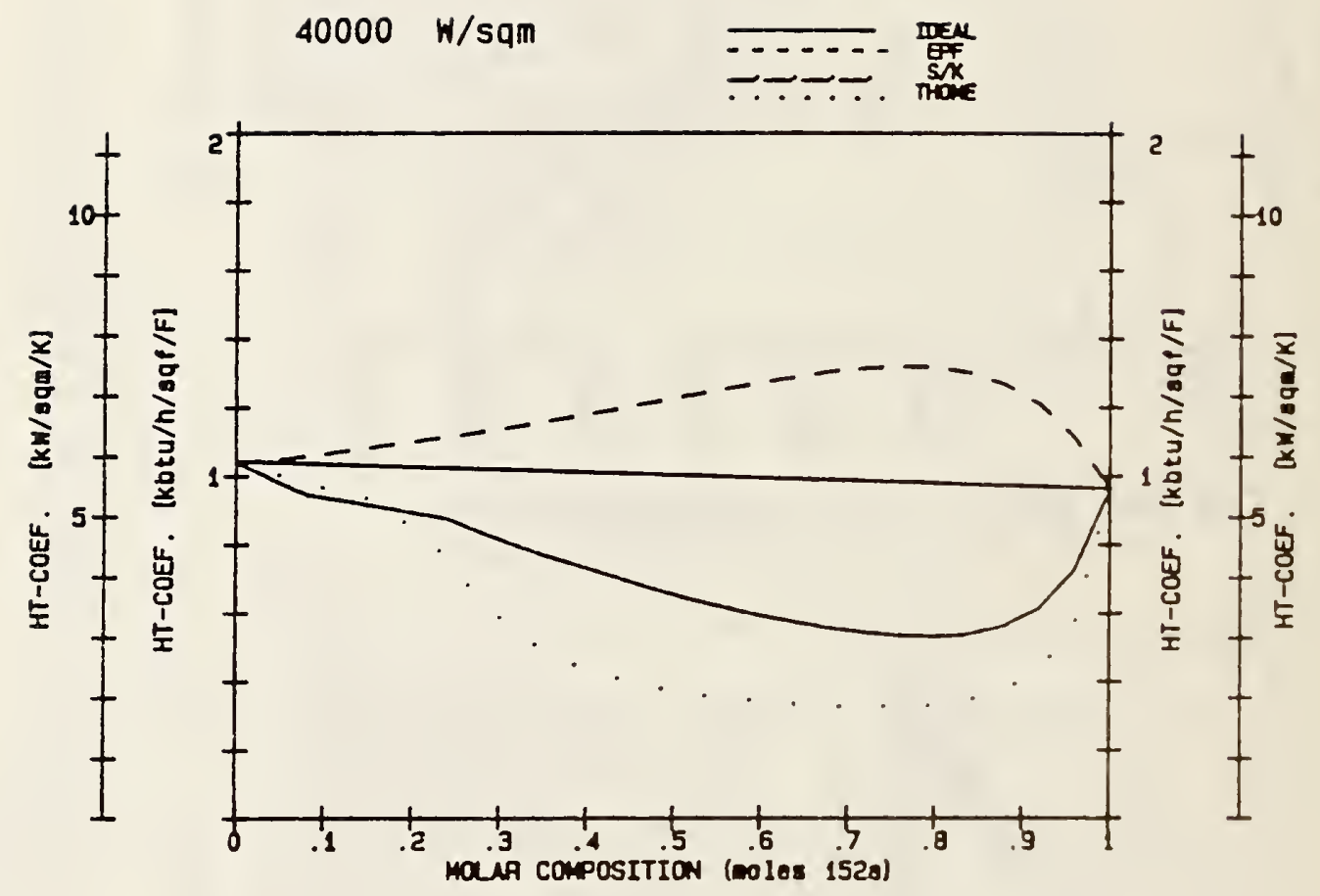

Figure 7-3: Comparison of mixture correction factors. Note the EPF model, which does not consider MTR sugrest: a higher heat transfer coefficient than ideal. 


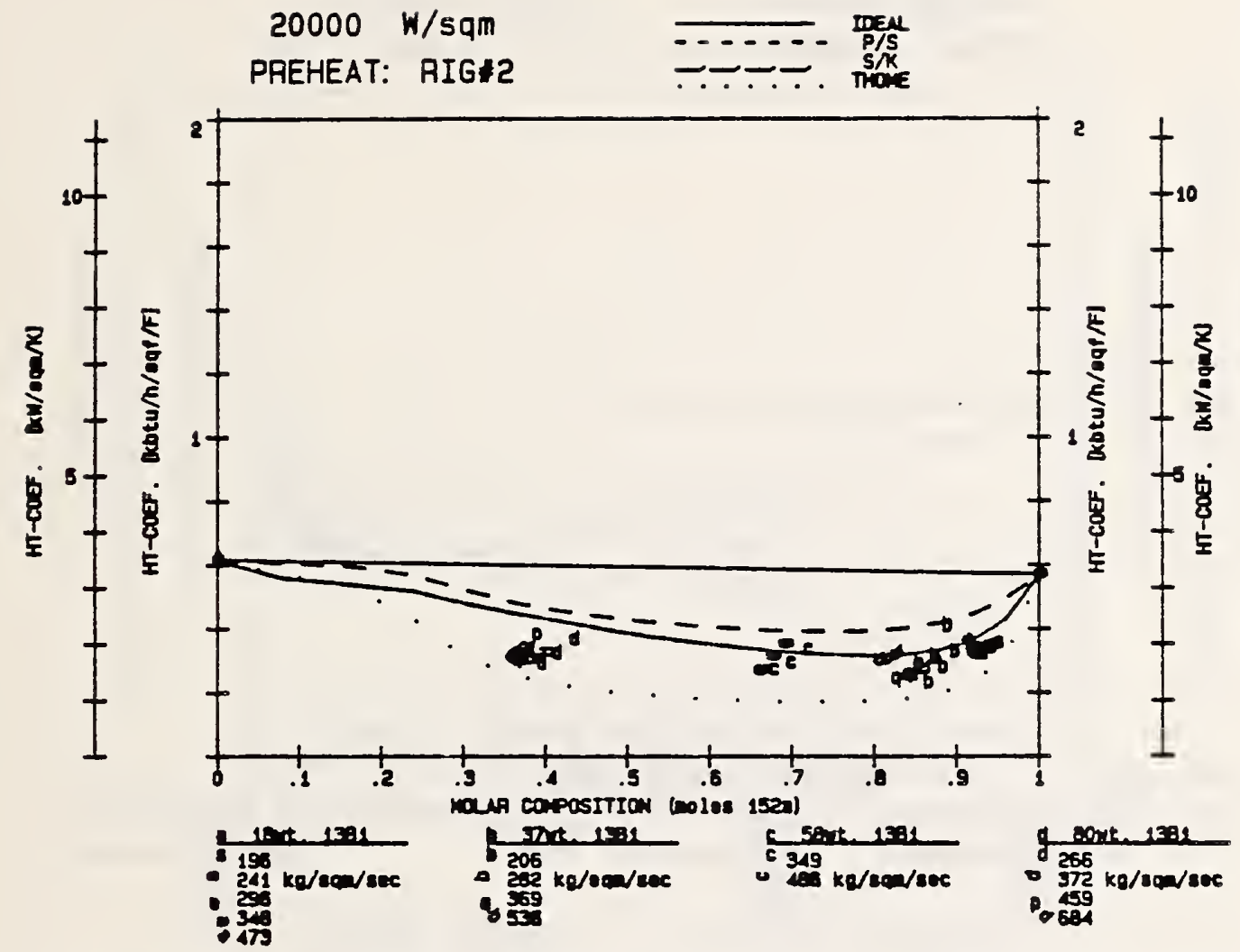

Figure 7-4: Comparison of Preheat Data to Pool Boiling Models

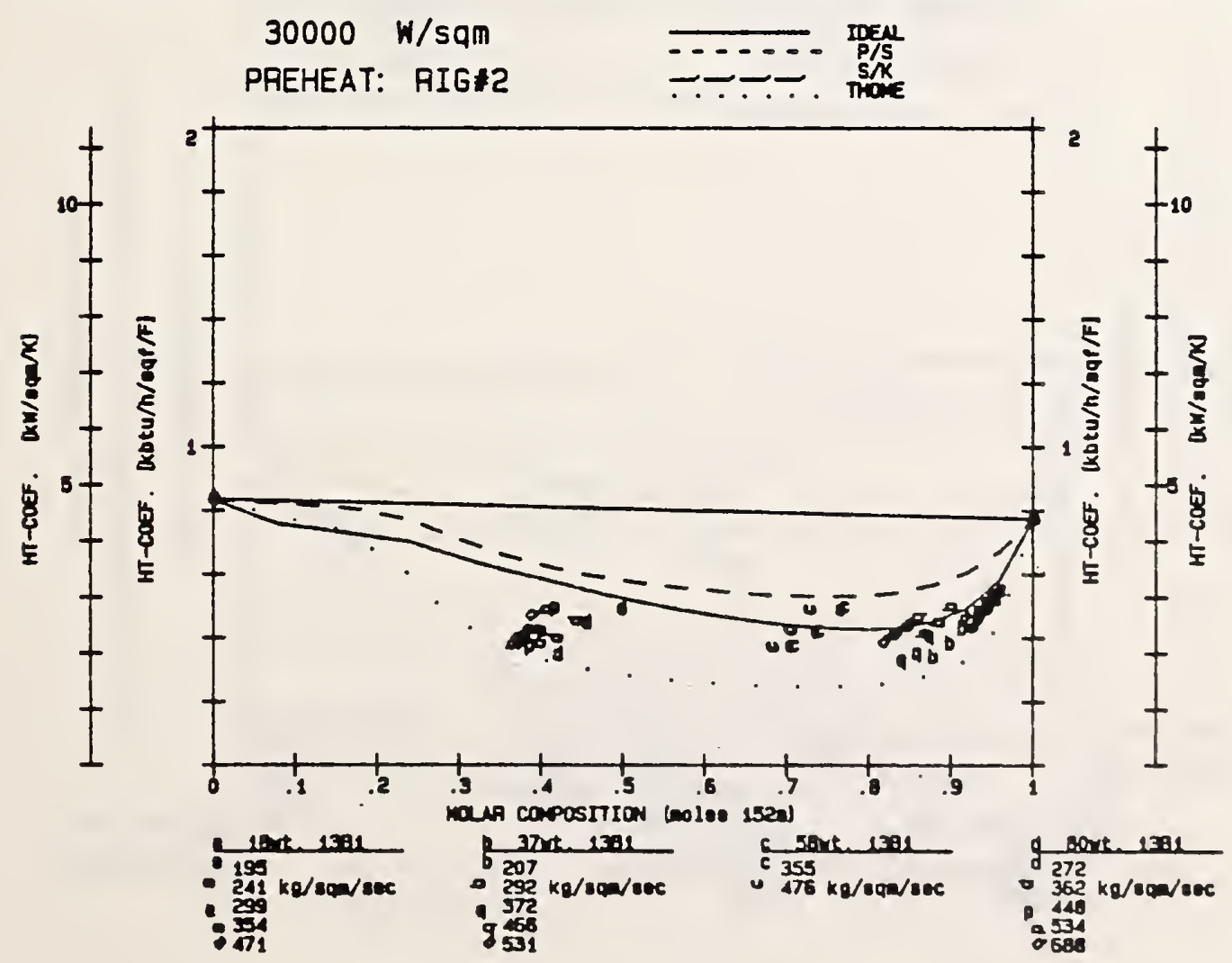




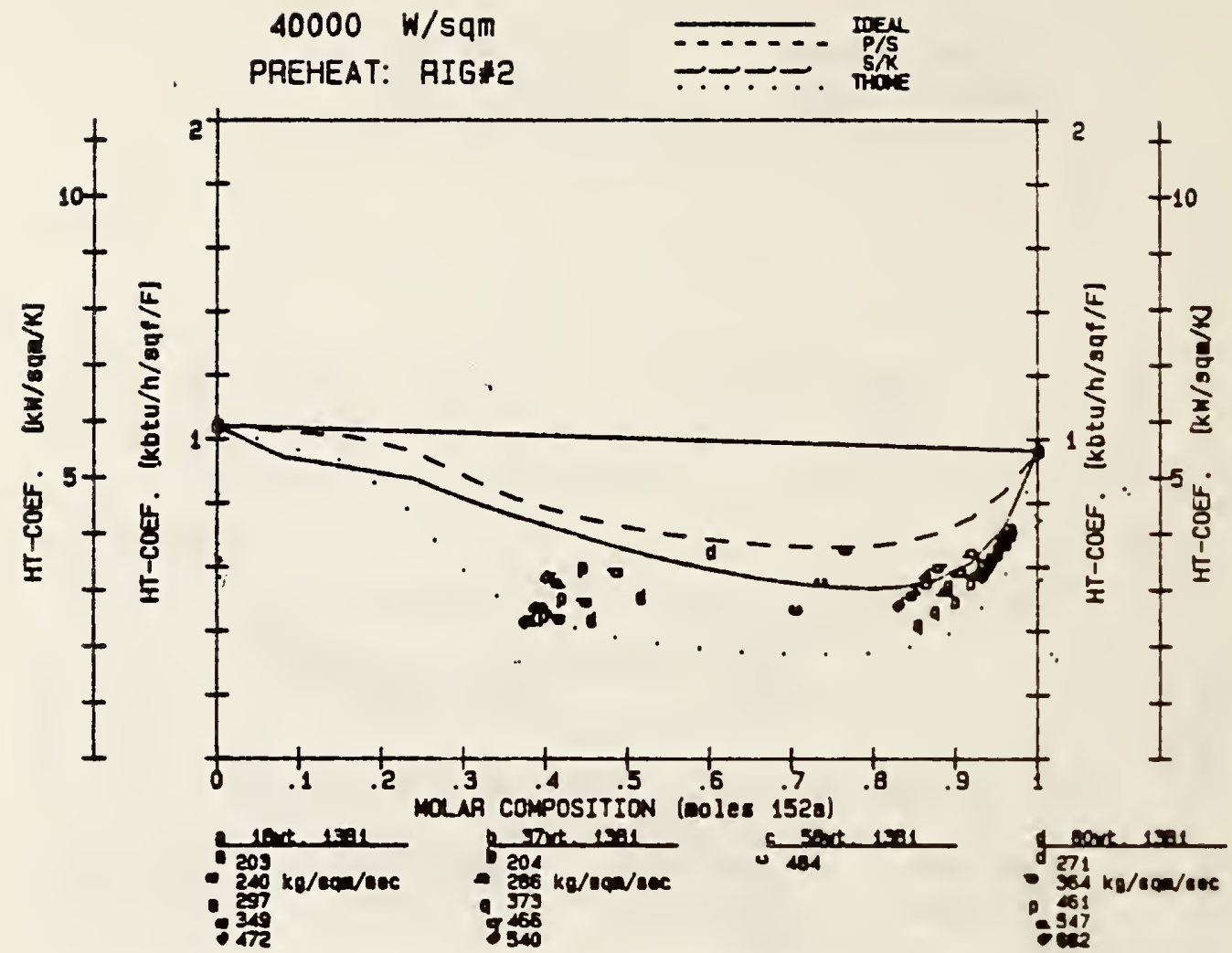

Figure 7-4 (cont): Comparison of Preheat Data to Models

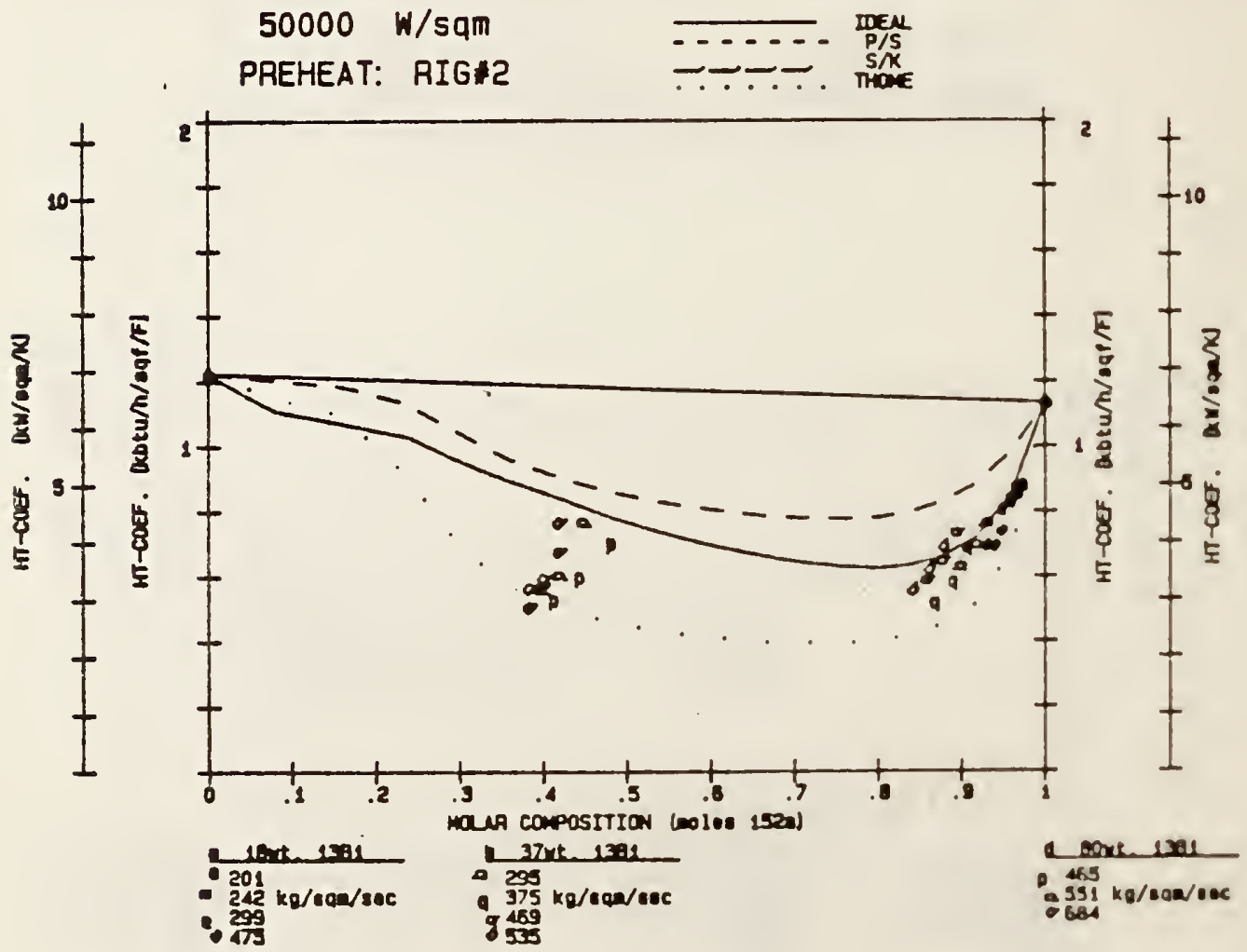



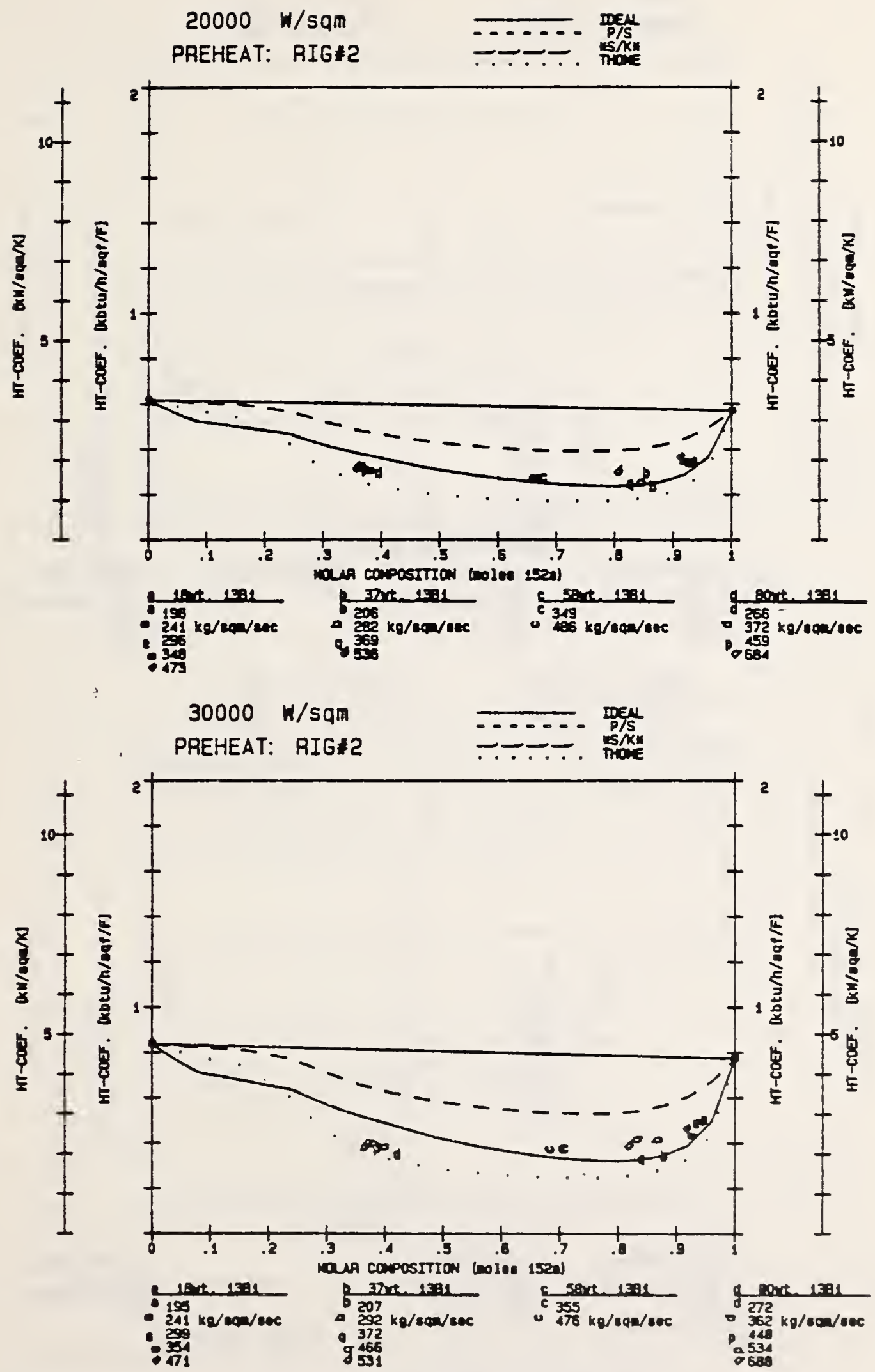

Figure 7-5: Comparison of lst Station Preheat Data to Pool Boiling Models 

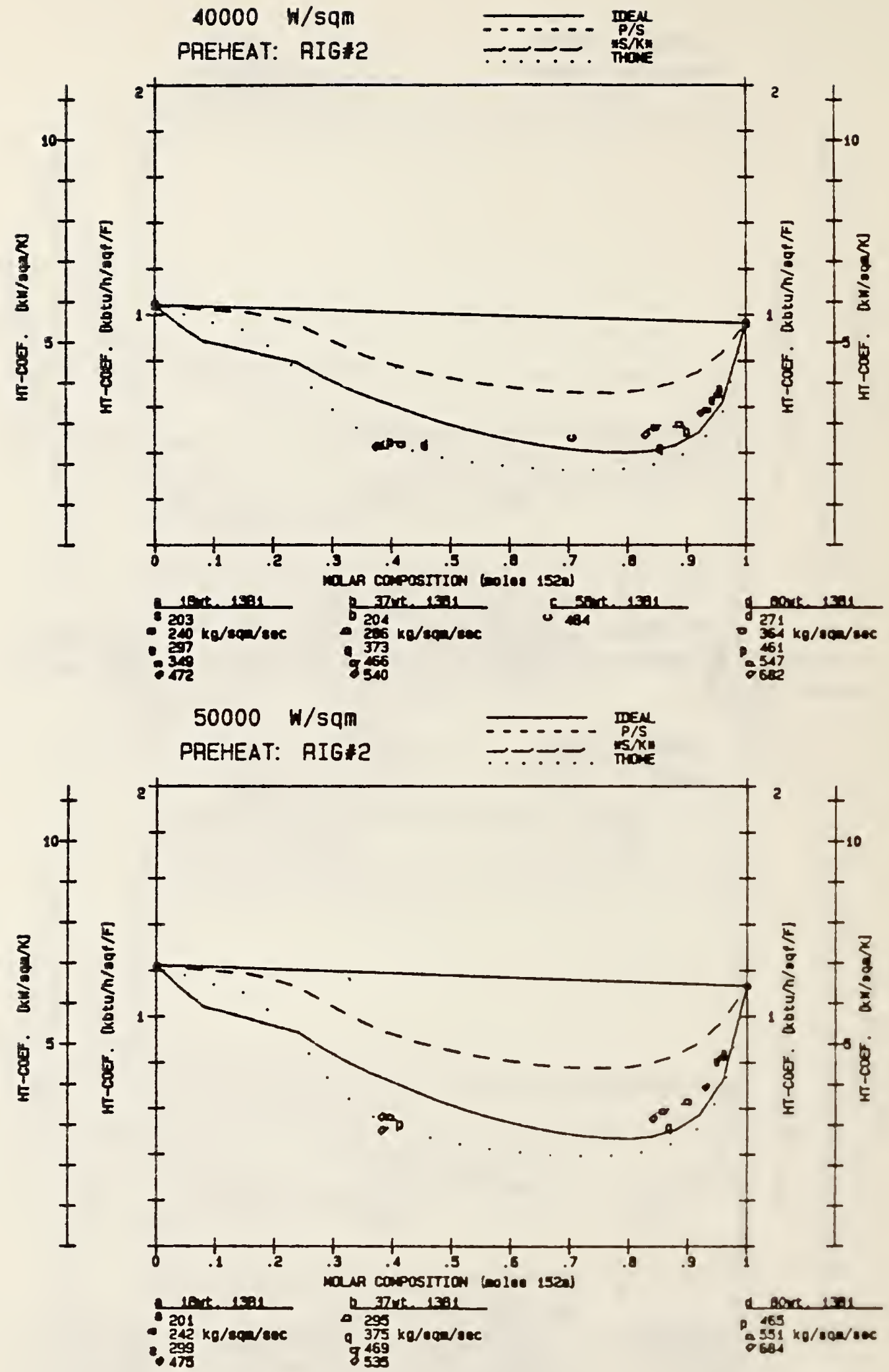

Figure 7-5 (cont): Comparison of lst Station Preheat Data to Pool Boiling Models 


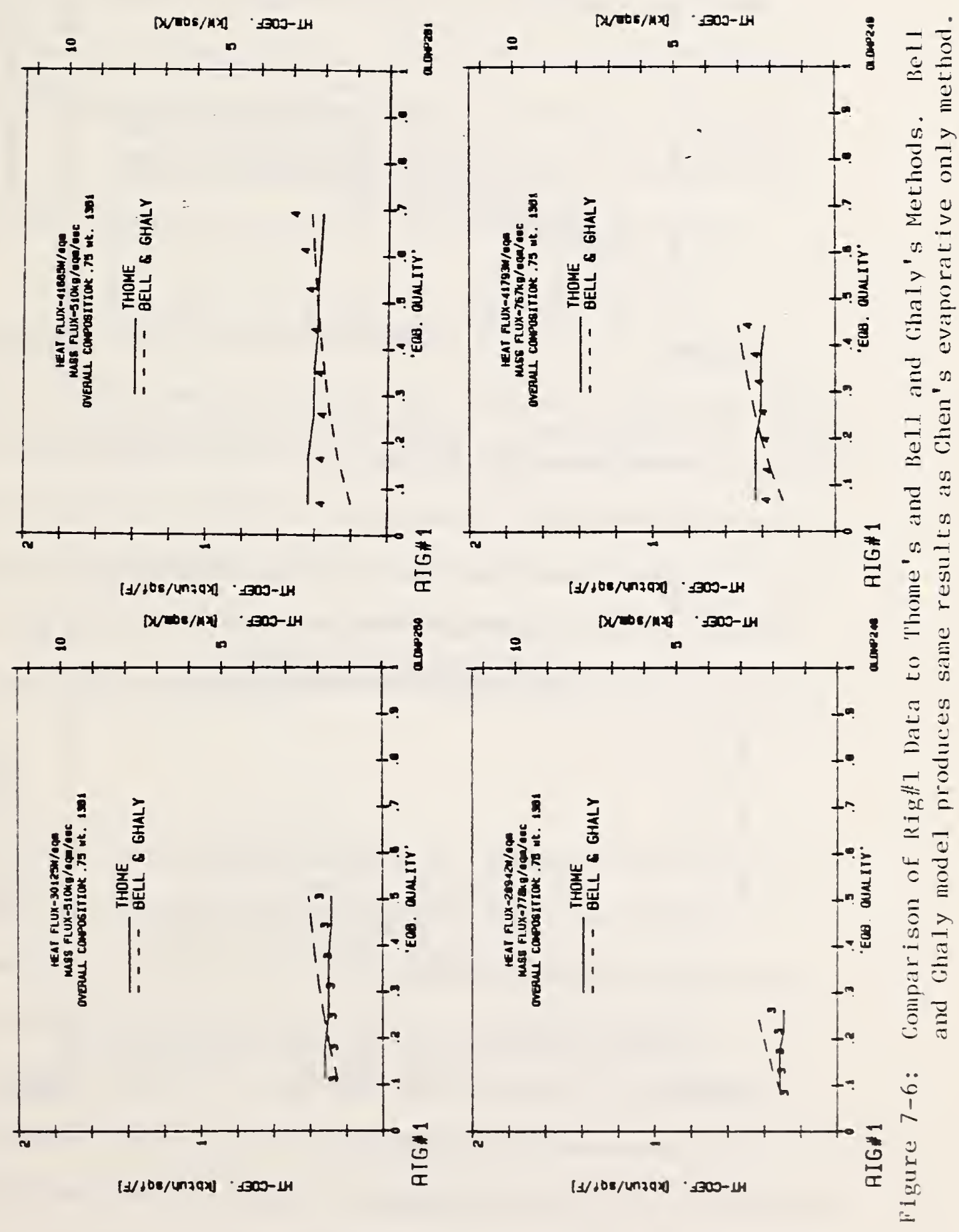




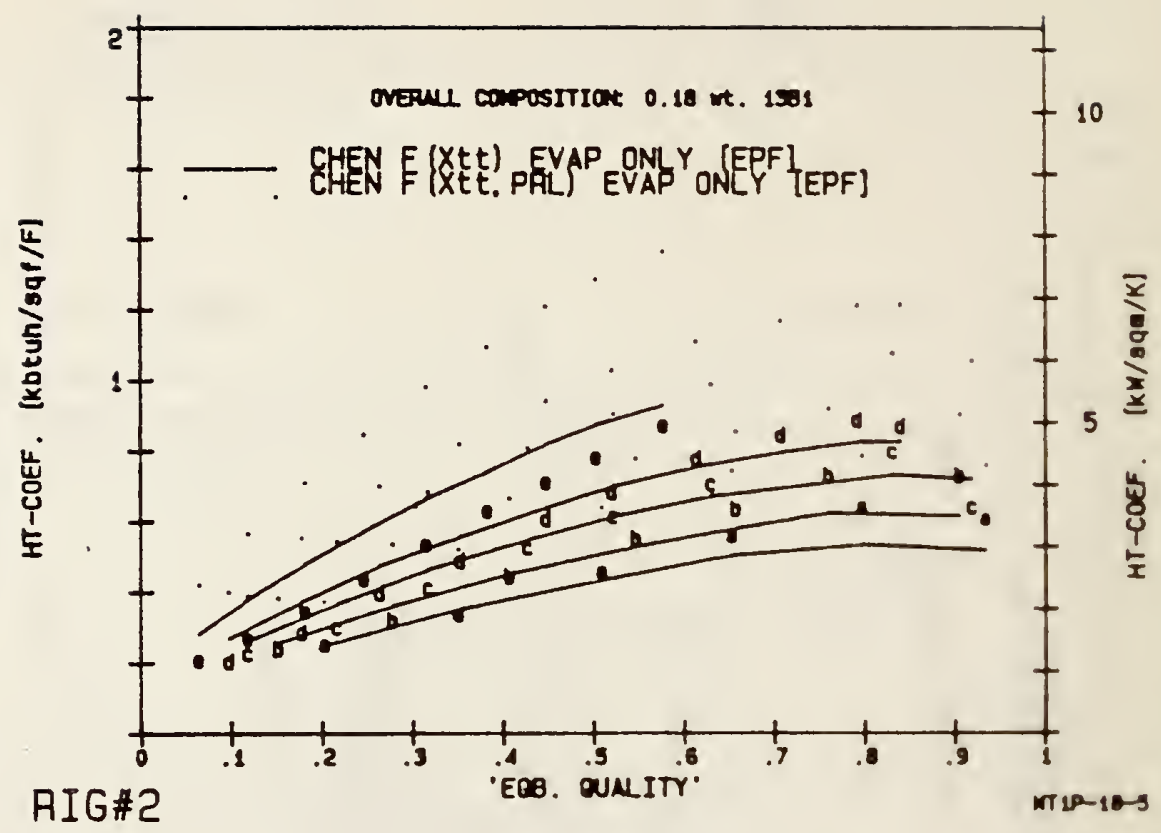

Figure 7-7: Comparison of lleasured Test Section Data to Evaporative Only Methods

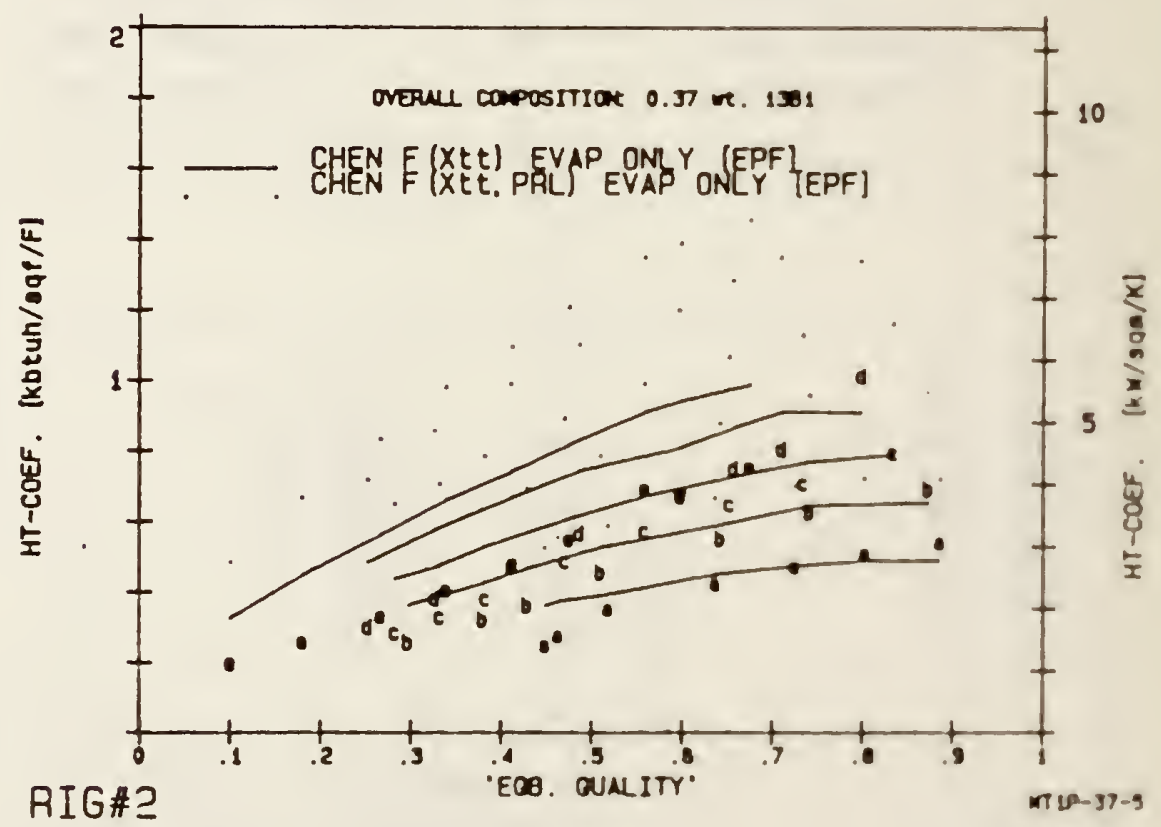


CHAPTER 8: CONCLUSIONS AND RECOMMENDATIONS FOR FURTHER RESEARCI

\subsection{Summary of Findings and Contributions}

The research described in this report involved determining experimental heat transfer coefficients, (BTC) examining the phenomena involved in the physical process, and analyzing the predictive ability of available models and correlations. This work was done for both pure and mired refrigerants. Over 1,000 data points were collected, covering a wide range of pressure, composition, quality, and beat and mass flux.

Several data collection and reduction factors were shown to influence the experimental heat transfer coefficients. The use of pressure taps and instream thermocouples may affect the wall temperature measurements (Chapter 3). The use of a preheater introduces concerns about entrainment and 1 iquid film superheat, though the effect was seen to be mini121. The use of equilibrium temperature in the definition of the HTC may itself cause a quality dependence to appear in the results (Chapter 5).

It is very important to be able to predict the dominant heat transfer regime and the point of complete suppression of nucleate boiling (Chapters 4 and 6). A controversy in the current 1 iterature regarding suppression of nucleate boiling was resolved by critical analysis in favor of traditional theory. It was seen that with pure refrigerants complete suppression occurs only at lower pressures than would occur in residential heat pump applications. A suppression criterion was applied to pore refrigerants at a selected cavity size, and shown to prodict 
quantitatively the quality at which suppression occurs for a given pressure, heat and mass flur. The method was extended to mired refrigerants via several hypotheses, and illuminated questions about predicting suppression with mistares. The method predicted that ebollition might be easier to achieve with mixtares than for pare R152a unless mass transfer resistance was included. In contrast, inclasion of ass transfer resistance term suggests that suppression would be easier to achieve. Various methods were suggested, and partially verified, for including a mixture effect in the suppression criterion.

Many pare fluid correlations were examined critically (27 in total). 01 der correlations proved to be inaccurate with data bases other than the ones with which they were originaly developed. The new method by Shah was shown to be inaccurate due to its treatment of the suppression point. The complete Chen correlation and its many variations were tested. The complete correlation predicts badly, generally overestimating the nacleate boiling contribation. The ase of the semi-analytic suppression factor which has been suggested recently agravated the overprediction. In the nucleate boiling regime, the new method of Stephan and Abdelsalam [St82] was validated. The inclusion of this method [St82] into Chen's correlation required special reformulation, and the method then predicted particularly well in the forced convection dominated region. A Prandt 1 number correction suggested by Bennett and Chen [Be80] as used in a new procedure to predict HTC's for pure refrigerants. The new procedure incorporates a pool boiling method [St82], the evaporative portion of the [Be80] method and uses the 
suppression criterion verified in Chapter 4. For these reasons it is better grounded in theory than recent regression-based correlations. It predicted the measured behavior better than calculations which have appeared in the 1 iterature, and can be used in a non-iterative manner with little loss in accuracy. The method was also checked against other independent data bases, and predicted the values well.

Sudden departare ficm nacleate boiling (DNB) events were observed in same of the pare fluid and mirtares' measurements. In examining other data sets of refrigerants, similar events coold be seen, though they remained unattributed as such by their original athors. These events may be the cause of same of the data scatter found in the literature; the data in the literature should be critically revieved for these events. The occurrence of DNB events suggest reduced heat transfer in the first rom of coils in heat pump evaporators; methods could be developed to prevent their occurrence (e.g., addition of a second component and/or modified).

In the case of mixtures, previously nnrecognized physical phenomena were noted: the circumferential variation in BTC may be opposite for mirtures than observed for pare fluids. This observation suggests the existence of a circuferential gradient in concentration and interfacial temperature (Chapter 5). Modelling then of mixtore heat transfer is fur ther complicated in that gradients exist in axial, radial and circum ferential directions. In the flow boiling of mirtures when the onset of nocleate boiling is more difficult to predict and when mass transfer 
resistance occurs, the actual quality may lag the 'equilibrium' quality in a different way than for pare flaids. This problem poses another difficulty for the correlations/models suggested to date.

For mixtures it was shown that the measured HTC would be different from ideal, even in the absence of mass transfer resistance, due to non-ideal property behavior. In the nucleate boiling dominated regime, $a_{E P F}$ is greater than $a_{i d}($ Chapter 7$)$. In contrast, in the forced convection/evaporation, regime, $a_{E P F}$ is less than $a_{i d}$ (Chapter 2). The measured $v a l$ ues in each regime showed a degradation in heat transfer over that predicted by equivalent pare fluid correlations, presumably due to mass transfer resistance (Chapter 7). The degradation compared to either pure fluid was seen to be sometimes very severe (greater than 50\%). In the nucleate boiling mode, this is due to mass transfer resistance restricting bubble growth. In the forced convection/evaporation mode, it may be due to mass transfer resistance suppressing the nucleate boiling for the mixture, but not for the pare components.

A total of 46 methods were examined for predicting heat transfer ith flow boiling of mixtures (Chapter 7). Mary methods were simple variations of the few existing techniques or were designed originally for pare fluids. Of the actual mixtures' models/correlations, some were found to be flawed on physical grounds. The regression-based correlation of Varma et al suggests that beat transfer is enhanced by the use of refrigerant mixtures over equivalent fluids; this has not been observed to date. The method of Bennett and Chen is also problematic at 
1arge Lewis numbers. Its correction term for mass transfer resistance in evaporative flow becomes negative (not physically possible) in this range. It should be excladed from further use.

None of the mirtures' calculation methods achieved closure with measured values to the same degree as was achieved with pore fluids. Closure, however, was typical of that reported in the literature for mirtures. In the nucleate boiling dominated regime, the method of Thame, suggested for pool boiling of mirtures, achieved the best agreement with the measured heat transfer coefficient. It however predicted the opposite qual ity dependence than was observed in the data. It was able to predict the average heat transfer coefficient in this regime very we11, and is thus recommended. In the forced convection evaporative regime, none of the methods predicted particularly well. The best fit to the data was achieved by the evaporative portion of Chen's original equation. This method then neglects any mirture effect, i.e., MTR, and suggests the absence of nucleate boiling. It does however tend to orerpredict, particularly at high mass flom rates. This tendency is opposite that observed for pure fluids. Unlike the case of pure refrigerants, no general complete correlation could be developed for mirtures. Chawla's original supposition, which worked very well for pore fluids, sometimes selected the less accurate predictive method. However, failing an al ternative, it is still recommended for use. This result, while disappointing, illustrates the difficalty with the prediction of mirtare behavior. It al so suggests the ide need for more experiments in the area. 


\subsection{Further Research Needs}

Several new efforts could be supported which would add to the understanding of flow boiling of mirtures. First the experimental rig could be redesigned in order to increase the speed ith which data could be collected. An example of such a rig is show in Figure 8-1. The rig, modular in orientation, could have removable tubes for special stadies (e.g. enhanced surfaces, artificial nucleation sites, effect of pressure and temperatore taps). It al so conld inclade one tabe which is designed for constant temperature operation and flow visual ization. Valving could be used to allow flow through the tubes in any order. Thermocouples could be placed to determine the onset of nucleate boiling point, film boiling occurrences, and instream temperatures (microthermocouples could be tried). O-tubes ith different radii of curvature could be installed betaen passes to allow stadies of the effect of evaporat or bends.

On a more fundamental basis, the principal efforts for pare flaids could be to nnderstand and predict: (a) entraiment and deposition rates, and (b) pressure drop. Data on the former is particularly scarce. For the latter, pressure drop should be measured in an experiment with simple evaporation (complete suppression) and again in an experiment ith nucleate boiling dominant. This might provide information to develop. more accurate pressure drop correlation. An improved $\Delta$ p correlation wond assist both the beat transfer and suppression predictions. 
For mirtures, there is a fundamental need to know concentration on both a bulk stream and local gradient basis. Because virtually no measurements have been taken to date, even instrusive measurements woula help. To this end, isokinetic sampling probles or hot wire techniques could be used in principle. In the immediate future, measurements of other mixtures should be done. If possible, an ideal mirture should be ased. The measurement program should vary parametrically, pressure, concentration, mass and heat flux, and cover the full quality range. The pressure levels should cover a range which suggests camplete suppression. This would provide information about possible mass transfer resistance and nor-equilibrium in the pore evaporative mode.

The issue of onset and suppression of nucleate boiling for mintures needs to be resolved. The literature offers few papers on experimentally determined ONB values and boiling site densities for mirtures. The new experimental rig could be designed to inclade boiling from artificial nucleation sites in a glass section (visualization studies would then be possible). If in this section, constant temperature, rather than constant heat flux, could be maintained, then ercellent resolution of the ONB point should be possible. Hysteresis stadies would also be assisted in the use of such a section.

In all cases, any future studies occur in parallel with measurements of transport properties of the mired fluids. Precise bnowledge of these properties would allow the soparate determination of the contribation of uass transfer resistance to each heat transfer regime. 
Even without further measurements, the existing data base and correlations can be examined further. There are near endless combination of portions of existing correlations which could be tested against the data. Data in the literature should be examined critically for experimental technique and data interpretation. In particalar, inlet conditions (subcooled liquid versus two phase) may have an effect on results. DNB events for mixed refrigerants al so needs to be studied further. Some of the scatter in the predictive ability in correlations may in fact be due to poor experimental technique or interpretation rather than a problem with the correlations. 


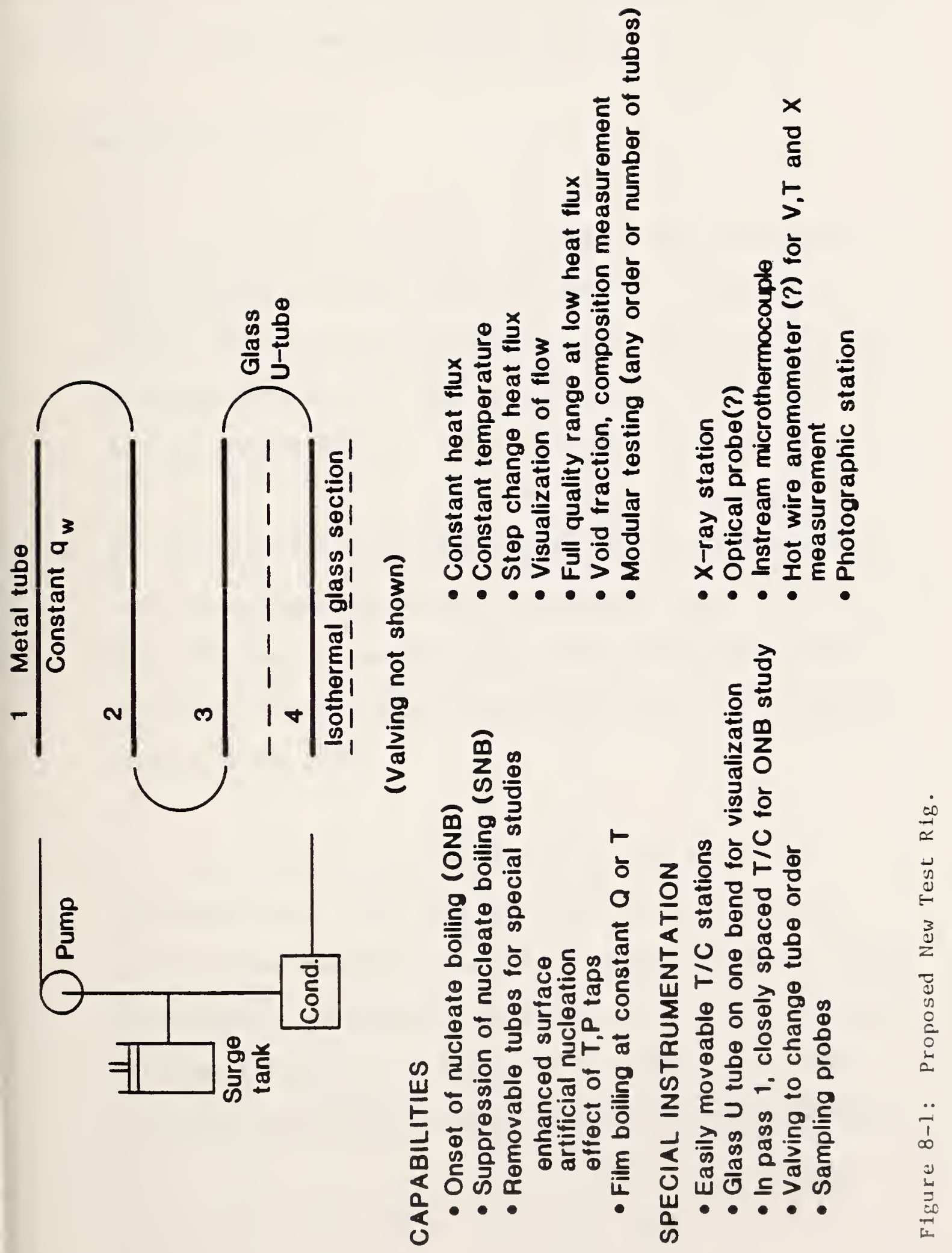



APPENDIX 3A: EXPERIMENTAL DATA SOMBLARY

The following 1 ists the data collected for this report. It is arranged so that other researchers may use it most easily.

The se items should be noted:

(a) The tests where film boiling occurred at the tube top are: $225,236,237,252,253,256,265,274,275$. Though the average heat transfer coefficient is only partially affected, this data should not be ased for comparison to annalar flow boiling correlations.

(b) The heat transfer coefficients, HTC, for val ues of $x=0.00$ may be incorrect The actaal thermodynamic quality was less than 0.00 , however in many cases, BTC was mistakenly calco1ated with the $T_{f}=T_{s a t}$, instead of $T_{f}=T_{s c}$. These values should not be used.

(c) 'Feed Comp' refers to the measured composition at the subcooled inlet. 'Mass Quality' refers to the calculated qua 1 ity based on weight, not moles. 'Liquid Comp' and 'Vapor Comp' Iefer to calculated compositions at the local pressure, enthal py and given feed composition 'Teqb' refers to the calculated fluid temperature assuming equilibrium conditions. 


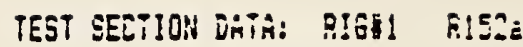

\begin{tabular}{|c|c|c|}
\hline $4 x$ & HET Fid: & QLLALIT \\
\hline
\end{tabular}

\begin{tabular}{|c|c|c|c|c|c|}
\hline \multirow{9}{*}{194} & $690^{\circ}$ & 4 -6es & 0.60 & 湎 & 257.5 \\
\hline & 399 & 42006 & 0.50 & $178 ?$ & 352.7 \\
\hline & 699 & 4âEล & .00 & $3 i ! i$ & 304.5 \\
\hline & 699 & +2068 &.$i j$ & 2370 & 254.0 \\
\hline & $i=\bar{T}$ & 4 cosos & .155 & 3120 & 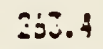 \\
\hline & เ? & 42069 & .08 & 3459 & $26 \div .8$ \\
\hline & 677 & $4=0003$ & .11 & JE:Z & 20000 \\
\hline & 659 & 4 4ंنo6 & .15 & $4: 97$ & 201.2 \\
\hline & 647 & $420 \leq 8$ &.$\AA E$ & 4670 & 250.4 \\
\hline \multirow[t]{9}{*}{195} & 703 & Eicjo & 0.00 & 18s: & 256.6 \\
\hline & $n j$ & 29056 & 0.00 & 1392 & 250.5 \\
\hline & 703 & 29536 & 0.00 & 2538 & 261.1 \\
\hline & $i \hat{Q j}$ & 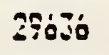 &.$\hat{v}_{z}$ & 2501 & 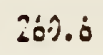 \\
\hline & $70 j$ & $93630^{\circ}$ &.$\hat{v} q$ & 250 & Sồ.: \\
\hline & 70 & ف: & .65 & 975 & 6.57. \\
\hline & 305 & 29536 & .07 & 3119 & 257.0 \\
\hline & 703 & 290ió & .09 & 3405 & $258 . j$ \\
\hline & 703 & 2SjJ\} & $.1 \mathrm{i}$ & $3 ? 69$ & 257.0 \\
\hline \multirow[t]{9}{*}{196} & 711 & 15795 & 0.00 & $: 759$ & 254.0 \\
\hline & $71 !$ & laits & 0.60 & 1776 & 250.3 \\
\hline & 711 & $! 8 ?: 25$ & 0.90 & $=0 \pm 0$ & $25: .5$ \\
\hline & 711 & $187: 5$ & $.0 !$ & 3010 & 257.2 \\
\hline & $i 11$ & 15725 & .02 & $=\hat{\because i 9}$ & $=0.7$ \\
\hline & 711 & !9745 & . $\therefore$ & 21:5 & :50.5 \\
\hline & ?il & 1E:? & .04 & $9: 39$ & IEj.i \\
\hline & $71:$ & $18: 25$ & .05. & $27 \leq 5$ & :Es.b \\
\hline & $7 i i$ & $18: 05$ & .06 & $3=51$ & 25J.? \\
\hline \multirow[t]{9}{*}{$: 97$} & $7: 3$ & 10250 & 0.20 & licj & 25:.? \\
\hline & 713 & $1035:$ & $\therefore .00$ & 1760 & 254.10 \\
\hline & 713 & $i 0750$ & 0.00 & $1 ? 3 !$ & 25\%.? \\
\hline & 713 & 10050 & .00 & 1045 & $: 54.5$ \\
\hline & 715 & 10350 & .01 & $10 \div 0$ & 254.3 \\
\hline & 313 & 10550 & .02 & 1631 & $\because 51.0$ \\
\hline & $7: 3$ & $10=50$ & .02 & $183 j$ & $253 . ?$ \\
\hline & 713 & 10550 & .03 & 2121 & 253.4 \\
\hline & $7 ! j$ & 10050 &. $.5 j$ & $\therefore 005$ & $25=0$ \\
\hline \multirow[t]{9}{*}{ i१: } & $4=5$ & 10030 & 9.00 & $!: 58$ & 257.7 \\
\hline & 405 & 11035 & $\hat{j} .00$ & !1ก? & 255.8 \\
\hline & $\$ 65$ & 11038 & .01 & $9 ! 5$ & 258.7 \\
\hline & 465 & $110 \div 3$ & .02 & 1135 & $: 58.6$ \\
\hline & $4 \dot{5}$ & $110=8$ & $.0 j$ & $15 j i$ & $2=8.5$ \\
\hline & 465 & 11038 & .04 & :7?E & $: 58.4$ \\
\hline & 405 & $110: 3$ & .05 & $19: 99$ & 258.2 \\
\hline & 965 & 1160 & .06 & 2095 & $\therefore 59.1$ \\
\hline & i65 & ¿1036 & .07 & 9279 & $25 \%$ ? \\
\hline
\end{tabular}




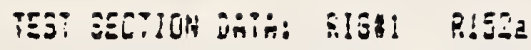

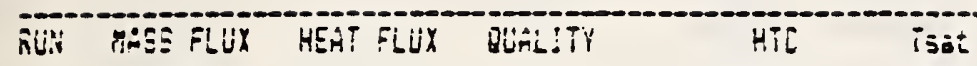

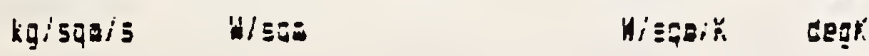

\begin{tabular}{|c|c|c|c|c|c|}
\hline \multirow[t]{9}{*}{159} & 402 & $1955 ?$ & $0.0 j$ & 1192 & ¿so. i \\
\hline & 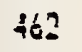 & 18592 & 0.60 & 1571 & 301.0 \\
\hline & 462 & 185: & $\therefore !$ & $20: 4$ & iş.: \\
\hline & 402 & 16592 & .05 & $21: 7$ & 201.9 \\
\hline & 402 & 185:? & .04 & 2991 & 201.9 \\
\hline & io? & iz:5? & 66 & $241 ;$ & áśd. \\
\hline & $i \varepsilon^{a}$ & $195=2$ & .08 & Zjosto & 261.4 \\
\hline & 402 & 1̊5:2 &. $\int \hat{v}$ & วละด & 261.1 \\
\hline & ite? & [559? & .12 & $3: 95$ & 260.9 \\
\hline \multirow[t]{9}{*}{ 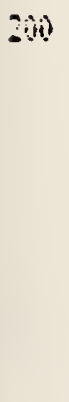 } & 40 & 29415 & 0.60 & $1 \leqslant 8$ & $26 \div .2$ \\
\hline & 4001 & 29415 & 0.06 & 2470 & ف 205.0 \\
\hline & 900 & 29415 &.$\hat{v i}$ & 2935 & 207.3 \\
\hline & 400 & 27415 & .64 & 2965 & 207.1 \\
\hline & $400 \hat{0}$ & $29 \$ 15$ & .07 & $3 \hat{4} 27$ & 260.00 \\
\hline & (j) & 29415 & .10 & 3184 & 260.00 \\
\hline & iso & 29415 & .13 & jEtá & $2 \$ 6.3$ \\
\hline & 450 & 29415 & .13 & $j: s ?$ & 250.0 \\
\hline & 460 & $994 ! 5$ & .19 & 4457 & 205.6 \\
\hline \multirow[t]{9}{*}{201} & 453 & 41920 & 0.000 & 2043 & 202.5 \\
\hline & 455 & $-1990 \hat{~}$ & 3.60 & 3402 & $=90 . j$ \\
\hline & q5j & $4: 920$ & .02 & 3993 & 272.1 \\
\hline & AE: & :บร20 & $.0 i$ & 3914 & $=7 ! .9$ \\
\hline & $45 j$ & 41720 & .11 & 3015 & $231 . i$ \\
\hline & $45 j$ & 41920 & .15 & $39: ?$ & $\rightarrow 1.5$ \\
\hline & $\{5 j$ & 41990 & .19 & 9450 & 971.2 \\
\hline & 455 & 41930 & .24 & 4997 & 270.7 \\
\hline & 450 & $4 ; 920$ & .20 & 5675 & 270.0 \\
\hline \multirow[t]{9}{*}{202} & 295 & ¿E?5j & .02 & $184^{\circ}$ & AES. 7 \\
\hline & 5 & $15 \div 50$ & .05 & 1955 & 258.5 \\
\hline & 205 & 18750 & .69 & 2120 & 258.4 \\
\hline & 235 & 18750 & .12 & 2168 & $258 . \hat{2}$ \\
\hline & 235 & 13750 & . ió & 2470 & 2Е8. \\
\hline & 235 & 19750 & .19 & 2735 & 957.7 \\
\hline & 235 & 23750 & $.2 j$ & 2750 & 257.4 \\
\hline & 235 & (20750 & .23 & 3253 & 257.1 \\
\hline & 655 & $: 8750$ & .29 & jöii & 250.7 \\
\hline \multirow[t]{9}{*}{$20 j$} & 237 & 10014 & .j1 & $\$ \$ 1 j$ & 257.3 \\
\hline & 257 & 10614 & $.0 j$ & Já7a & $2=7 . j$ \\
\hline & 237 & 10014 & .05 & 1703 & 257.? \\
\hline & 237 & 100 it & .07 & $1:: 2$ & $=57.2$ \\
\hline & $3 \bar{i}$ & lốlt & .09 & 1758 & 25.1 .1 \\
\hline & 293 & $106: 9$ & .11 & 1872 & 957.1 \\
\hline & 257 & 10619 & .13 & 3007 & 257.0 \\
\hline & 5 & 10514 & .15 & 2215 & 257.3 \\
\hline & 257 & 10614 & .17 & 2jỏ & 255.9 \\
\hline 204 & 229 & $467 !$ & 0.10 & 98: & โอบ..5 \\
\hline
\end{tabular}




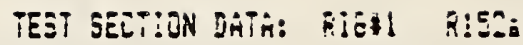

\begin{tabular}{|c|c|c|c|c|c|}
\hline FLi' & 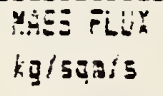 & $\begin{array}{c}\text { HET Fiji } \\
\text { WisQ }\end{array}$ & EUALJTY & $\begin{array}{c}\text { HIC } \\
\text { H/squik }\end{array}$ & $\begin{array}{l}\text { Tes: } \\
\text { de.jK }\end{array}$ \\
\hline \multirow{8}{*}{. } & 9 & $45:$ & .00 & 1357 & 350.0 \\
\hline & 299 & $467 i$ &.$\Delta 1$ & $.159 ?$ & 25E.9 \\
\hline & 9ac & $46 i 1$ & .02 & $15=$ & 250.6 \\
\hline & 297 & $48 i 1$ & .03 & 1794 & $=88.7$ \\
\hline & 395 & $467 i$ & .64 & $1 ? 28$ & 258. \\
\hline & 299 & 4671 & .05 & 1959 & 258.5 \\
\hline & 220 & 4671 & .00 & 1839 & 256.4 \\
\hline & 99 & tcil & .07 & $15 \%$ & 5:53. \\
\hline \multirow[t]{9}{*}{205} & 25 & aptisj & .02 & Q $E 9 ;$ & 251.2 \\
\hline & 27 & 29751 & .07 & 2491 & 281.5 \\
\hline & כَנنב & 29701 & .13 & 2457 & 251.2 \\
\hline & 235 & 2970 ใ & .19 & 2750 & 261.0 \\
\hline & 25 & 39701 & .24 & 3142 & $260 . \approx$ \\
\hline & 252 & E9Tol & .00 & jE!i & 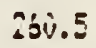 \\
\hline & 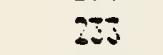 & 29761 & .35 & 3776 & 260.1 \\
\hline & Jiju & 29iol & .41 & $4 i j 5$ & $25: .7$ \\
\hline & 25 & 29761 & $.4 i$ & 4330 & 259.3 \\
\hline \multirow[t]{9}{*}{300} & 32 & 4:A9E: & $\hat{v} \mathrm{i}$ & $394:$ & $2: 5.4$ \\
\hline & 222 & 42257 & .09 & 3722 & 255.1 \\
\hline & 23 & 42957 & .18 & 3455 & 204.3 \\
\hline & 32 & 42357 & .26 & Z3tio & $26 \div .5$ \\
\hline & 332 & 42957 & .24 & $4: 15$ & 264.2 \\
\hline & $n=2$ & $4295 i$ & .42 & 4:2 & 23.7 \\
\hline & 232 & 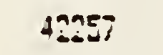 & $.5 i$ & 2375 & 365.2 \\
\hline & 329 & 4.-25: & .59 & $526:$ & 35.6 \\
\hline & 232 & $9: 257$ & ś & $55: 2$ & 262.0 \\
\hline \multirow[t]{9}{*}{$2: 10$} & 140 & 192E5 & .63 & 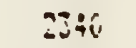 & $25 \div 3$ \\
\hline & $1 \div 0$ & 19955 & .09 & $2 \$ 50$ & 262.3 \\
\hline & $1+0$ & 17385 & .15 & anj: & $2 \notin \leq .6$ \\
\hline & 120 & 19385 & .21 & :38 & 202.5 \\
\hline & 140 & 19285 & .27 & $=115$ & 262.4 \\
\hline & 140 & 19285 & .55 & $247 !$ & $262 . j$ \\
\hline & 140 & ¡9285 & .40 & 2852 & 262.1 \\
\hline & $\$ 400$ & $19: 85$ & .96 & 3124 & $=61 .^{\circ}$ \\
\hline & 140 & 199.05 & .52 & ZXE? & $261 . \Xi$ \\
\hline \multirow[t]{9}{*}{211} & 141 & 29:19: & .32 & 70 & $\therefore: 5.5$ \\
\hline & 141 & $290 \hat{\mathrm{I}}$ &.$\therefore 1$ & $329 j$ & $2 \leq 8.4$ \\
\hline & 141 & 29021 &.$\therefore 1$ & 3027 & 268.3 \\
\hline & 141 & 29021 & .30 & 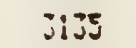 & $238 . i$ \\
\hline & 141 & 29021 & $\therefore 9$ & 30j4 & $2 \leq 3.0$ \\
\hline & $! \div 1$ & 29921 &.$\$ 7$ & 2500 & 257.8 \\
\hline & 141 & :902: & .58 & $40 \div 5$ & $26 i .0$ \\
\hline & $: 41$ & 29021 & .67 & 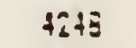 & 267.4 \\
\hline & 141 & 6?021 & .77 & 9591 & 263.2 \\
\hline \multirow[t]{2}{*}{212} & 250 & $417 \div 2$ & .03 & j:ذj & 365.9 \\
\hline & $27 \mathrm{c}$ & $\$ 1722$ & .10 & 3449 & 263.6 \\
\hline
\end{tabular}


TEST JEZTION UMTHÁ: RIEH! RISZZ

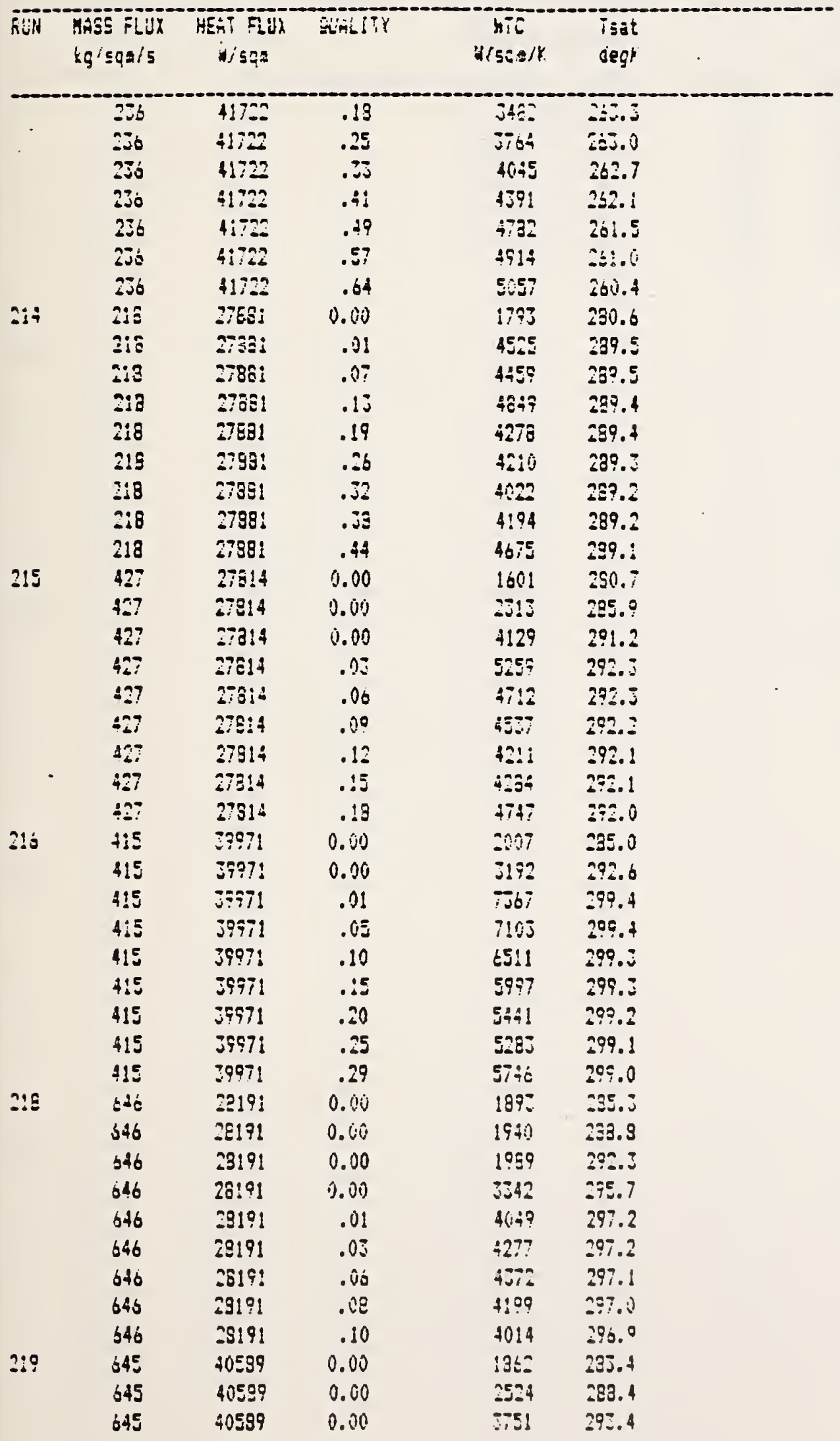


TEST GEETION DATÁ: R!Gal RiFì

\begin{tabular}{|c|c|c|c|c|c|}
\hline Rui & kg: squ/s & $\begin{array}{c}\text { W/sqa } \\
\text { W. }\end{array}$ & ULALITY & $\begin{array}{c}H T C \\
H_{i} \leq q \leq / k\end{array}$ & Tegat \\
\hline & 045 & 40539 & .6 & 5530 & 257.6 \\
\hline & 645 & 405E? & . & 5615 & 297.5 \\
\hline & b45 & $4059^{\circ}$ & . & 5417 & 297.1 \\
\hline & $\$ 45$ & drES:? & .10 & $5: 70$ & 297.4 \\
\hline & 645 & 40529 & .13 & $5 !=$ & $297 .:$ \\
\hline & 645 & 46899 & ذذ & $5 ! 5 i$ & 297.2 \\
\hline
\end{tabular}


TEST SECTIGN GATAA: R!GH! RIJ8!

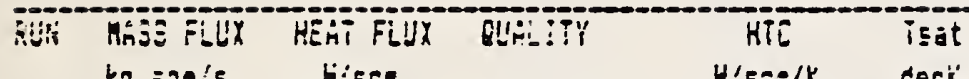

\begin{tabular}{|c|c|c|c|c|c|c|}
\hline \multirow[t]{9}{*}{25} & 364 & biti? & $i .00$ & 1497 & 259.5 & \\
\hline & 754 & 10547 & .00 & 201 & Soj.8 & \\
\hline & 754 & $1054 ?$ & .02 & 2602 & 251.3 & \\
\hline & 764 & 16549 & .04 & 2900 & 261.3 & \\
\hline & 754 & 10049 & .00 & 3125 & Z2:1.8 & \\
\hline & $\overrightarrow{i j 4}$ & 10049 & .96 & $340 !$ & ibj.i & \\
\hline & 764 & 10549 & .10 & 3619 & 251.7 & \\
\hline & 764 & 10849 & $.1 ?$ & 3497 & col.b & \\
\hline & 7É & 10049 & j & Sด̂E & 261.6 & \\
\hline \multirow[t]{9}{*}{293} & 1219 & 29494 & 0.000 & 4216 & 261.4 & \\
\hline & 1219 & 29494 & .12 & 5429 & $2 \pm 2.4$ & \\
\hline & 1219 & 290494 & .05 & 4785 & 262.2 & \\
\hline & 1219 & 25494 & .09 & 4451 & at5.0 & \\
\hline & 1219 & 29+94 & .12 & $410 ?$ & 261.9 & \\
\hline & 1219 & 29498 & .15 & 3990 & $20 \mathrm{si} 7$ & \\
\hline & $121 \%$ & 29494 & .19 & $410 j$ & 251.4 & \\
\hline & 1219 & 29494 &.$\hat{2}$ & $4 a ! j$ & 25่.2 & \\
\hline & 1219 & 29474 & .25 & 4416 & séi.o & \\
\hline \multirow[t]{9}{*}{234} & $12: 0$ & $422 ! 2$ & 0.00 & $5: 27$ & $20 \div .9$ & \\
\hline & 1210 & $\$ 2212$ & .03 & $7 ; 80$ & QSES.? & . \\
\hline & 1210 & 42212 & .40 & 6925 & 2.55.i & \\
\hline & 1210 & $4: 212$ & .13 & 0319 & 265.5 & \\
\hline & $12: 0$ & 42212 & .17 & Eg5:? & 2E5. & \\
\hline & 1210 & 4ä!n & .20 & 5025 & 265.0 & \\
\hline & 1210 & 42212 & .27 & 5597 & 254.1 & \\
\hline & !:10 & 22912 & .32 & 5990 & 264.5 & \\
\hline & 1210 & 420212 &.$\overline{3 i}$ & 5915 & 254.2 & \\
\hline \multirow[t]{7}{*}{295} & 802 & ใ22ิิ4 & .61 & $74: E$ & ij5. & \\
\hline & 302 & 430154 & .68 & TIST & ב̇E..A & \\
\hline & 802 & 422014 & .15 & $70 \hat{93}$ & 265.1 & \\
\hline & 802 & 422014 & .25 & 6900 & 264.9 & \\
\hline & 802 & $\$ 2304$ & .30 & 5383 & 254.9 & \\
\hline & 802 & 42204 & .37 & 5172 & 364.6 & \\
\hline & 802 & 42204 & .34 & 6500 & 204.4 & \\
\hline \multirow[t]{2}{*}{ • } & 802 & 125012 & .52 & bi:l & $=04.1$ & \\
\hline & 802 & $42: 94$ & .59 & $475 ?$ & $35=9 ?$ & \\
\hline \multirow[t]{9}{*}{ :ES } & 890 & 29530 & .01 & $5 \div 97$ & 261.6 & \\
\hline & E?! & 2QPON & .06 & 5590 & $26: .5$ & \\
\hline & 220 & 29530 & .11 & 5095 & 261.4 & \\
\hline & 920 & 29550 & .16 & $499 \mathrm{~J}$ & J.' & \\
\hline & 820 & 2૯:5:0 & .21 & 4003 & 231.2 & \\
\hline & E20 & 29500 & .25 & $451 i$ & 351.0 & \\
\hline & 320 & 29550 &.$j \hat{v}$ & 9055 & 2 วิธ. 8 & \\
\hline & $8 \div 0$ & 29330 & .25 & 4664 & 250.6 & \\
\hline & 320 & 29330 & .40 & 4644 & 200.4 & \\
\hline
\end{tabular}




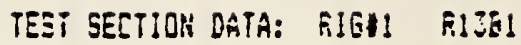

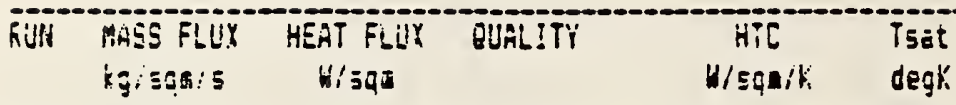

\begin{tabular}{|c|c|c|c|c|c|}
\hline \multirow[t]{9}{*}{387} & $4 \vdots \vdots$ & 69188 & .05 & $55: 1$ & 262.5 \\
\hline & 415 & 29189 & .15 & & $2 \in 2.4$ \\
\hline & 413 & 29188 & .25 & 3331 & 262.4 \\
\hline & 412 & 29189 & .34 & 3071 & 262.3 \\
\hline & 413 & 29188 & .44 & $359 ?$ & $2 \varepsilon 2.3$ \\
\hline & 413 & 29188 & .54 & 4895 & $26 \div 2$ \\
\hline & 413 & 29193 & .63 & $52: 1$ & $2 \$ 2.1$ \\
\hline & $41 j$ & 29199 & .73 & $59: 7$ & $26: . j$ \\
\hline & $+1 j$ & 29180 & .85 & 6554 & 261.9 \\
\hline \multirow[t]{9}{*}{252} & 414 & 18792 & $.0 ?$ & 4279 & 261.8 \\
\hline & 414 & 18:?? & .09 & 4336 & 201.7 \\
\hline & $4: 4$ & 18792 & .15 & $3 \varepsilon \div 5$ & 281.7 \\
\hline & 414 & 18792 & .29 & נדבגה: & 251.7 \\
\hline & 414 & $\cdot 15792$ & .28 & 3279 & 261.5 \\
\hline & 414 & 18792 & .34 & ت349 & 261.5 \\
\hline & 414 & 18792 & .20 & 4854 & 261.4 \\
\hline & 414 & 18792 & . tó & $5=09$ & 351.3 \\
\hline & 414 & 19792 & כ. & E:94 & 261.2 \\
\hline \multirow[t]{9}{*}{$29 ?$} & $40 j$ & 10522 & 0.00 & $: 775$ & 262.9 \\
\hline & 4ijj & 10532 & .02 & jis? & 2Eะ. \\
\hline & $40 j j$ & 10E:? & . & :En: & :อ́.ร.7 \\
\hline & 405 & 10522 & .07 & $452 !$ & 265.7 \\
\hline & 403 & 10522 & .12 & sis? & 265.6 \\
\hline & 403 & 1052? & ذ' & $=640$ & S.5.5. \\
\hline & $40 \bar{J}$ & $105=2$ & .20 & :29? & 365.5 \\
\hline & 403 & 10592 & .23 & $4 ! 70$ & $25=.4$ \\
\hline & 203 & 10522 & .27 & $\{: \hat{\imath} \hat{j}$ & ב \\
\hline \multirow[t]{9}{*}{$=50$} & 80 & 13753 & 0.000 & 1,709 & -50.t \\
\hline & 803 & i8753 & 0.00 & 2ラ:日 & 264.5 \\
\hline & $2 \hat{\theta}$ & :8358 & $.3 !$ & $478 i$ & Sojot \\
\hline & 805 & 16758 & .04 & 5679 & 267.4 \\
\hline & 803 & 18758 & .08 & 5297 & $2=i . j$ \\
\hline & 303 & 16758 & .11 & 5:19 & 367.3 \\
\hline & 303 & 19758 & .14 & 5520 & 257.2 \\
\hline & $80 j$ & 19758 & .17 & 0105 & 267.1 \\
\hline & 305 & 19758 & .21 & 59 iij & $26: .1$ \\
\hline \multirow[t]{9}{*}{$\$ 9:$} & 1230 & 19814 & 0.00 & 1226 & : 59.5 \\
\hline & 1220 & 16814 & 0.00 & $25: 17$ & $26 ! .2$ \\
\hline & $122 \mathrm{j}$ & 18214 & 6. 10 & 3010 & $2=4.0$ \\
\hline & 1220 & :S\$14 & .01 & $5 j i j$ & 255.0 \\
\hline & 1220 & !8914 & .04 & STRE & 25t.: \\
\hline & 1220 & $: 9914$ & .06 & 5253 & 354.3 \\
\hline & $12=0$ & 15914 & .09 & 5E:5 & $=09.7$ \\
\hline & 1920 & :3814 & .10 & $=i^{2} 5$ & $3: 24.6$ \\
\hline & 1990 & 18911 & 19 & $568 ?$ & 254.5 \\
\hline
\end{tabular}




\begin{tabular}{|c|c|c|c|c|c|c|c|c|c|}
\hline 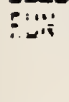 & 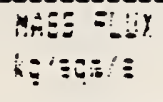 & 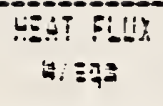 & $\begin{array}{c}\text { YA:SO } \\
\text { QUFL:TY }\end{array}$ & $\begin{array}{l}\text { HTi } \\
H / 543 / 6\end{array}$ & $\begin{array}{l}\text { Teqb } \\
\text { degik }\end{array}$ & $\begin{array}{l}\text { Conesio. } \\
\text { conse }\end{array}$ & $\begin{array}{l}\text { Comp. } \\
\text { Compens }\end{array}$ & 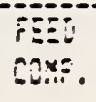 & $\begin{array}{c}\text { PPE:SU } \\
\text { ba: }\end{array}$ \\
\hline \multirow{9}{*}{ ניב } & 485 & 30:-is & .8 & liáa & 260.? & ذَذَ. &.$\dot{E}$ & .062 & 4.83 \\
\hline & $4 E 5$ & วอบง? & . & :ลอง & 250.4 & .64 & .86 & & 4.87 \\
\hline & $\because \hat{\text { Sa }}$ & 29709 & .17 & :IJ̃! & Bg0̂. $i$ &.$\therefore ?$ & .85 & & 4.65 \\
\hline & AES & $25: 19$ & .25 & 1374 & $2 \vdots 21.0$ & .51 & .95 & & 4.EZ \\
\hline & ใE & 20509 & $.3 j$ & 1457 & 201.3 & $.5 \bar{y}$ & .84 & & Q.E? \\
\hline & 486 & 29509 & oj & 1529 & 201.7 & .56 & .84 & & 4.56 \\
\hline & $48:$ & 29509 & .42 & :5:7 & 202.1 & .54 &.$\Xi Z$ & & q.छ̇ \\
\hline & $4 E t$ & 90:00 & .28 & $16 t !$ & $25 \ldots .5$ & .51 & .83 & & 4.53 \\
\hline & int & 29309 & .84 & 1769 & 35ว.!! & .45 & .92 & & 4.50 \\
\hline \multirow[t]{9}{*}{234} & $49 ?$ & 41398 & $.0 ?$ & $15 ! ?$ & 2క?.8 & .56 & .85 & $.0 \pm 2$ & 5.58 \\
\hline & 487 & 41508 & .12 & 1724 & $263 .:$ & .64 & .85 & & 5.10 \\
\hline & $95 i$ & มะวงด & .26 & 1399 & 2కड. 6 & $.6 !$ & .84 & & 5.02 \\
\hline & $\$ 87$ & 40308 & .31 & $19: 0$ & 267.0 &.$\equiv 8$ & .84 & & 5. is: \\
\hline & $48 i$ & 4iJje & $.4 \hat{v}$ & 2093 & 264.7 & .55 & .83 & & 4.79 \\
\hline & $48 ?$ & 4!:je & .43 & $2 x: t$ & 3ะE.. & $.5 !$ & .82 & & $4=5$ \\
\hline & 287 & HIŜE & .57 & 23 & $25 t .3$ & .47 & $.2 !$ & & 4.72 \\
\hline & 487 & Ұij0B & 5 & $250 ?$ & $26 ; .1$ & .45 & .99 & & $4 . \Xi \Xi$ \\
\hline & 487 & A!JUEE & .70 & $27 ! !$ & 263.1 & $.9 \hat{y}$ & ; & & 4.35 \\
\hline \multirow[t]{9}{*}{ MES } & 252 & IEs?! & $.0 S$ & !!n & $250 . !$ & .65 & . 86 & .52 & 4.54 \\
\hline & 252 & $2367 !$ & .20 & 1045 & 253.7 & .81 & .35 & & 4.5 \\
\hline & AE? & ZSbi! & $\therefore$ & 1129 & 251.5 & . & .84 & & $4.2=2$ \\
\hline & 252 & $2867 !$ & .44 & 1357 & $2=2.5$ & .53 & $.8 z$ & & $4.5 i$ \\
\hline & SE? & 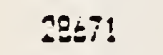 & .54 & 1994 & 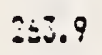 & .45 & $.8 !$ & & 4.60 \\
\hline & $25 ?$ & $2857 !$ & .54 & $16=0$ & 2EE.j & $.4 ?$ & . & & $4 . \Sigma \Xi$ \\
\hline & 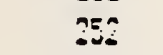 & $2 \sum b 71$ & .3 & diss & ใโะ. & .3 & .79 & & 㕸 \\
\hline & $=8$ & ZES?1 & .50 & !?? ? & $9: 3.2$ & Z & $.7 i$ & & 4.55 \\
\hline & 252 & 23571 & .97 & 2060 & 9.5 .5 & $\therefore$ & $\therefore$ & & ؛ \\
\hline \multirow[t]{9}{*}{ 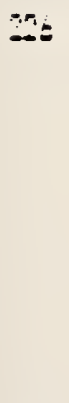 } & Ex? & $41194:$ & $.0 ?$ & 1570 & 252.7 & 4. & .25 & Z: & 5.19 \\
\hline & 259 & $4: 190$ & .97 & $1: 54$ & Rèv.? & $.0 \hat{\nu}$ & .34 & & $5.0:$ \\
\hline & 25 & $41: 50$ & .45 & i7j! & $2 \leq 5.0$ & .54 & . & & 5. 19 \\
\hline & 259 & 41190 & .58 & 20)84 & ¿to.9 & .97 & .30 & & 4.99 \\
\hline & 259 & 11190 & .70 & 2395 & 2Eะ.? & .40 & .77 & & 4.97 \\
\hline & 250 & $4 ! 180$ & .21 & EEE & $27 ! .0$ & .54 & . it & & 4.96 \\
\hline & 259 & $4 ! 190$ & .91 & 2548 & $27 \div 8$ & .29 & .70 & & 4.?4 \\
\hline & ?ב: & 41190 & 1.00 & 3130 & 274.4 & .25 & . só & & 4. $\because ?$ \\
\hline & 95 & $4 ! ! ? 90$ & $1.0 \hat{0}$ & 1355 & 278.7 & .20 & .08 & & 4. $=0$ \\
\hline \multirow[t]{9}{*}{900} & $?: 99$ & $29 ! 54$ & $0.0 \hat{0}$ & 1037 & AES.: & ذغ. & 1.05 & $.60=$ & ثن \\
\hline & 汇? & $29 ! 54$ & .05 & $1: 99$ & $=59.5$ & bt & $.3 b$ & & 4.50 \\
\hline & 799 & 25154 & .09 & 1227 & o. & .65 & . อิ & & 4.57 \\
\hline & PO? & $2 ? 154$ & .12 & $102 !$ & 2ES. I & . & כذ S. & & 4.55 \\
\hline & 725 & $29: 54$ & .17 & :SSO & $=5.8$ & .62 &.$\$ 5$ & & 4.52 \\
\hline & 79 & $29: 54$ & .21 & iq_! & 259.9 & $.5 !$ & .85 & & $\therefore .50$ \\
\hline & $?$ & $20: 54$ & S. & $14 i+$ & 260.0 & $.6 \hat{0}$ & .85 & 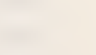 & $4.7 \%$ \\
\hline & 799 & 29:57 & .30 & 1528 & 2601.1 & .58 & .85 & & 4.44 \\
\hline & 799 & 29154 & .34 & 160ิ & 260.2 & .57 & .84 & & $4 . i t$ \\
\hline
\end{tabular}


TEET GETIIEN QÁTA: RIGA:

\begin{tabular}{|c|c|c|c|c|c|c|c|c|c|}
\hline SuY & $\begin{array}{l}\text { RÁS FIJU } \\
\text { kgisqnis }\end{array}$ & $\begin{array}{c}\text { HET F!UX } \\
\text { nisqa }\end{array}$ & MUÁ:IT & 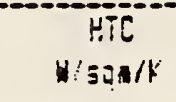 & degge & $\begin{array}{l}\text { LCOA: } \\
\text { Coms. }\end{array}$ & 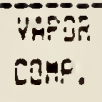 & 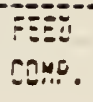 & $\begin{array}{l}\text { Gars } \\
\text { cons }\end{array}$ \\
\hline \multirow[t]{9}{*}{$Z^{\prime \prime}$} & 39 & $4: 15$ & 0.60 & 1901 & 201.0 & .00 & 8.097 & a & E. \\
\hline & 7月? & $4: 1 ! 5$ & .05 & 1710 & 202.5 & .65 & . & & 5 \\
\hline & $i 27$ & $4: 115$ & .12 & i780 & 205.0 &.$\leq 4$ & .95 & & 5.14 \\
\hline & 729 & $4: 1: 55$ & .18 & 1920 & 265.2 & .02 & .85 & & 5. $3 ?$ \\
\hline & 729 & 22915 & .25 & $20 \Omega$ & 253.4 &. \pm 0 & .34 & & 4.28 \\
\hline & $i_{6}$ & ia1:5 & $.3 !$ & A:AS & $2 \div \dot{0} .7$ & .53 & .9 & & 4. \\
\hline & $i n e$ & $4211 E$ & .37 & $219 !$ & 263.9 & .50 & . & & 9. \\
\hline & 99 & $4: 115$ & .43 & 2905 & 264.2 & .54 & ב3. & & 4.97 \\
\hline & 799 & $421: 5$ & .49 & $=46 j$ & 264.6 & $.5 !$ &.$\triangleq$ & & 4.85 \\
\hline \multirow[t]{9}{*}{34} & $4: ?$ & 29370 & .01 & 1110 & 358.? & .70 & .97 & .80 & :.65 \\
\hline & 497 & $29: 40$ & .68 & 1258 & 350.1 & .89 & $.3 i$ & & 9.53 \\
\hline & $49 i$ & 99340 & .15 & 1323 & ב.59.5 & .68 & . 36 & & 4.02 \\
\hline & 497 & 29340 & .22 & 1087 & 259.5 & ts & 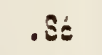 & & $4.5 \mathrm{i}$ \\
\hline & $4 \div 7$ & $295 \div 0$ & .29 & 1179 & 359.7 & .65 & S & & +.50 \\
\hline & 497 & $29-40 \hat{0}$ & .34 & 155 & 260.0 & .63 & .35 & & :. 5 \\
\hline & 497 & 90730 & $.4 \vdots$ & :5?\$ & 260.3 & 1 & ك. 25 & & 4.57 \\
\hline & 497 & 29340 & $.4 i$ & 1425 & 250.6 & .58 &.$S 4$ & & 4.5: \\
\hline & $49 i$ & 99542 & $.5 j$ & $15=1$ & $25 ! .1$ & .56 & .34 & & 4.49 \\
\hline \multirow[t]{9}{*}{295} & 507 & \$14!: & .01 & $1=20$ & $25 \%$ & .70 &. $\bar{Z}$ & . ins & 5.:4 \\
\hline & $50 ?$ & $414 ! !$ & .10 & :151 & 269.4 & .69 & 2. & & $5: 2$ \\
\hline & 507 & $4: 4 !:$ & .20 & $! 9 ! \vdots$ & ใดล. & .67 & .36 & & $5 .: 1$ \\
\hline & $30 i$ & $414 ! 1$ & .29 & 1990 & 265.0 & . 55 & . & & 三.id \\
\hline & $5:$ & $+14: 1$ &.$j a$ & 097 & 260.4 & .E: & .35 & & 5.0 \\
\hline & 507 & $4 ! 4 ! i$ & . $4 t$ & $2 ! \div 9$ & $26=99$ & .99 & .3: & & 5.6 \\
\hline & 507 & $4 i+11$ & .54 & $9=90$ & Bet.5 & .56 & . & & 4.3 \\
\hline & 507 & $41+11$ & .02 & $249:$ & 35E.: & 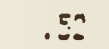 & .38 & & $\therefore \because \because$ \\
\hline & 沙7 & 4141: & .69 & ziti & $=36 . !$ & .97 &.$\Xi !$ & & (. 010 \\
\hline \multirow[t]{9}{*}{ نت } & $24 ?$ & בפogl & .09 & $: 15=$ & $=50.5$ & $.6 \%$ & .37 & $\therefore 6$ & 4.55 \\
\hline & 247 & 299?! & .29 & $10: 2$ & 250.1 &.$s c$ & . Sé & & $4.5:$ \\
\hline & 247 & $2927 ! 1$ & .35 & $:: 58$ & 259.3 & .65 & .35 & & 4.5 \\
\hline & $\therefore 77$ & 30.91 & .47 & $1: 39$ & 250.7 & .59 & .84 & & $4.3=$ \\
\hline & 247 & 39231 & .58 & is:0 & 3.:.?? & .55 & $.9 \Xi$ & & +.5: \\
\hline & $24 ?$ & 29931 & .09 & ióa & $2 \leqslant$ ¿. 4 & .47 & .81 & & $4.5 !$ \\
\hline & 247 & 29251 & .78 & IEב! & '55.: & $.4 i$ & .79 & & 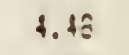 \\
\hline & $8+7$ & :923! & פיט. & 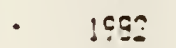 & $3: \leq .0$ & . & ic & & $\therefore: i$ \\
\hline & 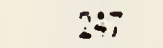 & | & .94 & $2: 30$ & : לร.: &.$\dot{\Delta}$ & .7 & & $i .45$ \\
\hline \multirow[t]{9}{*}{97} & 297 & 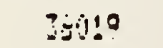 &.$: 0$ & בְּ: & 959.3 & ?: & 33. & . & 4. $\because$ \\
\hline & 247 & 36019 & .26 & 1299 & IEvi.s & .65 & $.5 t$ & & $\therefore .:$ \\
\hline & 297 & 36019 & .11 & i:i:j & -31.5 & .61 & .85 & & 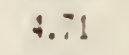 \\
\hline & 247 & 36019 & .56 & : & $26 \% ?$ & .54 & .83 & & $1 . \therefore$ \\
\hline & 247 & $360: 9$ & .69 & $\{\Phi t\}$ & 359.7 & $.4 i$ & .81 & & i.ci \\
\hline & 247 & $3 \$ 019$ & .50 & 3179 & 260.7 & .40 & .78 & & $\therefore .07$ \\
\hline & 217 & 35019 & .90 & $\Delta+1\rangle$ & 268.7 & .24 & .75 & & 4.65 \\
\hline & 247 & i60119 & 1.00 & $979 n$ & 570.7 & .99 & .il & & i.: : \\
\hline & 247 & 3001? & 1.00 & Masa & 372.6 & .27 & 6 & & $\therefore .6 !$ \\
\hline מב & 758 & 29?:१ & 0.00 & $: 2: 16$ & 257.5 & .71 & $1.00)$ & $\therefore=$ & 4. : \\
\hline
\end{tabular}


TEST SESTION LHATH: RIGI!

\begin{tabular}{|c|c|c|c|c|c|c|c|c|c|}
\hline SUE & 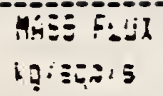 & $\begin{array}{c}\text { HETi FLUI } \\
\text { H/sqE }\end{array}$ & $\begin{array}{c}\text { MHES } \\
\text { BUALITY }\end{array}$ & $\begin{array}{c}\text { nistik } \\
\text { nisqaik }\end{array}$ & $\begin{array}{l}\text { Teqg } \\
\text { degl }\end{array}$ & $\begin{array}{l}\text { Cakp. } \\
\text { Caito }\end{array}$ & COMF. & 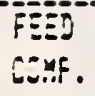 & $\begin{array}{c}\text { bars } \\
\text { bas }\end{array}$ \\
\hline & 5 & 2909 & . & $15+1$ & $26 ! .5$ & 0 &.$E^{5}$ & & $9.7:$ \\
\hline & 753 & 39ะจด1 & .07 & $14=0$ & 259.4 & .69 & .37 & & 4.68 \\
\hline & $75 E$ & $2999 !$ & .12 & $19=0$ & 25.9 .4 & .68 & .86 & & 4.80 \\
\hline & $i \equiv 8$ & $2959 !$ & .15 & 1479 & 259.5 &.$\$ 3$ & $.8 t$ & & 4.54 \\
\hline & 759 & ใหรโ1 & .3 & $15=0$ & 250.5 & .67 & .36 & & $4.6:$ \\
\hline & ies & 29791 & .35 & 1601 & $2 E 9.5$ & .45 & S & & 4.35 \\
\hline & 759 & $295 \div 1$ & O3. & $17 \mathrm{a}$ & 259.6 & .64 & . Ế & & 4.50 \\
\hline & 758 & ¿859! & .34 & libi & $59 . i$ & .55 & .85 & & $4.5 ?$ \\
\hline \multirow[t]{9}{*}{939} & 754 & $4 ! 485$ & 0.00 & !5e! & $25 \div .7$ & $. ?:$ & j.in? & $.70 t$ & 5.19 \\
\hline & 754 & $4148=$ & .84 & ¿Ẽ & $25=0$ & $.7 \mathrm{j}$ & . & & 5.: \\
\hline & 754 & $4 \mathrm{i} 485$ & .11 & $137 \dot{~}$ & โยล.. & .07 & . & & $\Xi . \vdots \vdots$ \\
\hline & 754 & $4 i 495$ & .17 & \$\$16 & $2 \hat{b i . j}$ & .67 & .80 & & $\equiv .12$ \\
\hline & 754 & $\$ 1465$ & .25 & 2029 & it2.E & 6 & .96 & & 5.!! \\
\hline & 754 & 41485 & .29 & 2190 & 262.9 & .65 & $.8 E$ & & 5.45 \\
\hline & $?: 4$ & $414 E^{2}$ & .35 & 2245 & $2 \leq \overline{3} .1$ & J. & .5 & & $5.0 \%$ \\
\hline & 754 & $4 ! 485$ & $.9 i$ & 2455 & $2 \leq 3.2$ & $.5 !$ & .85 & & 4. $: 9 g$ \\
\hline & 754 & 41495 & .47 & 2556 & 265.4 & .59 & .94 & & $\dot{A}_{\text {Q }}$ \\
\hline \multirow[t]{9}{*}{$2+2$} & 502 & 41\%3: & .01 & $1+30 j$ & 260.4 & . 洏 & .86 & .700 & $5.3 i$ \\
\hline & $5 \hat{x}$ ? & 4173? & . ij & 1797 & 2 & .09 & .85 & & $\Xi$ \\
\hline & $50 ?$ & 41732 & .19 & 1E:? & $26 \div .9$ & .67 & .36 & & $5 . .1$ \\
\hline & sng & $417=2$ & .23 & $192 \%$ & 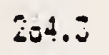 & .25 &.$\xi E$ & & $\Xi .29$ \\
\hline & 50. & 41732 & IT & 2110 & 264.3 & .82 & .85 & & 5.27 \\
\hline & 5 & $4173 \hat{~}$ & .46 & $2: 97$ & $2 \leq 5 . j$ & & .84 & & E.: \\
\hline & $50 ?$ & +1732 & . & 205 & 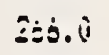 & .56 & .85 & & $\Xi .92$ \\
\hline & 502 & $417: 3$ & 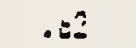 & 250 & aะs.8 & .52 & .22 & . & E.:1S \\
\hline & $50:$ & $417 \mathrm{O}$ & .70 & 2844 & $2.67 . i$ & .45 & $.3 \vdots$ & & $\Xi .: 3$ \\
\hline \multirow[t]{9}{*}{895} & $5 n !$ & 3608 & $.0 !$ & $1: 19$ & Cov. & . ij & .37 & o & 4.39 \\
\hline & Ej! & 30505 & $.3 E$ & i40s & 200.7 & $.6 ?$ & $.8 t$ & & 4.35 \\
\hline & $5: !$ & 90058 & .14 & :9ง̂์ & 250.7 & قُ & . 35 & & $\therefore$. \\
\hline & 501 & 30053 & $.2 !$ & 1927 & SGi.i & b́ & . 36 & & $\therefore .35$ \\
\hline & 501 & 30058 & .28 & $15: 2$ & 261.4 & .65 & .86 & & 4.24 \\
\hline & $50 !$ & ว0ิ05ล & .34 & $15=9$ & 261.6 & $.6 j$ & .35 & & $\dot{4} .8$ \\
\hline & 501 & 301458 & .41 & 169.2 & 262.0 & .01 & .35 & & 4.79 \\
\hline & 501 & BU5: & .47 & 17isi & $2 \leq 6.9$ & .58 & .84 & & $4 .: 7$ \\
\hline & 501 & Mótisâ & ذَّ .50 & $i \varepsilon_{0}:$ & 262.9 & $.50^{\circ}$ & .54 & & 4.95 \\
\hline \multirow[t]{9}{*}{394} & 297 & asios & .07 & $112 ?$ & 959.6 & . $8 ?$ & $.8 i$ & . 786 & 4.75 \\
\hline & 247 & $207 \div 6$ & رن & 1200 & $250 . !$ & .07 & .86 & & $4.7 !$ \\
\hline & 247 & ssiió & 3 & :!56 & 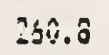 & .03 & . Eี & & $4.9 !$ \\
\hline & 297 & $207 \div 6$ & .45 & ¿395 & 261.7 & ד5 &.$\varepsilon$ & & $4.0 \%$ \\
\hline & $24 i$ & 23776 & .57 & $: 541$ & 262.9 & .54 & .33 & & 4.09 \\
\hline & 397 & $26 \bar{i} 76$ & .67 & 1673 & $2 \dot{E x}: 2$ & . & .32 & & $\therefore B_{i}$ \\
\hline & $i d i$ & 287 is & .77 & ¿Qิธด & 35. &.$i_{6}$ & . & & i.cs \\
\hline & 247 & 23776 & .39 & 9058 & 357.1 & .37 &.$\pi 7$ & & $\therefore .=:$ \\
\hline & 247 & 28770 & $.9 ?$ & 2547 & 369.0 & Jj. & .14 & & $4.8 j$ \\
\hline \multirow[t]{2}{*}{$\because \%$} & 779 & 29943 & 0.00 & 1049 & $\because 55.8$ & .75 & 1.60 & .750 & 0.75 \\
\hline & 779 & - 5943 & 0.00 & 1259 & 264.6 & .75 & 3.08 & & 0.75 \\
\hline
\end{tabular}




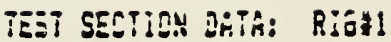

\begin{tabular}{|c|c|c|c|c|c|c|c|c|c|}
\hline hìn & $\begin{array}{l}\text { Minso Flijx } \\
\text { kgisasis }\end{array}$ & $\begin{array}{c}\text { HEAT FiUJX } \\
\text { Wis: }\end{array}$ & $\begin{array}{c}\text { MASS } \\
\text { QULLITY }\end{array}$ & $\begin{array}{l}\text { Hic } \\
\text { Wisçsik. }\end{array}$ & $\begin{array}{l}\text { Teqk } \\
\text { deghth }\end{array}$ & $\begin{array}{l}\text { Hiti:o } \\
\text { cone. }\end{array}$ & $\begin{array}{l}\text { Samf. } \\
\text { Safi }\end{array}$ & Conf. & $\begin{array}{c}\text { Fore } \\
\text { bars }\end{array}$ \\
\hline & 79 & 28943 & .05 & JEEj & कiv.4 & .74 & .97 & & 0.0 \\
\hline & $: 79$ & 28940 & .04 & .1570 & 290.3 & .35 & $.3 i$ & & 6.72 \\
\hline & 793 & $2374 j$ & .08 & 1648 & 230.7 & .74 & .97 & & 6.79 \\
\hline & 779 & 26945 & $.1 j$ & 1755 & 270.4 & .75 & .86 & & 9.7 \\
\hline & פידוד & $28 \div 43$ & .17 & 1794 & 270.5 & . & فَته. & & $0 . t 7$ \\
\hline & $7 ?$ & 25943 & $.2 ?$ & 1655 & 270.6 &.$i^{2}$ & . Is & & b. $\vdots 3$ \\
\hline & iis & 29943 & .20 & 2544 & 270.0 & .71 & ذ่ & & ง. $\vdots=$ \\
\hline 249 & $96 i$ & 41752 & 0.00 & $14: 5$ & $26: .0$ & .75 & 1.00 & .750 & o. o \\
\hline & $76 i$ & $41 ? 93$ & 0.00 & 15009 & 200.8 &.$E^{5}$ & .91 & & 6.45 \\
\hline & 757 & $4179 j$ & .07 & 2151 & Cto.s & .14 & .87 & & 0.14 \\
\hline & $7 \leq ?$ & 41793 & .15 & $219 j$ & วิะริ.ใ & .75 & .87 & & c. : : \\
\hline & $i b i$ & 41795 & .30 & 2185 & 669.1 & .32 & .86 & & $\therefore . \div 0$ \\
\hline & $73 i$ & 41793 & .26 & $22: 3$ & $2 \div 9.2$ & .7 & .86 & & S. \\
\hline & 767 & 41790 & .32 & 2372 & $20 \% .5$ & .0 & .36 & & j. \\
\hline & 967 & 417:3 & .39 & $3 \div 5$ & $26 \div .1$ & $.0 ?$ & .25 & & s.: \\
\hline & $76:$ & 11793 & .41 & $97 \div 9$ & :ร?. & .67 & .85 & & $9.2 ?$ \\
\hline 500 & 511 & 30125 & 0.00 & 1206 & 201.7 & .75 & 1.00 &.$; 50$ & E. ?: \\
\hline & 511 & 30125 & .04 & $15: 9$ & 265.3 & .74 & .97 & & 5. \\
\hline & E1! & सी:5 & .11 & 1 ISi & z $=5.5$ & .75 & .97 & & 5.9ij \\
\hline & $51 !$ & 30195 & .18 & נiنs & 265.6 & .12 & .57 & & $5 . i$ \\
\hline & 511 & $30 ! 25$ & .25 & 1570 & 265.0 & $.7 i$ & $\hat{B} \dot{C}$ & & $5.1 \vdots$ \\
\hline & 511 & 303125 & $.3 !$ & isji & 350.0 & .70 & .30 & & $5 . ?$ \\
\hline & 511 & 30125 & $\therefore 8$ & 泟! & 366.2 &.$t ?$ & E & & 5.75 \\
\hline & $5 ! 1$ & 20.25 & .44 & 180 & S50.5 & .55 & .85 & & $5 . i 3$ \\
\hline & 511 & 30125 & .51 & 197 & 200.9 & . &.$\subseteq 5$ & & E. $i i$ \\
\hline$=51$ & $5: 0$ & istsoo & 0.00 & 1630 & 265.2 & .75 & 1.00 & .75 & 6.40 \\
\hline & 5io & 4!tcob & .07 & 9094 & 268.0 & .it & $\bar{\varepsilon} \bar{i}$ & & ใ.ะ \\
\hline & 516 & $4: 500$ & .16 & 20097 & ZEE. & . & bु & & $\leq .: 3$ \\
\hline & 510 & $4 ! t=8$ & .26 & $\therefore 146$ & 269.1 & $.7 !$ & .36 & & $0 . \therefore=$ \\
\hline & 510 & tibbe & .35 & 2142 & 239.4 & .29 & os & & 0.85 \\
\hline & 510 & 4lises & .14 & 2281 & 269.3 & $.8 i$ & .05 & & $6 .:=$ \\
\hline & $5 ! n$ & qiób6 & J5. & $2 \$ 36$ & 230.5 & 55. & .84 & & $6 . j \hat{0}$ \\
\hline & 510 & 4joó & .51 & 2014 & 200.9 & $.6 \hat{~}$ & .09 & & $0.3 \hat{~}$ \\
\hline & $5 i t$ & $415 \leq 6$ & .69 & 2955 & 971.6 & .58 & I & & 6.35 \\
\hline 252 & 251 & 20202 & .05 & $1=8$ ? & $\therefore=4.6$ & .79 & .07 & . & E. . \\
\hline & $35 !$ & $9: 7: 22$ & .12 & 1100 & 265.0 & .72 & .97 & & इ.2i \\
\hline & 251 & 292272 & $.3 i$ & b.as & $: 55.5$ & .70 & .26 & & S.0i \\
\hline & 251 & $29:=2$ & .44 & $150 j$ & : $=5.1$ & $.0 i$ & .95 & & 5.04 \\
\hline & :5! & 909292 & .57 & $152 !$ & $367.1)$ & .05 & .84 & & 5.05 \\
\hline & 251 & 20292 & .63 & $: 575$ & :.52.! & .59 & .83 & & 8.64 \\
\hline & 251 & 25232 & .78 & $18: 4$ & 207.5 & .52 & $.8 i$ & & 5.05 \\
\hline & 51 & $\because 222$ & .87 & (2) 92 & 971.2 & . io & .70 & & E. 20 \\
\hline & 251 & 29929 & .95 & 2373 & $272 . ;$ & .40 & .77 & & 5.01 \\
\hline j. & 254 & 34447 & .195 & : 963 & 956.2 & .74 & $.8 ?$ & .750 & 5.95 \\
\hline & 954 & $3: 447$ & .21 & $1: 54$ & 256.6 & . ia & .86 & & E. $3 E$ \\
\hline & 354 & $3444 ?$ & t.je & !JE: & 20:.j & $.0 \%$ & .36 & & ع. \\
\hline
\end{tabular}




\begin{tabular}{|c|c|c|c|c|c|c|c|c|c|}
\hline 84 & $\begin{array}{l}\text { RHE: Filli } \\
\text { kgi Equ/s }\end{array}$ & 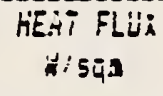 & $\begin{array}{c}\text { NASE } \\
\text { G.JALit }\end{array}$ & $\begin{array}{c}\text { HTE } \\
\text { Hisqa.K }\end{array}$ & $\begin{array}{l}\text { Te:p } \\
\text { dect. }\end{array}$ & $\begin{array}{l}\text { bisi:j } \\
\text { coms. }\end{array}$ & $\begin{array}{l}\text { IAFOE } \\
\text { COAKF. }\end{array}$ & 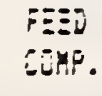 & $\begin{array}{c}\text { Fogajuine } \\
\text { bars }\end{array}$ \\
\hline & 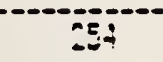 & $3+44 i$ & $.5 !$ & $155 i$ & 209.1 & jE & .85 & & $\equiv .94$ \\
\hline & 254 & 34447 & .64 & $\therefore 8 \leqslant 5$ & $2 \leq 53$ & ? & S & & 5.江 \\
\hline & 054 & 34447 & .77 & $2: 00$ & 270.7 & زَّ. & $.8 ?$ & & $5.5:$ \\
\hline & 254 & 39447 & .87 & $25 ! 3$ & 272.8 & .47 & .79 & & 5.91 \\
\hline & 25 & 34443 & .97 & 2959 & 274.8 & $.4 i$ & .76 & & $5.5 \%$ \\
\hline & 254 & 34447 & 1.00 & $3 \vdots 12$ & 375.9 & .24 & ב? & & E. \\
\hline \multirow{9}{*}{25} & 267 & $25: 15$ & .02 & $\$ 48 \div$ & $2 b i . \bar{i}$ & .85 &.$\overline{70}$ & .85 & e. $\equiv ;$ \\
\hline & $26 ?$ & 29215 & .15 & 1462 & 38.7 & .82 & .90 & & 0.55 \\
\hline & 269 & 249.15 & .28 & 1424 & 268.! & . El & .89 & & e. $5 ?$ \\
\hline & 369 & 29215 & .42 & 1355 & ¿QE. & .50 & .39 & & $\dot{0.5 j}$ \\
\hline & 269 & 29215 & .54 & 1415 & 268.7 & .78 & .88 & & 3.56 \\
\hline & 269 & ใร:15 & .56 & j68? & $26 \% .1$ & is & .87 & & 0.55 \\
\hline & 267 & 29215 &.$\pi$ & $181 i$ & $26 \div .0$ & .73 & St & & 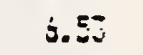 \\
\hline & $25 ?$ & 29215 & .99 & 2059 & $2: 0.5$ & .67 &.$E E$ & & ง. Eq \\
\hline & 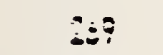 & $209 ! 5$ & פצ. & $85 ! ?$ & 371.8 & .65 & .34 & & A. $三 1$ \\
\hline \multirow[t]{9}{*}{ 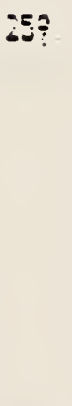 } & E:5 & 41410 & 0.00 & J:95 & 261.3 & J5. & 1.00 & & 7.26 \\
\hline & 825 & $+1+10$ & 0.60 & 2947 & 269.7 & J & 1.00 & & $\bar{i} .26$ \\
\hline & 925 & $4: 410$ & .05 & 2615 & 271.0 & 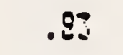 & .90 & & 7.95 \\
\hline & 275 & $41210^{\circ}$ &. .11 & 3450 & $91 . i$ & .85 & .90 & & i. \\
\hline & $E^{n} E$ & $414 i 0$ & .17 & 2400 & 271.1 & .82 & .39 & & $i .93$ \\
\hline & قี & $41+10$ & .24 & 2570 & $27 ! .1$ & .22 & 99. & & 7.20 \\
\hline & 9:E & $4+\$ 10$ & .29 & 2424 & 271.1 & .31 &.$\equiv$ ₹ & & $i .17$ \\
\hline & $8: 5$ & $41+10$ & jo & 2573 & 271.1 & .80 & $.8 ?$ & & 7.14 \\
\hline & 925 & $\$ 1110$ & .42 & 2763 & 27.00 & .80 & SE & & $\therefore . \therefore$ \\
\hline \multirow[t]{9}{*}{250} & $8:=$ & 28542 & 0.05 & 1147 & 2รฏ.ะ & . $\bar{E}$ & 1.00 & .335 & 3.51 \\
\hline & 326 & 28542 & 0.00 & $i 540$ & $2=2.4$ & .85 & 1.00 & & 0.01 \\
\hline & 896 & $235+2$ & .01 & $197 \%$ & 251.8 & $.8 \mathrm{~J}$ & .70 & & $\dot{3} .00$ \\
\hline & 325 & 25042 & .06 & 1527 & 367.5 & 35 & .90 & & 0.57 \\
\hline & S:S & 29642 & .10 & bis & íji.g & ذ3 & .90 & & $6.5:$ \\
\hline & 326 & 29542 & .14 & 1759 & 257.8 & .82 & .90 & & $\vdots . \Xi \Sigma$ \\
\hline & 926 & CEst2 & .15 & 1777 & $25 i . j$ & . E? & .39 & & 0.54 \\
\hline & 226 & 28542 & $.2 j$ & $: 816$ & 257.7 & .82 & . $5 ?$ & & 6.52 \\
\hline & 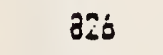 & 23642 & .27 & 1921 & $25 i .7$ & .81 & .59 & & s. $\Sigma_{0}$ \\
\hline \multirow[t]{9}{*}{262} & 528 & $22460^{\circ}$ & 0.00 & $!: 094$ & 260.7 & .93 & 1.00 & .835 & 7.29 \\
\hline & Eng & 29ษoj & 0.00 & $! 544$ & 357.9 & $.3 j$ & $\therefore .00$ & & $7.2 ?$ \\
\hline & E:E & 22460 & .02 & $2 a+1$ & $971 . !$ & 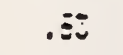 & .90 & & 7.8 \\
\hline & $5=3$ & $92 \leq 65$ & .00 & B:IE! & $\bar{a}: .2$ & $.3:$ & 界 & & $\bar{i} .3 \mathrm{~s}$ \\
\hline & S?9 & $2 a 46 j$ & .13 & 2075 & 271.2 & .32 & .89 & & 7.95 \\
\hline & 523 & $2940 j$ &. .8 & $195 !$ & 271.2 & . $2 ?$ & .89 & & 7.24 \\
\hline & $5: 8$ & 22400 & .24 & 1?:? & 271.2 & $.8 ?$ & .89 & & 7.32 \\
\hline & $5=9$ & $39+6 j$ & .20 & 2002 & 371.2 & $.8 !$ & . 59 & & $?: 20$ \\
\hline & 523 & $29 \$ 6 j$ & .34 & 3096 & 811.2 &.$\$ 1$ & .67 & & $\therefore$ is \\
\hline \multirow[t]{4}{*}{$3 k^{\circ}$} & 543 & j73ii & 0.00 & $140 ?$ & $3: 5.2$ & .35 & 1.00 & E5j & 3.25 \\
\hline & 543 & 37301 & 0.60 & 2237 & Miv.: & .97 & 89. & & 3..24 \\
\hline & 543 & 77301 & .09 & $287 j$ & 275.6 & .93 & .89 & & 9.33 \\
\hline & 543 & $5750 !$ & .19 & 3700 & 275.7 & $.8 ?$ & .89 & & 9.28 \\
\hline
\end{tabular}




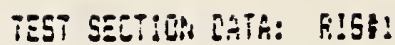

\begin{tabular}{|c|c|c|c|c|c|c|c|c|c|}
\hline SUN & 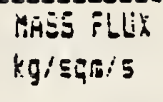 & $\begin{array}{c}\text { HEST } \\
\text { MíG }\end{array}$ & $\begin{array}{c}\text { BRSE } \\
\text { BLMLIII! }\end{array}$ & $\begin{array}{c}\text { HTC } \\
\text { W: } 342.6\end{array}$ & $\begin{array}{l}\text { Teat } \\
\text { OREL }\end{array}$ & $\begin{array}{l}\text { bials } \\
\text { cone. }\end{array}$ & 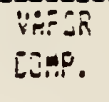 & $\begin{array}{l}\text { ESED. } \\
\text { COND. }\end{array}$ & $\begin{array}{c}\text { Gars } \\
\text { baje }\end{array}$ \\
\hline & $59 j$ & 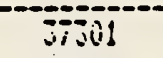 &. $\bar{s}$ & 年 & 行.9 &.$i_{i}$ & .59 & & 3.:-: \\
\hline & $5 ? 2$ & 37501 & .85 & .2424 & 275.9 & $. S:$ & .83 & & 2..ด \\
\hline & 545 & 实ill & .93 & asib & 276.0 & . So & .28 & & 8.:? \\
\hline & 595 & Sisit? & .52 & 2710 & 270.1 & .79 & .35 & & 3.15 \\
\hline & 5 & STOS & .57 & TYbo & 2it.: & $\therefore$ & .87 & & E.1J \\
\hline 267 & 590 & 235Eะ & 0.00 & $\dot{3 ! b}$ & 95:1.? & כ & 1.00 & בצבהם. & $i .5 ?$ \\
\hline & 546 & I85:E & 1). (10) & 1855 & 272.0 & . $\hat{E}$ & $1 . \cdots j$ & & i. \\
\hline & 540 & ก2:555 & .05 & $21 \leq 2$ & ZIấ. b́ & .83 & .96 & & i.5 \\
\hline & 546 & 2605 & .12 & Żoú & 272.0 & .92 & ? & & $: .57$ \\
\hline & 546 & $29=55$ & .19 & 20.45 & 272.7 & $.5 ?$ & .5 & & 7.5 \\
\hline & 5.96 & $365: 5$ & .25 & 1984 & 272.8 & $.8 !$ & . & & $7 . \equiv 4$ \\
\hline & 596 & $25 \in 55$ & .31 & 2005 & 272.8 & .81 & .39 & & ?. \\
\hline & 546 & 28655 & .38 & $2 \hat{959}$ & 272.8 & .80 & .83 & & $7 . . !$ \\
\hline & $\xi_{i j}$ & ใดิร5ร & .44 & 2992 & 272.9 & .77 & .95 & & 3.40 \\
\hline 265 & 236 & $185: 38$ &.$\hat{\imath} !$ & 1110 & 265.7 & .23 & .90 & .300 & s.av \\
\hline & 350 & 18599 & .09 & 1296 & วิธE. & .8 & .90 & & b. 09 \\
\hline & SEs & 13598 &. .8 & 1293 & ZLE.? & .92 & .90 & & $0 .: \div$ \\
\hline & 260 & 195:E & S & 1!:5 & 256.1 & .31 & .2? & & $6.17=$ \\
\hline & Ztbs & ¡3599 & .84 & 1067 & Sob.2 & .80 & .69 & & c. 19 \\
\hline & $20 \dot{C}$ & 19578 & .12 & i0ot & 200.4 & .79 & .59 & & 0.18 \\
\hline & 260 & iE5998 & 1. & 1130 & ás่ós & .78 &.$\varepsilon \hat{\varepsilon}$ & & $=.13$ \\
\hline & 206 & 19599 & .58 & iIJE & $=05.8$ & $.7 i$ & .83 & & $=.: 7$ \\
\hline & Se & 18578 & ó & 1350 & $2 \leq 7.1$ & .95 & $.3 ?$ & & b. $:=$ \\
\hline É & $5: 5$ & 41457 & 0.00 & $16=5$ & it: & تَ5. & 1.00 & I: & $\therefore 5=$ \\
\hline & 5ू5 & $4145 !$ & .05 & 2978 & $2=2.0$ & $.8 j$ & .60 & & $i . \equiv 9$ \\
\hline & 5.5 & $\{1957$ & .15 & $31: 5$ & $27 . .7$ & .30 & .39 & & 7.57 \\
\hline & 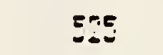 & 41457 & .25 & วЕอิ & aris.j & .81 &.$c^{a}$ & & -.55 \\
\hline & 525 & $4145 i$ & .35 & S5il & 272.5 & .30 & .39 & & i.5i \\
\hline & E.? & $4 i 457$ & .44 & 2050 & 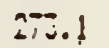 & .79 & .38 & & $7.5 ?$ \\
\hline & 5: & $4: 457$ & .53 & 2754 & 273.2 & .73 &.$\subseteq 5$ & & 7.3 \\
\hline & EnE & 41457 & .02 & $30 ! 4$ & $2 \pi .4$ &.$i$ & .27 & & $7.4 ?$ \\
\hline & 5.25 & 41457 & $.7 i$ & idui & 273.7 & .75 &.$\hat{\epsilon}$ & & $? .45$ \\
\hline 270 & 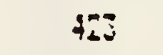 & 10555 & 0.00 & 745 & 250.4 & .45 & 1.00 & .454 & E. \\
\hline & 423 & 19557 & 0.00 & $9 \geq 9$ & 364.8 & .45 & 1.06 & & 5.7 \\
\hline & 425 & iSEZT & $.0 j$ & 1039 & 30.2 & .94 & . is & & 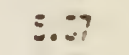 \\
\hline & $\because 9$ & ใด & .04 & $103 \pi$ & 270.2 & .44 & 99 & & 5.25 \\
\hline & 43 & $185 \div ?$ & .09 & $10 E 5$ & [7is.o & .45 & . & & E.:: \\
\hline & 423 & $18=39$ &.$i j$ & 1131 & 271.0 & .41 & .17 & & 5.:Z \\
\hline & 423 & 18539 & .17 & 11:4 & $=71.5$ & .89 & is & & 5.31 \\
\hline & 423 & $185 \mathrm{~S}^{7}$ & .2 & 1217 & $9 ? 1.9$ & ד & .78 & & 5.29 \\
\hline & 4 & 18537 & .25 & ling & $27 n_{0}: 2$ & . &.$i 5$ & & E. \\
\hline 372 & $: 28$ & 29:19: & 0.00 & $\because 29$ & SלU. E & .95 & 1.80 & $.45:$ & e.:A \\
\hline & 428 & ETig! & 0.00 & i¿ba & $=81.7$ & .45 & 1.00 & & 0.14 \\
\hline & 499 & ลดิ78! & .04 & $140 i$ & Siq.? & .44 & .78 & & 5.: \\
\hline & 425 & 28781 & .11 & 1543 & 275.7 & .12 & .77 & & 0.13 \\
\hline & 428 & 28781 & .17 & $16 ! 9$ & ??:อ.5 & .29 & .25 & & 0.12 \\
\hline
\end{tabular}




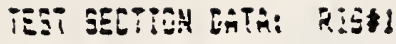

\begin{tabular}{|c|c|c|c|c|c|c|c|c|c|}
\hline rin: & 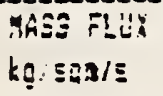 & $\begin{array}{c}\text { HEAT F:UI? } \\
\text { Hisq? }\end{array}$ & $\begin{array}{c}\text { MHES } \\
\text { QU:?:TH }\end{array}$ & $\begin{array}{c}\text { 4TL } \\
\| i s q 2 / R\end{array}$ & $\begin{array}{l}\text { Teqb } \\
\text { cegl" }\end{array}$ & $\begin{array}{l}\text { LIfUIS } \\
\text { COME. }\end{array}$ & $\begin{array}{l}\text { MAES } \\
\text { CAME. }\end{array}$ & $\begin{array}{l}\text { FEE } \\
\text { BUAPP. }\end{array}$ & $\begin{array}{c}\text { PSEEE:SE } \\
\text { UA: }\end{array}$ \\
\hline & 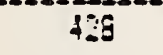 & 28731 & .35 & 1949 & $277 . j$ & $.3 i$ & .94 & & $6.1=$ \\
\hline & $4: 5$ & $2879 !$ & .25 & 1849 & 273. j & .35 & .72 & & e.22 \\
\hline & $4=8$ & $2878 \mathrm{i}$ & .34 & 2609 & 279.6 & .82 & .71 & & $6 .: 2$ \\
\hline & 420 & 28781 & .28 & 2905 & 279.3 & .30 & $.5^{2}$ & & s. $: 0$ \\
\hline-3 & 97 & 41167 & 0.00 & iะ5e & $2 \leqslant 2 . i$ & .45 & 1.00 & .954 & 0.31 \\
\hline & 450 & $4 ! 1 \leq ?$ & 0.00 & 1662 & 270.7 & 5j. & .82 & & . \\
\hline & 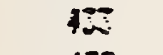 & $4: 30 \bar{i}$ & . & 2097 & 274.7 & .7 & $.7 \bar{i}$ & & 0.00 \\
\hline & 49 & 41167 & .18 & $21 \mathrm{E}$ & 275.3 & .37 & .75 & & 5.99 \\
\hline & $4 j 3$ & 41167 & .27 & 2345 & 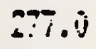 & .5 & .73 & & 5.72 \\
\hline & 45 & $411 \mathbf{s}^{\circ}$ & .34 & 2560 & 978.2 & .52 & $.7 !$ & & 5.97 \\
\hline & 430 & 41167 & $.4 !$ & 2795 & 299.4 & .29 & .68 & & 5.95 \\
\hline & 433 & $4 ! 1: ?$ & . & 512: & 290.4 & .27 & .05 & & $5.9 ;$ \\
\hline & 45 & $4: 137$ & .54 & jEg? & 281.4 & $\therefore 4$ &.$\varepsilon j$ & & 5.93 \\
\hline 974 & ilj & 40459 & 0.00 & !oij & 271.2 & .45 & 1.10 & $.45 \%$ & $0.2 \%$ \\
\hline & :3? & 40459 & .17 & :945 & 280.7 & $.4 \pi$ & .74 & & D.EE \\
\hline & $2 ! 3$ & 44459 & כני. & $21: 2$ & 295.0 & دََّْ. & .70 & & ง. ถิ \\
\hline & $2 ! 3$ & $4: 4959$ & .47 & 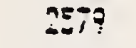 & AEE. 4 & .22 & .65 & & ¿. 38 \\
\hline & 213 & 40457 & .59 & 3:?: & 297.4 & .21 & .00 & & $6.8 \%$ \\
\hline & 213 & 40459 & .70 & 375 & 289.0 & . $2:$ & .5s & & 6. So \\
\hline & 263 & $4455 ?$ & .22 & :208 & $290 . \div$ & .15 & 53 & & ذ. \\
\hline & $21 j$ & 20459 & .72 & 5023 & 971.6 & .16 & $.4 \hat{z}$ & & $=.85$ \\
\hline & $3: j$ & +11459 & 1.00 & bE5: & 293.7 & . 19 &.$\div$ & & 6.09 \\
\hline 275 & 215 & 23745 & 4.90 & $: 015$ & 299.1 & .45 & $\therefore 00$ & $.45:$ & 5 \\
\hline & $1: 5$ & 29745 & .11 & 1215 & :3!.Z & .41 & .78 & & 5.5 \\
\hline & $2: 5$ & 29745 & .24 & :55 & 27.9 & 65 & $.7 E$ & & 5.28 \\
\hline & 215 & $28: 45$ & . & $147 !$ & 274.6 & $\therefore$ &. $\bar{i}$ & & 5 \\
\hline & 215 & $28: 45$ & .44 & 1751 & 276.4 & .23 & .69 & & 5.87 \\
\hline & 215 & 25745 & $.5 \bar{Z}$ & 2045 & $27.0 \hat{0}$ & .24 & .64 & & 5.3 \\
\hline & 215 & 28745 & . & 2980 & 299.5 & .22 & .61 & & 5.3 \\
\hline & 285 & 28745 & .59 & 2197 & 230.' & . & $.5 i$ & & E.jo \\
\hline & $2: 5$ & 23745 & .76 & 2707 & 291.6 & .17 & .54 & & 5. \\
\hline
\end{tabular}


PREHETT SECIION EATH: RJEL RISTO

\begin{tabular}{|c|c|c|c|c|c|}
\hline $\operatorname{sen}$ & $\begin{array}{l}\text { MASE FLUY } \\
\text { KG: } 52015\end{array}$ & 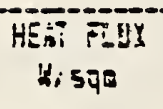 & QUALLIT & $\begin{array}{c}\text { HTC } \\
\text { Wistaik }\end{array}$ & $\begin{array}{l}\text { Tsat } \\
\text { deglit }\end{array}$ \\
\hline \multirow[t]{3}{*}{ 涪 } & EE & 10025 & .66 & 治E: & 290.0 \\
\hline & $! \vdots 3$ & (1)0is & .09 & $: 317$ & 290.6 \\
\hline & $: 5 E$ & 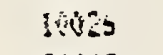 & .12 & jall & 2Sís.d \\
\hline \multirow[t]{3}{*}{283} & 157 & 20102 & .14 & $356 !$ & 290.4 \\
\hline & 157 & 20002 & $.2 !$ & SOET & $: 90 . ;$ \\
\hline & 157 & 200012 & .23 & 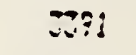 & 290.2 \\
\hline \multirow[t]{3}{*}{397} & IE: & $2=\mathrm{Vu}_{29}$ & .19 & ט & $2 ? 0.0$ \\
\hline & 154 & 25024 & 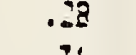 & 3819 & 2900.0 \\
\hline & 354 & $25 \hat{V}^{2} 24$ & .35 & 3997 & 230.0 \\
\hline \multirow[t]{2}{*}{ ja } & $\begin{array}{l}157 \\
157\end{array}$ & 3059? & .24 & 3249 & $\begin{array}{l}290.1 \\
200.1\end{array}$ \\
\hline & 157 & $30 \leq 97$ & .45 & 4004 & 290.3 \\
\hline \multirow[t]{3}{*}{ ES } & 104 & Hinis & .30 & 4675 & 290.4 \\
\hline & 164 & $3507 ?$ & .38 & 4338 & 290.5 \\
\hline & 167 & $3507 \%$ & .49 & $52 ! 9$ & 290.3 \\
\hline \multirow[t]{3}{*}{234} & dE? & 50996 & .32 & 5209 & 290.2 \\
\hline & $15 \%$ & 39995 & .45 & 5279 & ${ }_{2}^{2} \hat{v}_{0} 1$. \\
\hline & 159 & 39996 & .59 & 5884 & 290.1 \\
\hline \multirow[t]{3}{*}{235} & 159 & 45020 & $.3 ?$ & $5: 09$ & $290 . j$ \\
\hline & 158 & 45020 & .52 & 5707 & 290.3 \\
\hline & 158 & 450120 & .67 & 59:: & $29 \hat{9} .2$ \\
\hline \multirow[t]{3}{*}{320} & $2: 2$ & 200050 & .08 & 3076 & 290.5 \\
\hline & בצ? & $200 \div 6$ & .12 & Titis & 250.6 \\
\hline & 232 & 30035 &. .17 & 3:3: & 290.5 \\
\hline \multirow[t]{3}{*}{295} & ME & 30340 & jJ & 455j & :ริे.'0 \\
\hline & $2 \mathrm{JE}$ & $30024 i$ & .20 & itis & $290, .5$ \\
\hline & ใิธี & $302+00$ & .26 & $47 j 4$ & 290.5 \\
\hline \multirow[t]{3}{*}{259} & $\because 3$ & 40142 & .19 & $E=: 9$ & 270.4 \\
\hline & 198 & 40442 & .29 & $5: 56$ & 20.0 .3 \\
\hline & 293 & 60442 & .37 & 575! & 200.2 \\
\hline \multirow[t]{3}{*}{305} & $24 !$ & $\div 9890$ & .24 & 639! & 290.3 \\
\hline & $2 \div 0$ & 49390 & .35 & $625 !$ & 290.2 \\
\hline & 240 & $\$ 5390$ & .46 & Dinj & 290.1 \\
\hline \multirow[t]{3}{*}{3044} & 246 & 59991 & .29 & 7596 & 290.9 \\
\hline & 246 & $5999 !$ & .82 & in49 & 290.9 \\
\hline & 245 & 5997! & .54 & 7670 & 290.3 \\
\hline \multirow[t]{3}{*}{510} & Z75 & b泬电 & .35 & E::1: & $\because 90 \hat{v} 3$ \\
\hline & 246 & 59999 & .50 & $301 j$ & 350.7 \\
\hline & 246 & 67999 & .65 & $86 j 2$ & 390.0 \\
\hline \multirow[t]{3}{*}{322} & 282 & 20000 & .05 & jositi & 290.2 \\
\hline & 29? & agors & .09 & 5745 & 290.2 \\
\hline & 352 & gnód & .18 & 3990 & ISC.1 \\
\hline \multirow[t]{3}{*}{204} & 285 & DO 250 & .10 & 4015 & 290.0 \\
\hline & ละร & 30250 & .15 & $: 51 j$ & $25 \% .5$ \\
\hline & 295 & 3̂े250 & $.2 !$ & $4 ?: 6$ & 290.5 \\
\hline
\end{tabular}


PPEHEAT SECTIAN BRTA: RIGIS RISL

\begin{tabular}{|c|c|c|c|c|c|}
\hline ติบ & $\begin{array}{l}\text { MAEE FIJW } \\
\mathrm{kg} / 5 q R / \mathrm{s}\end{array}$ & $\begin{array}{c}\text { HEFi FLUX } \\
\text { W/squ }\end{array}$ & GLAL:TY & $\begin{array}{c}H T \\
N / 5 q 4\end{array}$ & $\begin{array}{l}\text { Tsat } \\
\text { deng:t }\end{array}$ \\
\hline \multirow[t]{3}{*}{809} & 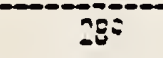 & 40094 & .14 & 5Eig & 290.6 \\
\hline & 287 & 201074 & .29 & $5 \$ 7 \hat{9}$ & 290.5 \\
\hline & 299 & $400 \div 4$ & .29 & 5771. & 290.4 \\
\hline \multirow[t]{3}{*}{$29 ! !$} & ลิ3 & 49985 & .19 & 0534 & 291.0 \\
\hline & 285 & 49990 & .29 & jE!? & 299.9 \\
\hline & J & 21558 & .33 & 6954 & 290.3 \\
\hline \multirow[t]{3}{*}{291} & 293 & $57999 ?$ & .24 & $75=5$ & 290.7 \\
\hline & 355 & 59992 & .35 & $74: 3$ & 290.5 \\
\hline & 295 & $5999 ?$ & . ft & 79014 & $290 \hat{. Z}$ \\
\hline \multirow[t]{3}{*}{292} & 277 & $7000 \mathrm{~J}$ & .30 & 8310 & 290.7 \\
\hline & $27 i$ & 70ิง & .25 & $6: 45$ & 290.6 \\
\hline & $\pi i$ & 700003 & .87 & Sód\& & 290.4 \\
\hline \multirow[t]{3}{*}{295} & 291 & $749 \div ?$ & .32 & 6782 & 290.9 \\
\hline & $28 !$ & 74967 & . 45 & Bteg & 290.3 \\
\hline & 281 & $7490^{-}$ & .60 & 8922 & 290.5 \\
\hline \multirow[t]{3}{*}{ jof } & $25 i$ & $80: 18$ &.$j 3$ & $\{9 a 1\}$ & $29: .1$ \\
\hline & 297 & 90118 & .47 & 9751 & 270.9 \\
\hline & $39 ?$ & 80119 &.$j \hat{z}$ & 9195 & 290.7 \\
\hline \multirow[t]{3}{*}{$3 \hat{~ 3: ~}$} & $i+i$ & 26005 & .04 & 365 & 390.6 \\
\hline & 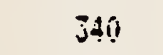 & 20039 & .07 & P7:5 & 290.5 \\
\hline & 240 & 20050 & $.1 \hat{v}$ & $4 \hat{n} 7 \dot{4}$ & 290.5 \\
\hline \multirow[t]{3}{*}{$2 \%$} & j54 & 3029:5 & .05 & $97 !:$ & 296.6 \\
\hline & $35:$ & 302ne & $.1 !$ & 405 & 250.6 \\
\hline & 94 & 30225 & .15 & $=0$ & 250.5 \\
\hline \multirow[t]{3}{*}{ ac: } & ت5 & $20 \div 47$ & .10 & Esaz & 290.5 \\
\hline & 65 & 40447 & .15 & 5417 & 290.4 \\
\hline & ز5ב & 4647 & .2 & 5715 & 290.4 \\
\hline \multirow[t]{3}{*}{ in! } & ?:5? & 99330 & .15 & 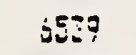 & 290.9 \\
\hline & is? & $\$ 5930$ & .21 & J'S & 290.3 \\
\hline & $35 ?$ & $495=0$ & .25 & 8697 & $2 \div 0.8$ \\
\hline \multirow[t]{3}{*}{$30 j$} & 356 & ร399: & .17 & 7398 & 250.5 \\
\hline & $25 j$ & 59981 & $.2 \dot{0}$ & $71: 0$ & 290.4 \\
\hline & 350 & 5:991 & E & 7792 & 200.2 \\
\hline \multirow[t]{3}{*}{ จิง } & 367 & 69795 & .20 & g:ant & 290.7 \\
\hline & $j \leqslant i$ & ל999? & .30 & Eûse & 390.5 \\
\hline & 357 & 69993 & .40 & $8=3$ & $\because \operatorname{siv.4}_{4}$ \\
\hline \multirow[t]{3}{*}{ j11 } & job & 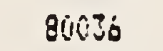 & .23 & $9 !: \bar{~}$ & โ90.? \\
\hline & Jab́ & 30050 & 55. & $88: 3$ & 290.7 \\
\hline & 356 & 80036 & .47 & 9070 & 290.5 \\
\hline \multirow[t]{3}{*}{$31 t$} & $36 j$ & 89967 & .23 & $100 \leqslant 3$ & 290.7 \\
\hline & ند & 89907 & .41 & ?:18 & 230.7 \\
\hline & $35{ }^{\circ}$ & 8990) & .54 & $971 ?$ & 290.4 \\
\hline \multirow[t]{3}{*}{323} & 419 & 2000 & .02 & BEST & 290.6 \\
\hline & 419 & 20020 & .04 & 3791 & 290.7 \\
\hline & $\$ 19$ & $200 \therefore 0$ & .07 & $4: 43$ & 670.7 \\
\hline
\end{tabular}


PREHEAT SELIION GATh: RiEł? RiSid

\begin{tabular}{|c|c|c|c|c|c|}
\hline Sü. & 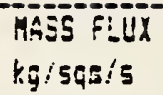 & $\begin{array}{c}\text { MEAT FLUI } \\
\text { W SGA }\end{array}$ & QUALTiY & $\begin{array}{c}\text { HIC } \\
n \leq 5=i k\end{array}$ & $\begin{array}{l}\text { Isat } \\
\text { deģi: }\end{array}$ \\
\hline \multirow[t]{3}{*}{3} & 418 & 30342 & .05 & $49 !$ & 990.7 \\
\hline & 418 & 3ivit2 & .09 & 4795 & 290.6 \\
\hline & 419 & 30942 & .13 & 4817 & 290.5 \\
\hline \multirow[t]{3}{*}{299} & 425 & 40416 & .07 & 5221 & 290.9 \\
\hline & 495 & 40110 & .82 & $57 ?$ & 209.18 \\
\hline & $4^{n z}$ & $404: 5$ & .17 & 5530 & 290.7 \\
\hline \multirow[t]{3}{*}{300} & $4:$ & 49945 & .10 & 6594 & $2=0.9$ \\
\hline & 427 & 49945 & .16 & $8: 99$ & 290.8 \\
\hline & 427 & 45045 & .2 & 6059 & 290.7 \\
\hline \multirow[t]{3}{*}{305} & 450 & 50975 & בZ: & T74 & $29 ! .3$ \\
\hline & 930 & bigis & .20 & 法9 & 251.1 \\
\hline & 430 & 60275 & .23 & $740 j$ & 290.7 \\
\hline \multirow[t]{3}{*}{308} & 450 & T001! & .16 & 2369 & 990.9 \\
\hline & 430 & $7001 !$ & .24 & 8127 & $290 . i$ \\
\hline & 40 & $7001 !$ & نבני. & $8: 42$ & .90 .5 \\
\hline \multirow[t]{3}{*}{312} & $42 ?$ & $30110 !$ & $.8 ?$ & 0959 & 290.9 \\
\hline & 420 & 80101 & .29 & 9995 & 290.3 \\
\hline & $42 ?$ & $8010 !$ & . 58 & 9275 & 290.6 \\
\hline \multirow[t]{3}{*}{ iE! } & $14 \hat{E}$ & 1005s & .19 & $89: 7$ & 350.5 \\
\hline & $: 98$ & 10096 & .12 & 3015 & 290.3 \\
\hline & 148 & 19086 & .16 & $300 ?$ & 290.5 \\
\hline \multirow[t]{3}{*}{$i d j$} & :5j & $2045 !$ & $.2 \hat{v}$ & E211 & 279.4 \\
\hline & is: & $2042 i$ & .27 & 3431 & 290.4 \\
\hline & $15:$ & 39421 & .34 & 3558 & 290.9 \\
\hline \multirow[t]{3}{*}{ :E: } & 145 & 25340 & .29 & $349 ?$ & 290.2 \\
\hline & 249 & 25346 & $.2 !$ & รEs9 & 999.1 \\
\hline & 149 & 25546 & .40 & 4180 & $290 \hat{.1}$ \\
\hline \multirow[t]{3}{*}{$j \Xi$} & 150 & 30731 & .30 & 4390 & 250.4 \\
\hline & 150 & 30731 & .41 & $i \in 1 i$ & 290.4 \\
\hline & 150 & 30731 & .52 & $993 \%$ & 290.3 \\
\hline \multirow[t]{3}{*}{ j\$? } & $: 50$ & 35105 & .36 & 4992 & 299.2 \\
\hline & 150 & 35105 & .49 & 5028 & 290.1 \\
\hline & 150 & 35105 & .61 & 5224 & 290.1 \\
\hline \multirow[t]{3}{*}{$522^{\circ}$} & 150 & 35937 & $.3 \hat{6}$ & $5: 02$ & 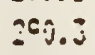 \\
\hline & 156 & 29957 & .46 & דִ & J. \\
\hline & $! 5_{i}$ & 39937 & 250 & 5555 & 230..2 \\
\hline \multirow[t]{3}{*}{339} & 280 & 20494 & .05 & jei: & 399.4 \\
\hline & EO & 20484 & .09 & 3712 & 290.4 \\
\hline & 250 & 204044 & .12 & $3 i a j$ & 3 \\
\hline \multirow[t]{3}{*}{ בד: } & 233 & jú700 & .09 & $\{a b ;$ & 290.8 \\
\hline & 288 & 30700 & .15 & $\$ 937$ & 290.8 \\
\hline & 288 & 30700 & . & 515i & 250.7 \\
\hline \multirow[t]{3}{*}{$3 j$} & 296 & ¿9891 & .14 & 5697 & $=90.2$ \\
\hline & 90 & $2: 891$ & .21 & 5559 & 290.1 \\
\hline & 296 & 39391 & .27 & $56-i 2$ & 290.0 \\
\hline
\end{tabular}


PREHEAT GECTION JATÄ: RIGHZ RISĔ

\begin{tabular}{|c|c|c|c|c|c|c|}
\hline RIN & $\begin{array}{l}\text { HASS FLLX } \\
\mathrm{kg} / \mathrm{sqa} / \mathrm{s}\end{array}$ & $\begin{array}{c}\text { HEAT FLUX } \\
\text { WisรZE }\end{array}$ & Guinlity & $\begin{array}{c}H T C \\
Y: 5 q=: K\end{array}$ & $\begin{array}{l}\text { Tsat } \\
\text { deghi }\end{array}$ & \\
\hline \multirow[t]{3}{*}{534} & 235 & 50014 & .19 & 6717 & 290.7 & \\
\hline & $\because \vdots 5$ & Siviga & $\therefore 3$ & 0046 & 250.6 & \\
\hline & 285 & Sision & .87 & .5716 & 290.5 & \\
\hline \multirow[t]{3}{*}{ iniz } & 287 & 60008 & .26 & $i t e$ & 250.9 & \\
\hline & 297 & bijovis & .37 & 7736 & 390.8 & \\
\hline & 237 & 50008 & .48 & 9927 & 296.7 & \\
\hline \multirow[t]{3}{*}{344} & 225 & 70701 & $.3 n$ & 汸乼 & $29: .9$ & \\
\hline & $2 \pm 5$ & Toiśl & .45 & $35: 2$ & 290.5 & \\
\hline & 295 & 70761 & .58 & $881 ?$ & 290.7 & \\
\hline \multirow[t]{3}{*}{546} & 295 & 80452 & . DE & 97 & 25.9 .9 & \\
\hline & 295 & 804:3: & .50 & $\$ 773$ & 290.7 & \\
\hline & \$ & $8045 ?$ & .65 & 19135 & 290.4 & \\
\hline \multirow[t]{3}{*}{$=\dot{0}$} & $\{93$ & 30684 & .02 & $505 ?$ & $290 \hat{.8}$ & \\
\hline & 423 & 30684 & .00 & 5050 & 290.8 & \\
\hline & 425 & 30634 & .10 & $5=97$ & 290.7 & \\
\hline \multirow[t]{3}{*}{ } & 4.5 & 39857 & .07 & 5893 & 290.2 & \\
\hline & 425 & 39997 & .12 & $5 \dot{00}$ & 290.2 & \\
\hline & $1: 9$ & j9389 & .19 & 5737 & 230.1 & \\
\hline \multirow[t]{3}{*}{ כנכני } & $42 ?$ & 50004 & .09 & 69.55 & $29 ! . !$ & \\
\hline & 429 & 500014 & .16 & Qizi & 291.0 & \\
\hline & 429 & 50004 & .92 & 6628 & 290.9 & \\
\hline \multirow[t]{3}{*}{$? 4 !$} & 926 & 59965 & .13 & $95 ! 9$ & 20.909 & \\
\hline & 426 & 53956 & .20 & $7 i$ is & 250.9 & \\
\hline & 225 & $5=560$ & .29 & 995 & :ริิ.i & . \\
\hline \multirow[t]{3}{*}{$\vdots: Z$} & 59 & 70981 & .10 & $3: 99$ & $99: .4$ & \\
\hline & 430 & $7 \hat{7}: 5\}$ & .85 & bovit & $29 \mathrm{i} .3$ & \\
\hline & 0 & 你海: &.$j q$ & 8795 & 291.6 & \\
\hline \multirow[t]{3}{*}{$\therefore 45$} & $4 i 4$ & 79979 & .20 & 5500 & 291.1 & \\
\hline & 424 & T9TYด & .30 & 9217 & 290.9 & \\
\hline & $4: 9$ & 79792 & .40 & $91: 3$ & 290.6 & \\
\hline \multirow[t]{3}{*}{$34 i$} & 924 & $897 ! 5$ & .35 & 10315 & $39 ! .4$ & \\
\hline & 224 & 89915 & .35 & livigt & 291.2 & \\
\hline & 424 & 39915 & .46 & 0994 & 290.9 & \\
\hline
\end{tabular}


PREHEÄT SECT:QK DATA: RIG:2 RIJEL

\begin{tabular}{|c|c|c|c|c|c|}
\hline 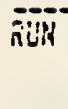 & $\begin{array}{l}\text { has } F L U X \\
\text { hg/इais }\end{array}$ & $\begin{array}{c}\text { HEAT F!UA } \\
\text { WISATA }\end{array}$ & GLALITY & 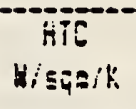 & $\begin{array}{l}\text { Tsat } \\
\text { degk }\end{array}$ \\
\hline \multirow[t]{3}{*}{ Sive } & 35 & 10169 & .10 & 289 & .54 .4 \\
\hline & 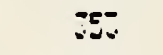 & 16099 & .14 & $29 \div 6$ & isti.4 \\
\hline & JE & 10039 & .18 & 1755 & $25 i .4$ \\
\hline \multirow[t]{3}{*}{5 iñ } & jóvi & 15178 & .10 & $299 \bar{J}$ & $25 \div .4$ \\
\hline & 360 & $151 ; 8$ & .22 & 2925 & $254 . j$ \\
\hline & 350 & $15: 78$ & .29 & 2231 & 254.3 \\
\hline \multirow[t]{3}{*}{505} & 357 & 19555 & .21 & 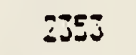 & $254 . j$ \\
\hline & 359 & 19955 & .29 & 2745 & 254.3 \\
\hline & 359 & 19955 & .37 & $318 ?$ & 254.2 \\
\hline \multirow[t]{3}{*}{504} & 357 & $25: 5$ & .97 & 25.05 & 255.0 \\
\hline & ? & 25015 & $.3 i$ & 3551 & 255.0 \\
\hline & 357 & $250 ! 5$ & .17 & 4174 & $2=4.9$ \\
\hline \multirow[t]{3}{*}{505} & 355 & 30765 & .34 & 3201 & 254.7 \\
\hline & 756 & 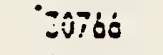 & .46 & 4519 & 254.6 \\
\hline & 350 & DOJiso & .57 & $47 \equiv 0$ & 954.5 \\
\hline \multirow[t]{3}{*}{491} & $4 \overline{J j}$ & 10006 & .08 & 2601 & 257.3 \\
\hline & 403 & 160016 & $.1 !$ & 3541 & $254 . j$ \\
\hline & $4=3$ & 10006 & .14 & $241 j$ & 254.2 \\
\hline \multirow[t]{3}{*}{ 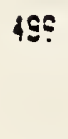 } & 434 & !5026 & .12 & 2955 & 754.2 \\
\hline & ivit & $150\{\vdots$ & .17 & .795 & 25ร. 2 \\
\hline & 454 & 15025 & .22 & 2700 & 254.1 \\
\hline \multirow[t]{3}{*}{490} & $44 i$ & 19973 & .17 & 2750 & 254.5 \\
\hline & $\{41$ & 19975 & .13 & :95: & 354.5 \\
\hline & $i+1$ & $: 9973$ & .30 & j:Ti & -54.4 \\
\hline \multirow[t]{3}{*}{$i E i$} & 4E! & 2529: &.$\therefore !$ & ilss & $\because E 4.2$ \\
\hline & $4 E i$ & פ525 & .29 & $339 !$ & $25 \div .8$ \\
\hline & 451 & 25ijio & .57 & $596 ?$ & 254.7 \\
\hline \multirow[t]{3}{*}{ PQj } & $4: ?$ & 30790 & .20 & 4270 & - 55.1 \\
\hline & $4: 2$ & 39786 & .37 & $\{710$ & $=55.0$ \\
\hline & 432 & 20735 & .50 & it!5 & 251.9 \\
\hline \multirow[t]{3}{*}{489} & 445 & 35215 & II & $45: 3$ & 255.2 \\
\hline & 445 & 35215 & .42 & siaj & 254.9 \\
\hline & 445 & 35215 & .54 & 5149 & .54 .5 \\
\hline \multirow[t]{3}{*}{975} & 451 & 41013 & .34 & 4705 & 254.9 \\
\hline & $45 !$ & 41015 & .47 & 557 & 252.9 \\
\hline & $45:$ & $4: 013$ & . 30 & 5::! & 254.7 \\
\hline \multirow[t]{3}{*}{492} & 557 & if747 & .08 & $31=2$ & :ET.8 \\
\hline & 55: & 19947 & .12 & Zlizi & 354.5 \\
\hline & 559 & $14 \div 4 i$ & .16 & $3 ! 97$ & $25 i .5$ \\
\hline \multirow[t]{3}{*}{480} & 555 & 29278 & Jj & jô:T & 54.5 \\
\hline & 558 & 20279 & .19 & $\Sigma \Sigma 30$ & 254.5 \\
\hline & 59 & 20278 & .34 & $3 \$ 5 j$ & $\therefore 54.4$ \\
\hline \multirow[t]{3}{*}{493} & 5 s.2 & $24 \div 49$ & .15 & 2245 & :54.8 \\
\hline & $5 \leqslant 4$ & $2 \div 949$ & .21 & $\$ 150$ & $2 E 4.9$ \\
\hline & 5 & 27849 & .28 & $+29 j$ & 254.7 \\
\hline
\end{tabular}




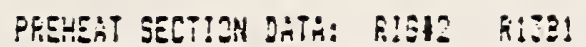

\begin{tabular}{|c|c|c|c|c|c|}
\hline Bi: & 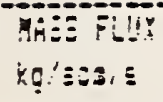 & 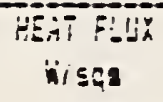 & Q:IS:TYY & $\begin{array}{c}\text { ATL } \\
W i \leq 92 ; K\end{array}$ & degt: \\
\hline \multirow{3}{*}{452} & 55 & 9615 & .19 & 4596 & CEE. \\
\hline & $5: 5$ & .9615 & .29 & PIÉ & 254.9 \\
\hline & 554 & jósis & .25 & 4645 & 254.2 \\
\hline \multirow[t]{3}{*}{$: 94$} & 58 & $\$ 4900$ & .2 & 5153 & EE. \\
\hline & $50:$ & $3: 700$ & $.3 !$ & इ⿻上丨 & 209.7 \\
\hline & $5: 2$ & 34910 & .40 & 5003 & $\therefore=5.9$ \\
\hline \multirow[t]{3}{*}{$4 i z$} & 500 & 40206 & ذَد.. & $45: 4$ & 254.9 \\
\hline & 550 & 40006 & jo & Etái & 251.3 \\
\hline & 550 & 40305 & .97 & 5105 & $5 E 4.6$ \\
\hline \multirow[t]{3}{*}{995} & 555 & 49960 & .30 & $5 \leq 6.9$ & $2 E \leq .4$ \\
\hline & $5:=$ & 44768 &.$+i$ & GIEt & $: 55.2$ \\
\hline & 585 & 44968 & .55 & $62 ! !$ & 255.1 \\
\hline \multirow[t]{3}{*}{$: 7$} & Est & 51255 & .j & 3091 & 255.3 \\
\hline & 5 & 51.993 & .44 & $530 !$ & $-5 E .1$ \\
\hline & 5 & $5: 295$ & .57 & 5081 & $25 \div .9$ \\
\hline
\end{tabular}


PREHEAT EETILN DATA: RIEA? .E0 nt R1:E!

\begin{tabular}{|c|c|c|c|c|c|c|c|}
\hline Ritis & 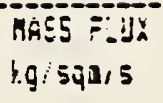 & 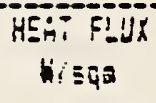 & $\begin{array}{l}\text { Mise } \\
\text { Gunlity }\end{array}$ & Wiscask & Jeģ: & $\begin{array}{l}\text { liau:o } \\
\text { Conp. }\end{array}$ & $\begin{array}{l}\text { CONE } \\
\text { COAF? }\end{array}$ \\
\hline \multirow[t]{3}{*}{ 晹 } & $\therefore 3$ & 16019 & .10 & 1159 & 25.0 & .79 & .95 \\
\hline & 25 & 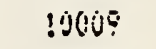 & .15 & $.1 ! 54$ & 259.1 & .79 & . $\$ ?$ \\
\hline & ¿ذ\$ & 10009 & .19 & $144 ?$ & 255.2 & .98 & . हिप \\
\hline \multirow[t]{3}{*}{535} & $2 t 9$ & 15020 & .17 & 1429 & 259.3 & .75 & .89 \\
\hline & 258 & 15020 & .24 & 1520 & 258.4 & .78 & .97 \\
\hline & 268 & 15020 & .31 & ל'? & 258. & .77 & .29 \\
\hline \multirow[t]{3}{*}{$5: 7$} & לb & 20041 & .24 & $16 i^{\circ}$ & ISE. & .? & F \\
\hline & 205 & $2(1941$ & .54 & $15 \pm 1$ & 259. & is & .38 \\
\hline & 250 & $2044 !$ &.$+j$ & 2: :I! & $259 . \varepsilon$ & .95 & .93 \\
\hline \multirow[t]{3}{*}{$5:$} & $2 \leqslant 8$ & 25001E & . ¿े & 1892 & $25 \equiv .8$ & .77 & . 58 \\
\hline & 239 & soite & .42 & $3 !: 3$ & 2E:.? & .55 & .95 \\
\hline & 258 & 25008 & .55 & $2 \div 45$ & 250.0 & 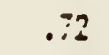 & .57 \\
\hline \multirow[t]{3}{*}{59} & 272 & 30055 & $.5 i$ & 1097 & 258.9 & .96 & .98 \\
\hline & 39 & SดิE? & .50 & 2588 & 257.4 & .78 & .37 \\
\hline & $3=$ & 30059 & ذَ. & $38 ! 5$ & $3=1.3$ & : & .27 \\
\hline \multirow[t]{3}{*}{5} & 290 & 35052 & .94 & $2=\div 5$ & 259.1 &.$i 9$ & . ÊE \\
\hline & 212 & j5it? & .59 & 2940 & 257.8 & .71 & .87 \\
\hline & $27:$ & - 50102 & $.7 j$ & $=2 ! 5$ & $20 \div 6$ & .05 & ò \\
\hline \multirow{3}{*}{550} & 271 & 4004: & .50 & $340 ?$ & $=59.5$ &.$T$ & .37 \\
\hline & $27 i$ & 40041 & .67 & 2375 & 250.4 & .65 & .96 \\
\hline & $27 !$ & 40041 & .35 & 5074 & $2 t \leq .1$ & . & .94 \\
\hline \multirow[t]{3}{*}{ En: } & 59 & มิดी & .04 & $112 \%$ & 257.0 & .9 .9 & . $2 \hat{q}$ \\
\hline & Do & $1002 \mathrm{i}$ & .07 & $12 ! 5$ & $25 \% .6$ & .9 & .39 \\
\hline & 30 & 1001 & $.1 \mathrm{i}$ & $1: 36$ & $: 3: 1.7$ &.$; 9$ &.$\tilde{z}^{6}$ \\
\hline \multirow[t]{3}{*}{$5: 5$} & ב": & $15: 97$ & .09 & : & 255.1 & .79 & .39 \\
\hline & 373 & :E02? & .14 & : & $=53.2$ & $\therefore$ & .27 \\
\hline & 57 & $150=7$ &.$i 9$ & $15 \dot{1}$ & J5: &.$i \Xi$ & . \\
\hline \multirow[t]{3}{*}{524} & נ:- & 206128 & .14 & ITIS & 255.0 &.$i 7$ & .39 \\
\hline & 39 & $300 ? 9$ & $.2 i$ & 1759 & 255.1 & $.7 j$ & .35 \\
\hline & 30 & 29029 & .27 & $: 971$ & $9=5.2$ & .7 & .30 \\
\hline \multirow[t]{3}{*}{ נים } & joó & 25.057 & $.2 \hat{v}$ & 1971 & 35.1 & . & . \\
\hline & Sob & 25057 & .29 & 3006 & 255.5 & .7 & .26 \\
\hline & كذذن & $25.05 i$ & D & & $=8.5$ & . & .32 \\
\hline \multirow[t]{3}{*}{$5=2$} & 362 & 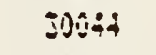 & .25 & 2170 & 258.5 & .73 & .30 \\
\hline & 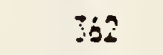 & 3ivi44 & . & Mà & $=53 . i$ & . ib & .38 \\
\hline & 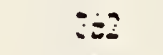 & $904:$ & .45 & 957 & :58.: & $\therefore:$ &.$\$ 8$ \\
\hline \multirow[t]{3}{*}{$5 a:$} & 800 & $:: 359$ & . & $\because 3:=$ & 255.3 & .77 & . \\
\hline & ito & $3: 959$ & .42 & $: 5 j 5$ & 259.1 & .75 & .23 \\
\hline & تלt & 54959 & .5 & $29: 45$ & 259.5 & .72 & .85 \\
\hline \multirow{3}{*}{$5: i$} & 304 & 39750 &.$j 4$ & $3: 21$ & 259.0 &.$i 6$ & .02 \\
\hline & Iót & 39750 & .97 & n?7a & :59.4 &.$i 4$ & . 98 \\
\hline & 364 & 35750 & .59 & 3บงิ & :E9.9 &.$i i$ & .87 \\
\hline \multirow[t]{3}{*}{523} & 302 & 45850 & .41 & $\because 3$ & 85.5 &.$i 5$ & .83 \\
\hline & 302 & 15890 & . $5 ?$ & $310:$ & 260.0 & $.7 !$ & .87 \\
\hline & 362 & 45850 & $.7 !$ & ¿EE0 & $=60.9$ & . & . sิó \\
\hline 547 & 372 & $5 \pm 110$ & .48 & $\therefore 89$ & 259.5 & . & .67 \\
\hline
\end{tabular}




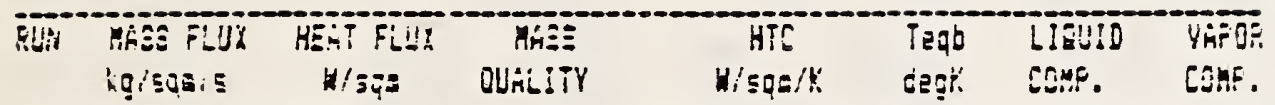

\begin{tabular}{|c|c|c|c|c|c|c|c|}
\hline & 淐 & $55: 10$ & .55 & $3 e \div 4$ & $z \leq 0.4$ & ?". & .36 \\
\hline & 372 & $551 i \hat{0}$ & $.8 i$ & i5:4 & 265.9 & .61 & .85 \\
\hline \multirow[t]{3}{*}{57} & 947 & 5954 &.$\hat{v} \hat{z}$ & 1:45 & 25: & .80 & .89 \\
\hline & 447 & 9954 & .85 & 1574 & 257.6 & .80 & .89 \\
\hline & $44 i$ & 9964 & .68 & leli & 257.6 & .79 & .89 \\
\hline \multirow[t]{3}{*}{53} & $45 ?$ & 19946 & .10 & $17: 2$ & 251.7 & .79 & .89 \\
\hline & 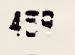 & 19940 & .15 & 1805 & 255.0 &.$i ?$ & .87 \\
\hline & $45 \bar{Y}$ & 19946 & .21 & 2152 & $259 . j$ & .78 & .87 \\
\hline \multirow[t]{3}{*}{539} & 49 & 29950 & .18 & 2036 & 258.5 & .78 & . 89 \\
\hline & 4dQ & 29950 & .27 & $235 i$ & $255 . ?$ & $.7 T$ & عَ \\
\hline & 499 & 29950 & .75 & 2977 & 258.9 & .70 & 8E. \\
\hline \multirow[t]{3}{*}{5} & 40! & 40909 & .25 & 3489 & :58. ? & .77 & .99 \\
\hline & 451 & 40949 & . 86 & $396 !$ & $=59.1$ & . is & .28 \\
\hline & $4 a !$ & 405099 & .46 & 5280 & $25 \div .4$ & .74 & .82 \\
\hline \multirow[t]{3}{*}{544} & 405 & 49831 & ל. & 2964 & 258.0 & .76 & $.3 E$ \\
\hline & 465 & $498=:$ & .45 & 3055 & $25 \hat{~}$ & .74 & .53 \\
\hline & 405 & 40931 & .58 & $994 E$ & 259.7 & $.7 !$ & .37 \\
\hline \multirow[t]{3}{*}{545} & $46 \bar{i}$ & 57935 & .33 & 3380 & 259.5 & .75 & .85 \\
\hline & 457 & 57903 & j5. & 59 & $2 \pm 0.0$ & .12 & .57 \\
\hline & $40 ;$ & 57963 & .07 & 4409 & 206.0 &.$\$ 3$ & .80 \\
\hline \multirow[t]{3}{*}{550} & $\therefore 00$ & Gi575 & 06. & 3992 & $25 \%$. & iti & .99 \\
\hline & 460 & siojs & J5 & itis & 2000.3 & .69 & .87 \\
\hline & 860 & bisis & .79 & 5107 & $3 \pm 1.0$ & .62 & .35 \\
\hline \multirow[t]{3}{*}{596} & 59 & 25050 & 6́ & 1913 & 258.0 & . & .89 \\
\hline & 590 & $2 E_{i} E_{j}$ & .12 & 1979 & 2EE.1 & .79 & .39 \\
\hline & 548 & 25055 & .18 & 2072 & 25E.1 & .73 & .89 \\
\hline \multirow[t]{3}{*}{$5: ?$} & 53 & 35031 & .13 & 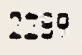 & IEล.4 & .79 & .89 \\
\hline & 535 & 250031 & .22 & 2412 & 955.5 & .78 & .89 \\
\hline & 5 & 30051 & .30 & 2759 & 258.7 & .77 & $.3 E$ \\
\hline \multirow[t]{3}{*}{$5+10$} & 534 & 29751 & .17 & $225 j$ & ใรE. & .78 & .39 \\
\hline & 534 & 29551 &.$\hat{64}$ & $25 \div 9$ & 259.5 & .78 & .87 \\
\hline & 504 & 29951 & . $3 !$ & $277 !$ & 258.6 & .77 & .33 \\
\hline \multirow[t]{3}{*}{ sie } & 597 & j9940 & .16 & 2435 & 259.气 & .79 & .59 \\
\hline & $54 i$ & 39946 & .26 & 2tsei & 258.9 & .77 & .89 \\
\hline & 547 & Sifís & .34 & $310 \%$ & $25 \div .0$ & $\therefore$ & . aE \\
\hline \multirow[t]{3}{*}{548} & $55 !$ & Evنli & בz & jisi & $=59.9$ & $.7 i$ & \% \\
\hline & EE! & socti & . & $\because:: !$ & בE:D: & .75 & .39 \\
\hline & 551 & Sôü17 & .48 & 4542 & 259.5 & .75 & .38 \\
\hline \multirow[t]{3}{*}{526} & $5 \Sigma 0$ & $5=035$ & $\therefore ?$ & $53: n$ & ES:0 0 & .77 & .88 \\
\hline & 550 & 55075 & .91 & 5046 & 259.2 & .75 & .88 \\
\hline & $55_{0}$ & 55035 & .55 & 4307 & $=59.5$ &.$\therefore 2$ & .37 \\
\hline \multirow[t]{3}{*}{$55 i$} & 540 & $3+953$ & J & EYSS & 250.4 & .76 & .98 \\
\hline & 540 & SA9ES & .50 & 4208 & 2Eะ.8 & . i & .57 \\
\hline & 540 & 64955 & .04 & 523 & 250.1 & .67 & .96 \\
\hline \multirow[t]{2}{*}{511} & izs & 20055 & .02 & $180 ?$ & 257.6 & . Sn & PQ \\
\hline & 685 & 20055 & .00 & $174 i$ & ذ. & .80 & .69 \\
\hline
\end{tabular}


FREHEAT SECTION DATA: RIGBZ .80 wt 91381

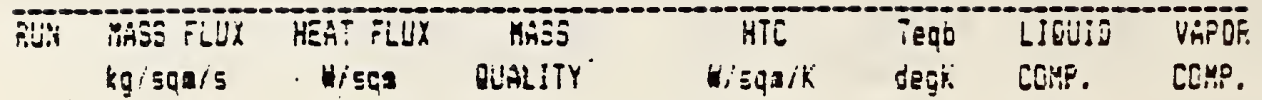

\begin{tabular}{|c|c|c|c|c|c|c|c|}
\hline & $66^{5}$ & $200 \mathrm{ES}$ & .10 & $194 i$ & 257.7 & .79 & .89 \\
\hline \multirow[t]{3}{*}{512} & EEt & 20134 & .07 & 1561 & $2=3.1$ & .90 & .89 \\
\hline & 684 & 20184 & $.0 s$ & 1800 & $=53.1$ & .79 & .85 \\
\hline & 654 & 20194 & .12 & 1956 & 259.2 & .79 & . 89 \\
\hline \multirow[t]{3}{*}{510} & SES & 30190 & .07 & 2184 & 258.1 & .90 & .89 \\
\hline & 085 & 30180 & .13 & 2191 & 258.1 & .79 & .59 \\
\hline & 685 & 20180 & .18 & 2429 & IEQ.1 & .79 & .89 \\
\hline \multirow[t]{3}{*}{$54:$} & 679 & 29911 & .10 & 2965 & 258.1 & .79 & .99 \\
\hline & 6iq & 29911 & .16 & 3503 & .58. 1 & .79 & 89 \\
\hline & 679 & 29911 & .21 & 2692 & 258.2 & .78 & .59 \\
\hline \multirow[t]{3}{*}{$5 ! 3$} & $\$ \$ 1$ & 35085 & .09 & ביט & 258.5 & .79 & .89 \\
\hline & 691 & i5ن85 & .15 & 2577 & 255.5 & .77 & $.8 ?$ \\
\hline & $67 !$ & 35085 & .22 & 2738 & AEE.b & .79 & .89 \\
\hline \multirow[t]{3}{*}{5} & te? & 40329 & .13 & 3445 & רES.J & .79 & .89 \\
\hline & $\varepsilon \hat{0} \tilde{z}$ & 40729 & .20 & $2 \leq 91$ & 258.4 & .76 & $.3 \bar{y}$ \\
\hline & bez & 903799 & .28 & 3240 & 258. 1 & . & .29 \\
\hline \multirow[t]{3}{*}{$5: 2$} & 695 & 44753 & .14 & 2907 & 258.4 & $.7 ?$ & .89 \\
\hline & 695 & 94758 & .23 & 2957 & 258.4 & .13 & .59 \\
\hline & 495 & 44758 & $.3:$ & 3525 & 258.5 & .77 & .58 \\
\hline \multirow[t]{3}{*}{509} & 682 & 49814 & .17 & 2977 & 259.5 &.$i a$ & .09 \\
\hline & $=84$ & 49314 & $.2 T$ & $204 j$ & .58 .9 & .77 & . \\
\hline & 604 & $4881:$ & S & $\because 2: 5$ & 259.0 &.$i t$ & .98 \\
\hline \multirow[t]{3}{*}{$54 ?$} & GE: & 49975 & .18 & $32 \hat{v}$ & =55.? &.$i s$ & .59 \\
\hline & $\triangle E Z$ & 99975 & .27 & 3090 & 257.0 & .77 & . \\
\hline & bEj & 19973 & cic & 4354 & =ะ. 1 & .76 &.$£ 9$ \\
\hline \multirow[t]{3}{*}{553} & 673 & SHEZG & .22 & $402=1$ & 257.4 & .73 & .89 \\
\hline & 595 & 64896 & .04 & 2112 & 959.1 & $\therefore 6$ & . 3ิ \\
\hline & $6 \div 5$ & 64826 & . iś & 5359 & 259.2 & it & .33 \\
\hline \multirow[t]{3}{*}{582} & dê! & j-92j &.$\therefore$ & 4035 & 259.7 &.$i$ & .57 \\
\hline & ละ) & $5492 j$ & .36 & $+1+\hat{v}$ & 357.9 &.$i s$ & 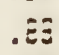 \\
\hline & SE! & 04928 & .49 & 5396 & $250.1)$ & .73 & $.8 i$ \\
\hline \multirow[t]{3}{*}{$5 \equiv:$} & 700 & 74948 & .27 & $4: 75$ & 258.3 &.$i$ & .88 \\
\hline & 700 & 74943 & .41 & 4719 & ^59.5 & .75 &.$S E$ \\
\hline & 730 & 14948 & .54 & 5940 & 259.7 & .72 & .87 \\
\hline
\end{tabular}




\begin{tabular}{|c|c|c|c|c|c|c|c|}
\hline חה & 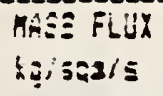 & $\begin{array}{l}\text { HEST FIUL } \\
W / 5 C S\end{array}$ & $\begin{array}{c}\text { nASE } \\
\text { SuLL! iY }\end{array}$ & $\begin{array}{c}\text { HTC } \\
W_{i} \leq q 9 / K\end{array}$ & $\begin{array}{l}\text { Tequ } \\
\text { JEgt: }\end{array}$ & $\begin{array}{l}\text { LIOUID } \\
\text { COKF. }\end{array}$ & $\begin{array}{l}\text { UAFĩ? } \\
\text { Canf. }\end{array}$ \\
\hline \multirow[t]{3}{*}{57} & उอ & $20^{103}$ & .12 & i4!6 & 279.6 & .32 & .73 \\
\hline & 205 & 25102 & Jَבמ. & 1553 & $5=2.4$ & .3 & . $e^{?}$ \\
\hline & 396 & $25: 1: 4$ & .32 & $\cdot: 750$ & $2 T: .1$ & .29 & .05 \\
\hline \multirow[t]{3}{*}{410} & 200 & 20081 & .22 & 1674 & 276.1 & .33 & .09 \\
\hline & 206 & 200a! & .30 & $18 \hat{5} 5$ & $2 \pi 0.6$ & .25 & 6́ \\
\hline & 206́ & 200981 & .37 & $25+2$ & -74.? & .22 & .65 \\
\hline \multirow[t]{3}{*}{ : } & 202 & $205 ? 2$ & .27 & $1: 25$ & 275.1 & $.2 \vdots$ & $.6 ?$ \\
\hline & 200 & $2035:$ & . I4 & de: & 299.5 & تבدב. & .34 \\
\hline & 200 & 29592 & $.4 ?$ & 1985 & ZTE.? & .30 & נن. \\
\hline \multirow[t]{3}{*}{ dil } & 202 & 2SEB4 & .32 & 1957 & 274.0 & .24 & .65 \\
\hline & 20? & -75e? & .42 & 2192 & $2 \div .3$ & .30 & נים. \\
\hline & 2.05 & 26534 & .51 & 2459 & 277.4 & .17 & j. \\
\hline \multirow[t]{3}{*}{415} & 205 & 3E1:a? & ט. & 2799 & $275 . j$ & .92 & .02 \\
\hline & 305 & 35122 & .49 & $j 12 i$ & $27 \bar{i} . \overline{3}$ & .18 & .57 \\
\hline & 205 & 35192 & $.5 ?$ & 3812 & 279.5 & .10 & $.5 ?$ \\
\hline \multirow[t]{3}{*}{432} & 205 & J㣙浞 & .42 & $230 j$ & 276.6 & $.2 \hat{0}$ &.$\hat{\mathbf{v}}$ \\
\hline & 205 & ร9858 & .54 & 3129 & 278.0 & .17 & .54 \\
\hline & 205 & & . & $347 !$ & 279.6 & .14 & $.4 \%$ \\
\hline \multirow[t]{3}{*}{ tió } & 394 & 49834 & .47 & $340 ?$ & $27 \% .:$ & .19 & .58 \\
\hline & 219 & i $48 \varepsilon:$ & .50 & 351 & 279.1 & .15 & $.5 !$ \\
\hline & 204 & ૧-884 & .75 & $449 \%$ & 290.6 & .13 & . 46 \\
\hline \multirow[t]{3}{*}{495} & 230 & $150 ! 5$ & .13 & 1220 & 270.2 & .32 &.$i 2$ \\
\hline & 230 & 15015 & .27 & 1250 & 271.0 & .50 & $.7 !$ \\
\hline & 230 & $: 5015$ & .32 & 1256 & 271.9 & .29 & .69 \\
\hline \multirow[t]{3}{*}{ 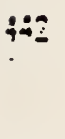 } & 20? & 20495 & .18 & 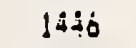 & 271.5 & .30 &.$i !$ \\
\hline & 295 & 20195 & .24 & 1558 & $2 \pi .5$ & .27 & ל? \\
\hline & 285 & 04496 & .99 & $17: 2$ & 975.5 & . & ob \\
\hline \multirow[t]{3}{*}{ 43E } & :29? & 2ะ $399 \%$ & .21 & $19: 9$ & 272.0 & .23 & .70 \\
\hline & 389 & โบ85? &.$\hat{0}$ & 1987 & SiJ.S & iśs & . \\
\hline & 399 & 24897 & .34 & 2126 & 274.3 & $\therefore$ & .84 \\
\hline \multirow[t]{3}{*}{455} & 292 & $500: 1$ & .27 & 2367 & 275.1 & فة & .57 \\
\hline & 292 & 30041 & .34 & 2585 & 274.5 & .23 & .64 \\
\hline & $39 ?$ & 30041 & .42 & 2842 & 275.8 & .20 & .30 \\
\hline \multirow[t]{3}{*}{452} & 297 & $402 \leq ?$ & .35 & 2973 & 274.7 & S & .64 \\
\hline & 297 & $4 \hat{a}=6 ?$ & .45 & $30: 5$ & 970.6 & .19 & .59 \\
\hline & $28 ?$ & 41252 & (5: & 3057 & 2i7.? & .17 & .55 \\
\hline \multirow[t]{3}{*}{443} & 295 & 50295 & .41 & $583 !$ & 27o.! &. .1 & . ¿́! \\
\hline & 995 & Sำโะ5 & .92 & 3956 & 397.9 & .17 & .55 \\
\hline & 275 & sigas & .62 & +541 & 279.3 &.$: 5$ & $.5 !$ \\
\hline \multirow[t]{3}{*}{$+4 i$} & 289 & bj5g? & .48 & $4 i 5 \dot{s}$ & 97.3 & .19 & .57 \\
\hline & 269 & 60989 & $.6 !$ & 9540 & 279.1 & .15 & $.5 i$ \\
\hline & 299 & 60559 & $.7 j$ & $490 ?$ & 280.5 & ¿ & . \\
\hline \multirow[t]{3}{*}{$44 !$} & 359 & 20375 & .11 & 1556 & $=69.9$ & $.3 \hat{z}$ & $.7 j$ \\
\hline & 399 & 20375 & .16 & 11:99 & 250.3 & .30 & .12 \\
\hline & 369 & 20575 & .21 & :000 & 271.6 & .29 & .70 \\
\hline $4: 9$ & 353 & คะ658 & .14 & 1855 & $30 . \hat{z}$ & . 11 & כי. \\
\hline
\end{tabular}




\begin{tabular}{|c|c|c|c|c|c|c|c|}
\hline ñivi: & 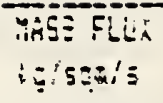 & $\begin{array}{c}\text { Hex } \\
\text { R }\end{array}$ & 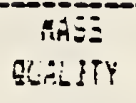 & nis & $\begin{array}{l}\text { TEzE } \\
\text { segr. }\end{array}$ & $\begin{array}{l}\text { CQYe. } \\
\text { chy. }\end{array}$ & $\begin{array}{l}\text { yE: } \\
\text { SInt }\end{array}$ \\
\hline & 50 & 39005 & .20 & 1950 & 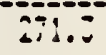 & .3 & .76 \\
\hline & $\because \vdots$ & $24 E \div 0$ & .35 & : $2 \hat{V} \measuredangle\}$ & $27,2.3$ & .26 & . ذ6 \\
\hline \multirow[t]{3}{*}{ S } & 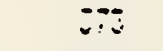 & X́viq4 & .15 & $134 !$ & 271.0 & $\therefore 0$ & .79 \\
\hline & $\because$ & $\because 7$ & $=0$ & 1965 & 99.3 & $.2 ?$ & $.6 ?$ \\
\hline & 335 & 301944 & .20 & $\therefore$ & 273.4 & .25 & ذڤ. \\
\hline \multirow[t]{3}{*}{307} & 374 & $: 9989$ & .21 & $2 \pi k$ & $\because \because 2.0$ & .23 &.$i 0$ \\
\hline & 574 & $3: 507$ & .20 & 2575 & 275.5 & $\therefore$ & . bt \\
\hline & $3: 2$ & 39939 & .77 & jü:? & 294.9 & $.2 ?$ & .02 \\
\hline \multirow[t]{3}{*}{ S:P } & $3 i t$ & 49060 & .27 & $289 \%$ & 275.4 & .28 & .07 \\
\hline & 376 & 49560 & .37 & İjJ & 275.1 & .22 & .63 \\
\hline & 376 & 49000 & .45 & $: 8 \leq 1$ & 276.7 & .19 & .56 \\
\hline \multirow[t]{3}{*}{$8 \div ?$} & 372 & .59445 & .3 & j56j & 274.7 & .24 & .35 \\
\hline & 372 & 59445 & .44 & 4007 & $=i b .3$ & .20 & ס 50. \\
\hline & 97 & 579745 & .54 & $460 \%$ & 27อ. 1 & .17 & .54 \\
\hline \multirow[t]{3}{*}{490} & $\therefore 74$ & 69055 & . 20 & 4159 & 235.8 & .2 &.$\dot{E} z$ \\
\hline & STi & 69055 & .50 & 4753 & 277.8 & .18 & $.5 t$ \\
\hline & Sit & 690.055 & .61 & 5276 & 279.3 & .15 & .51 \\
\hline \multirow[t]{3}{*}{401} & 372 & 79Sú: &.+3 & 4853 & $\hat{L} 7 \bar{T} \cdot \hat{v}$ & .20 & .57 \\
\hline & $3: 2$ & 793014 & .57 & 5471 & 279.1 & .16 & Jَّ. \\
\hline & 372 & 79804 & .69 & 5740 & 284.5 & .19 & $.9 i$ \\
\hline \multirow[t]{3}{*}{$541^{\circ}$} & 400 & 24819 & .09 & 1P:E & 209.7 & . & . iq \\
\hline & 260 & 24815 & .14 & 1937 & 230.5 &.$j !$ & .79 \\
\hline & 460 & 24918 & .13 & 1950 & $27 ! .5$ & .29 & $\because$ \\
\hline \multirow[t]{3}{*}{454} & $45 i$ & $5998 \mathrm{i}$ & .15 & 2050 & $=70.8$ & .Z! & .7 \\
\hline & $\div 67$ & 39081 &.$Z_{j}$ & 2455 & 27:.? & .27 &.$\hat{v}$ \\
\hline & $4 b i$ & $2709 \mathrm{i}$ &.$\therefore 5$ & $20 \mathrm{ii}$ & $=-.7$ & $\therefore 6$ & . \\
\hline \multirow[t]{3}{*}{$45 !$} & 407 & 40110 & .90 & 汪得 & ร:2.: & : & in \\
\hline & 407 & 20100 & $.3 !$ & ¿หลô & 江.; & .20 & .07 \\
\hline & $45 ;$ & 4): & בَבנ. & ت055 & 297.5 & $\therefore \div$ & .05 \\
\hline \multirow[t]{3}{*}{455} & 169 & :77? & .24 & & $97 . .2 ?$ & .27 & .08 \\
\hline & Ao? & $4979 \mathrm{~b}$ & .32 & 3648 & 274.5 & .24 & .05 \\
\hline & $4 j ?$ & $49790^{\circ}$ & .40 & $114 \%$ & 275.9 & .21 & .61 \\
\hline \multirow[t]{3}{*}{445} & 947 & 50525 & .32 & $35 \div 8$ & $274 . j$ & $\therefore 4$ & . \\
\hline & 417 & 600525 & .41 & $4 i 11$ & $\because \therefore .0$ & .21 & $.5 !$ \\
\hline & 497 & $605-5$ & .50 & 9045 & 971.2 & .12 &.$\Sigma$ \\
\hline \multirow[t]{3}{*}{ 6 } & 453 & 69050 & .35 & $\$ 1196$ & [:5.2 & $\therefore$ & .53 \\
\hline & 459 & ง & . 40 & 4752 & 277.0 & .19 &.$\equiv 9$ \\
\hline & $2 E S$ & 59950 & .55 & 5448 & 273.2 & .17 & .54 \\
\hline \multirow[t]{3}{*}{450} & $9 \leq 7$ & 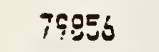 & & $400 \overline{3}$ & M… & $\therefore=$ &.$\vdots 2$ \\
\hline & $40 \bar{i}$ & 79650 & .50 & E:4: & 27.6 & $\therefore: 9$ & .55 \\
\hline & 407 & 7985s & .60 & suij & 97.7 & .15 & . \\
\hline \multirow[t]{3}{*}{$4 \$ 0$} & 445 & 90060 & .42 & Egid & 397.0 & .20 & .30 \\
\hline & 496 & giscoso & .55 & Lesj & :-E.S & $.1 i$ & .54 \\
\hline & 440 & 900150 & .67 & 6795 & 30.2 &.$\vdots 1$ & .48 \\
\hline \multirow[t]{2}{*}{$4 c a$} & 5? & 10252 & .02 & $11: 3$ & วิธ.7 & 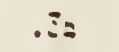 & .76 \\
\hline & 525 & 10052 & .04 & 1144 & $26 \% .0$ & .35 & .85 \\
\hline
\end{tabular}




\begin{tabular}{|c|c|c|c|c|c|c|c|}
\hline Bivin & 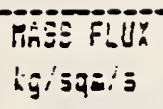 & $\begin{array}{c}\text { HERT Fijo } \\
\text { di }\end{array}$ & 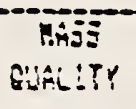 & $\begin{array}{l}\text { MiC } \\
\text { wisquK. }\end{array}$ & $\begin{array}{l}\text { Teqt } \\
\text { EEg: }\end{array}$ & han. & cints. \\
\hline & 53 & 1005 & $.0 \delta$ & iiso & SES & .75 & \\
\hline \multirow[t]{3}{*}{467} & 5 & 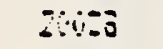 & . $\Delta E$ & .1720 & ZSF: & .5 & $i z$ \\
\hline & $5=$ & 2605 & . & bito & $25: .3$ & כ5 & \\
\hline & 59 & 305 & .23 & $13: 7$ & 70.3 & E & \\
\hline \multirow[t]{3}{*}{$45=$} & $5=2$ & 30078 & . & mi: & 270.0 & E & \\
\hline & $5=2$ & 30070 & .15 & EA & $8,-1.3$ & Dذ & \\
\hline & $5=?$ & 3090 & .3 & $\therefore$ & ذ. & 59. & \\
\hline \multirow[t]{3}{*}{465} & 541 & 4RĖ & $.8=$ & 0 & 59.7 & إذ. & \\
\hline & $\xi: !$ & $409 !$ & 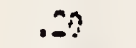 & $\because 1 T$ & 971.3 &.$Z$ & \\
\hline & $5 \$ 1$ & 2DIE: & .25 & $3:-5$ & $=\square .3$ & E & 0 \\
\hline \multirow[t]{3}{*}{$\doteqdot 4$} & $5: 5$ & $4955 i$ & .15 & $:: ? !$ & 27.9 &. & \\
\hline & $5: j$ & ใจ९ระT & ذ. & 959 & 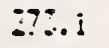 & $\therefore$ & , so \\
\hline & 5.5 & 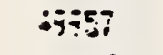 & $\bar{J}$ & לבים"ה & 278.3 & בَב & . \\
\hline \multirow[t]{3}{*}{ TE } & $5+3$ & $601=5$ & .2 & 20. & 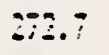 & E & $\therefore$ \\
\hline & E:⿱一𫝀口 & sU: & $\therefore$ & :I!: & 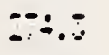 & $\therefore$ & $=$ \\
\hline & E: & S::E= & is & $4: 8:$ & $275 .=$ & $\therefore$ & ב. \\
\hline \multirow[t]{3}{*}{$=5$} & $j: \sum$ & 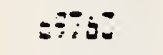 & 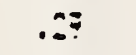 & 4016 & $\because-1.0$ & $\therefore$ & 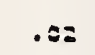 \\
\hline & $5: 9$ & 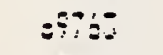 & 8.5 & ¿: & «J. & $\therefore$ & \\
\hline & $5: \vdots$ & $\vdots=-:=$ & 5 & $5 ?$ & 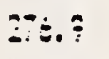 &.$: \$ ?$ & \\
\hline \multirow[t]{3}{*}{$\therefore:$} & Bu & ontot &.$\hat{ن}$ & 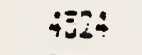 & 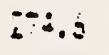 & & 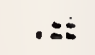 \\
\hline & $5:-5$ & $3: 4$ & $\therefore$ & 3265 & 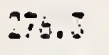 & .23 & o: \\
\hline & 引4 & 5004 & .30 & E2:- & $\because 7.2$ &.$\therefore$ & 12 \\
\hline \multirow[t]{3}{*}{$1::$} & $\because=$ & 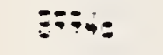 &.$\because$ & 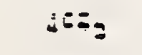 & 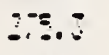 &.$:$ & 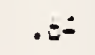 \\
\hline & E: & 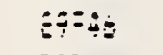 & $\therefore$ & $5=5$ & $\because !$ &.${ }^{2}$ & $\therefore$ \\
\hline & $E^{-}=$ & E: & .5 & $\therefore \div$ & 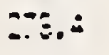 & S & \\
\hline
\end{tabular}




\begin{tabular}{|c|c|c|c|c|c|c|c|}
\hline 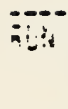 & 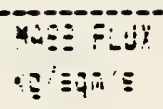 & 和和 & 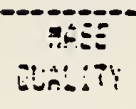 & 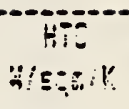 & $\begin{array}{l}\text { IEs: } \\
\text { tE: }\end{array}$ & 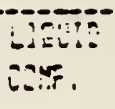 & 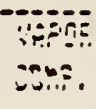 \\
\hline \multirow[t]{3}{*}{ 泣 } & jo & 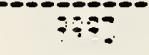 & $\therefore e$ & 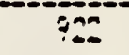 & Bis.: & .15 & .55 \\
\hline & $:$ & $5=95$ &.$a^{i j}$ & ivia & $=97.1$ & $.1:$ & .5 \\
\hline & $: 90$ & : & 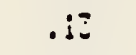 & 1207 & 59.7 & $\therefore:$ & $\therefore$ \\
\hline \multirow[t]{2}{*}{5} & $\begin{array}{l}0 \\
\vdots 95 \\
\vdots \vdots 0\end{array}$ & $\begin{array}{l}20190 \\
20140\end{array}$ & .15 & $\begin{array}{l}1955 \\
20=5\end{array}$ & $\begin{array}{l}279.7 \\
2 \leq ! . !\end{array}$ & 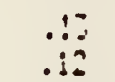 & .9 \\
\hline & ל广 & Za: & .80 & 9655 & CE: I &.$j i$ & .35 \\
\hline \multirow[t]{3}{*}{$5: 7$} & $\vdots=\vdots$ & 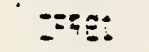 & 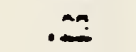 & ด:3: & 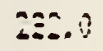 & .11 & . i: \\
\hline & i\%: & $2=5=81$ & .30 & ¿९E5 & 23:.2 & & .37 \\
\hline & غ?? & 29૯E! & .37 & 315 & 284.1 & .03 & .3 \\
\hline \multirow[t]{3}{*}{ : } & 20 & 40797 & $.2 ?$ & 2855 & 292.5 &.$\$ 0$ &.$ذ \hat{~}$ \\
\hline & OY & & $.4 !$ & 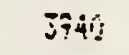 & IE:.ร & $.0 E$ & פת \\
\hline & 28 & 40729 & .51 & tos? & 394.7 & .07 & .98 \\
\hline \multirow[t]{3}{*}{595} & 202 & ใด99? &.$\pi$ & $\$ 779$ & 294.0 & 45. & .24 \\
\hline & 202 & 4799? & .500 & $i 58 \dot{0}$ & 285.2 & .07 & .89 \\
\hline & 292 & $4909 ?$ & . j4 & 5000 & 296.0 & .06 & $.2 \equiv$ \\
\hline \multirow[t]{3}{*}{590} & 205 & $600: 32$ & .84 & $504 j$ & โEะ.J & .08 & .51 \\
\hline & 205 & 60132 & .60 & 5705 & $2 \Xi 5 . j$ & .06 & .25 \\
\hline & 205 & OUิ15: & .75 & 3995 & 236.0 & .05 & .29 \\
\hline \multirow[t]{3}{*}{599} & 292 & 10023 & .04 & 854 & 279.4 & . lé & .53 \\
\hline & 29 & 10029 & .07 & $102:$ & 279.1 & .15 & .51 \\
\hline & 212 & $100 \div 3$ & .09 & $11: 5$ & 979.7 & .15 & $.5 \hat{v}$ \\
\hline \multirow[t]{3}{*}{57} & $\therefore \div$ & 20119 & 99. & 1390 & 270.9 &.$: 5$ & .50 \\
\hline & $29:$ & $20 ! 18$ & .35 & 1913 & 200.0 & $.8=$ & . \\
\hline & $21 \mathrm{i}$ & $20: 15$ &.$\hat{2} \hat{v}$ & zovi & $=20.9$ & .12 & .15 \\
\hline \multirow[t]{3}{*}{$5 \Xi \hat{c}$} & $24:$ & 29939 & s. & $=909$ & 930.7 & . & .45 \\
\hline & $2 \geq 1$ & 29539 & .24 & $205 !$ & 332.0 & $\therefore:$ &.+1 \\
\hline & $2+!$ & 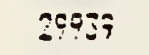 & .31 & $308 \%$ & 25:.? &. .99 &.$\because$ \\
\hline \multirow[t]{3}{*}{$59 \overline{ }$} & 246 & $40: 44$ & .24 & $j E=\dot{S}$ & วิว..2 &.$\vdots 1$ & $.4 !$ \\
\hline & 240 & 40744 & .34 & לת & $38 j .5$ & .07 & .85 \\
\hline & 210 & $407: a:$ & .42 & 5938 & $294 . j$ & .08 & $.3 !$ \\
\hline \multirow[t]{3}{*}{ فة } & $24=$ & 495:: & .27 & 4600 & E. E. T &.$\vdots 0$ & 涪 \\
\hline & 242 & 40913 & .41 & 4869 & 294.0 & .05 & .22 \\
\hline & 342 & 49813 & .52 & 4955 & 204.3 & .07 & .28 \\
\hline \multirow[t]{3}{*}{55} & 349 & 59915 & . 55 & 5404 & 253.7 & .09 & .25 \\
\hline & ?:? & $5 \div 915$ & . 98 & 5504 & 284.9 & .07 &.$\therefore 9$ \\
\hline & 399 & $509 ! 5$ & ít & 570 & $295 . i$ & . io & .25 \\
\hline \multirow[t]{3}{*}{ 5?: } & 35 & 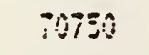 &.$i^{n}$ & S:Tis & -24.5 & .08 & .32 \\
\hline & 246 & 70750 & .59 & 5i94 & 295.6 & t! & $\therefore$ \\
\hline & 246 & 70750 & .73 & 3850 & 28j.J & .05 & .83 \\
\hline \multirow[t]{3}{*}{597} & 295 & $1000 \mathrm{j}$ & .02 & 880 & 275.0 & .17 & .55 \\
\hline & 295 & 10003 & . $\hat{H}$ & $: 05$ & 273.5 & . ló & .53 \\
\hline & 995 & luิต̣ติ & .07 & 113: & .979 .0 & .15 & .51 \\
\hline \multirow[t]{4}{*}{576} & 296 & 201099 & .05 & 19.15 & 273.9 & si & .52 \\
\hline & 295 & 20109 &.$: 1$ & $1990^{\circ}$ & 279.9 & .1: & .47 \\
\hline & $29 \mathrm{~s}$ & 30109 &.$! 5$ & 1929 & $290 . \bar{i}$ & .12 & .46 \\
\hline & $=00$ & 29960 & .11 & 2737 & 279.9 & .14 & a \\
\hline
\end{tabular}




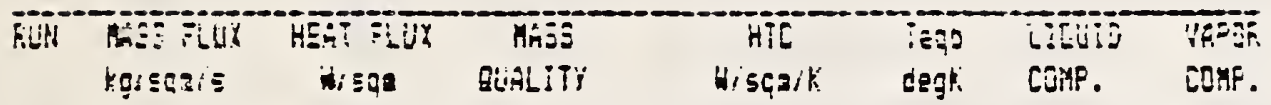

\begin{tabular}{|c|c|c|c|c|c|c|c|}
\hline & 86 & $=8000$ & $\therefore i$ & $=5$ & : & .12 & .44 \\
\hline . & 500 & - $9 \% 60$ & .24 & .2983 & $28: . \dot{v}$ & $.1 !$ & $.4 i$ \\
\hline \multirow[t]{3}{*}{$58:$} & 209 & 3991! & .17 & $351 ?$ & 280.9 & .12 & .45 \\
\hline & $2: 5$ & IY:11 & 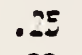 & ioit & 992.1 & .10 & .40 \\
\hline & 95 & 37911 & $\therefore$ & $39: 20$ & รaว่.! & .09 &.$j \dot{~}$ \\
\hline \multirow[t]{3}{*}{$58:$} & 235 & :9?35 & $\therefore$ & 4517 & $391 . ?$ & $.1 !$ & .41 \\
\hline & 299 & A9TES & .32 & 993 & 285.2 & $\hat{\theta}$ & S. \\
\hline & 299 & 49785 & $.4 !$ & fE:9 & EE4.0 & .08 & .32 \\
\hline \multirow[t]{3}{*}{593} & 299 & 59972 & 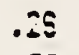 & EAET & 2B:. & .10 & .j3 \\
\hline & 298 & 59873 & .39 & 5499 & 254.2 & .08 & .33 \\
\hline & 298 & 59873 & .50 & 570 & ZEE.0 & .07 & .29 \\
\hline \multirow[t]{3}{*}{595} & 300 & 79923 & .80 & 6758 & 284.2 & .08 & .33 \\
\hline & 300 & 79923 & נE. & 678t & IES.T &.$\hat{i} 7$ & .23 \\
\hline & 200 & 790925 & $.6 \hat{~}$ & 7984 & 286.1 & .05 & .24 \\
\hline \multirow[t]{3}{*}{$5 \div 4$} & 305 & 89372 & .45 & 7549 & .94.3 & $.6 \bar{~}$ & .jI \\
\hline & 305 & s9a?: & .57 & 7740 & 295.6 & .06 & .35 \\
\hline & 205 & s9:?: & .75 & 8597 & 236.2 & .05 & .29 \\
\hline \multirow[t]{3}{*}{5} & 345 & 1005\% &.$\hat{u} j$ & 95: & 277.8 & .17 & .55 \\
\hline & 345 & 10069 & .03 & 700 & 290.2 & .17 & .54 \\
\hline & 345 & lo0E? & . & $76 a$ & 270.7 & $.1 \dot{0}$ & .52 \\
\hline \multirow[t]{3}{*}{575} & 349 & 20695 & .04 & 1955 & 296.3 & .16 & .55 \\
\hline & 349 & 20055 & .10 & 1796 & 209.2 & .15 & $.5 i$ \\
\hline & I49 & 20095 & .12 & isis & 250.0 & .14 & $.4 \varepsilon$ \\
\hline \multirow[t]{3}{*}{535} & I:: & 3075 & .08 & $275 \hat{u}$ & 279.3 &.$: 5$ & .51 \\
\hline & I: & 30743 & .14 & $271 !$ & 2E0.4 & .12 & .47 \\
\hline & Z54 & 30745 & $\therefore ?$ & 2755 & $2 \geq ! .4$ &.$i 2$ & .45 \\
\hline \multirow[t]{3}{*}{$5 \Sigma 5$} & $i=0$ & จ:Gร5 & .18 & วิنี่อ & 250.0 & $.1 j$ & . 97 \\
\hline & 8 & ¿9935 & .20 & 3484 & 281.2 & .12 & Jَ' \\
\hline & 350 & J9SEE & .27 & :5i: & 295.2 & .10 & .39 \\
\hline \multirow[t]{3}{*}{$50 \hat{0}$} & $j 5 !$ & $5119 t$ & .19 & $42 \div 5$ & 281.5 & .12 & .44 \\
\hline & i5! & 51190 & .77 & 4459 & 282.8 & .10 & $.3 ?$ \\
\hline & $35 !$ & 51196 & .35 & $40=0$ & 235.7 & .09 & .25 \\
\hline \multirow[t]{3}{*}{$5 b^{3}$} & -54 & $5990 \mathrm{y}$ & .32 & 4968 & 286.5 & .11 & .41 \\
\hline & 354 & $5590 i$ & .32 & 5150 & 35.7 & .199 & 6 \\
\hline & 35 & $599 \hat{q}^{i}$ &. $\mathrm{ii}$ & $5: 54$ & 294.0 & .06 & .32 \\
\hline \multirow[t]{3}{*}{5} & 泣! & ei34i & .27 & $5 i 1 ?$ & $23 \div .7$ & .10 & ? \\
\hline & SII & 09247 & .38 & 5EEt & 34.2 & .93 & Jَنَ. \\
\hline & jEl & $6984 ?$ & .49 & oljéc & นิร5. 0 & $.0 i$ & .29 \\
\hline \multirow[t]{3}{*}{504} & $3+8$ & 79720 & .36 & 6454 & $29 j .0$ & .09 & .36 \\
\hline & $34 \varepsilon$ & 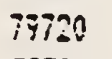 & .45 & 0.587 & วิล.ว & $.0 j$ & .31 \\
\hline & 340 & 79720 & .57 & $59 ! 9$ & 295.5 & .06 & .26 \\
\hline \multirow[t]{3}{*}{ S'E } & $35 !$ & 90237 & . 30 & 1995 & $: 84.7$ & .69 & .34 \\
\hline & 351 & 90235 & .51 & $73 \hat{0} 8$ & -25.5. & $.0 i$ & .28 \\
\hline & 551 & 90257 & .65 & 7099 & 256.2 & .06 & .24 \\
\hline \multirow[t]{2}{*}{ Ets } & If9 & 35147 & .39 & T5iE & 394.6 & .08 & .35 \\
\hline & 349 & 95147 & .54 & 7648 & 285.7 & .07 & .27 \\
\hline
\end{tabular}


FREETEAT SELTION OATH: RIGZ? . 18 nt Ri:3il

\begin{tabular}{|c|c|c|c|c|c|c|c|}
\hline BjW & $\begin{array}{l}\text { AHES FLUX } \\
\mathrm{kg} / \text { SQRO/S }\end{array}$ & $\begin{array}{c}\text { HEST FLUX } \\
\text { H/sqD }\end{array}$ & $\begin{array}{c}\text { MAES } \\
\text { QUAL:TY }\end{array}$ & $\begin{array}{l}\text { Hí } \\
\text { Wisqeik }\end{array}$ & $\begin{array}{l}\text { Teq: } \\
\text { deggi: }\end{array}$ & $\begin{array}{l}\text { LIUU:o } \\
\text { COMP. }\end{array}$ & $\begin{array}{l}\text { Comp. } \\
\text { Camo. }\end{array}$ \\
\hline & 349 & 55147 & .69 & 853 & 36.3 &.$j 5$ & .25 \\
\hline \multirow[t]{3}{*}{595} & 465 & $190 \hat{j Z Z}$ & .00 & 1506 & 277.4 & .18 & .56 \\
\hline & 408 & 16032 & .02 & 98? & $=97.7$ & .17 & .55 \\
\hline & 469 & $100 i 3$ & .03 & 941 & :73. 1 & .17 & $.5 t$ \\
\hline \multirow[t]{3}{*}{574} & $17 \Xi$ & 200165 & .01 & 2101 & 37.5 & i & .55 \\
\hline & $4: 3$ & 20065 & .04 & 1374 & 979.2 & .16 & .55 \\
\hline & 4:5 & $200 \leq 5$ & .07 & 1870 & ITE.S & .15 & $.5 !$ \\
\hline \multirow[t]{3}{*}{$5 \%$} & 492 & 30\%E? & .34 & $2 E=5$ & -TE.: & . 16 & .54 \\
\hline & $\$ 72$ & 30082 & .48 & 398: & 279.0 & . $! 5$ & $.5 j$ \\
\hline & 178 & 30092 & $.1 j$ & 2519 & 279.9 & .14 & .48 \\
\hline \multirow[t]{3}{*}{$5, ?$} & (7) & ร9975 & .07 & isaj & 979.2 & .15 & $.5 !$ \\
\hline & 473 & 3993E & .15 & נב:S & 280.2 & . if & .45 \\
\hline & 473 & 59938 & .18 & ¿38? & 20:.2 & .12 & .44 \\
\hline \multirow[t]{3}{*}{$57 !$} & 475 & Sijost & .10 & 3958 & ลว7. & .14 & .49 \\
\hline & 475 & 50054 & .17 & 3950 & $=21.0$ & .12 & .94 \\
\hline & 475 & $50006^{\circ}$ & .24 & 1139 & 332.0 & .11 & .10 \\
\hline \multirow[t]{3}{*}{590} & 4 & 600ิitd & .14 & (4ód & $23 ! .0$ & .12 & .96 \\
\hline & 472 & 80044 & .22 & $\$ 610$ & 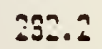 & . 1! & .41 \\
\hline & 475 & 60044 & 30 & $\$ 955$ & ¿Еะ.2 & .10 & .7 \\
\hline \multirow[t]{3}{*}{$5: 5$} & 479 & 70725 & .18 & 5274 & 231.6 & .12 & .44 \\
\hline & 479 & 70795 & $\therefore$ & $5:=9$ & 23..9 & .10 & .8 \\
\hline & 477 & ב3:707 & ذذَ. & 5655 & EE & .09 & . \\
\hline \multirow[t]{3}{*}{ SES } & 460 & Sô. 154 &. .1 & $=9$ & ZSล.: : & .11 & .82 \\
\hline & 480 & 30154 & .31 & 6545 & פב.4 & S5. & וֹ: \\
\hline & 480 & 30154 & $.41 j$ & 7158 & IE4... & .08 & $\therefore$ \\
\hline \multirow[t]{3}{*}{$5_{2 i}$} & $.47 j$ & 90326 & .25 & $5 \$ \$ 0$ & เэะ..9 & .11 &.$\because \hat{\theta}$ \\
\hline & $47 j$ & 90396 & .36 & .969 & $33 \div .2$ & .09 & . \\
\hline & 473 & $90 \%$ & .46 & $38 \mathrm{i} \hat{0}$ & $294 . ?$ & .07 & .00 \\
\hline
\end{tabular}




\begin{tabular}{|c|c|c|c|c|c|c|c|}
\hline RUK & $\begin{array}{l}\text { RASS FLUT } \\
\text { kọ:'sqais }\end{array}$ & $\begin{array}{c}\text { HETi FUX } \\
\text { Hisas }\end{array}$ & GUALITY & $\begin{array}{c}\text { HTC } \\
\text { WIsq8IK }\end{array}$ & degti. & $\begin{array}{l}\text { LiEUIO } \\
\text { COME. }\end{array}$ & $\begin{array}{l}\text { Come. } \\
\text { capose }\end{array}$ \\
\hline \multirow[t]{3}{*}{640} & 5 & 90045 & .20 & 1574 & 264.7 & $.8 ?$ & .82 \\
\hline & 350 & $2004 ?$ & o. & 1702 & క65.? & .29 & .82 \\
\hline & j50 & 20042 & .33 & 1937 & 255.9 & $.4 i$ & .81 \\
\hline \multirow[t]{3}{*}{637} & 355 & 30505 & .28 & 2147 & 265.7 & .47 & $.8 !$ \\
\hline & 355 & ¿Q̂̇OE & .37 & 2395 & $260 . \hat{\imath}$ & .45 & .80 \\
\hline & 355 & 30305 & .45 & 2799 & 257.3 & .41 & .79 \\
\hline \multirow[t]{3}{*}{699} & JE: & 20048 & .20 & 1578 & 054.7 & .52 & .92 \\
\hline & 351 & 20048 &.$\hat{6} \bar{i}$ & 1677 & 365.3 & .49 & .82 \\
\hline & IE! & 20048 & ذَذ. & 1954 & iะ5. 9 & .47 & .81 \\
\hline \multirow[t]{3}{*}{635} & $3+9$ & 30511 & .30 & 2158 & $2 \in 5.5$ & .48 & .81 \\
\hline & 399 & 30 s̄ll & .39 & $24: 8$ & 266.8 & .44 & .80 \\
\hline & 349 & 3031! & .47 & 2956 & $\leq \leqslant 8.0$ & . 40 & .78 \\
\hline \multirow{3}{*}{655} & 975 & 10002 & $.0 !$ & 987 & 252.5 & .55 & .84 \\
\hline & 475 & $1000 ?$ & 0.00 & 904 & $26: .3$ & .02 & $.2 \Xi$ \\
\hline & $4: 5$ & 10002 & 0.60 & 862 & 260.2 & $.6 i$ & .86 \\
\hline \multirow[t]{3}{*}{60} & 486 & 20404 & .14 & 1532 & 263.5 & .54 & .83 \\
\hline & 486 & $2 \hat{1} 404$ & .18 & 1801 & 260.9 & .52 & .83 \\
\hline & 435 & 20404 & $.2 \vdots$ & 2012 & 264.3 & . & .82 \\
\hline \multirow[t]{3}{*}{054} & 481 & 10000 & .07 & 1040 & 355.0 & .56 & .84 \\
\hline & 481 & $1000 E$ & .99 & $107 i$ & 235.1 & .55 & $.8 \Sigma$ \\
\hline & $46 !$ & 10000 & .12 & i1:4 & joj.j & .54 & .85 \\
\hline \multirow{3}{*}{ bij } & iti & $2040 \hat{s}$ & .15 & 1554 & T. & تد. & $.8 j$ \\
\hline & $4 T$ & $2040 t$ & .30 & :81! & ใด้.: & .52 & . $\hat{2} 2$ \\
\hline & 476 & ใิ่งใด & .35 & 2029 & 204.5 & .50 & .22 \\
\hline \multirow[t]{3}{*}{$E$} & 475 & 30040 & $.2 !$ & 2104 & $2 \leq 4.7$ & .51 & $.8 ?$ \\
\hline & 475 & 50046 & .25 & 2407 & $2 j 3 . j$ & .99 & .21 \\
\hline & 470 & 30104 & .34 & 9779 & $i=6.0$ & .46 & .30 \\
\hline \multirow[t]{3}{*}{$=25$} & 454 & 41) $\{29$ & .27 & 2540 & iss.i & .49 & .31 \\
\hline & 484 & tiviz? & . & iljl & 260.2 & .95 & .80 \\
\hline & $45:$ & 406399 & .44 & 3709 & 257.2 &.$\$ 1$ & .79 \\
\hline \multirow[t]{3}{*}{62} & 479 & 20407 & .14 & 1501 & 263.7 & .54 & .83 \\
\hline & 479 & 20407 & .19 & $\vdots ; 88$ & 264.1 & .59 & .82 \\
\hline & 479 & 20407 & .24 & 2008 & 204.5 & .50 & .52 \\
\hline \multirow[t]{3}{*}{287} & 479 & 30046 & .21 & 2124 & 269.5 & .51 & .32 \\
\hline & 999 & 30046 & .27 & 2769 & 265.0 & .49 & .62 \\
\hline & 179 & jồ̂ंí & .34 & נ:?:בר & 25s. & . tó & $.8 !$ \\
\hline \multirow[t]{3}{*}{624} & $47^{\circ}$ & : & .23 & 2050 & $265 . j$ & $.9 ?$ & $.3 !$ \\
\hline & 479 & 400436 & . & $3: 45$ & 266.2 & .45 & .80 \\
\hline & 479 & 40430 & .45 & STI:0 & 267.3 & .41 & .79 \\
\hline \multirow[t]{3}{*}{$6 ! 6$} & 176 & 19999 & .12 & 1774 & 275.3 & .55 & .81 \\
\hline & 975 & :9998 & .17 & 1779 & 3.75. & .5נ & .81 \\
\hline & $4 i 6$ & i9999 & .22 & $18+4$ & ?7o.1 & $.5 n$ & .80 \\
\hline \multirow[t]{3}{*}{ bil } & 475 & 50104 & .19 & $2 ! 21$ & Bic.l & $.5 i$ & .80 \\
\hline & 470 & 30104 & $.2 j$ & 2196 & 276.7 & .50 & .79 \\
\hline & dis & 30104 & בנב. & 2900 & 277.4 & .48 & .78 \\
\hline 017 & 476 & 70452 & $.4 J$ & 3598 & 278.9 & .14 & .77 \\
\hline
\end{tabular}




\begin{tabular}{|c|c|c|c|c|c|c|c|}
\hline RLIN & 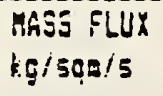 & $\begin{array}{c}\text { HERT FLUXX } \\
\text { H/SqE }\end{array}$ & $\begin{array}{c}\text { MASS } \\
\text { QUALITY }\end{array}$ & $\begin{array}{c}H T C \\
W / 5 q=/ K\end{array}$ & $\begin{array}{l}\text { Teqb } \\
\text { degk. }\end{array}$ & $\begin{array}{l}\text { LiQulo } \\
\text { CoMf. }\end{array}$ & $\begin{array}{l}\text { VAFBFF } \\
\text { COMF. }\end{array}$ \\
\hline & 278 & 70452 & .57 & 4157 & 280.8 & .58 & .75 \\
\hline & 478 & $7045=$ & .62 & 9694 & $282 . i$ & نَّن. & .70 \\
\hline \multirow[t]{3}{*}{315} & 475 & :001! & .12 & 1970 & 975.9 & .55 & .81 \\
\hline & 475 & 20011 & .16 & 1705 & 975.7 & J5 & .81 \\
\hline & 475 & zô0i1 & .21 & 1832 & 276.1 & .52 & .80 \\
\hline \multirow[t]{3}{*}{$51=$} & 475 & 30158 & .19 & 2148 & 270.1 & 55. & .30 \\
\hline & 475 & 30133 & .26 & 2204 & 276.9 & .50 & \\
\hline & 475 & 30130 & .3 & 3730 & 277.5 & .43 & .13 \\
\hline \multirow[t]{3}{*}{ ธิ์ } & $4 i \dot{o}$ & 401572 & .97 & 2400 & 277.0 & .50 & . i9 \\
\hline & 476 & $49 \div 7 ?$ & . 35 & 2619 & 277.9 & .97 & .75 \\
\hline & $4 i b$ & 40672 & .44 & jûcin & 279.0 & .45 & .76 \\
\hline \multirow[t]{3}{*}{818} & $48 ?$ & 70459 & .45 & $3: 14$ & 275.2 & .44 & .77 \\
\hline & 482 & 701459 & .55 & 4245 & 281.1 & .33 &.$T$ \\
\hline & 4E? & 700458 & .67 & $\$ 205$ & $23 j .0$ & Jנن. & .70 \\
\hline \multirow[t]{3}{*}{ blq } & 49 & - $2003 i$ &. .11 & 1776 & 275.5 & .55 & .81 \\
\hline & $4 i ?$ & 20037 & 'ś. & 1724 & 975.3 & .55 & $.8 !$ \\
\hline & 479 & 2ิง̂ิ37 & $.2 !$ & $162 j$ & $276 . j$ & .52 & . \\
\hline \multirow[t]{3}{*}{612} & $4 i 4$ & S01Jo & .19 & 2150 & 270.1 & J & .80 \\
\hline & 479 & 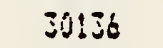 & $.2 \xi$ & $2: 17$ & 275.9 & .50 & . i9 \\
\hline & 474 & تóljó & .32 & 2452 & $27 i .5$ & $.4 a$ & .78 \\
\hline \multirow[t]{3}{*}{605} & 471 & $40 i 14$ & .27 & $269 i$ & $2 T i .1$ & .50 & is \\
\hline & $47 !$ & 40714 & .36 & 2554 & 278.1 & .40 & .13 \\
\hline & $4 i !$ & 40314 & .95 & 2399 & 979.2 & .45 & .ij \\
\hline \multirow[t]{3}{*}{520} & $5 i i$ & $995 \div 0$ & .30 & Bi? & 237.1 & $.4 E$ & $.3 !$ \\
\hline & El; & $4=540$ & .96 & 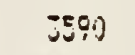 & 2tE.5 & . $\$$ & $\therefore 0$ \\
\hline & $\Xi !:$ & 29540 & .49 & $41: 8$ & 269.8 & .29 & .77 \\
\hline \multirow[t]{3}{*}{$52 !$} & 475 & 97502 & İ & $323 i$ & ibo.: & is & $.9 !$ \\
\hline & 475 & $4750 ?$ & $.4 j$ & 3074 & 200.5 &.$+\hat{6}$ & (c) \\
\hline & 475 & 49502 & $.5 j$ & $4: 98$ & 268.9 & .37 & . io \\
\hline \multirow[t]{3}{*}{607} & 482 & 60042 & .j6 & itis & ZIE. & .16 & .78 \\
\hline & $\$ 82$ & 600042 & .98 & $\$ 945$ & 279.7 & $.9 i$ & . \\
\hline & 422 & 60042 & .57 & $400 j$ & $2: 2.4$ & . 33 & . is \\
\hline \multirow[t]{3}{*}{619} & $4 i 7$ & 70430 & .42 & 3323 & 278.3 & .24 & $.7 i$ \\
\hline & $\{T i$ & 10450 & .56 & 4346 & 230.7 & .jS & .i: \\
\hline & 477 & 70450 & $.6 i$ & 4942 & 282.0 & j & . iv \\
\hline
\end{tabular}




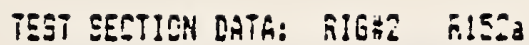

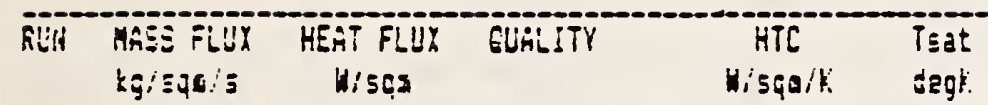

\begin{tabular}{|c|c|c|c|c|c|}
\hline $32 ?$ & 158 & 969 & .19 & 2875 & $29 \% .6$ \\
\hline $25 E$ & $15:$ & 10195 &.$\therefore$ & 3544 & ¿E: \\
\hline 257 & 159 & 10095 & .49 & 3750 & 2EST: \\
\hline 283 & 157 & 9954 & .59 & YE:? & 25.9. \\
\hline 285 & 154 & 1005 & .52 & 4400 & 204.2 \\
\hline 284 & $15 \%$ & 10011 & is & SOSOS & 250.0 \\
\hline 285 & $: 58$ & $10 j j !$ & . je & 4505 & 290.1 \\
\hline 320 & $2=9$ & 7759 & .24 & $321 i$ & 20.0 .5 \\
\hline בะะ & 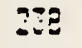 & 10195 & .36 & 4310 & $2 \div 0.4$ \\
\hline :ЕE & 200 & $10:=1$ & .48 & 4915 & 290.1 \\
\hline 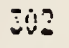 & 240 & $10 ! 15$ & .50 & $5 \$ 50$ & 289.9 \\
\hline 54 & 246 & 10102 & .70 & $0 \div 20$ & 29.5 .5 \\
\hline 30 & 336 & 9976 & .93 & $84: 3$ & :99.3 \\
\hline Ian & $2 \cong$ & 的浩 & .19 & $3: 50$ & 290.1 \\
\hline 994 & 2EE & 10905 & .29 & $43: 5$ & 29.9 .4 \\
\hline $3=9$ & $25 ?$ & 10155 & .87 & 5022 & $29 \dot{\mathrm{u}} . j$ \\
\hline 290 & İJ & 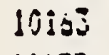 & .49 & 5359 & 290.06 \\
\hline :\$! & ¿כj & 10159 & .80 & $6=85$ & 299.0 \\
\hline $29 ?$ & 277 & 10199 & .12 & $730 \mathrm{i}$ & 290.1 \\
\hline 2E: & 28! & $1 \hat{10}: \Omega 5$ & .75 & 7105 & 290.2 \\
\hline$\therefore 4$ & L97 & Q:ंE & .79 & $7 \$ 25$ & 296.3 \\
\hline כai & 340 & 9700 & .15 & 河: & 230.4 \\
\hline 5 & i5i & 10130 &.$a^{n}$ & $95: 5$ & 990.4 \\
\hline $8:$ & 3 & 10191 & .30 & 5017 & 350.2 \\
\hline $3 j$ & 359 & 10101 & ? & 5990 & 290.5 \\
\hline 95 & 添ó & 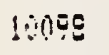 & .45 & E:10: & 290.0 \\
\hline :i: & IST? & $: 0017$ & .52 & 7374 & 290.0 \\
\hline 311 & joc & 9975 & . & 8418 & 250.1 \\
\hline 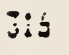 & $3 j:$ & lvitit & . $5 ?$ & $E \equiv 75$ & 289.9 \\
\hline$\overline{X I}$ & 419 & 9૯รด & .11 & उ:89 & 270.6 \\
\hline 39 & $4: 3$ & $956 ?$ & .18 & 4010 & 990.4 \\
\hline 299 & $4: 5$ & lôlct & .24 & $5: 23$ & 390.5 \\
\hline $30 j$ & 427 & 00096 & .30 & 5955 & 299.5 \\
\hline 115 & ใบิ & 9954 & .306 & î̃.5 & 290.6 \\
\hline 368 & 430 & 9993 & .43 & 7459 & 290.0 \\
\hline $3: 2$ & 420 & $90: 5$ & 50 & gE?0 & $2 ? 9.1$ \\
\hline$z:$ & $1: S$ & ¿9ioti & .27 & 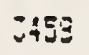 & 290.2 \\
\hline 340 & 158 & 29214 & .49 & \$01? & 290.3 \\
\hline 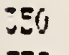 & $14 ?$ & 19660 & .57 & 3909 & 290.0 \\
\hline 58 & 150 & $=9248$ & .71 & 4640 & 290.2 \\
\hline 349 & 150 & $1: 438$ & .91 & 5105 & 290.0 \\
\hline 32 & 150 & 20037 & .81 & 5120 & 290.1 \\
\hline \$9? & $2 a b$ & 20261 & .30 & isal & 20.0 .2 \\
\hline 357 & 238 & 20202 & .50 & 4277 & 290.6 \\
\hline jil & ZBo & 6ิ057 & .40 & 5191 & :39.9 \\
\hline jut & 995 & 20003 & .51 & 2560 & ? \\
\hline
\end{tabular}


TEST EETION GHTH: RIER2 RIS2a

\begin{tabular}{|c|c|c|c|c|c|}
\hline 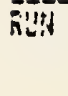 & MASE Fitjo & 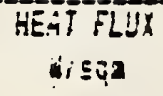 & GUnilit & $\begin{array}{c}H i 6 \\
W / s q 2 i k\end{array}$ & $\begin{array}{l}\text { Tiat } \\
\text { deghk }\end{array}$ \\
\hline 34 & 297 & 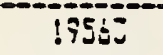 & .53 & $7 ! ! 9$ & 200.4 \\
\hline 344 & 285 & $18: 30$ & .75 & $79: 2$ & 990.4 \\
\hline 346 & 25 & $15: 91$ & .85 & 3159 & 290.10 \\
\hline فَنْز & 423 & 20183 & .17 & 4104 & 5.9.3. \\
\hline 30 & 429 & 199909 & .25 & $5 \div 50$ & 299.9 \\
\hline 353 & 429 & 20042 & .31 & 0927 & $26 j .0$ \\
\hline 341 & 425 & 19597 & .38 & 3904 & 290.4 \\
\hline 345 & 420 & 1 :505 & .45 & 7936 & 290.6 \\
\hline 345 & 424 & 19143 & .52 & $825 i$ & 290.1 \\
\hline iq? & 424 & 17356 & .50 & $1050 ?$ & 290.5 \\
\hline
\end{tabular}

TEST SECTION DATA: RIELE RISBI

\begin{tabular}{|c|c|c|c|c|c|c|}
\hline hivi & 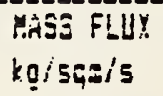 & $\begin{array}{l}\text { HEAT FLUV } \\
\text { WISGQ }\end{array}$ & GUALITY & $\begin{array}{l}\text { HTIF! } \\
\text { WISqMik. }\end{array}$ & $\begin{array}{l}H: E(i) \\
\text { Wisquik }\end{array}$ & $\begin{array}{l}\text { T三at!ff } \\
\text { deç: }\end{array}$ \\
\hline 5196 & S & 10048 & .37 & 晹定 & 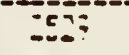 & 55.3 \\
\hline 502 & 350 & 10048 & ? & 9 & 8097 & 254.2 \\
\hline 503 & 589 & 10053 & .50 & ספ: & 3275 & 254.1 \\
\hline 5.4 & 359 & 10048 & $.5 ?$ & AIPJ & $3+5$ & 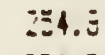 \\
\hline 505 & '5' & 16057 &.$i b$ & $45: 9$ & 注: & 254.3 \\
\hline 1 & 45 & $10:: 5$ & .22 & 5050 & 2753 & :54. \\
\hline 489 & 974 & 10107 & .31 & 2931 & $2=33$ & 354.0 \\
\hline 490 & i41 & $: 01: 3$ & .40 & Sill? & 5927 & $35+.3$ \\
\hline is? & 451 & 10104 & 99. & उ95 & 3513 & -54.5 \\
\hline $48 j$ & 432 & 10005 & .64 & 4795 & z8B8 & $254 . ?$ \\
\hline 498 & 445 & 10107 & .69 & $52 \leq ?$ & $411 j$ & $=54.0$ \\
\hline 40 & $45 !$ & 9938 &.$\pi$ & 5903 & 1679 & $254 .:$ \\
\hline 492 & 5E9 & lú1s7 & .25 & 3410 & $=0,05$ & $=54.4$ \\
\hline 480 & 553 & 9992 & .23 & -85? & उश?: & $\therefore 5+.=$ \\
\hline 405 & $\Sigma_{64}$ & lelói & .7 & ذزَ: & 注注 & $\therefore \Sigma: \Xi$ \\
\hline $4 \hat{e} 2$ & 554 & 9987 & . is & $48: ?$ & 5900 & $\therefore 4.5$ \\
\hline 494 & 502 & 10105 & .52 & 5 5jô & il:- & .54 .5 \\
\hline 979 & 550 & $2 \cos 1$ &.$\dot{0}$ & $50: 34$ & 46 i2 & $=54.9$ \\
\hline 495 & EE5 & !v̂!Do & .68 & 5798 & 4745 & 254.7 \\
\hline$\$ 77$ & Etó & $999 ?$ & .79 & 7258 & $5: 37$ & .5 \\
\hline
\end{tabular}




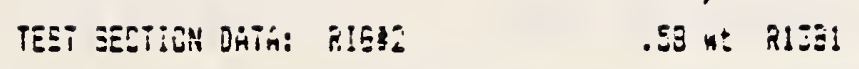

\begin{tabular}{|c|c|c|c|c|c|c|}
\hline FiLi & kg/Eqn/s & $\begin{array}{c}\text { HET SILU } \\
\text { disGs }\end{array}$ & $\begin{array}{l}\text { MÁ̄ं } \\
\text { QUALITY }\end{array}$ & $\begin{array}{c}\text { hit } \\
\text { Hijosit. }\end{array}$ & Jeg: & $\begin{array}{l}\text { bigl: } \\
\text { coms. }\end{array}$ \\
\hline
\end{tabular}

\begin{tabular}{|c|c|c|c|c|c|c|}
\hline 350 & 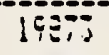 & .45 & $24: 4$ & SET & .41 & .75 \\
\hline :3ะ5 & 20253 & .59 & 5ذ' & 239.9 &.$j \dot{i}$ & .it \\
\hline $35 i$ & 30274 & .49 & 2800 & 201.9 & $.4 \dot{0}$ & 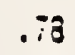 \\
\hline 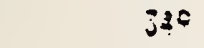 & 30230 & .03 & 3ial & 270.5 & בَבُ. & .75 \\
\hline $4: 5$ & 9998 & .17 & $15 i s$ & 263.0 & .53 & $.8 j$ \\
\hline $4{ }^{2}$ & 10067 & .30 & 1810 & 265.9 & 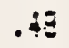 &.$\$ 1$ \\
\hline 481 & 20019 & .20 & 1793 & 25:.9 &.$\Sigma \overline{3}$ & .32 \\
\hline $4 \overline{i b}$ & 20019 & .34 & $24: 9$ & 265.5 & .40 & $.8 !$ \\
\hline 970 & 20015 & .45 & JIJ & 207.5 & $.4 !$ &.$i t$ \\
\hline 484 & 200015 & كذة. & iF & 269.0 & ت5 & .75 \\
\hline 479 & 30217 & S & $25: 3$ & $2 \cos ^{2} \mathrm{z}$ & .45 & .89 \\
\hline 479 & 20011 & .77 & jồ & $2 \leq 7.5$ & .40 & .78 \\
\hline 479 & 30609 & .59 & $45 i 98$ & 209.5 & .34 & .75 \\
\hline 476 & $999 ?$ & ? & 1949 & 270.7 & .49 & .79 \\
\hline 473 & 10000 & .42 & 2597 & 278.5 & .44 & .79 \\
\hline Ais & 10034 & .90 & 32 ?०ी & 2E4.5 & .29 & $.8 \Sigma$ \\
\hline 475 & 20013 & $.3 i$ & 2379 & 277.0 & .28 & .70 \\
\hline 475 & 20000 & .44 & 9077 & 278.9 & .45 & .76 \\
\hline 475 & 200148 & فj. & javid & 200.8 & . & .75 \\
\hline 482 & 19957 & .31 & $4=44$ & LEE. 2 & .29 & .85 \\
\hline 479 & 30109 & בَ'. & 2587 & 277.5 & .48 & .75 \\
\hline 474 & 5008 & .46 & 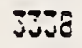 & 279.2 & .92 & $.7 j$ \\
\hline $4 i:$ & 29859 & .59 & $34 \div 1$ & 2Ei.j & 6 & .7 \\
\hline 517 & jug:! & .83 & $500:$ & 271.9 & J'ن. &.$i j$ \\
\hline 45 & ijogs & ? & 5280 & $27 i .2$ & .0 & .il \\
\hline 99 & 50024 & . & $8: 94$ & SS:.3 & .30 & $.3 i$ \\
\hline $47 i$ & 30976 & 5 & $5 ! 50$ & $255 . !$ & .27 & .64 \\
\hline
\end{tabular}




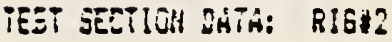

.18 wt in $138 \mathrm{~B}$

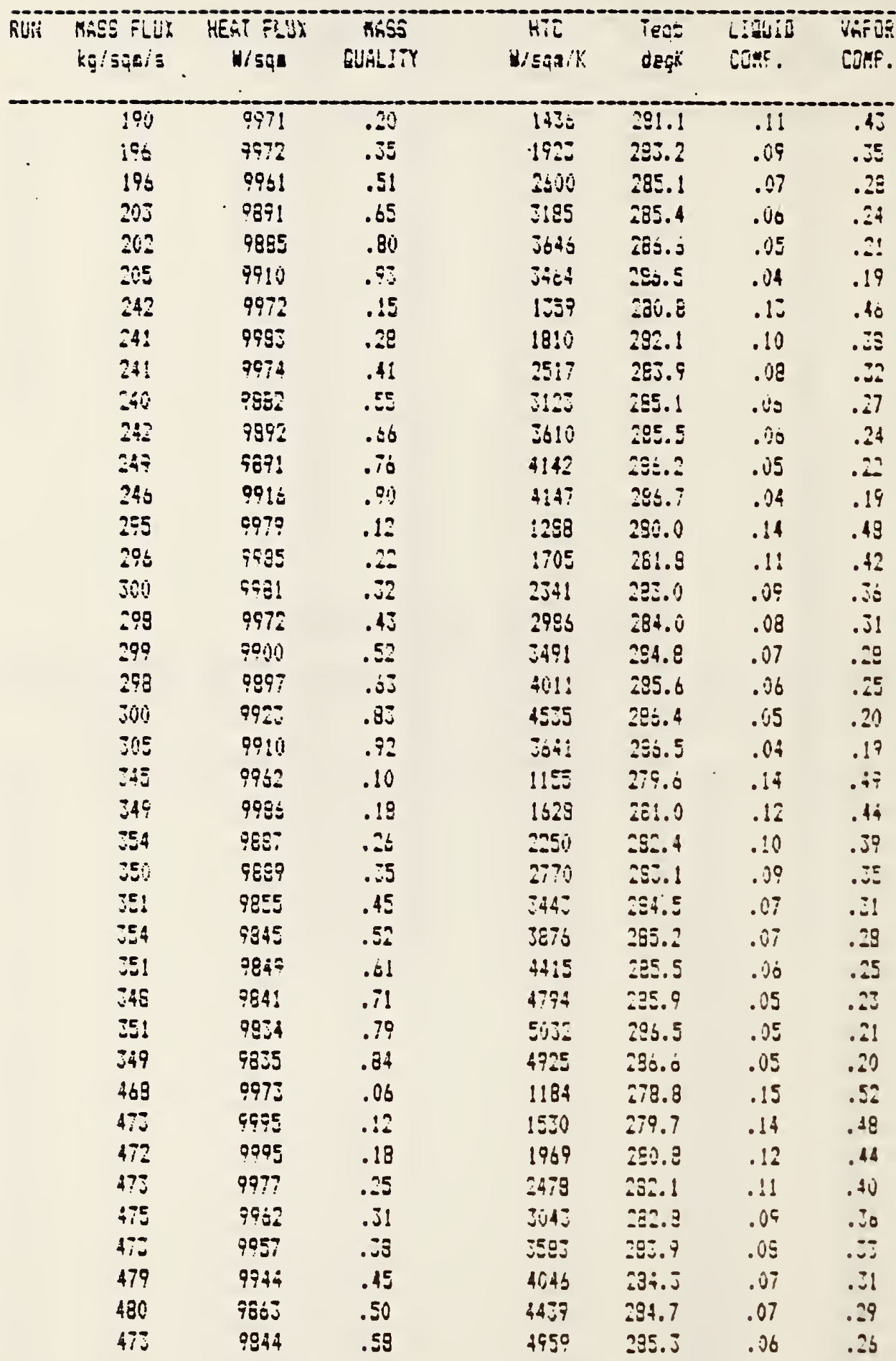




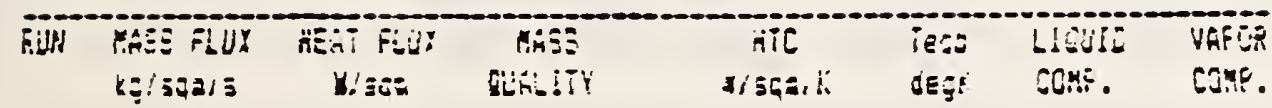

\begin{tabular}{|c|c|c|c|c|c|c|}
\hline 369 & 10075 & .29 & 1757 & 252.4 & .77 & .28 \\
\hline 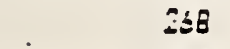 & $10 \hat{s}$ & E & $.18 \div 8$ & 258.7 & $.7 i$ & .88 \\
\hline iss & 16073 & .57 & 2000 & 959.4 & .71 & $.8 i$ \\
\hline $3 \dot{3}$ & GOHE & .09 & 2202 & 200.0 & .67 & .85 \\
\hline 27 & 10045 & .50 & 2550 & 201.5 & إ. & .85 \\
\hline$\because 2$ & liviou & $.9 j$ & 32 & 3ड்'. & .54 &.$E Z$ \\
\hline 271 & $1005 \div$ & .98 & 3457 & 265.4 & .45 & .21 \\
\hline 390 & $190: 0$ &. is & $14 t 4$ & 257.5 & .78 & . $三 ?$ \\
\hline 375 & $1000 !$ & .28 & $1: 95$ & 255.4 & .17 & . $\mathrm{E}$ \\
\hline 污 & $103 ! 1$ & .80 & 1950 & ZES.: & .75 &.$\subseteq$ \\
\hline Isó & 10157 & .49 & $219:$ & 25E.? & .73 &.$\therefore 5$ \\
\hline $\mathrm{Bez}$ & 10160 & .59 & $290 \%$ & בร:Y.5 & .71 & .87 \\
\hline $35 t$ & $10: 174$ & . & 2659 & 250.3 & .65 & . \\
\hline 304 & $: 00: 70$ & .75 & 29870 & 281.1 & .04 &.$\Xi \Xi$ \\
\hline Si:? & $1000=$ & .87 & 3407 & 2629 & .57 & .84 \\
\hline 32 & LONG4 & .97 & 5994 & CÉ4.9 & .45 & .51 \\
\hline$\$ 27$ & 10102 & .84 & 2065 & $25 i .7$ & .99 & .89 \\
\hline 459 & 10025 & .29 & 2500 & 250.2 & $.7 \%$ & . SE \\
\hline 449 & 10032 & .46 & 3505 & 258.1 & .74 & .88 \\
\hline 461 & $10: 27$ & .60 & $2: 75$ & 255.9 & .70 & $.3 i$ \\
\hline 4.55 & 10134 & .75 & 3051 & $2 \leq 0.6$ & .35 & s \\
\hline$\{c \bar{T}$ & 10123 & .93 & 5114 & $9 \leq \div .2$ & .59 & .84 \\
\hline $40 j$ & 10050 & .94 & 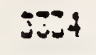 & 204.3 & .50 & E? \\
\hline $54 t$ & १९९! & $.3 i$ & 3099 & 25e.2 & . 汸 & .89 \\
\hline 55 & 10018 & .40 & 3 & ZEQ.j & . 污 &.$\approx 8$ \\
\hline 554 & 10244 & .41 & $265 !$ & ZES.S & .75 & .88 \\
\hline 547 & 16029 & .46 & $37 ! 9$ & 355.3 & .74 & . 35 \\
\hline $55 !$ & 10145 & .00 & JôE: & 257.7 & ה & $.3 i$ \\
\hline 550 & 10240 &.$j i$ & $3 ! 27$ & 2 ¿่งิ.0 & .28 & . St \\
\hline 540 & 20075 & .79 & 5995 & 231.5 & .32 & .95 \\
\hline 695 & 20145 & .15 & 1999 & $257 . i$ & .79 & .99 \\
\hline $084^{\circ}$ & 10265 & .18 & $2 !: 55$ & 258.2 &.$i 8$ & .89 \\
\hline เลอิ & 10129 & .26 & 35014 & 25E.? & .77 & .89 \\
\hline 579 & 10:?:! & $.20^{\circ}$ & 3728 & 2ธริ.2 & .77 & .39 \\
\hline โว! & 10150 & .jô & 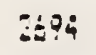 & 255.6 & $.7 !$ & . $\Xi E$ \\
\hline :งต: & 10059 & .37 & $2 \mathrm{sige}$ & 358.4 & .75 & .80 \\
\hline 5 & 10140 & . & $29 \leqq 8$ & 255.5 & .75 & .88 \\
\hline 54 & 10114 & .46 & 5102 & 259.0 & it & .98 \\
\hline SS3 & 10154 & .47 & 3132 & 253.1 & .74 & .53 \\
\hline نَ: & $101: 3$ & .59 & $345 !$ & 259.5 & .71 & .97 \\
\hline 621 & '005 & .ol & 5042 & $26 \hat{v} .3$ & . & .37 \\
\hline 700 & 10039 & .68 & 5586 & 250.2 & .62 & .86 \\
\hline
\end{tabular}


IEST SECIIOH DATH: SIGI?

.jE nt R!:E!

\begin{tabular}{|c|c|c|c|c|c|c|}
\hline $\begin{array}{c}\text { Sivi Mñs Finy } \\
\text { kg/sça!s }\end{array}$ & 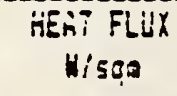 & $\begin{array}{l}\text { MAES } \\
\text { QUHLITY }\end{array}$ & $\begin{array}{l}\text { HTC } \\
\text { Wisosik' }\end{array}$ & ieqt & $\begin{array}{l}\text { Lienid } \\
\text { Comf. }\end{array}$ & $\begin{array}{l}\text { OAFCS } \\
\text { COME. }\end{array}$ \\
\hline 208 & 492 & .45 & 1217 & 30.4 & .19 & .59 \\
\hline Sot & $6=2:$ & .45 & $15 \div 2$ & 27.5 & .19 & .55 \\
\hline $\begin{array}{l}260 \\
-08\end{array}$ & $\begin{array}{r}9934 \\
101: 5\end{array}$ & .52 & 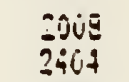 & $\begin{array}{l}279.0 \\
279.1\end{array}$ & $\begin{array}{l}.17 \\
.15\end{array}$ & . \\
\hline 245 & $10: 05$ & .72 & ?รE?: & 290.5 &.$: J$ & .10 \\
\hline 345 & 10106 & . & 395' & $2 \Xi 1 . !$ &.$\therefore$ & $.4 j$ \\
\hline 204 & [5.ง9! & .88 & jงi? & $282 \hat{.0}$ &.$\vdots 1$ & .90 \\
\hline 280 & 10192 & 0 & 1450 & ב?:. & 5 & . \\
\hline 253 & 10032 & .38 & 18:: & 27.2 & .22 & $.6 ?$ \\
\hline 299 & 9935 & .45 & 2051 & 970.1 & .20 & ji \\
\hline $29 ?$ & 10030 & .51 & 2573 & 277.3 &.$j a$ & 5. \\
\hline 297 & soits & .64 & ili! & 279.4 & .14 & .50 \\
\hline 295 & 10013 & .74 & 3557 & $2 \equiv 0.5$ & $.1 \vec{i}$ & $.4=$ \\
\hline 239 & ?9९5 & $.8 i$ & 3924 & $-3 ! .7$ &.$: !$ & $.4:$ \\
\hline$\dot{j} \dot{\theta}$ & 9955 & .93 & 1010 & $2: 9 ?$ & .95 & i ذ. \\
\hline $36 j$ & 959 ? & . & 1801 & 97.7 & $.2 j$ & .05 \\
\hline 393 & $390 !$ & .35 & 2159 & 275.0 & .99 & SO \\
\hline 374 & SSO0 & .47 & 2.70 & 270.5 & .19 & .55 \\
\hline Sis & 3771 & $.5 j$ & j=59 & วิธ.। & .10 & .5 \\
\hline $57 \%$ & 9750 &.$\$ 5$ & 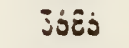 & 979.5 & .14 & .49 \\
\hline 374 & 9746 & .73 & 40ं:? & 250.5 & $.1 j$ & .10 \\
\hline Siin & 9791 & J5. & $i E \hat{u}$ & $=8 ! .6$ & .11 & .45 \\
\hline isi & 7957 & .95 & $15 \overline{5}$ & $27: 2.4$ & $\therefore$ & $.0 \bar{E}$ \\
\hline$A \dot{k} T$ & !OCist & .35 & $21 \leq 5$ & 2750 & .21 & .05 \\
\hline$t_{0} i$ & 10045 & $.4 !$ & 9575 & 275.3 &.$\therefore !$ &.$c !$ \\
\hline ici & 10045 & 40 & $22: Z$ & 97.8 & $\therefore \Xi$ & .5 \\
\hline $44 i$ & 10039 & .50 & 50.9: & 0.3 .5 &.$: 5$ & .52 \\
\hline 453 & soop! & os. & :@o! & 979.9 &.$i q$ & .49 \\
\hline 967 & i0035 &.$i !$ & $255 i$ & 230.00 & .15 & $.9 i$ \\
\hline$\$ 40$ & $=986$ & .30 & $5: 97$ & :2!.: &.$\therefore$ & . \\
\hline 523 & 10027 & .190 & $1: 18$ & 207.3 & jj. & .ii \\
\hline ?ִּ? & $1004 !$ & .12 & 1404 & 271.2 & . &.$i !$ \\
\hline 592 & 10054 & .27 & 1579 & $29 . .7$ & $.2 b$ & . \\
\hline Ei! & 10031 & . it & 307 & 379.1 & .93 & .64 \\
\hline $53 \overline{5}$ & 10029 &.+1 & 2728 & 275.0 & $\therefore 9$ & $.6 i$ \\
\hline 543 & 20010 & .47 & $\forall !: \varepsilon$ & $\because 7 \div$ ? &.$: 9$ & .5 \\
\hline $5: 5$ & 100079 & o & 3990 & $5: 8.19$ & $\therefore 0$ & .55 \\
\hline 520 & 9990 & . 50 & $-25=$ & $=i \Xi .9$ & .15 & $.5 !$ \\
\hline 530 & 100:2 & . & :235 & $=79.6$ & .14 & .48 \\
\hline
\end{tabular}




\section{APPENDIX 4A: ALTERNATE SUPPRESSION CRITERION}

Three alternate suppression criterion were found in the literature. All are based somewhat on Chaw la's original suggestion: to decide on the paper heat transfer regime one should calculate the heat transfer coefficient based on an accurate pool boiling relation, and again on an accurate forced convection/evaporative relation (e.g.. one based solely on m or $X_{t t}$, bot not heat flux). The larger a determines the correct beat transfer regime.

Collier has used Dengler and Addoms relation:

$$
\alpha / a_{L 0}=A\left(1 / X_{t t}\right)^{B}=q / \Delta T \quad A=3.5, B=0.5
$$

and combined it with an 'onset of nucleate boiling' criterion of Davis and Anderson similar to that derived by Hsa:

$$
\Delta T={\frac{8 \sigma q T_{s a t}}{\Delta h_{v} \lambda_{L} \rho_{v}}}^{0.5}
$$

to yield

$$
q=\frac{49 a_{L O}{ }^{2}}{\lambda_{I} x_{t t}} \frac{2 \sigma T_{s a t} a_{L O}}{\Delta h_{v}}
$$

Polley employed a similas approach; however, be used Cben's equation combined $\nabla i$ th $(4 \mathrm{~A}-1)$ to yield: 


$$
\Delta T_{i}=\frac{2 \sigma T_{s a t}}{\Delta h_{v}}\left(V_{v}-V_{L}\right) \frac{1}{I_{c}\left(1-\frac{F \alpha_{L O}}{\lambda_{L}} I_{c}\right)}
$$

If the given $\Delta T<\Delta T_{i}$, the flow is considered to be absent of nucleate boil ing.

Shab has recentig correlated a large amount of refrigerant data with a new correlation described in Chapter 6. He otilizes Chavla's suggestion exactly and employs the dimensionless number

$$
C_{0}={\frac{1-I}{I}}^{0.8}{\frac{\rho_{\nabla}}{\rho_{L}}}^{0.5}
$$

and relates co to an evaporative heat transfer coefficient. By a separate relation, he calculates a nucleate boiling a. The larger of the two determines the flow regime. His method therefore oquates dominant evaporative heat transfer regime with the complete suppression regime. This may not be true. As show in Chapter 6, the criterion frequently selected the rrong flow regime and badly predicted the experimental data. 
APPENDIX 4B: REVIET OF CORRELATIONS $a / a_{L}$ WITH $x_{t t}$

A common form of a correlation of the heat transfer coefficient witi fluid flow parameters is

$$
\frac{a}{a_{L}} \nabla s f\left(\frac{1}{x_{t t}}\right)
$$

Hesler (9) has examined such a form noting:

$$
\begin{aligned}
& a_{L}=0.023 \frac{\lambda_{L}}{D}\left(\frac{4 G}{\pi D_{\mu_{L}}}\right)^{0.8}\left(\frac{\rho_{L}}{\lambda_{L}}\right) 0.4 \\
& \left(\frac{1}{X_{t t}}\right)=\left(\frac{x}{1-x}\right)^{0.9}\left(\frac{v_{v}}{v_{L}}\right) 0.5\left(\frac{\mu_{V}}{\mu_{L}}\right) \cdot 1 \\
& a=\frac{q / A_{c}}{\Delta T}
\end{aligned}
$$

He then approximates:

$$
\left(\frac{x}{1-x}\right)^{0.9} \cong x
$$

$$
x G \Delta b_{v}=\int_{0}^{z} \frac{g}{A} \pi D d z
$$




$$
=\frac{\int_{0}^{z}(q / A) \pi D d z}{G \Delta h_{v}}
$$

where $z$ is the distance from the point of interest and the saturated BPL.

Equation 4B-6 assumes saturation conditions at $z=0$. Substituting equations $4 \mathrm{~B}-2$ and $4 \mathrm{~B}-4$ int 0 the 1 eft side of $4 \mathrm{~B}-1$, and $4 \mathrm{~B}-6$ into $4 \mathrm{~B}-5$ and $4 \mathrm{~B}-5$ into $4 \mathrm{~B}-3$, one gets:

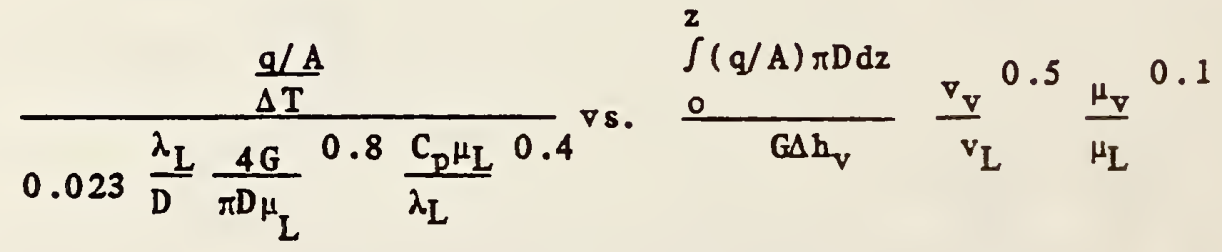

If one now assmes slowly varying properties, the equation reduces to:

$$
\frac{\frac{q / A}{\Delta T}}{G^{0.8}} \cong \frac{\int^{2}(q / A) d z}{G} c_{1}
$$

where $C_{1} \cong a$ constant made op of fluid properties. Mesler then approximates $G^{0.8}$ as $G$ to get

$$
\frac{1}{G} \frac{g / A}{\Delta T} \cong C_{1} \frac{1}{G} \int_{0}^{z} \frac{g}{A} d z
$$

The right side must remain in integral form since in the Denglor and Addoms experiment with which Mesler is concerned, there was a axial 
variation of heat flax. 1 If the integral does not varg sharply in the axial direction, one should get a strong correlation between the 0 sides of equation 4B-9.

This is as far as Mesler took the derivation. If one has a constant arial heat flux, then the last equation reduces to:

$$
\Delta \mathrm{T} \approx \mathrm{ct}=\mathrm{c}_{2}
$$

where the constant includes the presupposed slowly varying fluid properties. Yet, temperature differences do not remain constant in evaporating flows. This derivation leads then to an apparent contradiction: on the one hand, $a / a_{L}=f\left(1 / X_{t t}\right)$ yields good agreement since many of the same parameters appear on both sides of the equation. On the other hand, when the derivation is extended to constant heat flur, resuling in equation 4B-10, disagreement appears between experimental data and the form of the equation.

The contradiction in fact resuls, not from the extension to constant heat flax, but fram the overabundance of rounding and approximation. The assumption pot forth in equation 4B-5 is valid only at relatively 10r qualities. At a quality of .5, the error in the assumption is 100\%, at large qualities the error is even greater. Thus, the $(x /(1-x))^{0.9}$ parameter should not be approximated as stated in (4B-5) except at qualities less than 0.2 . This accounts for the variation one sees in

'In protesting Mesler's analysis, Standiford points out that (q/A) varied arially, and that Hesler did not consider this fact. Actually, Hesler is very careful in this rogard, as seon above. 
heat transfer coefficient in evaporating flow, and helps oxplain the legitimacy of using $\left(1 / X_{t}\right)$ as a correlating parameter. 
APPENDIX 4C: VISUAL EVIDENCE: REVIET OF LITERATURE

Hewitt et al [He63] boilt an experimental rig designed specifically to observe nacleation on a steam core-rater film vertical flow, as show in Figure 4C-1. A double annalus was formed with an inner metal rod and two glass tubes. Water could be introduced as a film attached around the rod and steam introduced in parallel with the water in the remainder of the inner annulus. The glass tube wall was kept clear and free of condensation by forcing hot air through the outer annulas. Both steam and water vere introdaced near saturation conditions, and heat applied directly through the inner metal rod. The experimental arrangement allowed film thickess, flow rate and heat flux to be varied. High speed films were used to observe rapid processes. The authors observed qualitatively that bubble nucleation depended on the flow Iates involved. At high steam velocity, as occur in flow boiling processes, no bubbles were seen; this study therefore supports the notion of a complete suppression of nucleate boiling. The authors al so noted that, for loweI steam velocities, when nucleate boiling was observed, that the heat fluz determined the number of sites and activity level of nacleation. The high speed film was shown recently [He84] and one cond observe nucleation at a particular site whenever a liquid wave passed over it. When the wave passed and the film thicbess receded, the nocleation disappeared.

Tippets [Ti62] attempted to observe flow petterns ấ vertical upward flow of high pressure boiling water at various heat flures. A rectangular channel was boilt with heater strips on two sides. High spood 
films ( 4300 frames/sec) were taken through the unheated sides (Figure 4C-1b). In the annalar flow regime and qualities of 45-60\% (i.e., very thin films), the anthor noted less but clear agitation of the 1 iquid film on the wheated surfaces as compared to the heated sarfaces. He considered this agitation the result of bubble growth within the liquid film; calculations revealed there to be sufficient wall superhest for bubble formation.

Hosler [Ho63] noted that the Tippetts study suffered from a 1 ack of depth perception. He constructed a horizontal rectangular channel with the bottan surface being electrically heated. The two sides were made of quartz prisms, allowing the sides as well as the top to be viewed simaltaneously (see Figure 4C-1c). He filmed medim pressure boil ing water at $4000 \mathrm{frames} / \mathrm{sec}$ as $\mathbf{\text { well }}$ as took still photographs of $0.5 \mu-$ soc exposure duration. His still photographs were mach clearer than those of Tippets. An annular flow pattern was observed at a los calculated quality. Very few bubbles were observed, and be conclades, 'when the vapor colmn nearly fills the channel, the mochanism of heat transfer apparently changes fram bubble generation to surface evaporation.' There may, however, be a bias in Hosler's study: he mentions, in pass ing, that the heater strip was 'machinod' to ensure uniform heat goneration. The machining process may have eliminated mang nucleation sitos, restricting potential babble growth.

The study of Berensen and Stone [Be63] differs fram those previously mentioned in several interesting was. They observed tho vaporization 
of $\underline{\text { R113 }}$ in a horizontal tube using constant temperature air as the heat source. It therefore differs from the studies in terms of fluid type, flow orientation, and boundary condition (constant temperature, Iather than constant heat flux). The refrigerant flowed inside a pyrez tube which was surrounded by a rectangular quartz duct (Figure 4C-1d).

Inside the annulus, $800^{\circ} \mathrm{F}$ air passed in connterflow to the refrigerant. Subcooled refrigerant entered the heated chamber and exited at moderate to high quality, depending on the amount of subcooling. Initially high speed films ( 7000 frames/sec) had poor resolution between the 1 iquid and vapor; the authors then added a refrigerant-coloring agent (used in leak detection) in a concentration of about $1 \% \nabla t$. They considered resolntion to be excellent. Berenson and Stone observed few bubbles in the film but considered the effect on the rate of vapor generation to be negligible. The authors conclude:

[Al thongh] nucleation of bubbles on the wall was observed whenever the wall was wet, in all flow regimes, (. . .) the dominant heat transfer mechanism in annular flow is conduction and convection through the 1 iquid $f$ il m on the wall. The vapor formation process occurs primarily at the interface between the 1 iquid annul us and the vapor core, and not by the formation of bubbles within the 1 iquid annulus.' 
Onfortunstely this study, like that of Hosler, suffers fran the potential lack of nucleation sites in that a pylex tabe was osed. Also Rl13 has a very small contact angle $\left(4^{\circ}\right)$ [Be84], so that very few cavities were unetted in all likelihood.

Staub and Zuber [St66] al so observed flow patterns with $R 22$ flowing vertically in a glass tube (quality range 0.14 to 0.22 ). An electrically conducting transparent coating was bonded to the inside of the tube. Observations and photographs were made with the conclusionabot the annolar flow regime: 'this well-defined mechaniso consists of a vapor core with or without entrained droplets and a liquid annus on the wall that is of ten quite thick and wavy and sometimes still contains very $\sin a 1$ vapor bubbles.'

Gouse and Common [Go65] used a similar arrangement as [St66] with Rl13 except that the coating $w a s$ placed on the outside of the tube. They observed suppression of nucleation whenever an annalar flow was observed. Again, their glass tubes did not contain the full range of activation sites, and Rl13 has a very small contact angle.

Mesler [Me77] reviews several studies of nucleate boiling in stationary and moving thin liquid films. In his ow study, high speed movies were taken of boiling of stationary water on metal surface. An artificial nucleation site was created in the surface; very near the site, rapid response small thermocouple was installed and polished flush with the surface. Films were taken which photographed simulaneously the bobbles 
growing and departing the surface and the thermoconple reading on at oscilloscope face. With this arrangement he was able to monitor local cooling (i.e., heat transfer) rates and bubble position. He observed high heat transfer Iates in the small area under the bubble for short periods. It is the high heat transfer rate which led him to consider boiling as the principal mechanism with all thin films. 

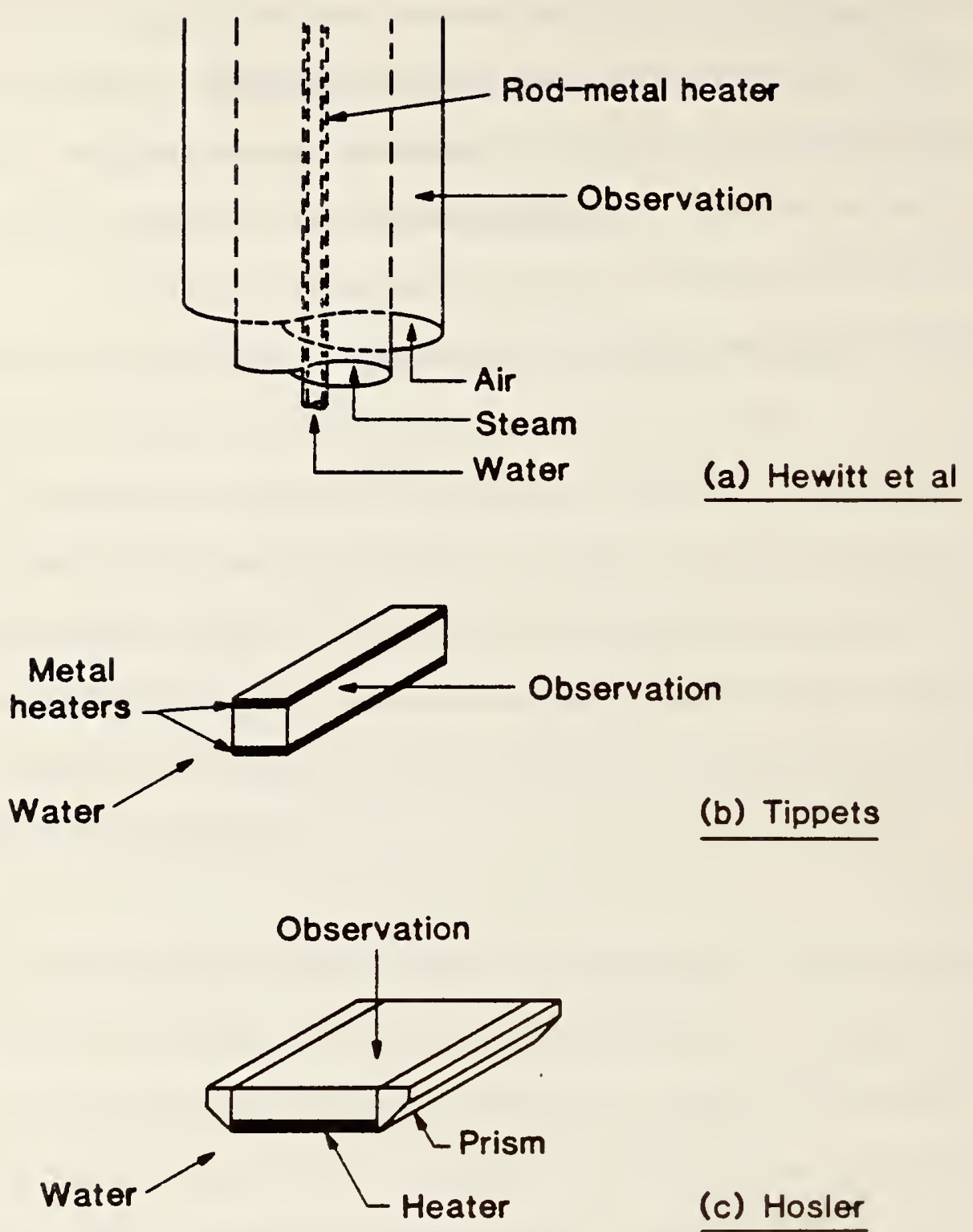

(c) Hosler

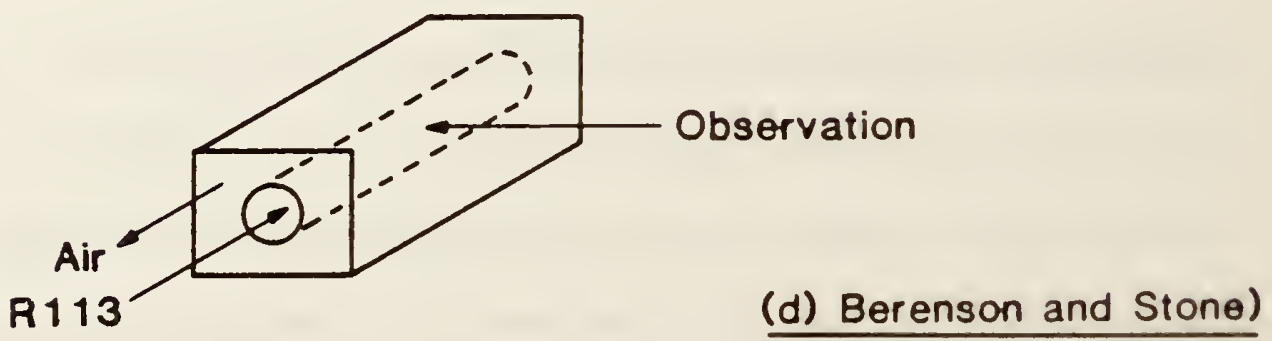

Figure 4C-1: Visualization Methods 
APPENDIX 4D: DEPENDENCE ON HEAT AND MASS FLUX Nacleate boiling suppression was first proposed by Dengler and Addams [De56] based on an experiment with vaporizing water which flowed inside 1 in. OD copper tubes. Steam was used as a heat source in five separate sections of a 20 foot copper tabe. Tall thermocouples were embedded in the tube wall, and pressure taps were installed at the entrance and exit of each section. Of note, the void fraction was measured to within $10 \%$ asing a radioactive tracer. The idea of suppression was advanced on the following experimental and theoretical observations:

(1) Heat flas increased sharply along the tabe; the quality increased as well but the wall-steam temperature difference remained constant, i. e., heat transfer coefficient increased sharply with quality.

(2) Both 1 iquid and vapor velocities could be obtained fram the measured void fraction and mass flor rates. An average velo city of the tro phase mirture was calculated and ased in a single phase convection heat transfer correlation to predict the heat transfer rate. Good agreement was achieved over mach of the tube, excepting the 10 quality portion.

(3) The use of a flow parameter, $1 / \mathbb{Z}_{t t}$, correlated the data fairly we11, except at 10\% quality.

(4) Previous studies showed that forced comection raised the value of the wall superheat necessary to initiate nacleate boiling. Thas, if one raised the velocity safficiently, nacleation should cease. 
At 1 ow quality, Dengler and Addoms considered both convection and nacleate boiling to be important, and their effects to be superposed. This conclusion is derived from the anderprediction of heat transfer at low quality by the correlation techniques described in observations and (3) above.

Mesler [Me77] examined in detail the data of Dengler and Addams. Dengler and Addoms claimed no correlation between measured heat flax and wall superheat (observation (1) above). Mesler reviewed the raw data fran Dengler's report, and rejects for various reasons (dryout, highly fluctuating wall temperatures, erratic thermocouples) over 100 of Dengler's original 185 data points. The original Dengler and Addams plot of $a / a_{L}$ versus $1 / X_{t t}$ showed a few points at 1 ow quality to have relatively high values of $a / a_{\mathcal{L}}$, and the authors attributed these points to have been caused by the presence/addition of nacleate boiling. Mesler replots their data with only his 'acceptable' points, and shows that nacleate boiling, if it is the proper explantion, contributes at a11 qualities. Using only the acceptable data, Mesler also plots heat

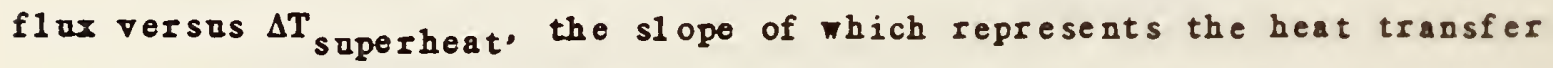
coefficient. He then plots in the same fashion the data of fivo pool boiling experiments and show them to have a similar, general shape as the flow boiling data of Dengler and Addams. He thas establishes a general relation between heat flar and wall superheat and dras fraw the data precisely opposite conclusions of the physical process than the authors who first collected and explained the data. In a brief letter of response, Standiford (15) points out an error in one of the five pool 
boiling experiments, which Mesler accepts but points to the remaining experiments.

Mesler al so considers observation (3) of Dengler and Addoms, the correlation of $a / a_{L}$ with $\left(1 / X_{t t}\right)$ on a $\log -\log$ basis. He argues about two potential pitfalls of such an approach. First the use of $10 \mathrm{~g}-10 \mathrm{~g}$ plots tends to reduce the appearance of variation. Secondly, he points out that there are several common variables in $a / a_{L}$ and $X_{t}$, and that with slowly varying fluid properties, one should antamatically expect a strong correlation, as Dengler and Addams achieved. His analysis is Ieviewed in more detail in Appendix 4B.

Hesler goes on to note that as convection of the vapor increases, $\left(1 / X_{t}\right)$ must increase. At the same time, with stronger convection one would expect a smaller $\Delta T$. Hesler then states 'This, in turn, predicts that when $a / a_{L}$, which tends to increase $\nabla i t h 1 / X_{t t}$, is maltiplied by $\Delta T$, which tends to decrease $\mathbf{i t h} 1 / \mathbb{X}_{t t}$, the quatity obtained should vary less with $1 / \mathbb{X}_{t}$ than did $a / a_{L}$. The actual data... contradict this prediction.' Since convection does not explain the data trends sufficiently. Hesler argues that nucleate boiling may be the phenomens, and compares the Dengler and Addams data to pool boiling data as previously discussed.

In $f$ urthor argaing for nucleate boiling as the dominant mechanism for all flow boiling situations, Mesler cites several studies of boiling with thin moving films. 
Beattie and Lawther have presented briefly evidence to support the notion of bubble existence in thin turbulent flowing films [Be79]. They maintain that attached babbles exist and serve to enhance surface rougb ness. The babble contribution to surface roughness is dependent on surface tension and shear forces. They then find agreement between a flow model which includes these forces ( $r$ ather than say, a model including Reynolds Nmber) to experimental data of velocity profile and friction factor in annular flows with thin films. This agreement, they conclude is ' consistent with the existence of attached wall bubbles in the film.. [and] that the nucleate boiling mechanism can contribute to heat transfer in thin film annular flows.' The notion of attached bubbles is not new: Lacey et al discuss the possibility, suggesting that bubbles might remain fired within the viscous sublayer [La62].

Turning to refrigerants, Chaw la [Ch67] noted that vaporization data taken with many flowing refrigerants indicated two separate regimes for beat transfer. The first regime showed near independence of the heat transfer coefficient from mass flow rate bat a strong dependence on beat flax. This regime in behaving similarly to pool boiling experiments was considered to be daminated by nucleation. A second regime showed com plete independence from heat flux but a strong dependence on mass flow. This second regime was characterized as being convection-dominated. A small region between these two general regimes is considered to be of transitive nature, with nacleation and evaporative mechamisns suppressed. Chawla's correlation of his data actually suggests a sup pression criterion (see Appendir 4A). Additionally, an independonce of 
the heat transfer coefficient fram heat flax has been observed ith benzene, toluene and in at least one case R12 [Da65,St65]. These studies suggest then an absence of nucleate boiling phenomena. Others have previously observed similar behavior with vertical flow of water; several studies are cited in Lacey, Hewitt, and Collier [La62].

In the most definitive study to date, Aounallah et al [A082] have recently bail an apparatos to determine if two phase heat transfer coefficients can be indeed completely independent of heat flur for a given flow rate, film thickness and quality. The comparison is potentially difficul since the amount of droplet entraiment is a weak fanction of heat flur; entraiment affects the thickness of the 1 iquid film. All previous experiments cited above used a uniform heat flar. For a given inlet condition and mass flow rate, the point at which a certain quality is reached is related directly to this heat flar (as shown in Chapter 3); with a larger heat flax, the same vapor quality vill be achieved at a different location in the flow. A comparison of the effect of heat flux would require then a comparison of heat transfer coefficients at different positions along the tube and is complicated by the fact that boiling is a fanction of local surface conditions. To avoid this problem, Aounallab et al bailt an apparatus similar in many ways to the second test rig used in this report. They used a vertical tube comprised of three sections: a preheat section, a 'calming' adiabatic section, and a test section. The preheat section was used to bring the flow to the desired qality. The calming section was included to bring the flow to estimated 'hydrodynamic equilibrium' at the inlet 
of the test section. Hydrodyamic equilibriom is achieved when the rate of entraiment exactly equals the rate of droplet deposition under adiabatic conditions. In this manner, not only the quality but the film thickness could be controlled at the test section inlet. The test section was outfitted with several closely spaced wall thermocouple stations; at each station, four thermoconples were mounted circumerer tially. Fluid temperatore was assumed as satorated and calculated from pressure measurements al ong the test section.

The athors then determined heat transfer coefficients for water at a fired flow rate and quality for five heat flux values. Tho heat transfer coefficients were found to be constant, i.e., independent of heat flux. These results were reported for four different qualities (.05 to .42), showing that as quality incressed, so did the heat transfer. Their results therefore support the notion of the complete suppression of nucleate boiling. It should be noted that their measured values vere not predicted $\mathbf{x}$ ell by a detailod film flow model but were predicted to about $\pm 20 \%$ by the forced convective portion of the Chen correlation [Ch66].

Beattie and Green [Be84] responded to the publication of Aonallah et al by examining in detail an old experiment by Bertolletiet al wose erperimental apparatus closely resembled that of Aounallah. The Bertoletti data is at mach higher pressure, flow rates, and heat fluxes though al so with flowing water. The data of Bertoletti did not show constant heat transfer coefficient, bat instead one which 'varied 
significantly with heat flux, having negligible dependent on mass flux, quality, tobe diameter, and distance from the inlet of the heated section'. Beattie and Green also campared the Bertoletti data to the pool boiling correlation of Aladiev et al. Agreement, as shown on a log-log plot is excellent. 

Little work has been done specifically addressing suppression or incipience of nacleate boiling of mistures. Howerer, the general study of nucleate boiling of nonazeotropic mirtures is itself a large and growing field major portions of tests have been devoted to this subject. As sach a comprehensive review of all featores of nacleate boiling of mixtures is beyond the scope of this report. In this apper dix certain critical features of bobble grow th as applied to the suppression question are introdaced.

Equation (4-1) for the soperheat requirement for a vapor nucleas of radius $I_{c}$ to exist in pure fluids is al so applicable for mirtures. However there are important differences in the $\nabla$ al aes of the terms of equation (4-1). First the term $d P_{s a t} d T$ for a mirture differs from that of a pore fluid or an ideal mixture as given by Collier:

$$
\left.\left.\left.\frac{d P_{s a t}}{d T}=\frac{\partial P}{\partial T}\right)_{\bar{X}}+\frac{p}{R T} \frac{\partial \bar{X}}{\partial T}\right)_{P}+(R-1) \bar{Y} \frac{\partial^{2} g}{\partial \bar{X}^{2}}\right)_{P, T}
$$

where $X$ is the equilibrium constant $(\bar{Y} / \bar{X})$ and $g$ is the Gibbs free energy. The term $\partial P / \partial T) \bar{X}$ is identical to what would appear for a pure flaid. The second term on the right side is always negative so that $d P_{s a t} / d T$ for a mixtare is always less than that for an ideal wixed flaid. On applying this finding to equation (4-1), the incipient super beat requirements for mirtares is increased over that of an ideal mired fluid. 
This change, however, has been shown by Shock (Sh77) to be less important than the behavior of the mirture's sorface tension Small additions of a second component may have drastic consequences on sarface tension, such that the superheat requirement may decrease substantial iy over that of one of the pore fluids or that presupposed of an ideal mixtore.

Three studies of the onset of nacleate boiling (onb) with binary mirtures rere found in the 1 iterature. Thame, Shakir, and Mercier [Th82] performed a careful study of the activation of a single first boiling site on a polished heated surface with mixtures of 1 iquid nitrogen-argon and ethano1-water. The composition of the cyrogenic mixture had no effect on the activation of the single site. Bowever, composition yielded a strong effect on their results with ethanol water. The results in both cases are due to the wetting characteristics of the mixtures. The cryogenic mixtare components have similar contact angles, whereas the addition of slight amounts of ethanol to water has drastic effect on surface tension and therefore the contact angle. The athors did not consider the effect of mass transfer resistance (mtr) in their results. In fact, the cryogenic mirture results suggest no effect of mir. However, the onb point for ethanol water is anderpredicted by treating the analysis as an equivalent pare fluid.

Shock eval uated binary mintures of ethano1-water and e thano1-benzene, with similar conclusions regarding the inf 1 uence of wetting characteristics [Sh77]. The onb point was found by wall temperatare 
measurement in his flow boiling experiments. He suggests that suppression of boiling of mixtores might not be strictly treated as the same as the onb problem, due to the possible existence of local concentration gradients around established naclei. He leaves open the possibility of mtr effects.

Toral studied ethanol-cyclohezane, which behaves in a nore ideal fashion than the previons ethanol mixtares, in a flom boiling apparatos similar to Shock's [To79]. He differentiates between activation of an isolated cavity and the sudden transition to multiple cavity activation. The 1atter mechanism, more relevant to the rork of this report, is called the onb by Toral. He concludes that composition has a considerable inf luence on multiple cavity activation, 'indicating the presence of mtr effect and suggesting that onb is governed by bubble growth dynamics.. ' If Toral's conclusions are correct, then mtr should be considered in subsequent prediction methods for mixtures.

Toral [To79] al so attempted a basic theoretical study of the potential for nucleate boiling in thin film flow of two non-azeotropic mixtures: an aqueons solntion of methanol and one of ethanol-cycloherane. He posed the following problem:

'A thin 1 iquid film flows in upward direction on a flat plate of infinite depth by the action of shear stress imposed upon it by vapor flowing concurrently. Thermodynamic equilibrim is assumed between 1 iquid and vapor phases with uniform temperature 
distribation dee to the adiabatic condition at the boundary with the flat plate. At time $t=0$ heat flux is applied at this boundary over the axial length $x=0$ to $x=L$. The temperature profile begins to develop. A net rate of evaporation begins when the heat flux reaches the interface. It is assumed that for $t>0$ heat flax at the wall remains constant at $q_{\mathbb{w}^{*}}$ '

As time goes on, the thickness of the layer decreases due to evaporatior, the film thickness al so decreases with downtrean distance.

The basic equations governing the problem are:

\section{Momentum}

$$
\rho \varepsilon_{m} \frac{\partial^{2} u}{\partial y^{2}}=-\rho g+\frac{d p}{d x}=c t
$$

$$
\begin{aligned}
& \text { Energy or Mass Transfer } \\
& \frac{\partial \emptyset}{\partial t}+a \frac{\partial \emptyset}{\partial x}=\varepsilon_{D} \frac{\partial^{2} \emptyset}{\partial y^{2}} \\
& D=T \text { in energy equation } \\
& D=C \text { in mass equation }
\end{aligned}
$$

The boundary conditions he used are discussed later.

Toral investigates the effect of assuning various thermal and mass diffusivities $\left(\varepsilon_{p}\right)$ in the above expression. Most researchers have assumed a single phase eddy diffusivity throughout the layer, L.e., 
growing tarbulence as one proceeds toward the interface. Some Iesearch has show that the turbulence may be damped in the vicinity of the interface, providing an increase of thermal and mass transfer resistance. ${ }^{1}$ The damped and undamped diffusivity models produced widely different results. Dsing an undamped profile, a smal1 wal1 superheat devel oped for the nor azeotropic mirtare, one which would be insafficient for nucleation. Using a damped profile, the calculated wall superheat increased by a factor of 6 , to the point that nucleation was likely. For the aqueous mirtare, wall superheat was too 1 ow to initiate bubble growth in either case. He concludes that only with high conductivity fluids, such as with aqueous mirtures, can one anticipate the suppression of nucleate boiling, if in fact turbulence damping occurs at the interface. It is important to note that the conductivity of rater or aqueous mirtures is roughly 100 times that of many other fluids, including most common refrigerants.

Toral's work is most relevant to this report since it is concerned $\mathbf{w}$ ith nor-azeotropic mirtures. A detailedreview of his posed problem reveals same potential difficul ties with his analysis.

The parabolic form of the energy and mass transfer equations requires the ase of only one boundry condition on $x$. Toral however uses:

${ }^{1}$ In the vicinity of the interface, the eddy diffusivity in the energ equation reduced to the thermal diffusivity, $\lambda / \rho C_{p}$. 


$$
\begin{array}{ll}
T(0, y, t)=T_{b u b}\left(C_{b a l k}\right) & c(0, y, t)=C_{b a l k} \\
d T(L, y, t) / d x=0 & d c(L, y, t) / d x=0
\end{array}
$$

The ase of two $x$ boundary conditions converts the solution technique from a 'forward marching' one to a closed form type. The effect of specifying the downstream condition propogates upstream in the solution technique. Toral even notes $\partial T / \partial x=0$ shortly after the inlet. Thas, the use of two boundary conditions is mathematically incorrect.

The physical meaning of $\partial T / \partial x=0$ can be interpreted simply with the use of a control volume heat is transferred into the control vol une by diffusion away fram the wall and by convection It is transferred out by convection and by diffusion toward the interface. The difference must be the amount of heat stored. If $\partial \mathrm{T} / \partial \mathrm{I}=0$, there is no sensible heating of the 1 iquid, and net flow of heat must either be to storage or toward the interface. A similar explanation is valid for the mas transfer equation.

The mathematical error might not have serious consequences, a marching type solution could produce the same result, since the specification of 'no sensible heating' may or may not be valid. Many condensation researchers consider the term to be soll, and that, at steady state, al 1 wall heat $f(n x$ is transferred to the interface (e.8., [Co37]). Bennett and Chen in their flow bo:ling study of an ethylene-glycol/water mirture considered sensible beating of the 1 iquid layer, and achieved only a slightly improved agreement with data [Be80]. On the other band, 
Toral called the stored heat 'sensible heat', and noted it represented 40-50\% of the total heat flux in the damped cases.

Despite its potential difficulties, Toral's posed problem is of substantial interest. An al ternate means of soltion rould be to assume steady-state and appoximate an initial film thickness $\delta(x=0)=\delta_{0}$. and march downstream ontil the film is depleted. In the case of refrigeration cycles, the initial condition in the x-direction can be simply equil ibrium vapor and 1 iquid compositions, since a two phase mixture in fact enters an evaporator from an isenthalpic expansion device. At the entering vapor quality of about $20 \%$ the phases separate into an annular flow pattern very near the evaporator inlet. At each $\Delta x$ step, the film thickness must be calculated ficm energy and species balances. Such a solution requires as inpat the same $y$-boundary conditions and $x$ initial condition as Toral assumes. 
APPENDIX 6A: MISCELLANEOUS HEAT TRANSFER FORMS

The graphical method of Shah, like Chen's method, attempts to predict a in either heat transfer regime. Mass flar and quality effects are considered in the nucleate boiling regime through the single phase heat transfer coefficient where

$$
a=a_{n b c}=a_{L O}(f(B O)
$$

Like form 6-1a equations, the nucleate boiling has a dependence on tabe diameter (explicit in $a_{L 0}$ ). The forced convection dominated regime, called by Shah the 'fully suppressed nucleate boiling regime,' yields

$$
a=a_{F C}=a_{L 0} f(\mathrm{Co})
$$

where

$$
C_{0}=\left(\frac{1-x}{I}\right)^{0.8}\left(\frac{\rho_{\nabla}}{\rho_{L}}\right)^{0.5}
$$

and Co is called the convection nmber. Co is similar to $\mathbb{X}_{t t}$ without the dimensionless viscosity term.

The correlation was compared to 810 data points from RI 1 , R12, and R22 experiments with a mean fractional deviation of $23 \%$ and a tendency to underpredict [De78]. Shah recently computerized the method [Sh82], and the algorithm was compared to the experimental data of this report. In general, the method predicted poorly (figare 6-1). Tho suppression 
criterion was incorrect, so that the algorithm selected a beat transfer coefficient calculated from equation (6-2b) instead of the more appropriate equation (6-2a). All of the Rig \#2 data tended to be underpredicted, less severely for the forced convection regime. The Rig \#1 data was al so poorly predicted (figare 6-1), though the mean deviation is reduced.

After analyzing Shah's correlation and others; Dembi et al. introdaced a new correlation for the forced convective/evaporative regime of the form [De7 8] :

$$
\alpha=0.115 \frac{\lambda_{L}}{D}\left(x^{4}\left(1-x^{2}\right)\right)^{0.11}\left(\frac{G^{2} \Delta h_{v}}{8 \rho_{L} \sigma}\right)^{0.44}\left(P_{L}\right)^{0.7}
$$

It correlated the same 810 point data base to a mean deviation of 0.15 . However, the coefficients were determined by regression analysis of the data base, so good agreement might be expected. It is interesting to note that beat flur does not appear in the equation though a weak dependence on heat flax was observed in the experimental data. No attempt was made to apply this correlation. It is al so interesting to note that the Prandtl number dependence is to the 0.7 power, similar to Bennett and Chen's correlation.

Most resently, Kandilikar [Ka84] has developed, via regression anlysis, a correlation which yields good agreement with a large body of experimental data on refrigerants, water, and organic flaids in both 
horizontal and vertical orientations. The method involves the classic superposition of convective/evaporation and nucleate boiling. It contains seven empirically determined constants, one of which is dependent on fluid type (and therefore fitted to the individal experiment ith that f1 nid). Test $w i$ th R152a and R13B1 are not incladed in this data base, and therefore the correlation could not be checked. 

Po1 1 ey has recently modified Chen's method and saggested a different pool boiling relation due to Cooper [P082]:

$$
a_{n}=a_{p 001}=c_{1}\left(\frac{P}{P_{c}}\right) \cdot 126\left(1-\frac{T}{T_{c}}\right)-.70 \dot{q}^{.69}
$$

where $C_{1}$ varying between 3 and 4.1 for refrigerants, and other val ues for different fluids. Also, he modified the suppression factor:

$$
\begin{aligned}
s & =1.0 \text { if } \frac{a_{e}}{a_{p 001}}<0.15 \\
& =-.5271 \ln \left(\frac{a_{e}}{a_{p 001}}\right) \text { if } 0.15<\frac{a_{e}}{a_{p 001}}<1.0 \\
& =0 \text { if } \frac{a_{e}}{a_{p 001}}>1.0
\end{aligned}
$$

The justification for the changes were given as:

(a) The pool boiling relation is simpler to apply, as it requires fewer properties;

(b) The suppression factor is based on heat transfer contributions directly; and most importantly,

(c) The revised form fit a very large data bank of steam-water data to a higher degree of accuracy than Chen's equation. 
Polley included an additional criterion to determine if a nacleate boiling contribution should be included. He suggested that the Davis and Anderson incipient superheat, $\Delta T_{i}$, be calculated (see Appendir 4A). If the given $\Delta T$ is less than that calculated, then no nacleate boiling should be included. It is, however, not clear how to apply it in the case of constant wall flux where $\Delta T$ is not bown a priori. 
APPENDIX 7: DEVELOPMENT OF CCON (MASS TRANSFER EFFECT ON LIQUID SIDE) IN BENNETT AND CHEN'S METHOD

Bennett and Chen postulated mass transfer does not affect $a$, but does effect the driving force.

For a pare fluid

$$
q_{\text {evap }}=a_{L 0}\left(T_{W}-T_{\text {eqb }}\right) \quad T_{\text {eqb }}=\underset{\text { fluid } T_{\text {eqb }}=T_{\text {sat }}}{\text { equilibrimm temp, for pare }}
$$

For a binary

$$
\begin{aligned}
& q_{B I N}=a_{L 0}\left(T_{W}-T_{i}\right) \quad T_{i}=\text { interfacial temp. } \\
& =a_{L 0} \frac{T_{W}-T_{i}}{T_{W}-T_{\text {eqb }}}\left(T_{W}-T_{\text {eqb }}\right) \\
& =a_{L 0} C_{C O N}\left(T_{W}-T_{\text {eqb }}\right)
\end{aligned}
$$

Since $T_{i}$ is anknown, Bennett derived a way of eliminating it from $(7 A-1)$, as fol 10 s (steps not shown in Bennett's report):

Define a mass transfer coefficient, $\beta_{L}$ :

$$
\dot{m}_{\mathrm{mv}}=\rho_{\mathrm{L}} \beta_{\mathrm{L}}\left(\mathrm{X}_{\mathrm{e}_{\mathrm{I}} \mathrm{b}}-\mathrm{X}_{\mathrm{i}}\right)
$$

$$
\text { mv }=\text { more volatile }
$$


Now, assune all heat input results in evaporation

$$
\dot{q}_{e v a p}=\Delta h_{v}=\frac{\dot{m}_{m v}}{Y} \Delta h_{v}
$$

and assume $Y=Y *$, i.e., $Y *=Y *\left(X_{\text {eqb }}\right)$, not $Y_{i}($ this is not strictly correct) and since

$$
\frac{T_{W}-T_{i}}{T_{W}-T_{e q b}}=\frac{T_{W}-T_{b q b}\left(X_{i}\right)}{T_{W}-T_{b q b}\left(X_{e q b}\right)}
$$

equations $(7 A-2),(7 A-3)$ and $(7 A-4)$ can be combined to give $C_{C O N}$ as foll ow :

From $(7 A-2)$ and $(7 A-3)$,

$$
\mathrm{x}_{\mathrm{eqb}}-\mathrm{x}_{\mathrm{i}}=\frac{\dot{\mathrm{m}}_{\mathrm{mv}}}{\rho_{\mathrm{L}} \beta_{\mathrm{L}}}=\frac{\dot{q}}{\Delta \mathrm{h}_{\mathrm{v}} \beta_{\mathrm{L}}}
$$

Al so

$$
X_{e q b}-X_{i}=\frac{d X_{e q b}}{d T_{b u b}}\left(T_{B}-T_{i}\right)
$$

combining $(7 A-5)$ and $(7 A-6)$

$$
\frac{d X}{d T_{b u b}}\left(T_{e q b}-T_{i}\right)=\frac{\dot{q}{ }^{*}}{\Delta b_{v} \rho_{L} \beta_{L}}
$$


$T_{e q b}-T_{i}=\frac{\dot{q} F \frac{d T_{b q b}}{d X}}{\rho_{L} \beta_{L} \Delta h_{v}}$

Add and subtract $T_{w}$, and multiply by -1

$$
\left(T_{W}-T_{e q b}\right)-\left(T_{W}-T_{i}\right)=\frac{-q Y * d T_{b u b} / d \Sigma_{e q b}}{\rho_{L} \beta_{L} \Delta h_{V}}
$$

Divide by $\left(T_{W}-T_{e q b}\right)$

$$
1-\frac{T_{T}-T_{i}}{T_{W}-T_{e q b}}=\frac{-q Y * d T_{b q b} / d X}{\rho_{L} \beta_{I} \Delta h_{V}\left(T_{W}-T_{e q b}\right)}
$$

$$
\frac{T_{W}-T_{i}}{T_{W}-T_{e q b}}=1+\frac{q Y * d T_{b q b} / d X_{e q b}}{\rho_{L} \beta_{L} \Delta h_{V}\left(T_{W}-T_{e q b}\right)}
$$

Note that (+) sign. In Bennett and Chen's paper they havea (-) sign. The difference is dee to definition. In the development here, $X$ is defined in terms of the more volatile component. Bennett and Chen used $X$ as the less volatile component. If defined in that manner, a $(-)$ is correct. 

$\dot{Q}=\dot{Q}_{\text {evap }}+\dot{Q}_{\text {sens }}+\dot{Q}_{\text {sens }}$

$\dot{Q}_{\text {evap }}=\dot{m b}_{v}$

$\dot{Q}_{\text {sensL }}=\frac{1}{\pi D} M_{L} C_{P L} \frac{d T_{b u b}}{d z}$

so

$\dot{Q}=m \Delta h_{v}+\frac{1}{\pi D} \dot{S}_{L} C_{P L} \frac{d T_{b u b}}{d z}$

Mass balances give

tota1 mass: $\quad-d\left(\dot{M}_{L}\right)=\dot{m}_{\pi D} d z$

component mass: $\quad-d\left(\dot{M X}_{L}\right)=m M^{*} \dot{\mathrm{D}} \mathrm{dz}$

$$
-Z_{B} \dot{d}_{L}-\dot{u}_{L} Z_{B}=\dot{m} \dot{H}^{* \pi D} d z
$$

Rearranging $(7 \mathrm{~B}-3)$

$$
\frac{d X_{B}}{d z}=\frac{-\dot{m} Y * D}{M_{L}} \frac{X_{B}}{M_{L}} \frac{d \dot{H}_{L}}{d z}=\frac{-m Y * D}{M_{L}}+\frac{\pi D \dot{m}}{M_{L}} X_{B} \text { from }(7 B-2)
$$


so, rearranging the last equation

$$
\frac{M_{L}}{\pi D}=\dot{m}\left(X_{B}-Y *\right) \frac{d z}{d X_{B}}
$$

Substitating $(7 B-4)$ into $(7 B-1 b)$

$$
\dot{Q}_{\text {sens }_{L}}=\dot{m C}_{\mathrm{PL}_{L}}\left(X_{B}-Y *\right) \frac{d T_{b n b}}{d X_{B}}
$$

and $(7 B-1 c)$ becames

$$
\dot{Q}=\dot{m L h}_{v}+\dot{m C}_{\mathrm{PL}}\left(\mathrm{X}_{\mathrm{B}}-\mathrm{Y} *\right) \frac{d \mathrm{~T}_{\mathrm{b} \mathrm{b}}}{\mathrm{d} \mathrm{X}_{\mathrm{B}}}=m \mathrm{~h}_{\text {ef } f}
$$

or

$$
\Delta h v_{\text {eff }}=\Delta h_{v}-C_{P L}\left(Y *-X_{B}\right) \frac{d T_{b a b}}{d X_{B}}
$$

where again XB and $y$ are in terms of more volatile component. Bennett and Chen ased less volatile so for their eqution:

$$
\Delta h v_{\text {eff }}=\Delta h_{v}+C_{P L}\left(X_{L V}-Y_{L V}\right) \frac{d T_{b u b}}{d X_{L V}}
$$




\section{APPENDIX 7C: MI CRQAYYER EVAPORATION MODELS}

Van Owerkerk [Va72] analyzed a hemispherical brbble with a thin microlayer of 1 iquid under it (see Figure 7C-1). The assumed physical properties were independent of both composition and temperature. The transient analysis of bobble grow th showed that brbble grow th rates were greatly reduced over an EPF fluid due to the depletion of the more volatile component in the microlayer. The depletion caused the usual rise in bubble point temperature reducing the evaporation rate. He noted that the Marangoni effect (i.e., surface tension gradient around a brbble influencing the grow th rate) could theoretically assist evaporation by drawing the more volatile component fram the bobble cap region to the microlayer. Upon calculation, he noted the effect is negligible for real istic brbble sizes.

Toral [To79] examined microlayer evaportion, bot allowed thermal properties to vary with composition. His numerical analysis treated the microlayer as a 1-D transport problem (see figare 7C-1). His conclusions are similar to Van Ounerkerk. He further noted that all evaporation may cease, so that a dryout condition might never bo reached. In this situation, much higher critical heat flures would be possible with a mirture. Recent1y, Stephan and Preuber [St82] developed a mode1 which suggests that, while microlayer evaporation occurs, condensation might occur simol taneously at the brbble cap. They assume the microlayer has a lower 1 iquid composition then the balk 1 iquid (see figare 7C-2). However, vapor is produced in this region due to its proximity to the heated wal surface. The vapor produced in microlayer evaporation is in equilibrium 
with the 1 iquid in this region, and once produced mires completely with the vapor in the bubble (assuned). The vapor therefore bas a composition no longer in equilibrium with the liquid at the bubble cap. To move toward equilibrium, the less volatile component condenses ont at the same time the more volatile component evaporates. They note that, if this modelled process indeed is accurate, the mass transfer resistance between the bolk and microlayer increases, i.e., the boundary layer bas more difficulty in finding available more volatile component. 

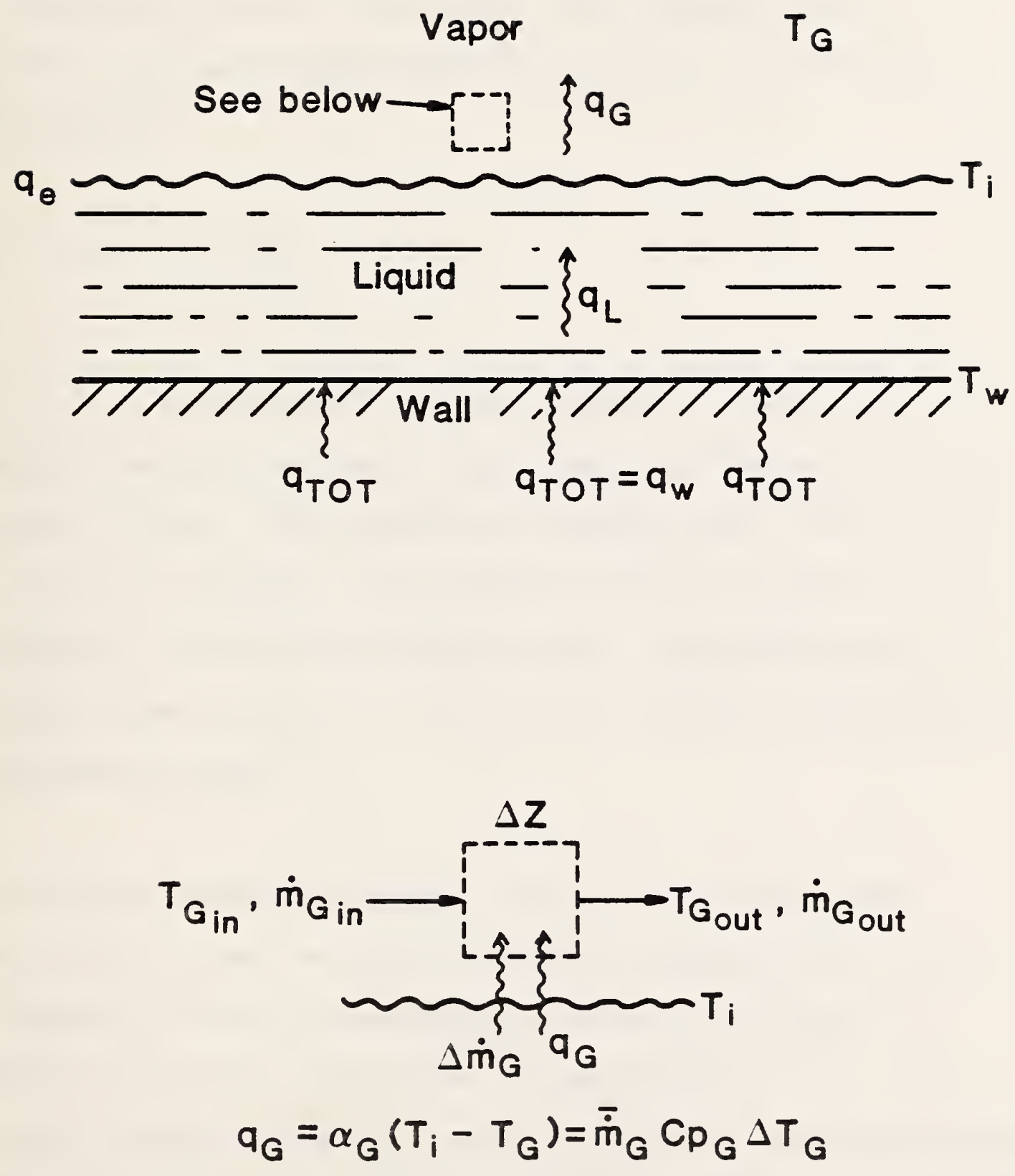

Figure 7E-1: The Bell and Ghaly Method 

APPENDIX 7D: FURTHER COMMENTS ON SHOCE'S ANALYSIS

There are some concerns regarding the analysis. Turbalence damping near the interface was neglected (this might affect finding (a) in particalar); eddy mass diffusivities are generally related to thermal diffusivities by

$$
\varepsilon_{D}=\varepsilon_{\mathrm{B}}^{\mathrm{n}}, \quad \mathrm{n}=\frac{1}{4} \text { to } \frac{1}{2}
$$

not $\varepsilon_{D}=\varepsilon_{H}$ as assmed in the analysis. However, finding (a) is supported by analjtic modelling of multicomponent condensation by Webb and Sardesai [We82]. They examined two 1 imiting cases: one in the rate of mass transfer in the condensate film was assmed to be infinitely slow, and the other infinitely fast. Condensation rates of individnal components agreed to within 15\% of each other and within $10 \%$ of experimental results.

Shock's initial condition, selected to maximize the vapor-liquid composition difference, was only $7^{\circ} \mathrm{C}$ from the boiling point of water. This suggests that with the maximum mass transfer resistance, the interfacial temperature could differ from equilibrium by $7^{\circ} \mathrm{C}$. Since the total temperatore drop across the 1 iquid film was calculated $40-70^{\circ} \mathrm{C}$, the largest possible reduction in heat transfer coefficient was 10-15\%. Shock does note that the findings may vary for mirtores $\rightarrow$ ith a wider boiling range than the considered $20^{\circ} \mathrm{C}$ of ethanol-water (R152a/R13B1 at $4.75 \mathrm{have}$ about a $30^{\circ} \mathrm{C}$ boiling Iange). In a related problem Price and Be11 [Pr74] compared a simplified condensation mode1 which neglects mass 
transfer resistance to a more ezact model. Agreement was good for a mixtare of methanol-water (small boiling range), but not for an r-botane/ n-octane mirture (wide boiling range). 
APPENDIX 7E: THE BELL AND GHALY WODEL

Figure 7E-1 shows simple heat transfer across a film and to a vapor core. Neglecting any sensible heating of the 1 iquid $\left(Q_{s_{L}}=0\right.$ in equation $\left.1-1\right)$,

$$
q_{T O T} \cong q_{L}=a_{L}\left(T_{W}-T_{i}\right)
$$

Similarly on the vapor side,

$$
q_{G}=a_{G}\left(T_{i}-T_{G}\right)
$$

Combining the previous equtions, and eliminating $T_{i}$ yields

$$
q_{T O T}=a_{L}\left(T_{W}-T_{G} \frac{q_{G}}{a_{G}}\right)
$$

and dividing both sides by q TOT $_{\text {TO }}$

$$
1=\frac{a_{L}}{a_{T O T}}\left(T_{W}-T_{G}\right)-\frac{a_{L}}{a_{G}} \frac{q_{G}}{q_{T O T}}
$$

$$
q_{T O T}=\frac{a_{L}}{\frac{a_{L}}{a_{L}} \frac{q_{G}}{a_{T O T}}}
$$

so that defining an effective heat transfer coefficient, 


$$
a_{\text {eff }}=\frac{1}{\frac{1}{a_{L}}+\frac{q_{G} / q_{T O T}}{a_{G}}}
$$

The ratio $q_{G} / q_{T O T} r e p r e s e n t s$ the ratio of the heat gained by the vapor to the total heat. Over a length $\Delta Z$,

$$
q_{G}=\grave{\dot{m}}_{G} C_{P_{G}} \Delta T_{G}
$$

where the mass flow rate of vapor is a mean quantity over the interval. The total heat supplied is over this same length,

$$
\mathrm{q}_{\mathrm{TOT}}=\dot{\mathrm{m}}_{\mathrm{TOT}}^{\Delta \mathrm{h}}
$$

so that

$$
\frac{q_{G}}{q_{T O T}}=\frac{G}{\min _{T O T}} C_{P_{G}} \frac{\Delta T}{\Delta h}
$$

over an infinitesmal length $(\Delta \mathrm{Z} \rightarrow 0)$,

$$
\frac{q_{G}}{q_{T O T}}=I c_{P_{G}} \frac{d T_{G}}{d b}
$$

To this point, the derivation is exact. The quatity $d T_{G}$ is now approrimated as

$$
d T_{G}=d T_{i}=d T_{e q b}
$$


so that equation $(7-4)$ becomes

$$
\frac{q_{G}}{q_{T O T}}=x c_{P_{G}} \frac{d T_{e q b}}{d H}
$$

The term $\mathrm{dT}_{\mathrm{eqb}} / \mathrm{dh}$ is referred to commonly as the 'condensation curve'. Typical curves for R13B1/R152a mirtures are shown on Figure 3-13. The effective heat trausfer coefficient of equation (7E-3b) is then

$$
a_{\text {eff }}=\frac{1}{\frac{1}{a_{G}}+\frac{x C_{p_{g}}\left(d T_{e q b} / d h\right)}{a_{G}}}
$$

The problem remains to eval ua te the heat transfer coefficients $a_{L}$ and $a_{G}$. Bell and Ghaly recommended the classic single phase relations:

$$
a_{G}=\left(\frac{G I D}{\mu_{G}}\right)^{\cdot 8}\left(\mathrm{PI}_{G}\right)^{4} \frac{\lambda_{G}}{D}=a_{G 0}
$$

and

$$
a_{L}=\left(\frac{G(1-I) D}{\mu_{G}}\right)^{.8}\left(P_{g}\right)^{-4} \frac{\lambda_{L}}{D}=a_{L 0}
$$

The stated philosophy behind their approach was to anderestimate the heat transfer coefficients to compensate for the ersor of ignoring mass transfer resistance. There is however no assurance that the two errors (neglecting both two phase flow effects and most transfer resistance) are of the same magnitude. 
In a later paper, Price and Bell [Pr74] suggest modifying equation (7-8) to include a two phase flow effect $\nabla$ ia the Martinelli parameter, tacitly assuming a Reynolds analogy:

$$
a_{G}=a_{G O}\left(D_{\mathrm{Vtt}}^{2}\right) \cdot 445
$$

In a separate paper, Chisholm [Ch81] suggests using the two phase moltiplier on the 1 iquid phase as wel $1:^{1}$

$$
a_{L}=a_{L 0}\left(D_{L t t}^{2}\right) \cdot 445
$$

At this point, one can analyze the method in light of the provious section's discussion. First, Shock found little difference between $T_{i}$ and $T_{G}$, and evez less difference between $d T_{i}$ and $d T_{G}$. Thas the assuption of (7-5) is minor for evaporation, though for condensation of a bighly superheated vapor fl ow one might suspect problems.

Finding (c) of section 7.3 suggested that mass transfer resistance might be neglected without serious error. The basic philosply of canpensating errors ith the original method is then undermined; it is not surprising that the modification suggested in equations (7E-10) and (7E-11) vere required.

\footnotetext{
${ }^{1}$ Chisholm credits Price and Bell, but no reference to this step could be found in their paper.
} 
In still another paper, McNanght [Mc79] attempted to modify the whole approach to inclade a mass transfer effect on the vapor side. If, however, the finding (c) of section 7.3 is valid, this final change is unnecessary except for laminar flow.

Finding ( $f$ ) of section 7.3 soggested that the sensible heating required on the vapor side is small when compared to the total heat (7-46).

Taking this to an extreme, eqution (7-4d) yields

$$
\frac{\mathrm{q}_{\mathrm{G}}}{\mathrm{q}_{\mathrm{TOT}}} \approx 0
$$

or, on examining the condensation curves $d T / d h$ is small. Equation (7-3b) becomes then

$$
a_{e f f}=a_{L O}\left(D^{2} L_{t t}\right) \cdot 445
$$

or, the heat transfer coefficient is descibed completely by the 1 iquid film! The suggestion by Chisholm to include a two phase effect is then we11-placed. Equation $(7-12)$ is only valid in the case of turbolent vapor flor. If the vapor heat transfer coefficient becomes soll, as in stagemant or solall laminar flow, then

$$
\frac{q_{G} / q_{\text {TOT }}}{a_{G}} \approx \frac{1}{a_{L}}
$$


and the vapor side beat transfer must not be neglected. It is interesting to note that Price and Bell found the 1 iquid side to control the process in several test cases. The implication of (7E-13) and in fact the findings of the previous section is that forced convection/ evaporation of mirtures may be treated exactly as for pare flaids, requiring a good estimate, however, of the 1 iquid properties. 


\section{MICROLAYER EVAPORATION MODELS}

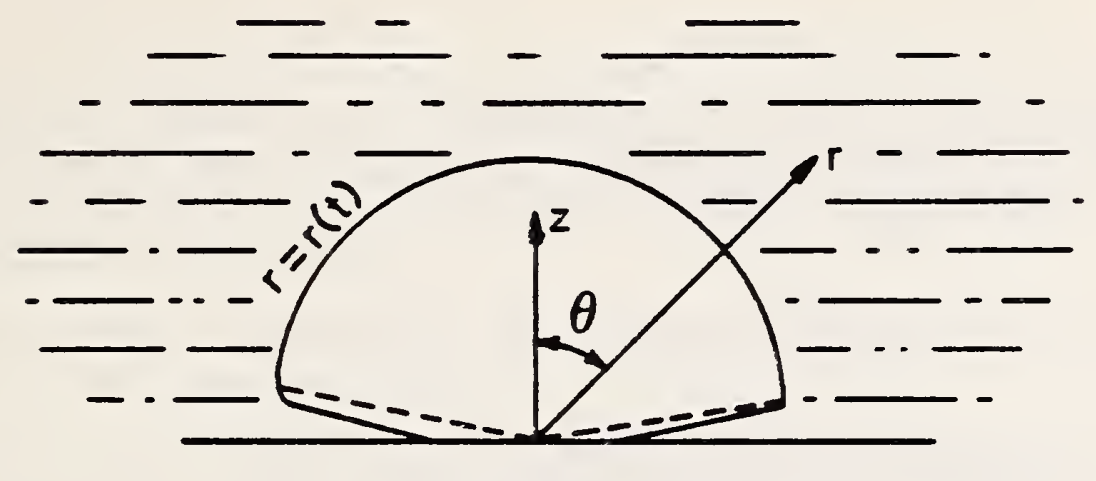

a) Van Ouwerkerk

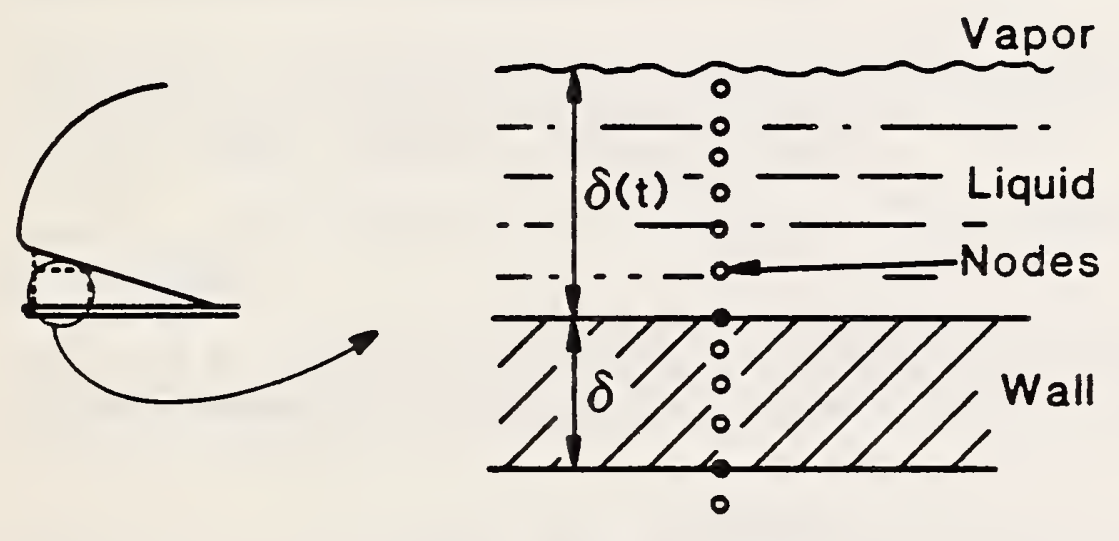

b) Toral

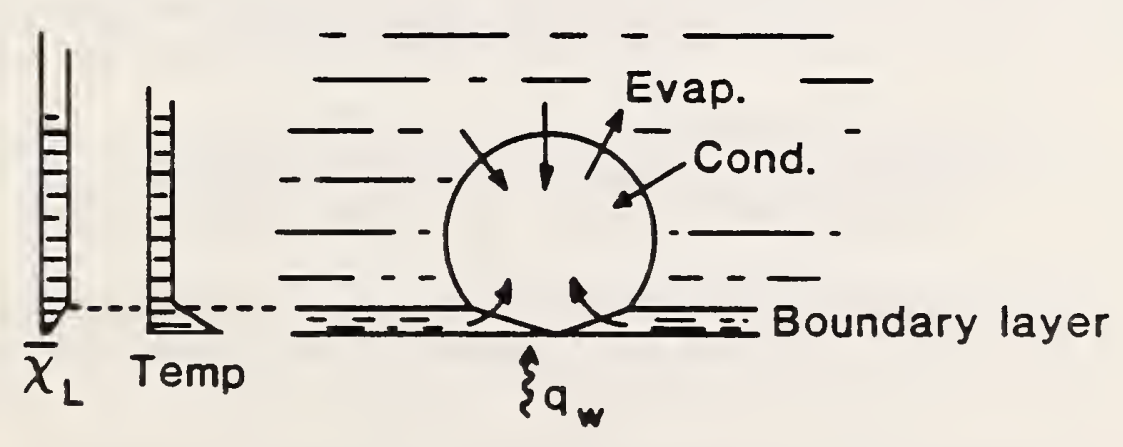

c) Stephan and Preuber

Figure 7C-1: Microlayer Evaporation Models 



\section{REFERENCES}

A160 Altman, H., Norris, R.H. and Staub, F.N., Local and Average Heat Transfer and Pressure Drop for Refrigerants Evaporating in Horizontal Tubes, J. Heat Transfer, 189-98, Angust 1960; ASME 59-A-278, November 1959.

Al77 Aljarrah, M.S. and Duminil, H., Rev. Gen. Froid, vo1. 68, No. 7, pp. 489-508, Julg/Ang. 1977 .

An66 Anderson, S. W., Rich, D.G., and Geary, D.F., Evaporation of Refrigerant 22 in a Horizontal $3 / 4$ in. OD Tube, ASHRAE Transactions, Vol. 72 , pp. 28-41.

As81 ASHRAE Handbook of Fandamentals - 1981.

A082 Aounal1ah, Y., Kenning, D.R.B., Whalley, P.B., and Hewitt, G.F., Boiling Heat Transfer in Annalar Flow, Proc. 7 th Int. Heat Transfer Conf., Monich, paper FB3 (1982).

A157 Aladier, I.T., Dodonov, L.D. and Udalor, D. S., Teploenergetika 4,9 (1957).

Af66 Afgan, N.H., Boiling Heat Transer and Burnout Heat Flur of Ethano1-Benzene Mirtures; 3rd Int'1 Heat Transfer Conf. (Chicago) (1966).

Bu79 Burkhardt, J. and Hahne, E., Influence of 0 il on the Nucleate Boiling of R-11; Int'1. Congress of Refrigeration (Venice), pp. 539-544, Proceeding, Vol. 2; (1979).

Bo5 1 Bonnet, W.E. and Gerster, J.A., Boiling Coefficients of Heat Transfer-C 4 Hydrocarbon/ furfural Mirtures Inside Vertical Tubes, Chem. Engrg. Prog., 77(3), 151-158 (1951).

Be80 Bennett, D.L. and Chen, J.C., Forced Convective Boil ing in Vertical Tubes for Saturated Pure Components and Binary Mirtures, AIChE Journal (Vol. 26, \#3), Pg. 454 (1980).

Br55 Bryan, T.L. and Siege1, L.G., Heat Transfer Coefficients in Horizontal Tube Evaporators, Refrig. Eng. 63, \#5, 36-45, 120 , 1955 .

Ba53 Baker, M., Touloukian, Y.S. and Hawkins, G. A., Heat Transfer Film Coefficients for Refrigerants Boiling Inside Tubes, Ref. Eng., 986-91, september 1953 .

Br5 1 Bryan, W.L. and Quaint, G.W., Heat Transfer Coefficients in Horizontal Tobe Evaporators, Ref. Eng., 67-73, January 1951. 
Ba74 Bande1, J. and Schlnder, E. U., Frictional Pressure Drop and Convective Heat Transfer of Gas Liquid Flow in Horizontal Tubes, Proceedings, Fifth International Heat Transfer Conforence, 1974, PP. 190-194.

Be82 Bejan, A., Entropy Generation

Bo75 Bennett, D.L., A study of Internal Forced Convective Boiling Heat Transfor for Binary Mirtures, Ph.D. Thesis, LeHigh University; 1975.

Be72 Boll, K.J. and Ghaly, M. A. , An Approximato General ized Design He thod, for Multicomponent/Partial Condensers, Chom. Eng. Prog. Symp. Series No. 131, 69, 72-79 (1972).

Bo67 Bogdanov, S.N., Determination of Heat Transfor Coefficients for Boiling Halocarbon Inside Horizontal Tubes, Engl ish Translation in ASHRAE J., p. 59, July 1967.

Be80a Bennett, D.L., David M.T. and Hertzlor, B.L., AIChE Symp. Ser., Vo1. 76, pp. 91-103, 1980 .

Be79 Beattie, D.R.H. Lawther, K.R., Letter to the Editor, AIChE J./25, 384 (1979).

Bo84 Beattie, D.R.H. and Green, The Existence of Nocleate Boiling in Diabatic Tro Phase snnular F10w, Int. J. Hoat Kass Transfor, Vo1. 27, \#2, pp. 315-317 (1984).

Be63 Berenson and Stono, Chom. Eng. Progress, AIChE National Meoting, 1963, Paper \#21.

Be64 Bertoletti, S., Lomberdi, C. end Silvesti, M. , Hoat Transfor to Steam-Water Mirtore, CISE report R-78 (1964).

Bu82 Butterworth, D., and Shock, R.A.T., Fl on Boil ing, Paper RK15, pg. 11, Proc. 7 th Int. Heat Trans. Conf., Monich (1982): Homisphere Publishing Corp.

Ch79 Chaddock, J. and Mathor, A.P., Heat Transfer to 0il-Rofrigorant Mirtures Evaporating in Tubes; Multiphaso Transport, Vol. 2. Homisphero Publishing Corp, Pg. $861 \mathrm{ff}$.

Ch67 Charla, J.M., Narmoubergang und Drackabfall in Maagrochton Rohren Bei Der Stromnag Von Verdampfonden Kaltemittelo, VDIForschungsh oft $523,1967$.

Co64 Collier, J.G., Lacoy, P. X.C. and Pulling, D.J., Heat Transfor to Two-Phase Gas-Liquid Mirtures in the Liquid Dispersod Rogion in an Annalus, AERE R-3809, 1964. 
Ch66a Chaddock, J. and Noerager, J.A., Evaporation of Rofrigezant 12 in a Horizontal Tube with Constant Wall Heat Flux; ASHRAE Transactions, January 1966, pg. $90 \mathrm{ff}$.

Co37 Colburn, A.P. and Drew, T.B., The Condensation of Mired Vapours, Trans. Am. Inst. Chem. Engng., 33, 197-215 (1937).

Ca72 Calus, W.F. and Rice P., Pool Boiling-Binary Liquid Miztures, Chem. Engng. Scionce, 27, 1687-1697 (1972).

Ch67a Chisholm, D. A Theoretical Basis for the Lockhart-Martinelli Correlation for Tro Phase F1ow: Int' $1 \mathrm{~J}$. Heat and Mass Transfer, Vol. 10, pp. 1767-1778.

Ch66 Chen, J.C., A Correlation for Boiling Heat Transfer to Saturated Flaids in Convective F1or, Ind. Eng. Chem. Process Design Derelop, 5, 322 (1966).

Co80 Collier, J.G., Competive Boiling and Condensation, MeGrav-Hill, 2nd Edition (1980).

Ch81 Chisholm, D., Modern Developments in Marine Condenser: Non Condensible Gases: An Orervier, pg. 95-142 in Porer Condenser Heat Transfer Technology, ed. Marto, P.J. and Nunn, B.H., Hemipshere Pablishing Corp., (1981).

Da66 Davis, E.J., and Anderson, G.H., The Incipience of Nac1eate Boiling in Forced Corvection Flow: AIChE Journal, pp. 774-780, 1966 .

Di66 Dickson, A.J. and Gonse, S. W., Jr., Heat Transfer and Flaid Flow in Horizontal Tube Evpa orator-Phase III, MIT Eng. Proj. Lab. Report DSR 9649-3, Angust 15, 1966, Trans. ASHRAE 1967.

De56 Deng1er, C.E. and Addoms, J.N., Heat Transfer Mechanism for Vaporization of Water in Vertical Tube, Chemical Engineering Progres s Symposinm Series, Vo1. 52, No. 18, 1956, pp. 95-103.

De7 8 Dembi, N.J., Dhar, P.H., and Arora, C.P., Lett. Heat Mass Transfer, Yo1. 5, pp. 287-296, 1978.

Da69 Danilova, D.N. , Heat Transfer to Boiling Refrigerants, pp. 107130 of Problems of Heat Transfer and Hydradics of Two PHase Media, Ed. S.S. Katateladze, Pergammon Press, Oxford, 1969.

Da66 David, E.J. and Anderson, G.H., AIChE J., Vo1. 12, pp. 774-780, 1966. 
Di84 Didion, D.A. and Mulroy, T.J., The Performance of a Residential Heat Pump Operating with a Non-Azeotropic Binary Refrigerant Mizture-An Interim Report, DoE/ORNL Heat Pump Conference, December 11-12, 1984 .

Fo55 Forster, H.K. and Zuber, N., Dynamics of Vapor Bubbles and Boiling Heat Transfer; AIChE Journal, December 1955 (Vo1. 1, \#4), p8. 531-535.

F170 F1orschaltz, L. W. and Rashid Khan, A., Growth Rates of Free Vapor Bubbles in Binary Liquid Mixtures at Oniform Superheta, 4 th Int'1 Heat Transfor Conference (Paris), Paper B.7.3. (1970).

Go65 Gouse, S. W., Jr. and Comor, R.G., Heat Transfer and F1 aid Flow Inside a Horizontal Tube Evaporator, Phase I, MIT Eng. Proj.

Lab. Report DSR 9649-1, June 1964; Tran ASHRAR Part II 71, 152$161,1965$.

Go66 Gouse, S.W., Jr. and Dickson, A.J., Heat Transfer and F1 nid F1ow Inside Horizontal Tube Evaporator, Trans of ASHRAE, Part I, 1966, See Discussion.

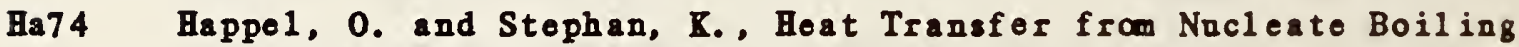
to the Beginning of Filn Boiling in Binary Mirtures, Paper B7.8 presented at 5 th Interastional Heat Transfer Conference, Tokyo, Sept ember (1974).

He63 Aewitt, G.F., ot al, Barnout and Nacleation in Climbing Fila F1 or, AERE-R4374 (1963).

Ho63 Hosler, E.R., Visual Study of Boiling at High Pressore, Chem. Eng., Progress, AIChE National Moeting (1963).

Hs76 Hsu, Y.Y. and Graham, R.X. , Transport Processes in Boiling and Tro Phase Systems, McGraw-Hil1, Chapter 1, 1976.

He84 Hewit, G.F., Workshop on Two Phase F1ow Processes, (1984).

Ht83 Heat Transfer and Fluid Flow Service (HTFS), data base (subscription), as of 1983 .

Ja74 Jallouk, P.A., Tro Phase Flon Pressure Drop and Beat Transfor Characteristics of Refrigerants in Vertical Tubes, Ph. D. Dissertation, University of Tennessee, 1974.

Ba75 Kandiker, S.G., Bijiani, C.A., and Sukhatme, S.P., Predicting the Properties of Mirtures of $\mathrm{B} 22$ and R12-Part II--Transport Properties, ASBRAE Transactions, Vol. 81, 1975, P8. $285 \mathrm{ff}$. 
Ka84 Kandliker, S.G., An Improved Correlation for Predicting Two Phase F1or Boiling Heat Transfer Coefficient in Horizontal and Vertical Tubes; 21 st National Heat Transfer Conference (Seattle) ASME $1983(639-84-21)$.

La66 Lavin, J.G. and Young, E.H., Heat Transfer to Evaporating Refrigerants in Two-Phase F1 ow, AIChE J. 11, \#6, 1124-32, November 1966.

L049 Lockhart, R. W. and Martine11i, R. C., Proposed Correlation of Data for Isothermal Tro-Phase, Two Component F1 ow in Pipes, Chem. Eng. Progress, Vol. 45, \#1, pp. 39-48 (1949).

La62 Lacey, P.M.C., Hewitt, G.F., and Collier, J.G., C1 imbing Film F1 ON, AERE-R3 962 (1962).

Ma83 Mattingly, G., NBS F1uid Measurements Group, private communications.

Mc42 McAdams, W.H., et a1, Vaporization Inside Horizontal Tubes-II, Benezene-0il Mirtures; Trans ASME, pp. 193-200 (1942).

Ma76 Mathur, A.P., oat Transfer to 0il-Refrigerant Mirtures Evaporating in Tubes; Duke University, (1976), (Ph.D. Thesis).

Mi81 Mishra, M.P., Varma, H.K., and Sharma, C.P., Heat Transfer Coefficients in Forced Convection Evaporation of Refrigerant Mixtures; Letters in Heat and Mass Transfer, Vol. 8, Pp. 127-136 (1981).

Mo82 Morrison, G., (draft paper), An Equation of State for Refrigerant Mirtures,

Mo84 Morrison, G., private communications.

Mo85 Morrison, G., The Inportance of Including the Liquid Phase in Equations of State for Non-Azeotropic Refrigerant Mirtures, ASHRAE Trans, Pt. 1 (1985).

Me76 Mesler, R.B. A Yechanism Supported by Extensive Experimental Evidence to Explain High Heat Flares Observed Daring Nacleate Boiling, AIChE J/22, 246-252 (1976).

Me77 Mesler, R.B.. An Alternate to the Dengler and Addoms Convection Concept of Forced Convection Boiling Heat Transfer, AIChE, J/23, 448-453, (1977).

Mc79 McNaght, J. Mass Transfer Correction Terms in Design Methods for Malti-Component Partial Condensers, 18 th National Heat Transfer Conference, (San Diego), pp. 111-118 (1979).

Mo7 8 Mori, S., Sakitani, K., and Isoaji, A., Reito, Vo1, 50, p8. 1-6. 
Ma48 Martinelii, R.C. and Nelson, D.B., Prediction of Pressure Drop During Forced Circulation Boiling of Water, ASME Trans., PP. 695-702, August 1948.

Mu72 Murphy, R.W. and Bergles, A.E., Proceedings of the 1972 Heat Transfer and Fluid Mechanics Institute, R.B. Landis and G.J. Hordemann, eds. Stanford University Press, Pp. 400-416, 1972.

Pi56 Pierre, B., Varmeovergangen $\nabla$ id Rokande Roldmedier Horizontella Rortekn, Ryltenick Tidakrift $\$ 3$, 129, May 1957; al so 12, 76, 1953; 16, \#, 225, December 1957. Warmeii Bergangazahl Bei Verdampfenden F 12 in Horizontalen Rohren, Raltertecknik 7, heft 6, 163-66, 1955; al so S.F. Review 2, \$1, 55-68, 1955; The Coefficient of Heat Transfer for Boiling Freon 12 in Horizontal Tube 8, Heating and Air Treatment Engineer, 302-310, December 1956 .

Pe70 Pethukov, B.S., Heat Transfer and Friction in Tubrulent Pipe Flow; Advances in Beat Transfer, Pergamon Pres8, 1970 , pg. $503 f f$.

Pr74 Price, B.C. and Bel 1, R.J., Design of Binary Vapor Condensers using the Colburn-Drew Equations, AIChE Symp. Series, 70 (138) 163-171 (1974).

Pu74 Purcipile, J.C., Riedle, R., and Schmidt, F.R., AIChe Symp. Ser., No. 138, Vo1. 70, Pp. 91-97, 1974.

Po82 Polley, G., Application of the Chen Correlation to Water, (1982).

Ra83 Radermacher, R., Ross, H., and Didion, D., Experimental Determination of Forced Convection Evaporative Heat Transfer Coefficients for Non-Azeotropic Refrigerant Mixtures, AS ME National Heat Transfer Conference, ASME 83-WA/HT54 (1983).

Ro78 Rohsenow, W.M. and Hartnett, J.P., Handbook of Heat Transfer, McGraw-Bill, New York, 1978.

Ri73 Riedle, R. and Purcupile, J.C., ASHRAE Tran8., Vol. 79, Pp. 142156,1973 .

Rh74 Rhee, B.W. and Young, E.H., Int. J. Heat Mass Transer, Vol. 23, PP. 73-87, 1980 .

Re79 Reid, Prausnitz and Sherwood, The Properties of Gases and Liquid, Mcgraw-Hill, (1979).

Sh73 Shock, R.A.W., The Evaporation of Binal Mixtures in Forced Convection (Ph.D. The si s), AERE-R7593 (1973). 
Sh82 Shock, R.A.W., Boiling in Multicomponent Fields, Multiphase Science and Technology - Vol. 1 by Bewitt, et al., Eemisphere Publishing Corp., 1982.

Sh77 Shock, R.A.W., Nucleate Boiling in Binary Mixtures, Int'l. Journal of Heat Kass Transfer, Pol. 20,701-709, (1977).

Sh83 Shock, R.A.W., Workshop Notes, Multicomponent Boiling and Condensation, NBS. (1983).

St78 Stephan, R. and Preuber, P., Heat Transfer in Natural Convection Boiling of Polynary Miztures. Proceedings of 6 th International Heat Transfer Conference, I, 187-192, Toronto, 7-11 August ( 1978 ).

St69 Stephan, R. and Rorner, M., Calculation of Heat Transfer in Evaporating Binary Liquid Mixtures, Chemie-Ingenieur Technik, 4l(7), 409-417 (1969).

St81 Stephan, R. and Auracher, B., Int. J. Heat Mass Transfer, Pol. 24, pp. 99-107, 1981.

St 80 Stephan, $R$. and Abdelsalam, M., Int. J. Heat Mass Transfer, Pol. 23, PP. $73-87,1980$.

St82 Stephan K., Boiling of Mirtures, Pol. 1, Proceedings Seventh International Heat Transfer Conference, Munich (1982);

Hemisphere Publishing Corp.

Si83 Singal, L.C. Charma, C.P., and Varma, H.R., Experimental Het Transfer Coefficient for Binary Ref rigerant Mixtures of R 13 and R12; ASERAE Transactions, Pt. 1, p. 175, (No. 2747) (1983).

Sa6 1 Sachs, P. and Long, RA.R., A Correlation for Heat Transfer in Stratified Two-Phase Flow with Paporization, Int. J. Heat and Mass Transfer 2, 223-30, 1961 ; NSA 15-19520.

St66 Staub, R.W. and Zuber, N., Poid Praction Profiles, Flow Mechanisms and Heat Transfer Coefficients for R22 Evaporating in a Vertical Tube, ASHRAE Transactions, (1966), Pg. $130 f f$.

Sh76 Shah, M.M., A New Correlation for Heat Transfer During Boiling Flow Through Pipes, ASHRAE Transactions, Vol. 82, Part II, 1976 , P . $66-86$.

Sh82a Shah, M.M., Chart Correlation for Saturated Boiling Beat Transfer: Equations and Further Study, ASHRAE Transactions, Vol. 88, Part I, 1982.

Sc62 Schrock, R.E. and Grosman, L.M., Forced Convection Boiling in Tubes, Nuclear Science Engineering, Vol. 12, 1962, Pp. 474-481. 
Sc59 Scriven, L.D., On the Dynamics of Phase Growth, Chem. Engng. Science, $10(1 / 2), 1-13$ (1959).

S170 Slipcevic, B., ASHRAE J., PP. 65-78, June 1970.

St7 8 Standiford, F.C., Letter to the Editor, AIChE J/24, 750 (1978).

St 85 Stoecker, W.F., Condensing Coefficients for Refrigerant

Mixtures, ASHRAE Tran8., Part 2, (1985).

Sa7 8 Sauer, H.J., et al., Influence of $0 i l$ on the Nucleate Boiling of Refrigerant 8, 6 th Int' 1 Heat Transfer Conf., (Toronto), paper B.12, (1978).

Si83a Singel, L.C., Sharma, C.P., and Varma, H.K., Pressure Drop During Forced Convection Boiling of Binary Refrigerant Mixtures, Int'l Journal Multiphase Flow, Vol. 9, \$3, Pp. 309-323 (1983).

St 81 Stoecker, W.F., Energy Characteristics of a Two Evaporator Refrigerator Using Refrigerant Mixture; ORNL/Sub/81-7762/2801 (1981).

Sc85 Schulz, J.W., The Characteristics of Fluid Mixtures and their Utilization in Vapor Compression Refrigeration Systems; ASHRAE Transactions, Vol. 2, (1985).

Sa82 Sardesai, Shock and Butterworth, Heat and Mass Transfer in Multicomponent Condensation and Boiling, Heat Transfer Engineering, Vol. 3, \$3-4, Jan.-June 1982.

Th82 Thome, J.R., Shakir, S., and Mercier, C., Effect of Composition on Boiling Incipient Superheat 8 in Binsry Liquid Mixtures; PB14, PP. 95-100; Proceeding8 7 th Int' 1 Heat Transfer Conference, Munich (1982).

To79 Toral, H., Flow Boiling Heat Transfer in Mixtures (Ph.D. Thesis), University of Oxford, Department of Engineering Science, 1979.

To73 Toda, S. and Dchida, B., Study of Liquid Film Cooling with Evaporation and Boiling, pg. 44-62, Heat Transfer - Japanese Research, (1973).

Tr78 TRAC-Pla: An Advanced Best-Estimate Computer Program for PWR LOCA Analysis, Volume I: Los Alamos Scientific Laboratory, 1978 ; Los Alamo 8, New Mexico.

Ti62 Tippet8, F.E., Am. Soc. Mech. Engr8. Paper No. 62-WA-162 (1962). 
Th70 Thorsen, R.S., Dobran, F., and Alcorta, J.A., A Comparative Study of Vertical Upflow and Downflow in a Uniformly Heated Boiling Fluid, paper B4.3, Int' 1 Heat Transfer Conference, (Toronto) (1970).

Uc66 Uchida, H. and Yamaguchi, S., Heat Transfer in Two-PHase Flow of Refrigerant-12 Through Horizontal Tube, Proceeding 3rd International Heat Transfer Conference, Vol. V, 1966, p. 69.

Va67 Van Stralen, S.T.D., Bubble Growth Rates in Boiling Binary Mixtures, Brit. Chem. Engng. 12(3), 390-394, March (1967).

Va77 Vaihigen, D., The Influence of Nucleate Boiling Heat Transfer with Pressure; Heat Transfer in Boiling led. Hahne, E. and Grigill, 0.](1977), Hemisphere Publishing Corp.

Va72 Van Ouwerkerk, H.J., Hemipsherical Bubble Growth in a Binary Mixture; Chem. Eng. Sci. vol. 27; Pp. 1957-1967, (1972).

Va7 9 Varma, H.R., Sharma, C.P,, and Mirkra, M.P., International Congr. Refrig. Paper Bl-46, Venice, 1979.

Wo60 Wor soe-Schmidt, P., Some Characteristics of Flow Pattern and Heat Transfer of Freon 12 Evaporating in Horizontal Tubes, Ingenioren (Demark) \$3, September 1959; ref. Zh. Mekh. 11793 , 1960 .

We 80 Webb, R.L. and Wanniarachchi, A.S., The Effect of Noncondensible Gases in Water Chiller Condensers--Literature Survey and Theoretical Predictions, ASHRAE Trans. p. 142, (1980).

We 81 Webb, D.R. and Sardesai, R.G., Verification of Multicomponent Mass Transfer Models for Condensation Inside a Vertical Tube; Int'l Journal Multiphase Flow Vol. 7, \$5, Pp. 507-520, (1981). 

NBS.114A (REV. 2-8C)

U.S. DEPT. OF COMM.

BIBLIOGRAPHIC DATA

SHEET (See in structions)

1. PUBLICATION OR
REPORT NO.
NBSIR-86/3450

2. Performing Organ. Report No. 3. Publication Date

INOVEMBER 1986

4. TITLE AND SUBTITLE

An Investigation of Horizontal Flow Boiling of Pure and Mixed Refrigerants

\section{AUTHOR(S)}

Howard D. Ross

6. PERFORMING ORGANIZATION (If joint or other than NBS, see instructions)

NATIONAL BUREAU OF STANDARDS

DEPARTMENT OF COMMERCE

WASHINGTON, D.C. 20234

9. SPONSORING ORGANIZATION NAME AND COMPLETE ADDRESS (Street, City, State, ZIP)

National Bureau of Standards

U.S. Department of Energy

Building Equipment Division, CBT

(via Oak Ridge National Laboratory)

Thermal Machinery Group

Washington, D.C. 20585

Gaithersburg, MD 20899

10. SUPPLEMENTARY NOTES

7 Document describes a computer program; SF-185, FIPS Software Summary, is attached.

11. ABSTRACT (A 200-word or less factual summary of most significant information. If document includes a significant bibliography or literature survey, mention it here)

The research involved determining experimental heat transfer coefficients (HTC), examining the phenomena involved in the physical process, and analyzing the predictive ability of available models and correlations. This work was done for pure RI52a and RI3BI and for mixtures of these refrigerants. The mixtures yielded sharply lower heat transfer coefficients than either pure refrigerant.

With pure refrigerants full suppression of nucleate boiling (FSNB) occurs only at rather low pressures. Correlative evidence suggests that suppression is easier to achieve with mixtures than pure fluids.

In the evaporation-dominated heat transfer regime, Chen's correlation was successfully applied to our refrigerants with and without the occurrence of FSNB conditions. A Prandtl number correction is needed when some nucleation occurs. For mixtures, mass diffusion may not complicate the problem substantially under FSNB conditions, and the same correlation may be used with success.

In the nucleate boiling dominated regime, the Stephan and Abdelsalam method was validated for pure fluids, and used successfully with Thome's method for mixtures. Pressure drop correlations for pure fluids were also extended to mixtures without modification.

12. KEY WORDS (Six to twelve entries; alphabetical order; capitalize only proper names; and separate key words by semicolons) evaporative flow; flow boiling; nonazeotropic mixtures; refrigerants heat transfer; two phase flow

13. AVAILABILITY

X Unlimited

For Official Distribution. Do Not Release to NTIS

Order From Superintendent of Documents, U.S. Government Printing Office, Washington, D.C. 20402.

14. NO. OF

PRINTED PAGES

358

X Order From National Technical Information Service (NTIS), Springfield, VA. 22161

15. Price

$\$ 28.95$ 


